U.S. Department of the Interior

U.S. Geological Survey

\title{
Statistical Summaries of Water-Quality Data for Selected Streamflow-Gaging Stations in the Red River of the North Basin, North Dakota, Minnesota, and South Dakota
}

By Kathleen M. Macek-Rowland and Valerie M. Dressler

Open-File Report 02-390

In cooperation with the Bureau of Reclamation 


\title{
U.S. DEPARTMENT OF THE INTERIOR \\ GALE A. NORTON, Secretary
}

\author{
U.S. GEOLOGICAL SURVEY \\ CHARLES G. GROAT, Director
}

Any use of trade, product, or firm names is for descriptive purposes only and does not imply endorsement by the U.S. Government.

For additional information write to:

\section{District Chief}

U.S. Geological Survey

Water Resources Division

821 East Interstate Avenue

Bismarck, ND 58503-1199

Copies of this report can be purchased from:

U.S. Geological Survey

Information Services

Box 25286

Denver, CO 80225-0286 


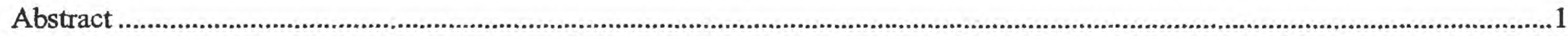

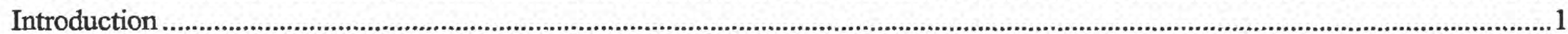

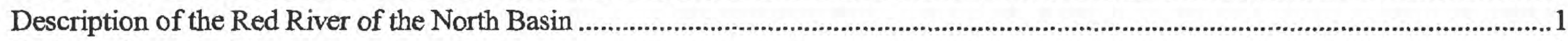

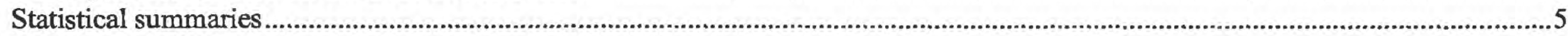

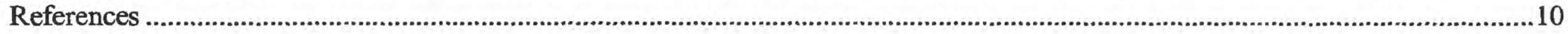

Supplements:

1. Statistical summary of water-quality data for the Otter Tail River below Orwell Dam near Fergus Falls, Minn.,

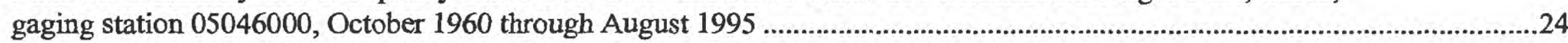

2. Statistical summary of water-quality data for the Bois de Sioux River near White Rock, S. Dak., gaging station

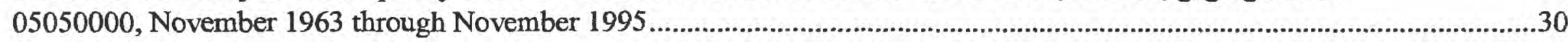

3. Statistical summary of water-quality data for the Bois de Sioux River near Doran, Minn., gaging station 05051300, March 1993 through August 1995

4. Statistical summary of water-quality data for the Red River of the North at Wahpeton, N. Dak., gaging station

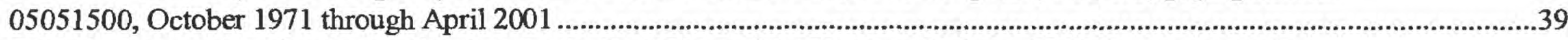

5. Statistical summary of water-quality data for the Red River of the North at Hickson, N. Dak., gaging station 05051522, November 1975 through April 2001.

6. Statistical summary of water-quality data for the Wild Rice River near Abercrombie, N. Dak., gaging station 05053000, June 1966 through April $2001 .$.

7. Statistical summary of water-quality data for the Red River of the North at Fargo, N. Dak., gaging station 05054000, May 1949 through July 2001

8. Statistical summary of water-quality data for the Red River of the North below Fargo, N. Dak., gaging station 05054020, July 1969 through July 2001

9. Statistical summary of water-quality data for the Sheyenne River above Harvey, N. Dak., gaging station 05054500 , October 1971 through July 2001.

10. Statistical summary of water-quality data for the Sheyenne River near Warwick, N. Dak., gaging station 05056000 , January 1951 through July 2001

11. Statistical summary of water-quality data for the Sheyenne River near Cooperstown, N. Dak., gaging station 05057000, October 1959 through April 2001

12. Statistical summary of water-quality data for Baldhill Creek near Dazey, N. Dak., gaging station 05057200, October 1971 through April 2001

13. Statistical summary of water-quality data for Lake Ashtabula at Baldhill Dam, N. Dak., gaging station 05057500, February 1960 through March 2001

14. Statistical summary of water-quality data for the Sheyenne River below Baldhill Dam, N. Dak., gaging station 05058000 , June 1959 through July 2001

15. Statistical summary of water-quality data for the Sheyenne River at Valley City, N. Dak., gaging station 05058500, November 1971 through April 2001

16. Statistical summary of water-quality data for the Sheyenne River at Lisbon, N. Dak., gaging station 05058700 , August 1956 through April 2001 ..

17. Statistical summary of water-quality data for the Sheyenne River near Kindred, N. Dak., gaging station 05059000, October 1971 through April 2001.

18. Statistical summary of water-quality data for the Sheyenne River at West Fargo, N. Dak., gaging station 05059500, September 1969 through July 2001.

19. Statistical summary of water-quality data for the Maple River near Enderlin, N. Dak, gaging station 05059700, October 1971 through April 2001

20. Statistical summary of water-quality data for the Maple River below Mapleton, N. Dak., gaging station 05060100, March 1995 through April 2001

21. Statistical summary of water-quality data for the Rush River at Amenia, N. Dak., gaging station 05060500, November 1971 through August 2000.

22. Statistical summary of water-quality data for the Buffalo River near Dilworth, Minn., gaging station 05062000, April 1962 through March 1991

23. Statistical summary of water-quality data for the Elm River near Kelso, N. Dak., gaging station 05062200 , February 1981 through April 1989

24. Statistical summary of water-quality data for the Wild Rice River at Twin Valley, Minn., gaging station 05062500, September 1974 through August 1998.

25. Statistical summary of water-quality data for the Wild Rice River at Hendrum, Minn., gaging station 05064000 , July 1978 through September 1999. 
26. Statistical summary of water-quality data for the Red River of the North at Halstad, N. Dak., gaging station 05064500, July 1961 through July 2001

27. Statistical summary of water-quality data for the Goose River at Hillsboro, N. Dak., gaging station 05066500 , September 1969 through April 2001

28. Statistical summary of water-quality data for the Marsh River near Shelly, Minn., gaging station 05067500 , July 1975 through September 2000.

29. Statistical summary of water-quality data for the Sand Hill River at Climax, Minn., gaging station 05069000 , November 1966 through September 2000

30. Statistical summary of water-quality data for the Lower Red Lake near Red Lake, Minn., gaging station 05074000, May 1962 through April 1965

31. Statistical summary of water-quality data for the Red Lake River at High Landing near Goodridge, Minn., gaging station 05075000, April 1979 through September 2000

32. Statistical summary of water-quality data for the Clearwater River at Red Lake Falls, Minn., gaging station 05078500 August 1992 through March 2001

33. Statistical summary of water-quality data for the Red Lake River at Crookston, Minn., gaging station 05079000, April 1962 through September 2000.

34. Statistical summary of water-quality data for the Red River of the North at Grand Forks, N. Dak., gaging station 05082500, June 1949 through July 2001

35. Statistical summary of water-quality data for the Turtle River at Manvel, N. Dak., gaging station 05083000 , October 1971 through October 1991

36. Statistical summary of water-quality data for the Forest River at Minto, N. Dak., gaging station 05085000 , October 1971 through April 2001

37. Statistical summary of water-quality data for the Snake River at Warren, Minn., gaging station 05085500 , April 1979

38. Statistical summary of water-quality data for the Middle River at Argyle, Minn., gaging station 05087500 , April 1968 through September 2000.

39. Statistical summary of water-quality data for the Park River at Grafton, N. Dak., gaging station 05090000 , September 1969 through April 2001

40. Statistical summary of water-quality data for the Red River of the North at Drayton, N. Dak., gaging station 05092000, October 1971 through April 2001

41. Statistical summary of water-quality data for the Pembina River at Neche, N. Dak., gaging station 05100000 , October 1971 through April 2001

42. Statistical summary of water-quality data for the Tongue River at Akra, N. Dak., gaging station 05101000, October 1971 through April 2001

43. Statistical summary of water-quality data for the Red River of the North at Emerson, Manitoba, gaging station 05102500, July 1974 through July 2001

\section{FIGURES}

1. Map showing location of selected streamflow-gaging stations in the Red River of the North Basin, North Dakota, Minnesota, and South Dakota

\section{TABLES}

1. Selected streamflow-gaging stations in the Red River of the North Basin, North Dakota, Minnesota, and South Dakota

2. Selected sources for water-quality data in the Red River of the North Basin, North Dakota, Minnesota, and South Dakota.

3. Common sources and environmental significance of selected water-quality properties and constituents 


\title{
Statistical Summaries of Water-Quality Data for Selected Streamflow-Gaging Stations in the Red River of the North Basin, North Dakota, Minnesota, and South Dakota
}

\author{
ByK. M. Macek-Rowland and V. M. Dressler
}

\begin{abstract}
The quantity and quality of current and future water resources in the Red River of the North Basin in North Dakota, Minnesota, and South Dakota are concerns of people who reside within the basin. Additional water resources are needed because of recent growth in population, industry, and agriculture. How the management of current and future water-resources will impact water quality within the basin is a critical issue. Water-quality data, particularly for surface-water sources, will help water-resources managers make decisions about current and future water resources in the Red River of the North Basin. Statistical summaries of water-quality data for 43 streamflow-gaging stations in the Red River of the North Basin in North Dakota, Minnesota, and South Dakota are presented in this report. Statistical summaries include sample size, maximum, minimum, mean, and values for the 95 th, 75 th, 50 th, 25 th, and 5 th percentiles.
\end{abstract}

\section{INTRODUCTION}

The Red River of the North, located in the north-central plains of the United States, plays an important role in regional development. The Red River of the North and its tributaries are used for water supply, irrigation, industry, livestock, and recreation. Additional water resources are needed because of recent growth in population, industry, and agriculture in the basin. The Red River of the North and its tributaries are affected by the quantity and quality of water within the basin. Therefore, the quantity and quality of current and future water resources in the Red River of the North Basin in North Dakota, Minnesota, and South Dakota are concerns of people who reside within the basin. How the management of current and future water-resources will impact water quality within the basin is a critical issue.

The Dakota Water Resources Act was passed by the U.S. Congress on December 15, 2000. The Act authorized the Secretary of the Interior to conduct a comprehensive study of the future water-quantity and quality needs of the Red River of the North Basin in North Dakota and the possible options to meet those needs. To provide needed information for the comprehensive study, the U.S. Geological Survey (USGS) conducted a study in cooperation with the Bureau of Reclamation. The study will provide water-quantity and quality information needed for the comprehensive study.

This report presents statistical summaries of water-quality data collected at selected USGS streamflow-gaging stations in the Red River of the North Basin in North Dakota, Minnesota, and South Dakota. Statistical summaries are provided for 43 streamflow-gaging stations (table 1). The data will help water-resources managers make decisions about current and future water resources in the Red River of the North Basin.

\section{DESCRIPTION OF THE RED RIVER OF THE NORTH BASIN}

The Red River of the North begins at the confluence of the Ottertail and Bois de Sioux Rivers in Wahpeton, N. Dak., and Breckenridge, Minn. (fig. 1). The river flows northward for about 394 miles to the United States-Canadian boundary. From the international boundary, the Red River of the North flows north about 155 miles and discharges into Lake Winnipeg. Drainage area at the Emerson, Manitoba, gaging station, which is about 0.8 mile downstream from the international boundary, is about 40,200 square miles (U.S. Geological Survey, 2002, p. 184). Excluding the Assiniboine River Basin, an additional 5,000 square miles of drainage area is located in Canada. The basin is relatively flat and has a shallow river channel. The flat portions of the basin were caused by sediment deposition from an ancient glacial lake, Lake Agassiz, that existed between 7,000 to 12,000 years ago. 


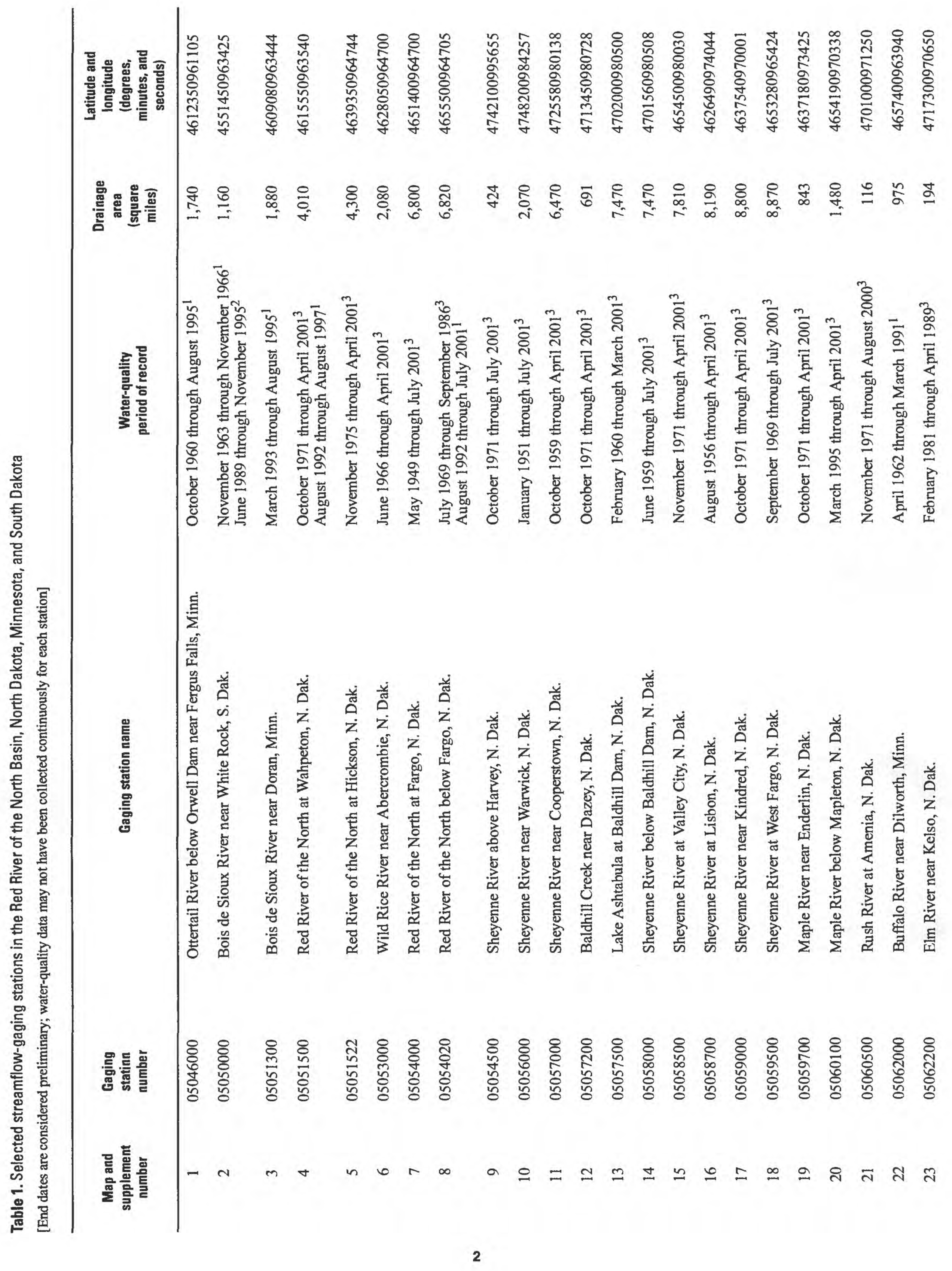




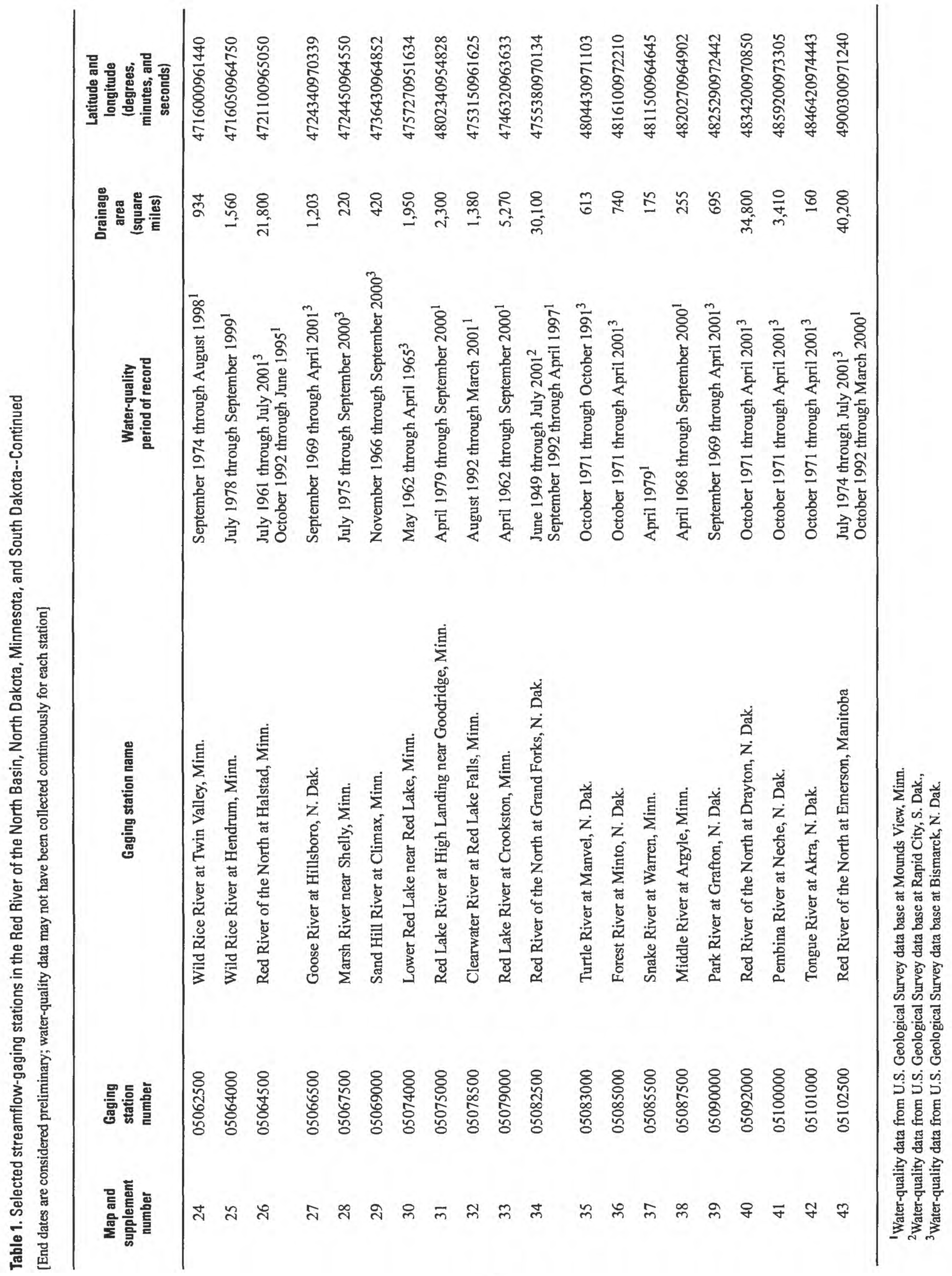




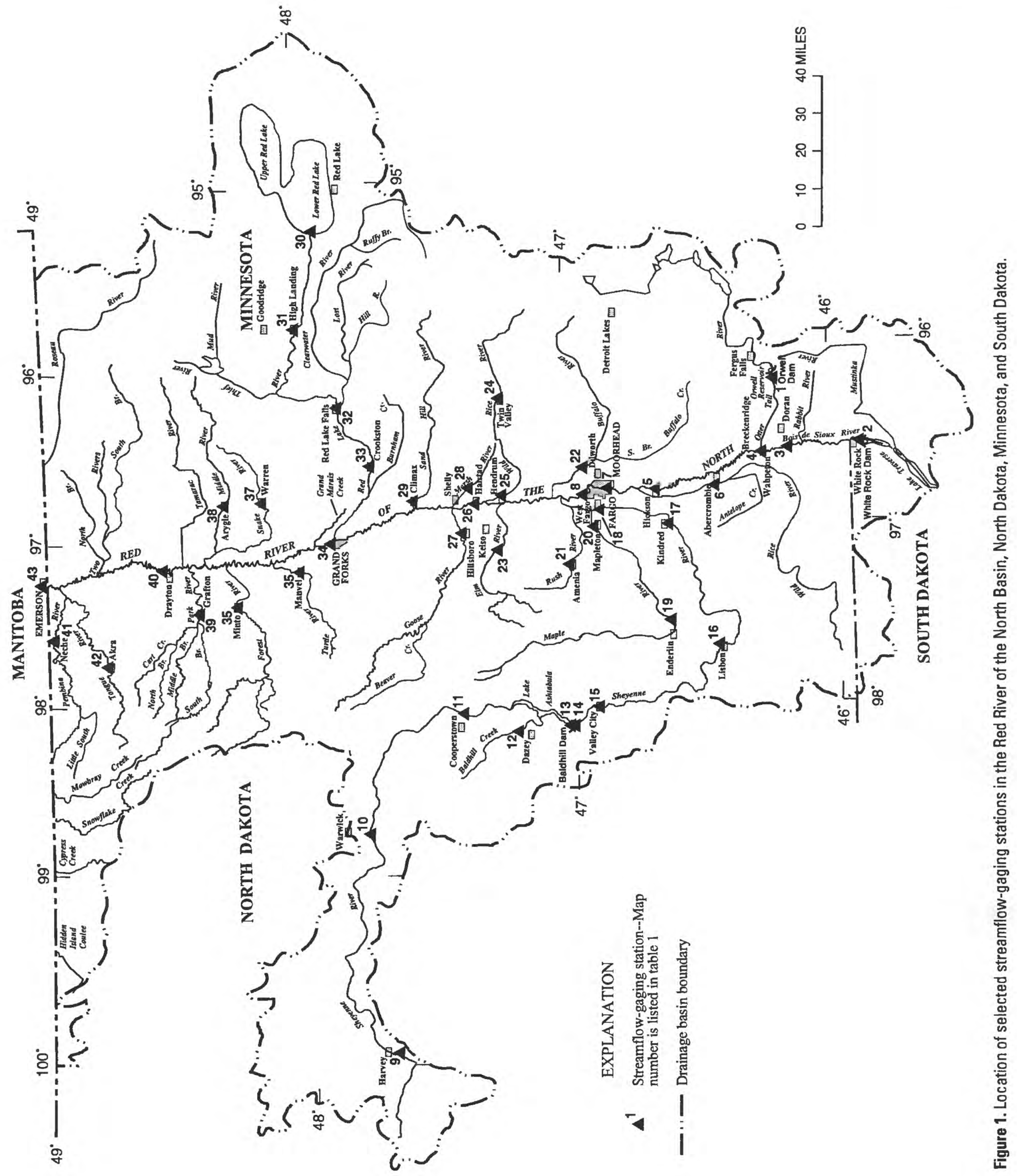


In the Red River of the North Basin, streamflow is composed of base flow from ground-water seepage and of runoff from within the basin. Streamflow throughout the basin can vary from virtually no flow in dry years to extensive flooding in wet years. During the most recent dry period, 1988-92, smaller tributaries to the Red River of the North had no flow and the Red River of the North had daily mean flows of less than 100 cubic feet per second at Fargo, N. Dak. Daily mean flows for the Red River of the North at Emerson, Manitoba, ranged from 0.97 cubic feet per second in 1937 to 133,000 cubic feet per second in the spring of 1997. The variability of flows, particularly during extremely dry and wet periods, can impact the quantity and quality of surface-water resources within the Red River of the North Basin. During extremely dry and wet periods, water-quality monitoring is critical to water-resources managers.

Water quality in surface-water sources is affected by the combination of ground water and runoff and by the stream channel composition. The water quality also is affected by water lost through evaporation and through transpiration from vegetation. The natural factors that affect water quality may be coupled with anthropogenic factors caused by people living and working within the basin. Some anthropogenic factors include the use of agricultural chemicals, the release of wastewater into rivers or streams by industries or water treatment facilities, and the changes made to land surface topography.

\section{STATISTICAL SUMMARIES}

Statistical summaries were compiled for water-quality data that were collected at 43 streamflow-gaging stations in North Dakota, Minnesota, and South Dakota. Streamflow-gaging stations were selected on the basis of their location, availability of data, and period of record. Selected streamflow-gaging stations, corresponding map numbers, gaging-station numbers, water-quality periods of record, drainage areas, and latitudes and longitudes are listed in table 1. An eight-digit number (e.g., 05051500) has been assigned to each streamflow-gaging station using the downstream-order numbering system of the USGS. The downstream-order number is unique to each station and is used to enter, store, and retrieve data from USGS data bases. The statistical summaries are given in supplemental format at the end of this report (supp. 1-43).

Statistical summaries were developed from water-quality data stored in the U.S. Geological Survey's National Water Information System (NWIS) data base as of October 2001. Water-quality data reported in the statistical tables were collected by USGS personnel and analyzed by USGS laboratories according to established data collection and analysis standards and methodologies of the USGS. Water-quality data for selected streamflow-gaging stations in this report may be obtained from the USGS district office in the state that the streamflow-gaging station is located in or from the NWIS data base maintained on each district's individual web site (table 2). Because of various data-collection programs and projects, additional water-quality data collected by USGS personnel or personnel from other agencies and analyzed by non-USGS laboratories may be included in the USGS water-quality data base. However, the water-quality data analyzed by non-USGS laboratories were not retrieved for this report but may be obtained at the USGS offices in North Dakota, Minnesota, and South Dakota or at other offices and agencies involved in water-resources monitoring in the Red River of the North Basin (table 2).

Descriptive statistics include: (1) the five-digit parameter code that is used to identify the corresponding parameter or constituent in the USGS National Water Information System and the U.S. Environmental Protection Agency Data Storage and Retrieval System; (2) the sample size, which is the number of data values from samples used in computing the statistics for a property or constituent; (3) the maximum, which is the largest data value in a group of data values from the sample; (4) the minimum, which is the smallest data value in a group of data values from the sample; and (5) the mean, which is the sum of individual data values in a sample size divided by the sample size number. Sample size will vary for each property and constituent depending on the data collection programs or projects involved with the station. Because the properties and constituents vary from station to station, a full descriptive term for each property and constituent is in a list at the beginning of the statistical summaries. Reference to this list may be needed when reviewing the individual properties and

constituents for each station in order to get a better understanding of the type of data collected at each location. Several of the more common properties and constituents are the same at many streamflow-gaging stations. Common sources and environmental significance of the selected water-quality properties and constituents are given in table 3 (Berkas, W.R., 1993, p. 429-436). 
U.S. Geological Survey

North Dakota District

821 East Interstate Avenue

Bismarck, ND 58503-1199

http://nd.water.usgs.gov/

North Dakota Department of Health

1200 Missouri Avenue

Bismarck, ND 58502-5520

http://www.health.state.nd.us/ndhd/environ/wq/index.htm

North Dakota State Water Commission

900 East Boulevard Avenue

Bismarck, ND 58505-0850

http://www.swc.state.nd.us/dataresources.hrml

\section{U.S. Geological Survey}

Minnesota District

2280 Woodale Drive

Mounds View, MN 55112

http://mn.usgs.gov/

Minnesota Pollution Control Agency

520 Lafayette Road

St. Paul, MN 55155-4194

http://www.pca.state.mn.us/water/basins/redriver/index.html

U.S. Geological Survey

South Dakota District

1608 Mt. View Road

Rapid City, SD 57702

http://sd.water.usgs.gov/

Manitoba Conservation

Water Quality Management Section

123 Main Street, Suite 160

Winnipeg, MB, Canada R3C 1 AS

http://www.gov.mb.ca/natres/watres/water_quality.html

Environment Canada

Chief, Ecological Research Division

Room 300, 2365 Albert Street

Regina, SK, Canada S4P 4K1

http://www.pnr-rpn.ec.gc.ca/water/science

\section{North Dakota}

USGS water-quality data for surface- and ground-water resources in North Dakota is stored on a data base accessed through the Internet. Contact the USGS office in Bismarck, N. Dak., for more site-specific data information.

http://waterdata.usgs/nd/nwis/qw

State water-quality standards and water-quality data from various surface- and ground-water monitoring programs. Contact agency for more site-specific data information.

Provides access to well data that is available in North Dakota. This includes site information, lithologic data, water-level data, and water chemistry. Contact agency for more site-specific data information.

\section{Minnesota}

USGS water-quality data for surface- and ground-water resources in Minnesota is stored on a data base accessed through the Internet. Contact the USGS office in Mounds View, Minn., for more site-specific data information.

http://waterdata.usgs/mn/nwis/qw

Water-quality standards and classifications, watershed stream waterquality assessments, and water quality for lakes. Contact agency for more site-specific data information.

\section{South Dakota}

USGS water-quality data for surface- and ground-water resources in South Dakota is stored on a data base accessed through the Internet. Contact the USGS office in Rapid City, S. Dak., for more site-specific data information.

http://waterdata.usgs/sd/nwis/qw

\section{Canada}

Manitoba water-quality standards. Contact agency for type of waterquality data available at Canadian sites.

Information on water-quality monitoring and aquatic-quality science. Contact agency for more site-specific data information. 
Tabla 2. Selected sources for water-quality data in the Red River of the North Basin, North Dakota, Minnesota, and South Dakota--Continued Internet addresses accessed on October 2, 2002; USGS, U.S. Geological Survey; STORET, storage and retrieval; NWIS; National Water Information System]

\section{Colorado}

Environmental Protection Agency

\section{Region 8}

999 18th Street, Suite 300

Denver, CO 80202-2466

http://www.epa.gov/region08/water/
Maintains two data bases populated by field water monitoring results from various offices and agencies in the United States. STORET Legacy Data Center holds data prior to 1999 and Modernized STORET receives new data on a regular basis.

http://www.epa.gov/STORET/dbtop.html

Note: USGS data have been removed from STORET files and are maintained on the USGS NWIS data base. 


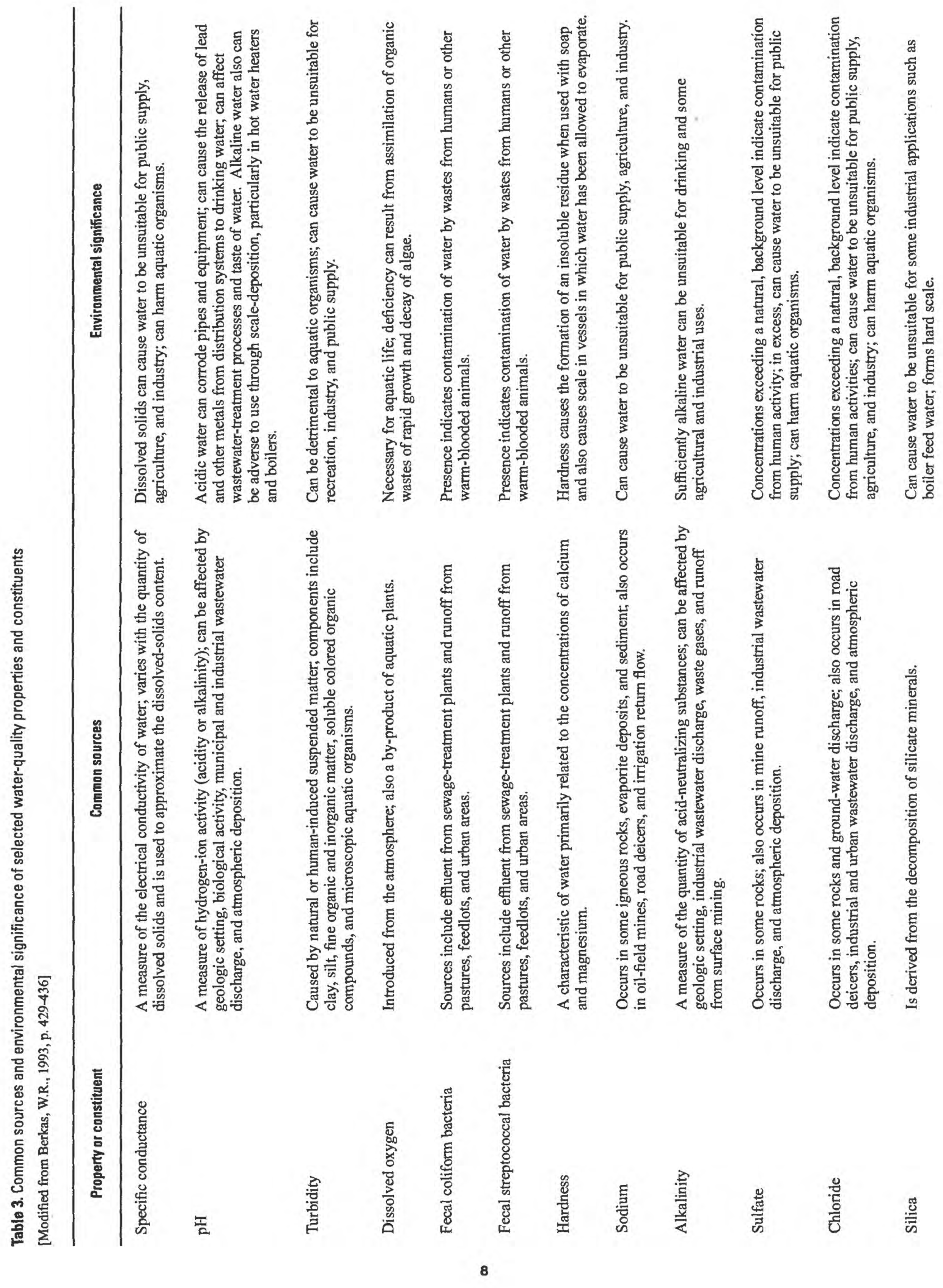




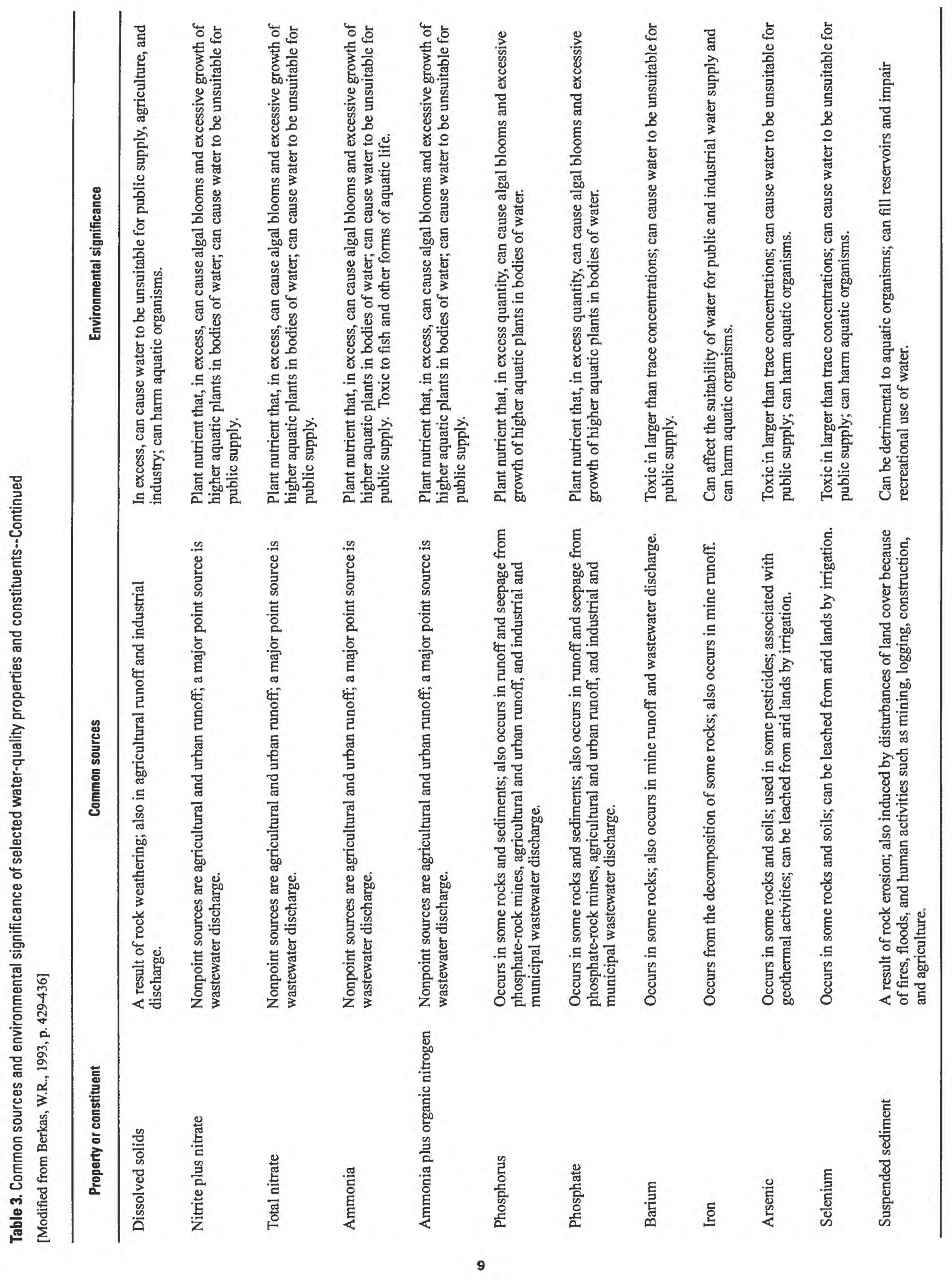


Values for the 95th, 75th, 50th (median), 25th, and 5th percentiles also are included in the statistical tables. The percentiles are the percentage of samples in which the values were less than or equal to those shown in the statistical table. The 50th percentile, or median, represents the middle value of an uneven sample number or the mean of two middle values of an even sample number. Only maximum and minimum values are given when there is more than one but less than five values for a property or constituent. A property or constituent may have more than five samples but no other statistics because the data for that property or constituent are calculated values, not actual values collected at a station. Calculated values need actual values from certain properties and/or constituents in order to be calculated. The calculated value cannot be computed and will show only the number of samples if one or more of the properties or constituents needed for the calculation are missing values, if one or more of the constituents are censored (less than a predetermined value), or if a property or constituent is missing or was not collected. USGS water-quality data may be obtained from the USGS WaterQuality Home Page on the Internet at http://nwql.usgs.gov (accessed on September 23, 2002).

\section{REFERENCES}

Berkas, W.R., 1993, North Dakota Stream Water Quality in Paulson, R.W., Chase, E.B., Williams, J.S., and Moody, D.W., comp., National Water Summary 1990-91, Hydrologic Events and Stream Water Quality: U.S. Geological Survey Water-Supply Paper 2400 , p. 429-436.

U.S. Geological Survey, 2002, Water resources data for North Dakota, water year 2001: U.S. Geological Survey Water Data Report, ND-01-1, 467 p. 


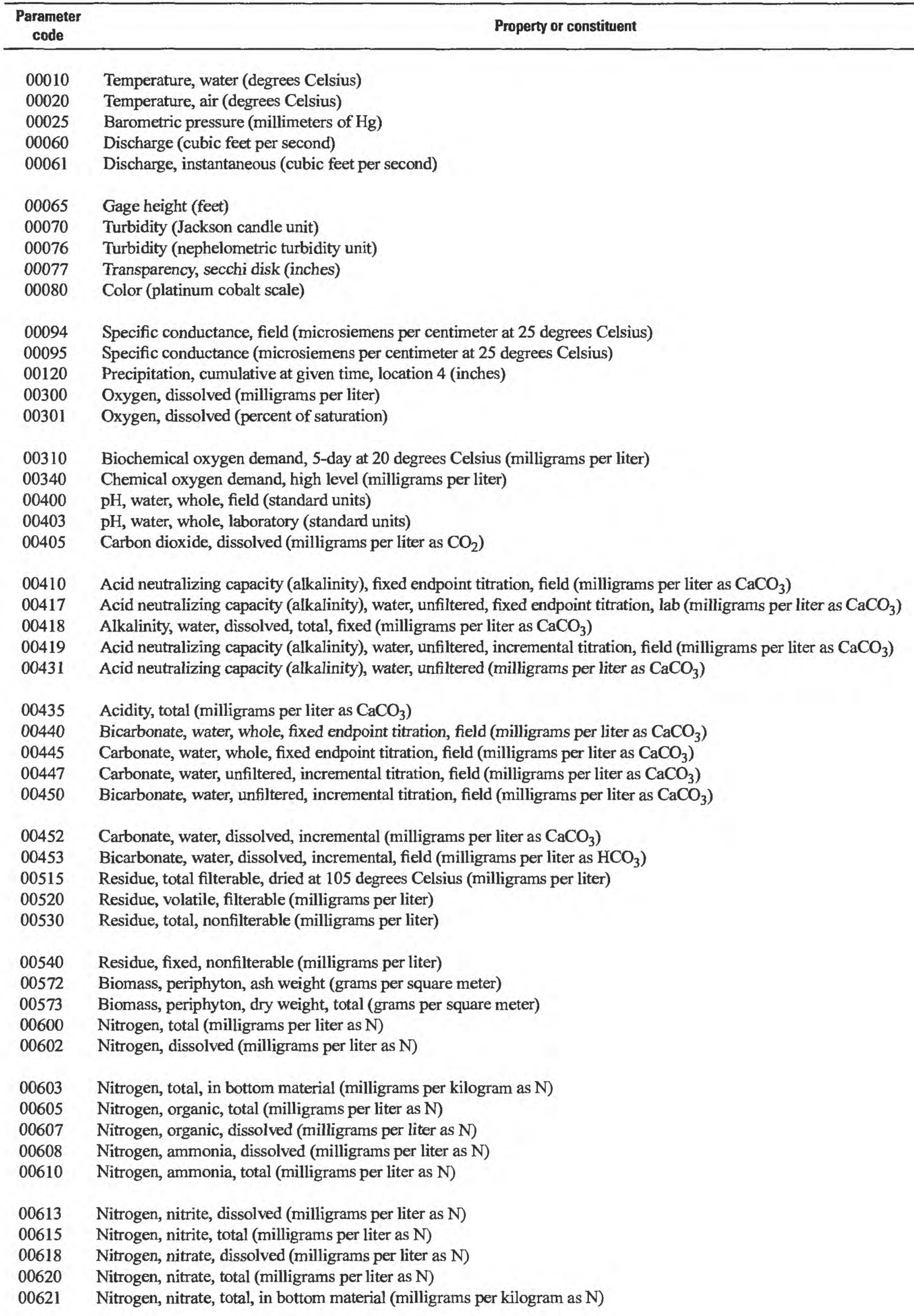




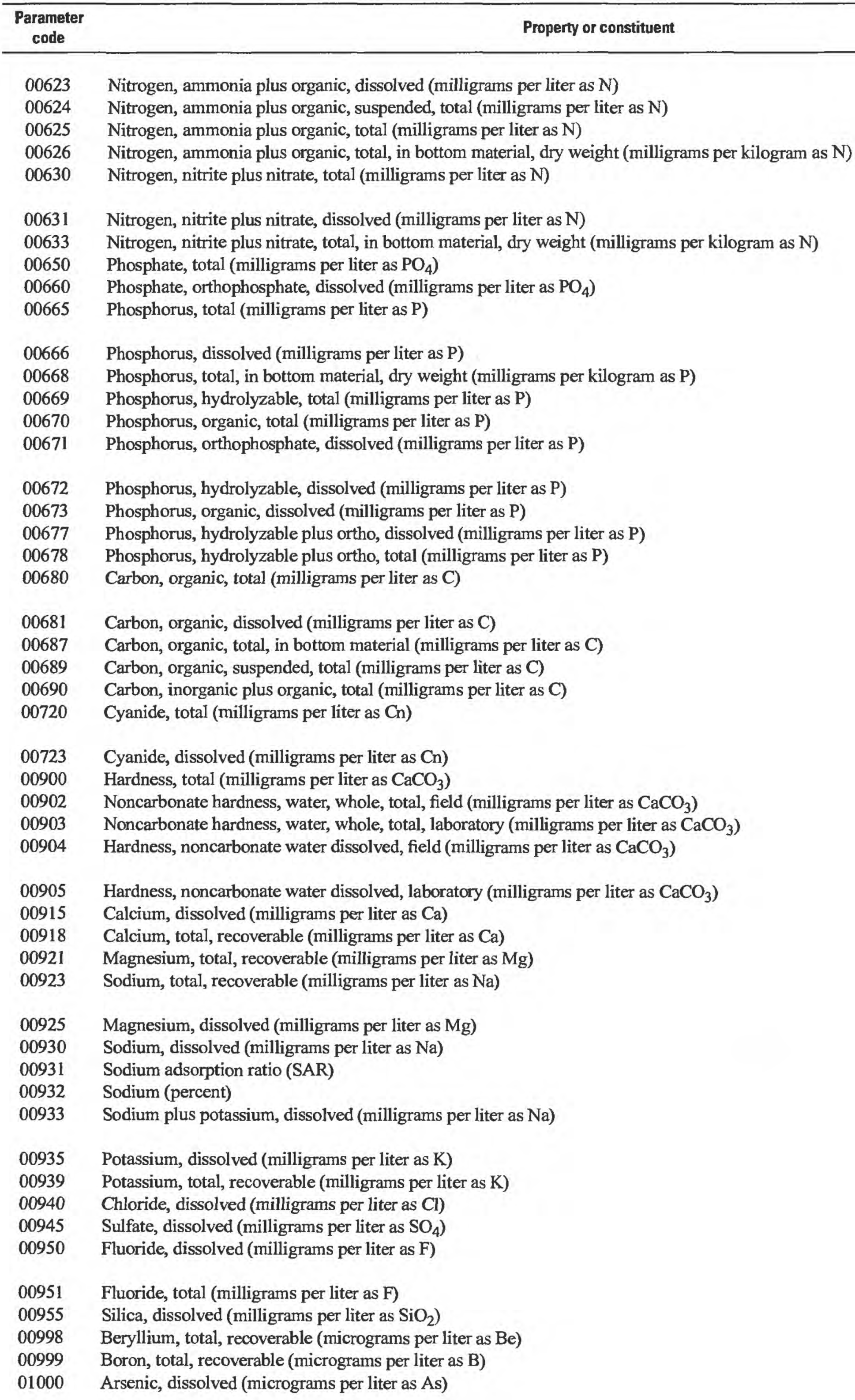




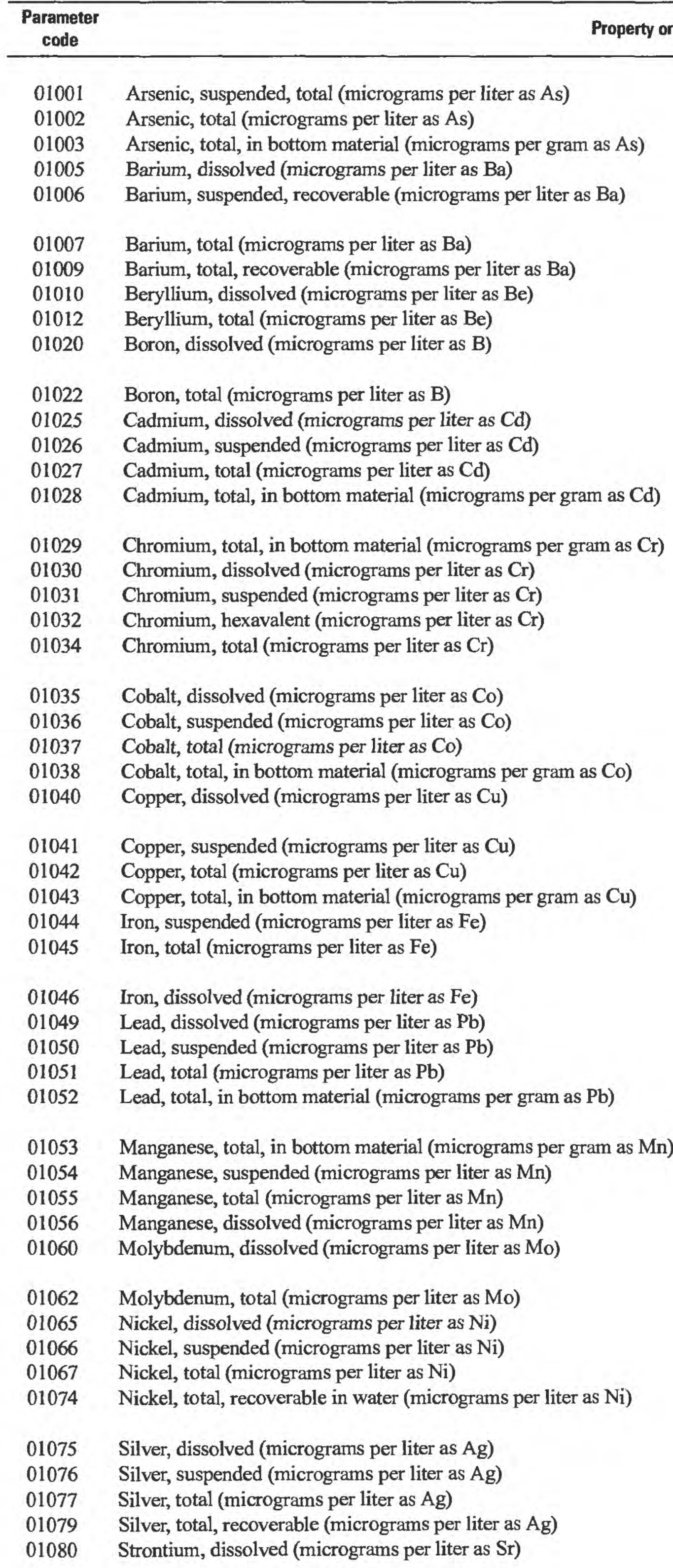




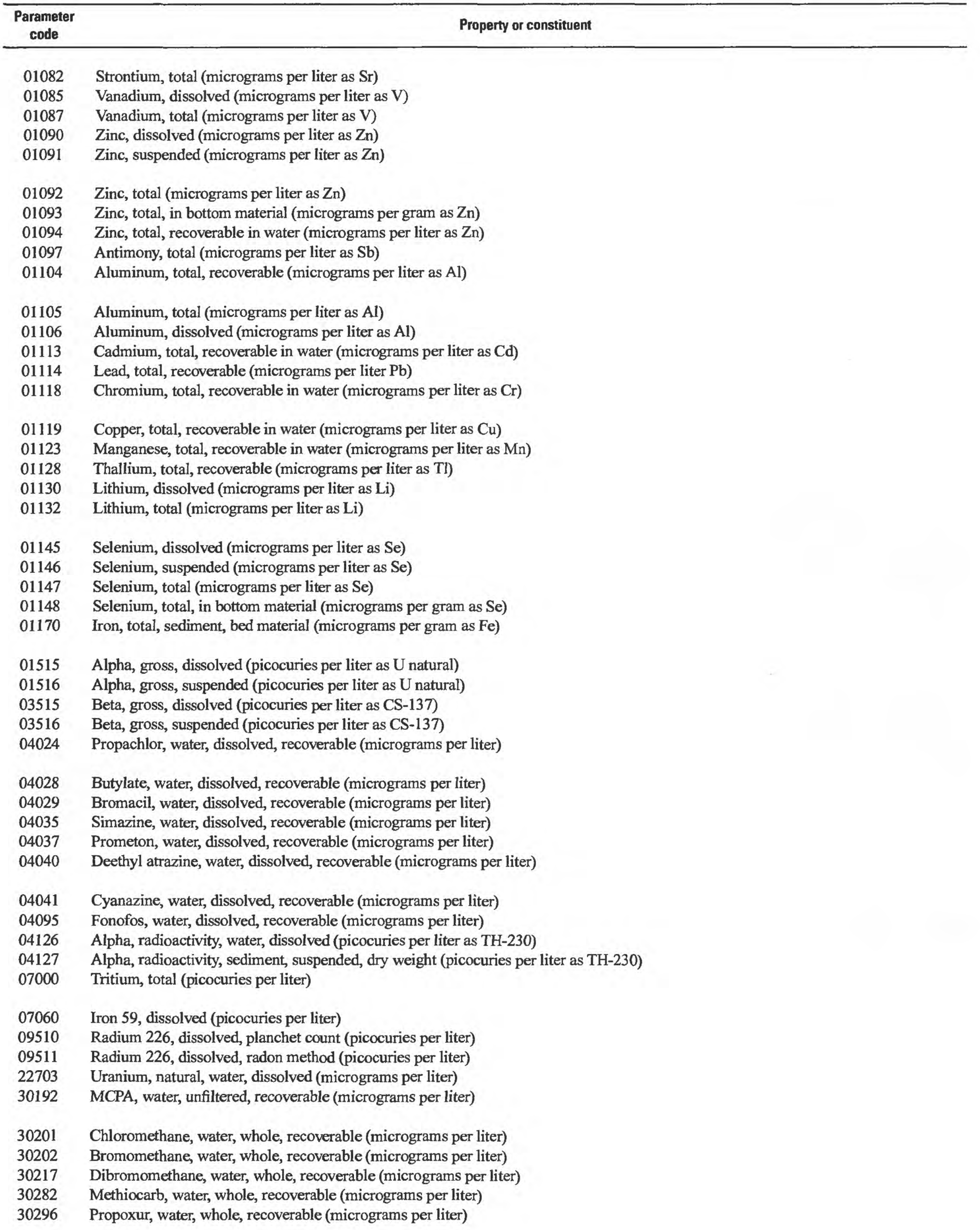




\begin{tabular}{l|l}
\hline $\begin{array}{c}\text { Parameter } \\
\text { code }\end{array}$ & Property or constituent \\
\hline
\end{tabular}

31501 Total coliform, water, (colonies per 100 milliliters)

31616 Fecal coliform, water (colonies per 100 milliliters)

31625 Fecal coliform, 0.7 micrometer-membrane filter (colonies per 100 milliliters)

31673 Streptococci, fecal, membrane filter (colonies per 100 milliliters)

31679 Streptococci, fecal, membrane filter (colonies per 100 milliliters)

32101 Bromodichloromethane, water, unfiltered, recoverable (micrograms per liter)

32102 Carbon tetrachloride, water, unfiltered, recoverable (micrograms per liter)

32103 1,2-Dichloroethane, total (micrograms per liter)

32104 Bromoform, total (micrograms per liter)

32105 Chlorodibromomethane, total (micrograms per liter)

32106 Chloroform, total (micrograms per liter)

32226 Chlorophyll b, periphyton, spectrophotometric, uncorrected (milligrams per square meter)

32228 Chlorophyll a, periphyton, spectrophotometric, uncorrected (milligrams per square meter)

32230 Chlorophyll a, phytoplankton, spectrophotometric, uncorrected (micrograms per liter)

32231 Chlorophyll b, phytoplankton, spectrophotometric (micrograms per liter)

32730 Phenols, total (micrograms per liter)

34010 Toluene, total (micrograms per liter)

34030 Benzene, total (micrograms per liter)

$34210 \quad$ Acrolein, total (micrograms per liter)

34215 Acrylonitrile, total (micrograms per liter)

34253 Alpha BHC (micrograms per liter)

34301 Chlorobenzene, total (micrograms per liter)

34311 Chloroethane, total (micrograms per liter)

34351 Endosulfan sulfate, total (micrograms per liter)

34356 Endosulfan II, water, unfiltered, recoverable (micrograms per liter)

34361 Endosulfan I, water, whole, recoverable (micrograms per liter)

34366 Endrin aldehyde, total (micrograms per liter)

34371 Ethylbenzene, total (micrograms per liter)

34396 Ethane, hexachloro-, water, unfiltered, recoverable (micrograms per liter)

34413 Methylbromide, total (micrograms per liter)

34418 Methylchloride, total (micrograms per liter)

34423 Methylene chloride, water, unfiltered, recoverable (micrograms per liter)

34475 Tetrachloroethylene, total (micrograms per liter)

34488 Trichlorofluoromethane, total (micrograms per liter)

34496 1,1-Dichloroethane, total

34501 1,1-Dichloroethylene, total (micrograms per liter)

34506 1,1,1-Trichloroethane, total (micrograms per liter)

34511 1,1,2-Trichloroethane, total (micrograms per liter)

34516 Ethane, 1,1,2,2-Tetrachloro-, water, unfiltered, recoverable (micrograms per liter)

34536 Benzene, o-dichloro-, water, unfiltered, recoverable (micrograms per liter)

34541 1,2-Dichloropropane, total (micrograms per liter)

34546 Trans-1, 2-Dichloroethene, total, in water (micrograms per liter)

34551 Benzene, 1,2,4-Trichloro-, water, unfiltered, recoverable (micrograms per liter)

34566 Benzene, 1,3-Dichloro-, water, unfiltered, recoverable (micrograms per liter)

34571 Benzene, 1,4-Dichloro-, water, unfiltered, recoverable (micrograms per liter)

$34653 \quad$ P,P'-DDE dissolved (micrograms per liter)

34668 Dichlorodifluoromethane, total (micrograms per liter)

34696 Naphthalene, total (micrograms per liter)

34699 Trans-1,3-dichloropropene, total (micrograms per liter)

34704 CIS-1, 3-Dichloropropene, total (micrograms per liter) 


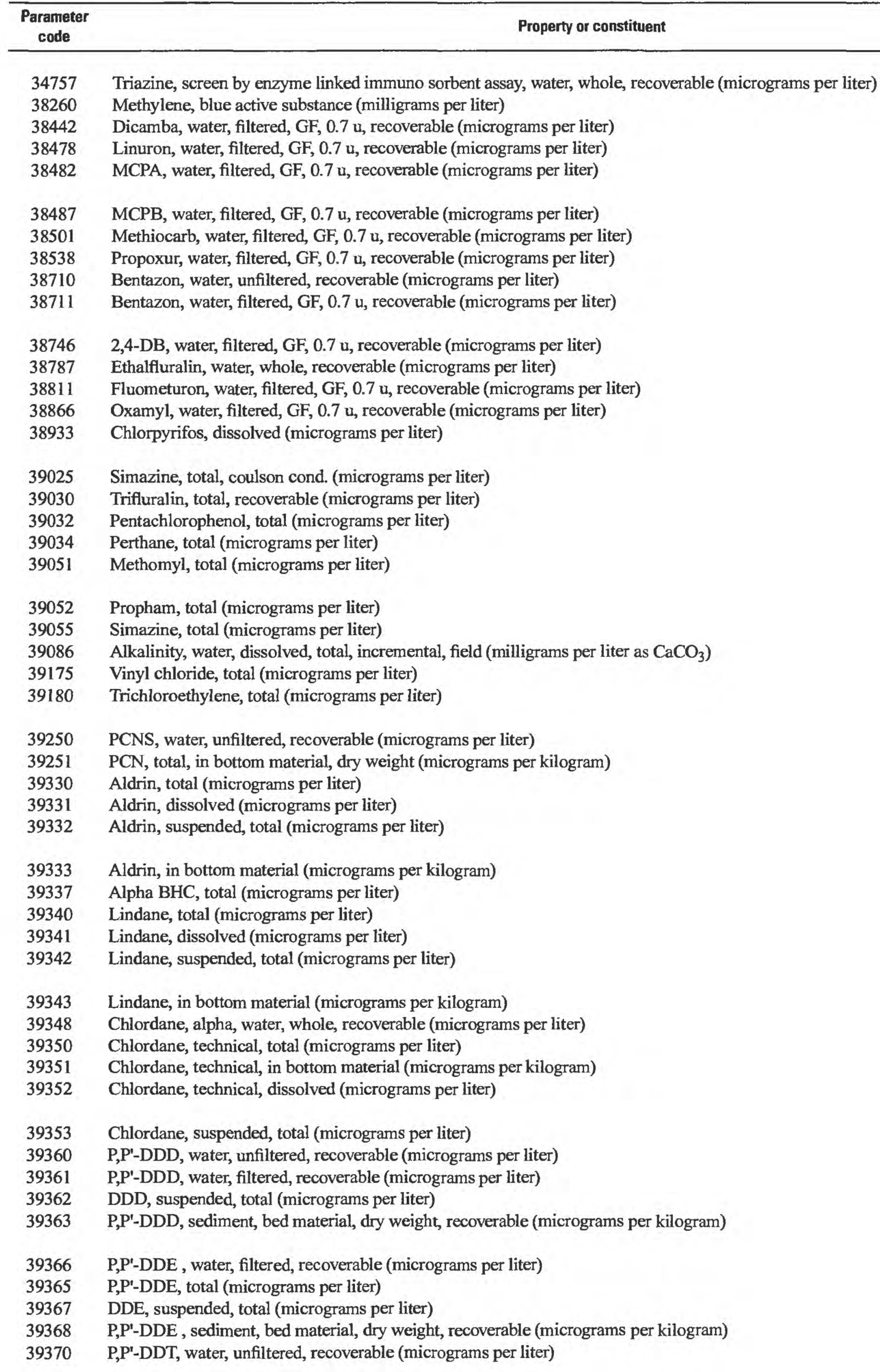




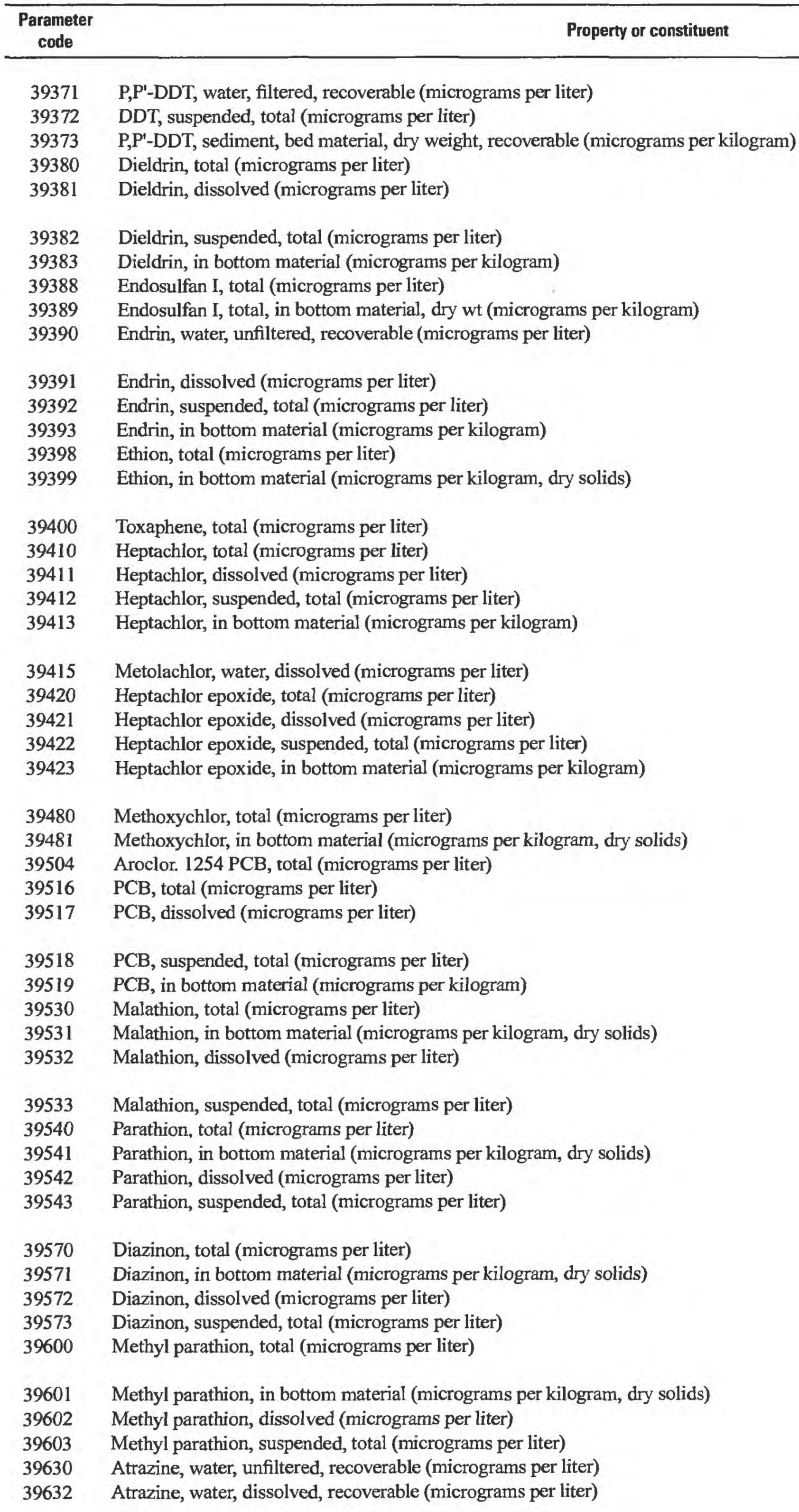




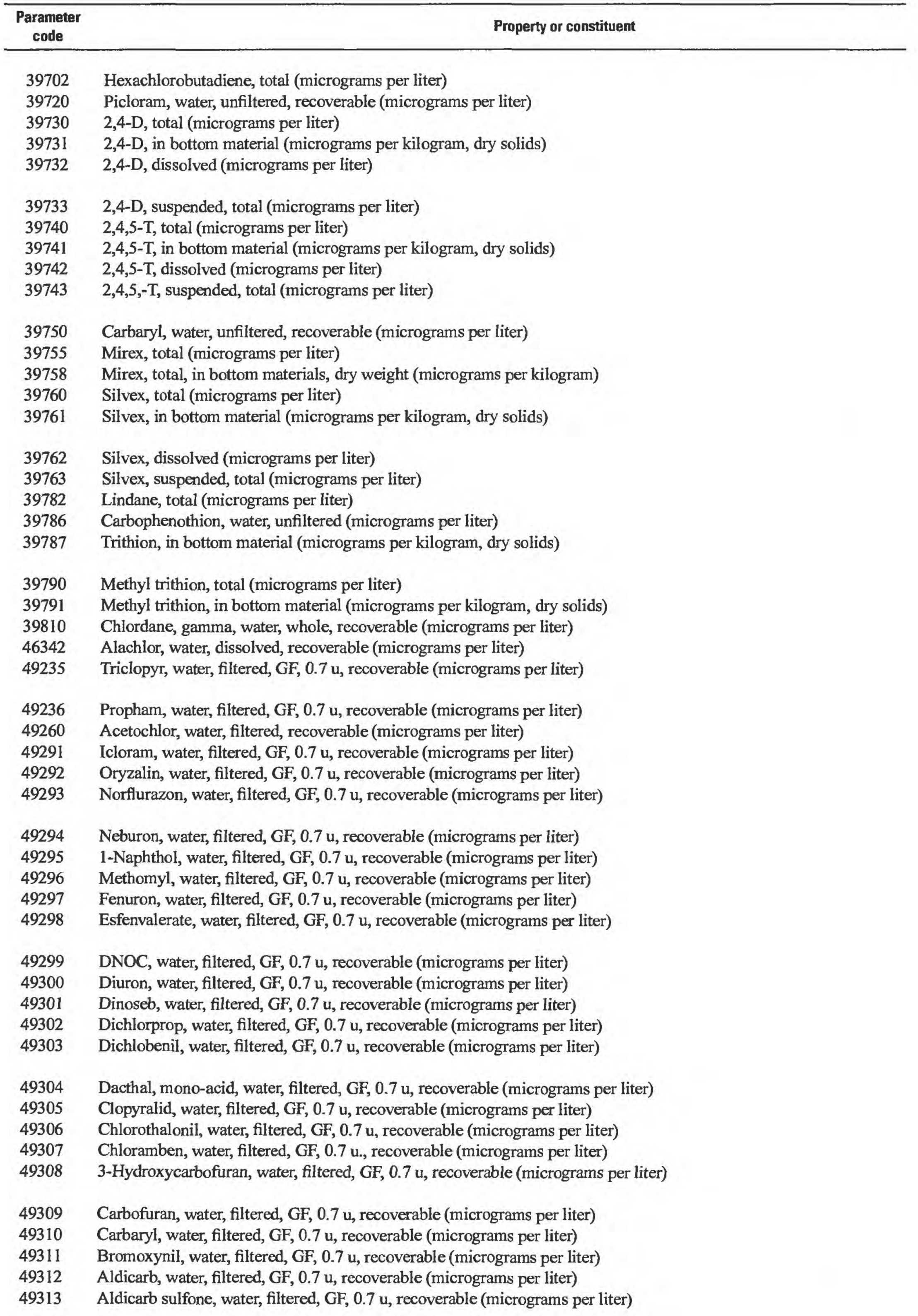




\begin{tabular}{cc}
\hline $\begin{array}{c}\text { Parameter } \\
\text { code }\end{array}$ & Property or constituent \\
\hline
\end{tabular}

49314 Aldicarb sulfoxide, water, filtered, GF, $0.7 \mathrm{u}$, recoverable (micrograms per liter)

49315 Acifluorfen, water, filtered, GF, $0.7 \mathrm{u}$, recoverable (micrograms per liter)

49991 Methyl acrylate, water, unfiltered, recoverable (micrograms per liter)

49999 Benzene, 1,2,3,4-tetramethyl, water, unfiltered, recoverable (micrograms per liter)

50000 Isodurene, water, unfiltered, recoverable (micrograms per liter)

50002 Bromoethene, water, unfiltered, recoverable (micrograms per liter)

50004 Ether, Tert-butyl Ethyl, water, unfiltered, recoverable (micrograms per liter)

50005 Ether, Tert-pentyl Methyl, water, unfiltered, recoverable (micrograms per liter)

60050 Phytoplankton, total (cells per milliliter)

61028 Turbidity, field, water, unfiltered (nephelometric turbidity unit)

61188 Chloramben, methyl ester, water, filtered, recoverable (micrograms per liter)

70299 Suspended solids at 110 degrees Celsius (milligrams per liter)

70300 Dissolved solids, residue at 180 degrees Celsius (milligrams per liter)

70301 Dissolved solids, calculated, sum of constituents (milligrams per liter)

70302 Dissolved solids (tons per day)

70303 Dissolved solids (tons per acre-foot)

70331 Sediment, suspended, sieve diameter (percent finer than 0.062 millimeter)

70332 Sediment, suspended, sieve diameter (percent finer than 0.125 millimeter)

70333 Sediment, suspended, sieve diameter (percent finer than 0.250 millimeter)

70334 Sediment, suspended, sieve diameter (percent finer than 0.500 millimeter)

70335 Sediment, suspended, sieve diameter (percent finer than 1.00 millimeter)

70336 Sediment, suspended, sieve diameter (percent finer than 2.00 millimeters)

70337 Sediment, suspended, fall diameter, distilled water (percent finer than 0.002 millimeter)

70338 Sediment, suspended, fall diameter, distilled water (percent finer than 0.004 millimeter)

70339 Sediment, suspended, fall diameter, distilled water (percent finer than 0.008 millimeter)

70340 Sediment, suspended, fall diameter, distilled water (percent finer than 0.016 millimeter)

70341 Sediment, suspended, fall diameter, distilled water (percent finer than 0.031 millimeter)

70342 Sediment, suspended, fall diameter, distilled water (percent finer than 0.062 millimeter)

70343 Sediment, suspended, fall diameter, distilled water (percent finer than 0.125 millimeter)

70344 Sediment, suspended, fall diameter, distilled water (percent finer than 0.250 millimeter)

70345 Sediment, suspended, fall diameter, distilled water, percent finer than 0.500 millimeter)

70346 Sediment, suspended, fall diameter, distilled water, percent finer than 1.00 millimeter)

70347 Sediment, suspended, fall diameter, distilled water, percent finer than 2.00 millimeters)

70507 Phosphorus, orthophosphate, total (milligrams per liter as P)

70949 Biomass-chlorophyll ratio, plankton (units)

70950 Biomass-chlorophyll ratio, periphyton (units)

70953 Chlorophyll a, phytoplankton, chromotographic-fluorometric (micrograms per liter)

70954 Chlorophyll b, phytoplankton, chromotographic-fluorometric (micrograms per liter)

70955 Chlorophyll a, periphyton, chromotographic-spectrophotometric (milligrams per square meter)

70956 Chlorophyll b, periphyton, chromotographic-spectrophotometric (milligrams per square meter)

70957 Chlorophyll a, periphyton, chromotographic-fluorometric (milligrams per square meter)

70958 Chlorophyll b, periphyton, chromotographic-fluorometric (milligrams per square meter)

71830 Hydroxide, water, whole, fixed endpoint titration, field (milligrams per liter as $\mathrm{OH}$ )

71845 Nitrogen, ammonia, total (milligrams per liter as $\mathrm{NH}_{4}$ )

71846 Nitrogen, ammonia, dissolved (milligrams per liter as $\mathrm{NH}_{4}$ )

71850 Nitrogen, nitrate, total (milligrams per liter as $\mathrm{NH}_{3}$ )

71851 Nitrogen, nitrate, dissolved (milligrams per liter as $\mathrm{NH}_{3}$ )

71855 Nitrogen, nitrite, total (milligrams per liter as $\mathrm{NO}_{2}$ )

71856

71865

Nitrogen, nitrite, dissolved (milligrams per liter as $\mathrm{NO}_{2}$ )

Iodide, dissolved (milligrams per liter as I) 


\begin{tabular}{l|l}
\hline $\begin{array}{c}\text { Parameter } \\
\text { code }\end{array}$ & Property or constituent \\
\hline
\end{tabular}

71870 Bromide, dissolved (milligrams per liter as $\mathrm{Br}$ )

71883 Manganese (milligrams per liter as Mn)

71885 Iron (micrograms per liter as $\mathrm{Fe}$ )

71886 Phosphorus, total (milligrams per liter as $\mathrm{PO}_{4}$ )

71887 Nitrogen, total (milligrams per liter as $\mathrm{NO}_{3}$ )

71890 Mercury, dissolved (micrograms per liter as $\mathrm{Hg}$ )

71895 Mercury, suspended, recoverable (micrograms per liter as $\mathrm{Hg}$ )

71900 Mercury, total, recoverable (micrograms per liter as $\mathrm{Hg}$ )

71921 Mercury, recoverable from bottom material (micrograms per gram as $\mathrm{Hg}$ )

73547 2-Butene, Trans-1, ,4-Dichloro-, water, unfiltered, recoverable (micrograms per liter)

73570 Methacrylate, ethyl-, water, unfiltered, recoverable (micrograms per liter)

75985 Tritium, 2 sigma precision estimate, water, whole, total (picocuries per liter)

75986 Alpha, radioactivity, 2 sigma precision estimate, water, dissolved (micrograms per liter as U natural)

75987 Alpha, radioactivity, 2 sigma precision estimate, water, dissolved (picocuries per liter as TH-230)

75988 Beta, radioactivity, 2 sigma precision estimate, water, dissolved (picocuries per liter as SR-90/y-90)

75989 Beta, radioactivity, 2 sigma precision estimate, water, dissolved (picocuries per liter as CS-137)

75990 Uranium, natural, 2 sigma precision estimate, water, dissolved (micrograms per liter)

76001 Radium-226, 2 sigma precision estimate, water, dissolved (picocuries per liter)

76004 Alpha, radioactivity, 2 sigma precision estimate, sediment suspended, total, dry weight (picocuries per liter as TH-230)

76005 Beta, radioactivity, 2 sigma precision estimate, sediment, suspended, total, dry weight, (picocuries per liter as CS-137)

77041 Carbon disulfide, water, whole, total (micrograms per liter)

77057 Acetate, vinyl, water, unfiltered, recoverable (micrograms per liter)

77093 CIS-1, 2-Dichloroethene, water, whole, total (micrograms per liter)

77103 2-Hexanone, water, whole, total (micrograms per liter)

77128 Styrene, total (micrograms per liter)

77135 O-xylene, water, whole, total (micrograms per liter)

77168 1,1-Dichloropropene, water, whole, total (micrograms per liter)

77170 2,2-Dichloropropane, water, whole, total (micrograms per liter)

77173 Propane, 1,3-dichloro-, water, whole, total (micrograms per liter)

77220 Toluene, o-ethyl-, water, unfiltered, recoverable (micrograms per liter)

77221 Benzene, 1,2,3-Trimethyl-, water, unfiltered, recoverable (micrograms per liter)

77222 Benzene, 1,2,4-Trimethyl-, water, unfiltered, recoverable (micrograms per liter)

77223

77224

77226

Benzene, isopropyl-, water, whole, recoverable (micrograms per liter)

Benzene, n-propyl-, water, unfiltered, recoverable (micrograms per liter)

Benzene, 1,3,5-Trimethyl-, water, unfiltered, recoverable (micrograms per liter)

O-chlorotoluene, water, whole, total (micrograms per liter)

Toluene, p-chloro-, water, unfiltered, recoverable (micrograms per liter)

Methane, bromochloro-, water, unfiltered, recoverable (micrograms per liter)

Benzene, n-butyl-, water, unfiltered, recoverable (micrograms per liter)

Benzene, sec-butyl-, water, unfiltered, recoverable (micrograms per liter)

77353

77356

77424

77441

77443
Benzene, tert-butyl-, water, unfiltered, recoverable (micrograms per liter) P-isopropyltoluene, water, whole, recoverable (micrograms per liter) Methyl iodide, water, unfiltered, recoverable (micrograms per liter) 1-Naphthol, water, whole (micrograms per liter) 1,2,3-Trichloropropane, water, whole, total (micrograms per liter)

Ethane, 1,1,1,2-Tetrachloro-, water, unfiltered, recoverable (micrograms per liter) Benzene, 1,2,3-Trichloro-, water, whole, recoverable (micrograms per liter)

1,2-Dibromoethane, water, whole, total (micrograms per liter)

Freon 113, water, unfiltered, recoverable (micrograms per liter)

Alachlor, total, recoverable (micrograms per liter) 


\begin{tabular}{lc}
\hline $\begin{array}{c}\text { Parameter } \\
\text { code }\end{array}$ & Property or constituent \\
\hline
\end{tabular}

78032

78109

78133

79190

79193

80010

80030

80040

80050

80060

80154 Sediment, suspended concentration (milligrams per liter)

80155 Sediment discharge, suspended (tons per day)

80156 Sediment discharge, total, suspended plus bed material (tons per day)

80157 Sediment, bed material, fall diameter, distilled water (percent finer than 0.004 millimeter)

80158 Sediment, bed material, fall diameter, distilled water (percent finer than 0.062 millimeter)

80159 Sediment, bed material, fall diameter, distilled water (percent finer than 0.125 millimeter) 80160 Sediment, bed material, fall diameter, distilled water (percent finer than 0.250 millimeter) 80161 Sediment, bed material, fall diameter, distilled water (percent finer than 0.500 millimeter) 80162 Sediment, bed material, fall diameter, distilled water (percent finer than 1.00 millimeter)

80164 Sediment, bed material, sieve diameter (percent finer than 0.062 millimeter)

80165 Sediment, bed material, sieve diameter (percent finer than 0.125 millimeter)

80166 Sediment, bed material, sieve diameter (percent finer than 0.250 millimeter)

80167 Sediment, bed material, sieve diameter (percent finer than 0.500 millimeter)

80168 Sediment, bed material, sieve diameter (percent finer than 1.00 millimeter)

80169 Sediment, bed material, sieve diameter (percent finer than 2.00 millimeters)

80170 Sediment, bed material, sieve diameter (percent finer than 4.00 millimeters)

80171 Sediment, bed material, sieve diameter (percent finer than 8.00 millimeters)

80172 Sediment, bed material, sieve diameter (percent finer than 16.0 millimeters)

80173 Sediment, bed material, sieve diameter (percent finer than 32.0 millimeters)

80184 Sediment, total, fall diameter, distilled water (percent finer than 0.016 millimeter)

80282 Bed material, fall diameter, distilled water (percent finer than 0.016 millimeter)

80283 Bed material, fall diameter, distilled water (percent finer than 0.031 millimeter)

80293 Bed material, fall diameter, distilled water (percent finer than 0.008 millimeter)

80294 Bed material, fall diameter, distilled water (percent finer than 0.002 millimeter)

81403 Chlorpyrifos, total (micrograms per liter)

81408 Metribuzin (sencor) in whole water sample (micrograms per liter)

81551 Xylene, water, unfiltered, recoverable (micrograms per liter)

81552 Acetone, water, whole, total (micrograms per liter)

81555 Bromobenzene, water, whole, total (micrograms per liter)

81576 Ether, Ethyl, water, unfiltered, recoverable (micrograms per liter)

81577 Di-isopropylether, water, unfiltered, recoverable (micrograms per liter)

81593 Methacrylonitrile, water, unfiltered, recoverable (micrograms per liter)

81595 Methylethylketone, water, whole, total (micrograms per liter)

81597 Methacrylate, methyl, water, unfiltered, recoverable (micrograms per liter)

81607 Furan, tetrahydro-, water, unfiltered, recoverable (micrograms per liter)

81757 Cyanazine, total (micrograms per liter)

81886 Perthane, in bottom material (micrograms per kilogram)

82052 Dicamba, total (micrograms per liter)

82068 Potassium 40. dissolved (picocuries per liter as K40)

82079 Turbidity, lab (nephelometric turbidity unit) 


\begin{tabular}{cl}
\hline $\begin{array}{c}\text { Parameter } \\
\text { code }\end{array}$ & \multicolumn{1}{c}{ Property or constituent } \\
\hline 82082 & Hydrogen (2 to 1 ratio per million) \\
82085 & Oxygen (18 to 16 ratio per milion) \\
82183 & 2,4-DP, total (micrograms per liter) \\
82226 & Dinoseb, water, unfiltered, recoverable (micrograms per liter) \\
82584 & 3-Hydroxy carbofuran, water, whole, total recoverable (micrograms per liter) \\
& \\
82586 & Aldicarb sulfoxide, water, whole, total, recoverable (micrograms per liter) \\
82587 & Aldicarb sulfone, water, whole, total, recoverable (micrograms per liter) \\
82612 & Metolachlor, water, whole, total, recoverable (micrograms per liter) \\
82613 & Oxyamyl, water, whole, total, recoverable (micrograms per liter) \\
82615 & Carbofuran, water, whole, total, recoverable (micrograms per liter) \\
82618 & Carbaryl, water, whole, total, recoverable (micrograms per liter) \\
82619 & Aldicarb, water, whole, total, recoverable (micrograms per liter) \\
82625 & Dibromochloropropane, water, whole, total, recoverable (micrograms per liter) \\
82630 & Metribuzin, (sencor), water, dissolved (micrograms per liter) \\
82660 & 2, 6-Dietrhylaniline, water, filtered, glass fiber, 0.7 u, recoverable (micrograms per liter)
\end{tabular}

82661 Trifluralin, water, filtered, glass fiber, $0.7 \mathrm{u}$, recoverable (micrograms per liter)

82663 Ethalfluralin, water, filtered, glass fiber, $0.7 \mathrm{u}$, recoverable (micrograms per liter)

82664 Phorate, water, filtered, glass fiber, $0.7 \mathrm{u}$, recoverable (micrograms per liter)

82665 Terbacil, water, filtered, glass fiber, $0.7 \mathrm{u}$, recoverable (micrograms per liter)

82666 Linuron, water, filtered, glass fiber, $0.7 \mathrm{u}$, recoverable (micrograms per liter)

82667 Methyl parathion, water, filtered, glass fiber, $0.7 \mathrm{u}$, recoverable (micrograms per liter)

82668 EPTC, water, filtered, glass fiber, $0.7 \mathrm{u}$, recoverable (micrograms per liter)

82669 Pebulate, water, filtered, glass fiber, $0.7 \mathrm{u}$, recoverable (micrograms per liter)

82670 Tebuthiuron, water, filtered, glass fiber, $0.7 \mathrm{u}$, recoverable (micrograms per liter)

82671 Molinate, water, filtered, glass fiber, $0.7 \mathrm{u}$, recoverable (micrograms per liter)

82672 Ethoprop, water, filtered, glass fiber, $0.7 \mathrm{u}$, recoverable (micrograms per liter)

82673 Benfluralin, water, filtered, glass fiber, $0.7 \mathrm{u}$, recoverable (micrograms per liter)

82674 Carbofuran, water, filtered, glass fiber, $0.7 \mathrm{u}$, recoverable (micrograms per liter)

82675 Terbufos, water, filtered, glass fiber, $0.7 \mathrm{u}$, recoverable (micrograms per liter)

82676 Pronamide, water, filtered, glass fiber, $0.7 \mathrm{u}$, recoverable (micrograms per liter)

82677 Disulfoton, water, filtered, glass fiber, $0.7 \mathrm{u}$, recoverable (micrograms per liter)

82678 Triallate, water, filtered, glass fiber, $0.7 \mathrm{u}$, recoverable (micrograms per liter)

82679 Propanil, water, filtered, glass fiber, $0.7 \mathrm{u}$, recoverable (micrograms per liter)

82680 Carbaryl, water, filtered, glass fiber, $0.7 \mathrm{u}$, recoverable (micrograms per liter)

82681 Thiobencarb, water, filtered, glass fiber, $0.7 \mathrm{u}$, recoverable (micrograms per liter)

82682 DCPA, water, filtered, lass fiber, $0.7 \mathrm{u}$, recoverable (micrograms per liter)

82683 Pendimethalin, water, filtered, glass fiber, $0.7 \mathrm{u}$, recoverable (micrograms per liter)

82684 Napropamide, water, filtered, glass fiber, $0.7 \mathrm{u}$, recoverable (micrograms per liter)

82685 Propargite, water, filtered, glass fiber, $0.7 \mathrm{u}$, recoverable (micrograms per liter)

82686 Methyl azinphos, water, filtered, glass fiber, $0.7 \mathrm{u}$, recoverable (micrograms per liter)

82687 Permethrin, cis, water, filtered, glass fiber, $0.7 \mathrm{u}$, recoverable (micrograms per liter)

85795 M-xylene/P-xylene, water, unfiltered, recoverable (micrograms per liter)

90095 Specific conductance (microsiemens per centimeter at 25 degrees Celsius)

90410 Acid neutralizing capacity (alkalinity), titration to $\mathrm{pH} 4.5$, laboratory (milligrams per liter as $\mathrm{CaCO}_{3}$ )

95410 Acid neutralizing capacity (anc), water, unfiltered, titration to $\mathrm{pH} 4.5$, laboratory (milligrams per liter as $\mathrm{CaCO}_{3}$ )

95440 Bicarbonate, titration to $\mathrm{pH} 4.5$, laboratory (milligrams per liter as $\mathrm{CaCO}_{3}$ )

95445 Carbonate, titration to $\mathrm{pH} 8.3$, laboratory (milligrams per liter as $\mathrm{CaCO}_{3}$ )

95902 Hardness, noncarbonate (milligrams per liter as $\mathrm{CaCO}_{3}$ )

99430 Acid neutralizing capacity, water, unfiltered, carbonate, incremental titration, field (milligrams per liter as $\mathrm{CaCO}_{3}$ )

99440 Bicarbonate, incremental titration, field $\left(\mathrm{mg} / \mathrm{L}\right.$ as $\left.\mathrm{HCO}_{3}\right)$ 
List of parameter codes and corresponding property or constituent--Continued

\section{Parameter}

Carbonate, incremental titration, field $\left(\mathrm{mg} / \mathrm{L}\right.$ as $\left.\mathrm{CO}_{3}\right)$

Phosphorus, total, water, whole, modified jirka method, total (milligrams per liter as P)

99892

Nitrogen, ammonia plus organic, water, whole, modified jirka method, total (milligrams per liter as $\mathrm{N}$ )

99893

Phosphorus, total, water, dissolved, modified jirka method (milligrams per liter as P)

99894

Nitrogen, ammonia plus organic, water, dissolved, modified jirka method (milligrams per liter as $\mathrm{N}$ ) 


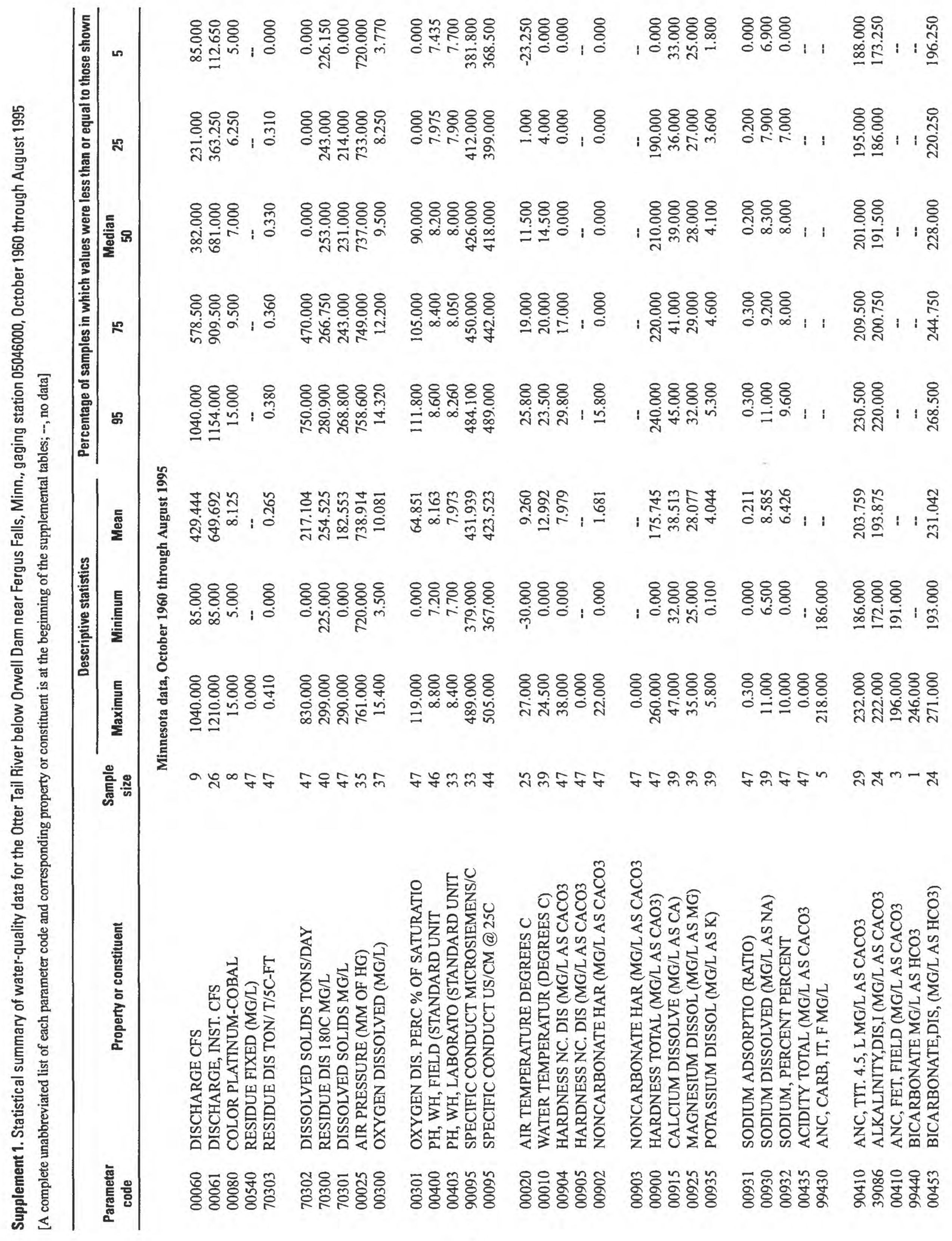




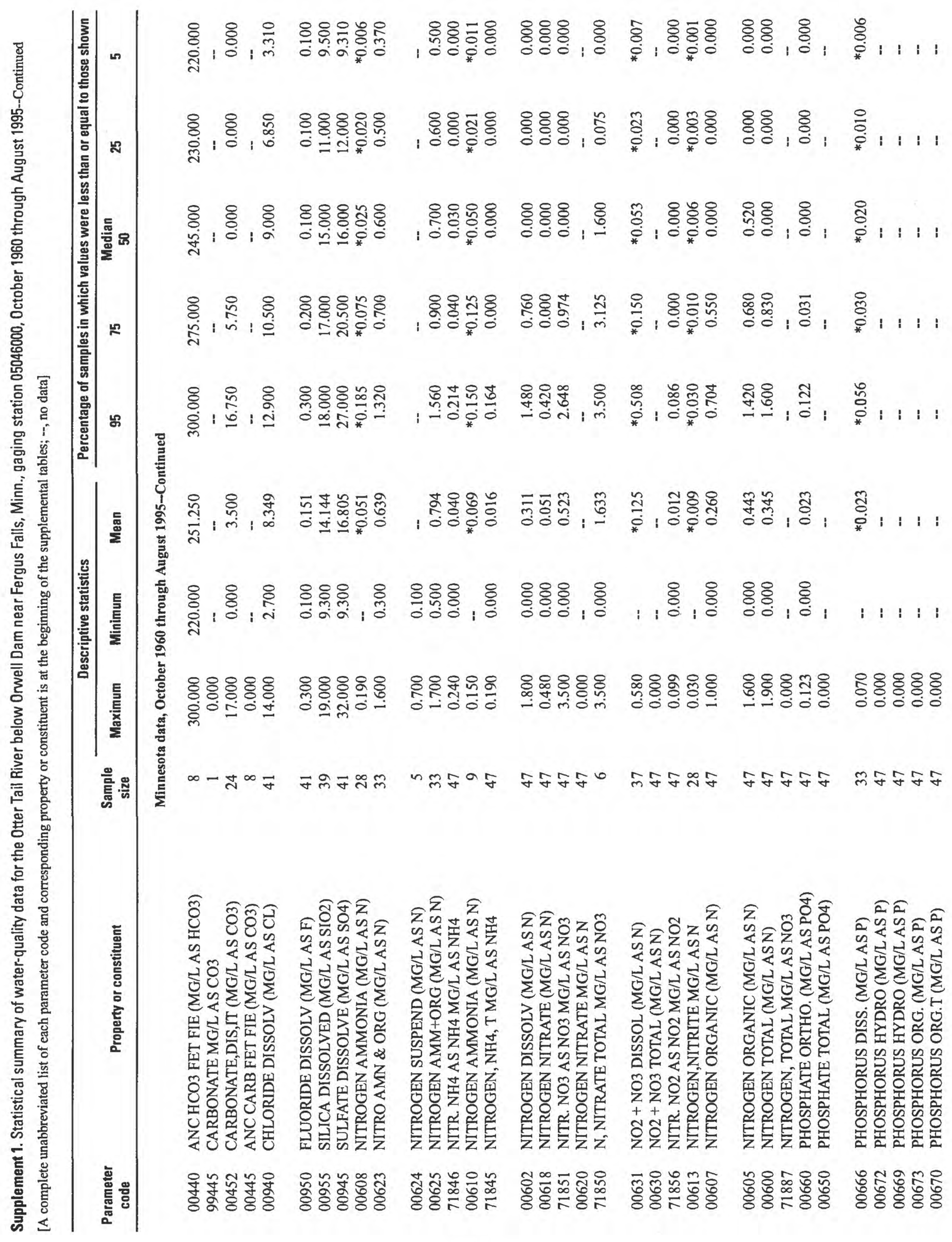




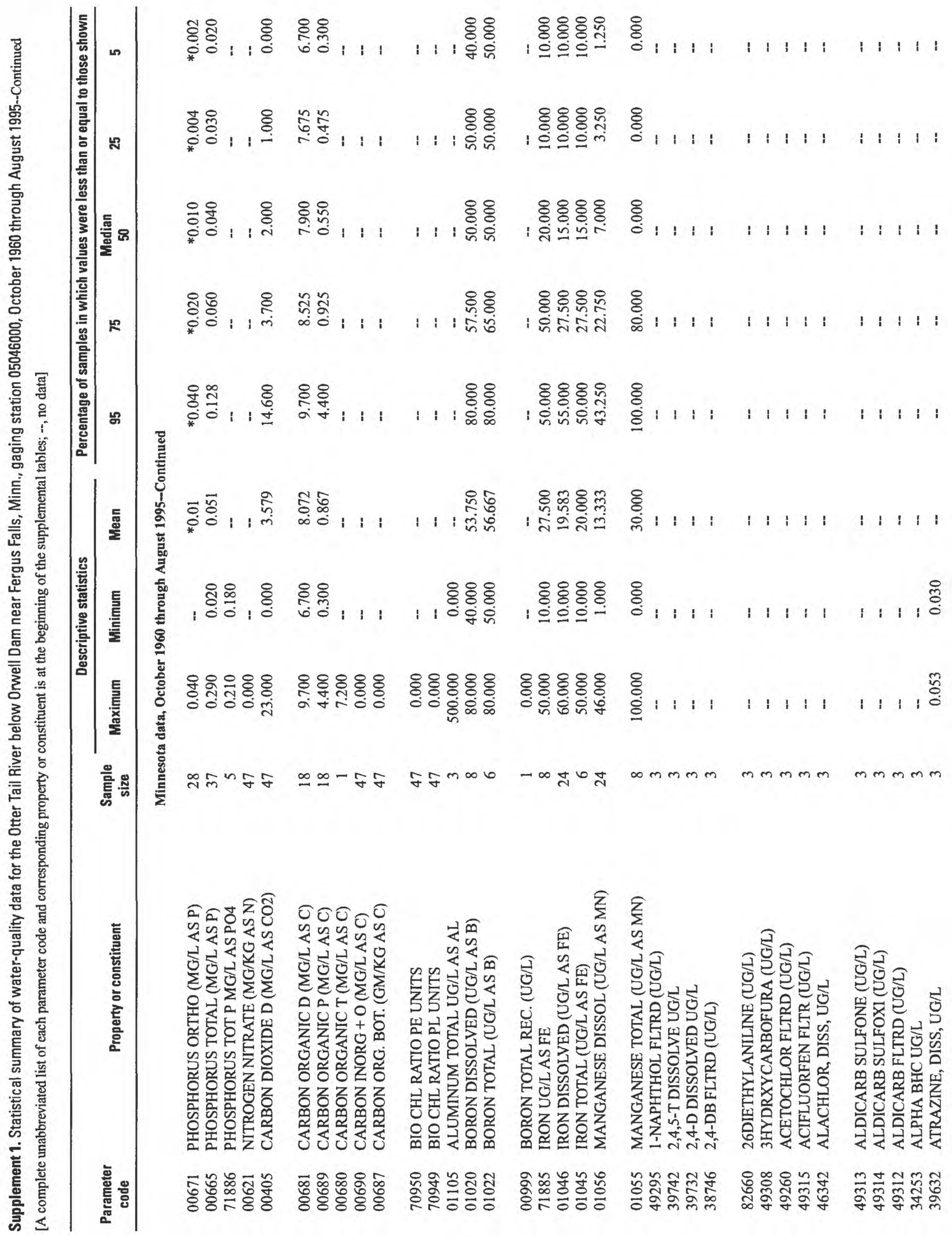




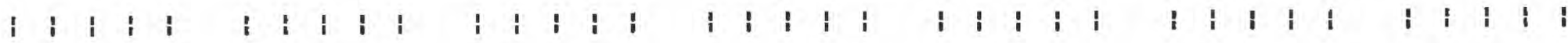

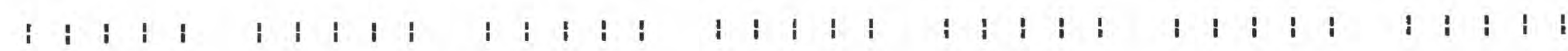

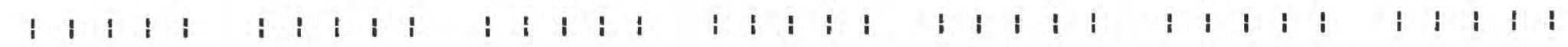

i i i i

i i i

; i

; ;

1 1 i i

1 1 1

1 1 i

总

1111

1 1 1

1 1 i 1 i

1 1 i i

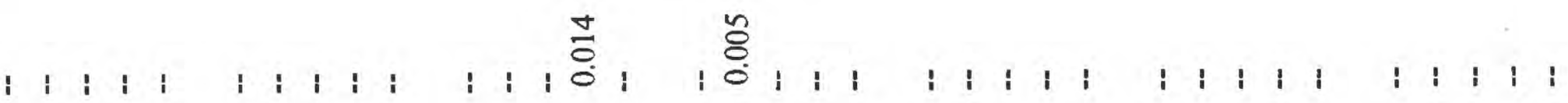
i i i i i i i i i i mmmmm mmmmm 吾 


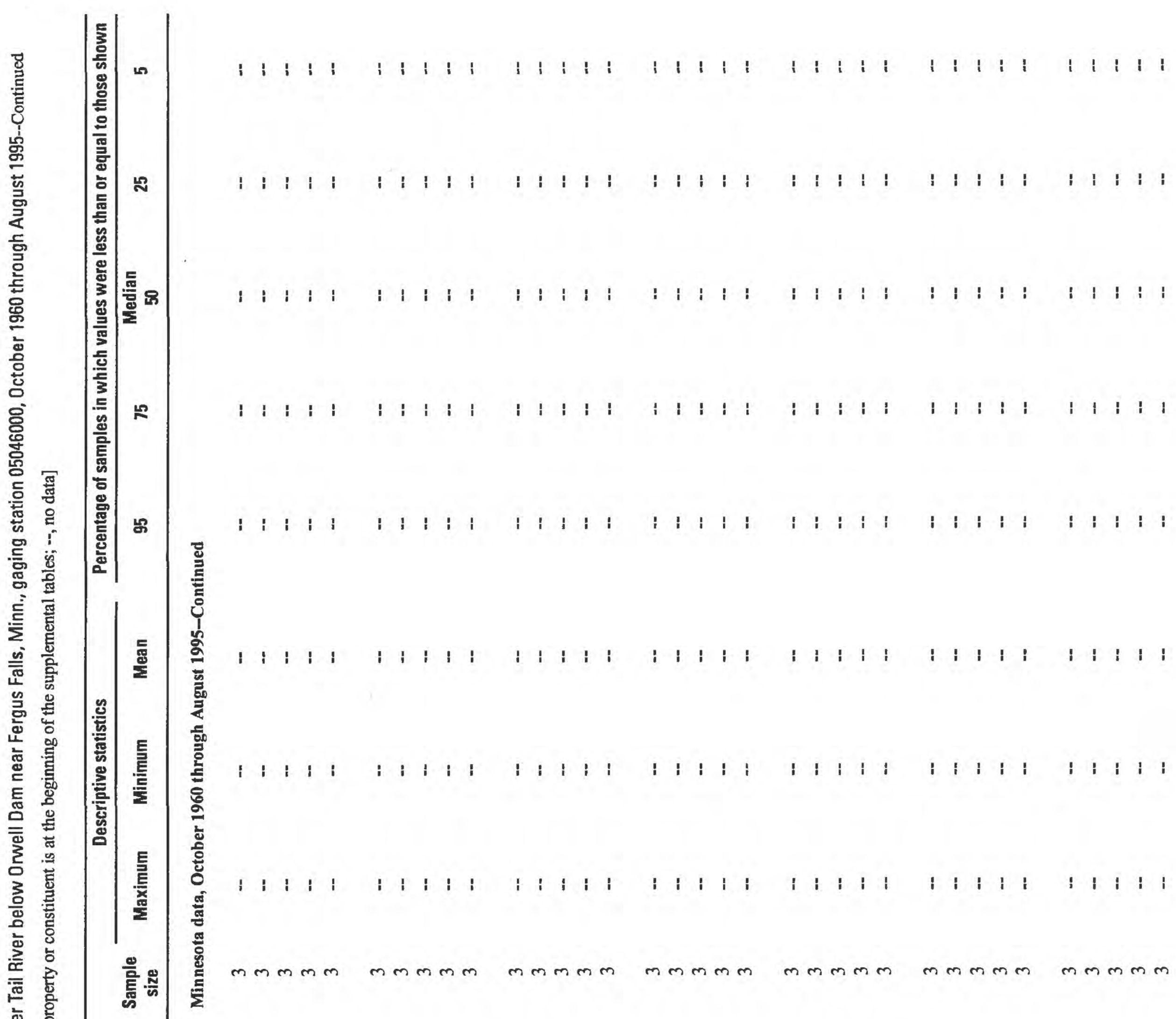

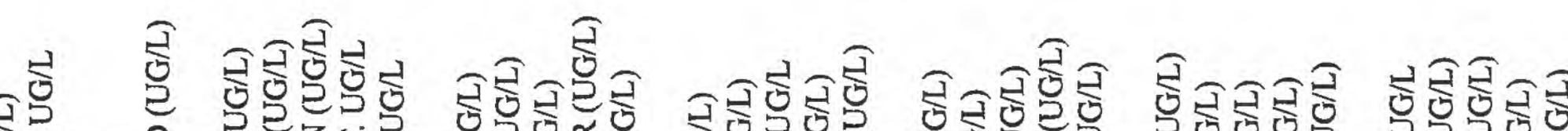

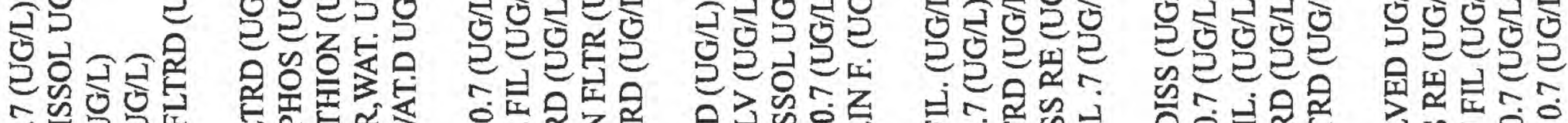

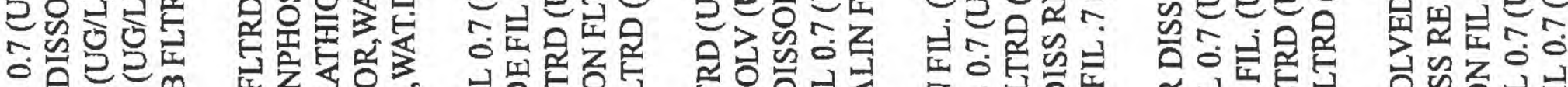

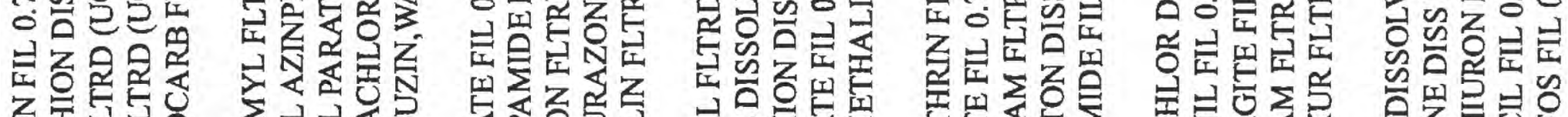

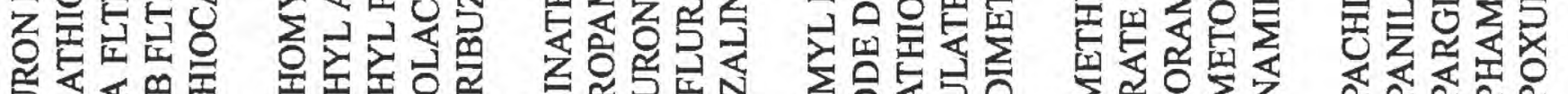

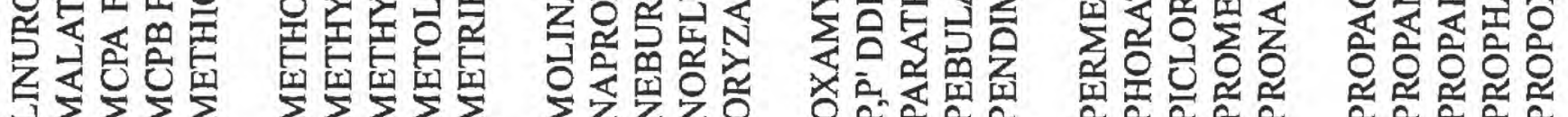

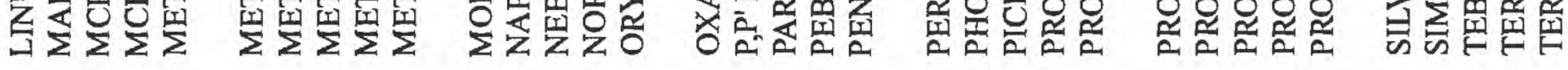




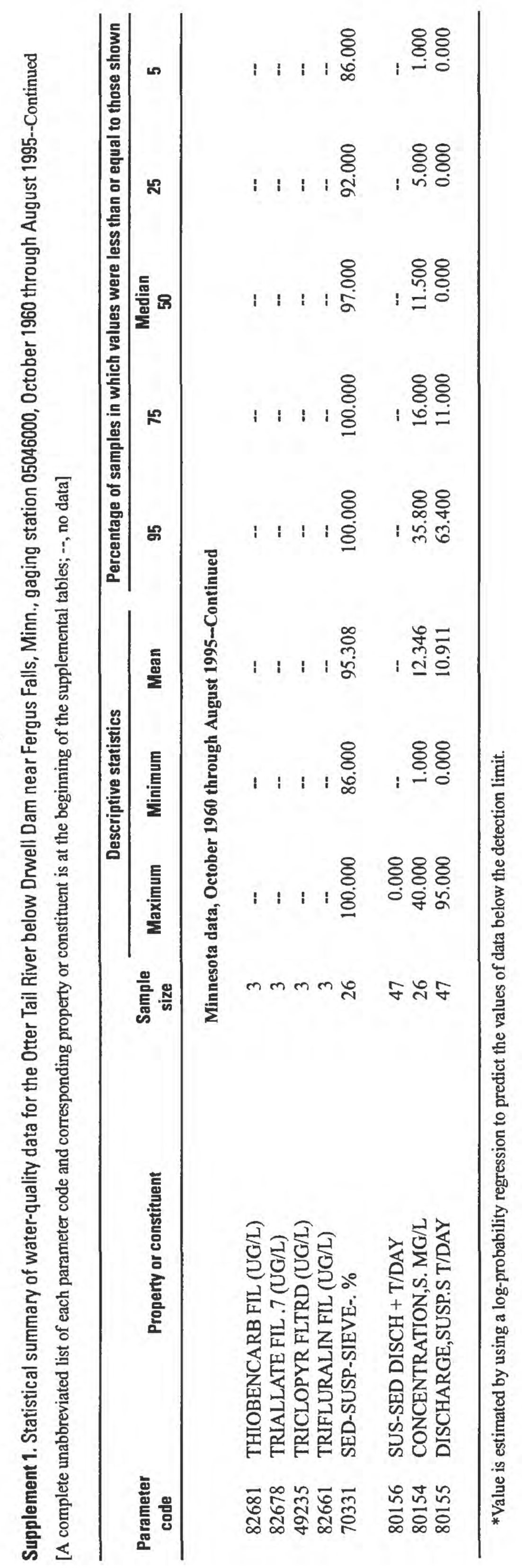




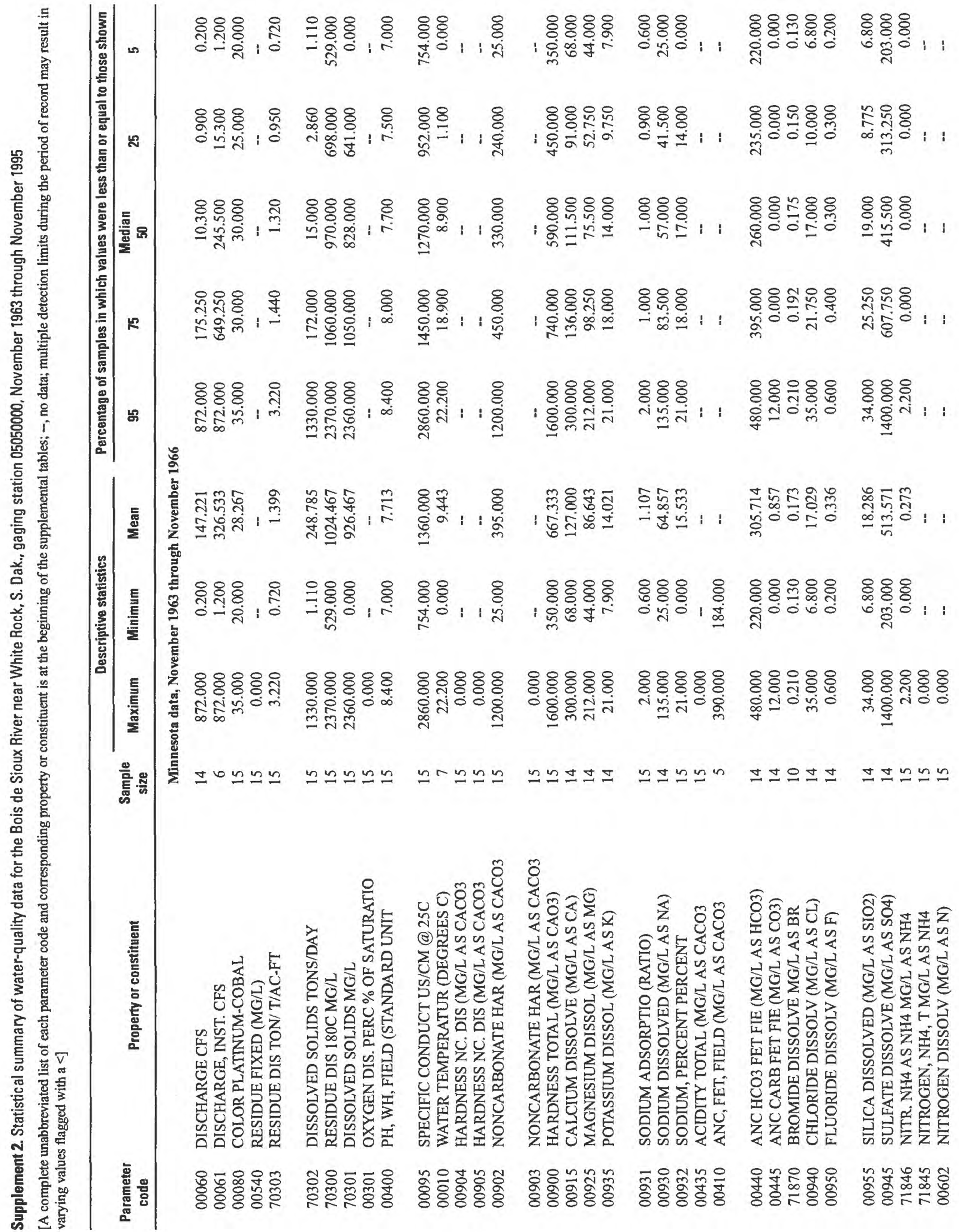




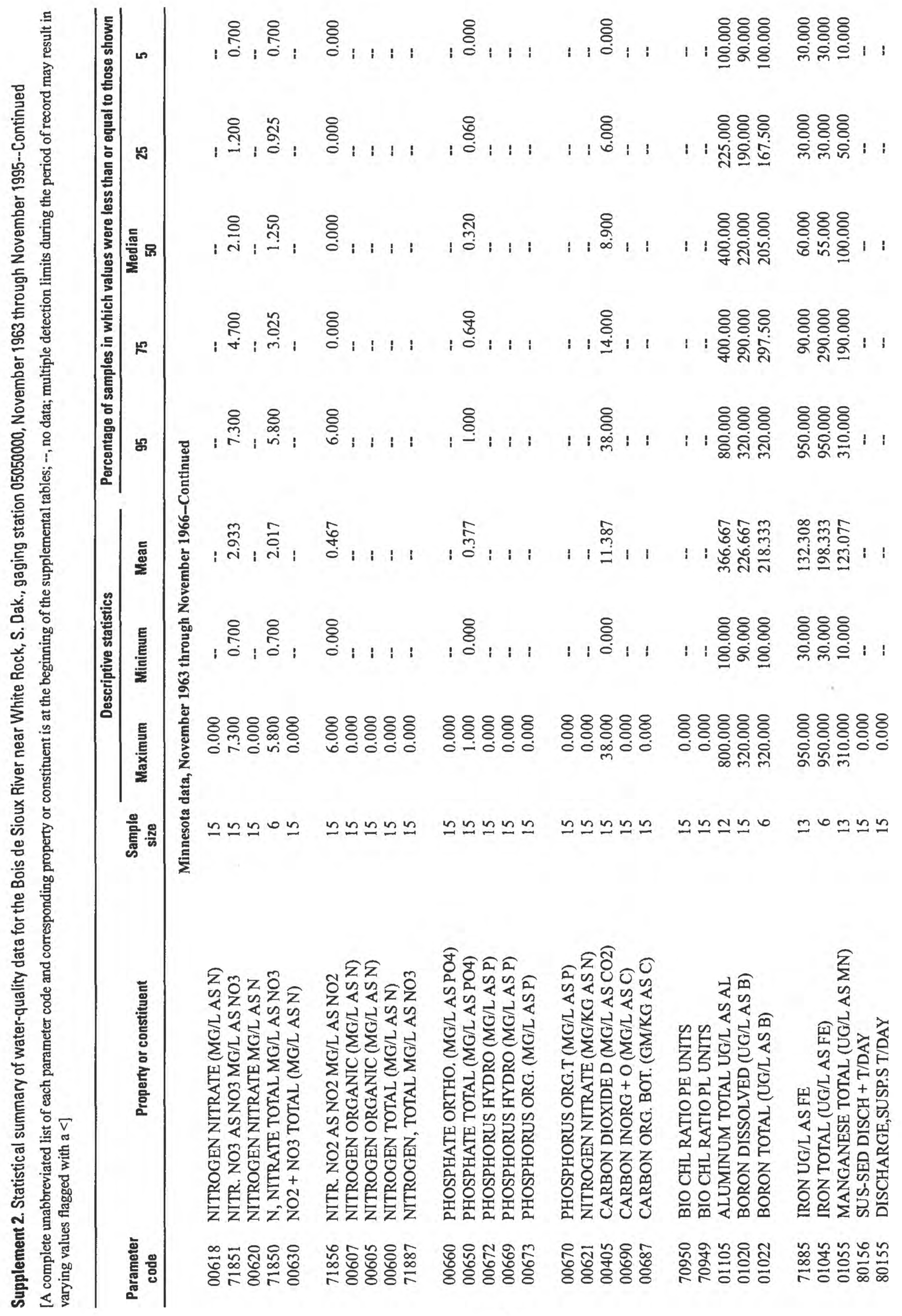




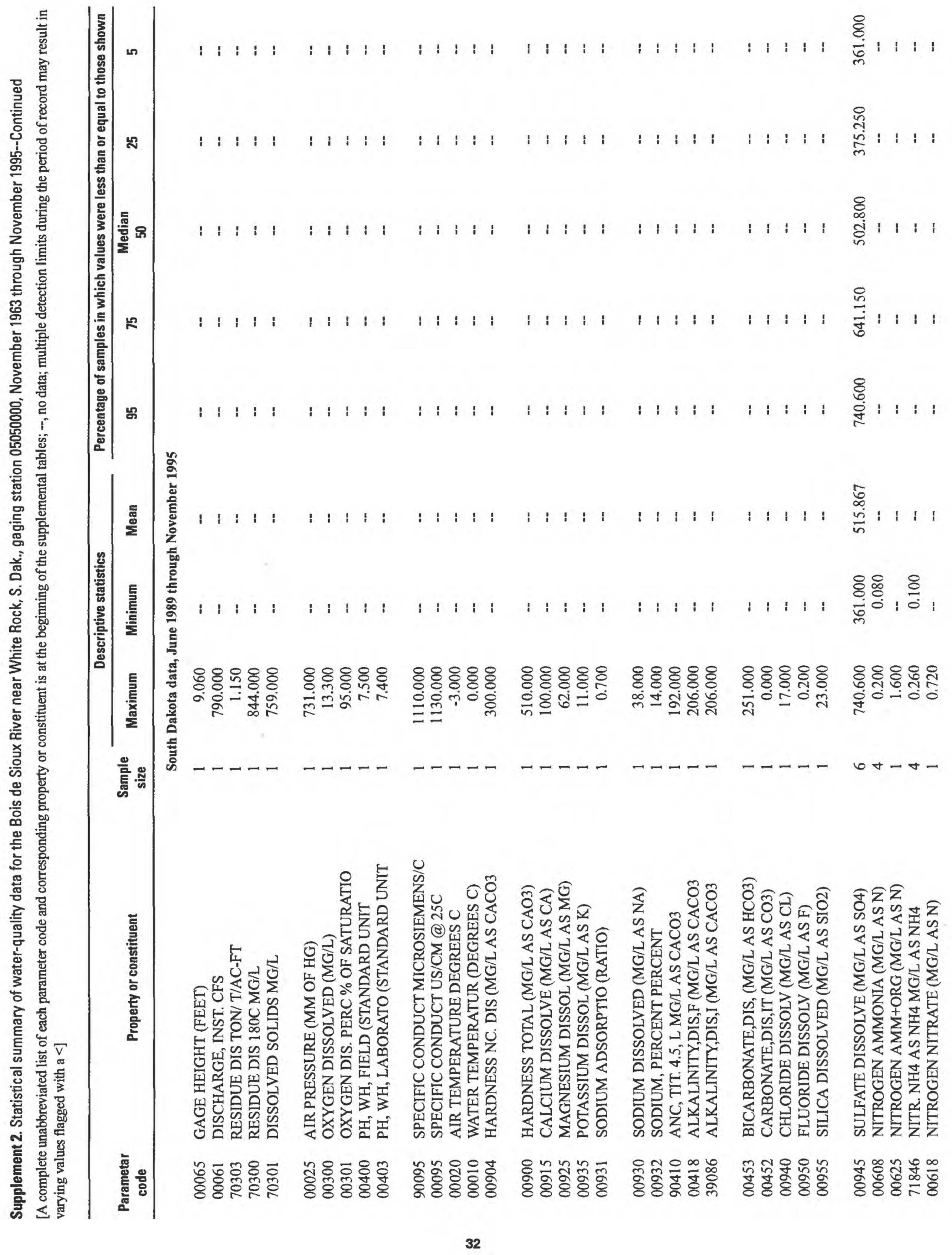




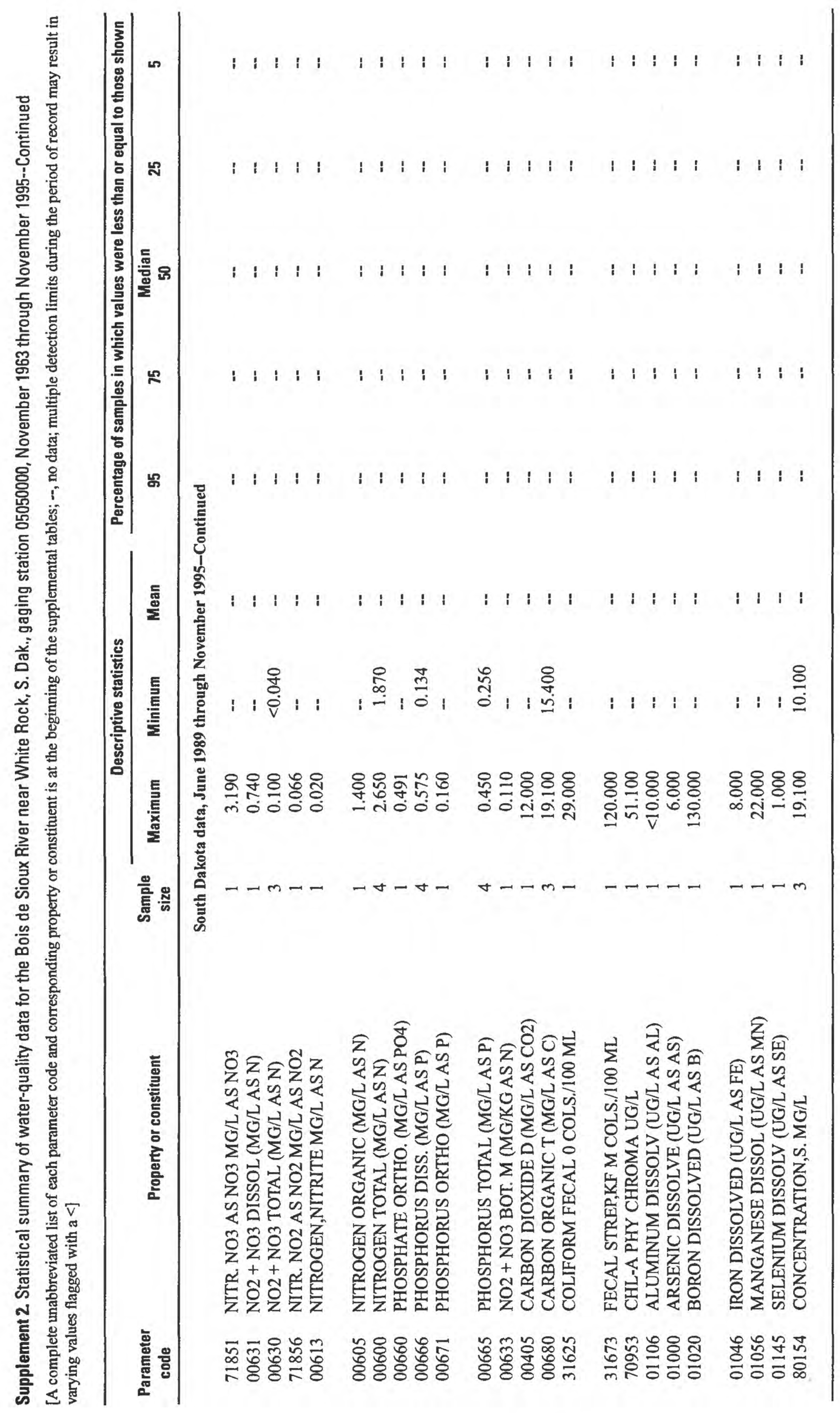




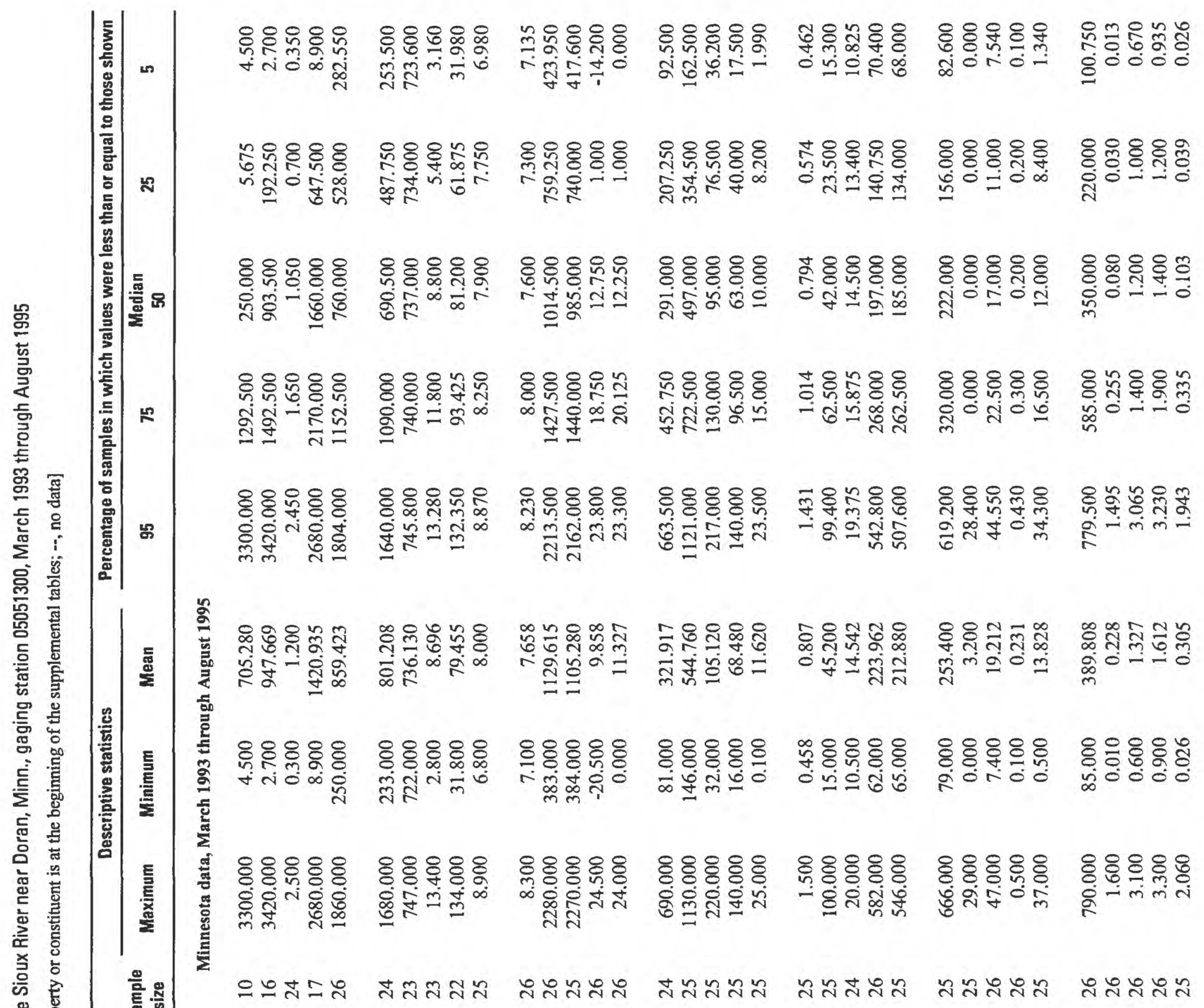
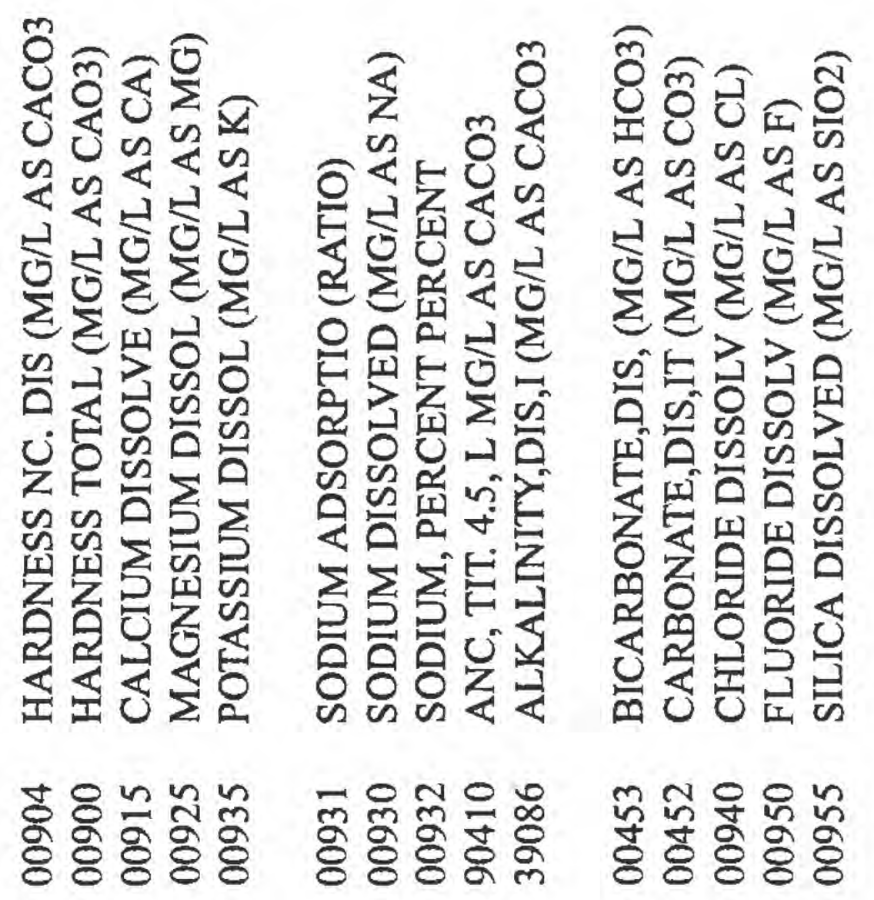

娄云

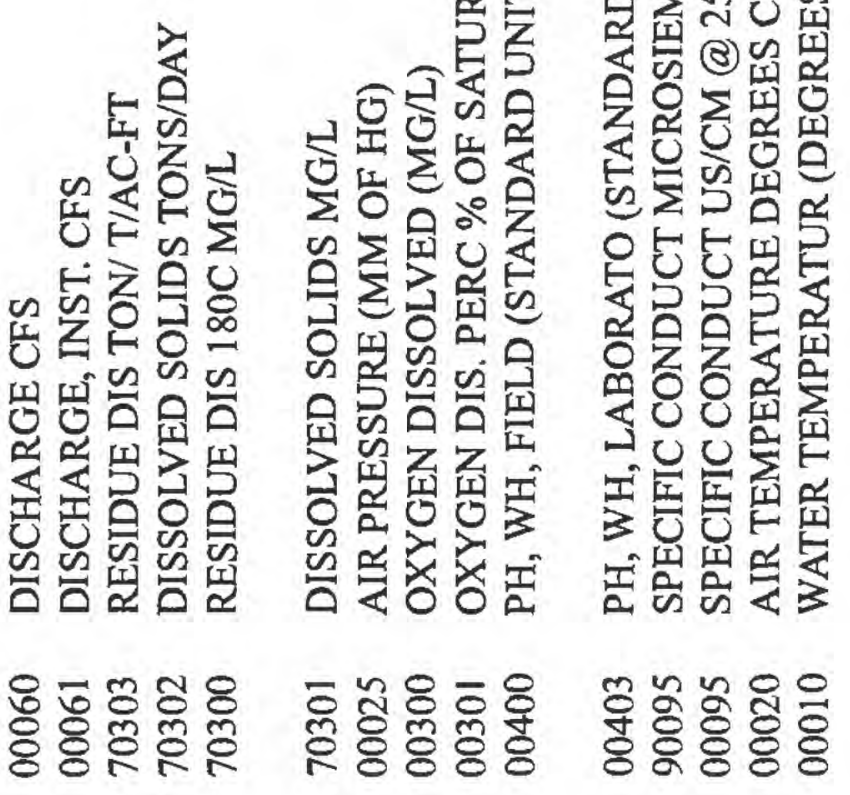
U यू क्ष这 어엃옳엉 띠임 延识出 z은

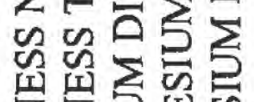
光记贸 空这

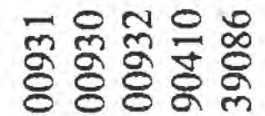

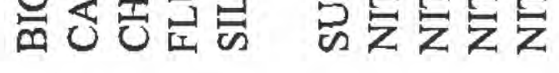



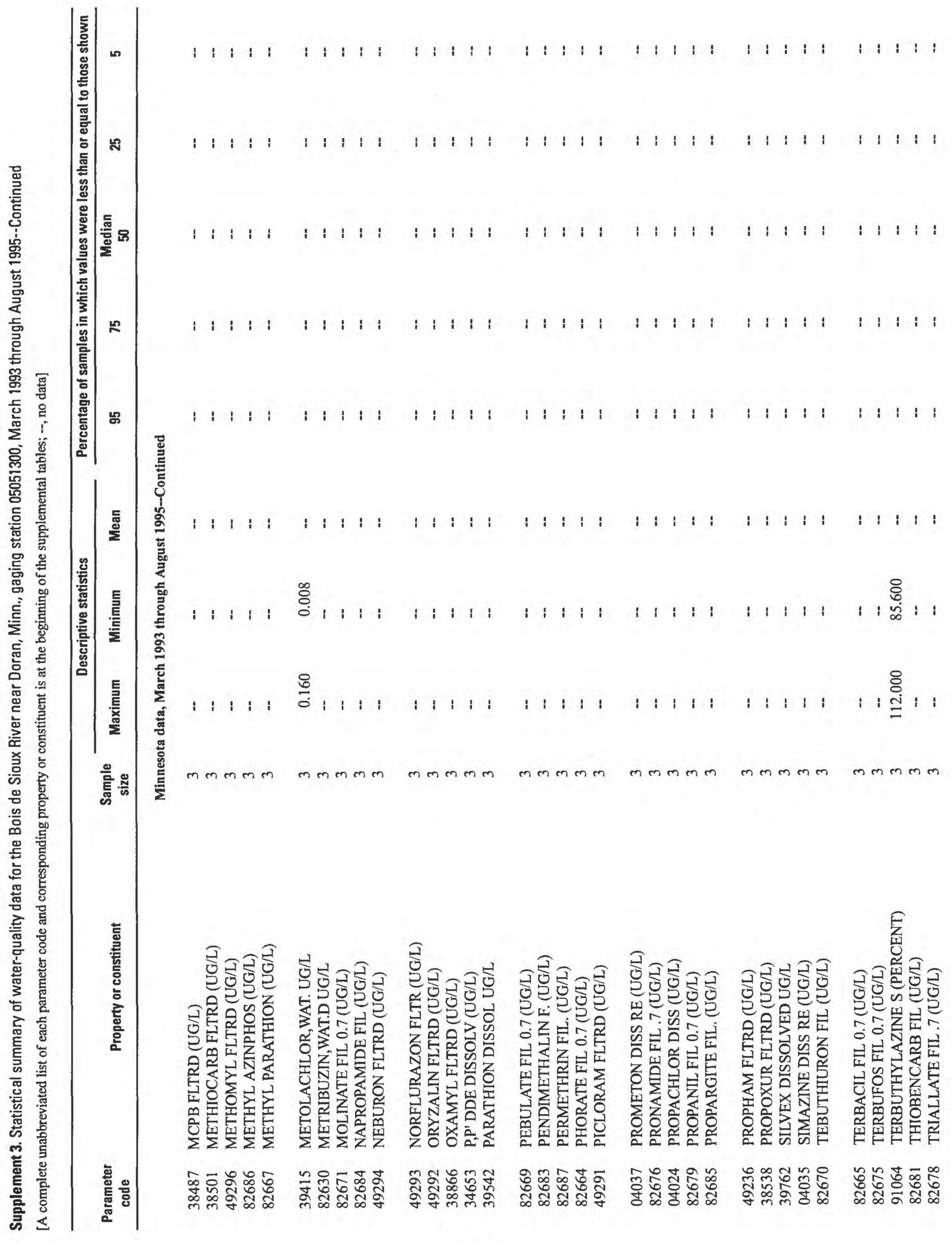


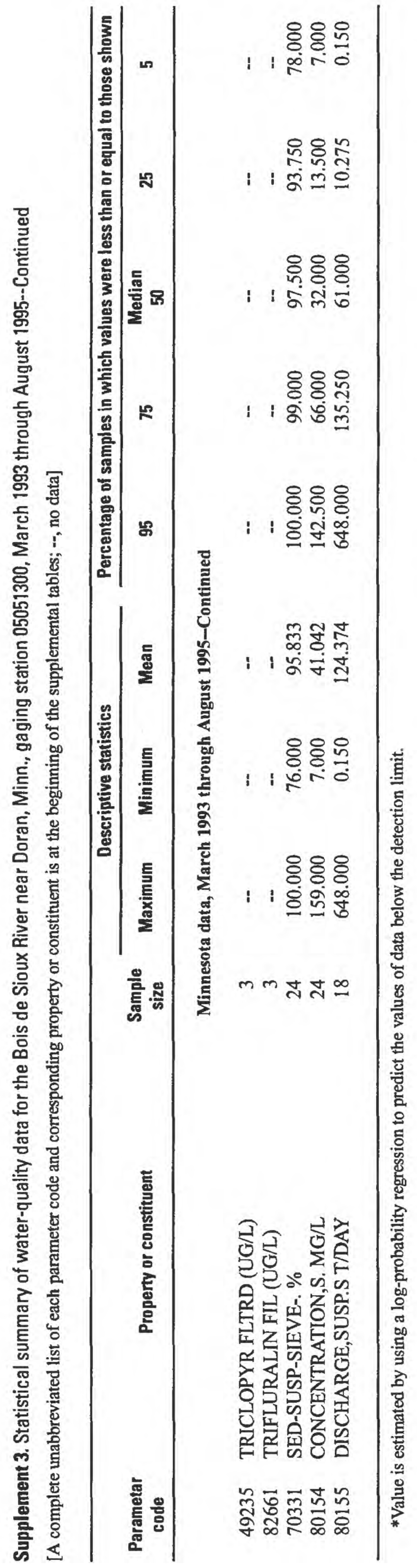




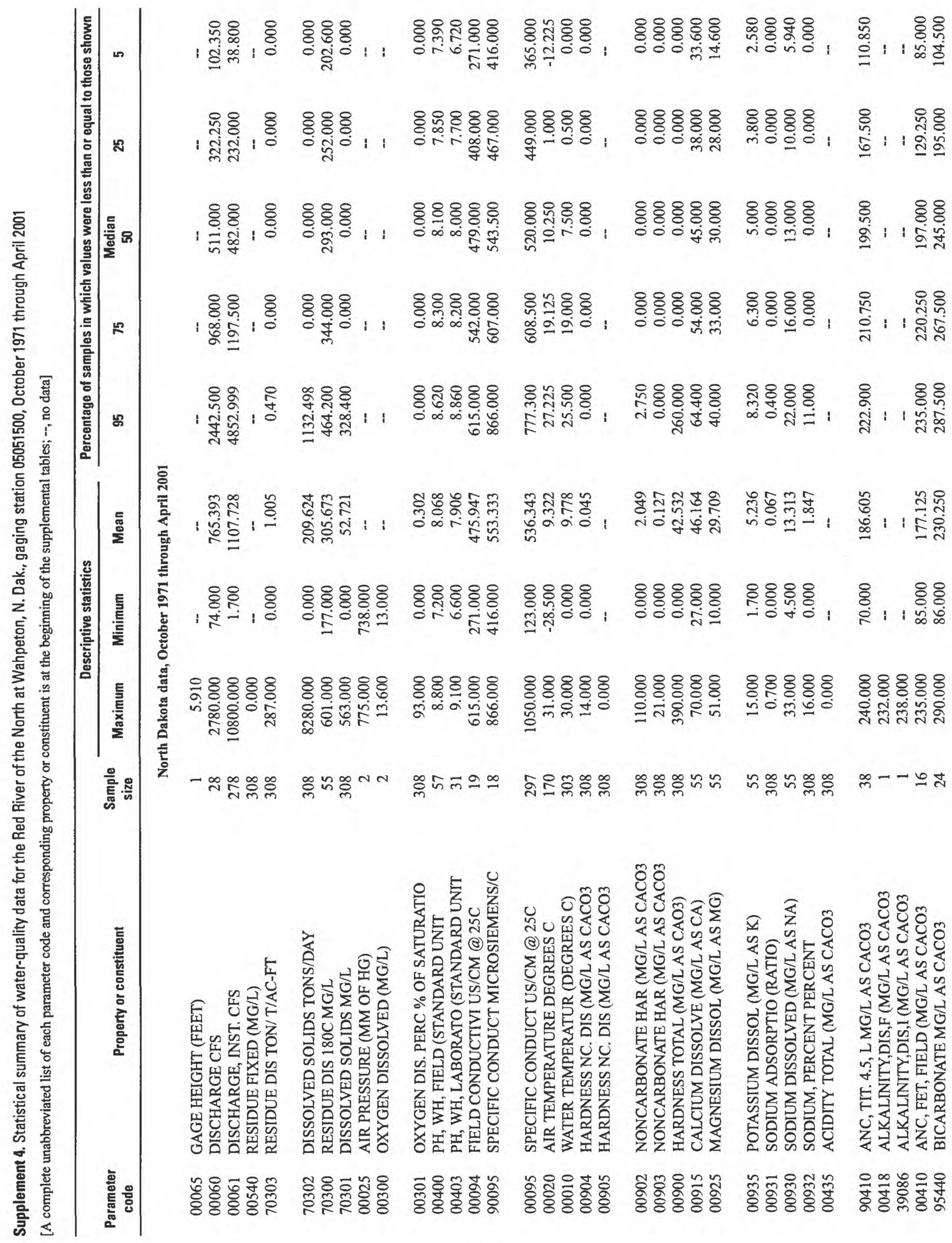




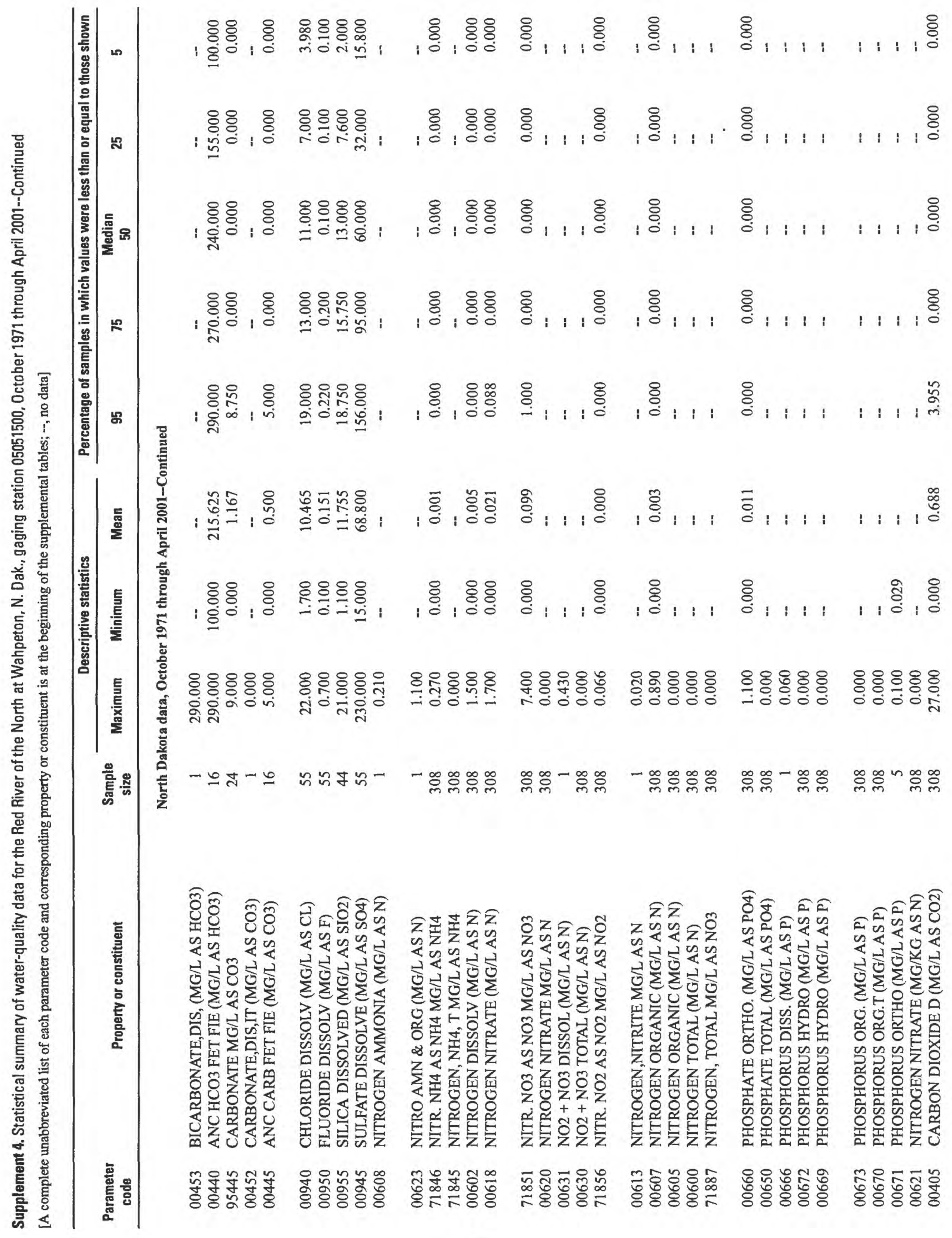




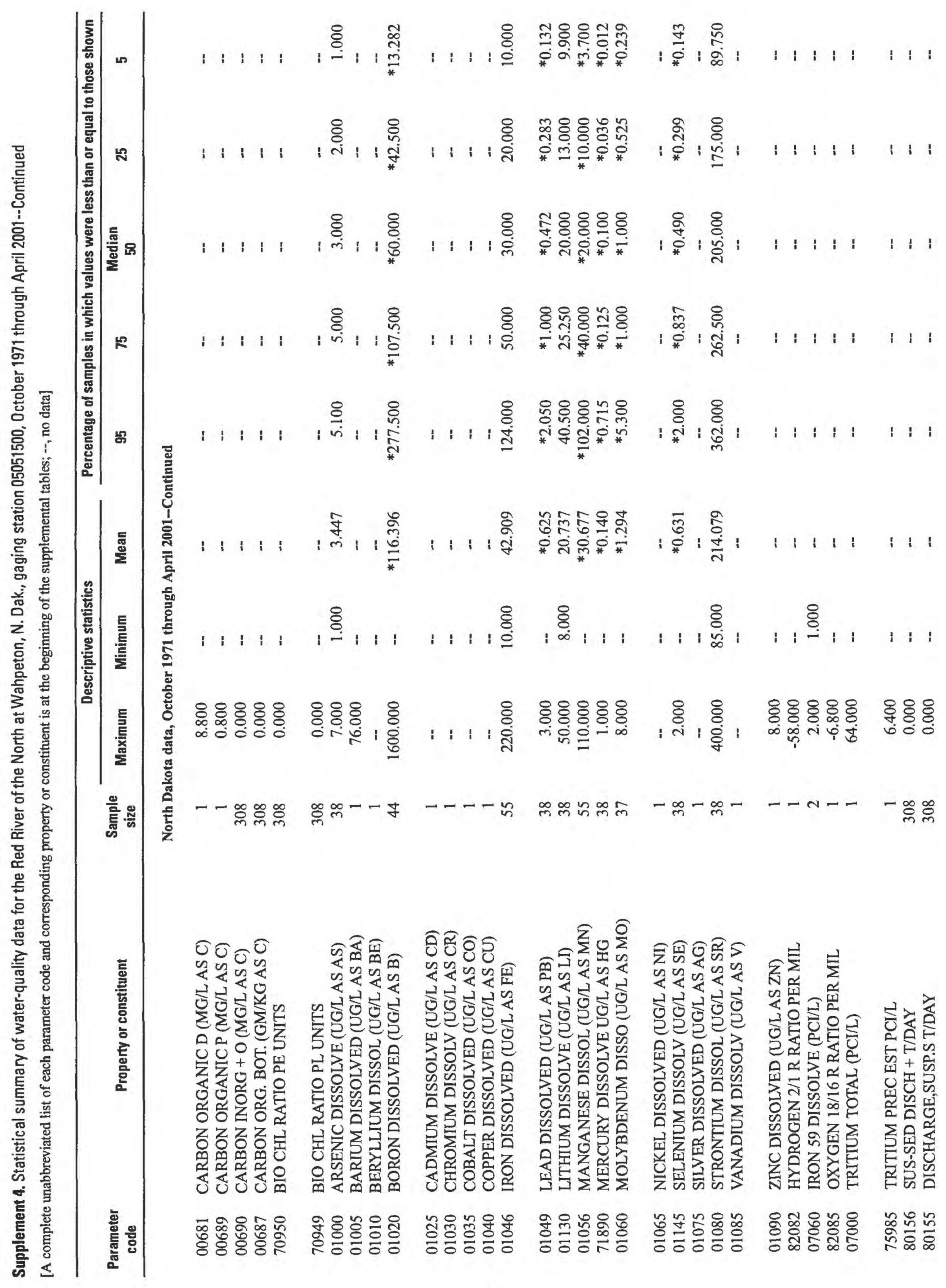




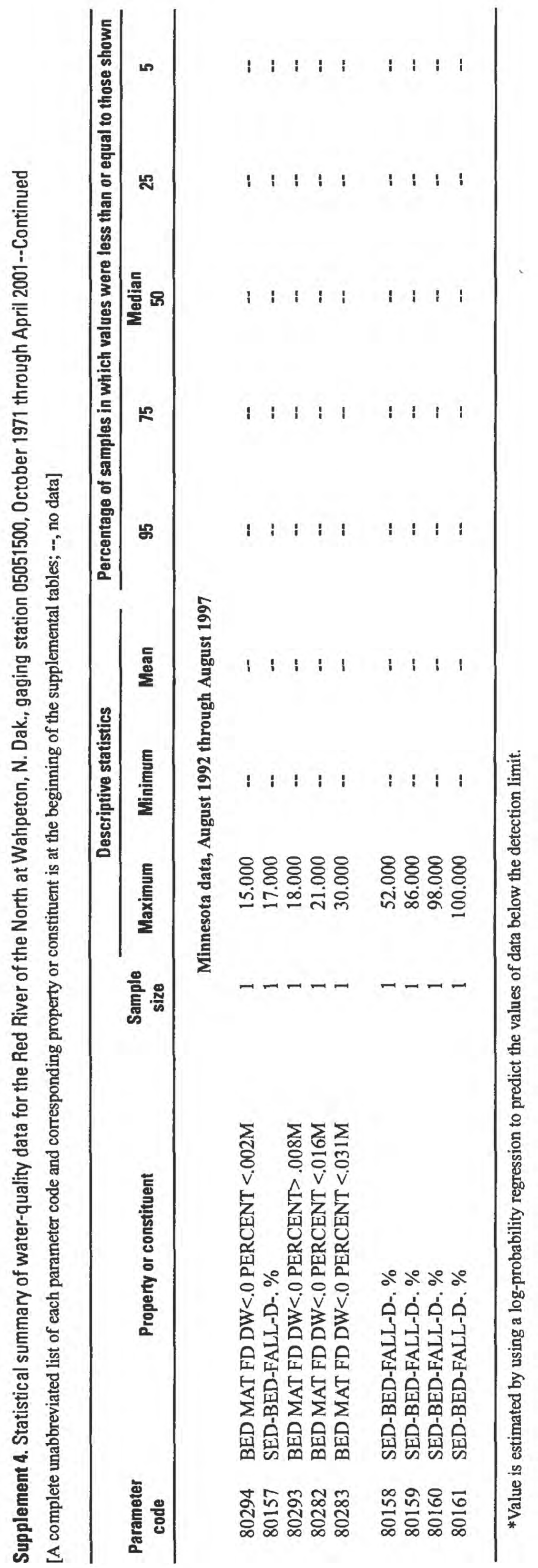




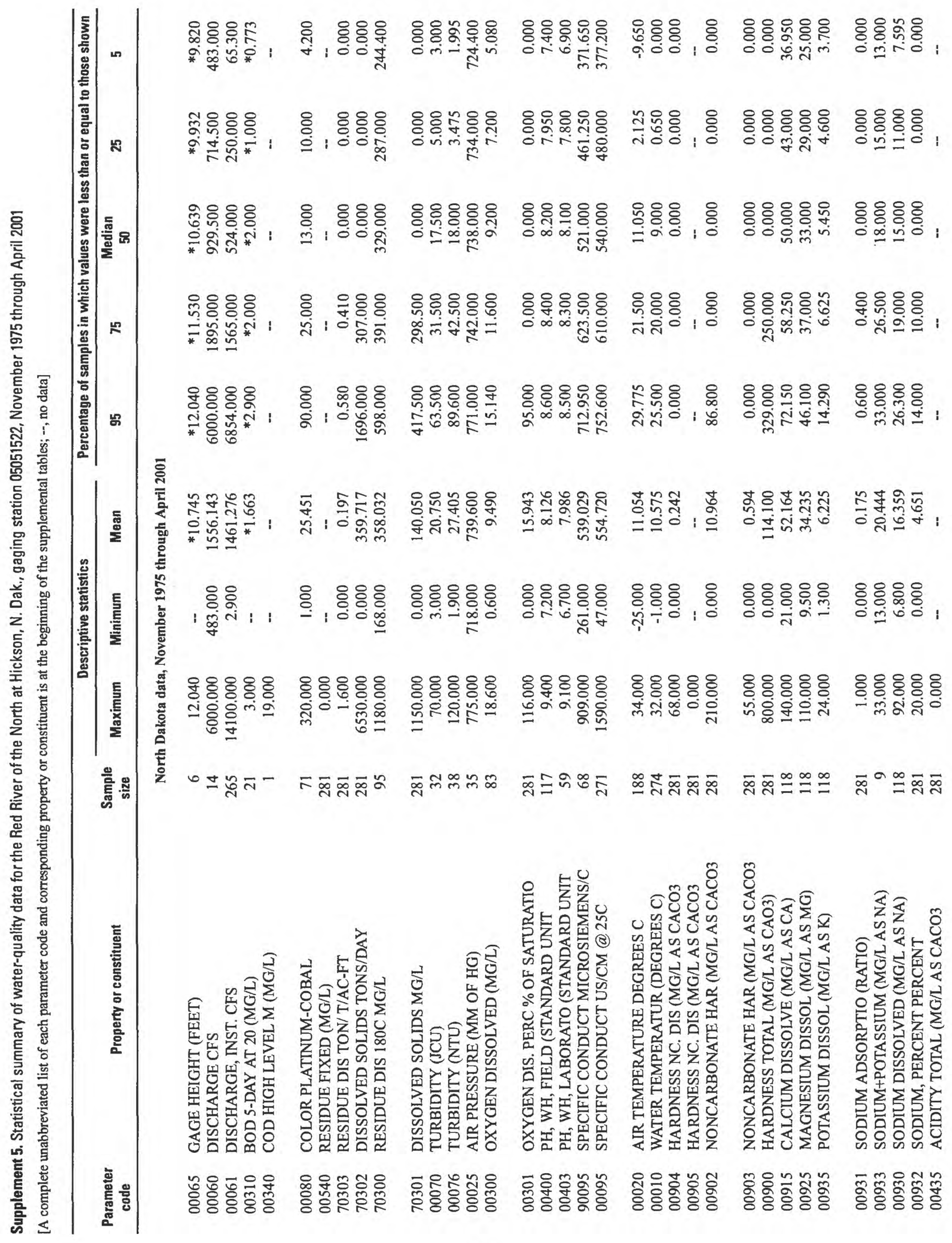




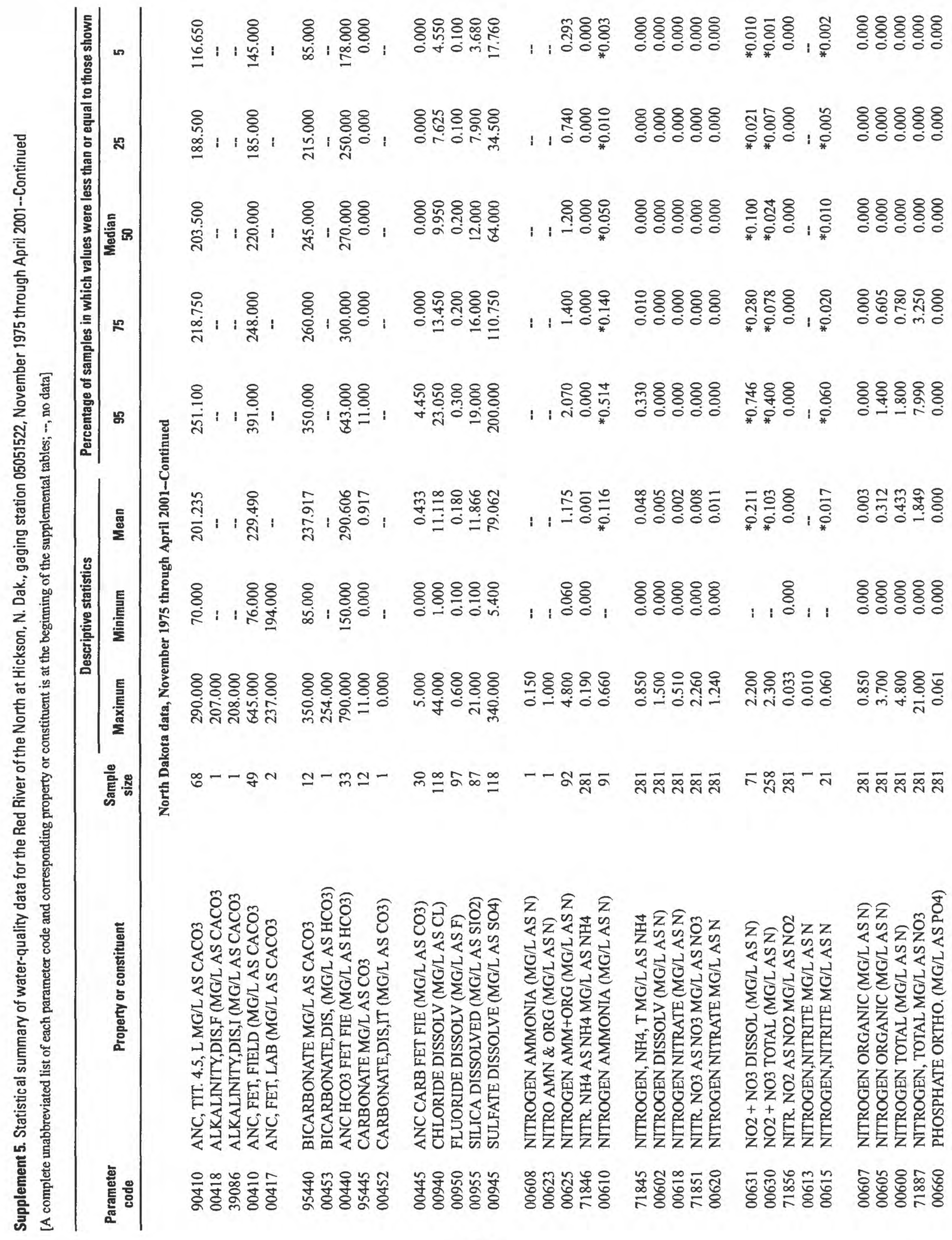




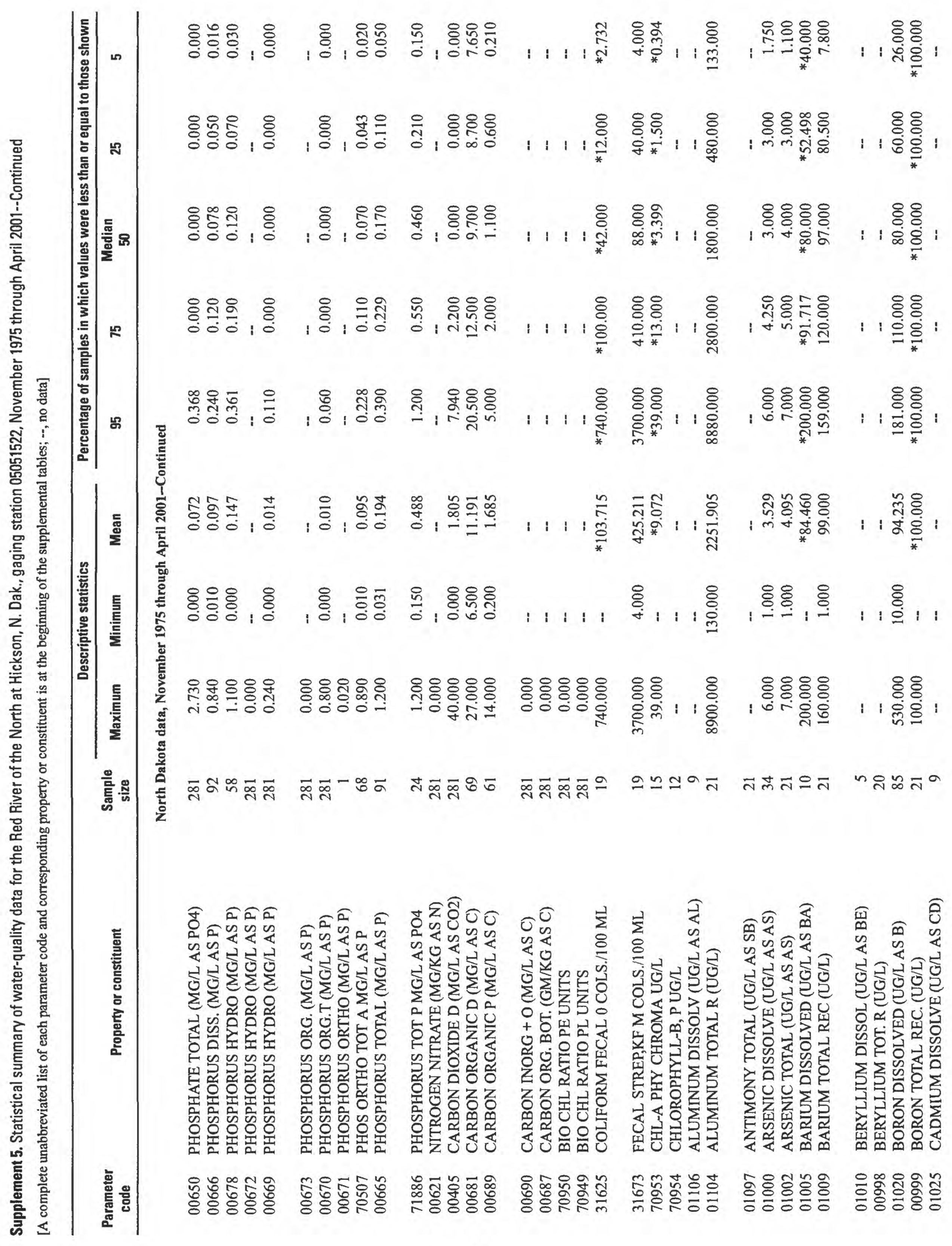




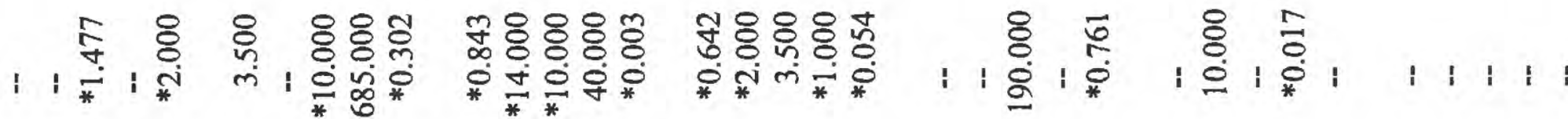

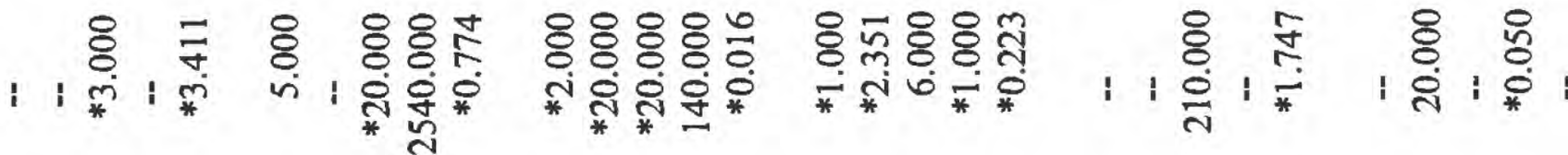

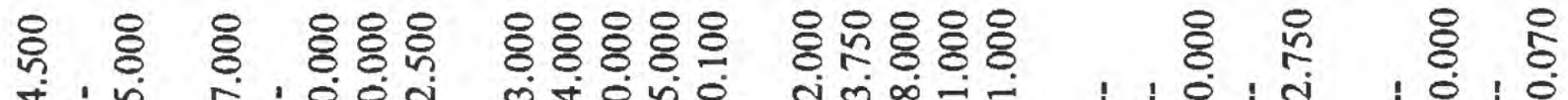

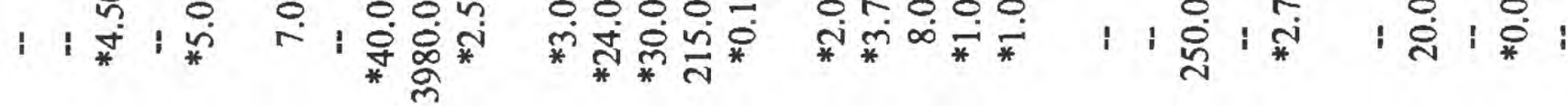

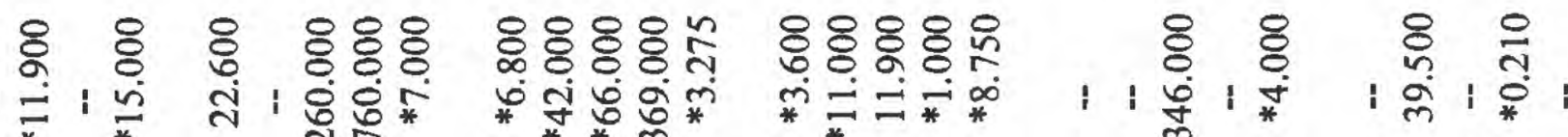
1

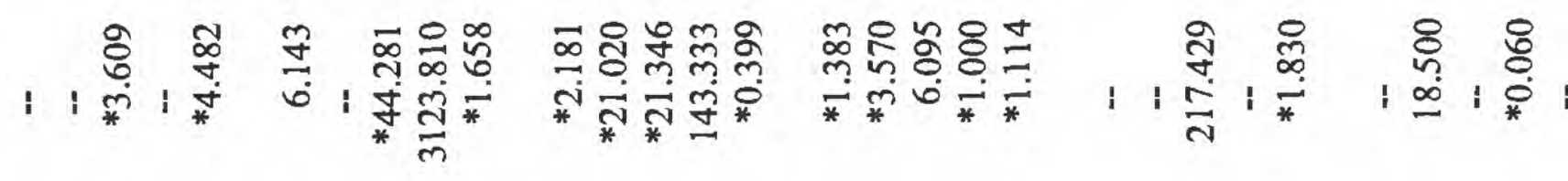
i : 1 :

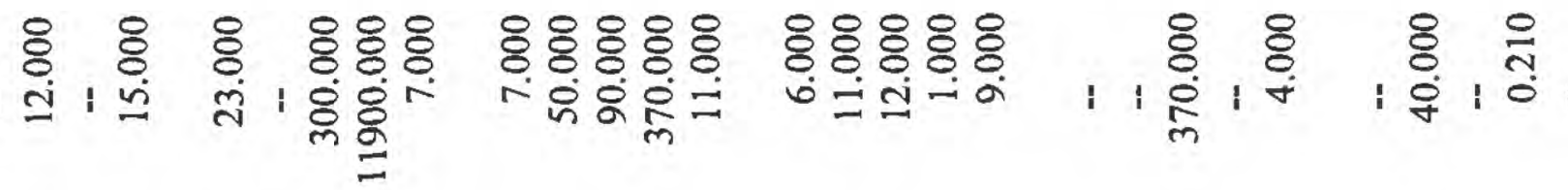

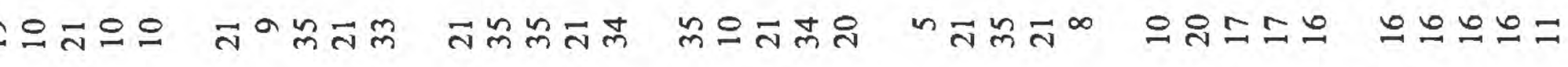
总

ชิ 85

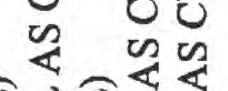

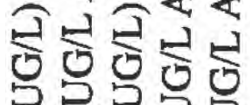
包芑起

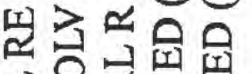
连统完

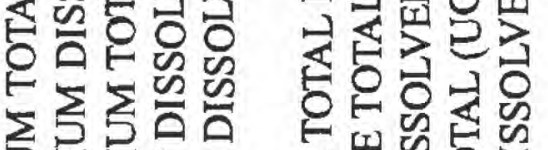

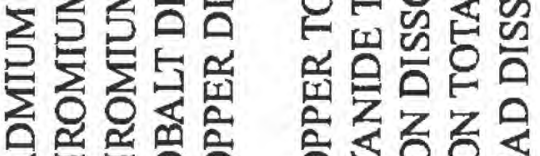

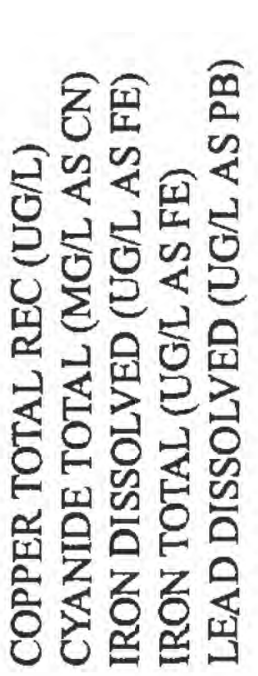

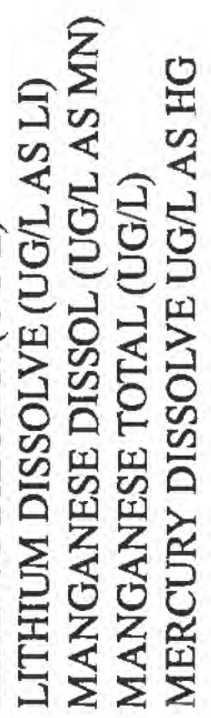

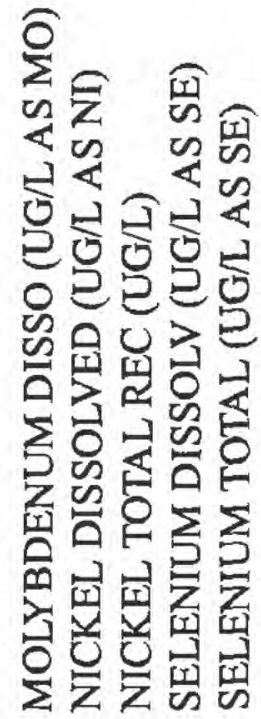

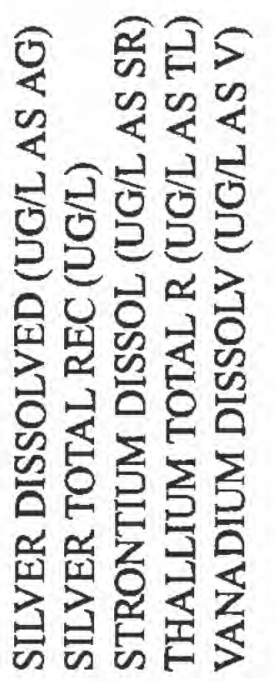
$\sum_{1}$ 넌엉요

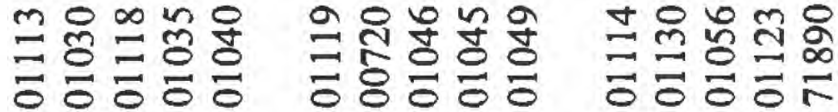

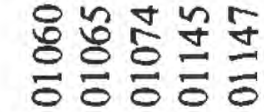
농옹융 웡 응응응 

(1)

\section{列}
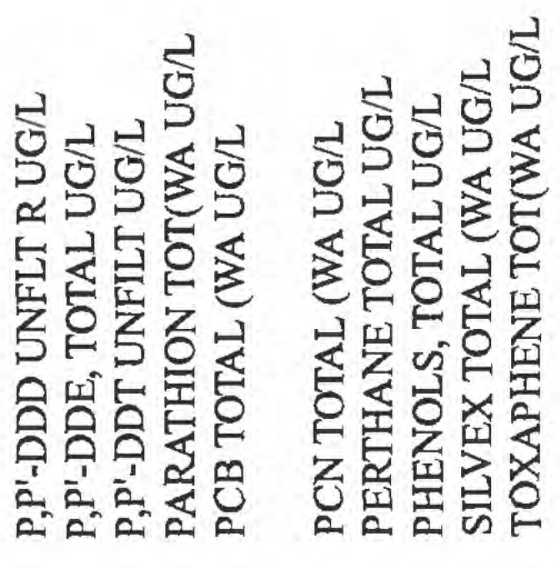

空㔯赑

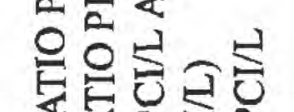

延昰它 뜬대

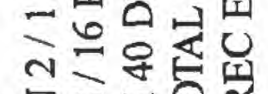

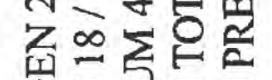

깅존

뭥류

釜是是

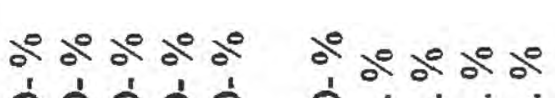

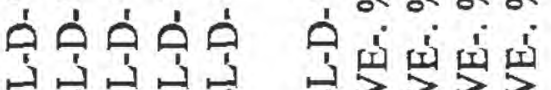

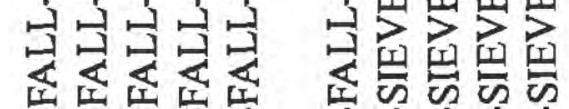

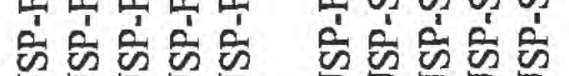

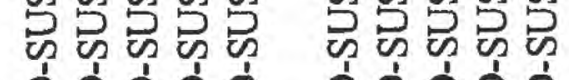

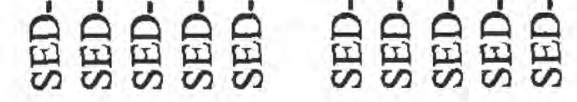

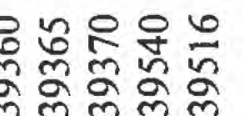

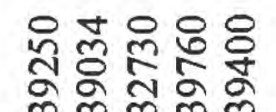

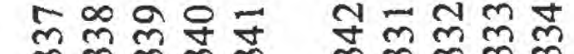
대융ㅇ

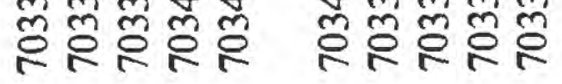




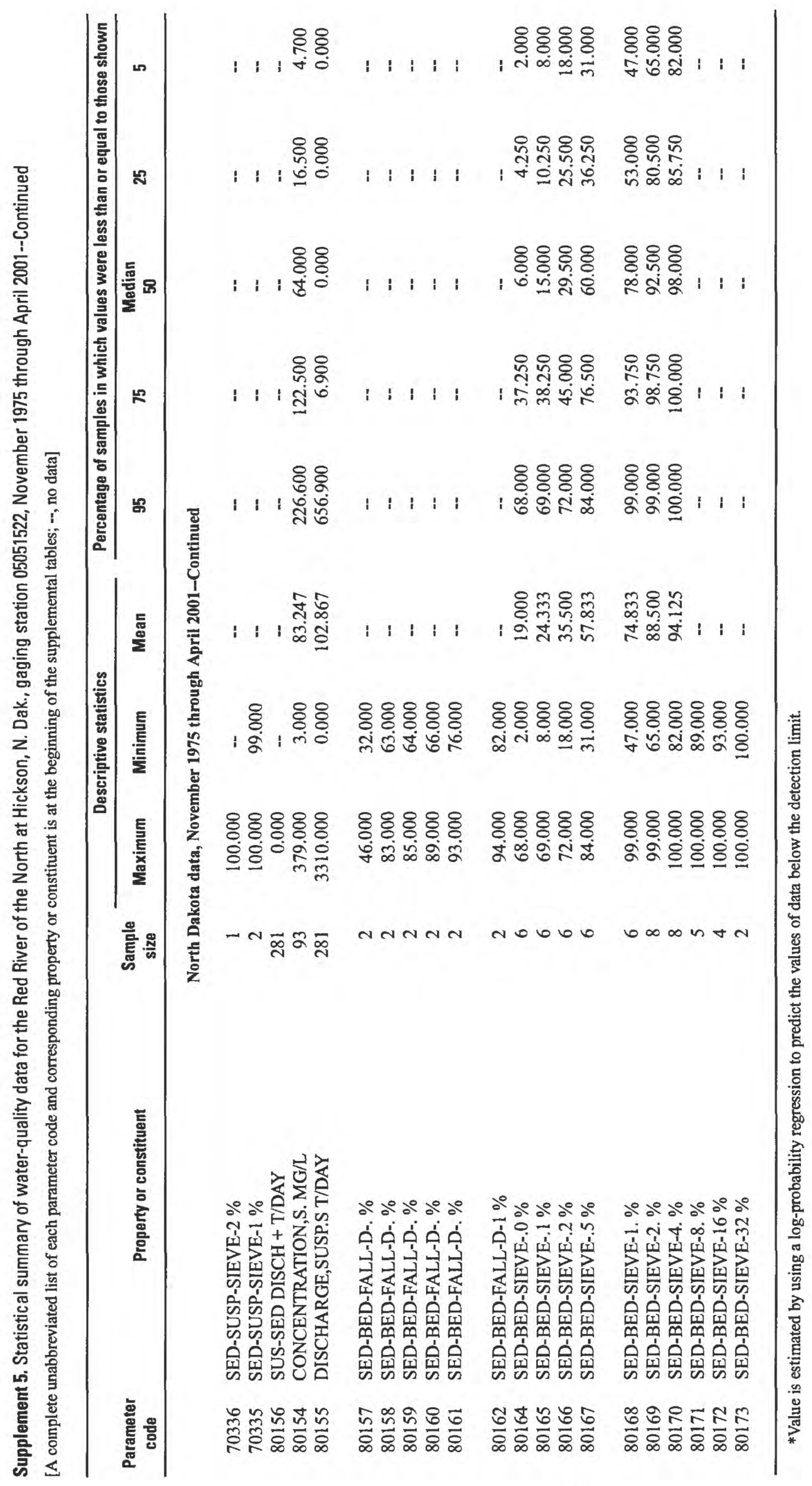




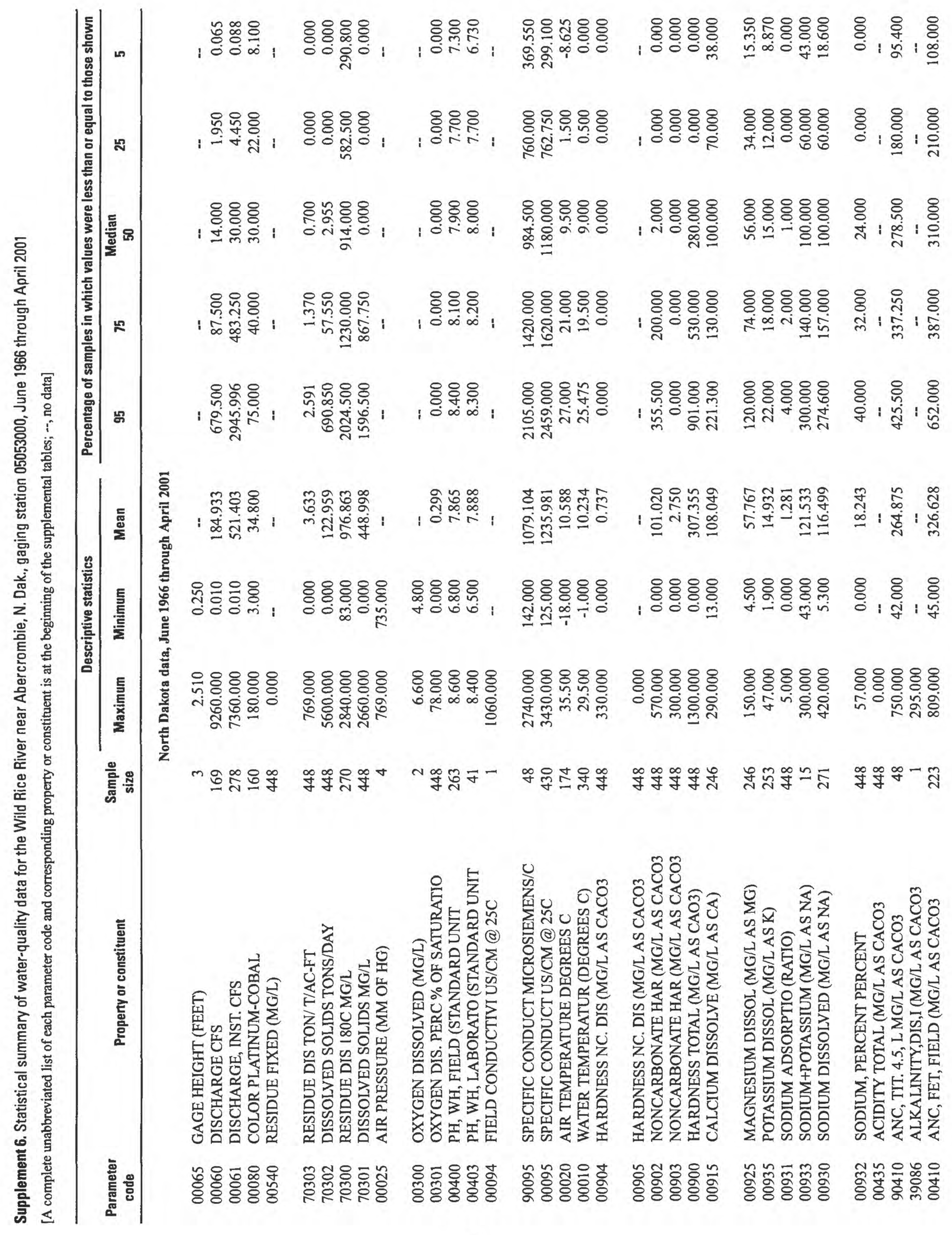




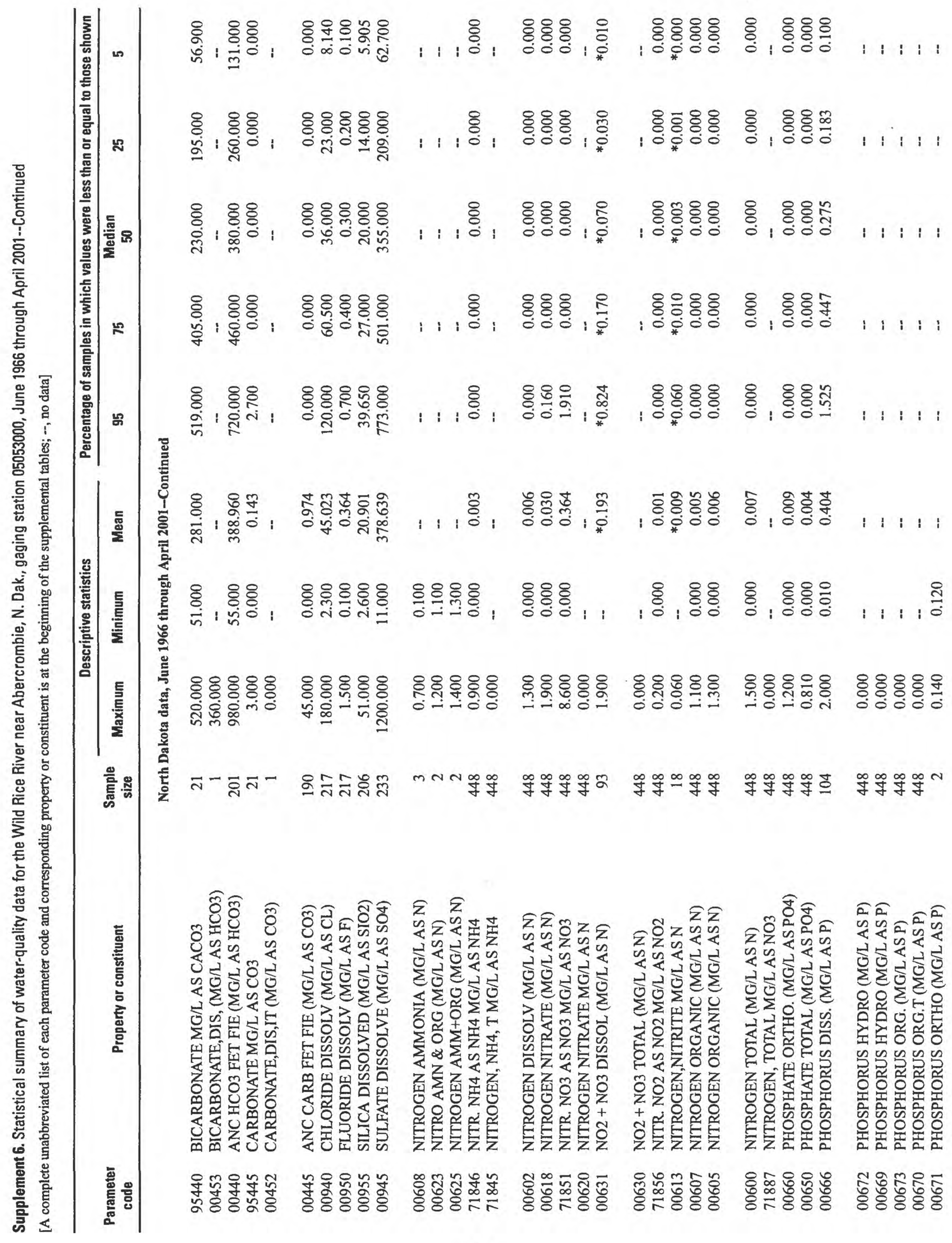




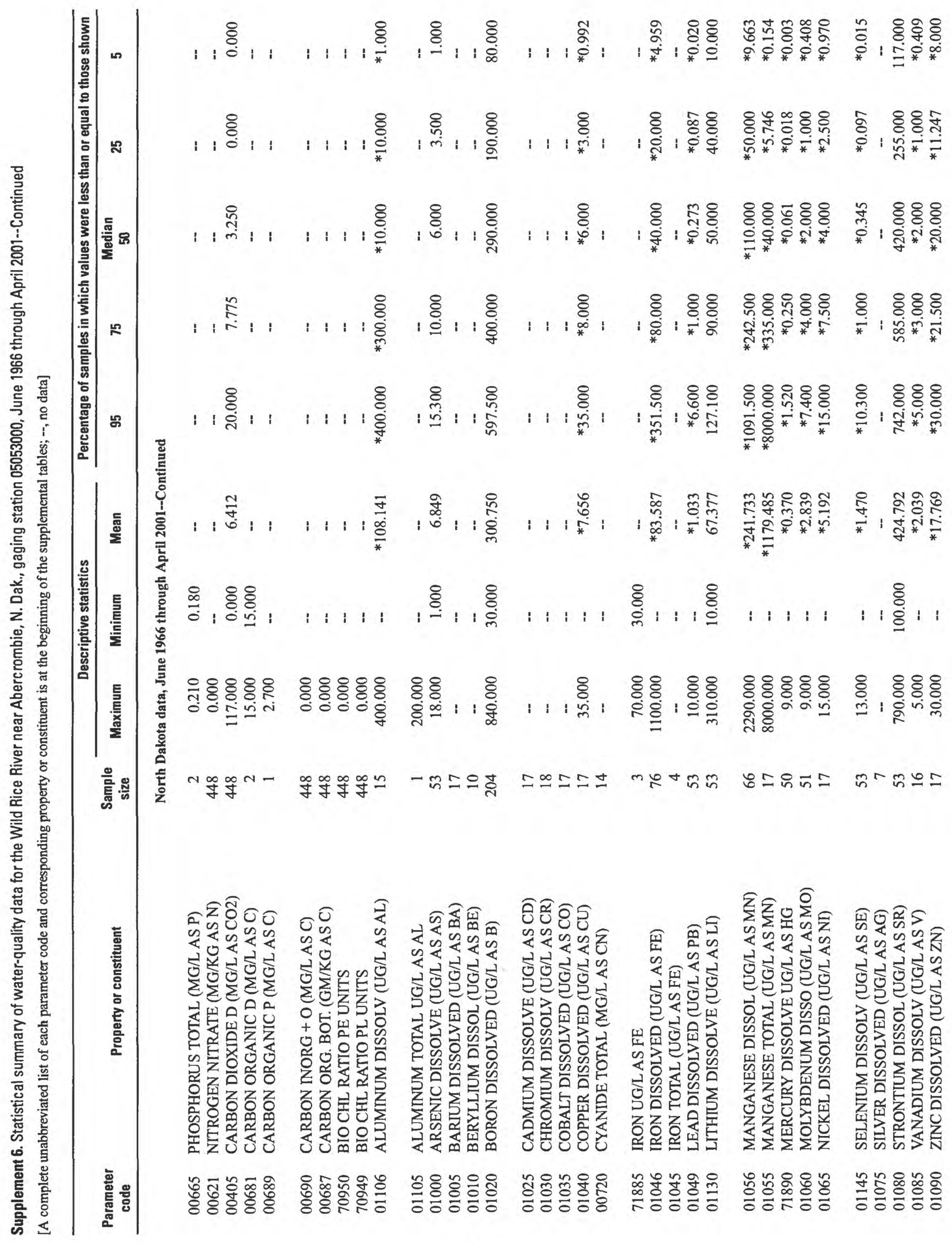




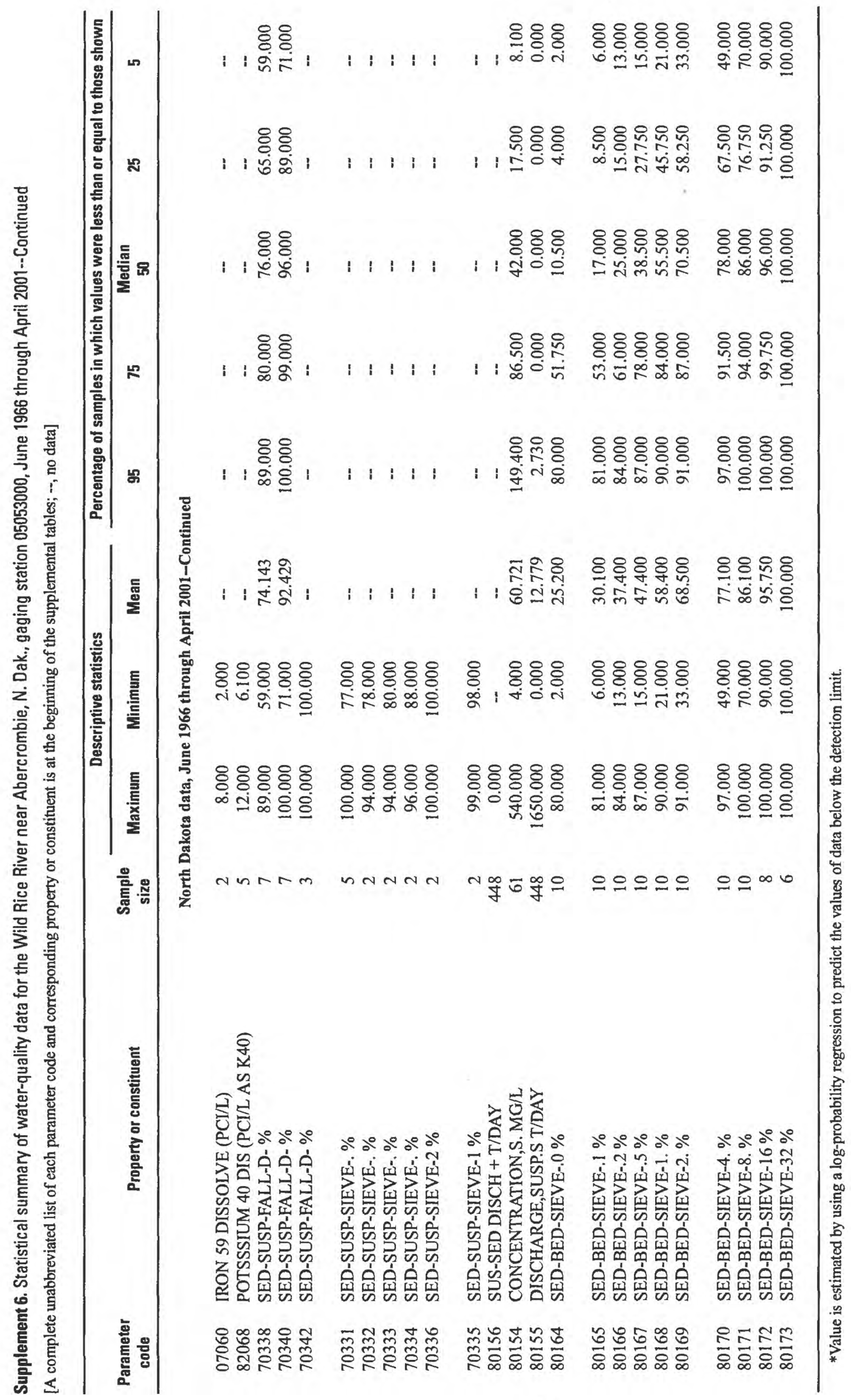




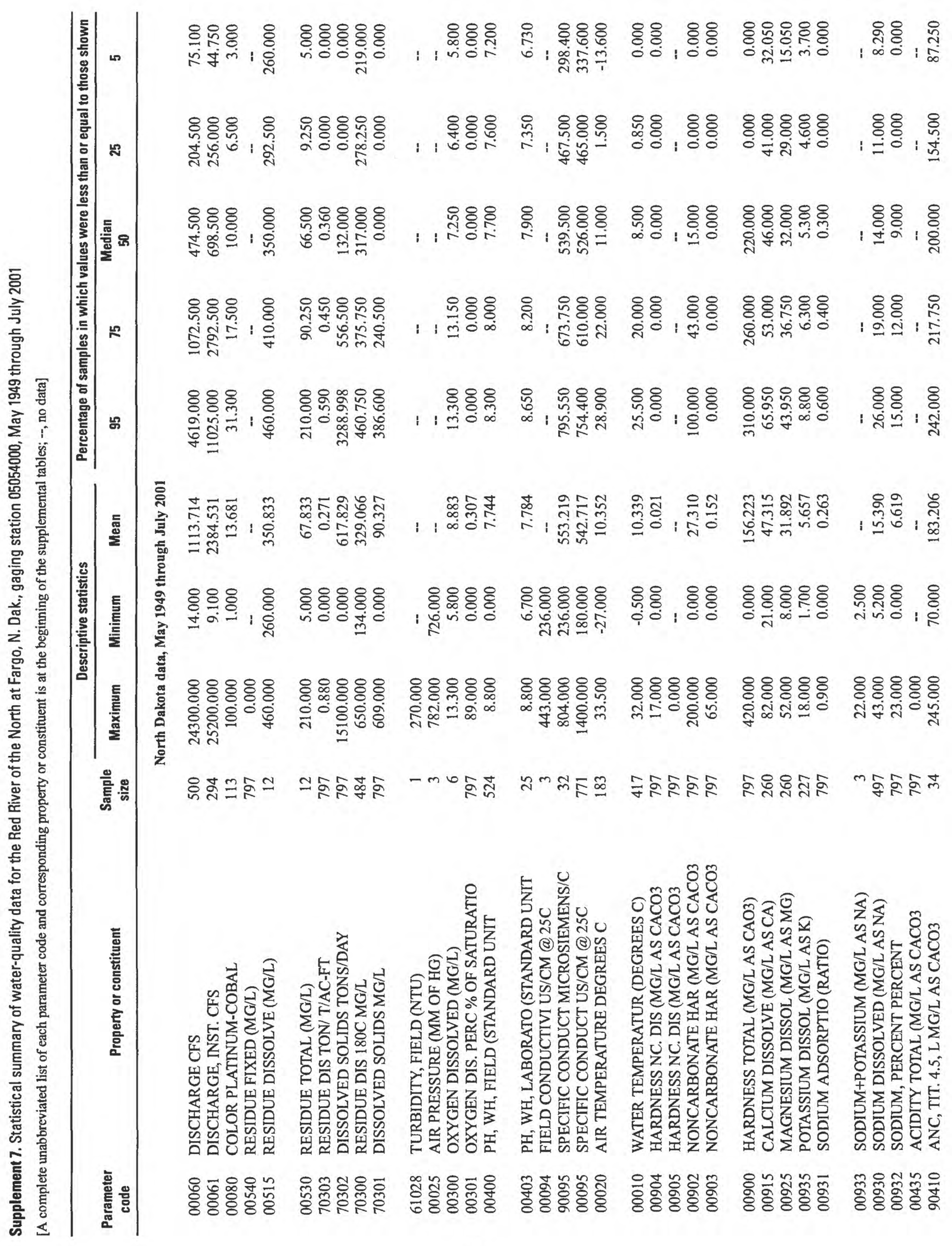




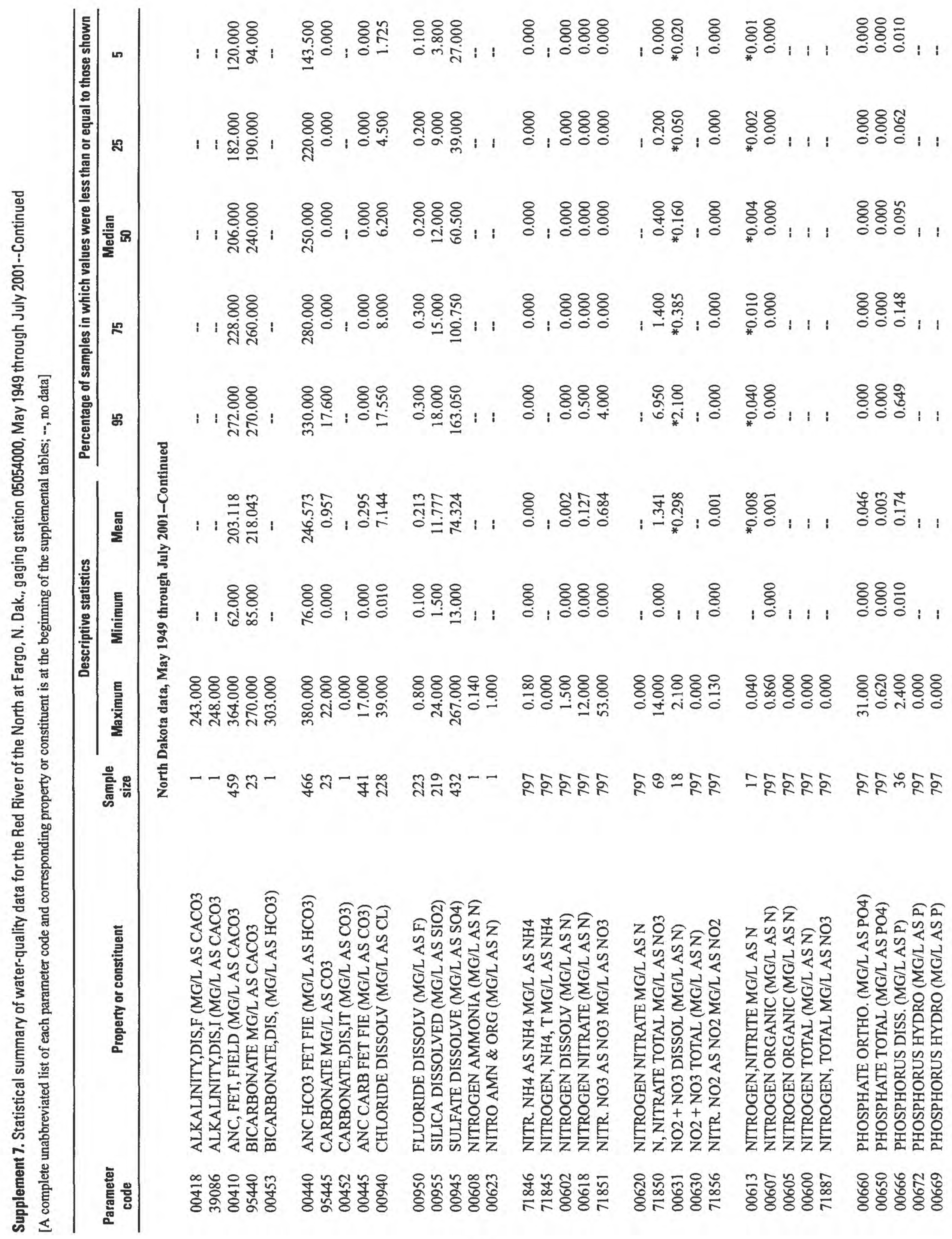




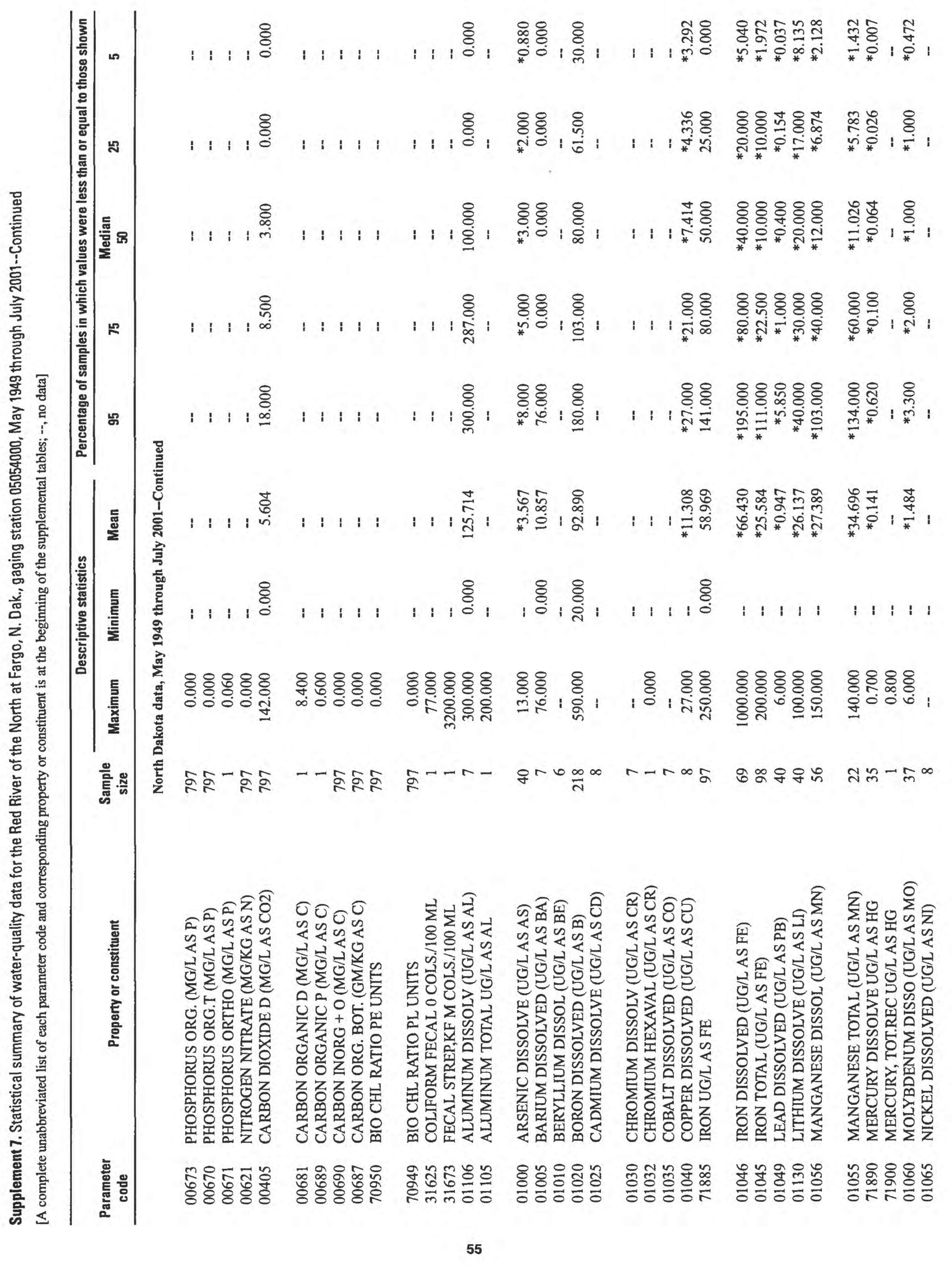




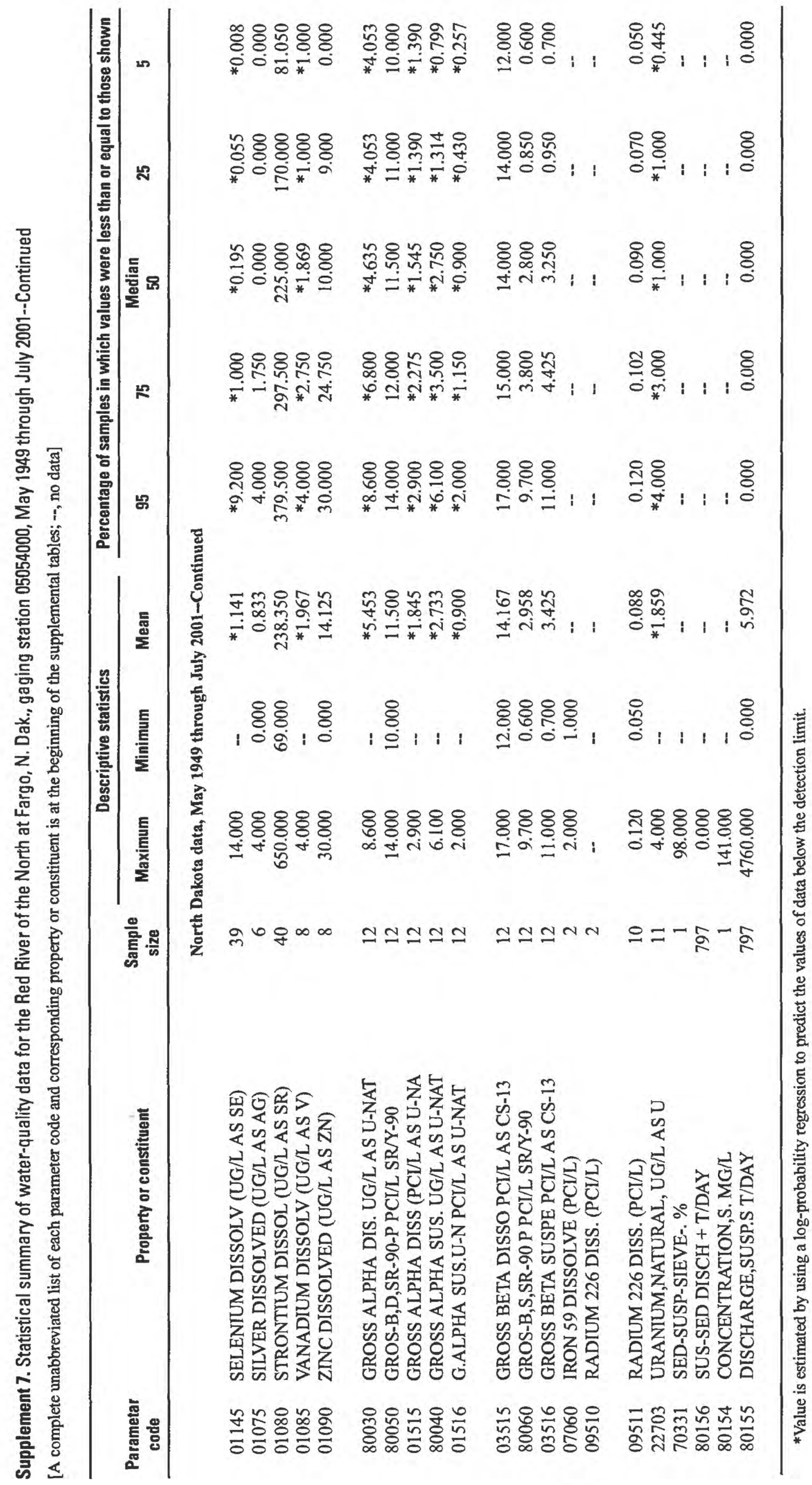




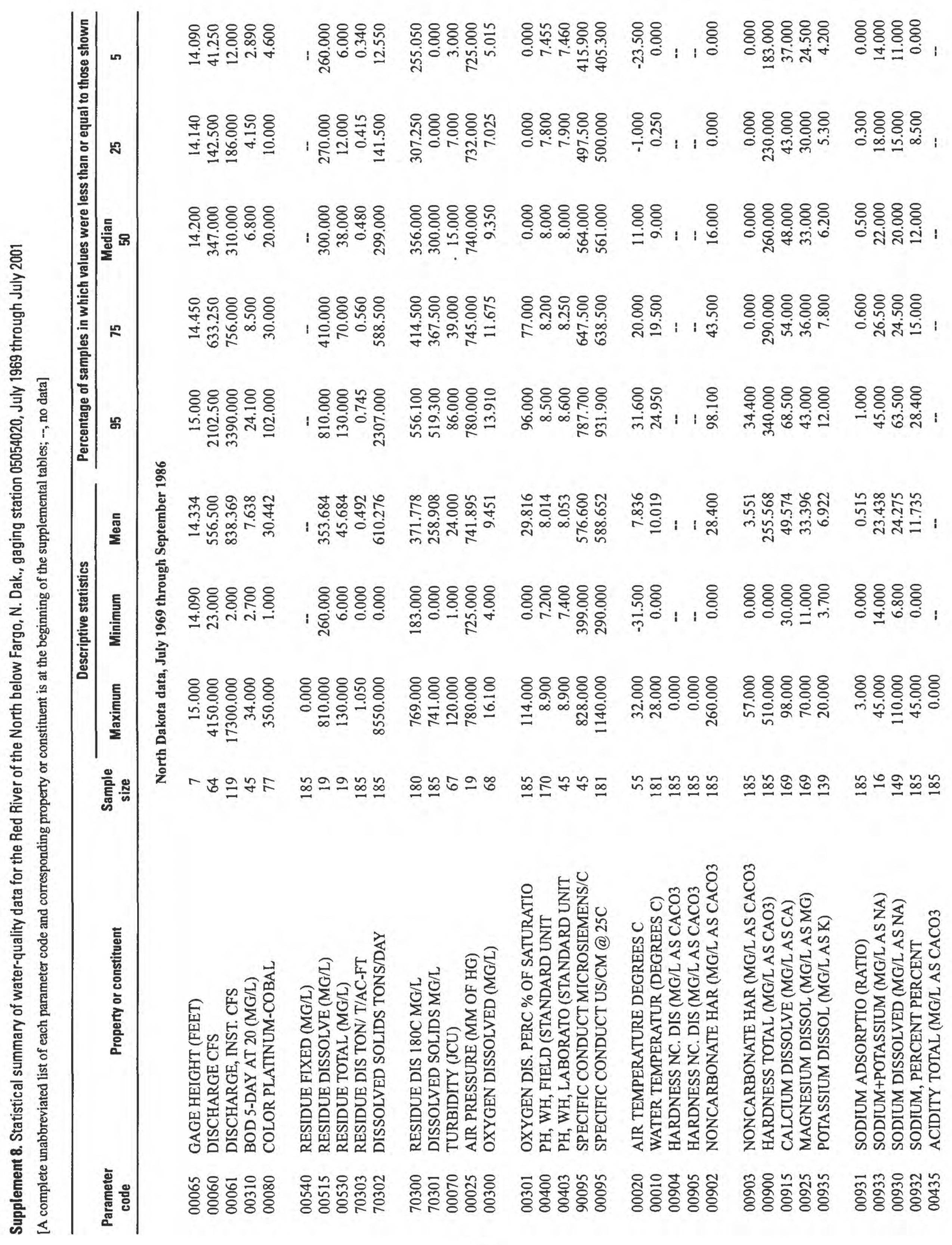




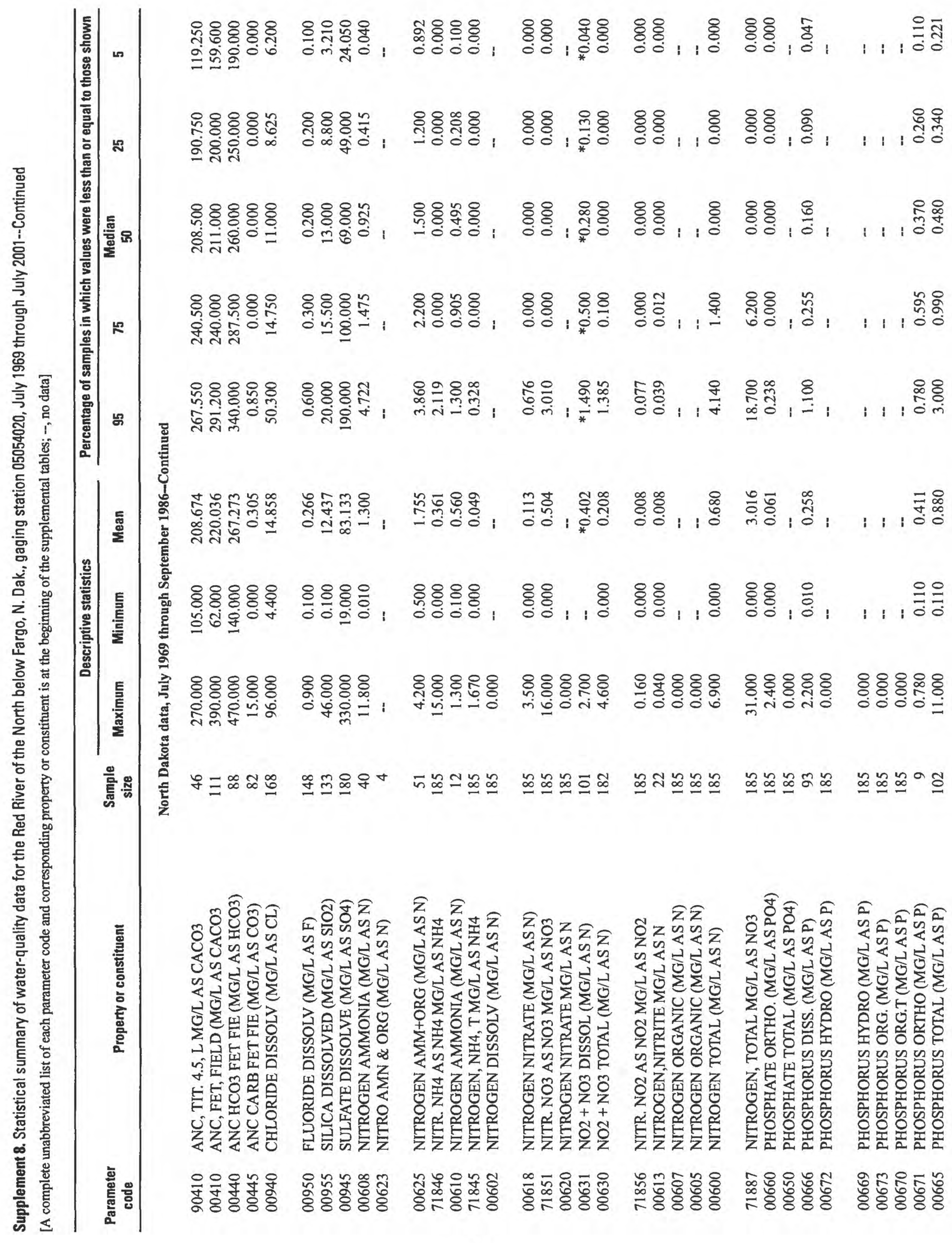




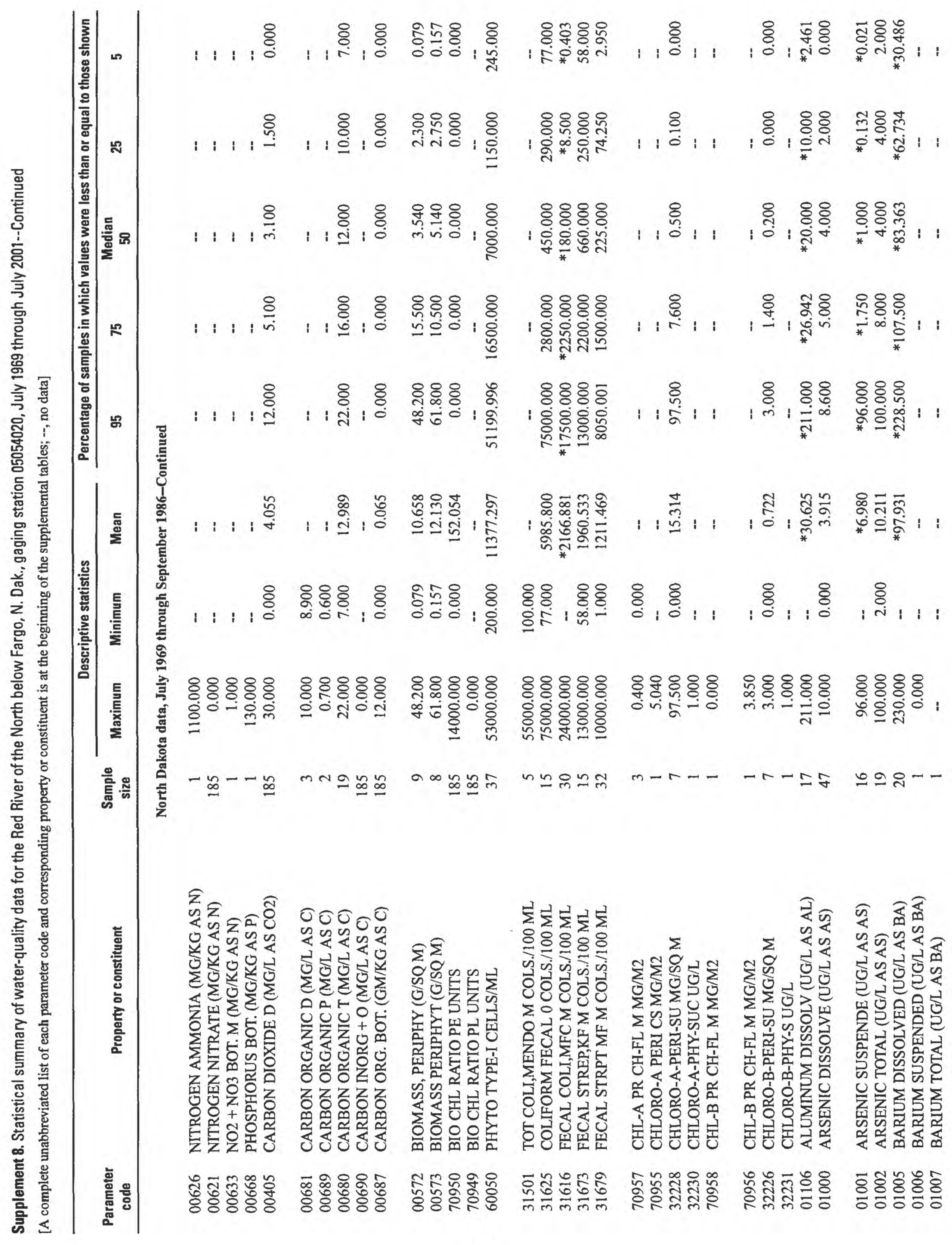




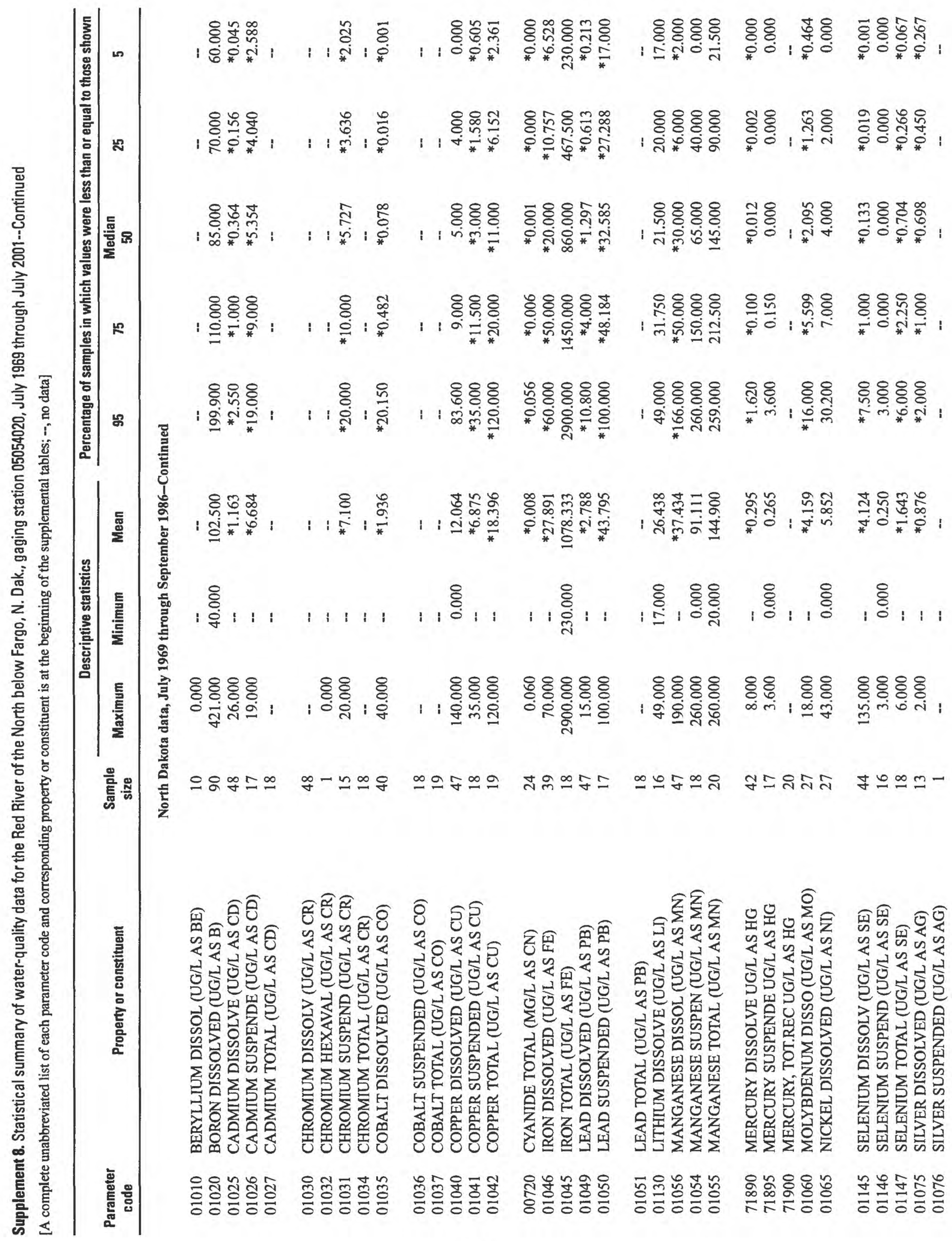




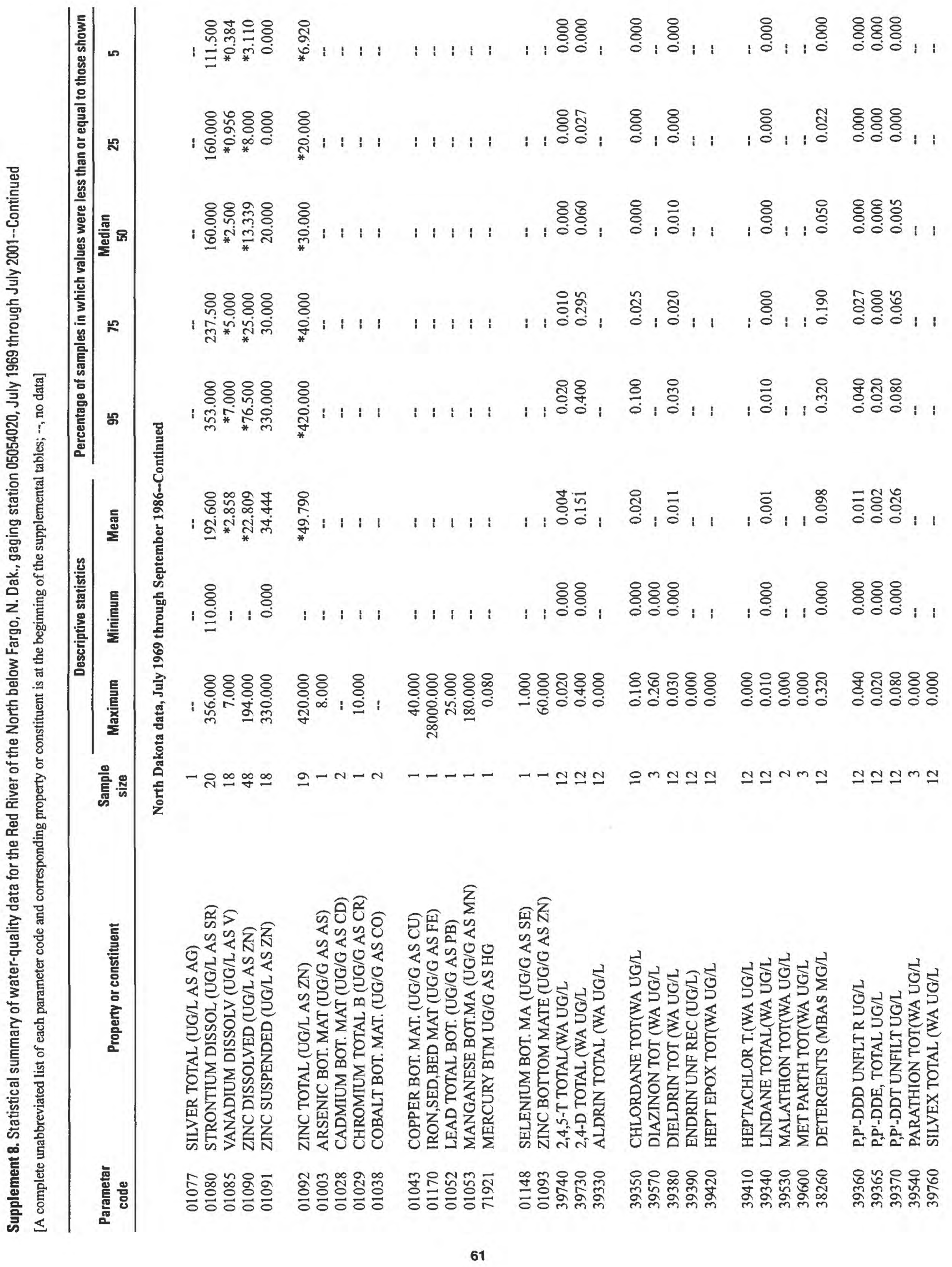




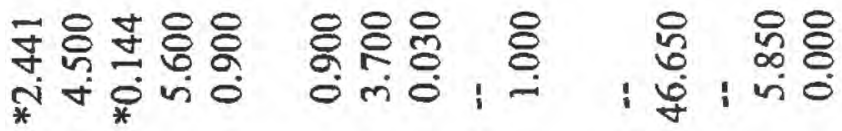

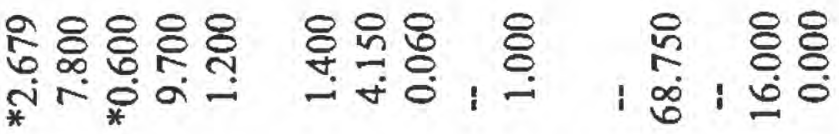

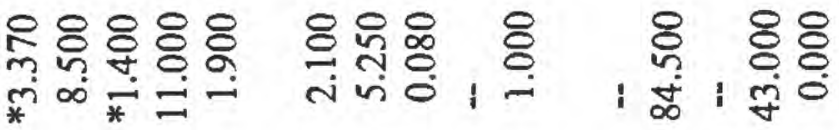

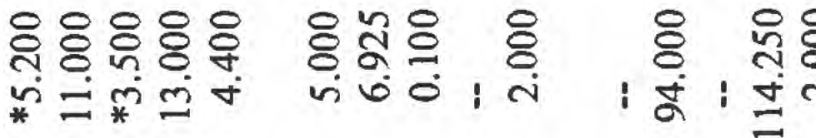

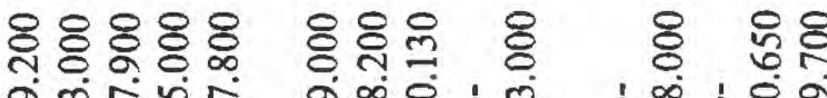

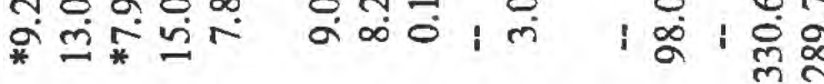

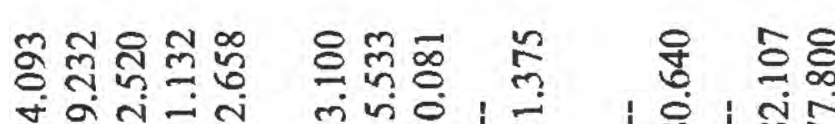

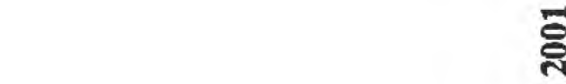

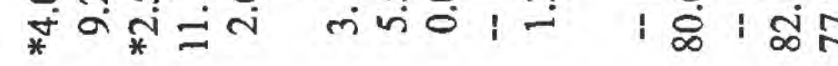

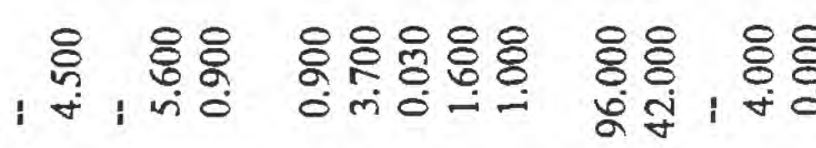

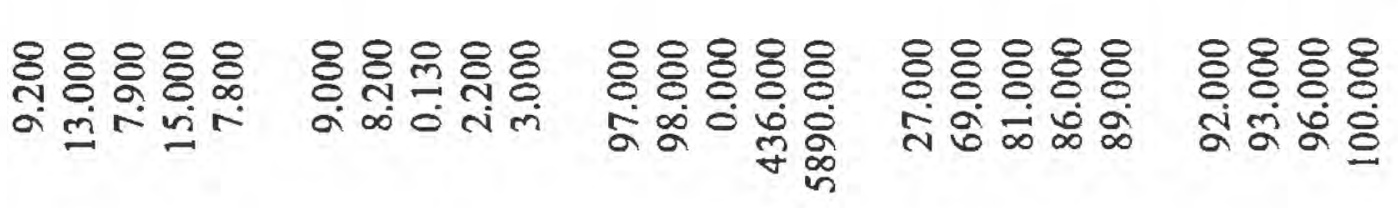

商

i i i i i

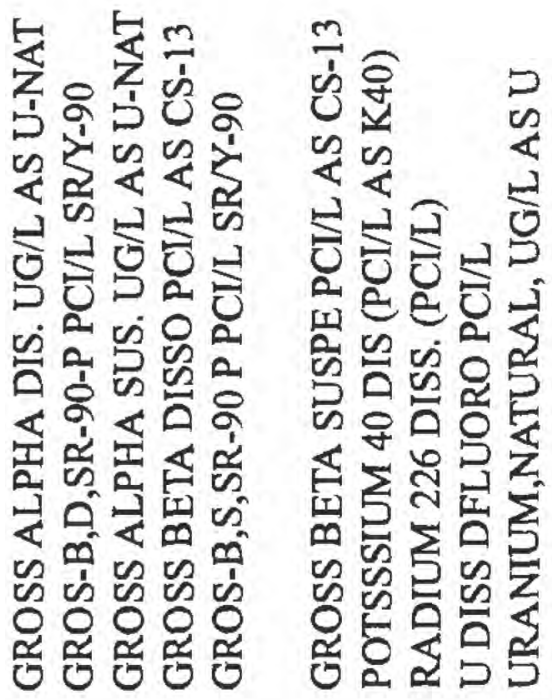

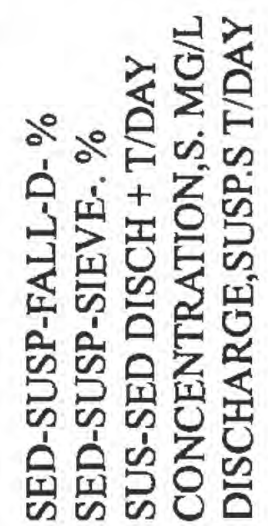

$\therefore \circ \div 50$

1ि官官

这是是是

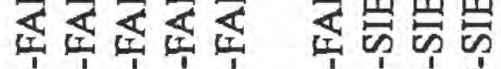

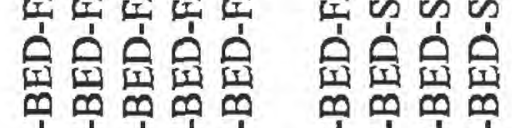

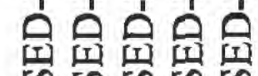

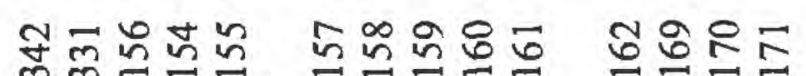

ํㅠㅇㅇㅛ

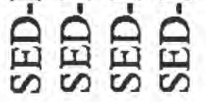

soso a

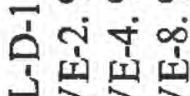

$>>$

車官

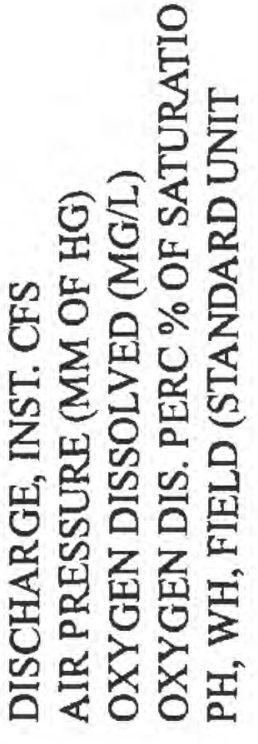

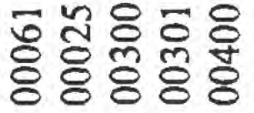




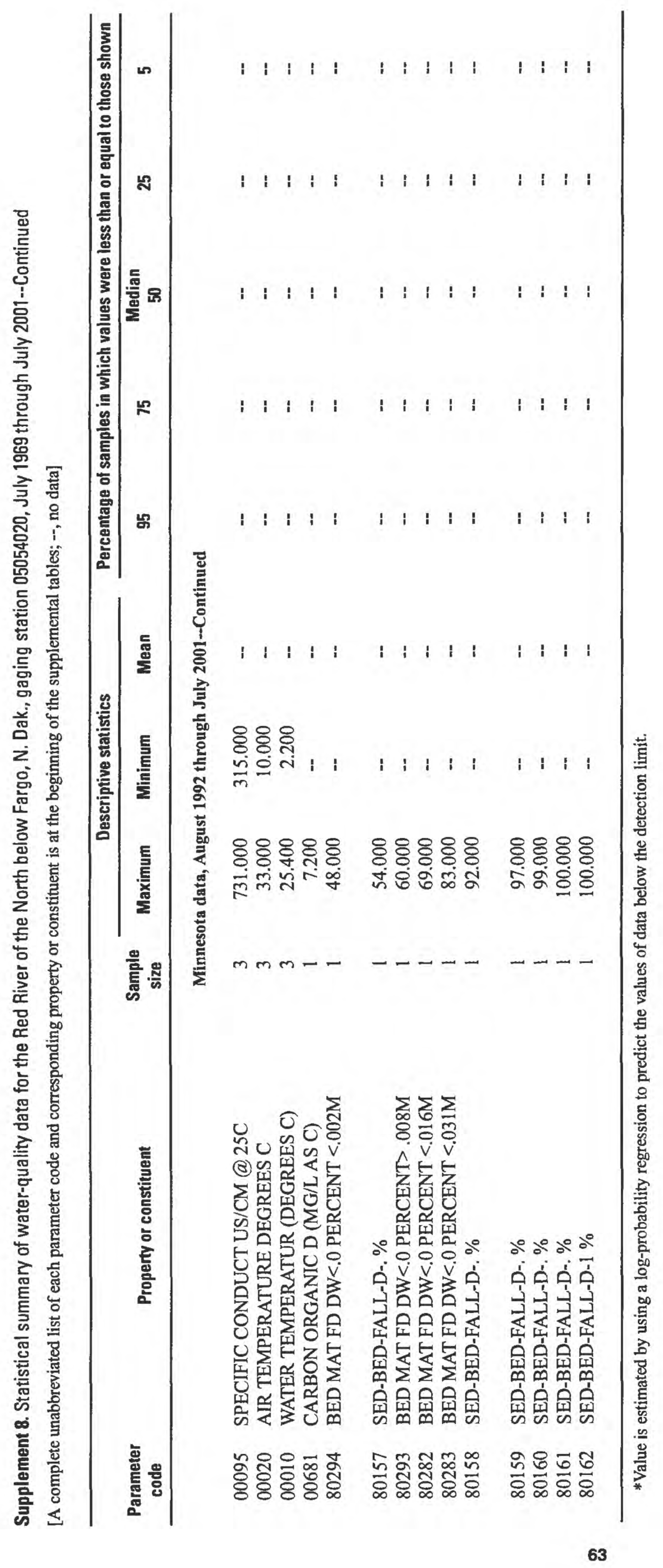




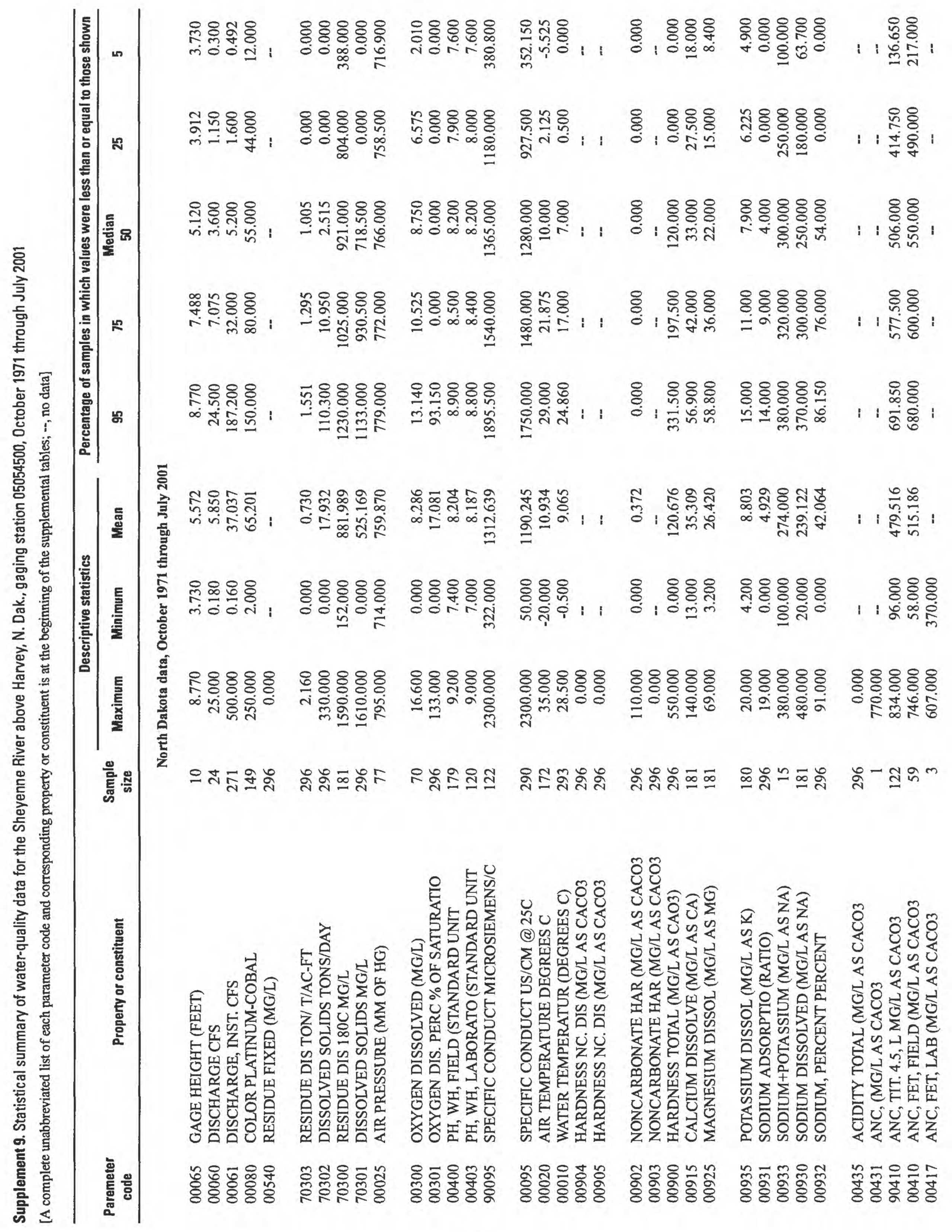




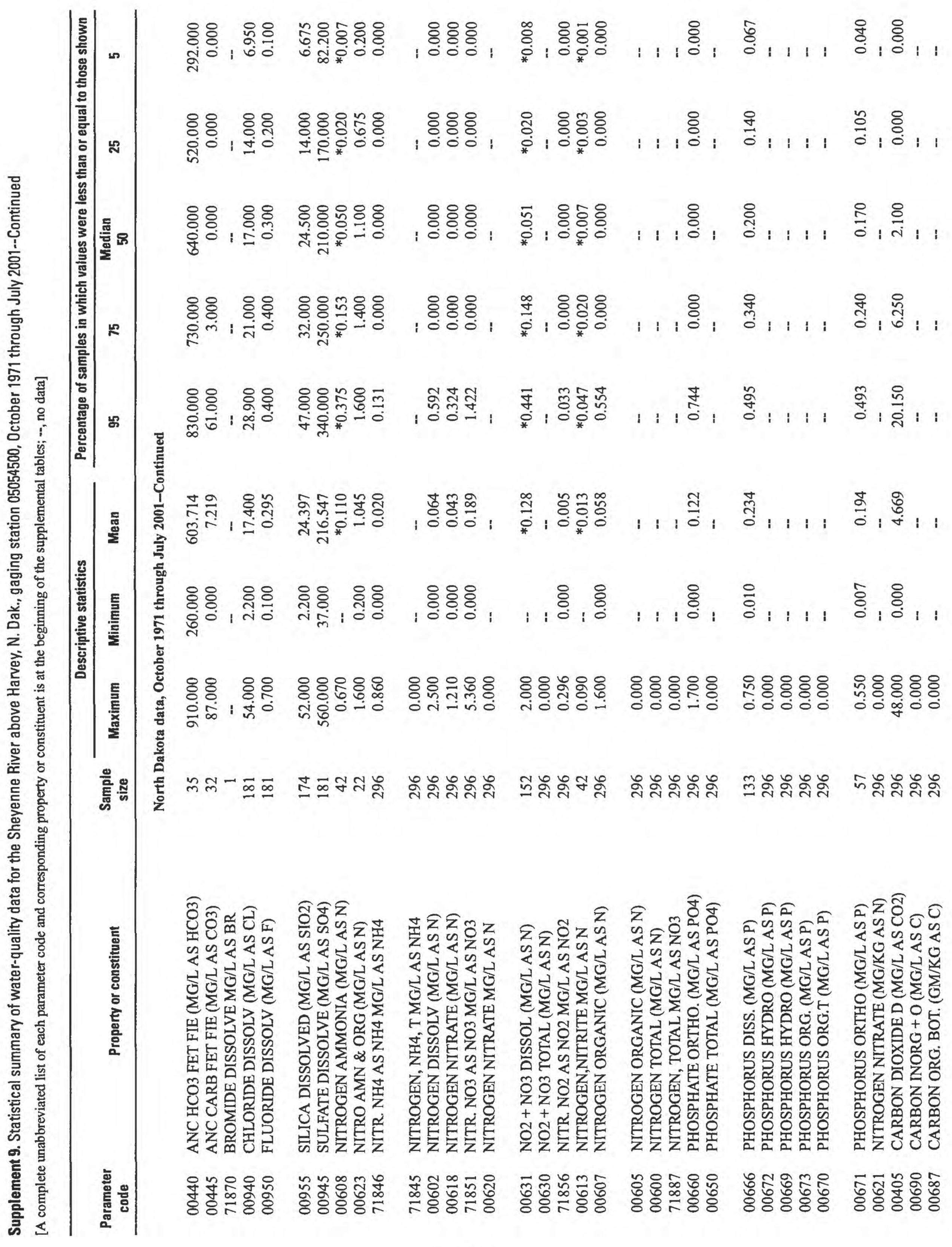




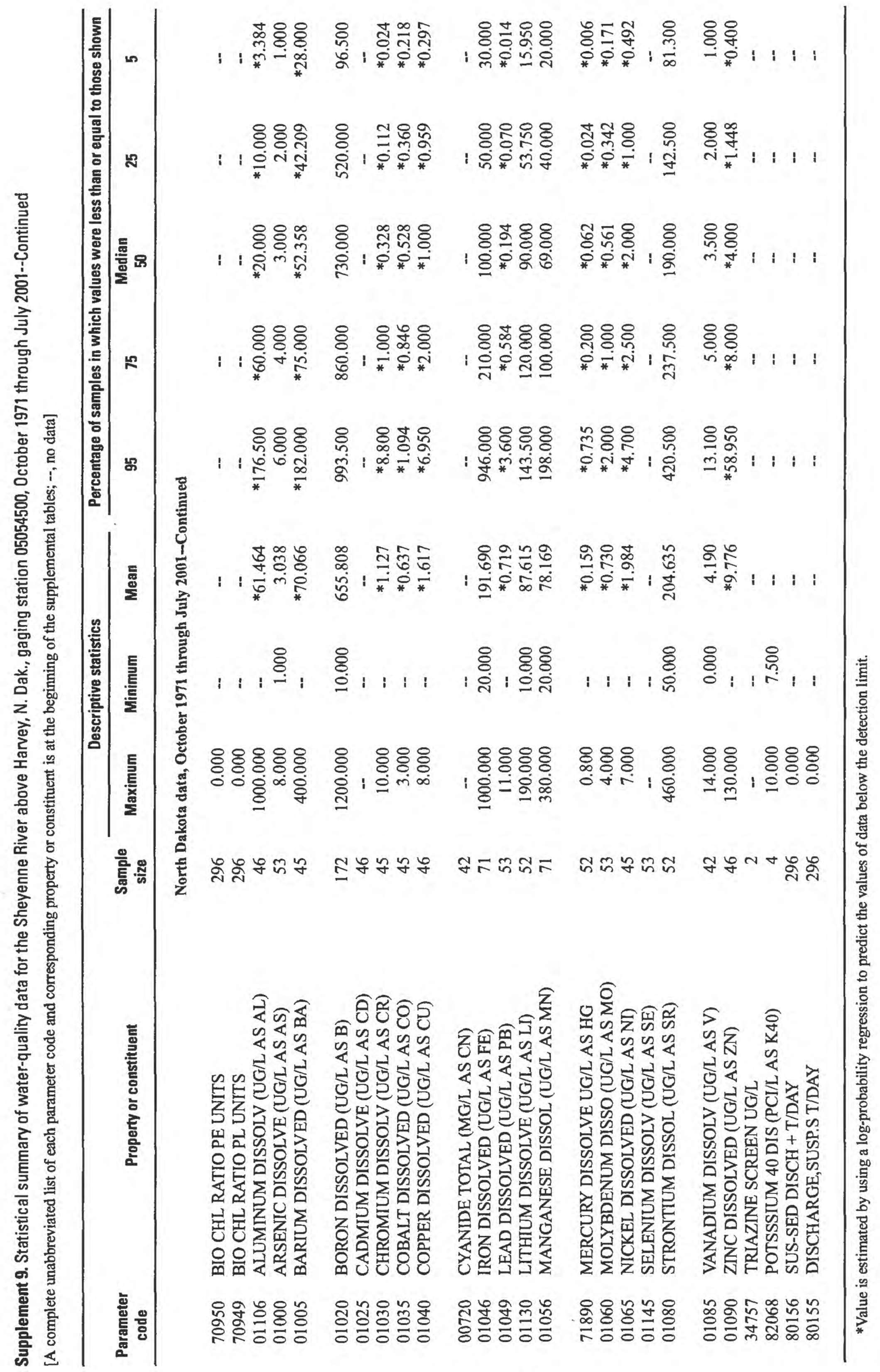




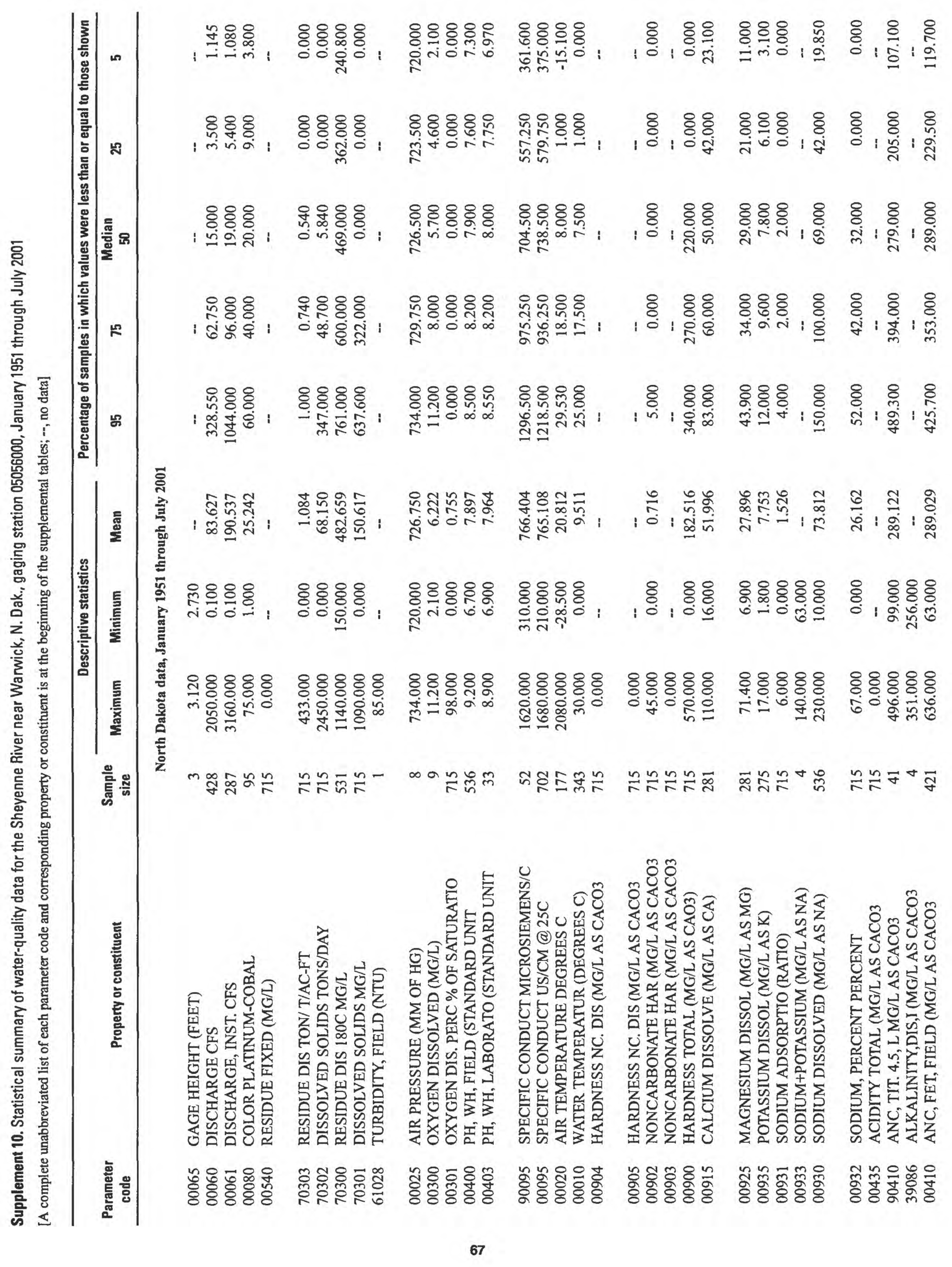




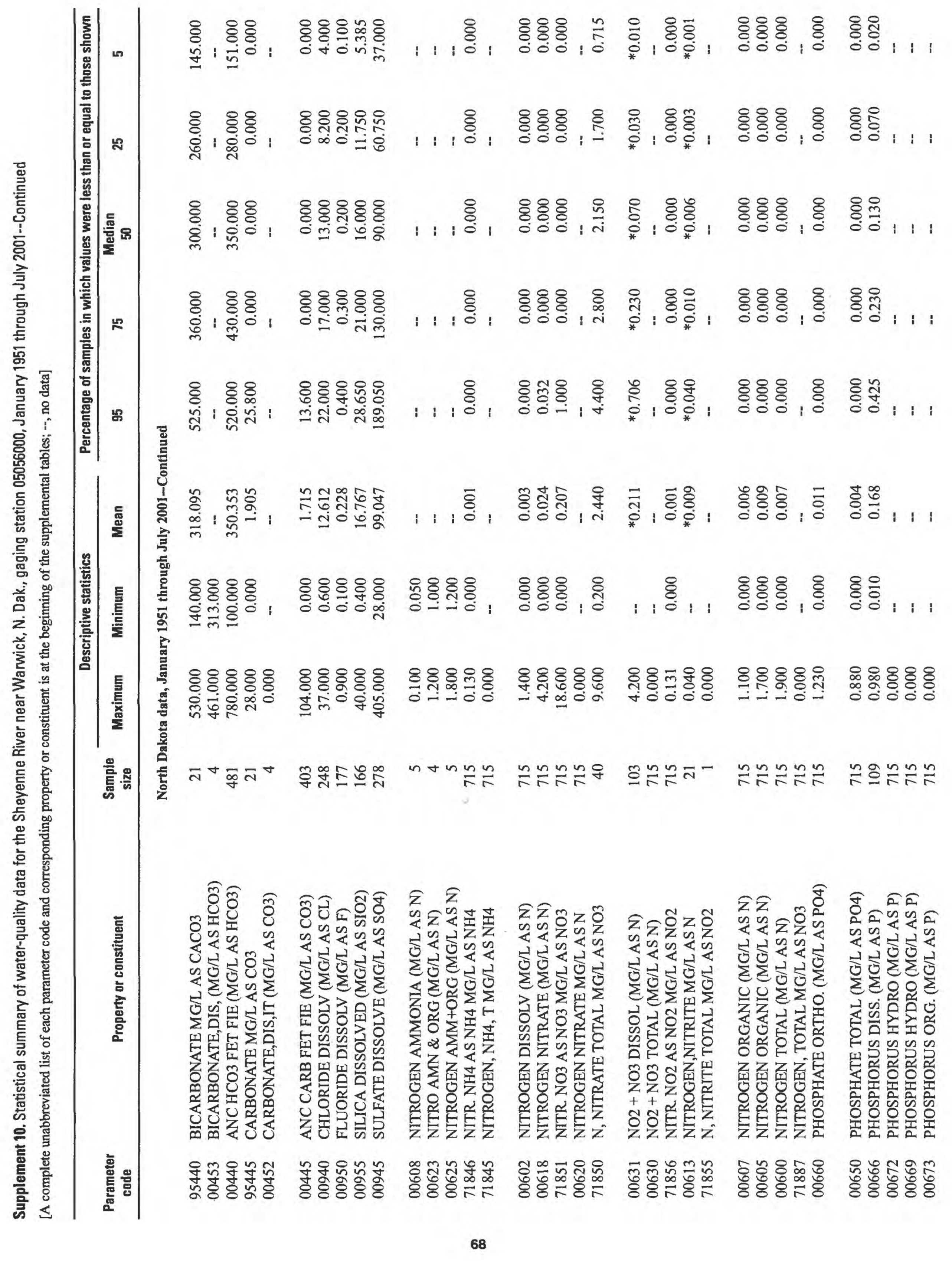




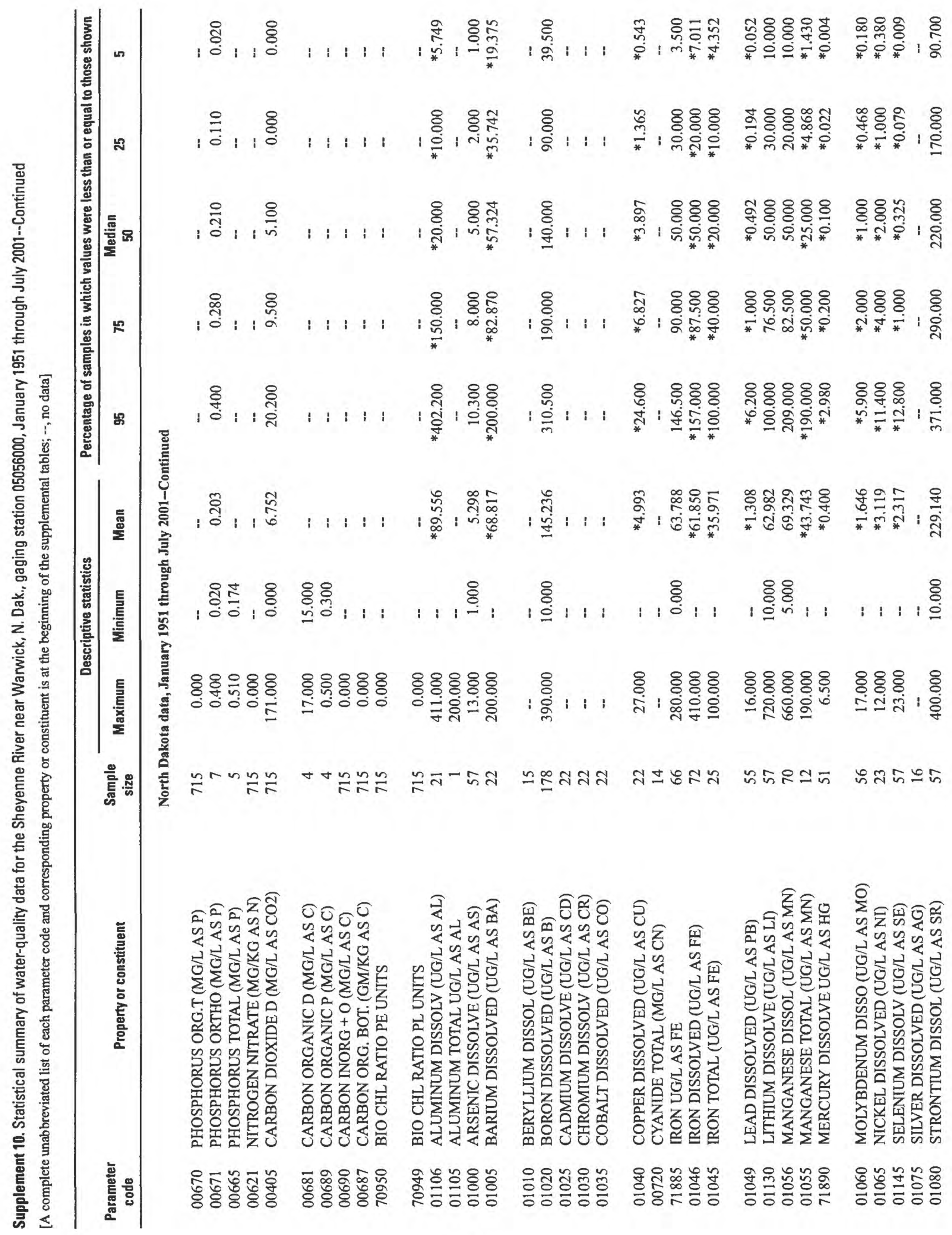




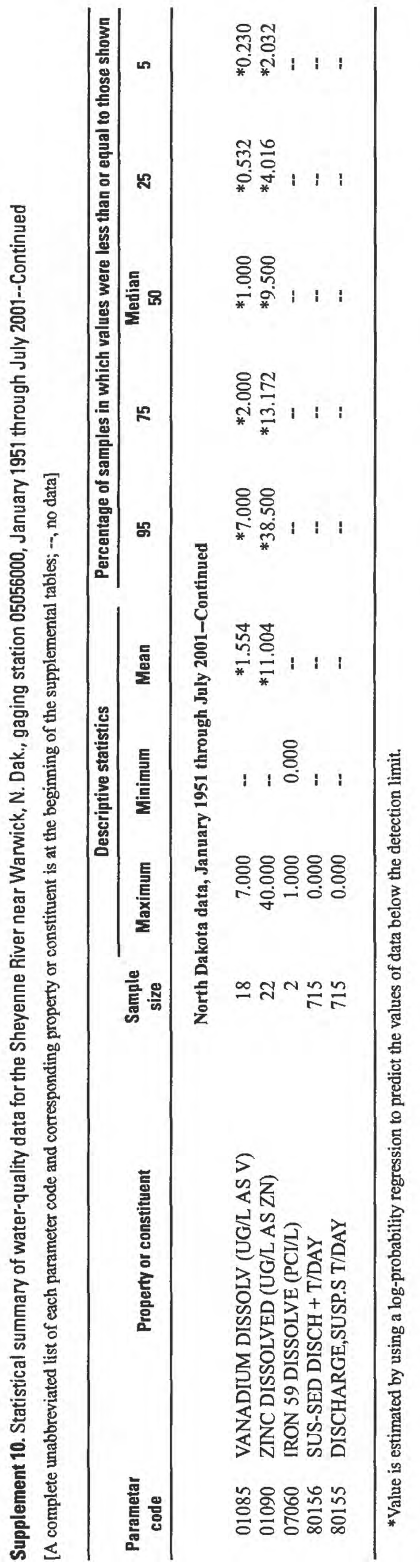




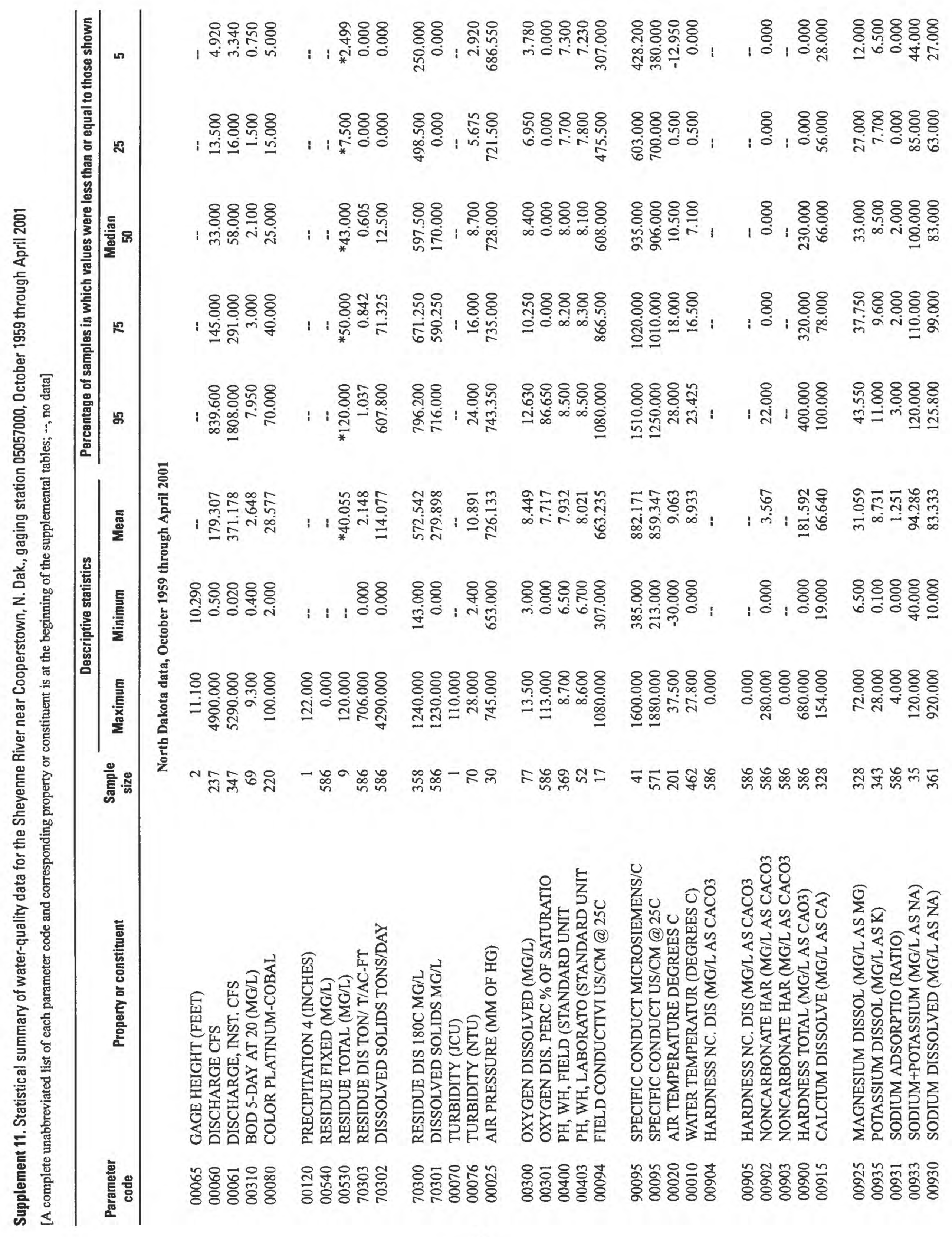




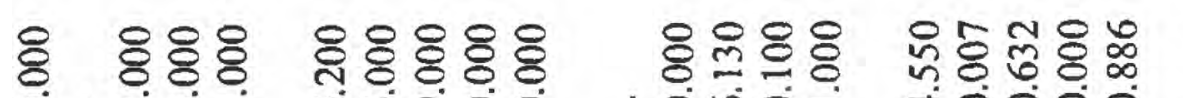

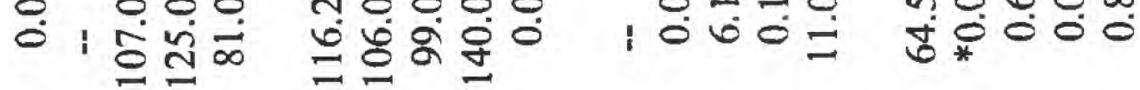

용응융용

용유용 ธิ:

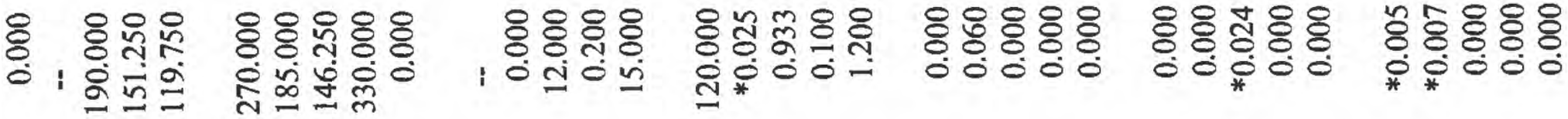

\& $8: 8$

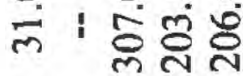

$8080 \%$

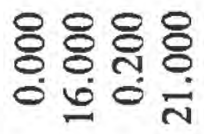

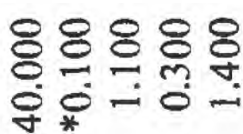

융ํㅇㅇㅇㅇㅇ

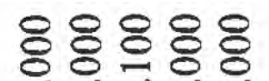

응 융융유.

$\circ 8$ ले तु

लं

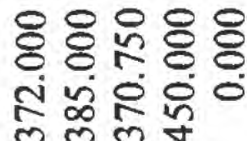

양유요

ธิํำำํำ

ㅇํㅇ융용

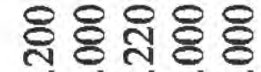

웅-

0000

00 \%

응 훙휴용

* 000

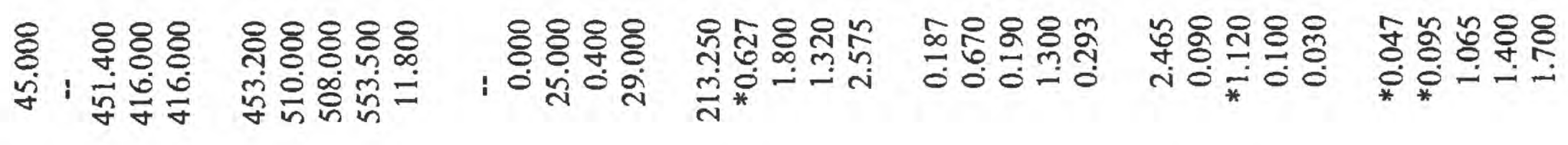

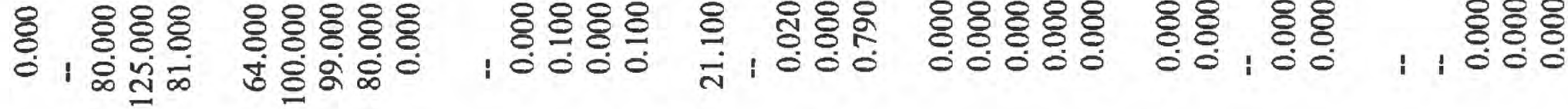

$\infty$ ิํㅇ 8

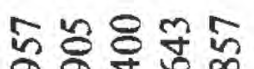

충ำ

뜽ํㅇำ

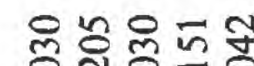

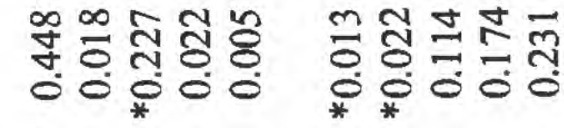
สं่ कूण

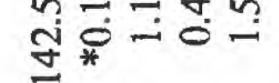
잉ㅇㅇ

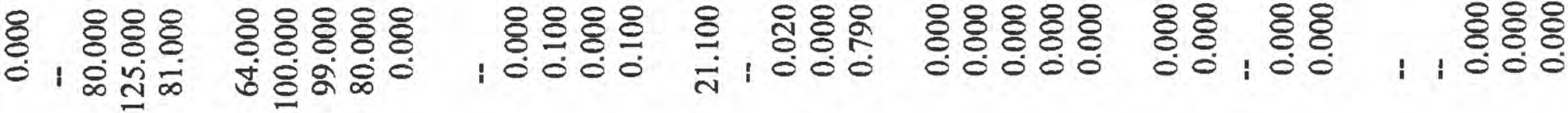

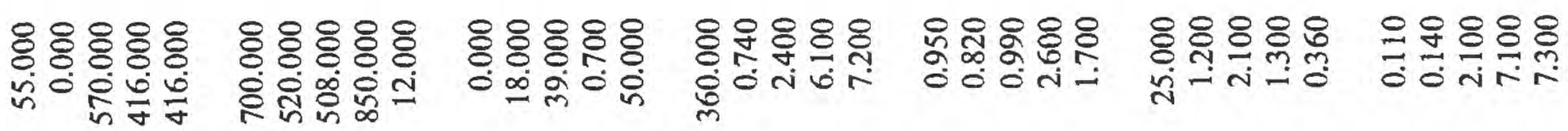

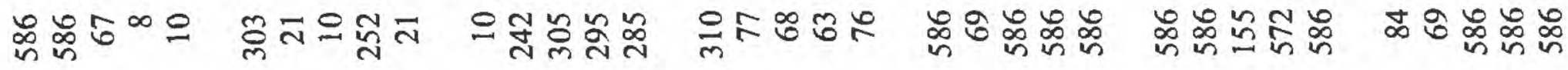

ఇ:

至论得罂

证牙

정

ज代远

之运出

记

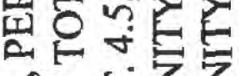

他它

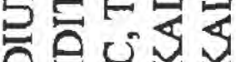

碞安选

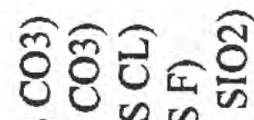

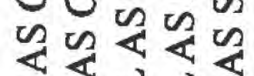

넝성어ㅇㅝㅓㅇ

$\sum \sum \sum \sum$

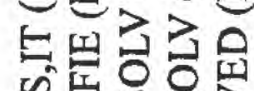

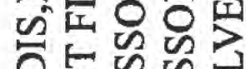

뙤

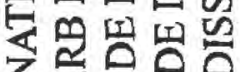

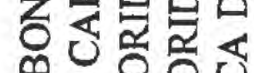

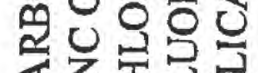

过年青届昆

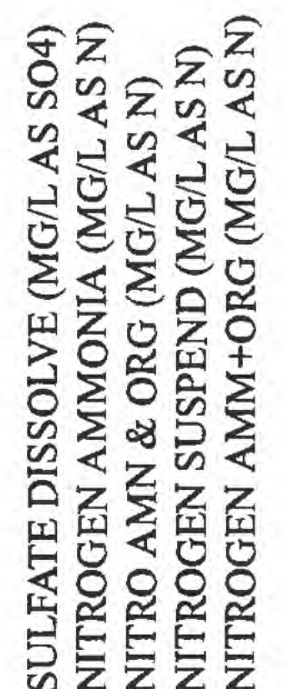

究运云

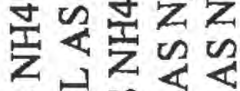

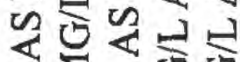

हु일

$\sum \leqslant \Sigma>$ 띠

$\forall$ 낭

z进员

《<得

可面廷要

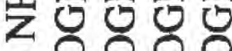

논

点言最

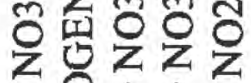

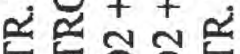

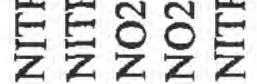

象管영영

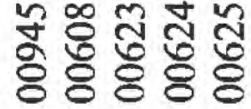

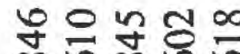

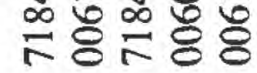

చెิ $\overline{\mathrm{n}}$

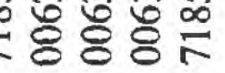

正

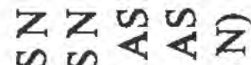

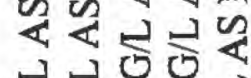
엉엉로옹 $\sum \sum_{1=1} U$

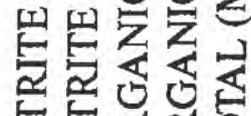
톤융

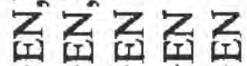
넝엉용

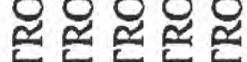
気武侌

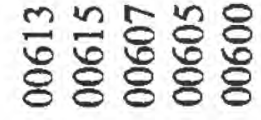




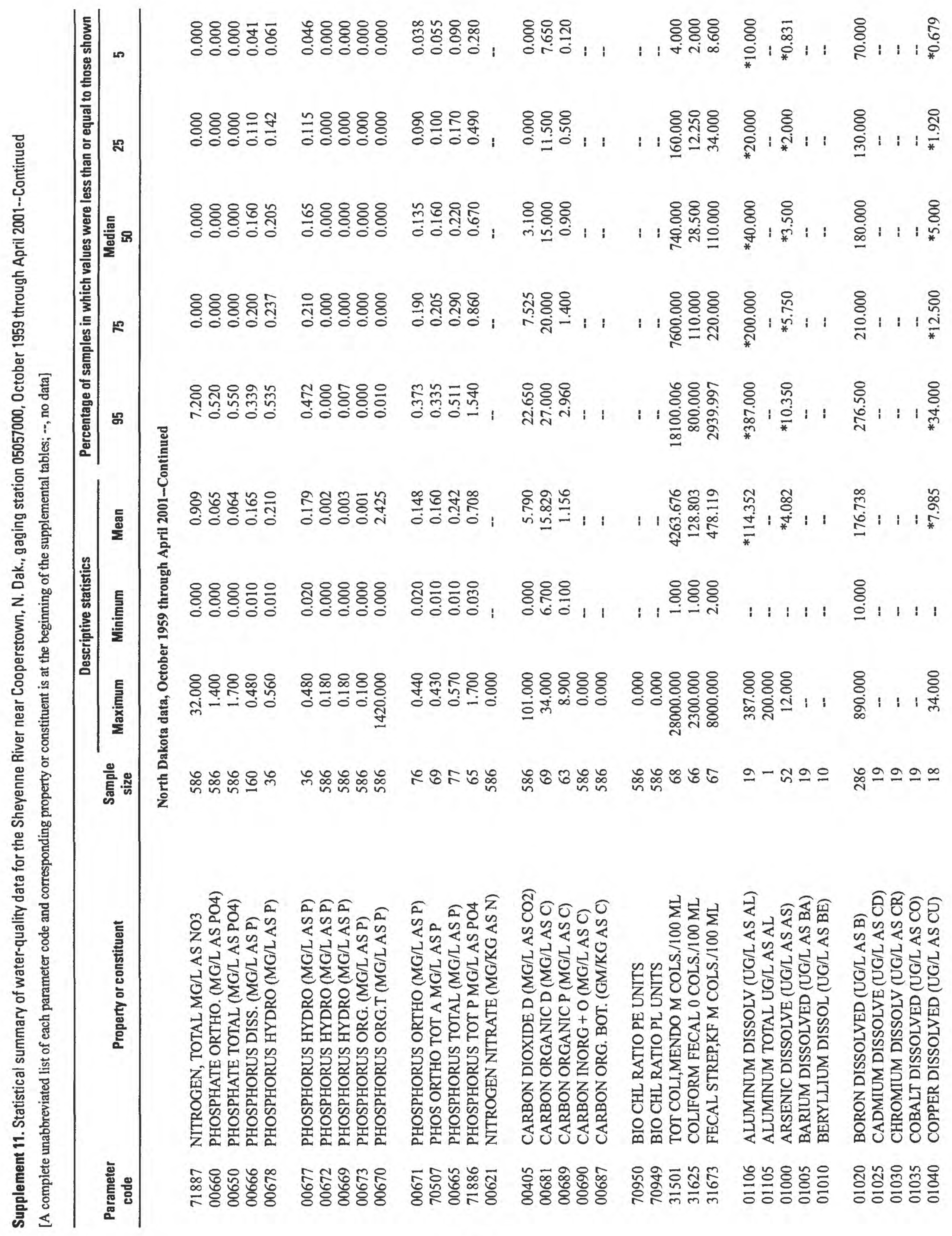




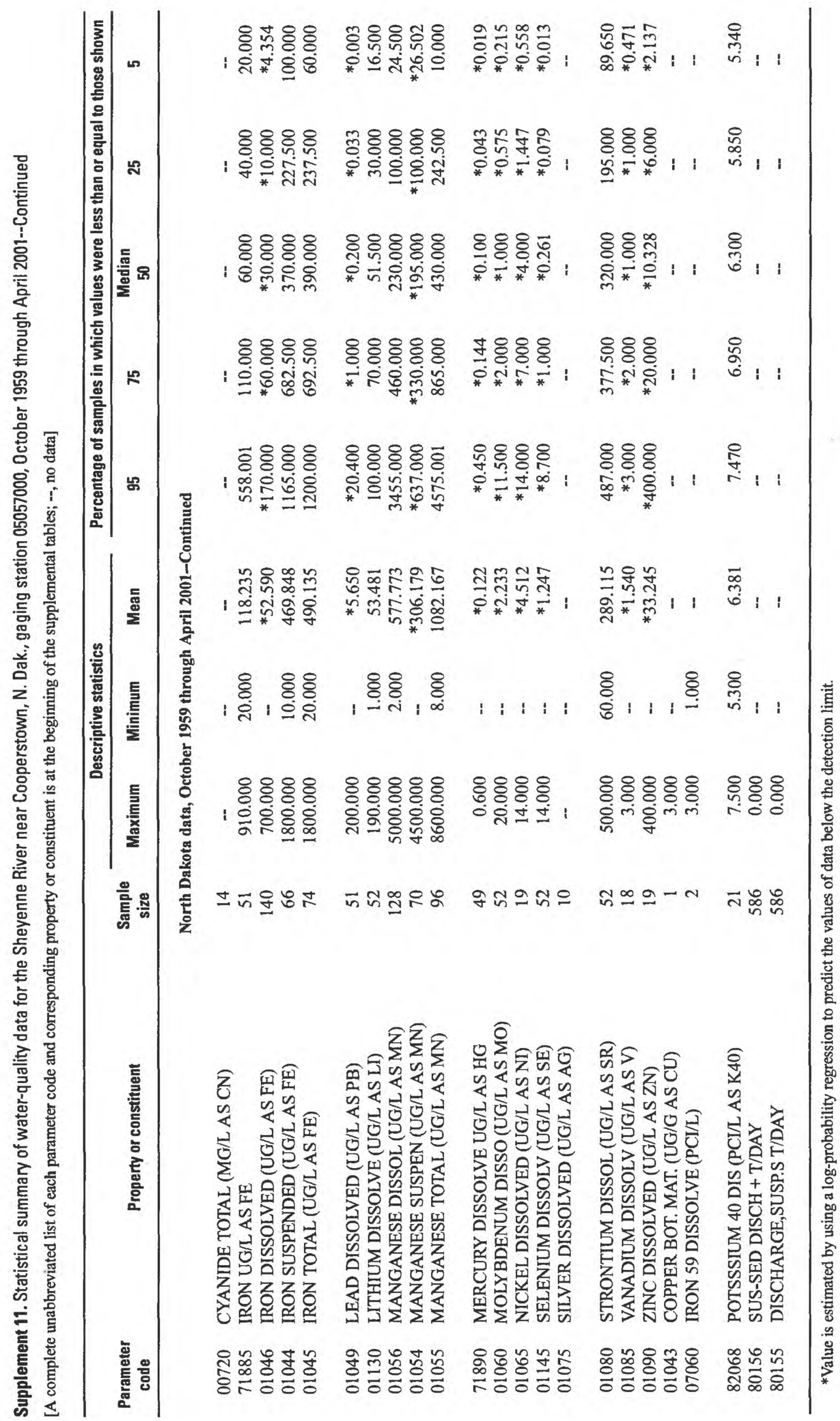




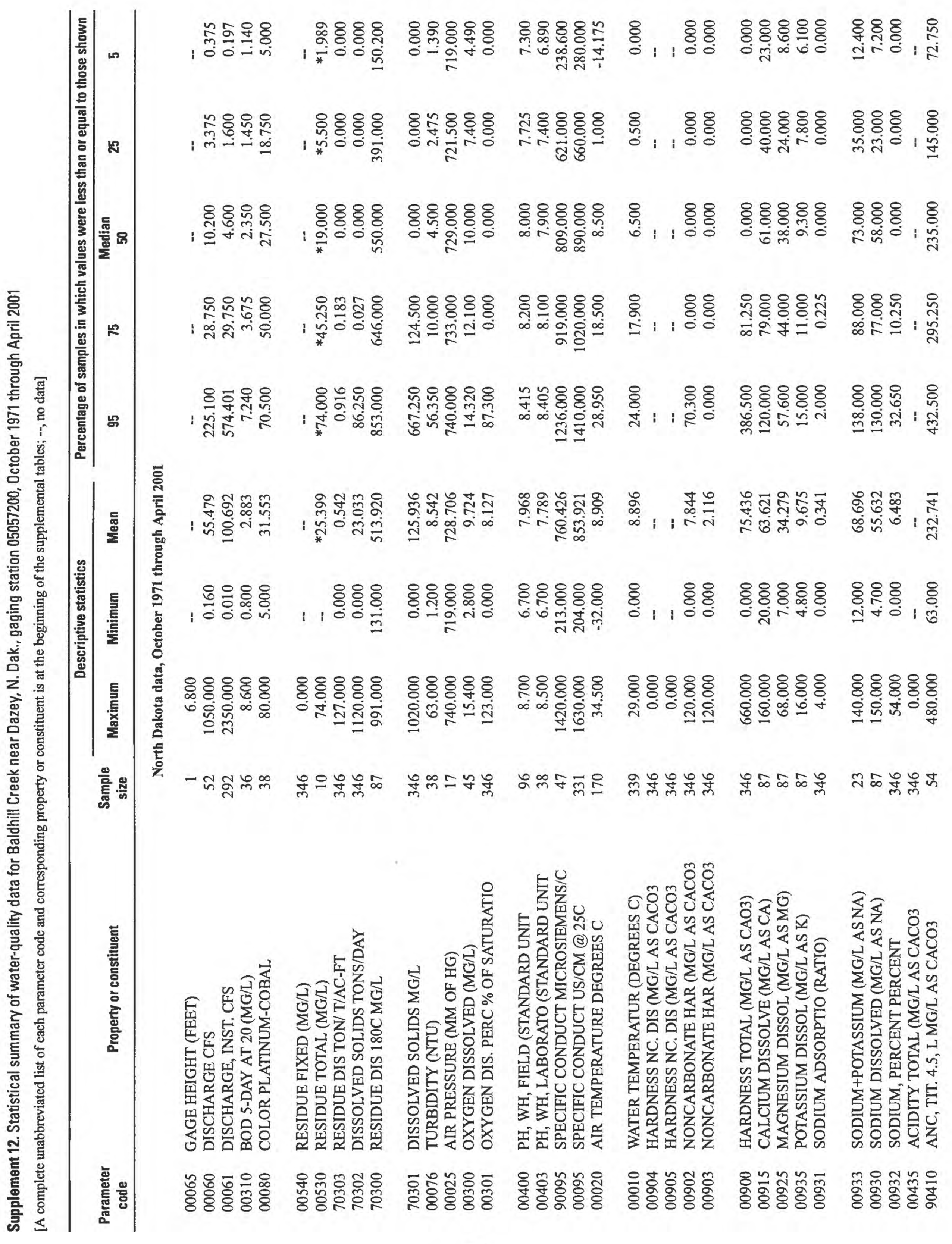




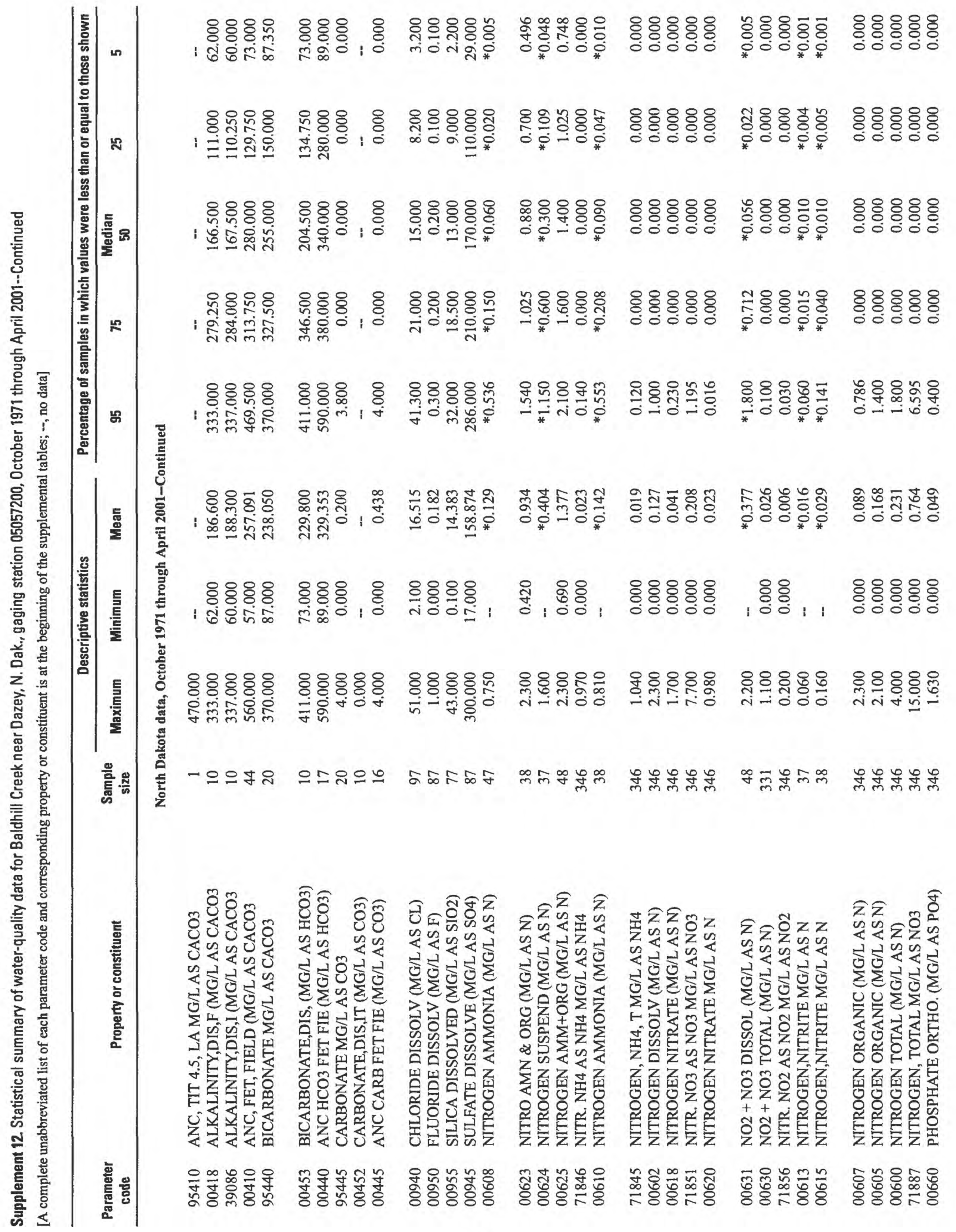




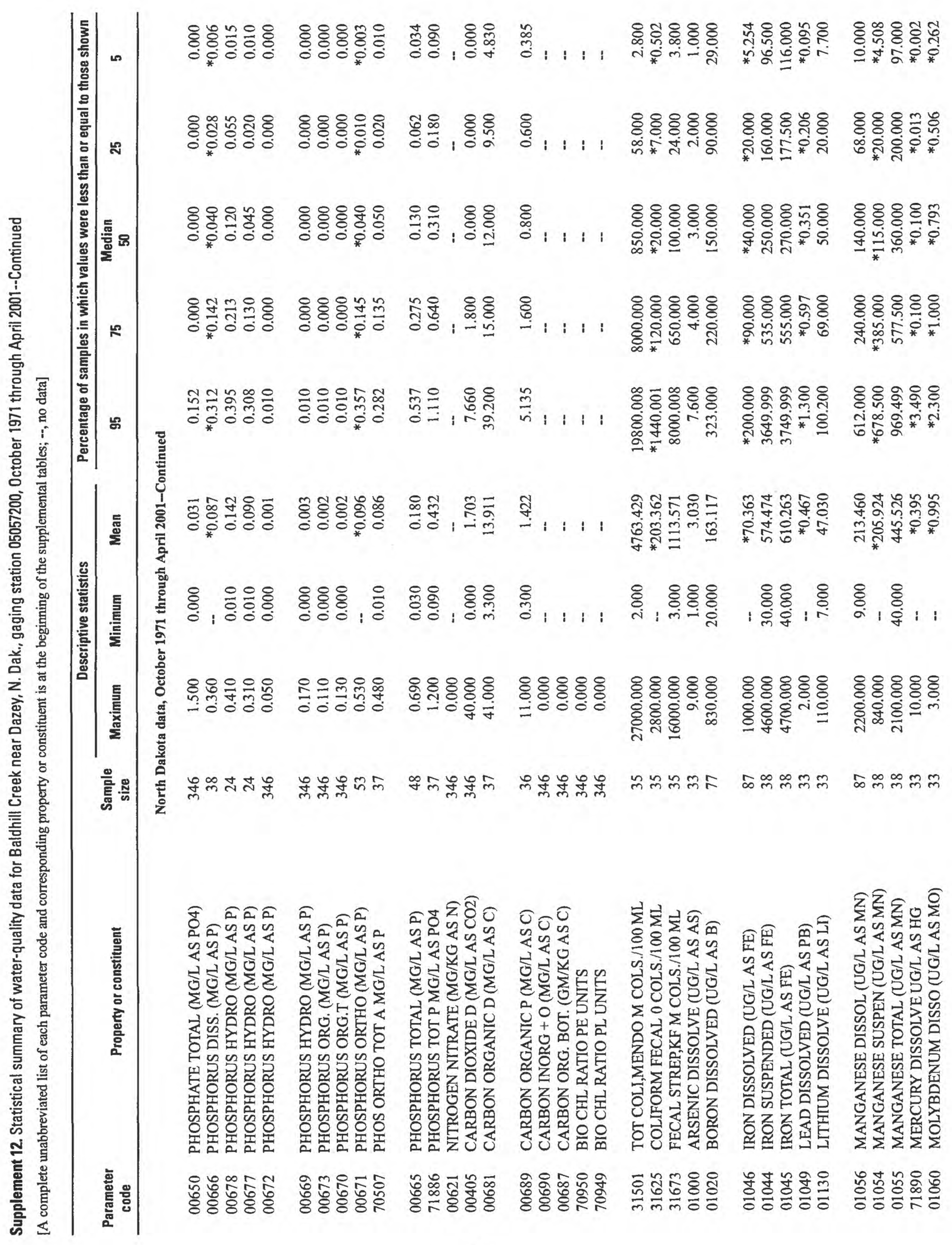




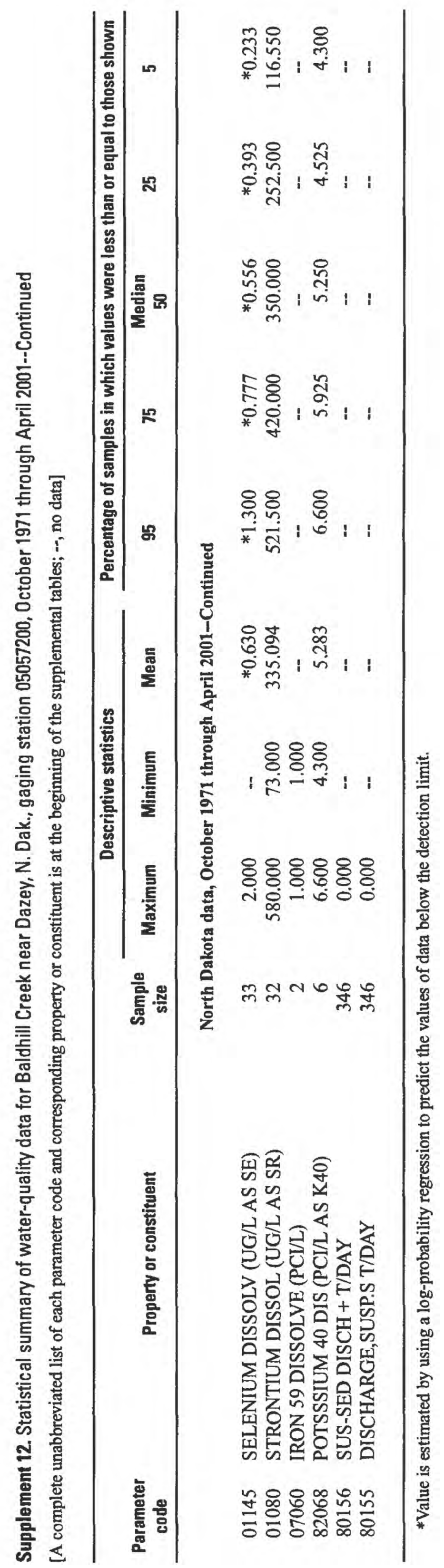




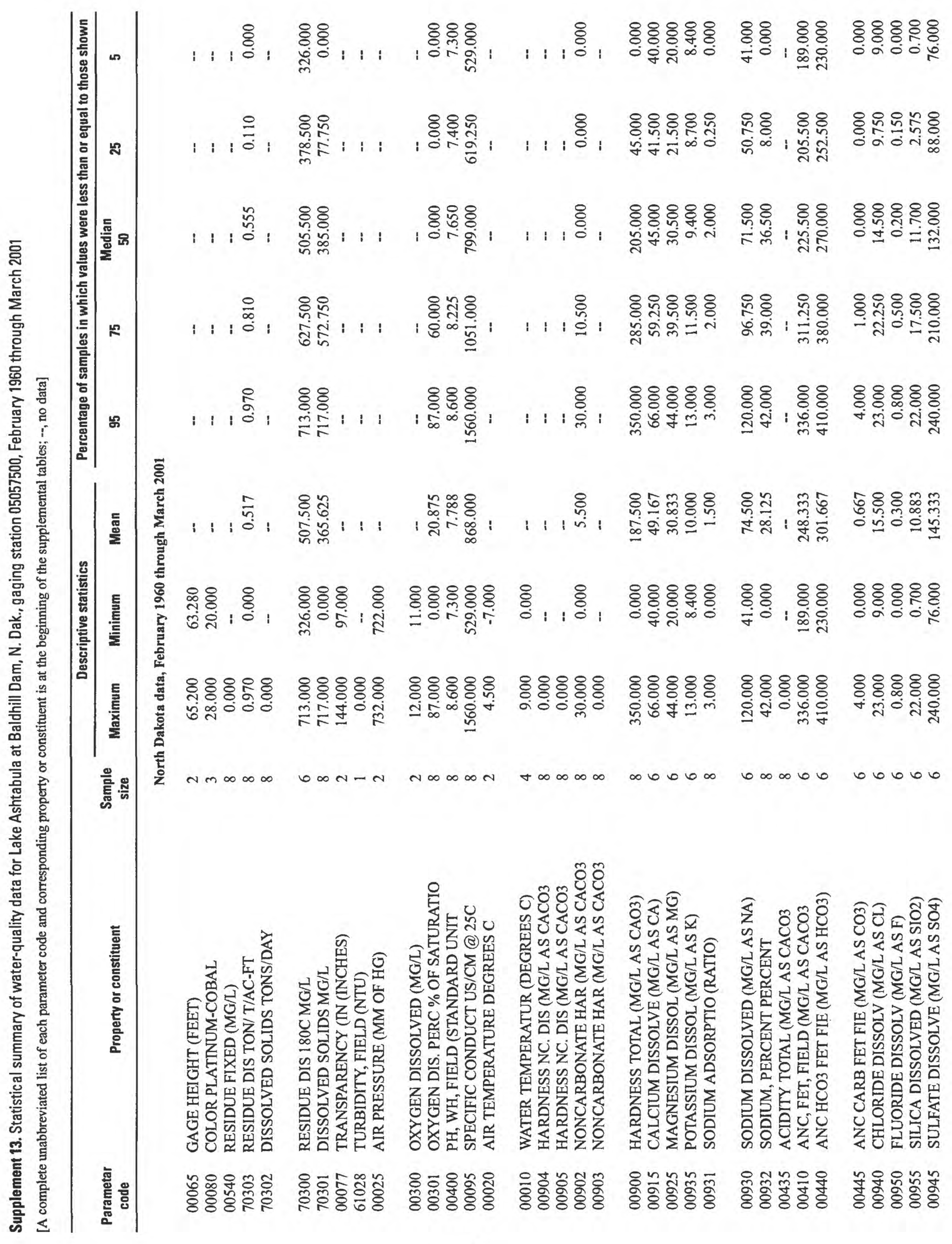




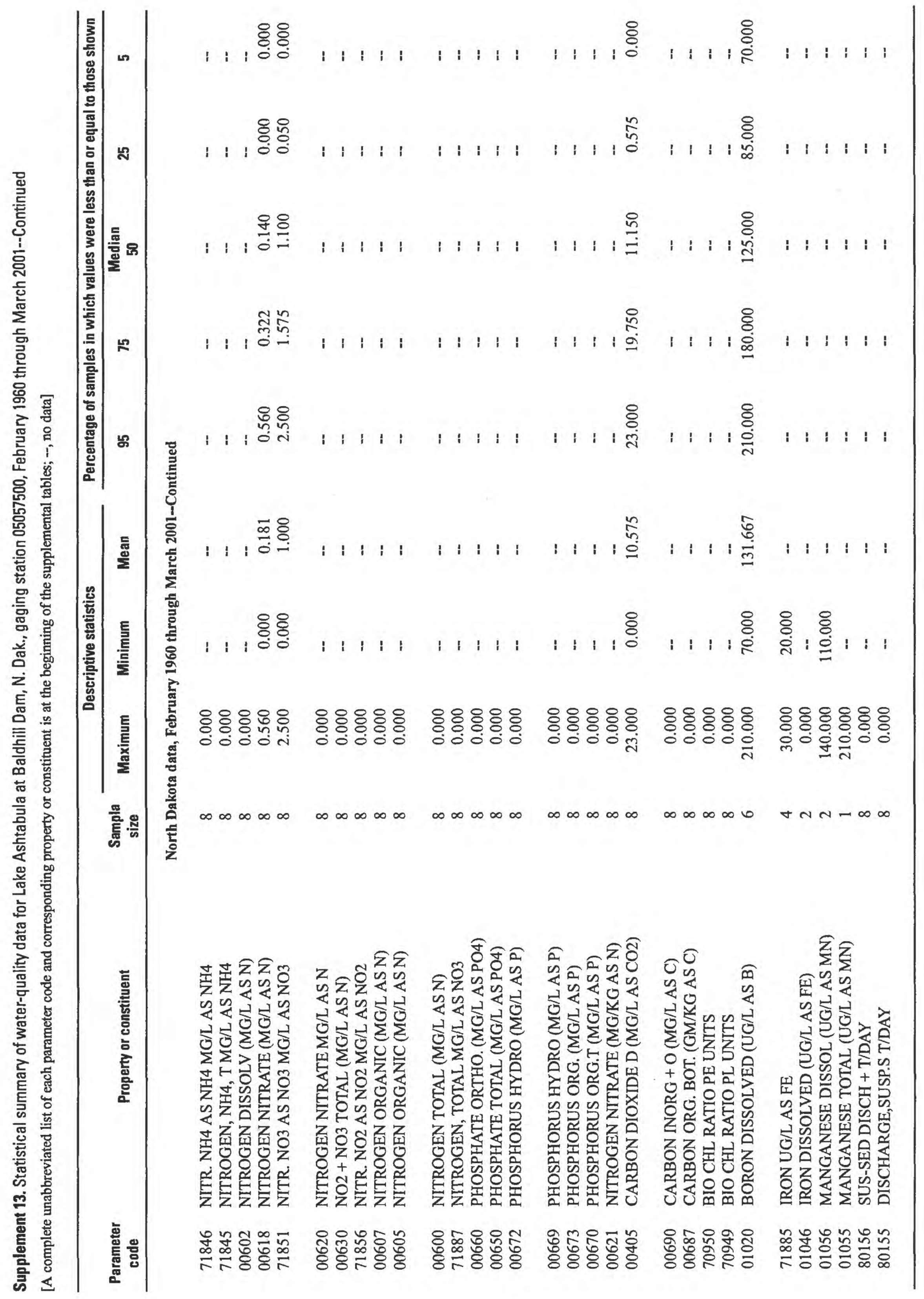




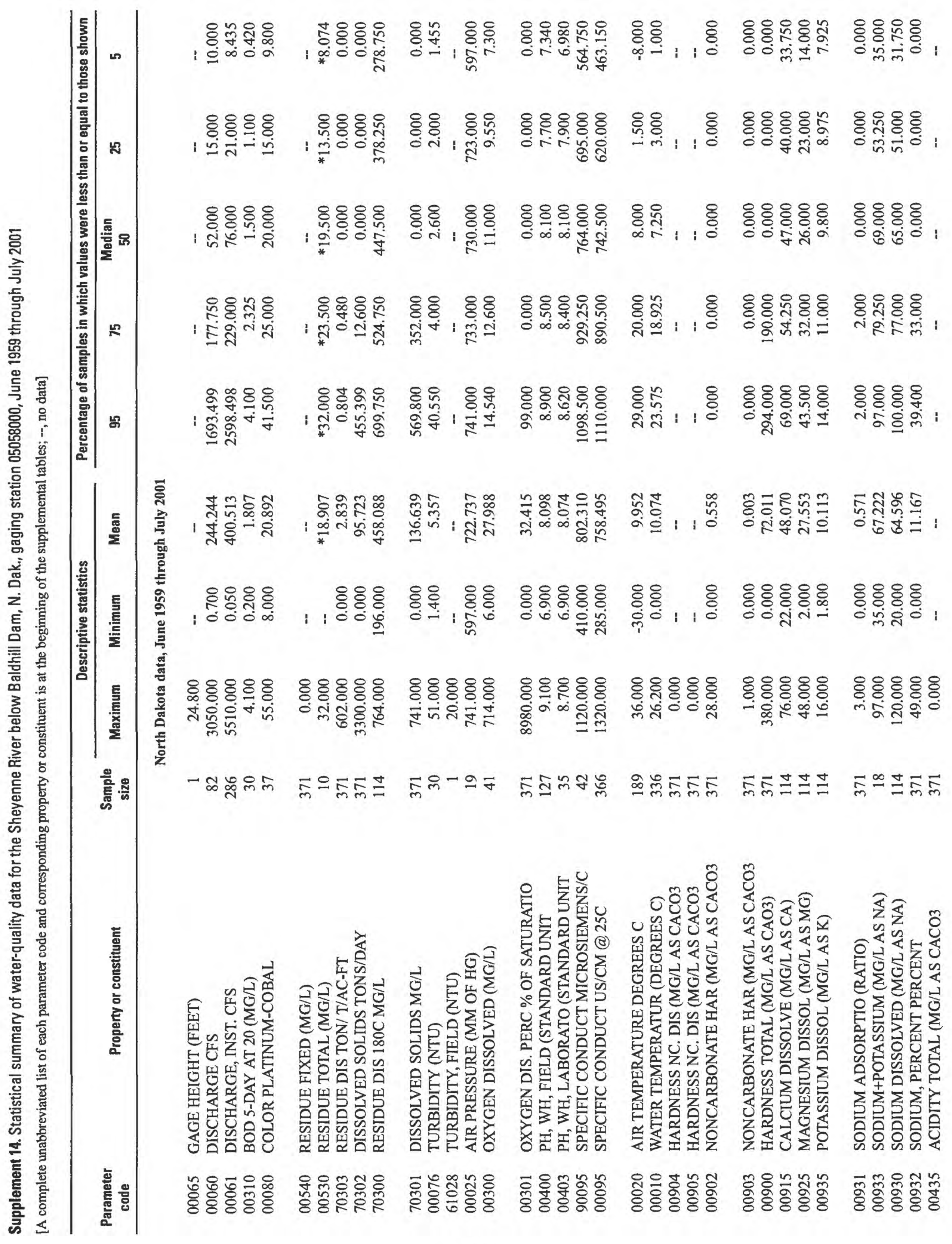




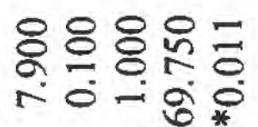

$\infty$
0
0
0
0
0

융ㅇㅇㅇ

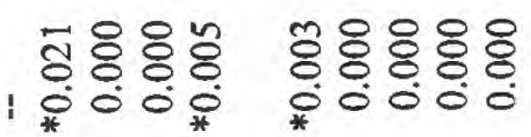

$8: 8 \%$

สิ่ป็

늉유융

$88 ㅇ ㅡ ㅇ ㅇ ㅛ$

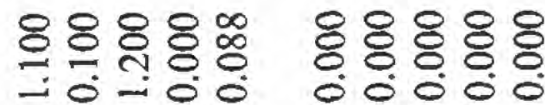

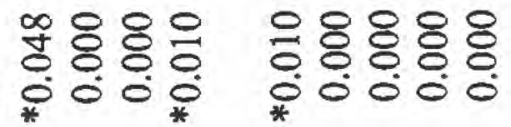

융융유.

穴 㝏

ำ

ㅇㅇㅇ유뉴.

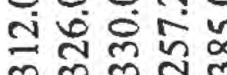

8888

$888 \%$ 융

융유유.

융ㅇㅇㅇㅇㅇ

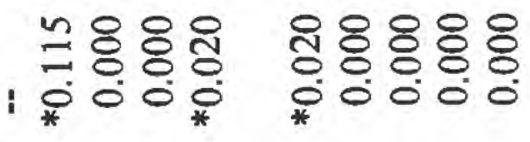

每品:

ल어요.

-

$\circ 0000$

융ㅇㅇㅇㅇㅛ

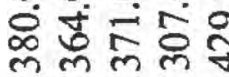

듕유용

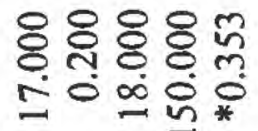

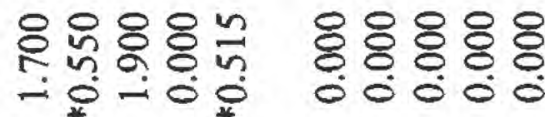

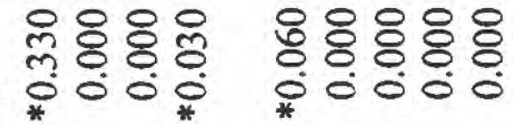

¿유융

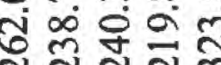

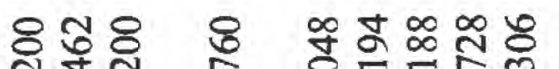

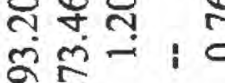

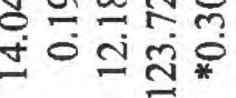

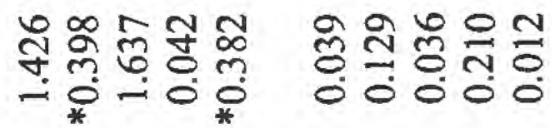

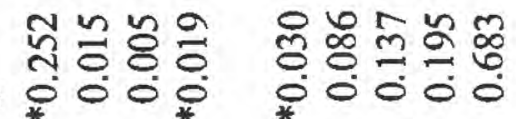

융융요

俕

융융ㅇㅇ

ㅇㅇㅇ용

융유융유.

88

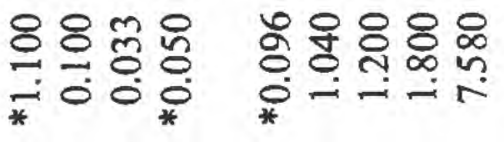

88.8

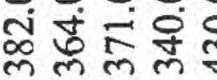

융융유융유

तुं

양융유융

융유융이

inmin-

ininio

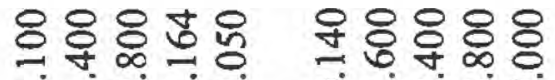

n으옹

으는조응

吉さす巳

윳ำ중

FFEF

一 유ㅇㅠㅛ

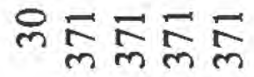

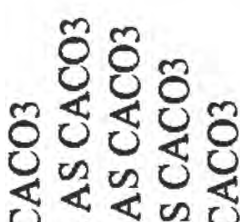

की

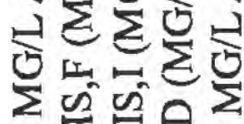

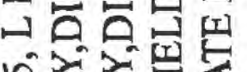

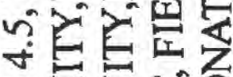

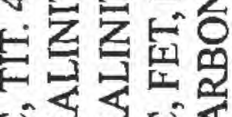

割论

这安它

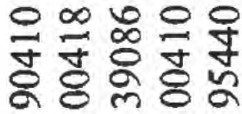

ชิซิ

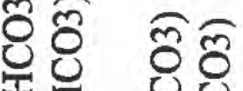

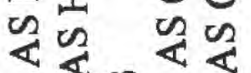

넝성엉던겅

$\sum \sum \approx \sum \Sigma$

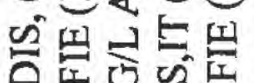

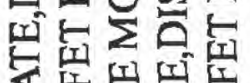

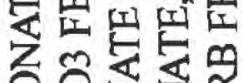

유원

定泡

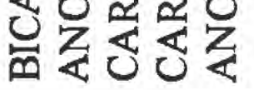

管露等筞等

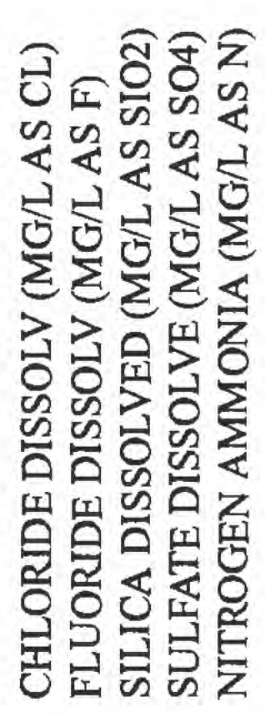

옿ํำ

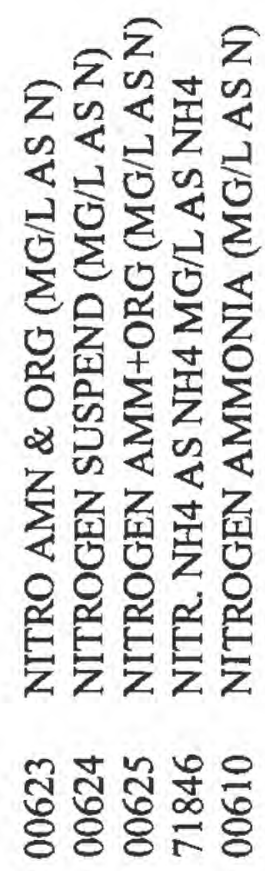

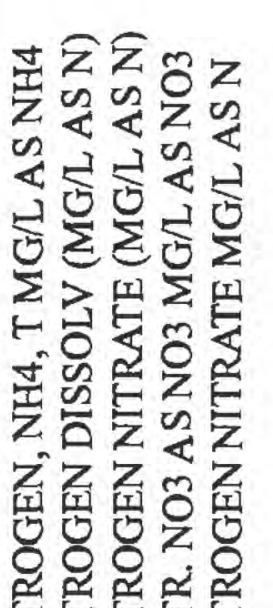

参急它总

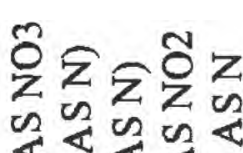

$<\ll<$

렁성렁어

$\sum \sum \sum \sum \sum_{i=1}$

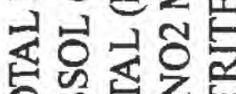

은은?

띱

解界

$++2$

zกิ์

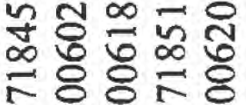

员대윰

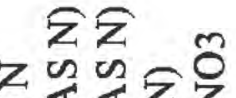

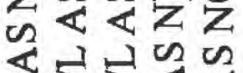

《러엉ㄴ

엉로엉벙

$\sum \cup 0 \sum \Sigma$

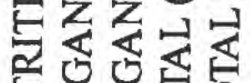

㫐论数

Zo요.

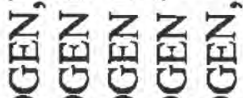

웅요

穵它芸

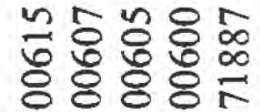




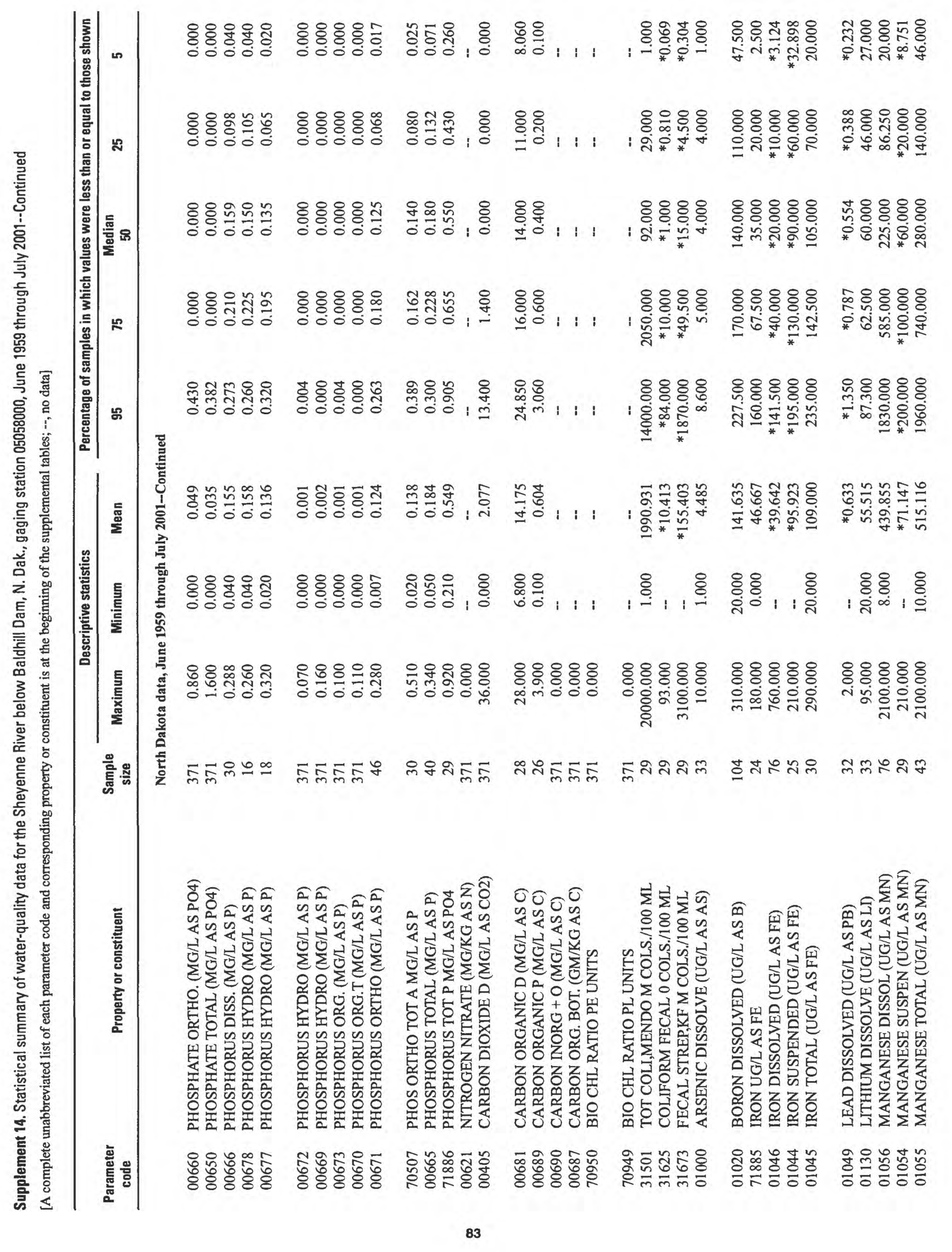




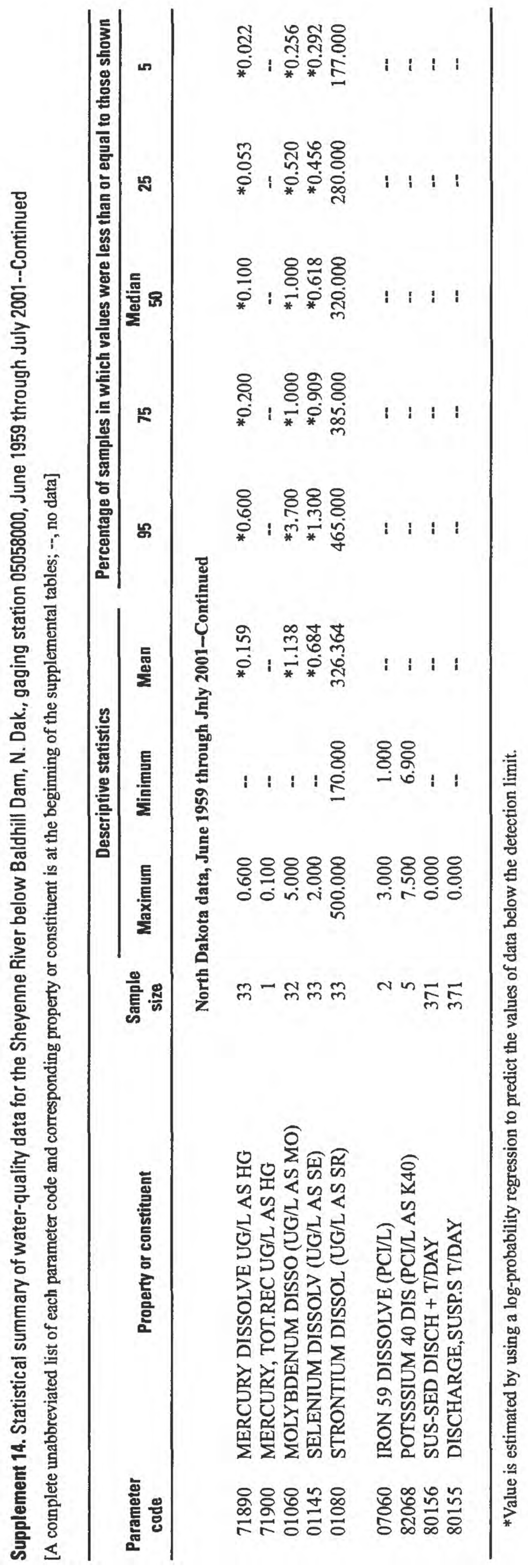




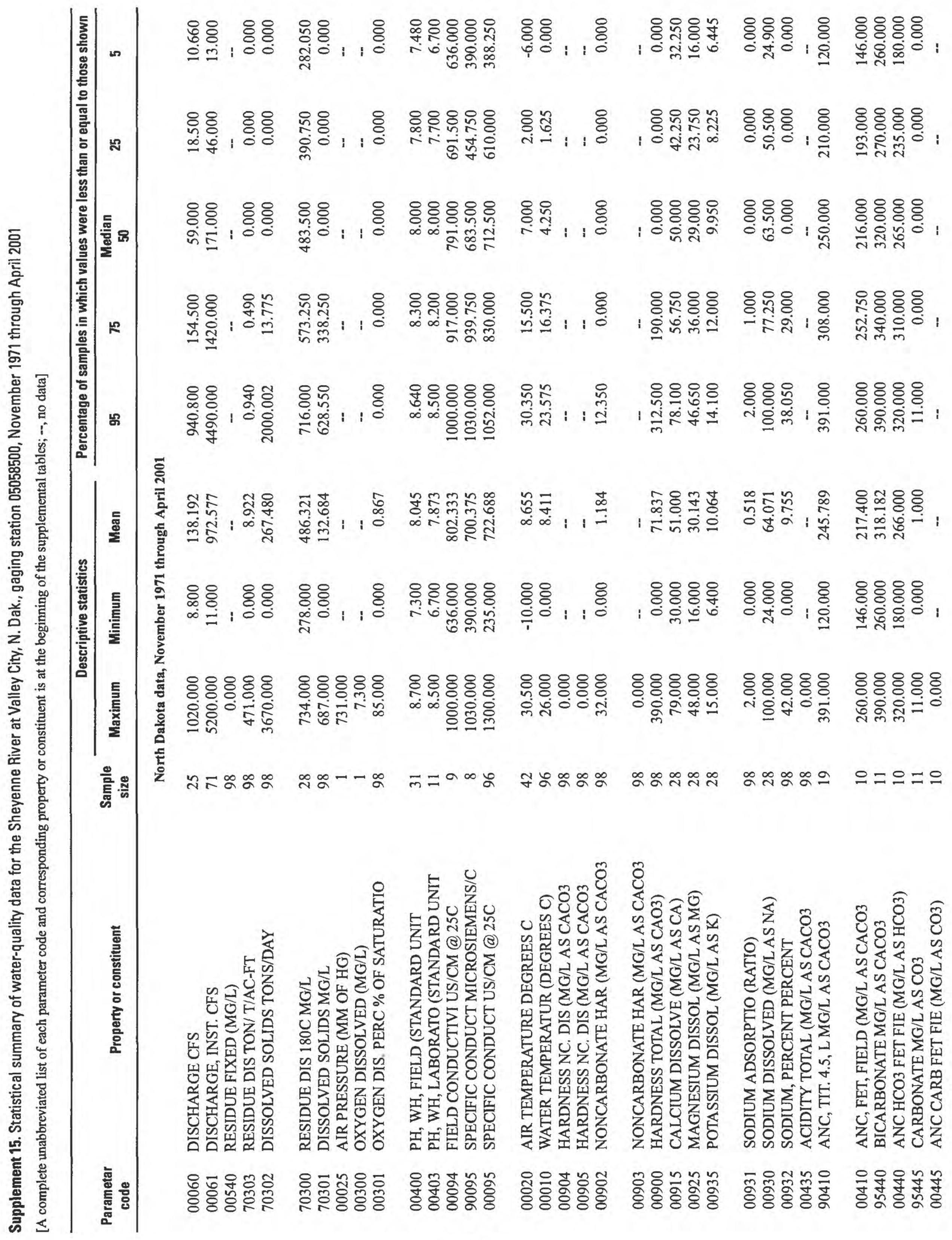




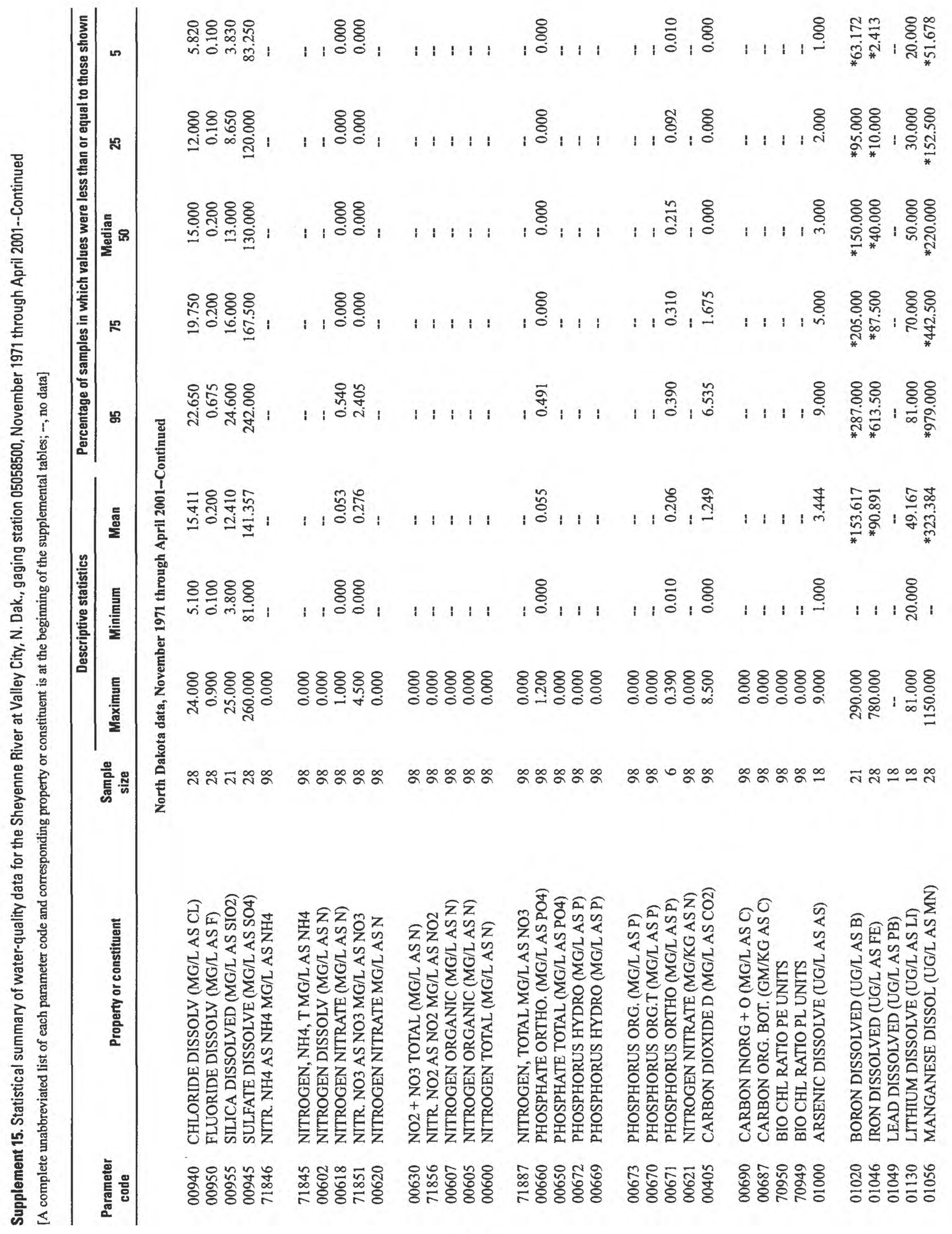




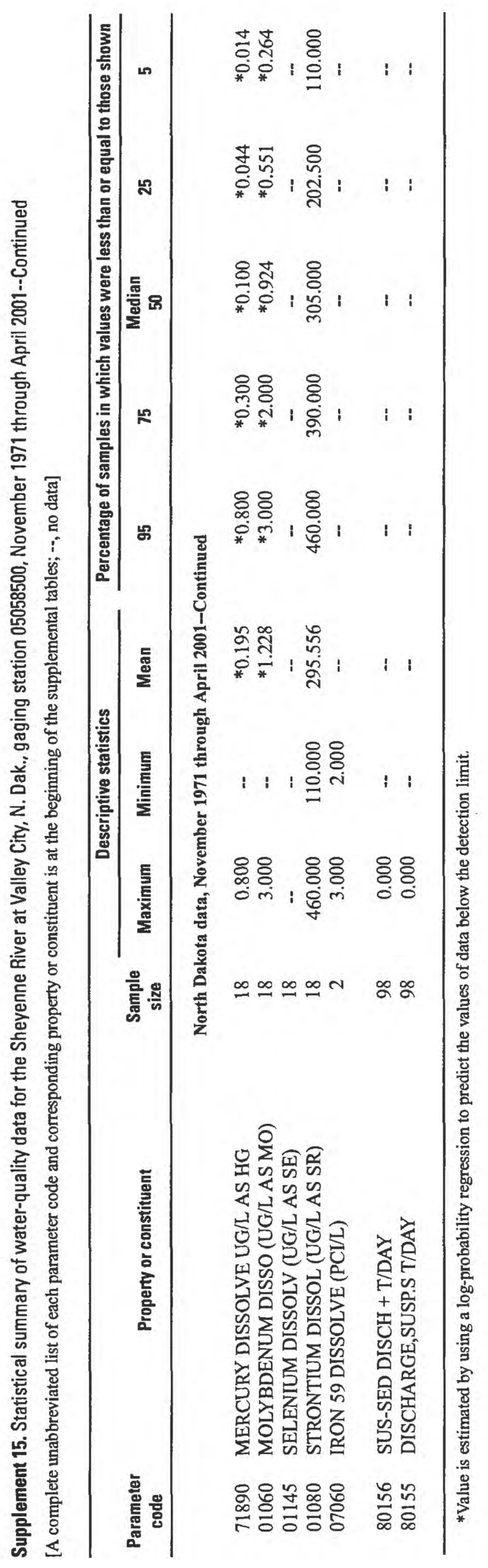




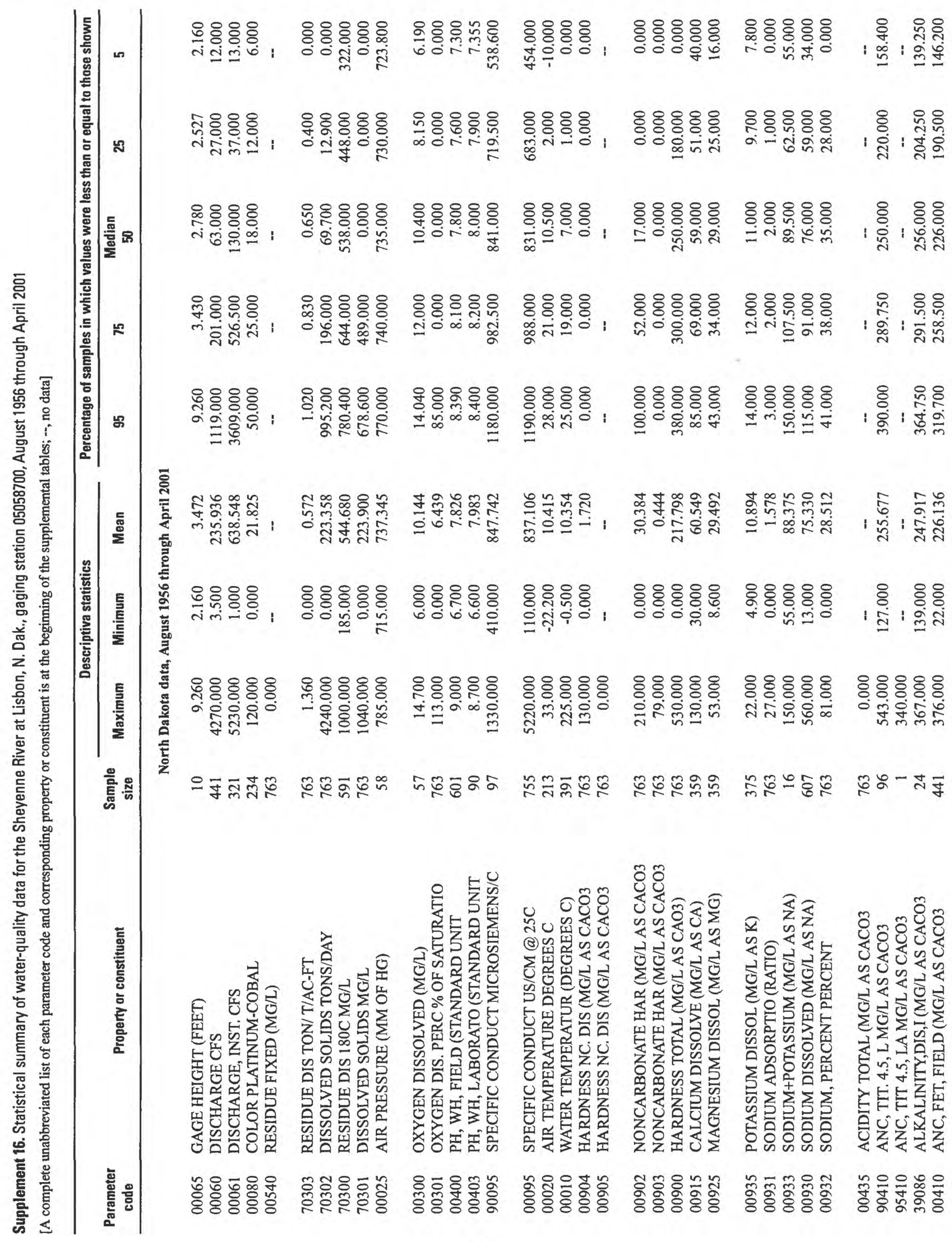




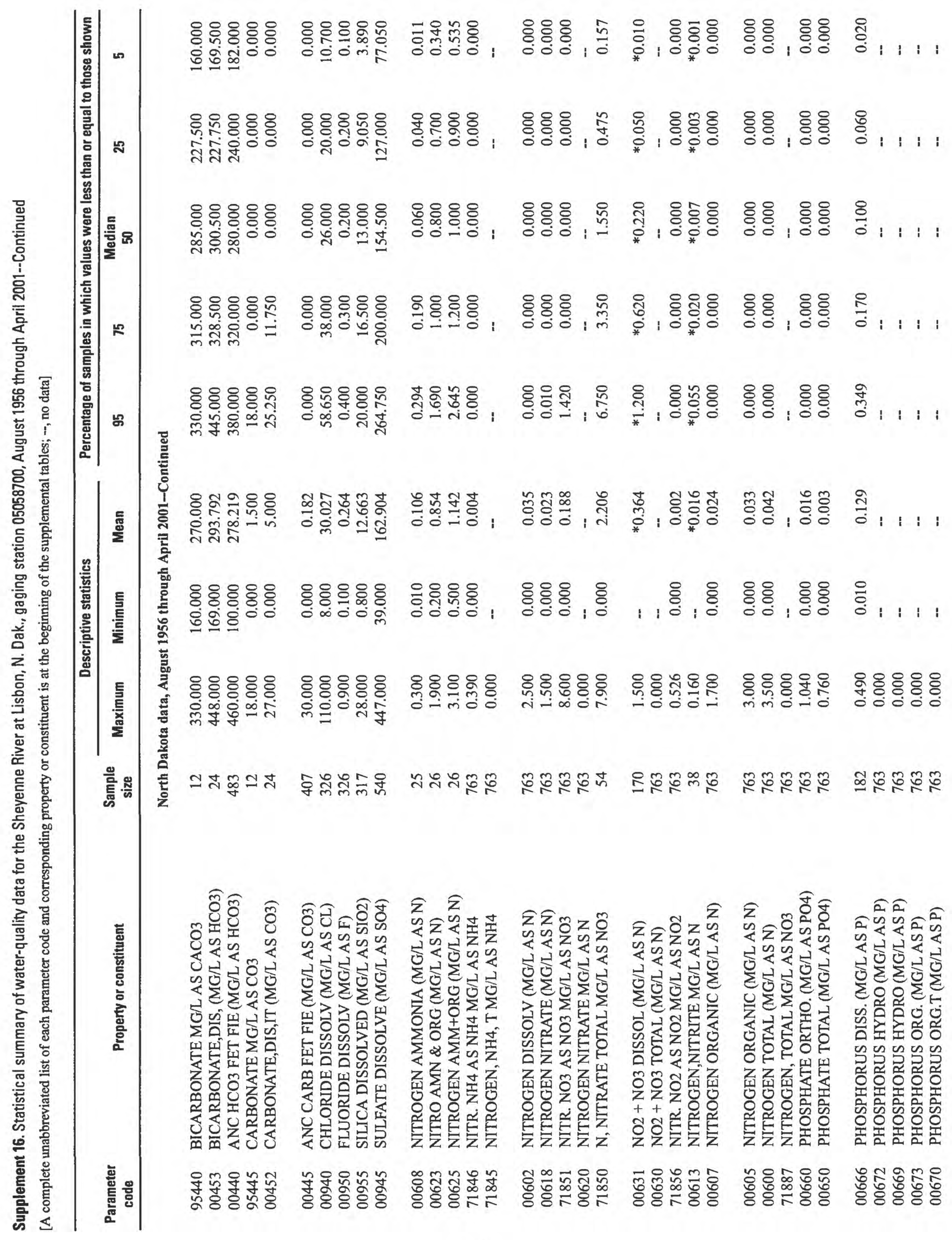




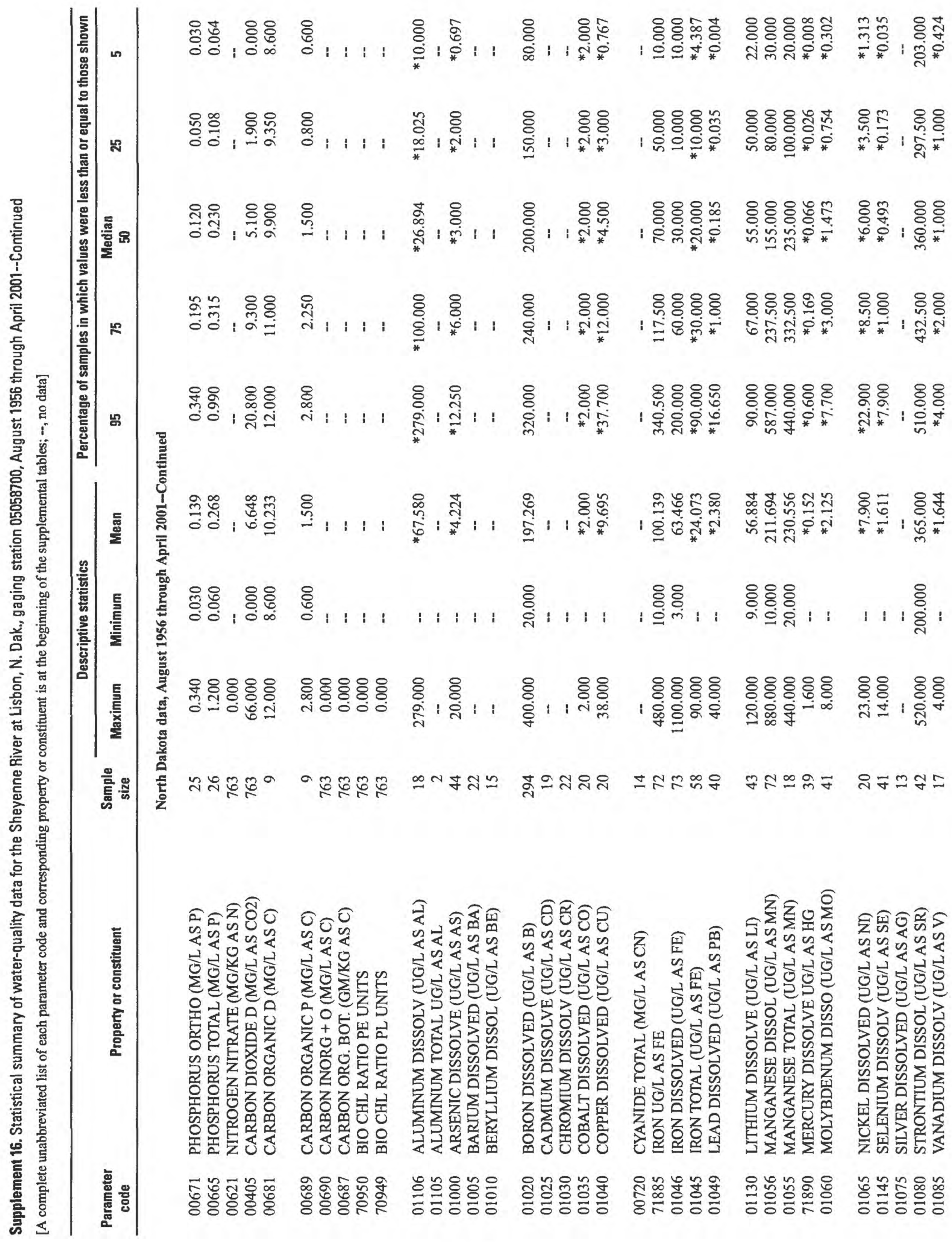




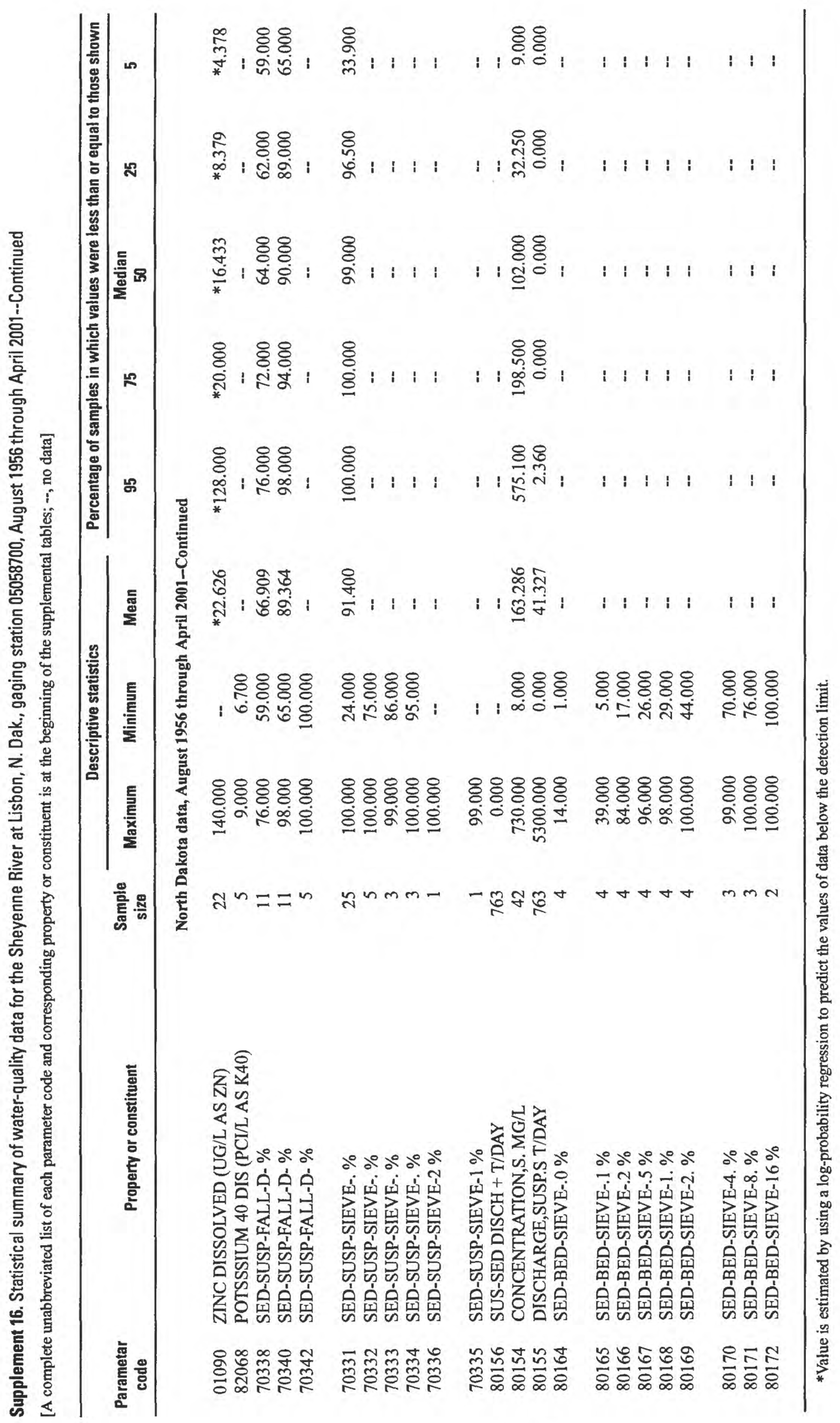




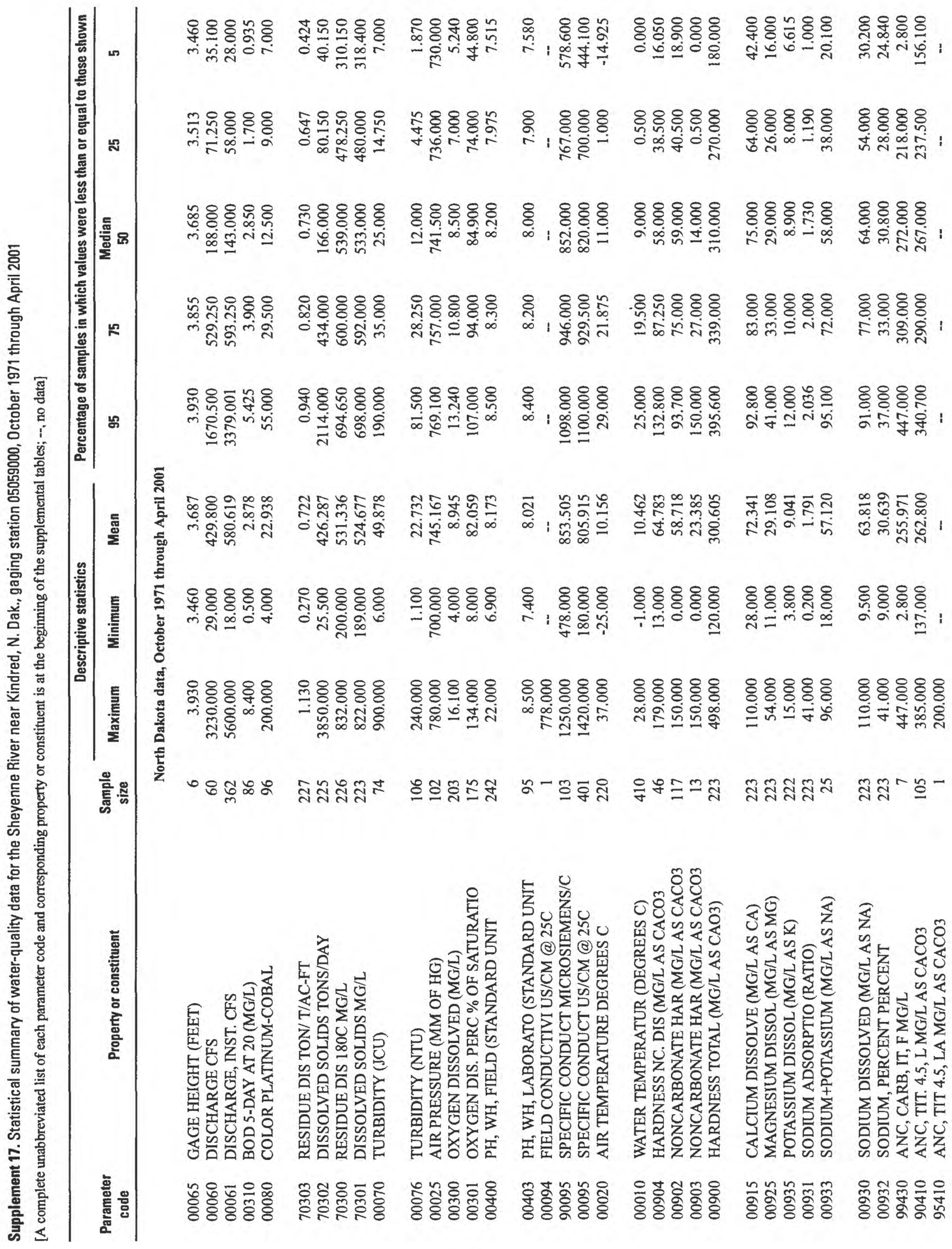




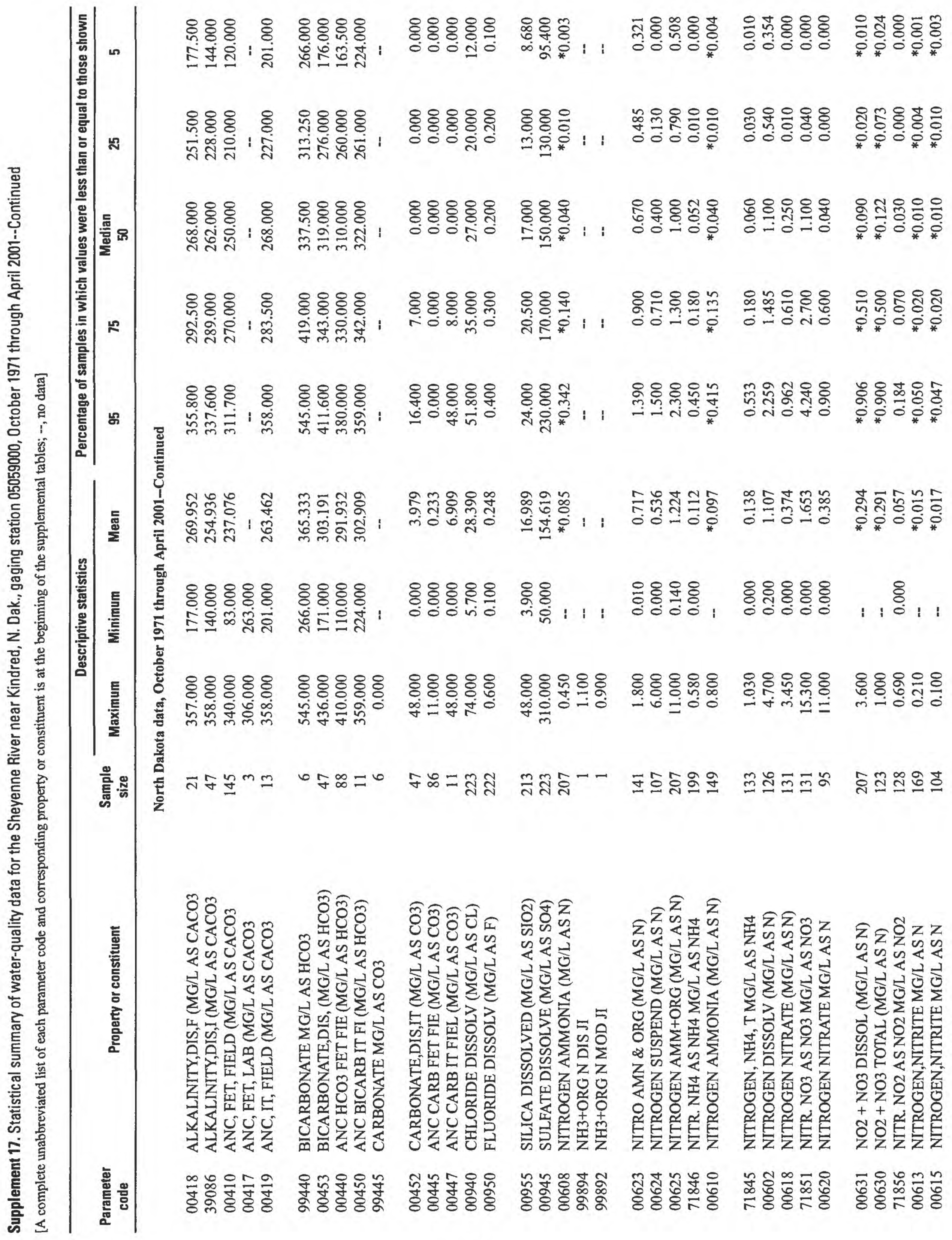




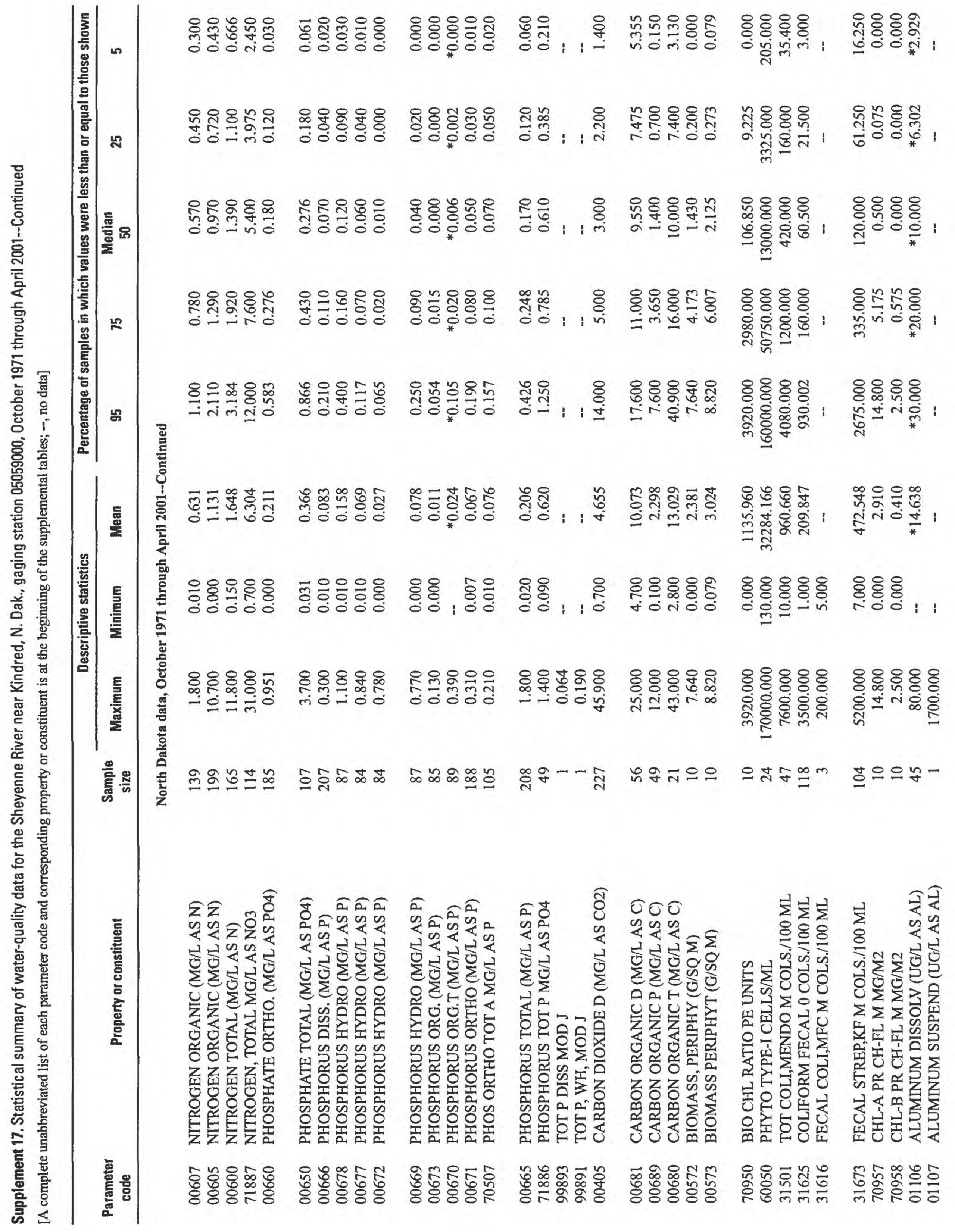




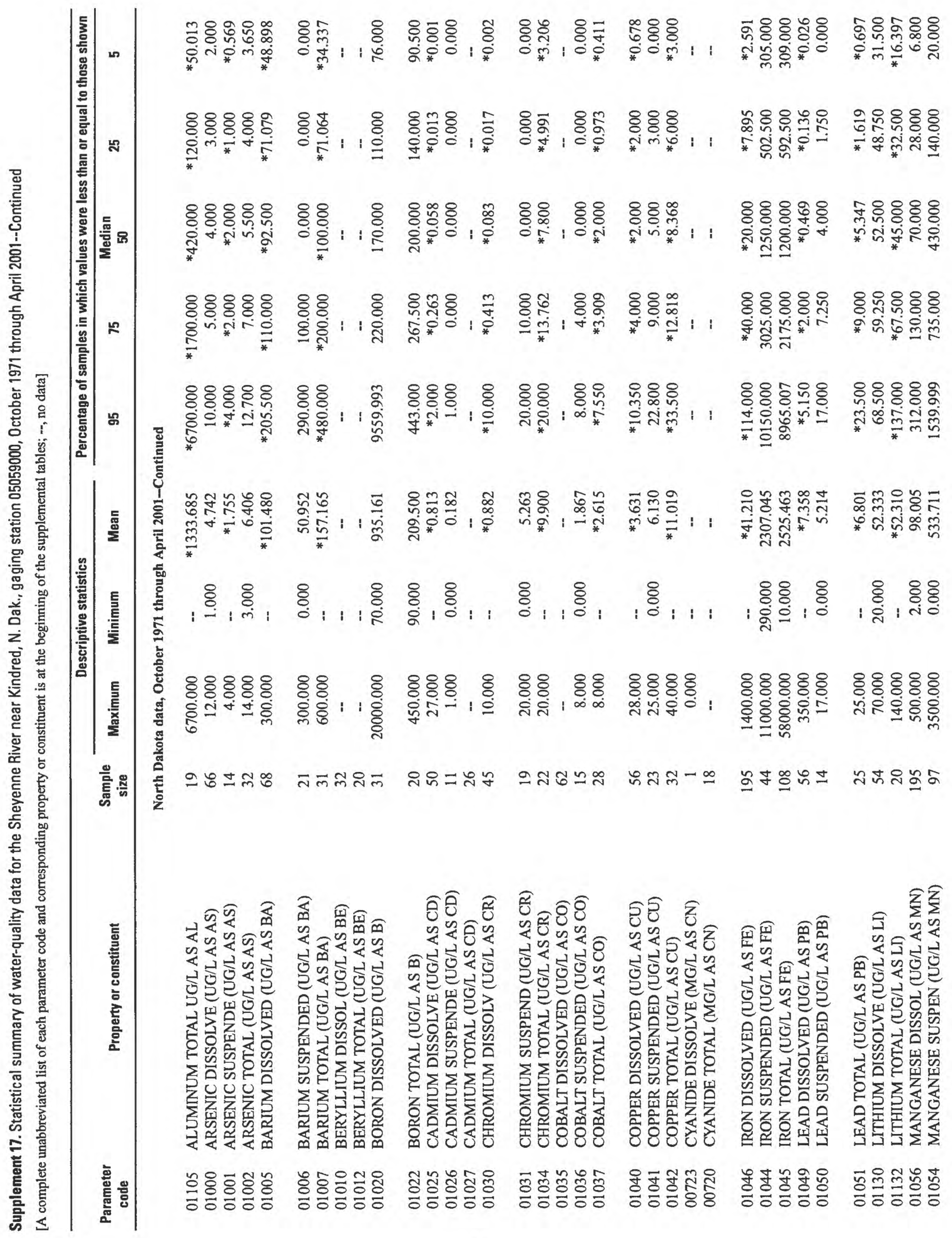




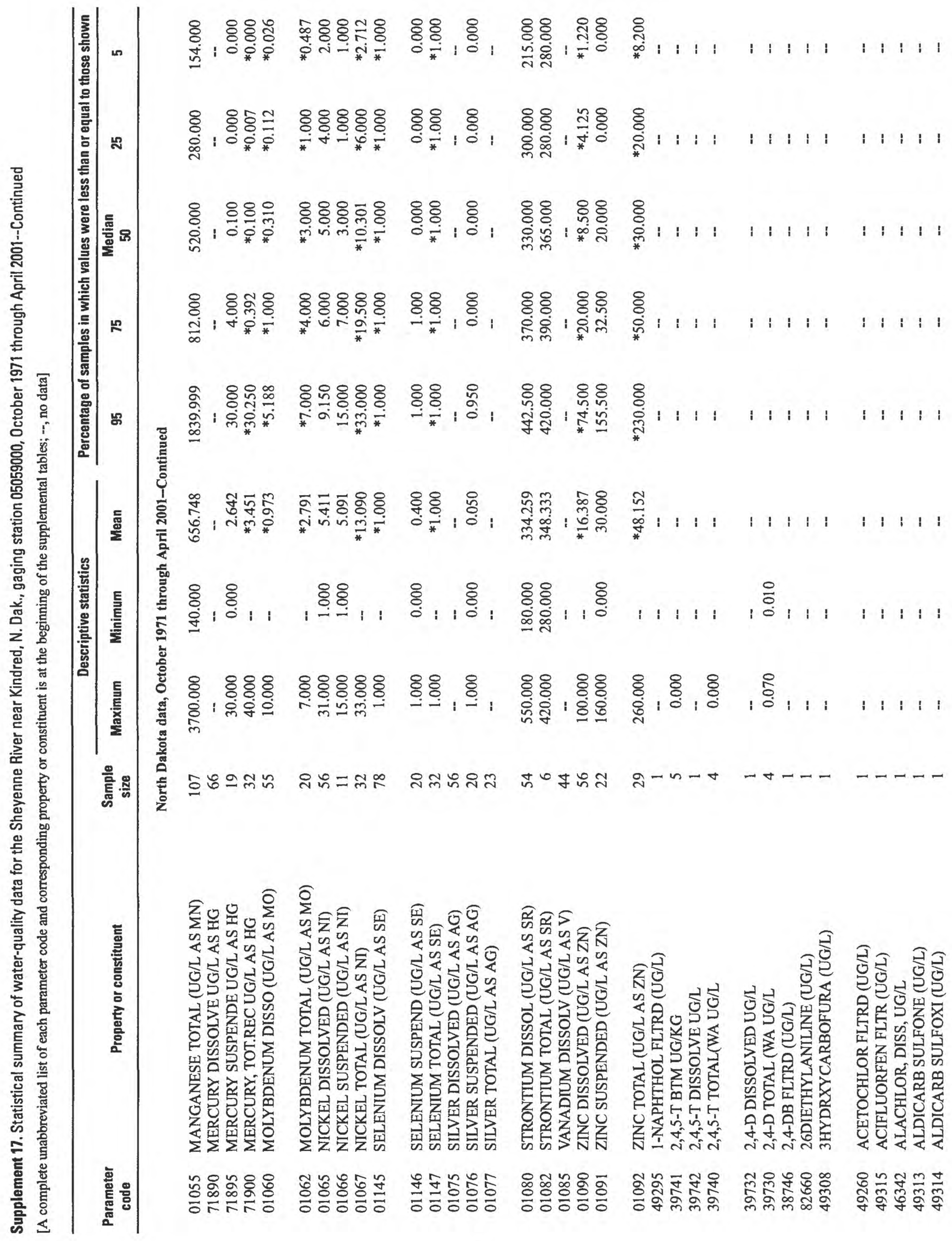




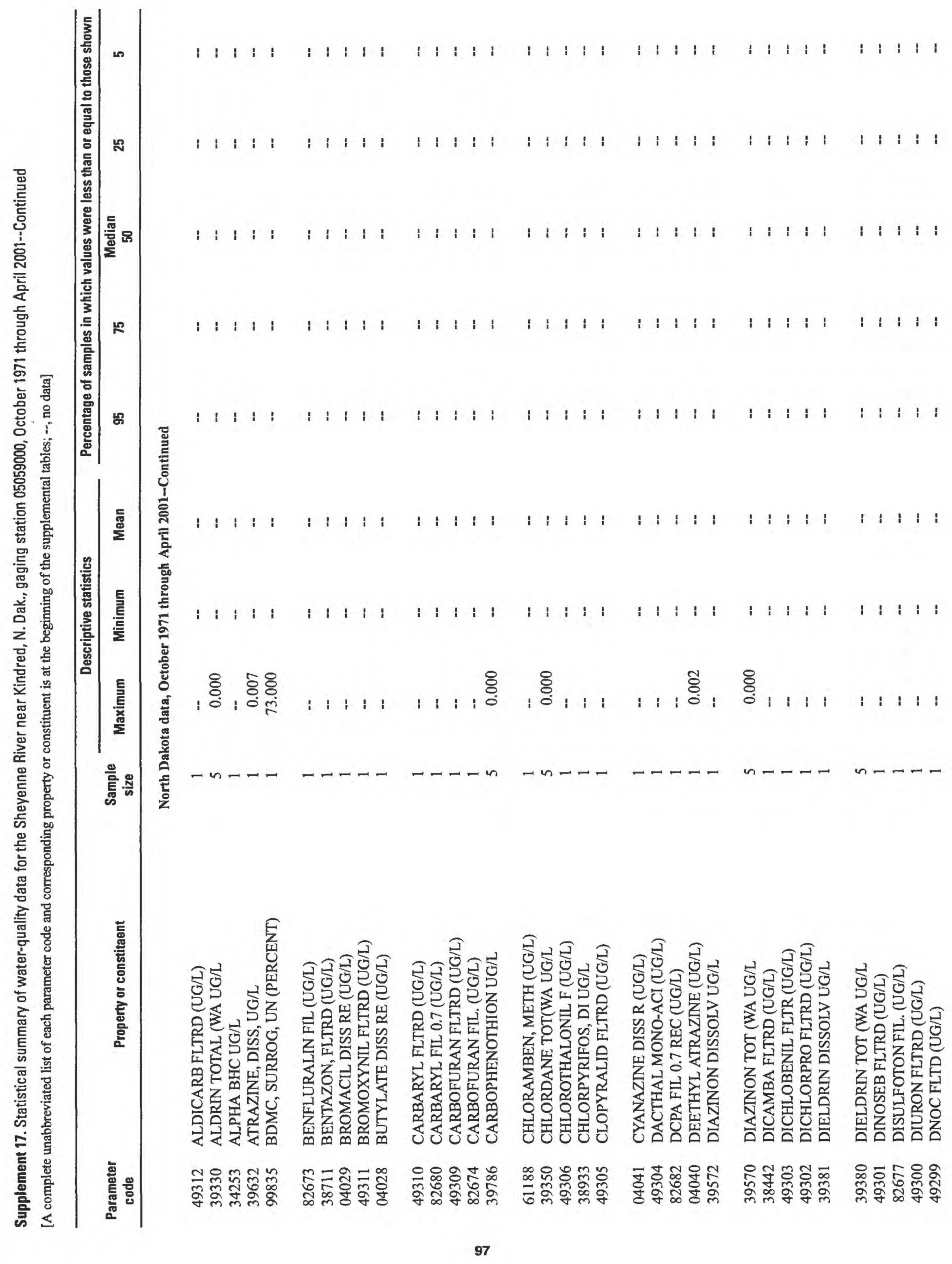




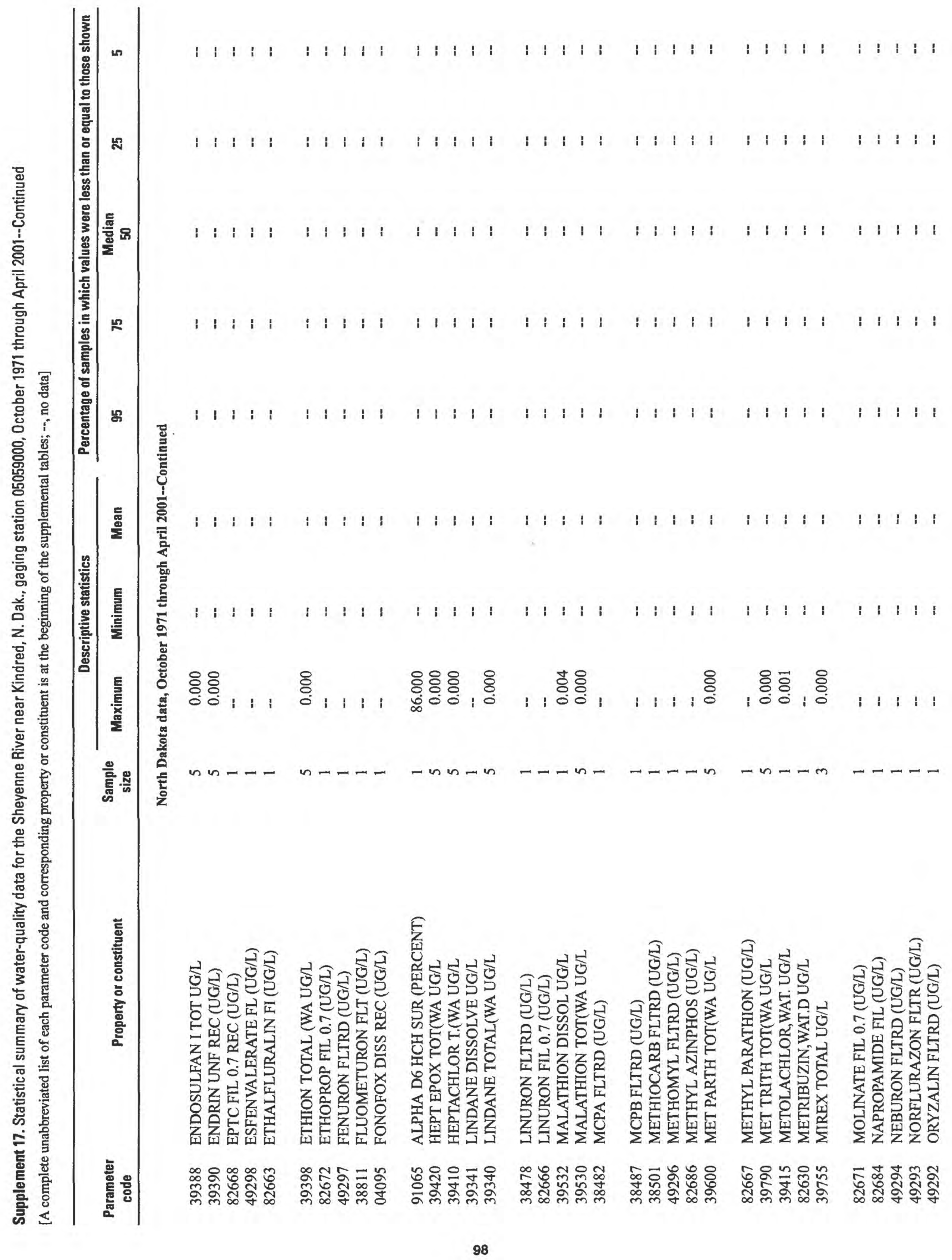




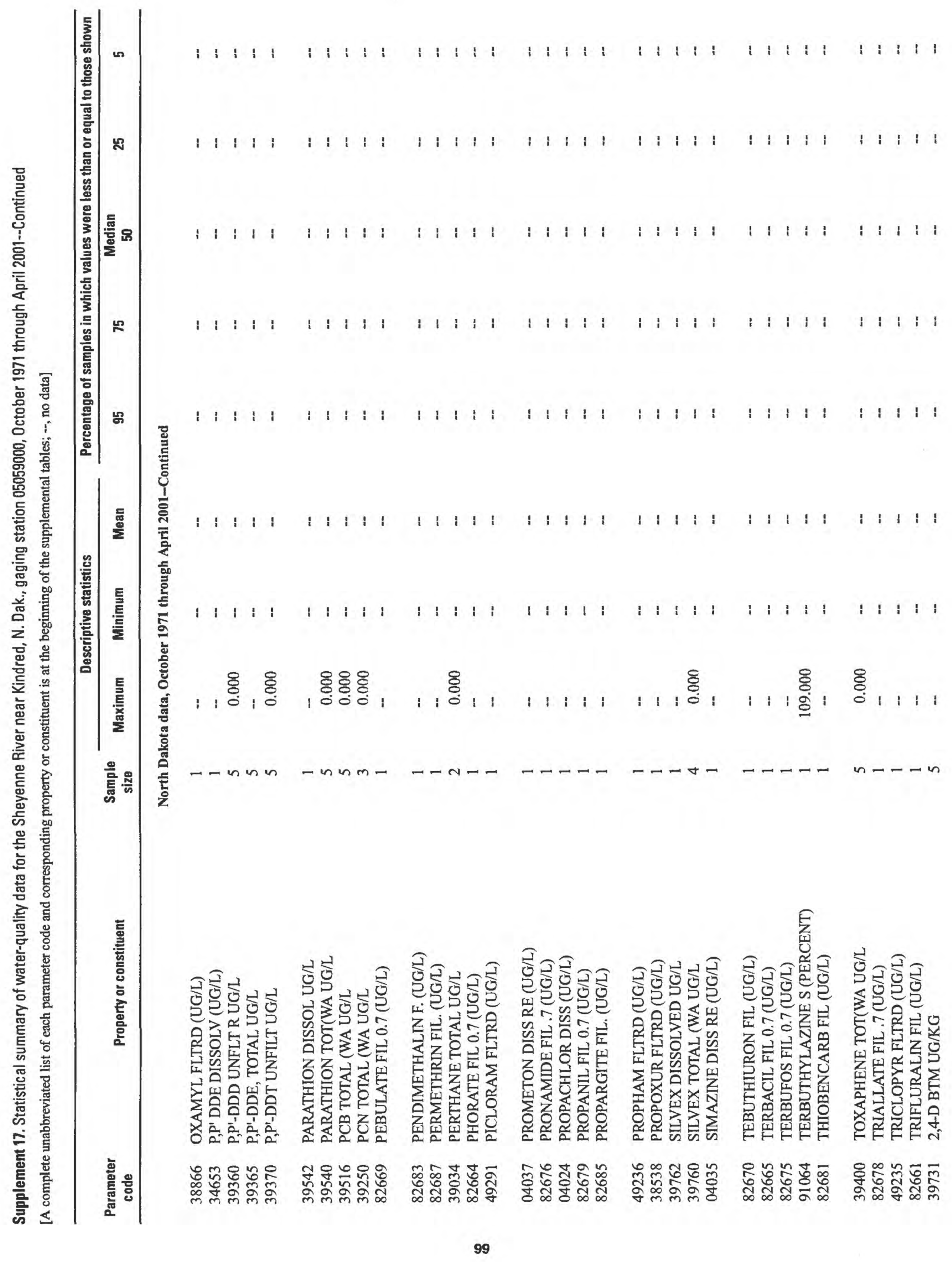




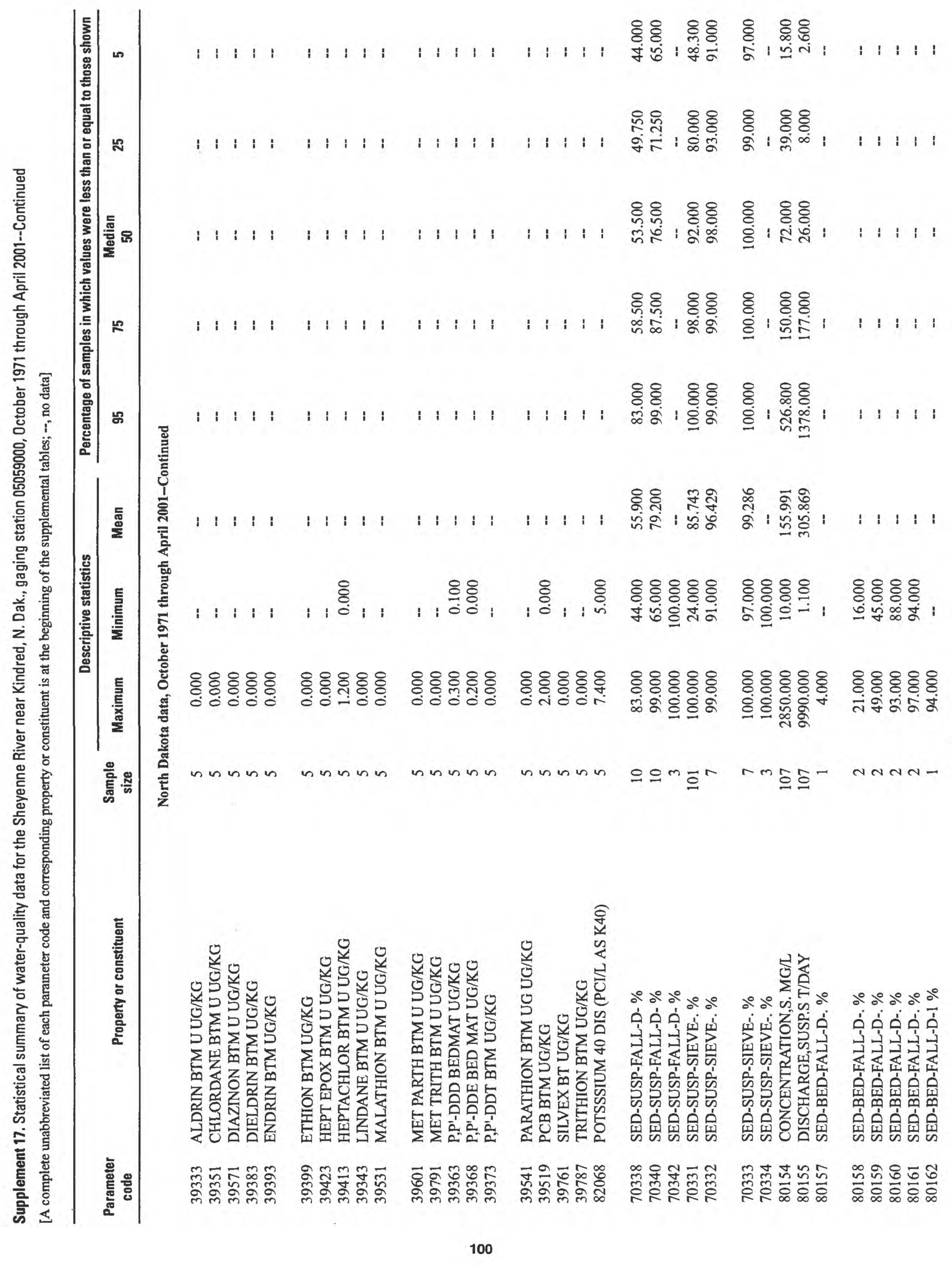




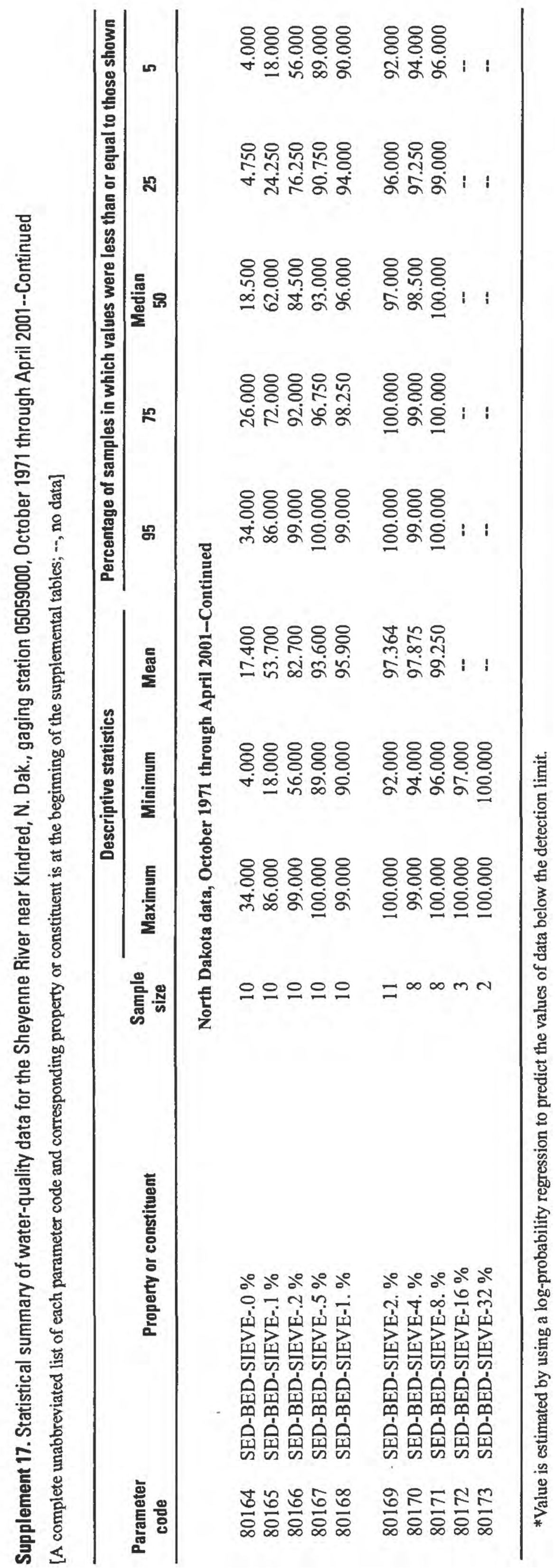




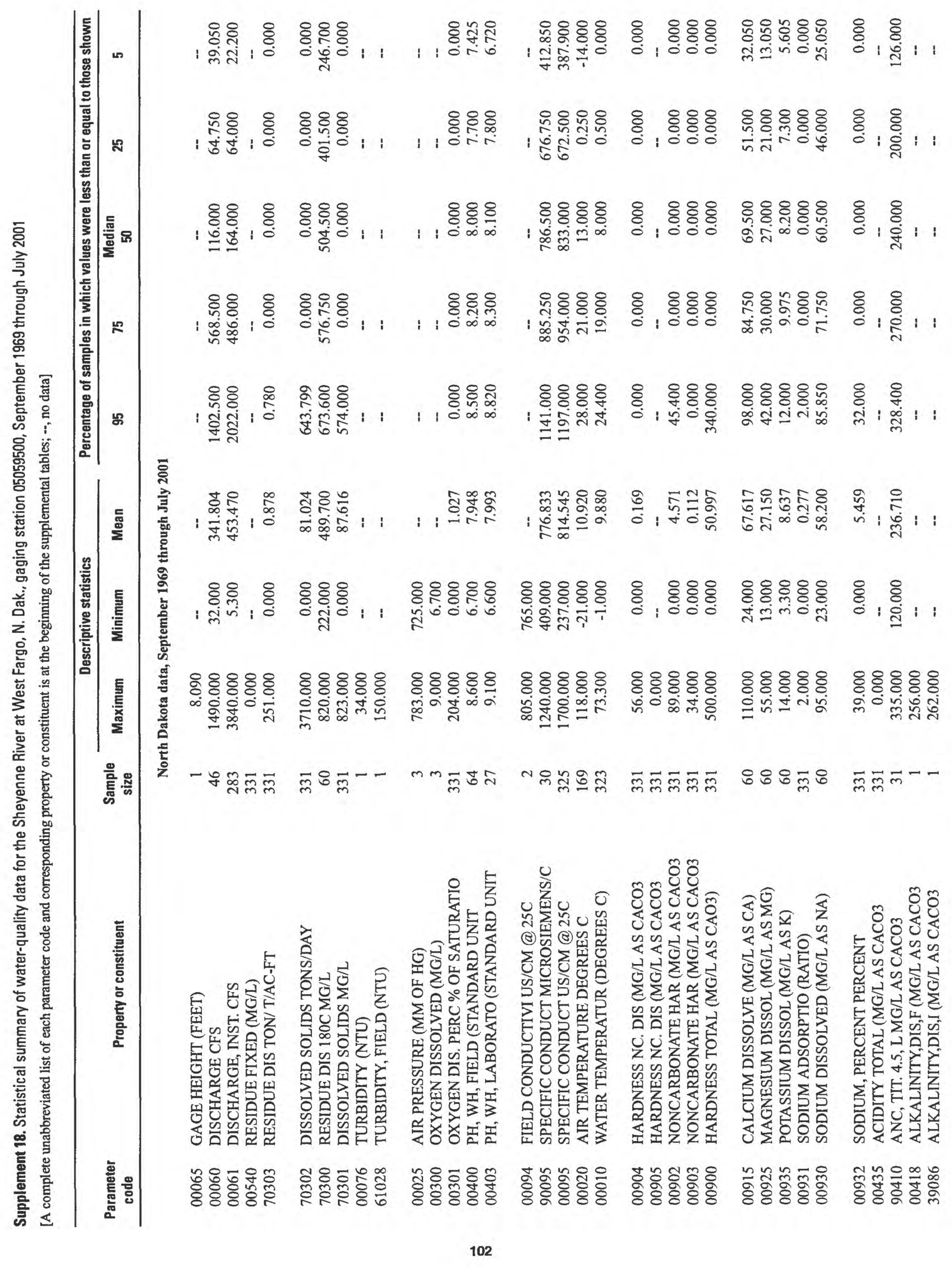




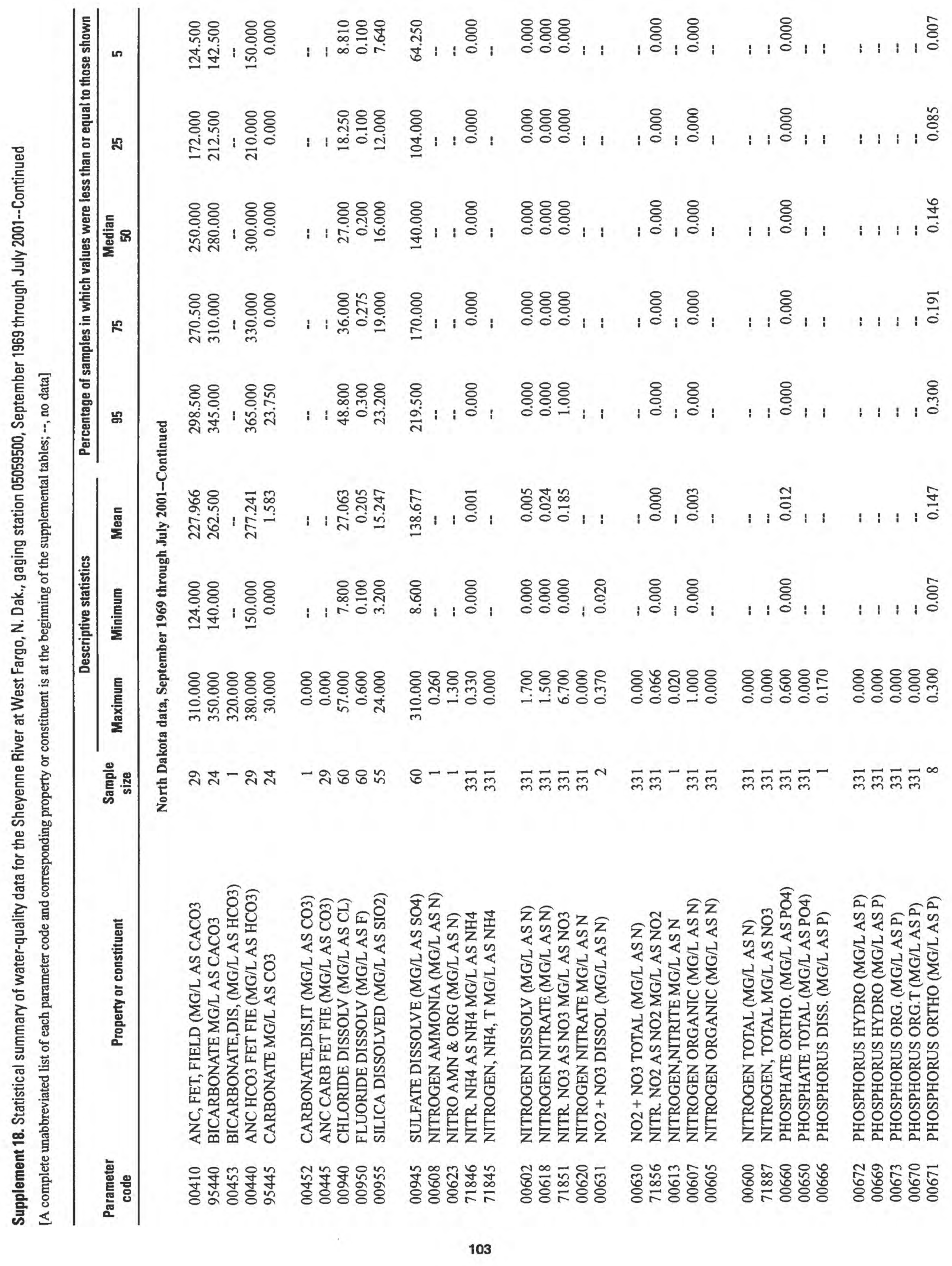




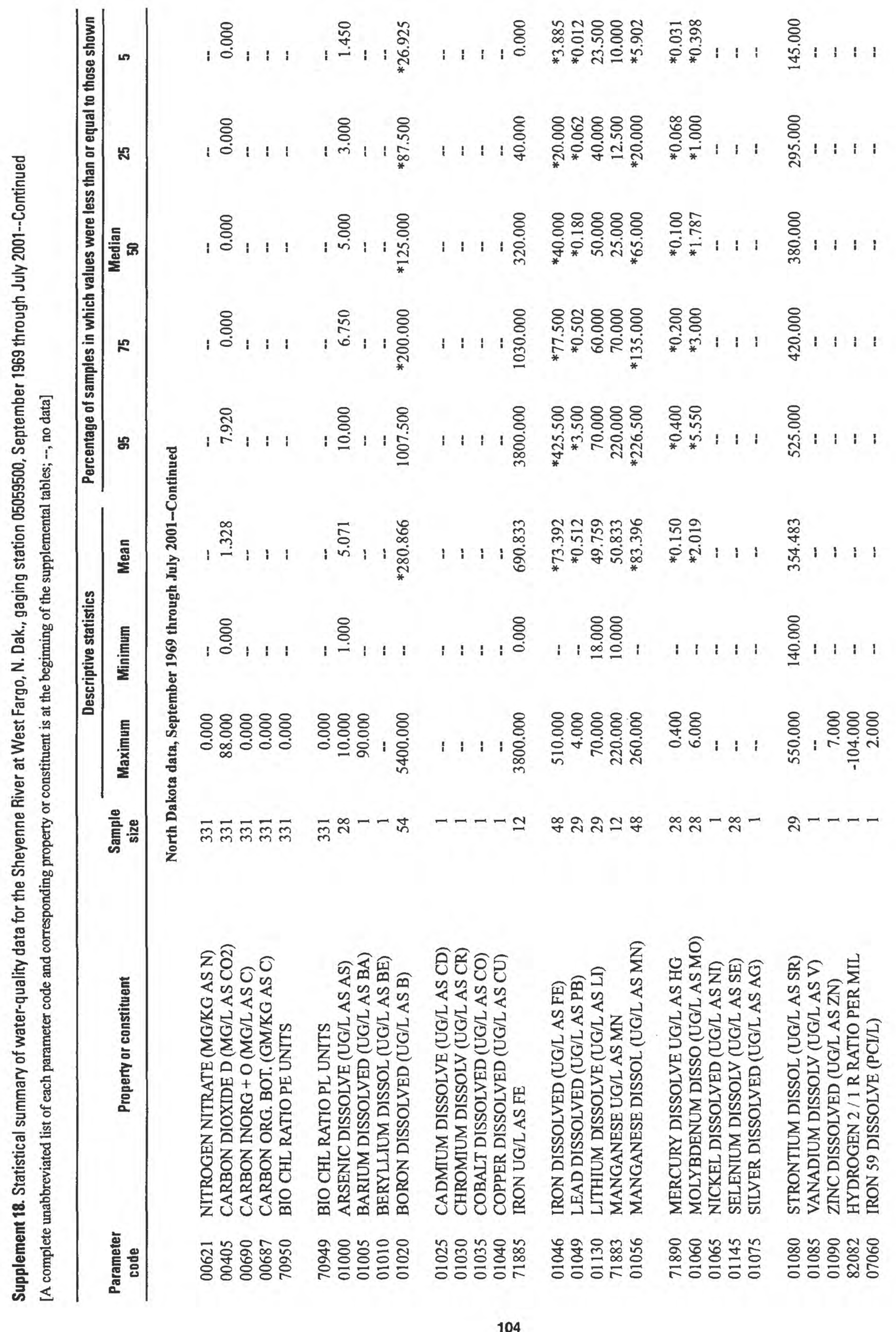




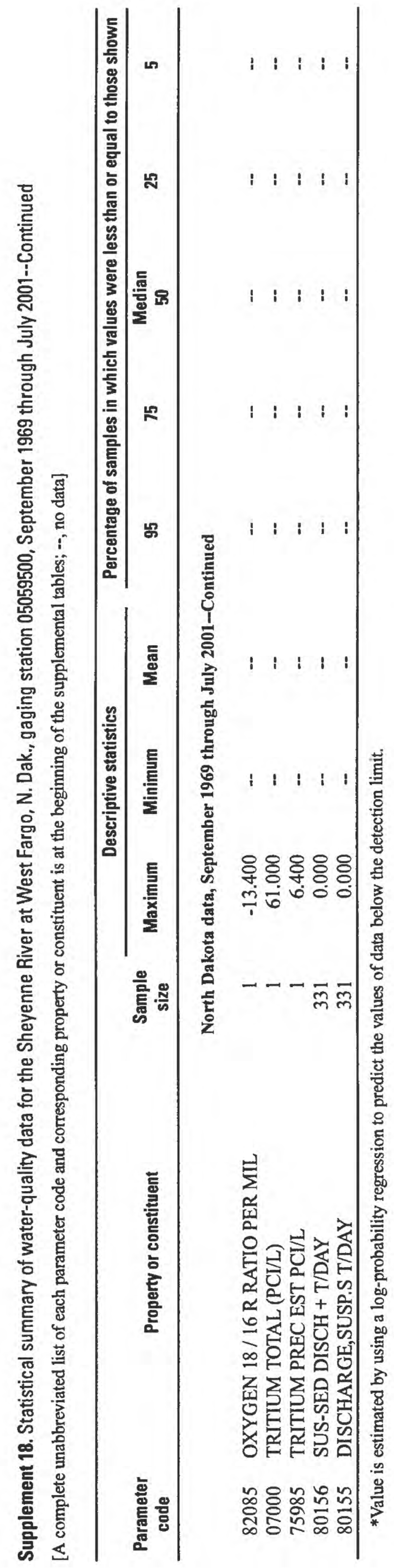




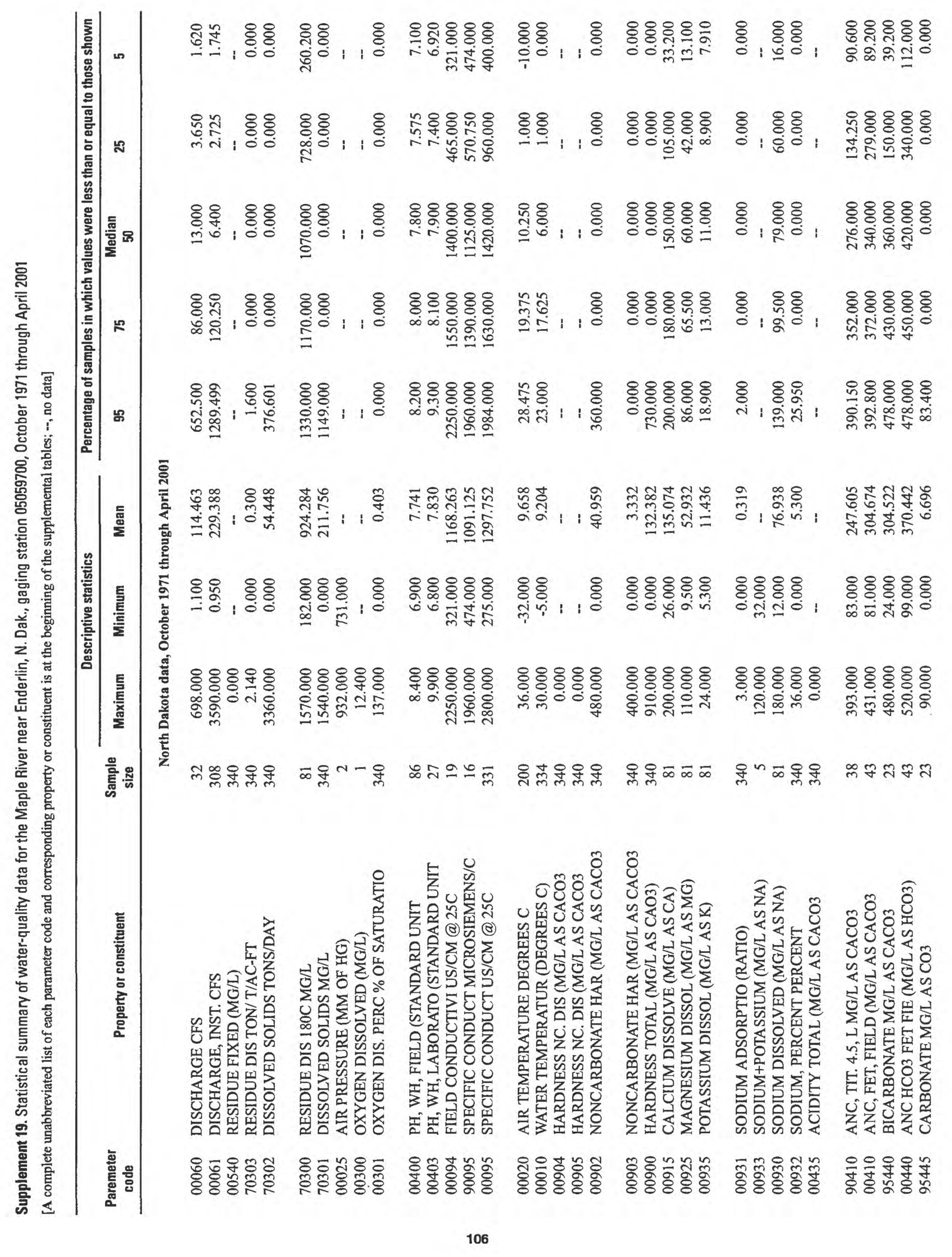




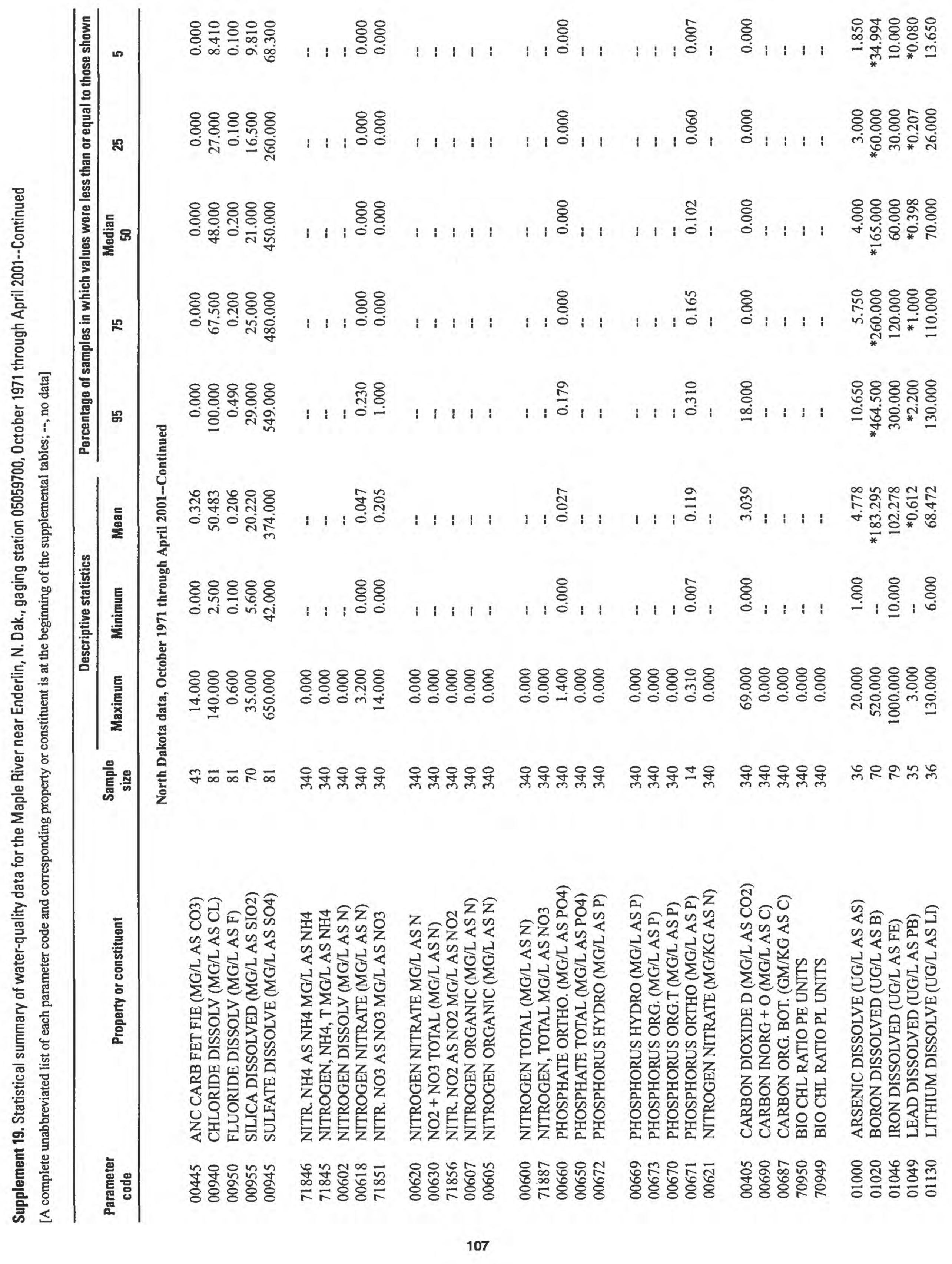




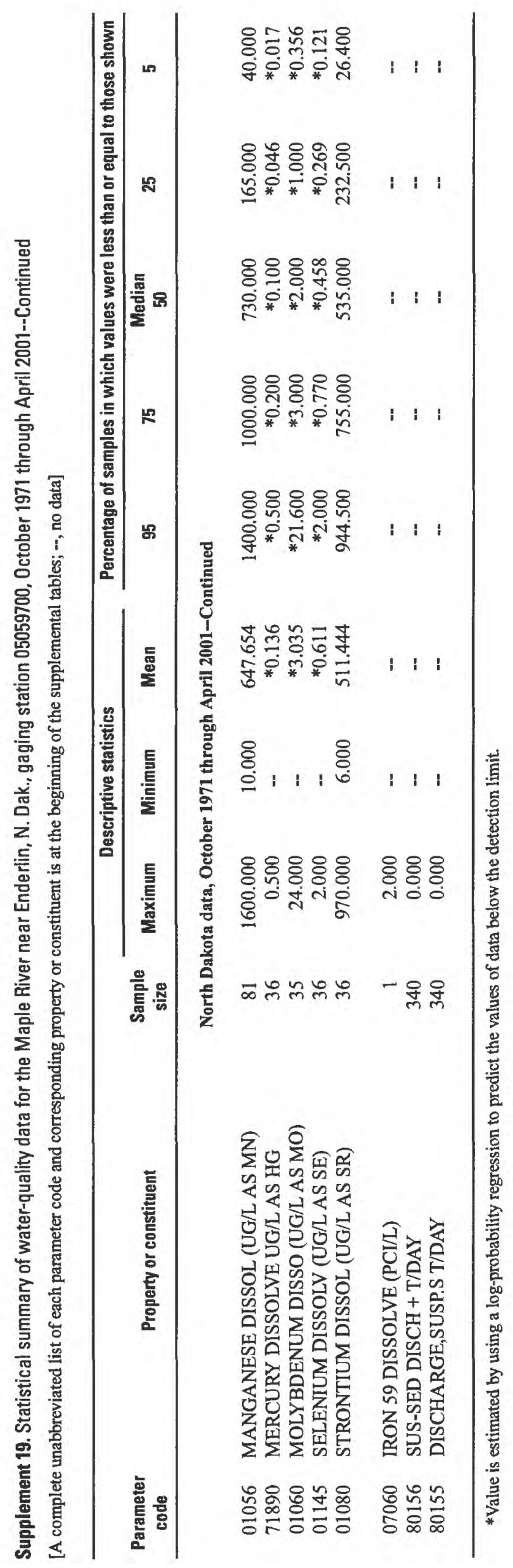




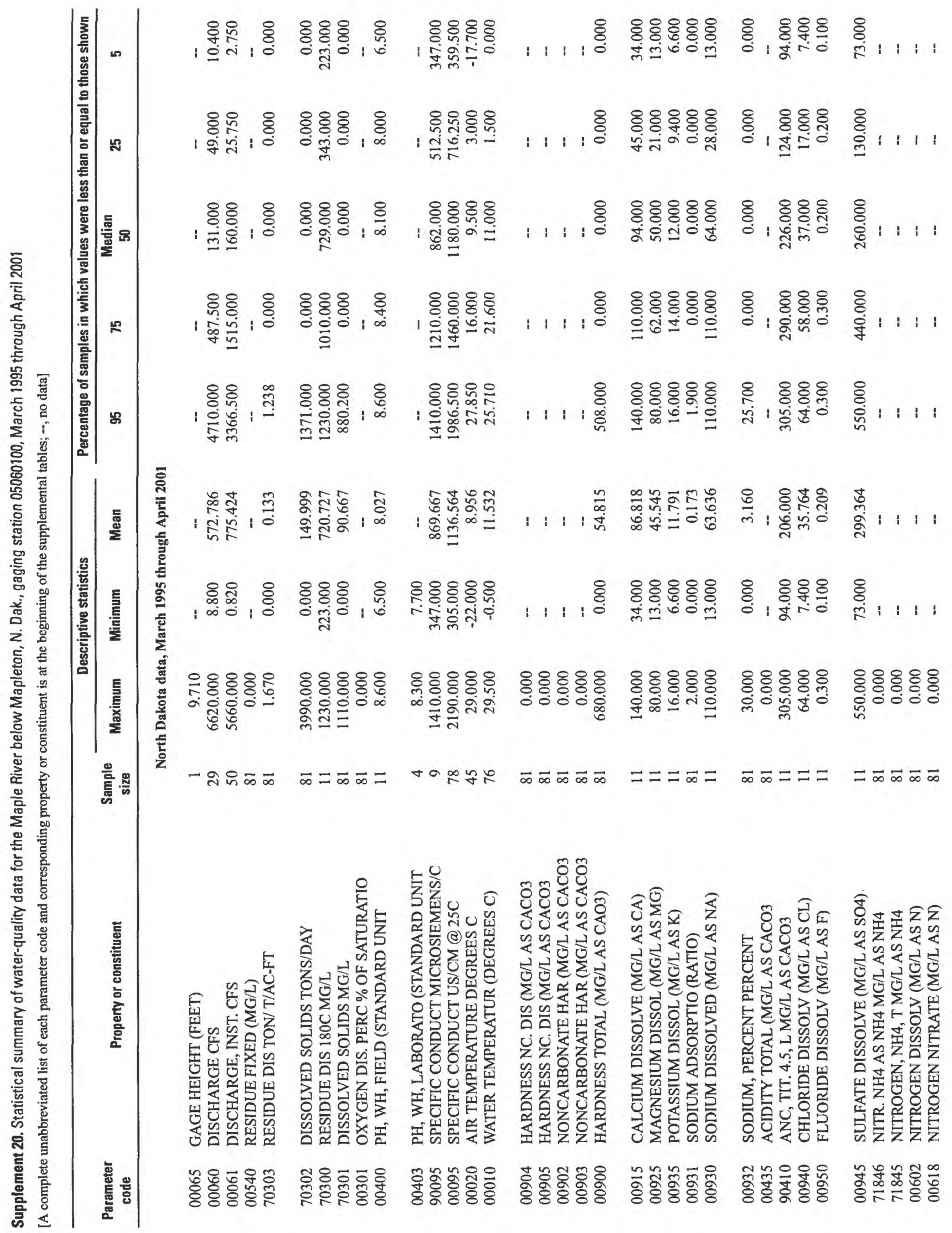




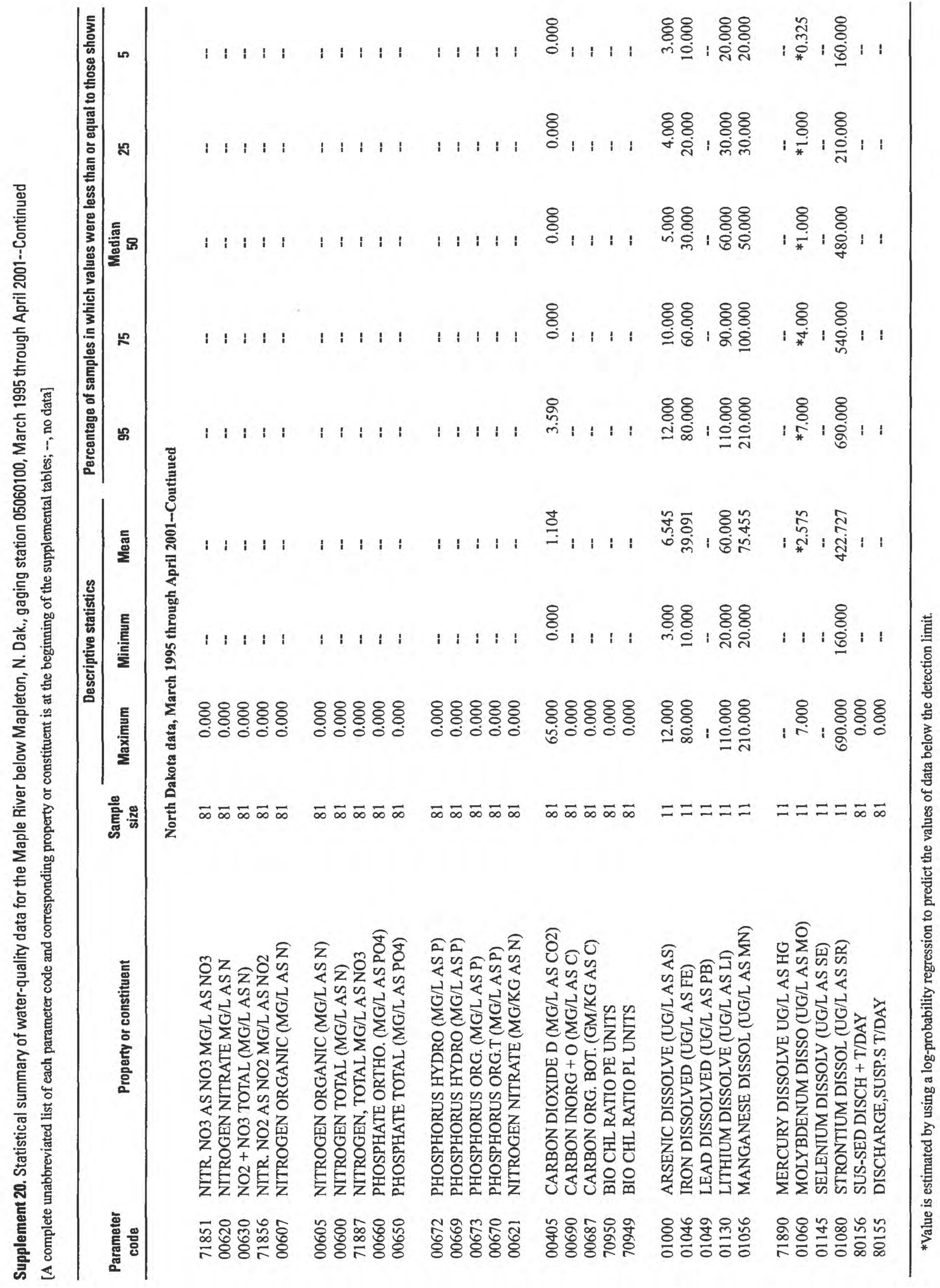




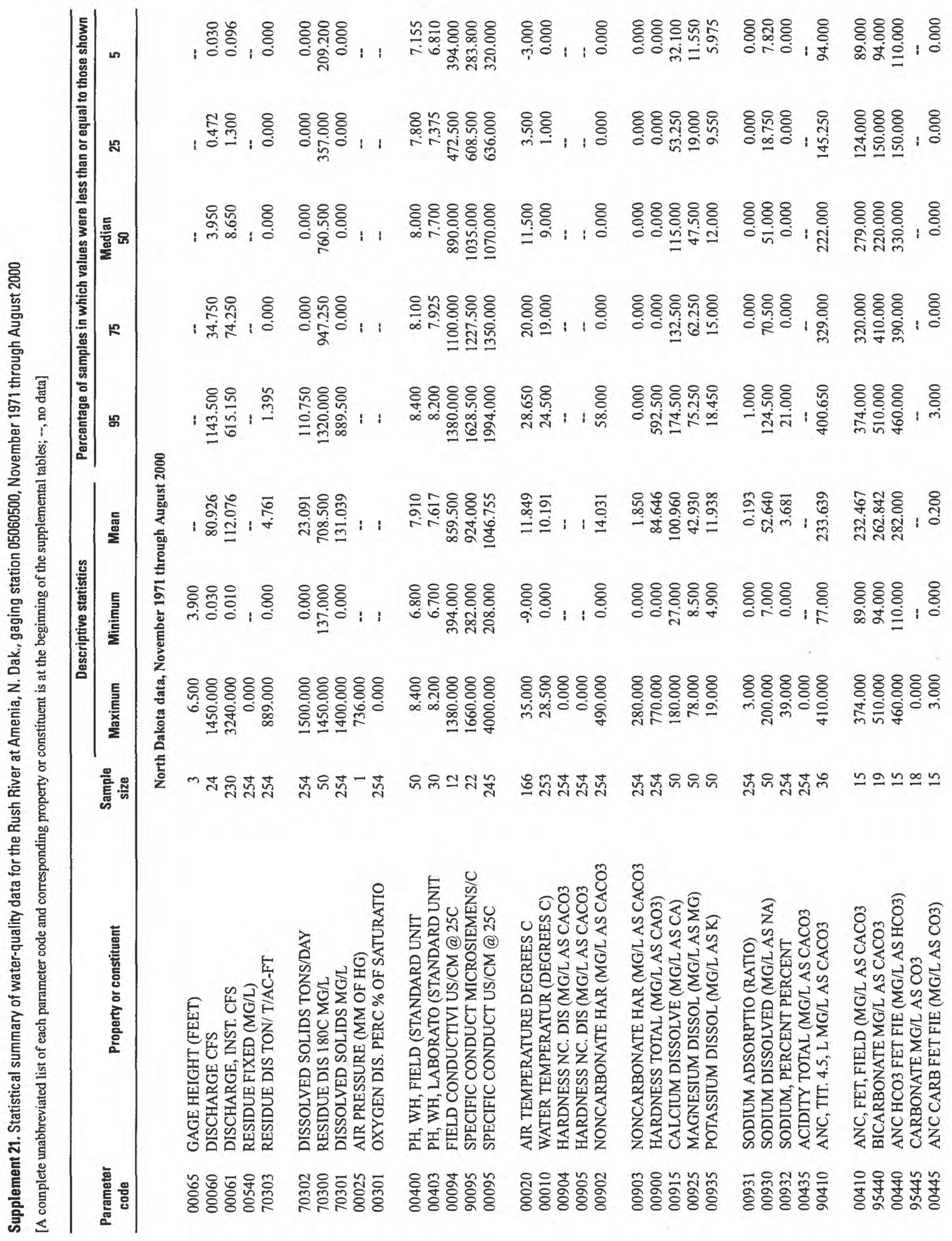




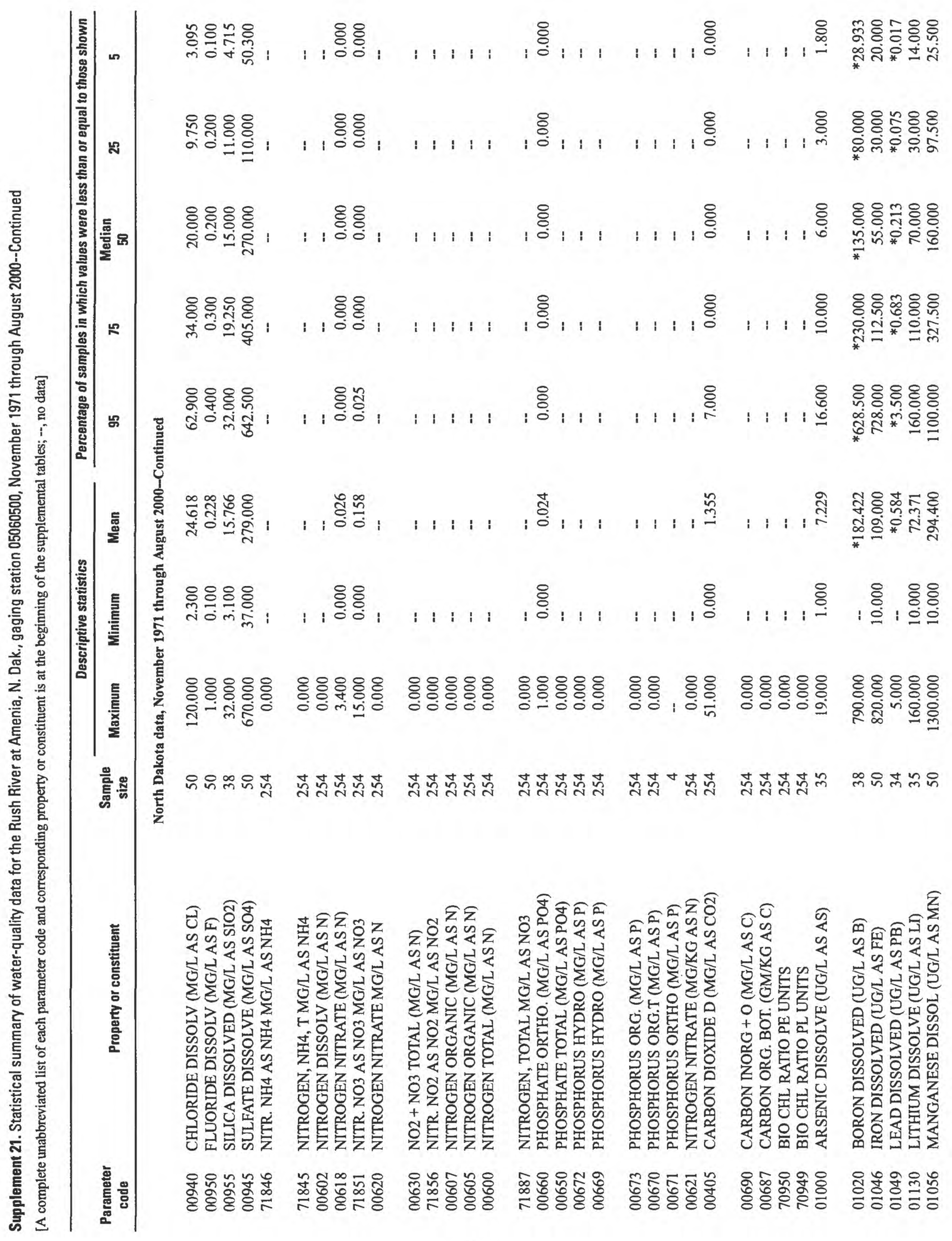




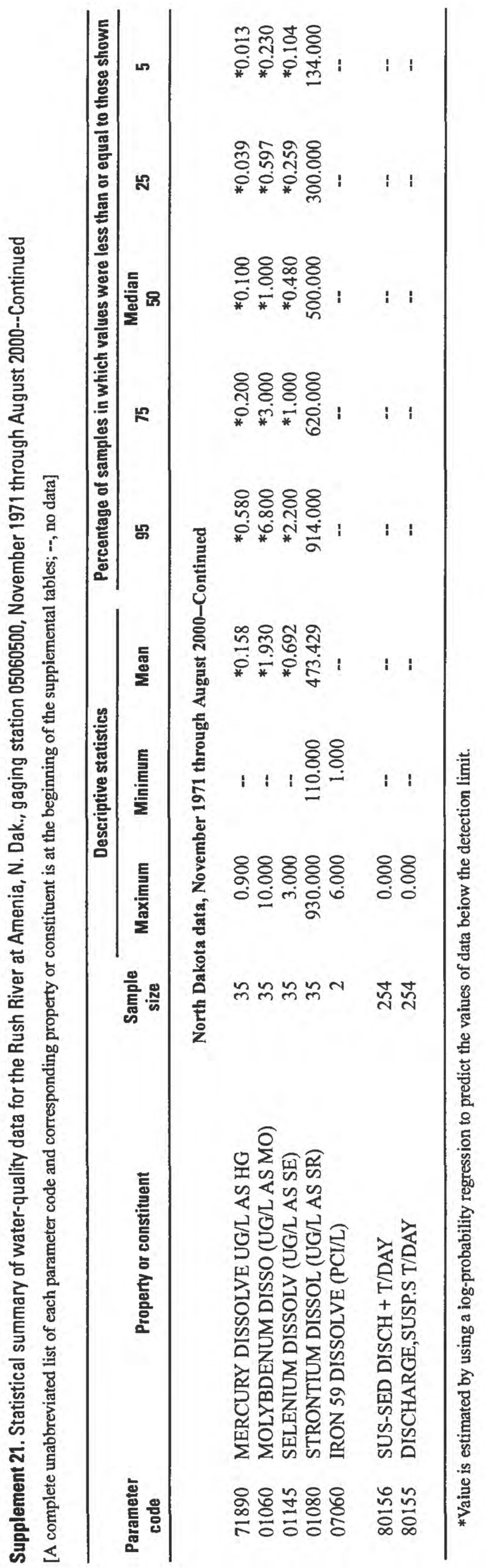




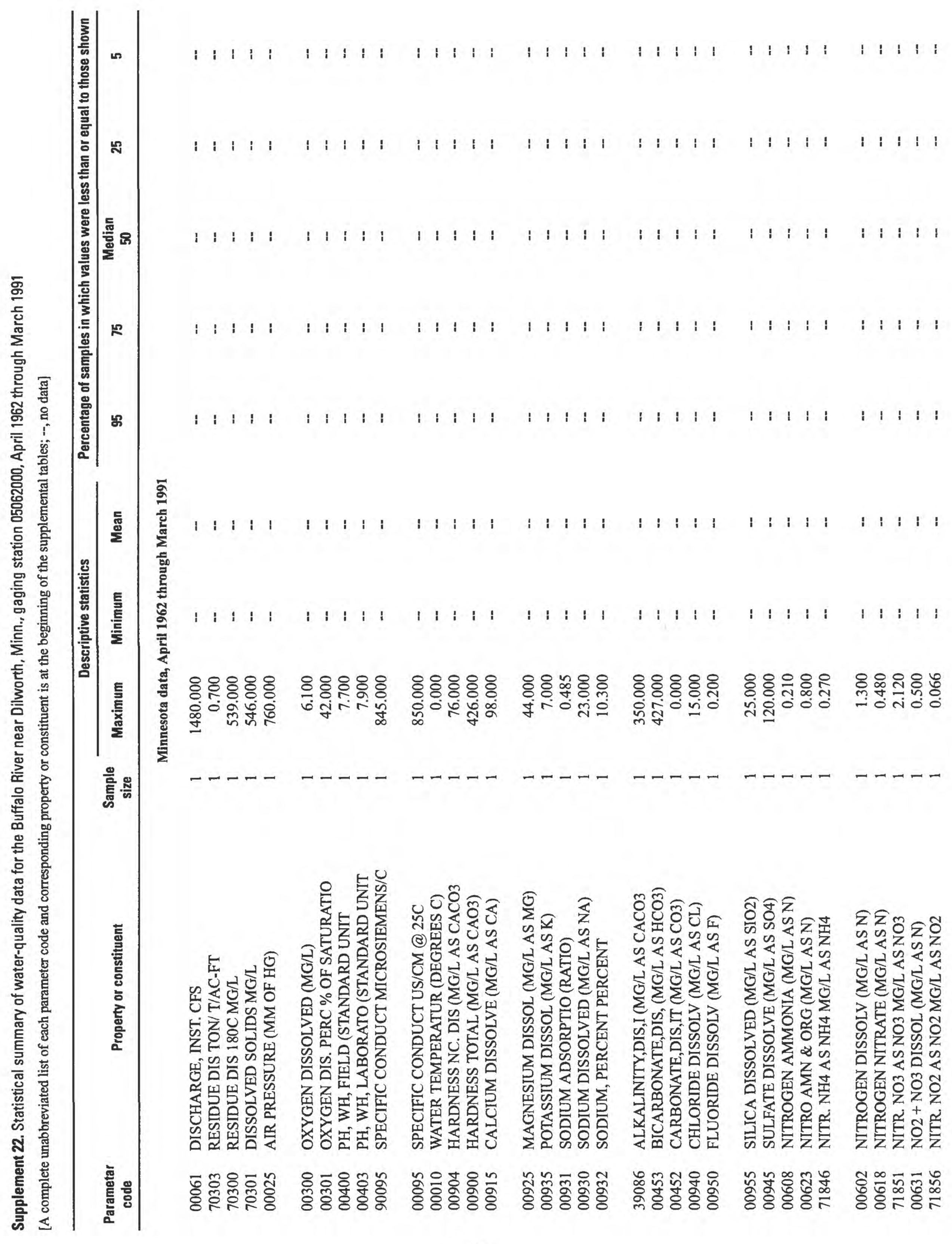




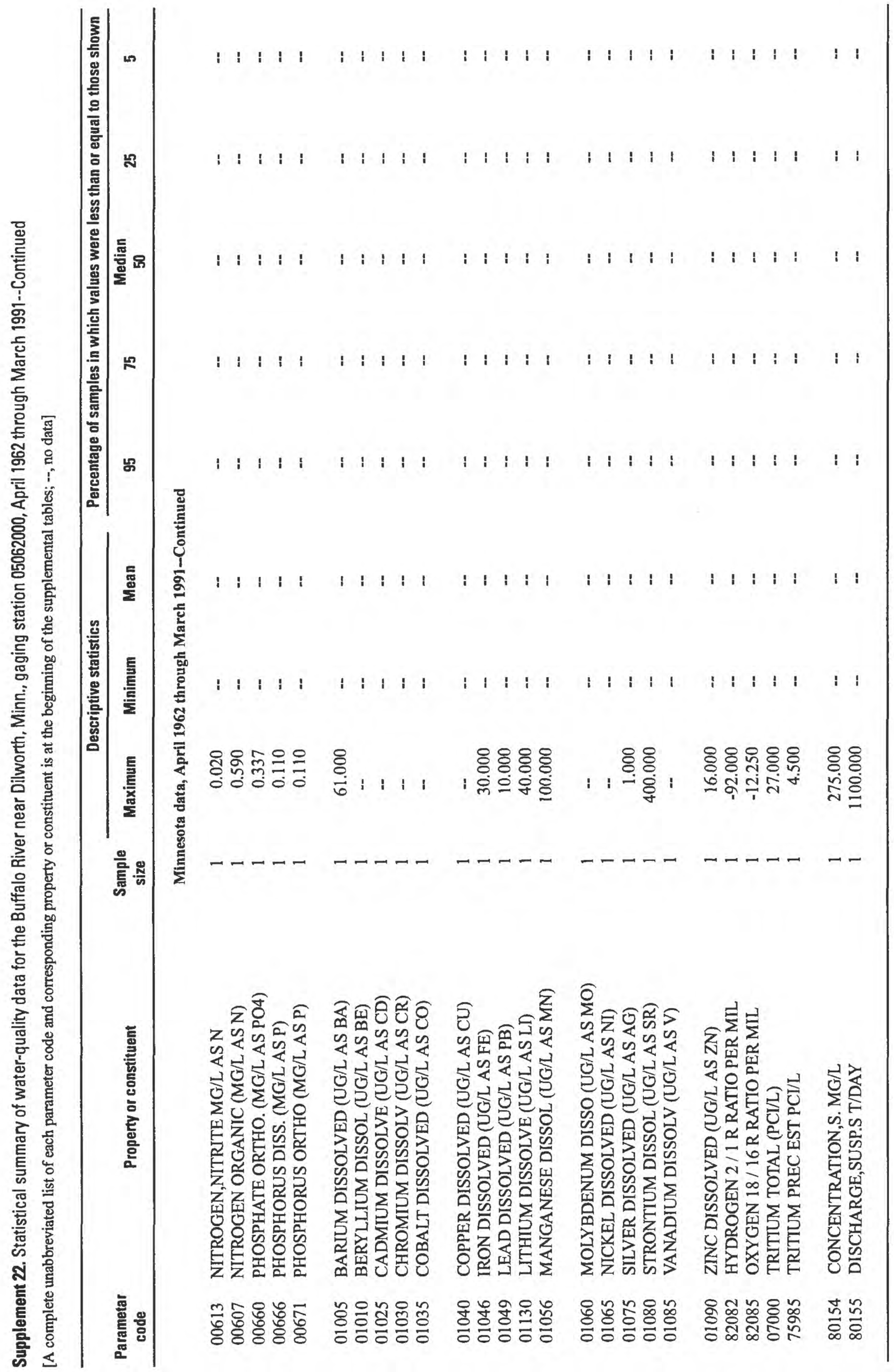




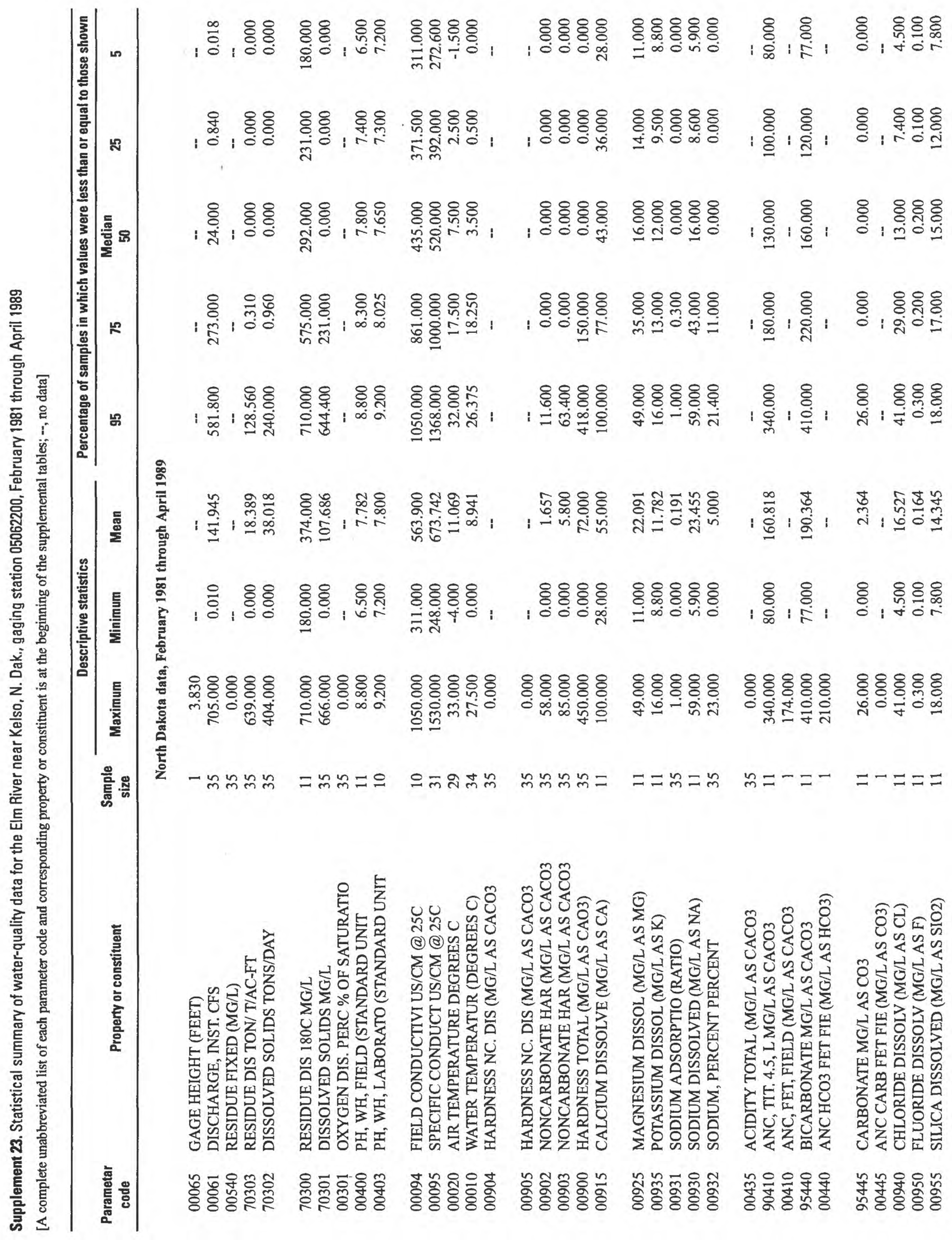




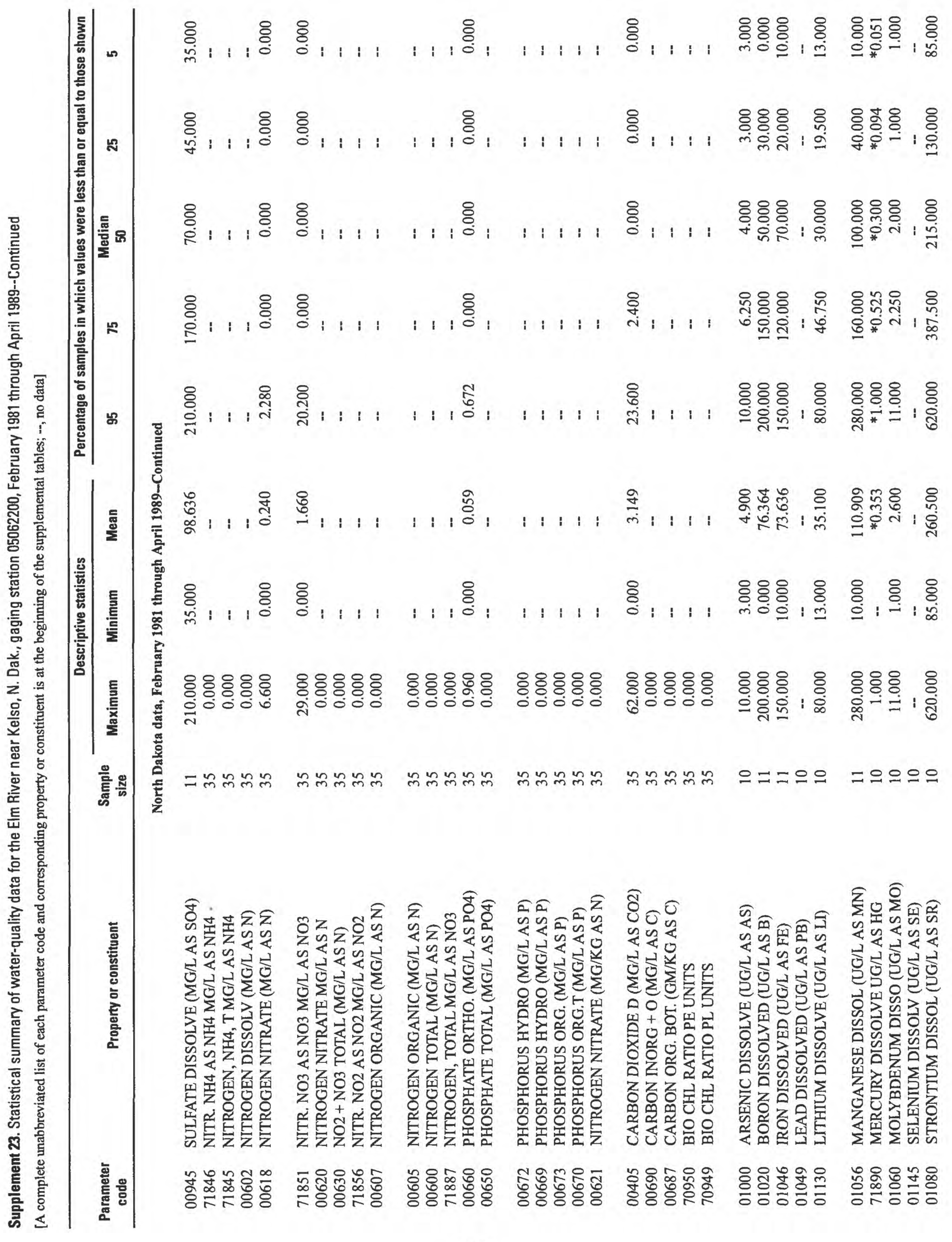




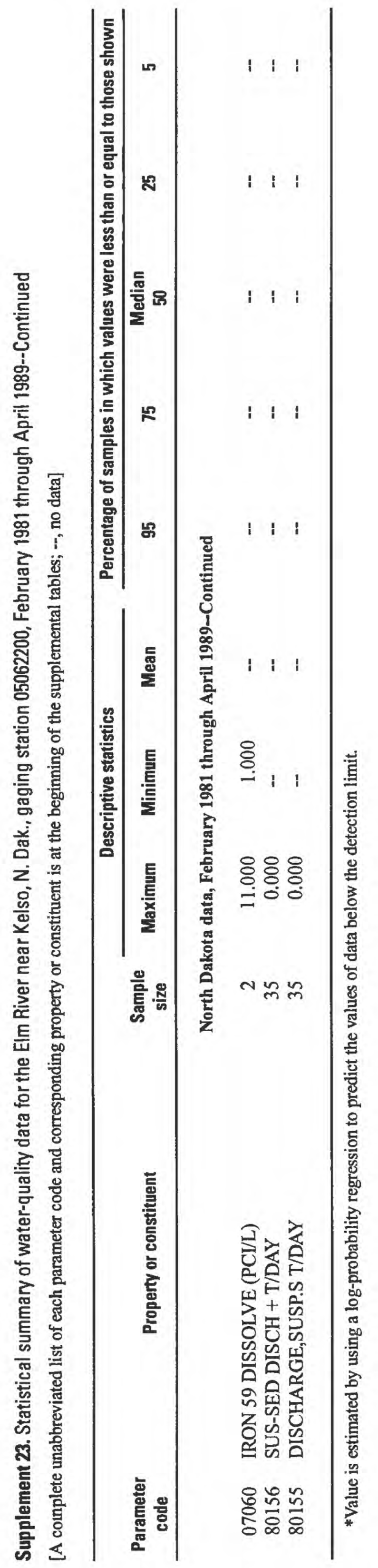




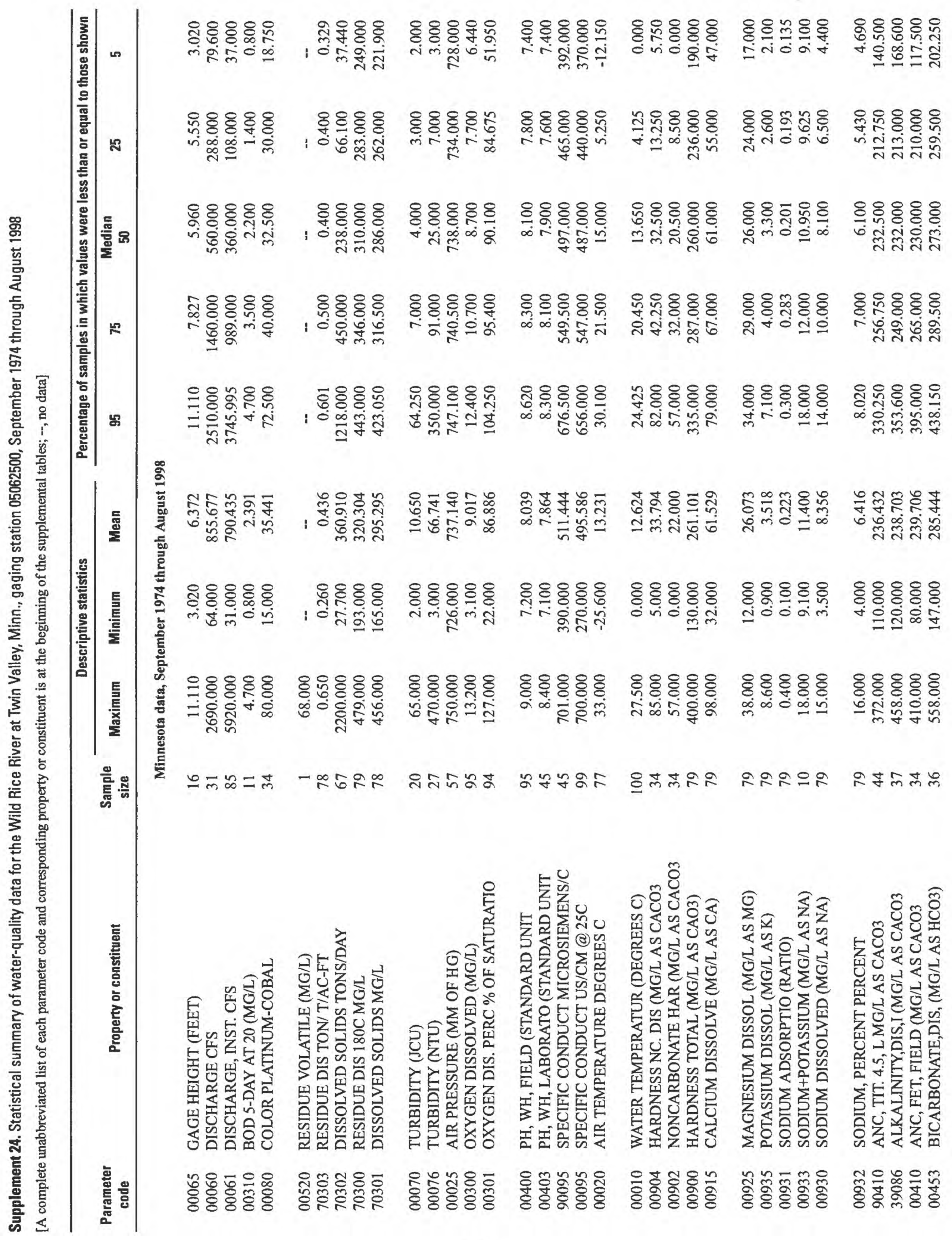




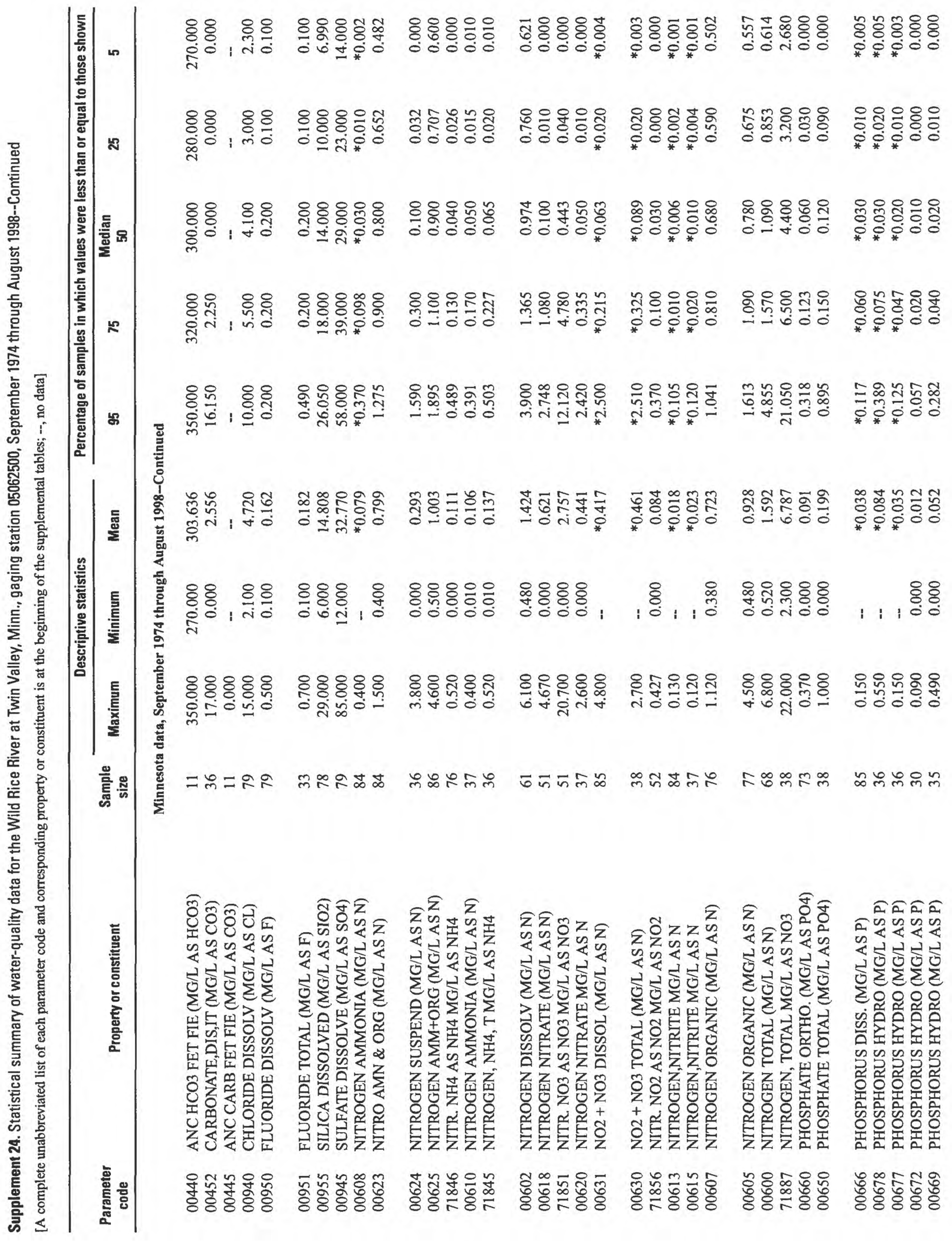




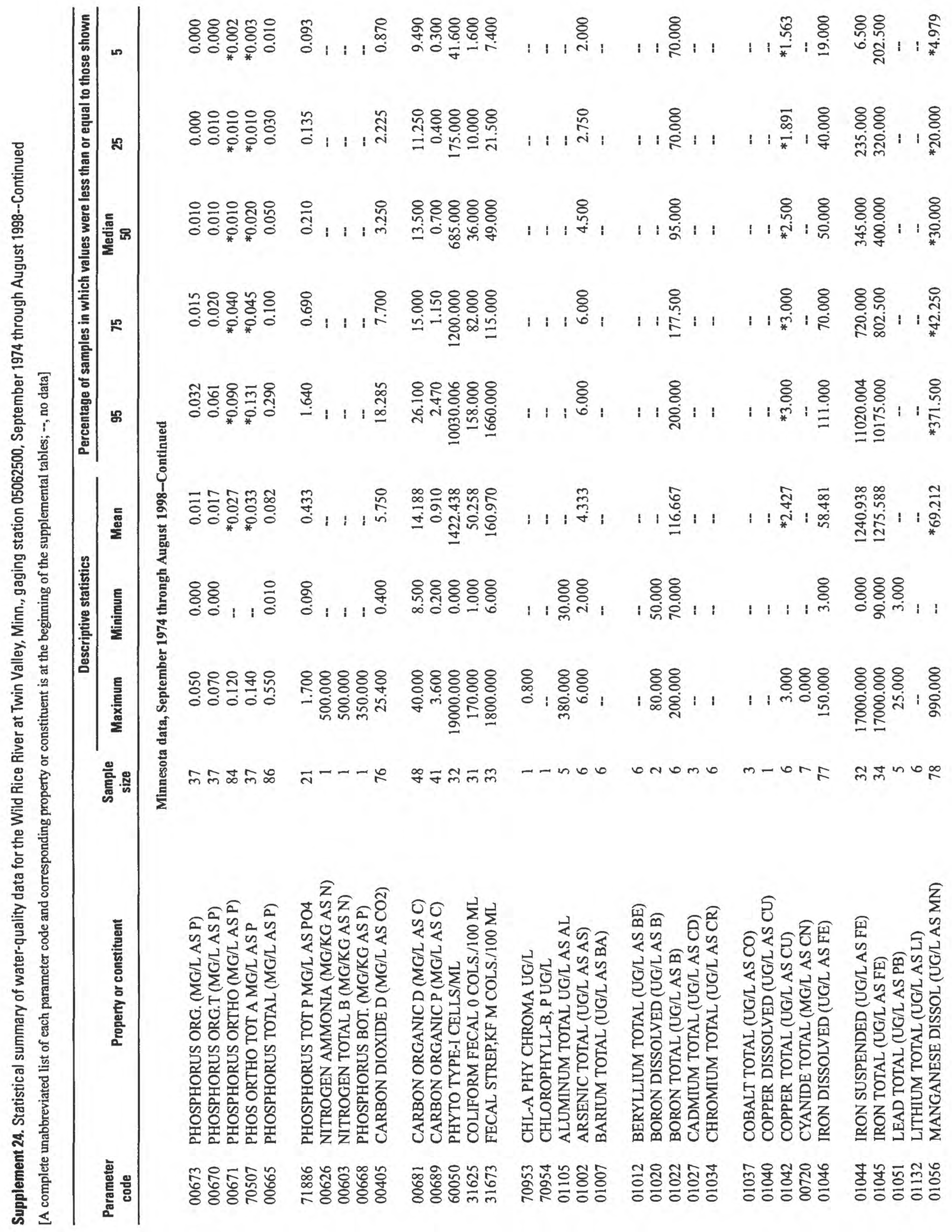




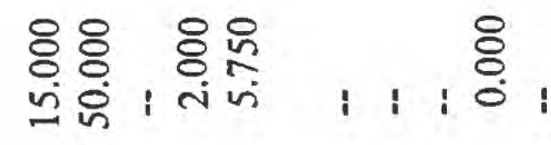

\&̊ㅇㅇㅇㅛ

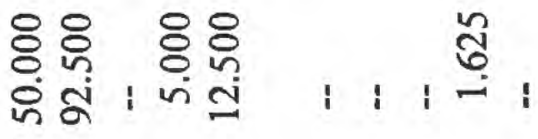

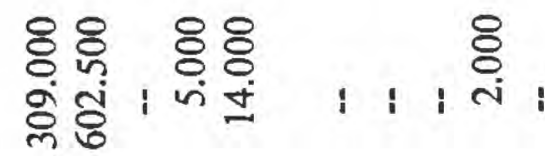

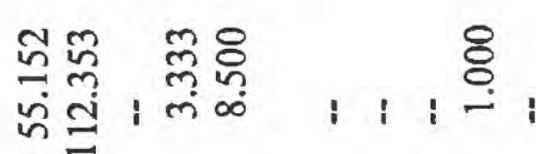

各

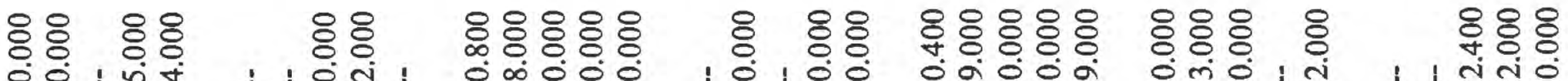

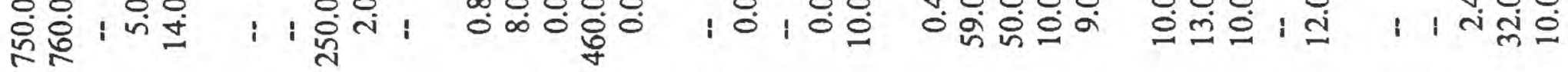

mुJ

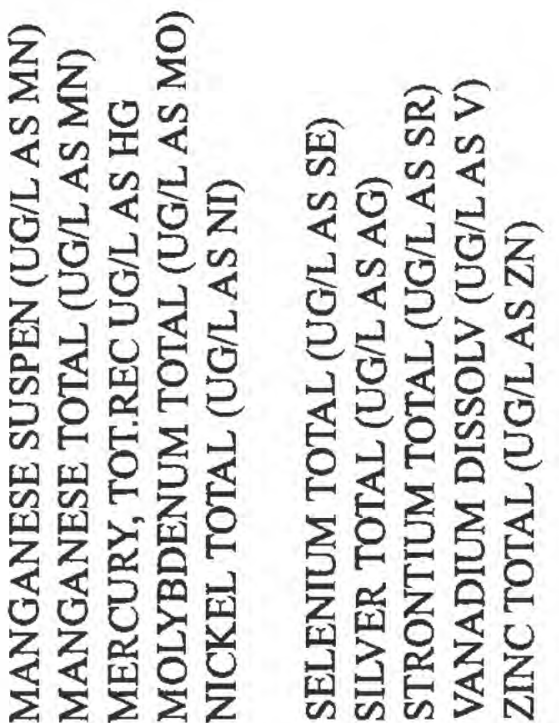

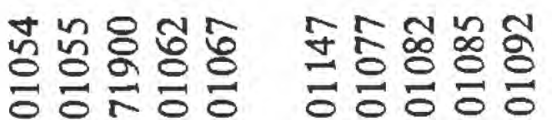

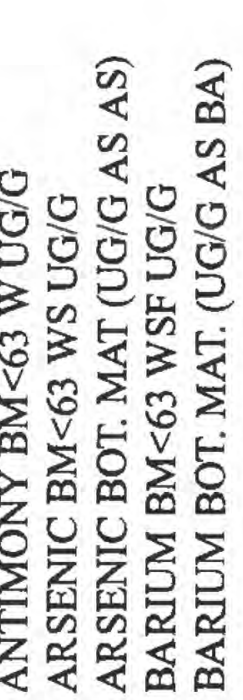

مै
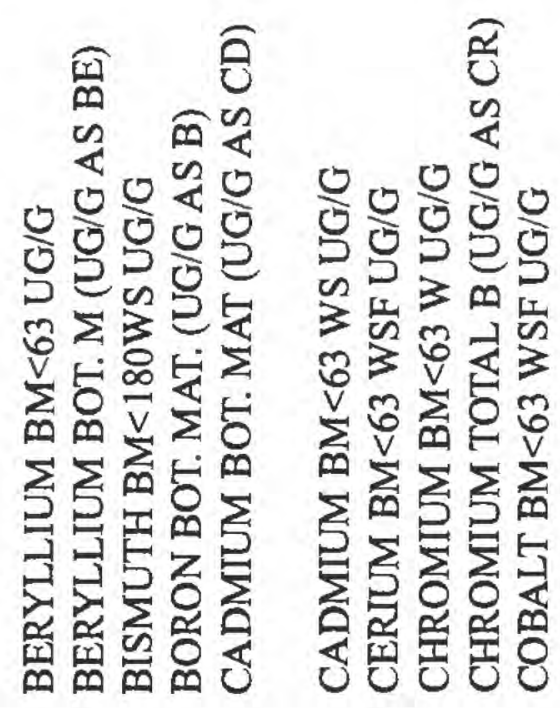

ช 8

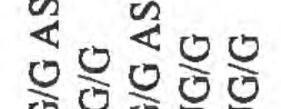

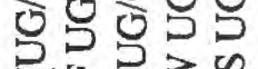

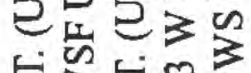

安安落

$\sum \hat{O} \sum_{i} \sum_{i}^{v} \sum_{i}^{v}$

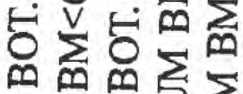

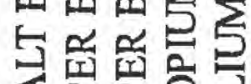

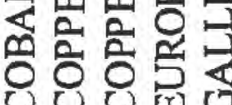

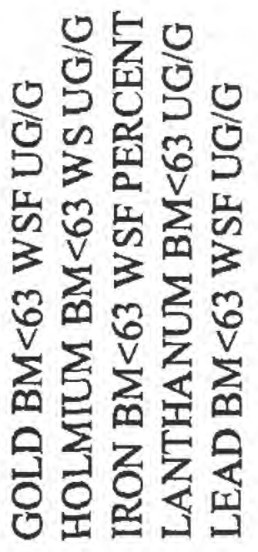

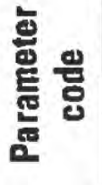

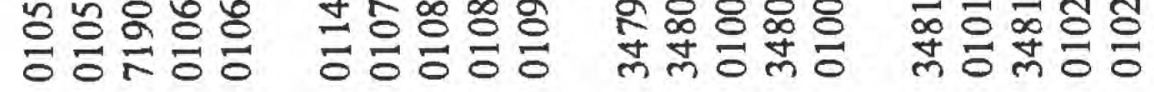

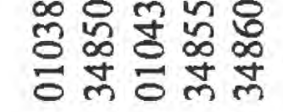

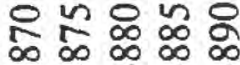

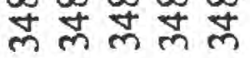




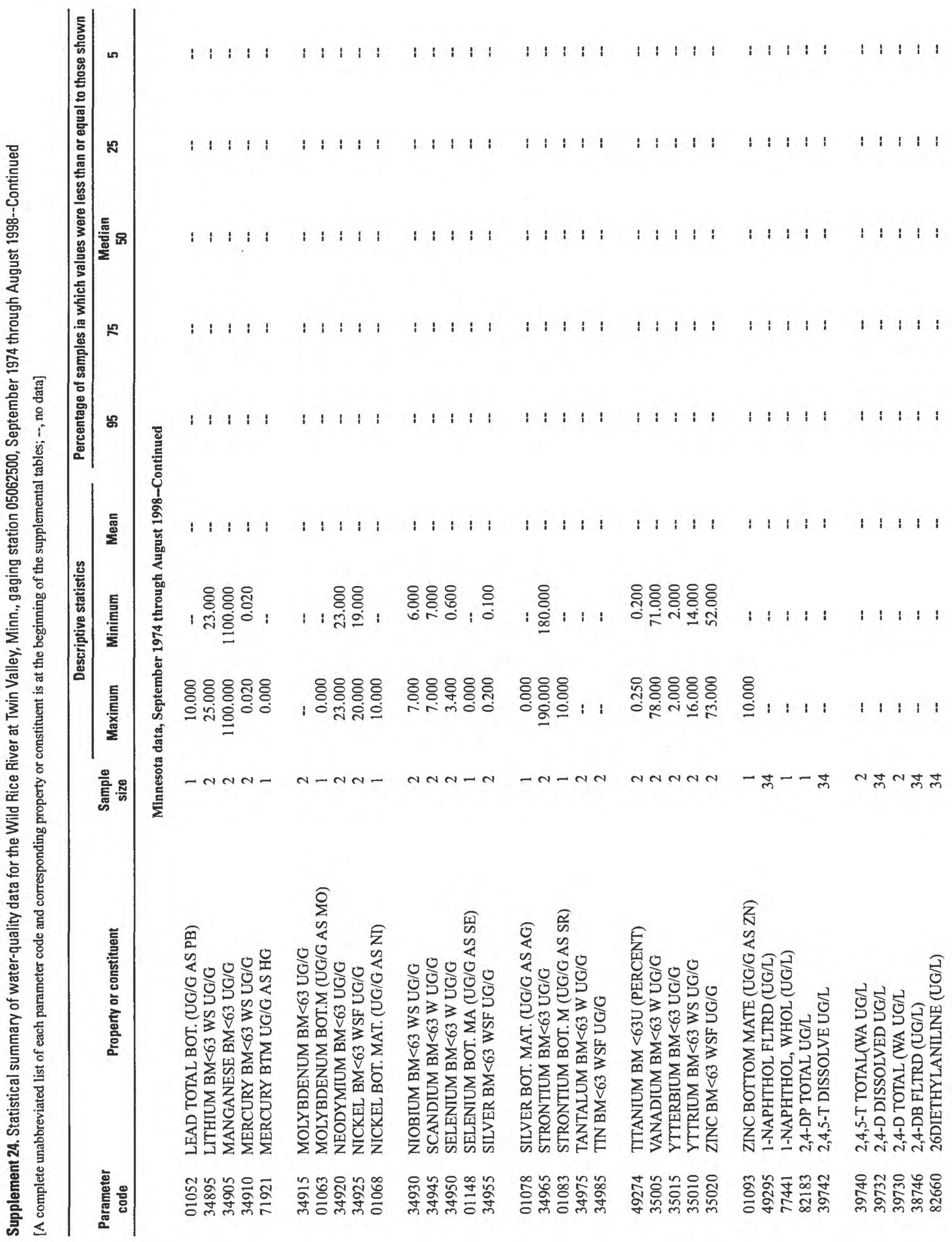




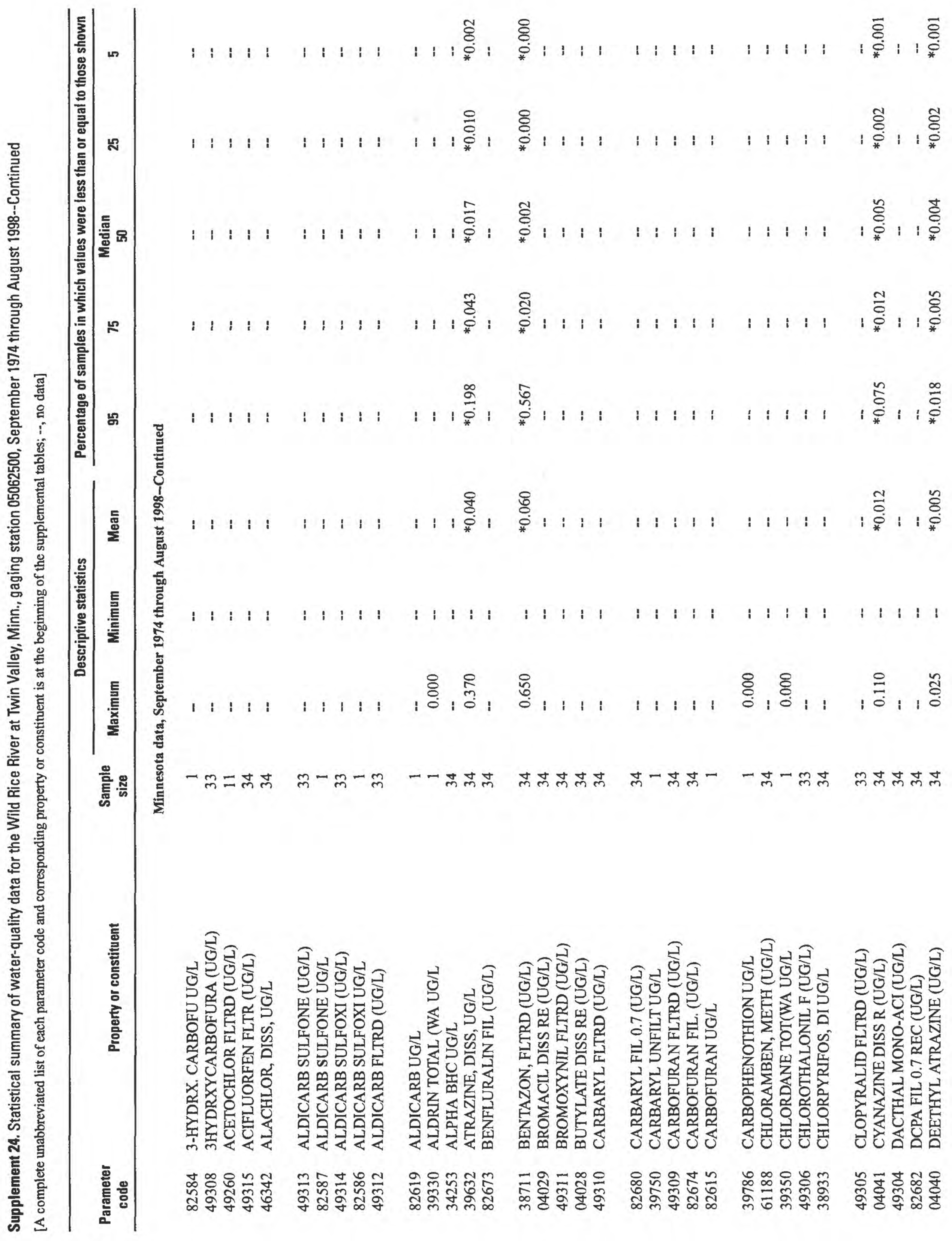




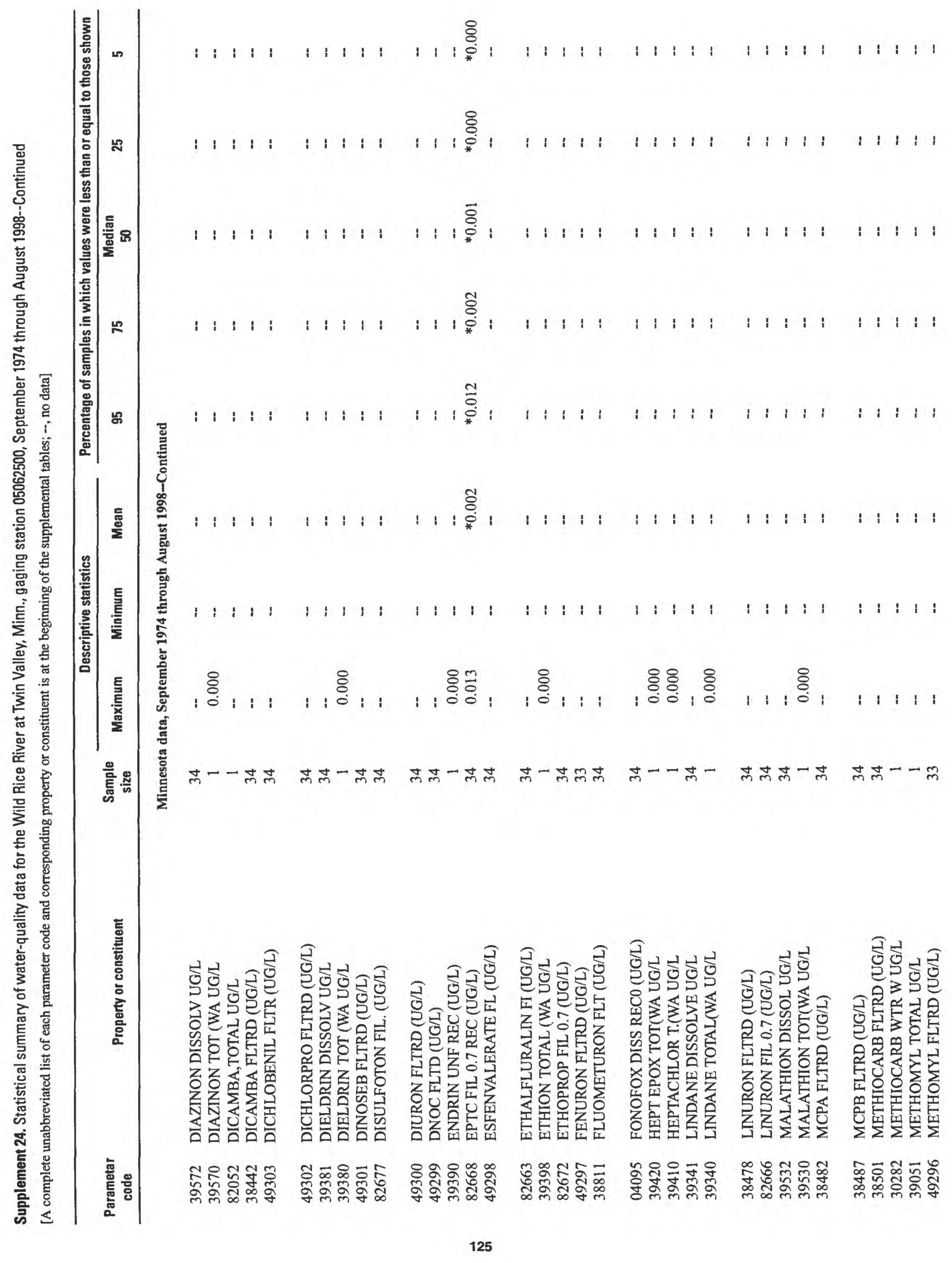




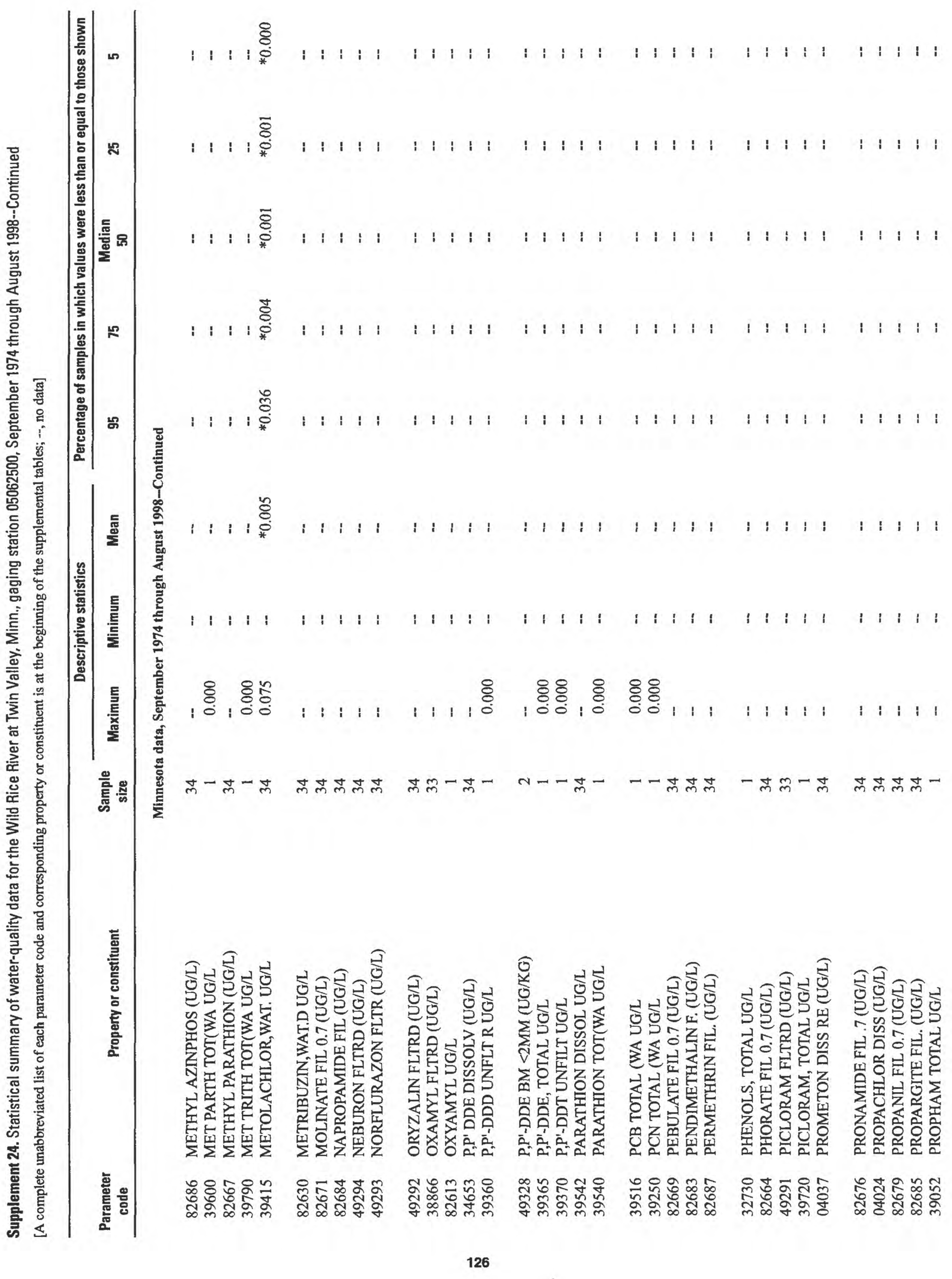




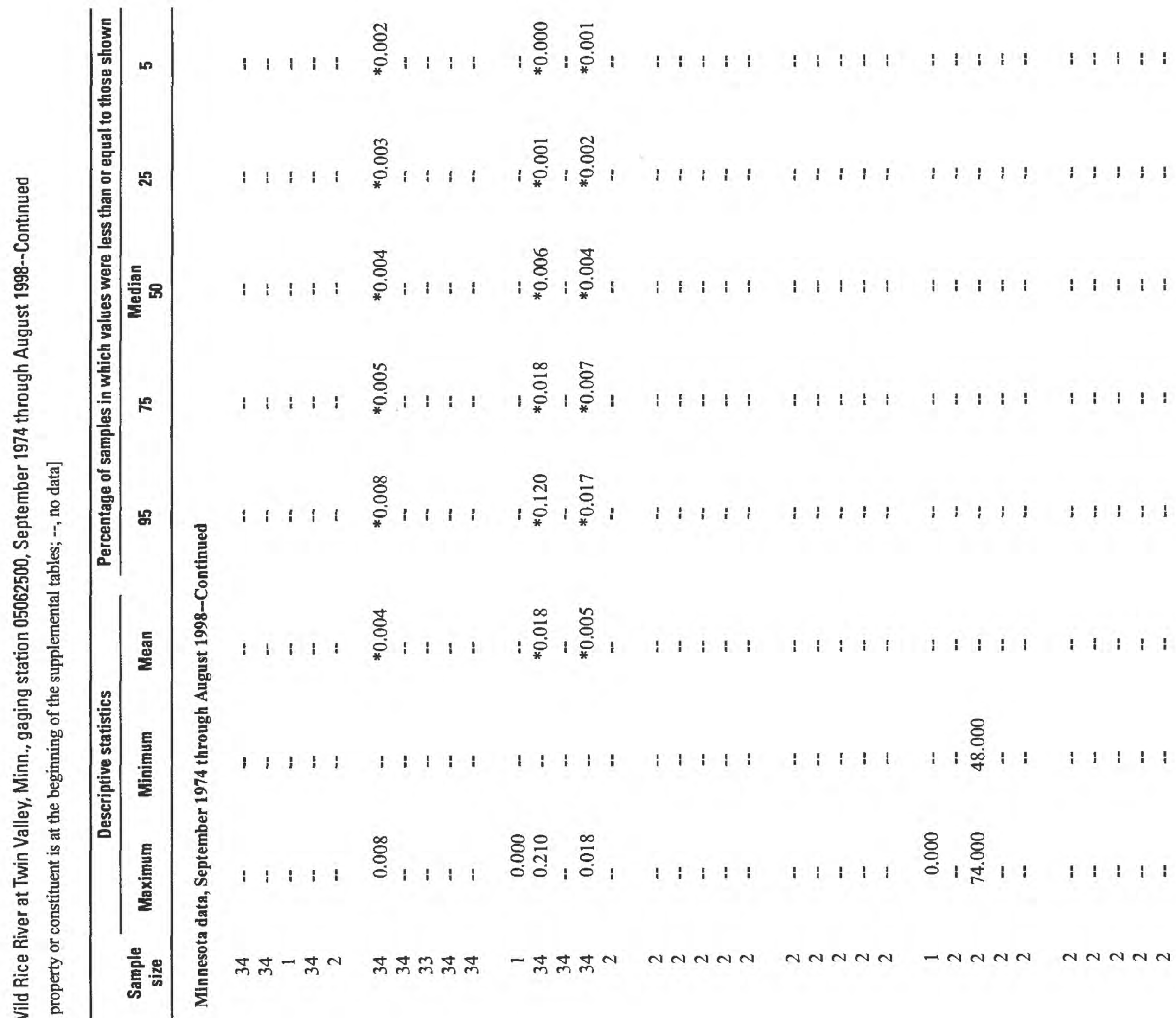

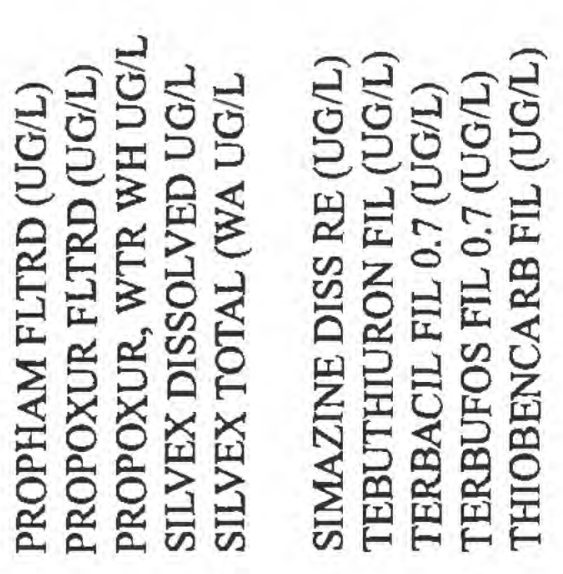

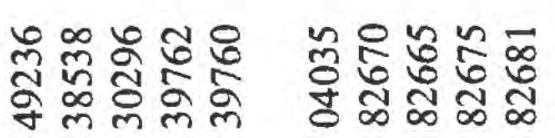

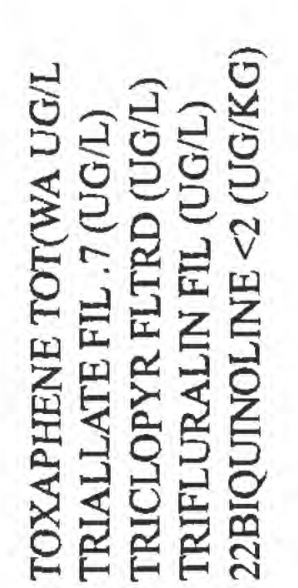

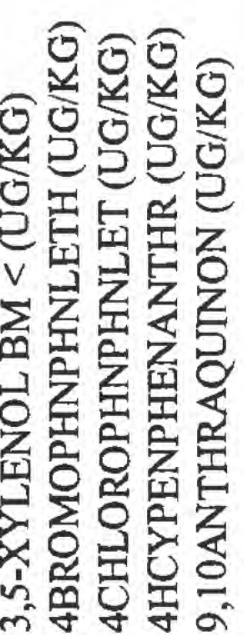

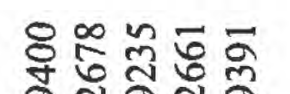

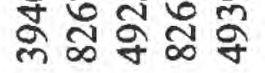

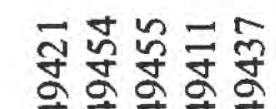
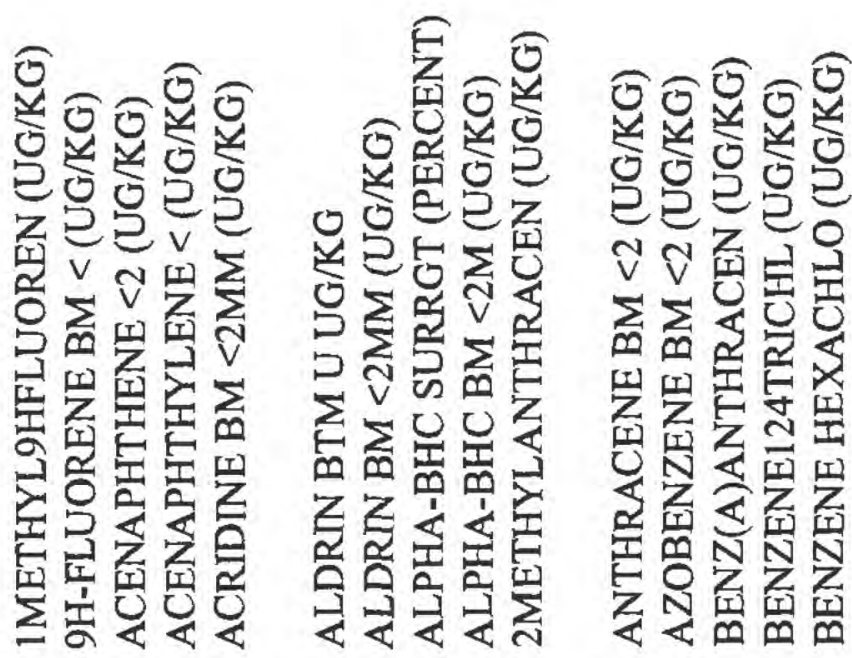

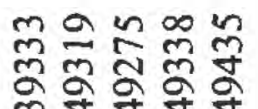

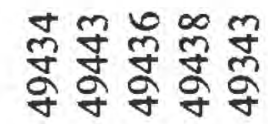




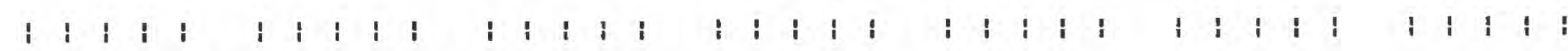

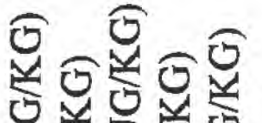

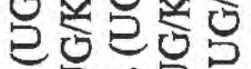
닐ㄹㄹㄹ 证空

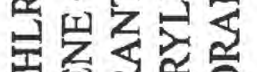

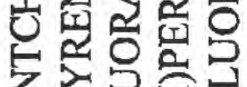
衣完星

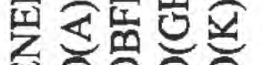
선선선

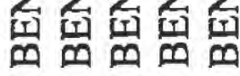

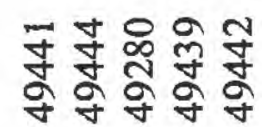

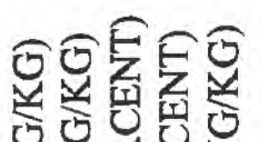
起学是

m 를

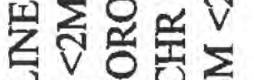

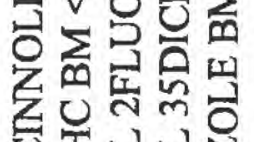

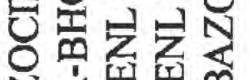

선될

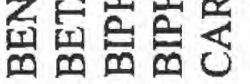
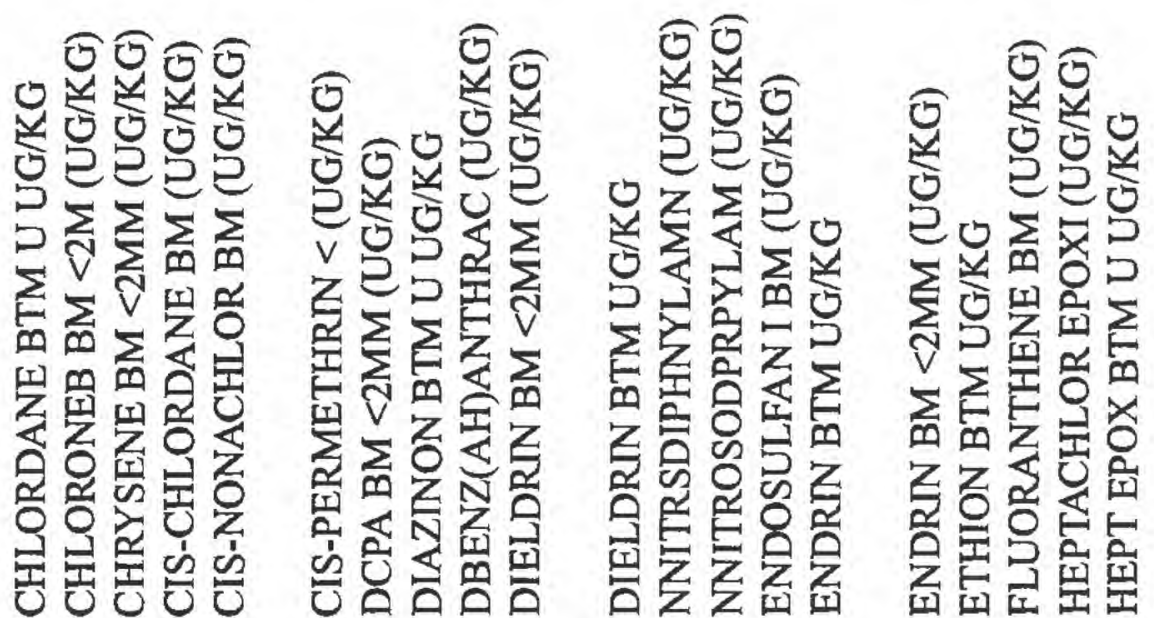


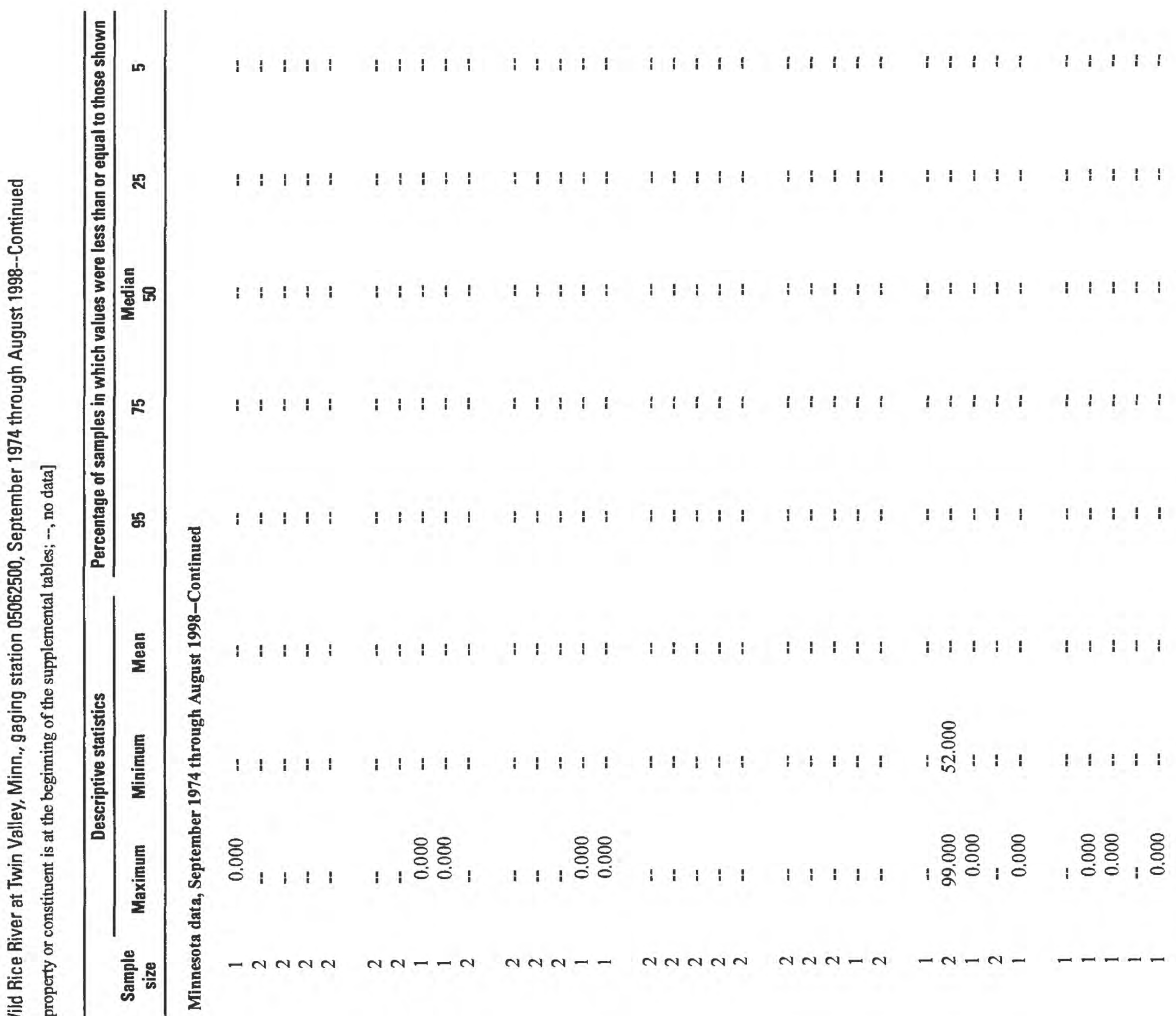

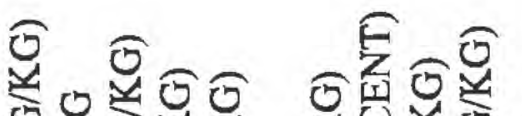

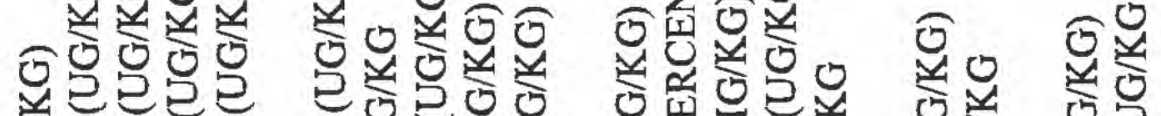

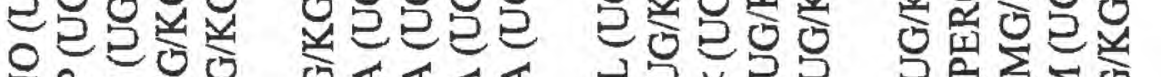

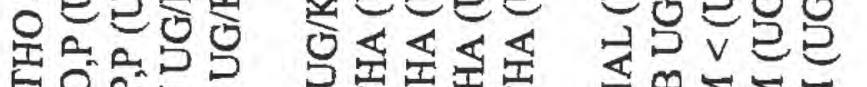

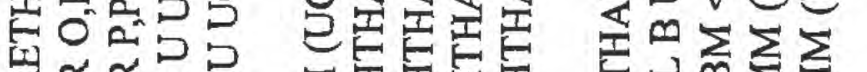

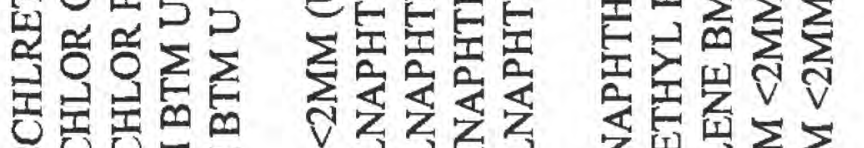


; 1 1

i 1 i i

I I I i

I 1 i

1 1 i

I 1

잉유유.

क्ष ni :

융ㅇํำ

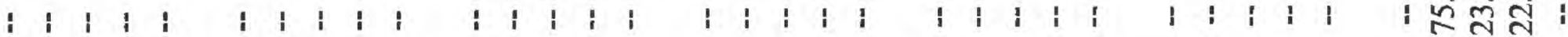

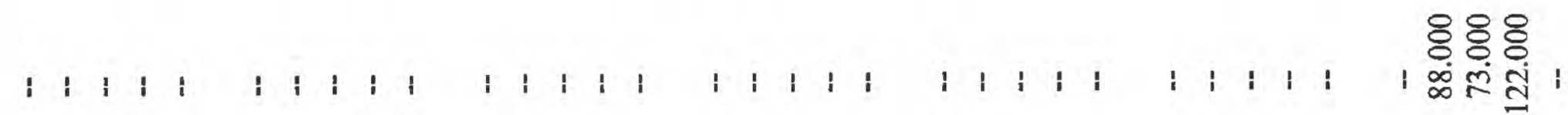

88

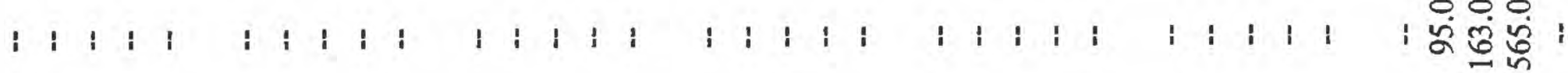

88.8

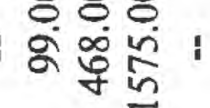

i i i

i i i

$1+1$

1111

1 1 1

1 11

th

|

, 1 i

,

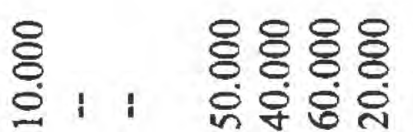

옹

888.

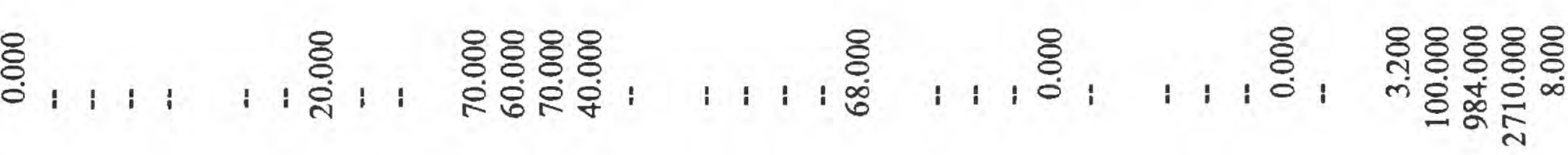

mene

$\operatorname{chn} 4$

$\operatorname{4nNen~}$

NNNNN

$44 n-4$

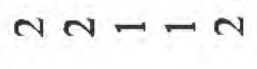

ㄱํำ
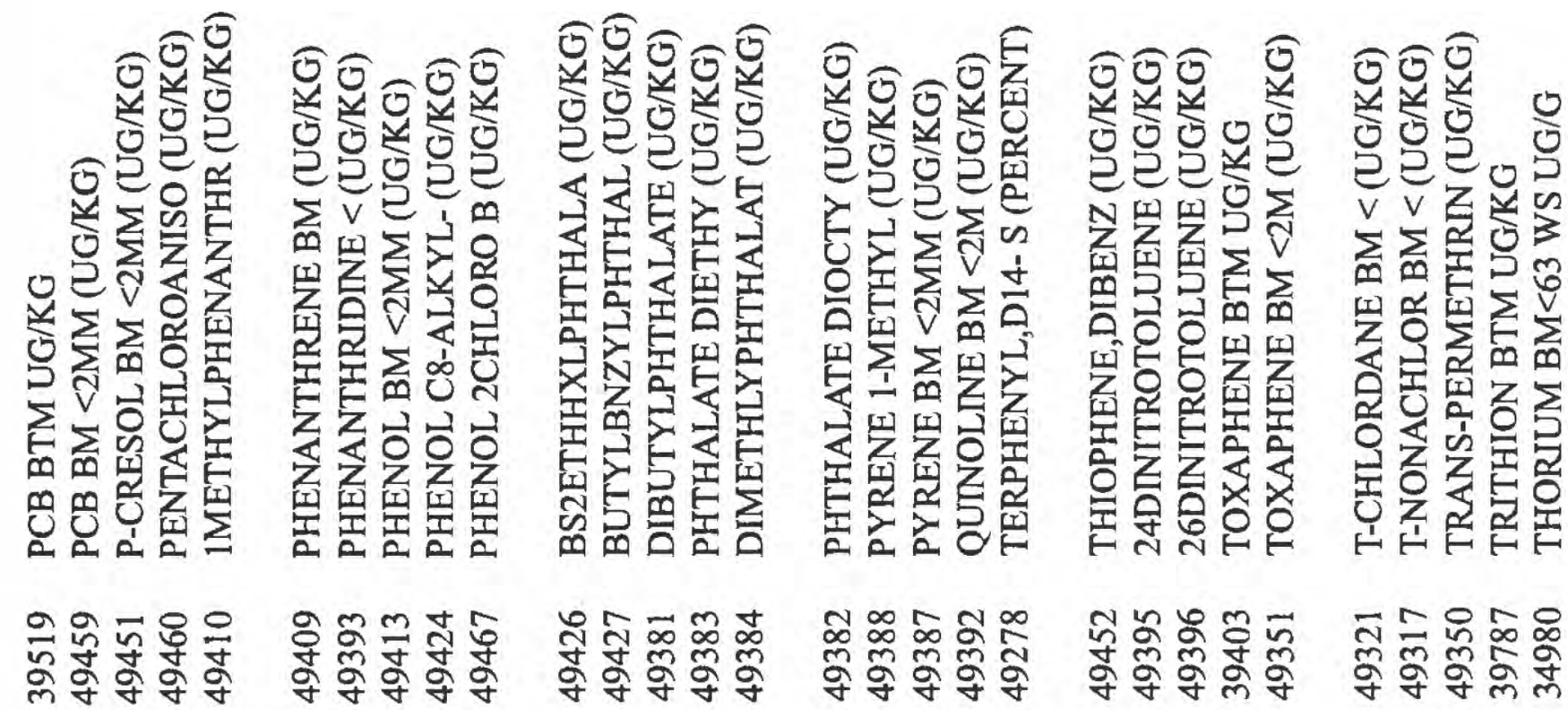

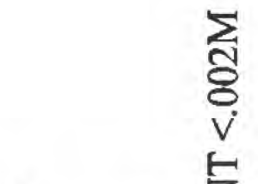

동ㅇㅇㅇㅝ

กู่ 


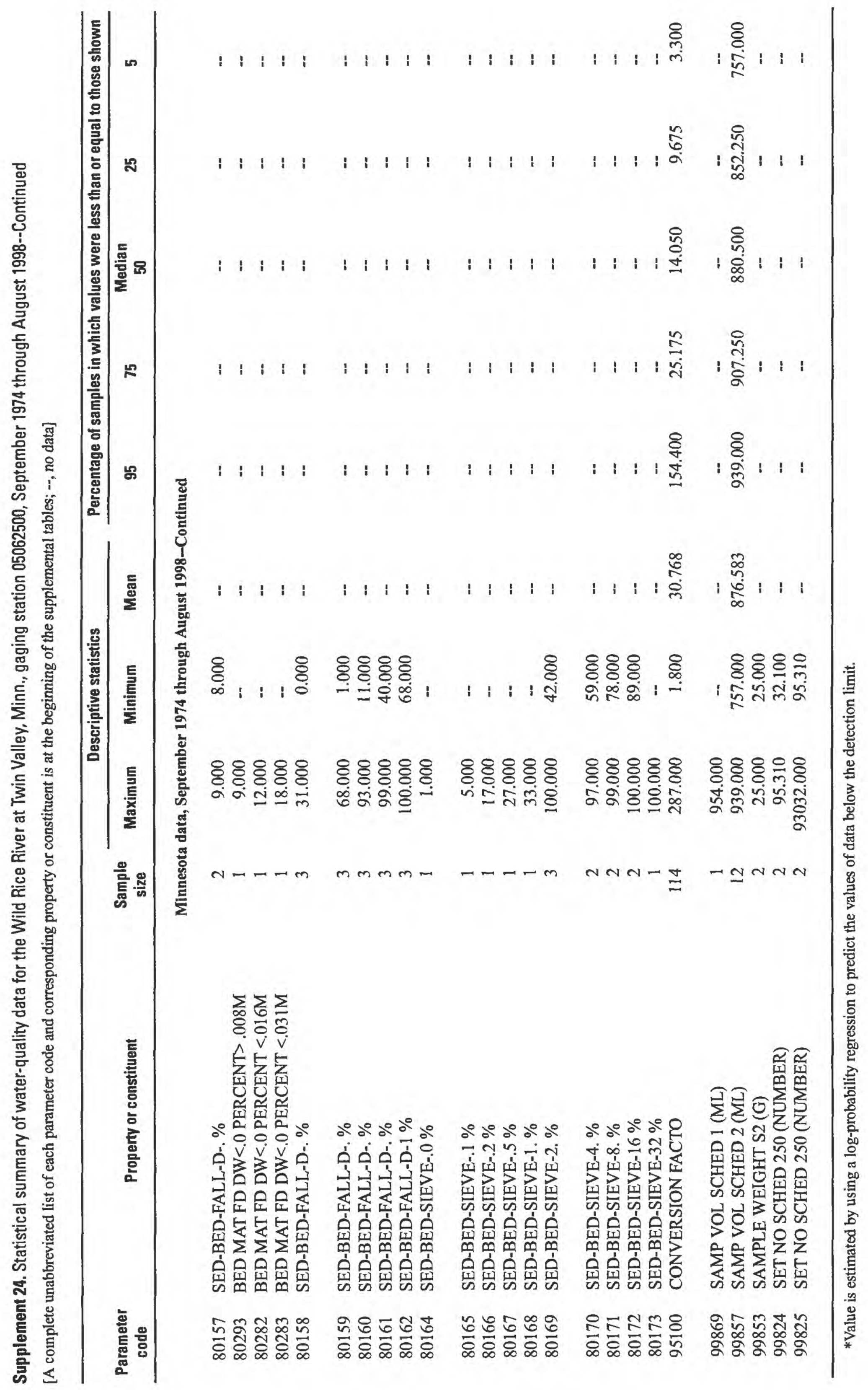




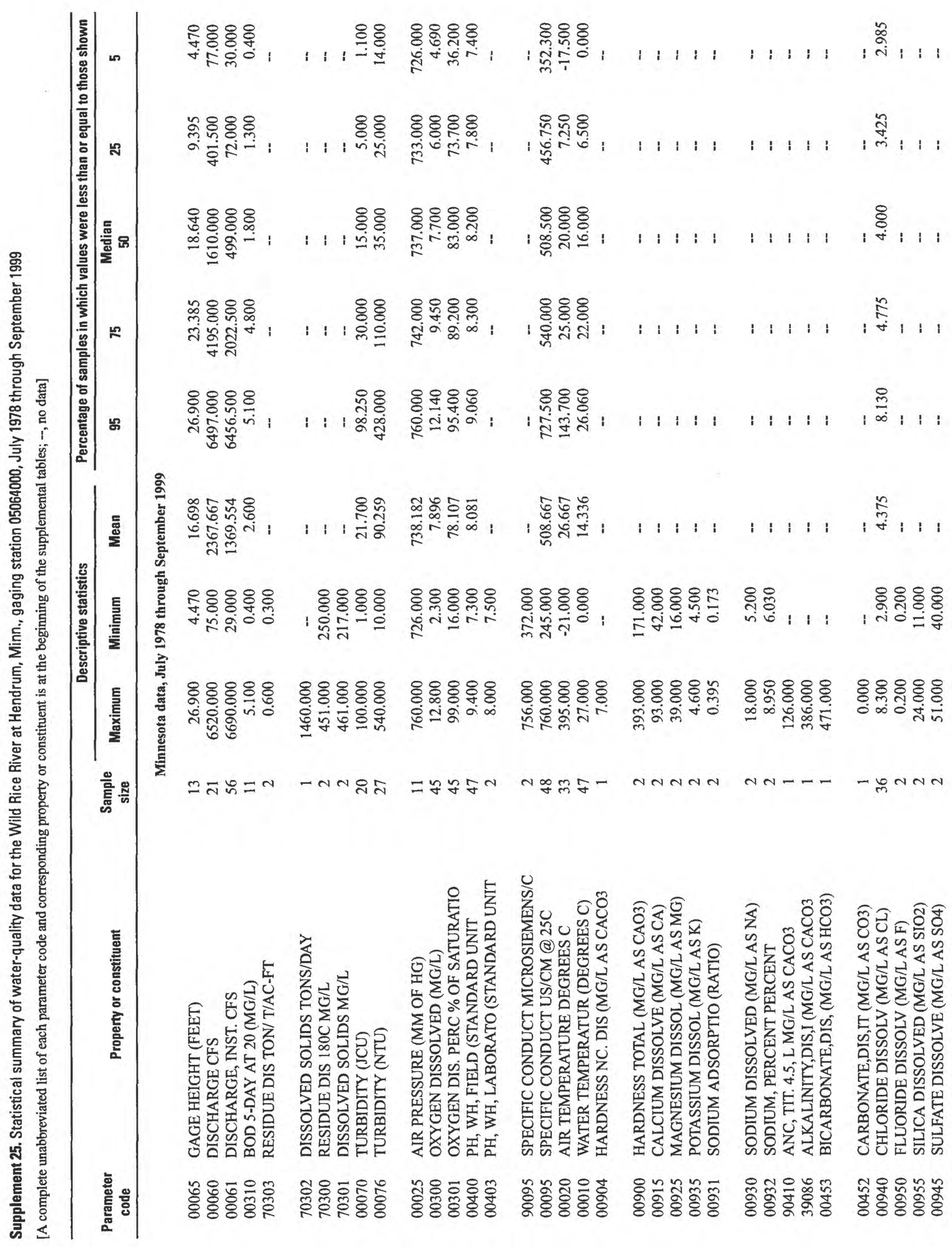




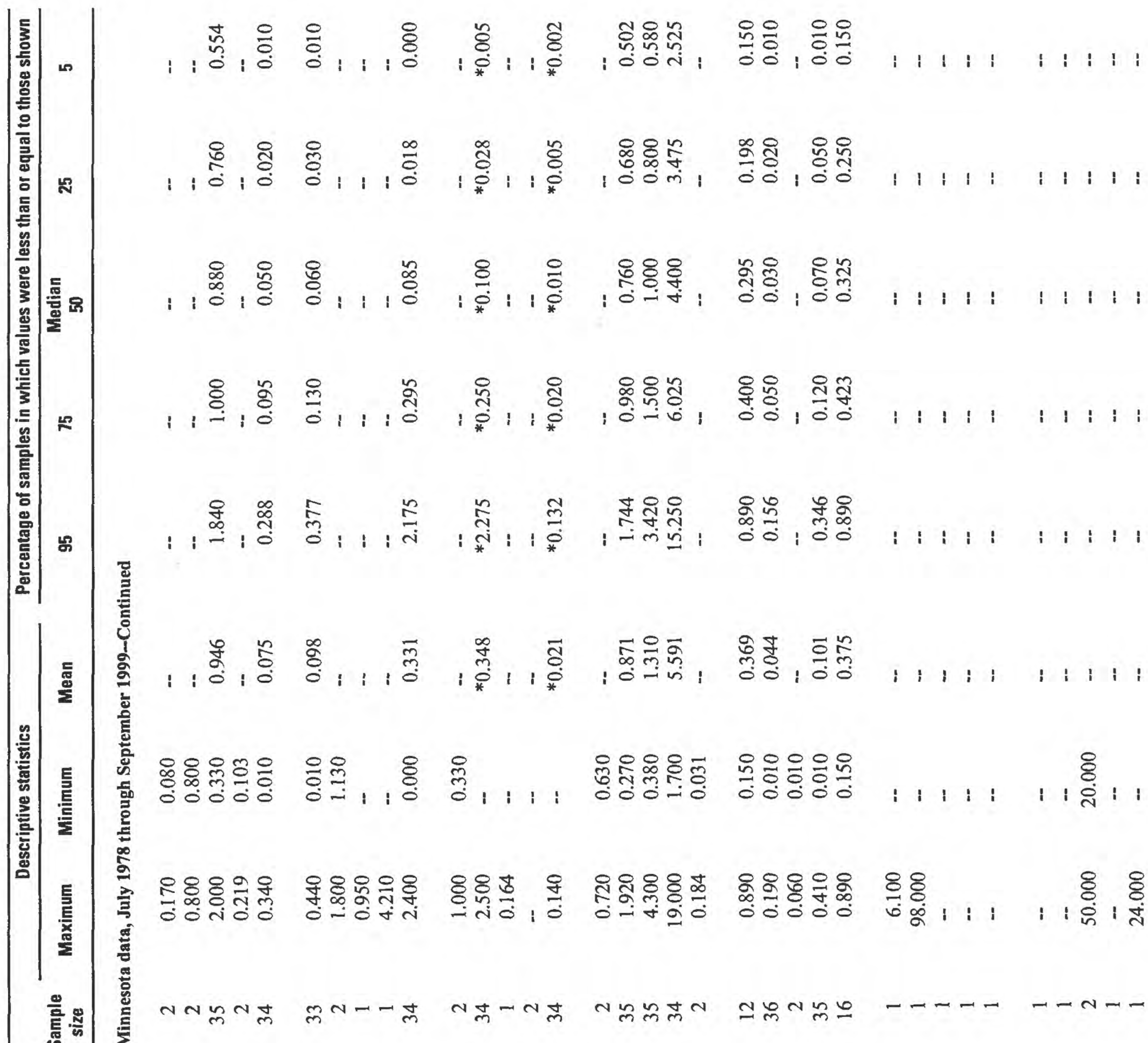

z $\bar{z}$

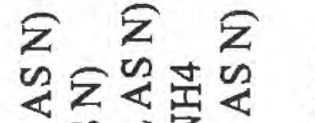

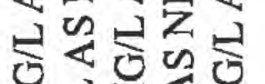

过造造

运旸

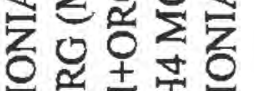

$\sum_{\sum}^{0} 0 \sum_{i}^{+} \sum_{i}$

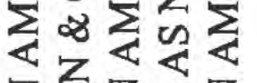

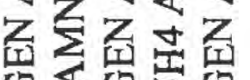

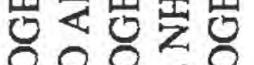

웅요

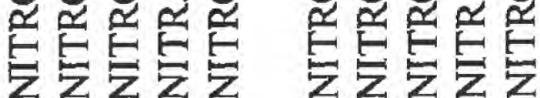

离

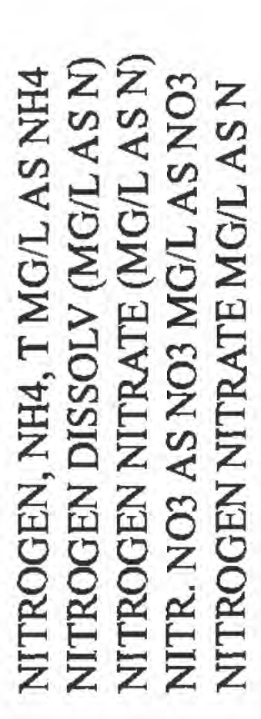

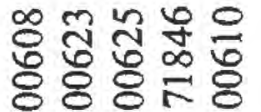

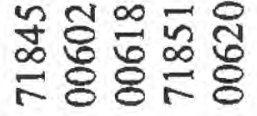

\section{蛋

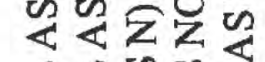

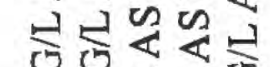 \\ 运定 \\ U⿺辶乞 \\ 棸运过 \\ 过它要

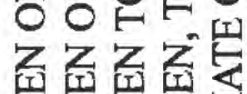 \\ O웡영

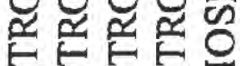 \\ 乬侌是是}

$++\alpha$

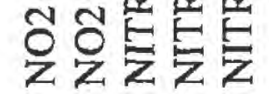

귱유ㅇㅠㅠ융

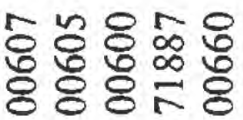

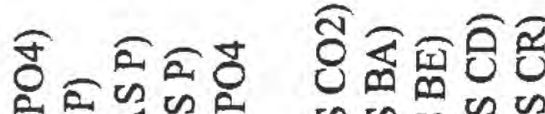
कूष्य

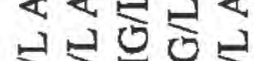

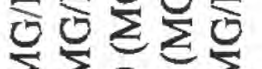
运运是

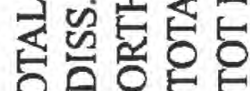
은

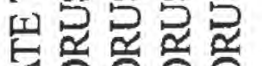

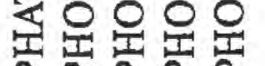

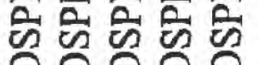
원언온원원 융유융유

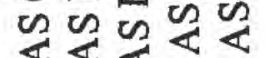

러넝엉

엉엉엉 ¿e?巳e 넘댕

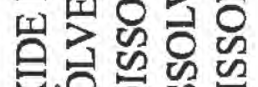

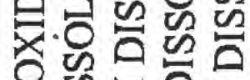
员的定

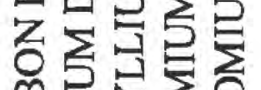

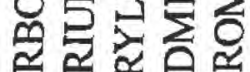

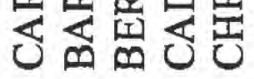

ชิ 约国㝵 《रा 的 서워 어엉엉 ㄹㄹㅇㅇㅇㅇ 외될ㄹ됟

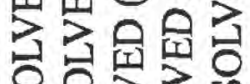

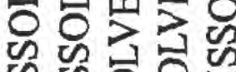
的。음 合旸充 凷向虽 视只是 ㅇㅇㅇ웝

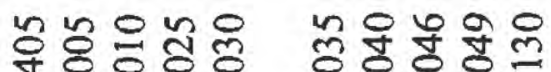
홍응응응 응융휴 


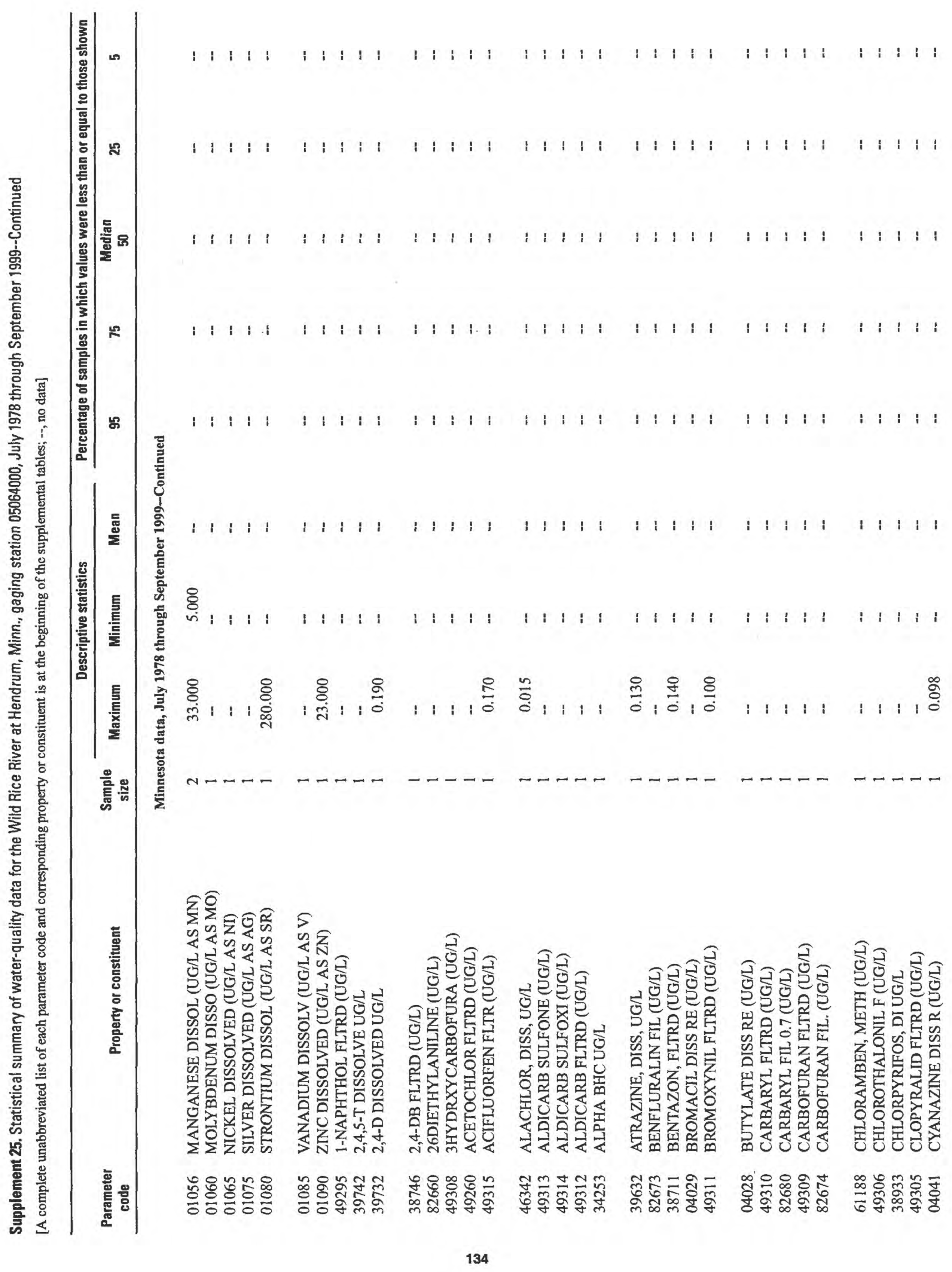




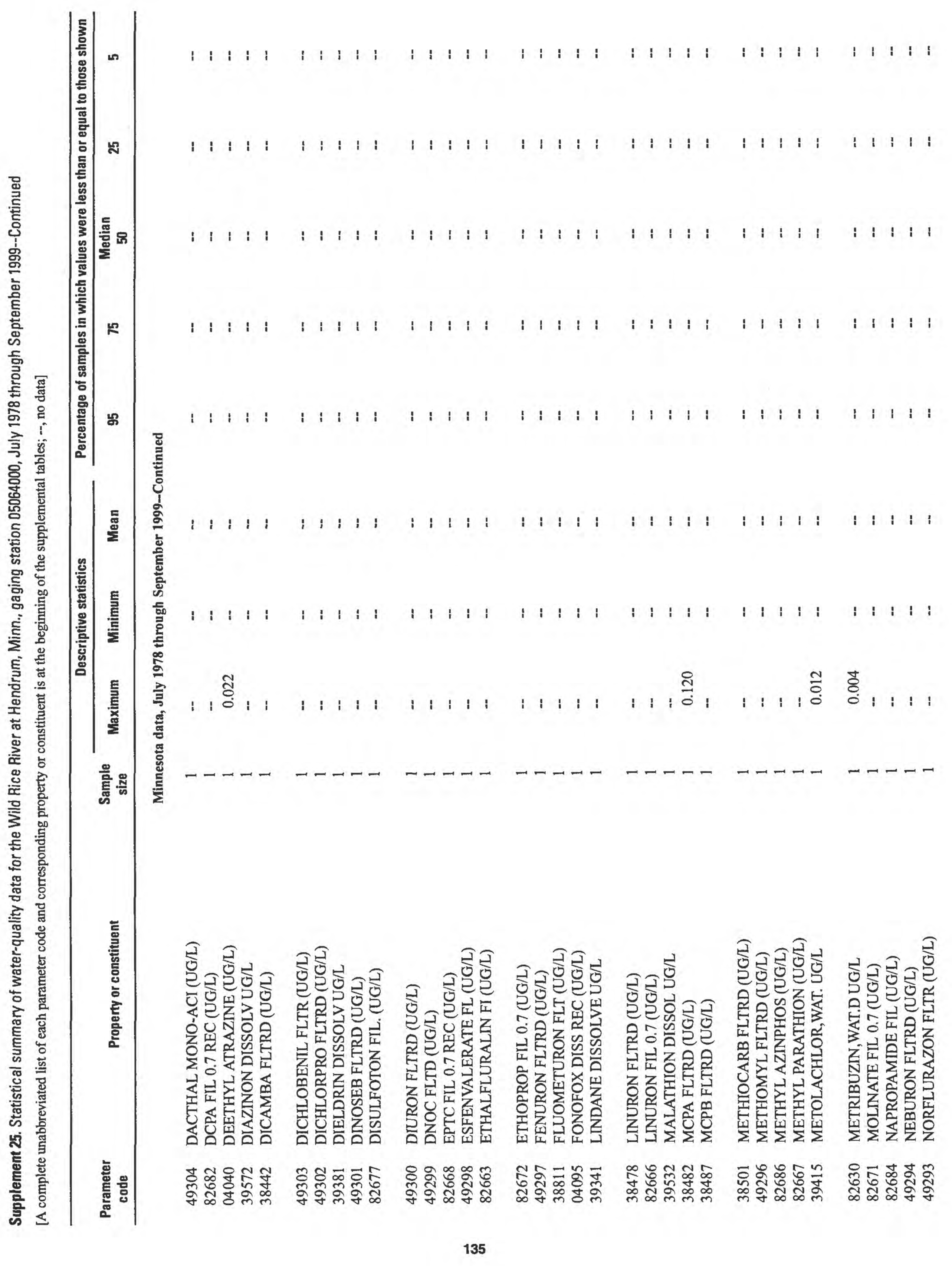




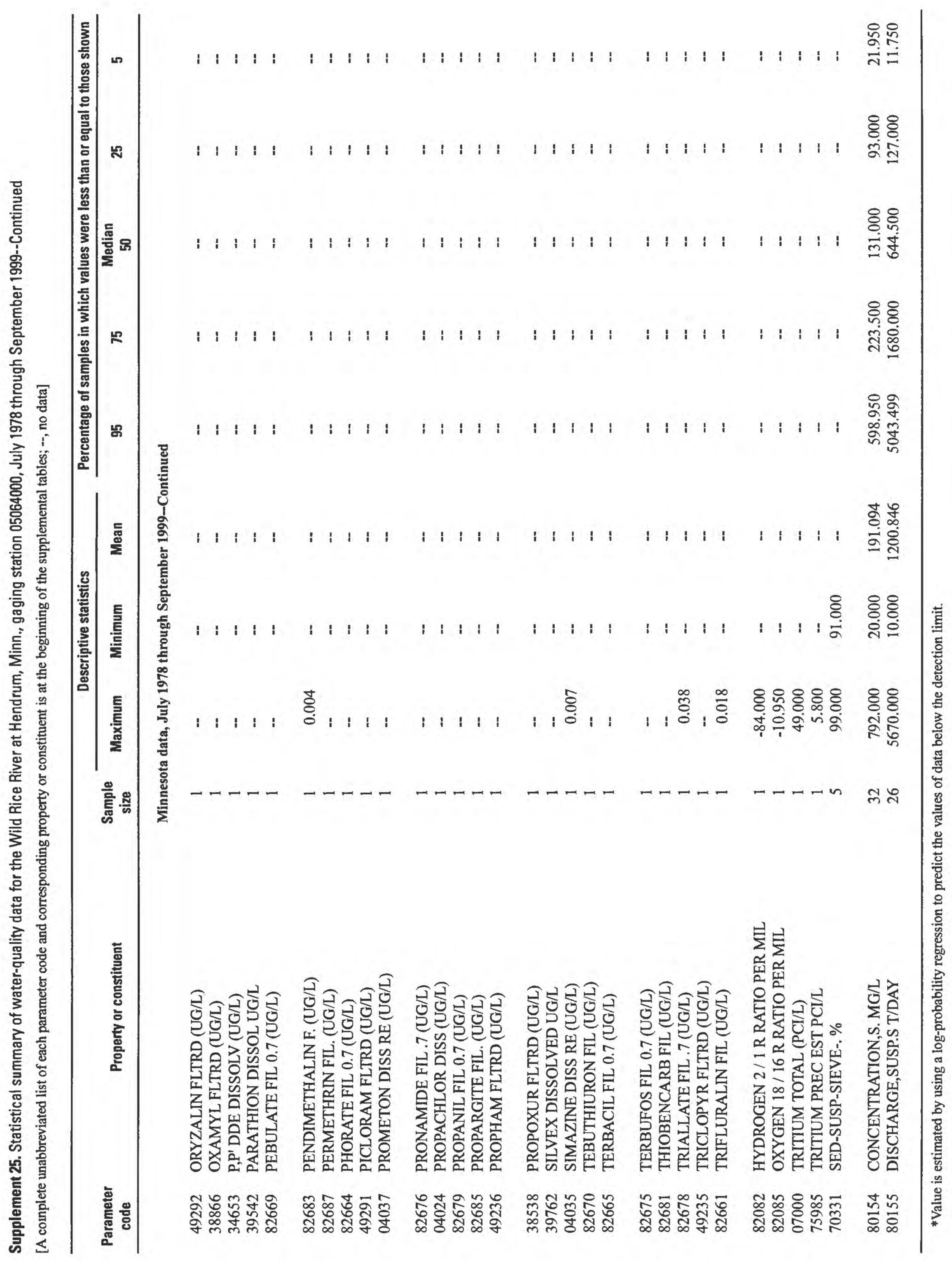




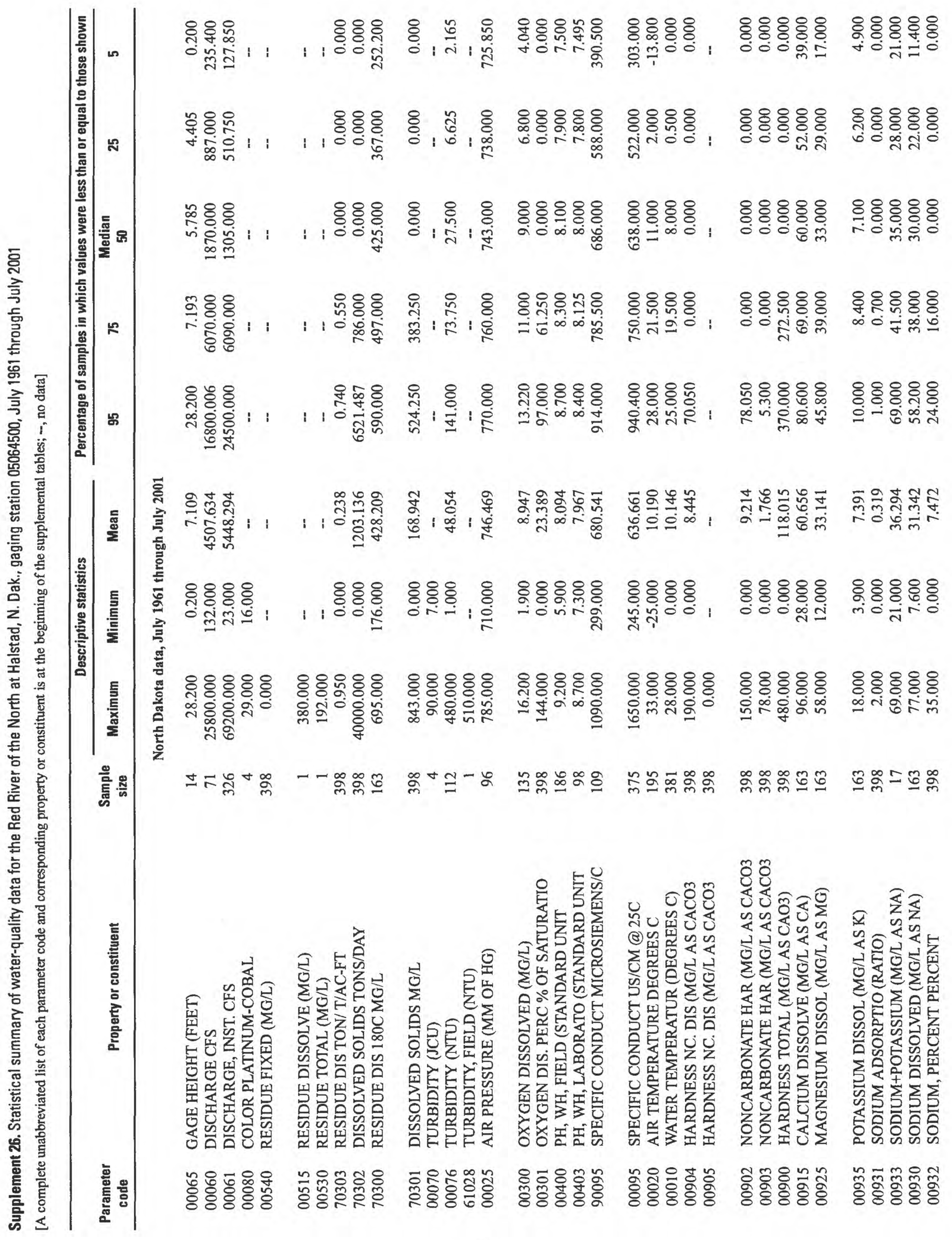




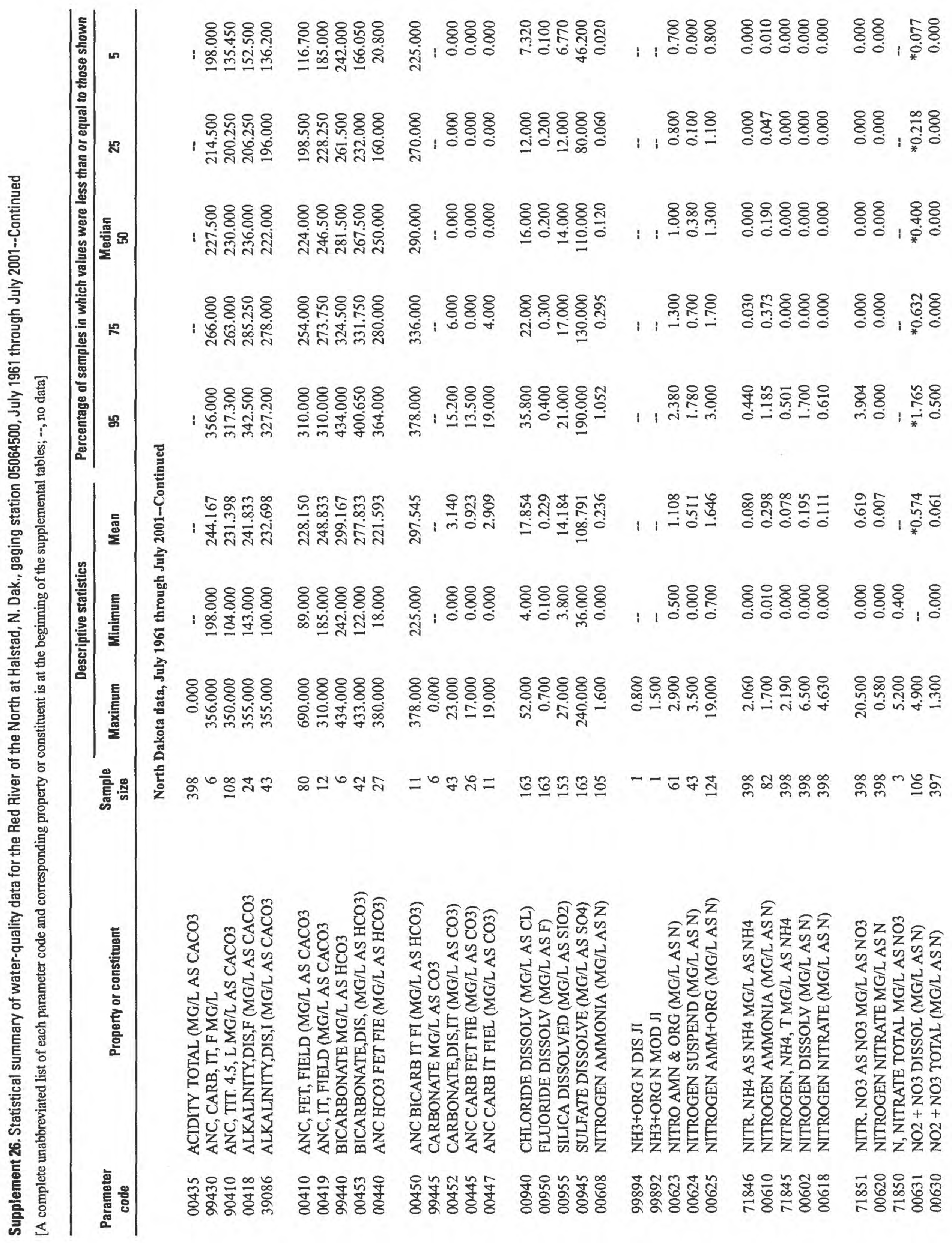




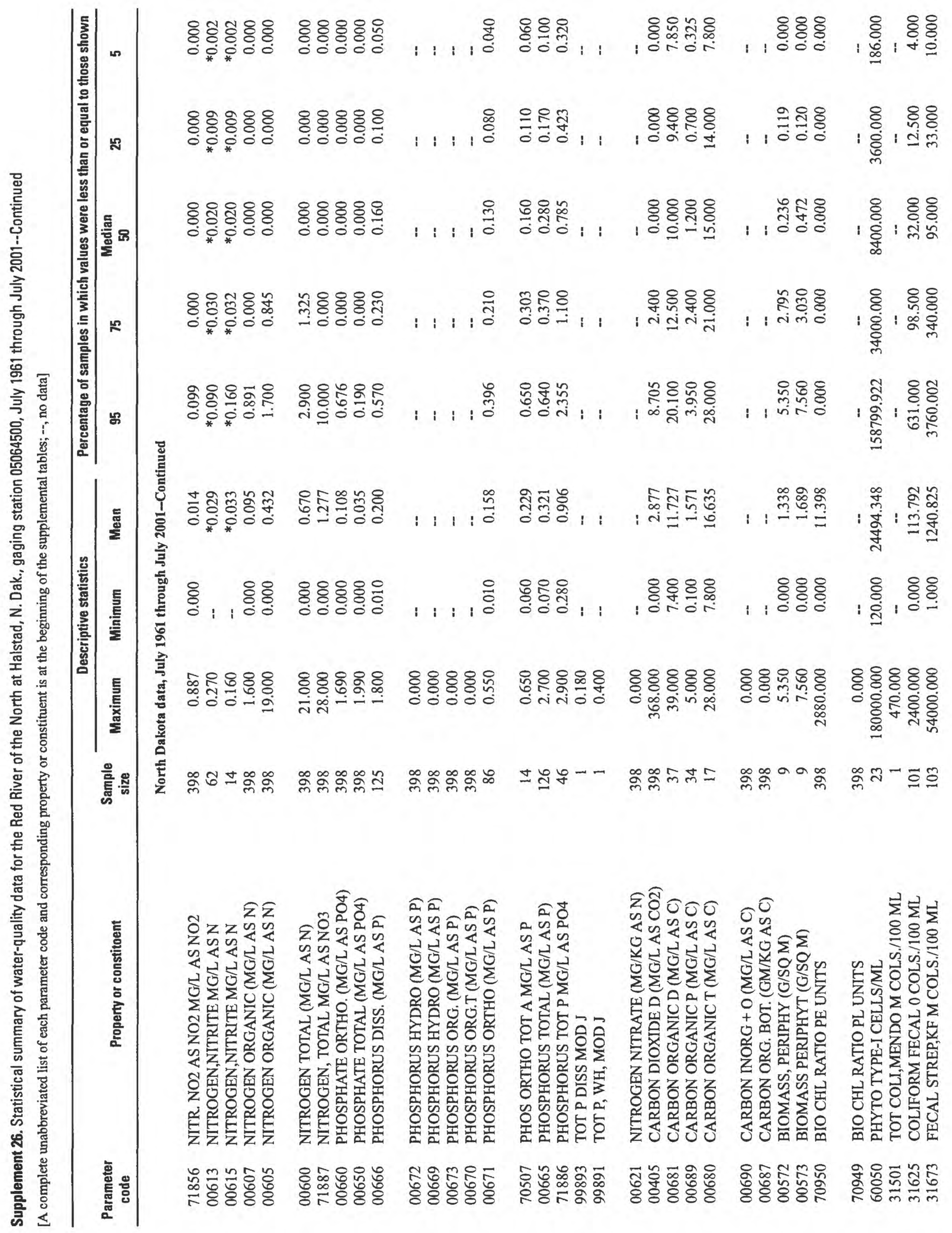




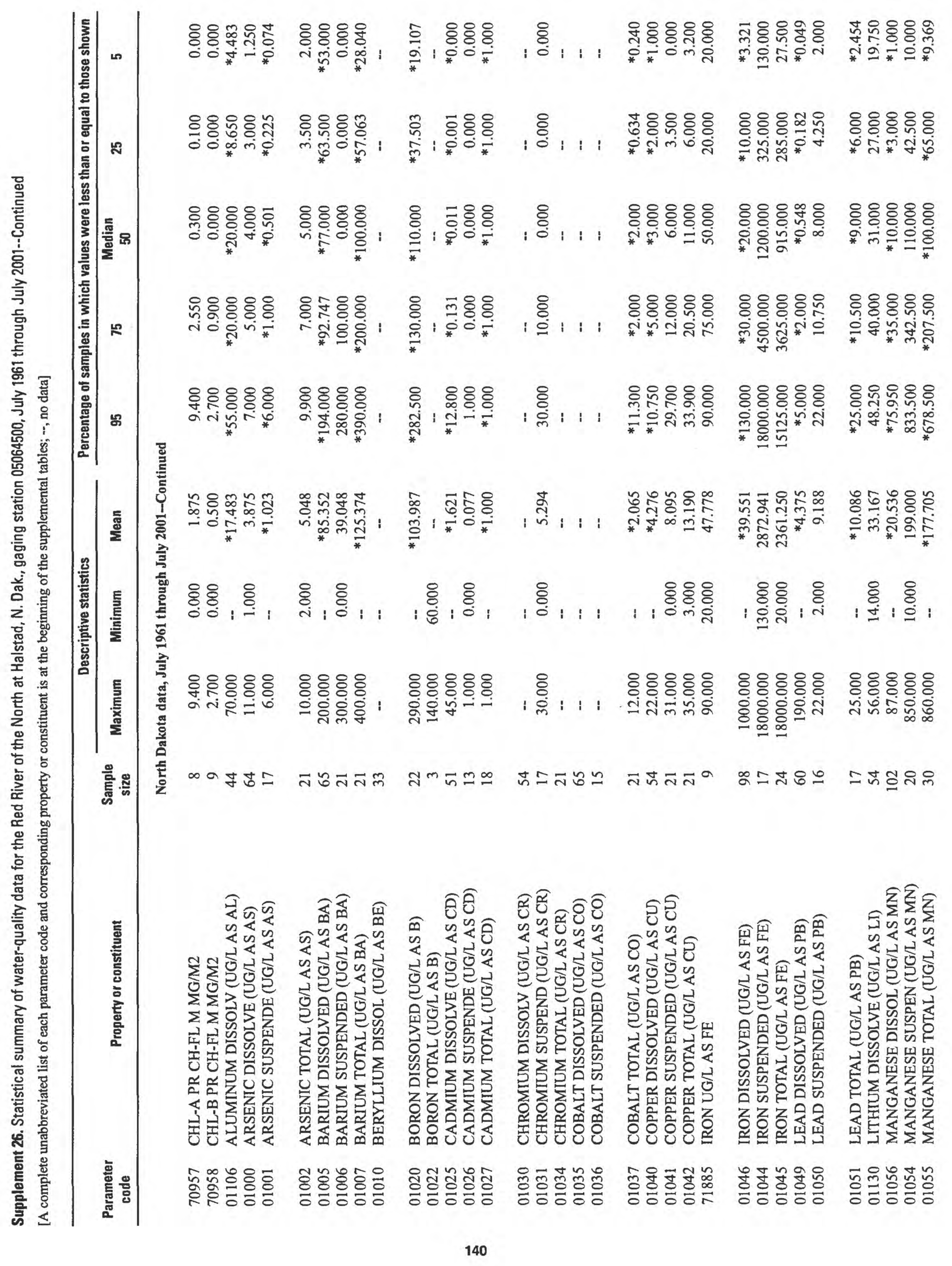



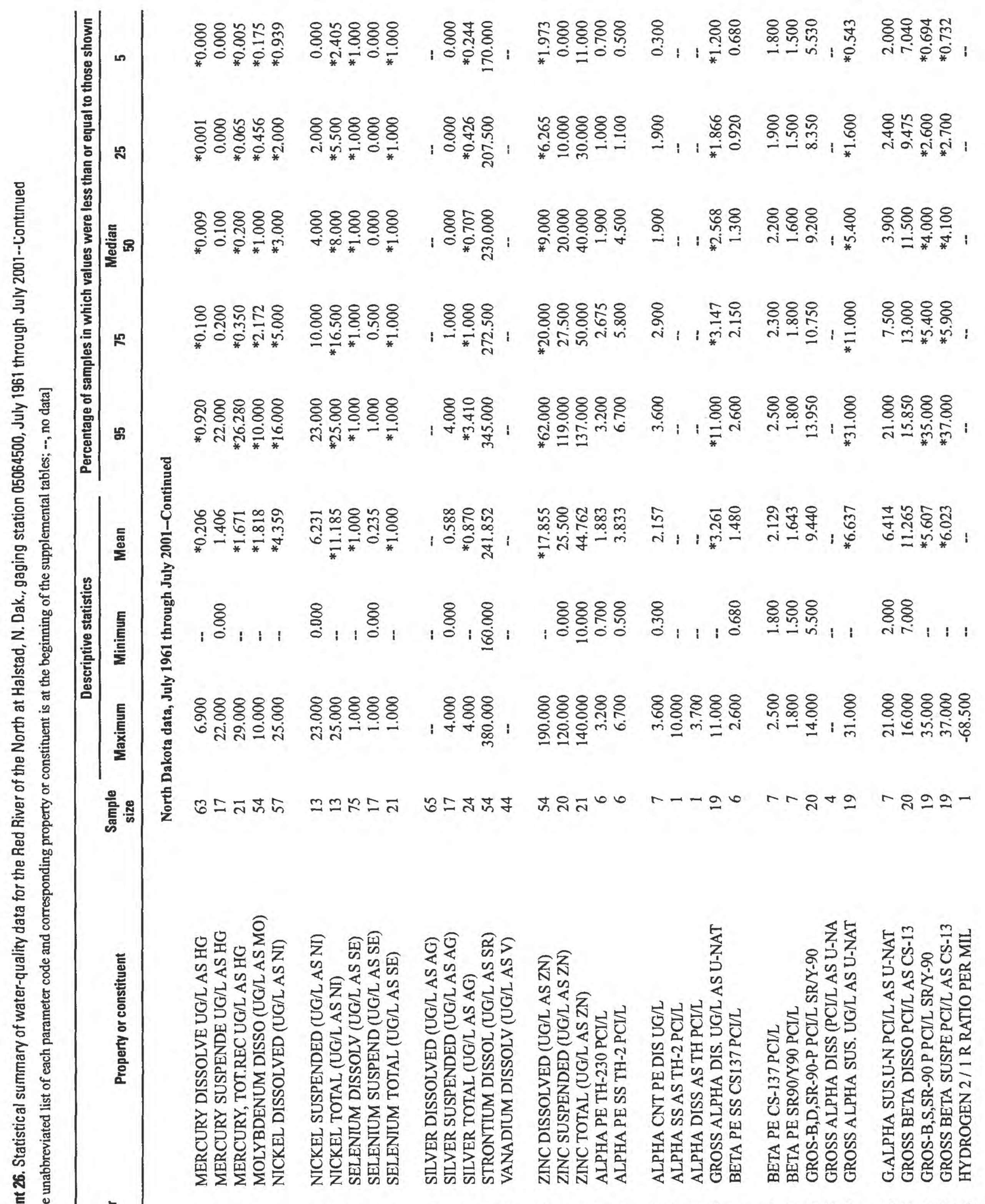

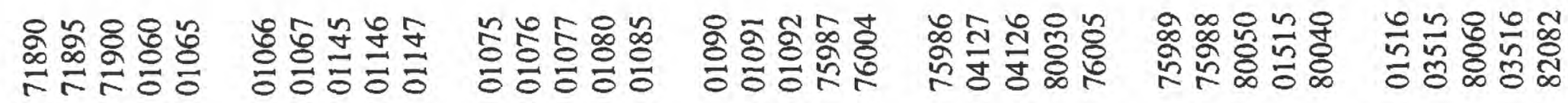


,

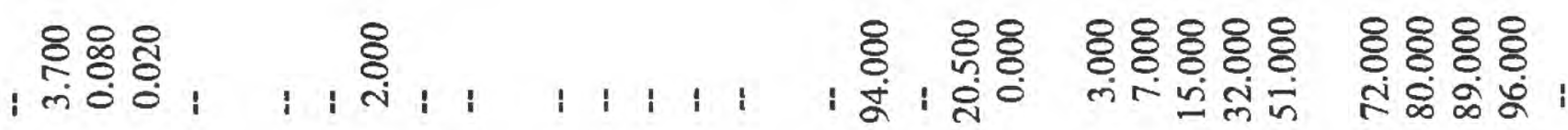

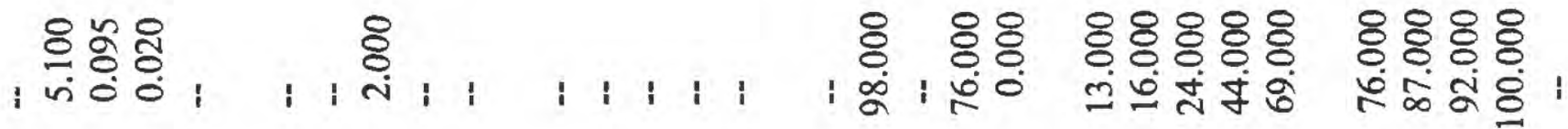

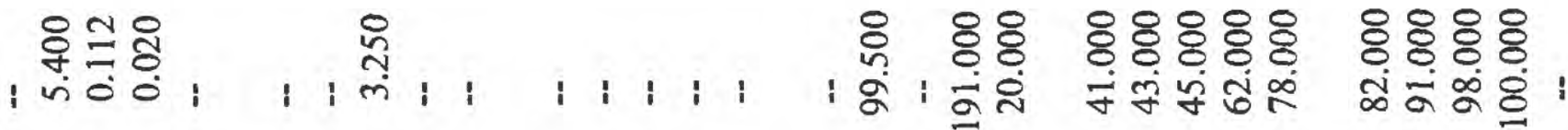

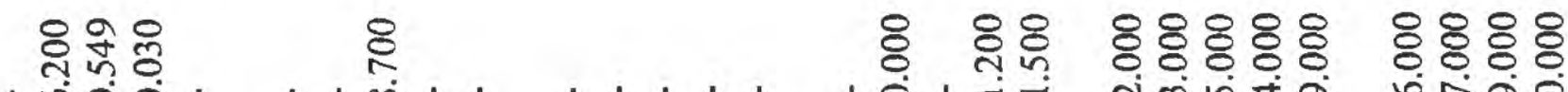

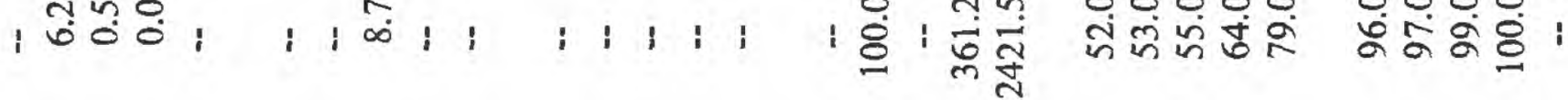

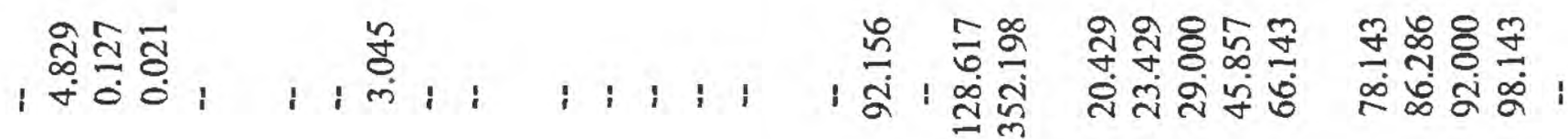

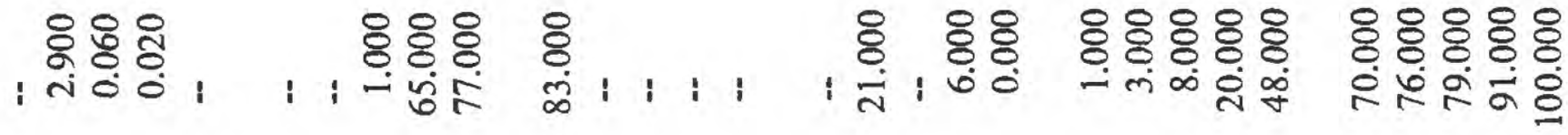

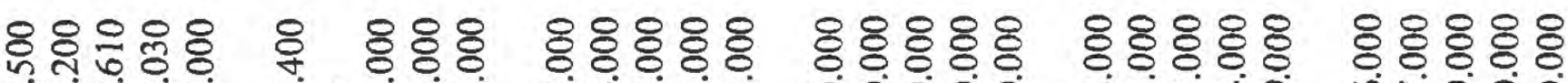

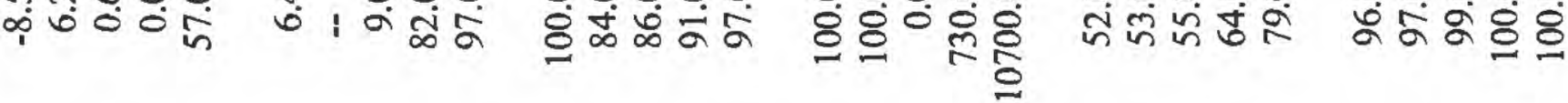

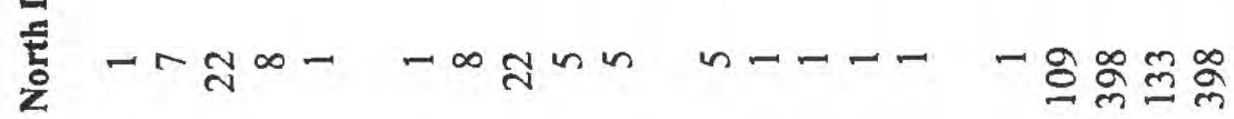

볼운

产早

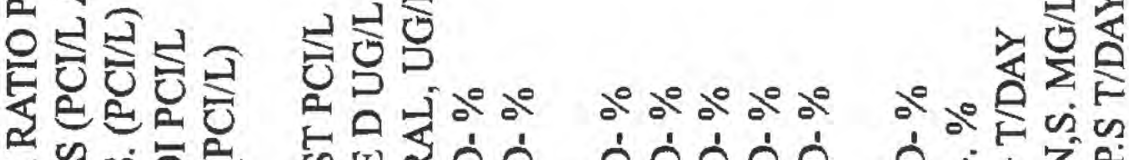

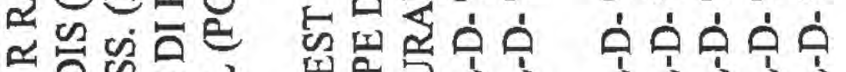

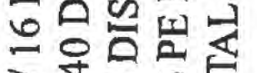

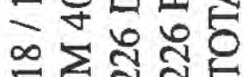

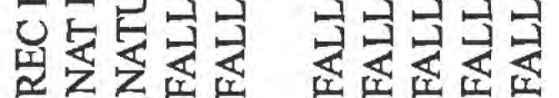

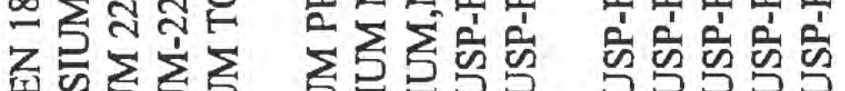

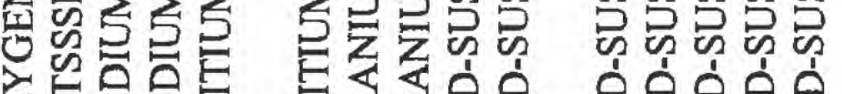

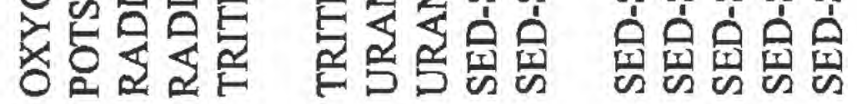

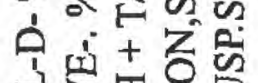

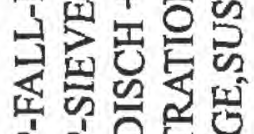

放记

品罡兒

宫它各

deàdede de aejodo

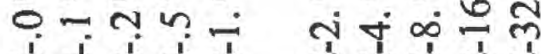

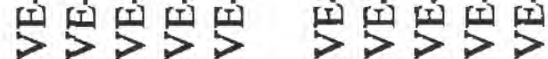

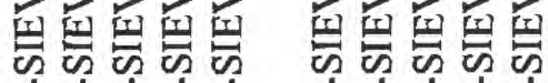

定

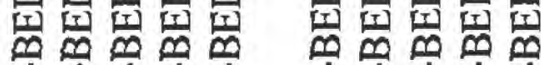

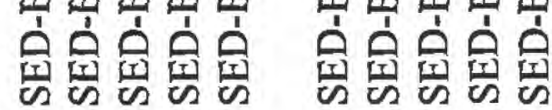

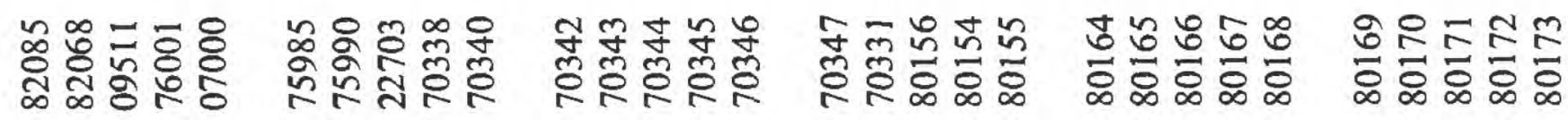




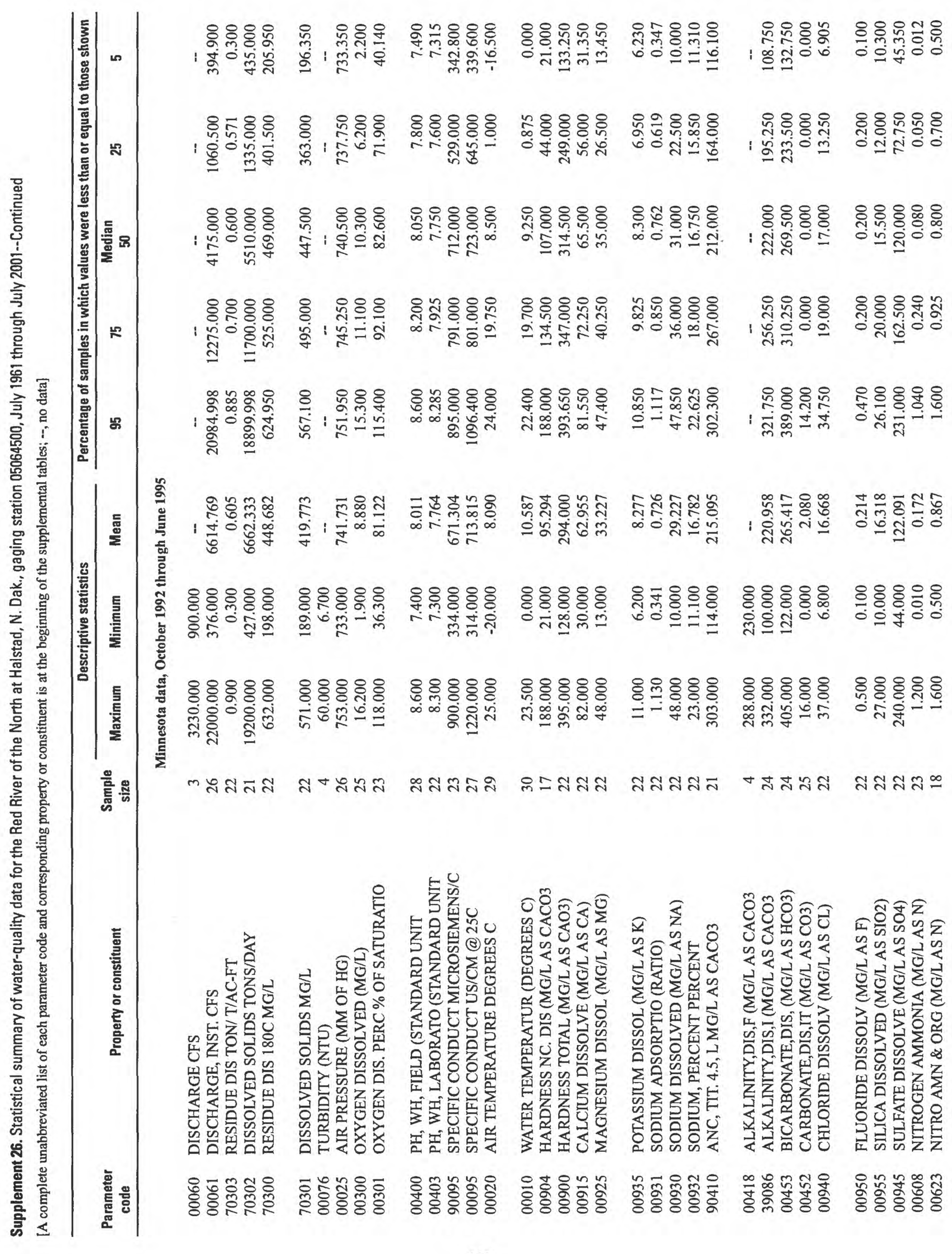




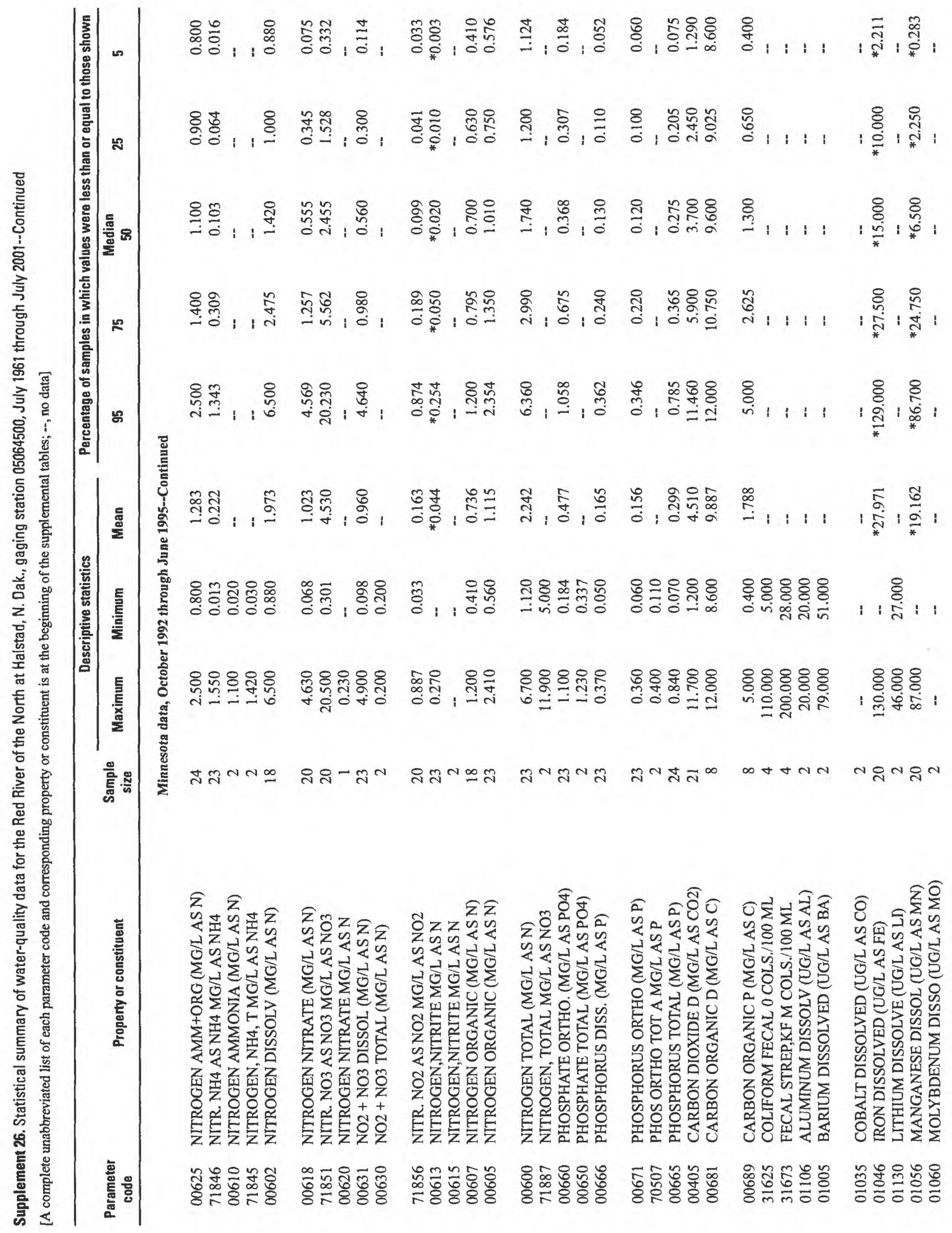




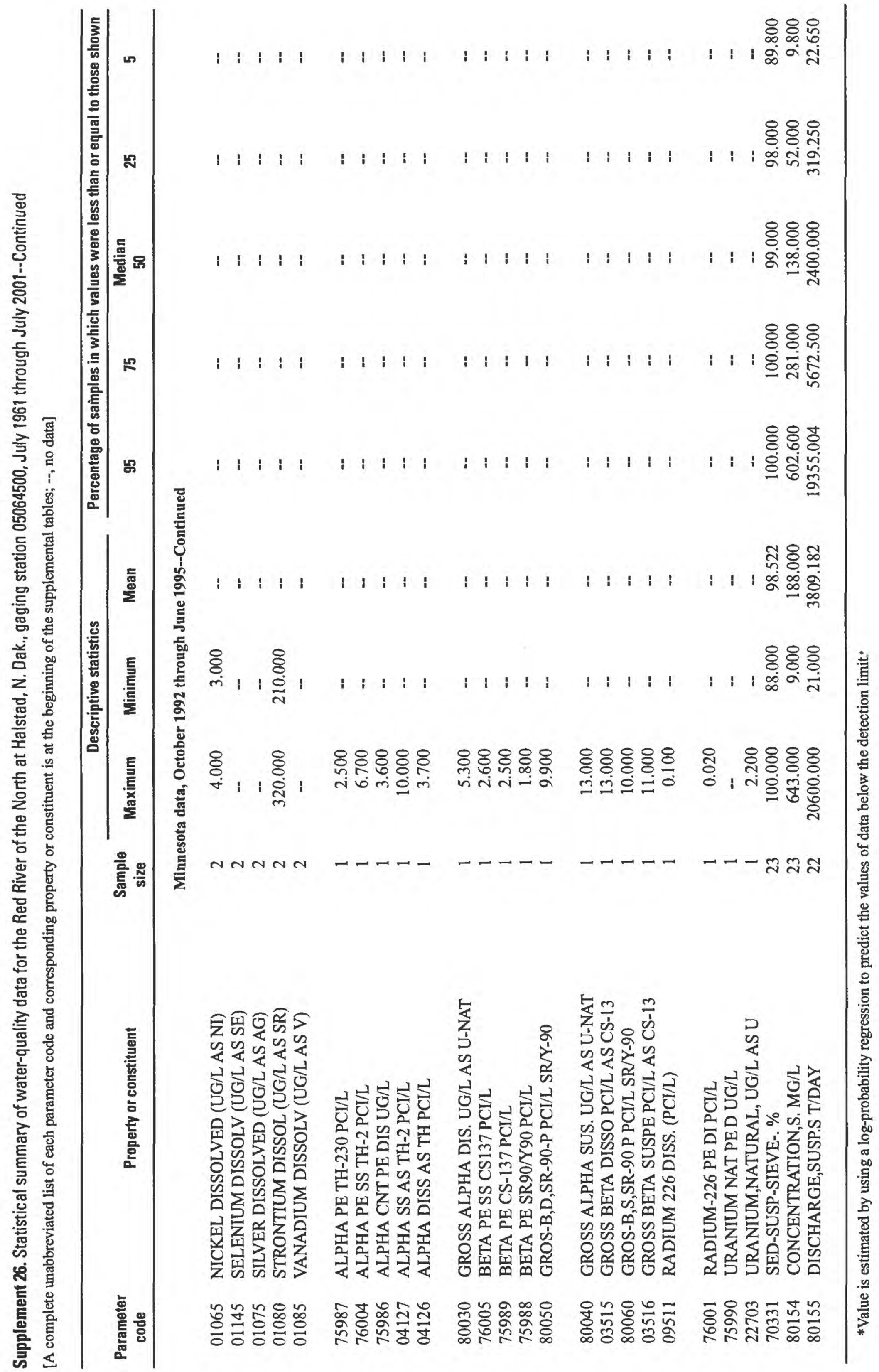




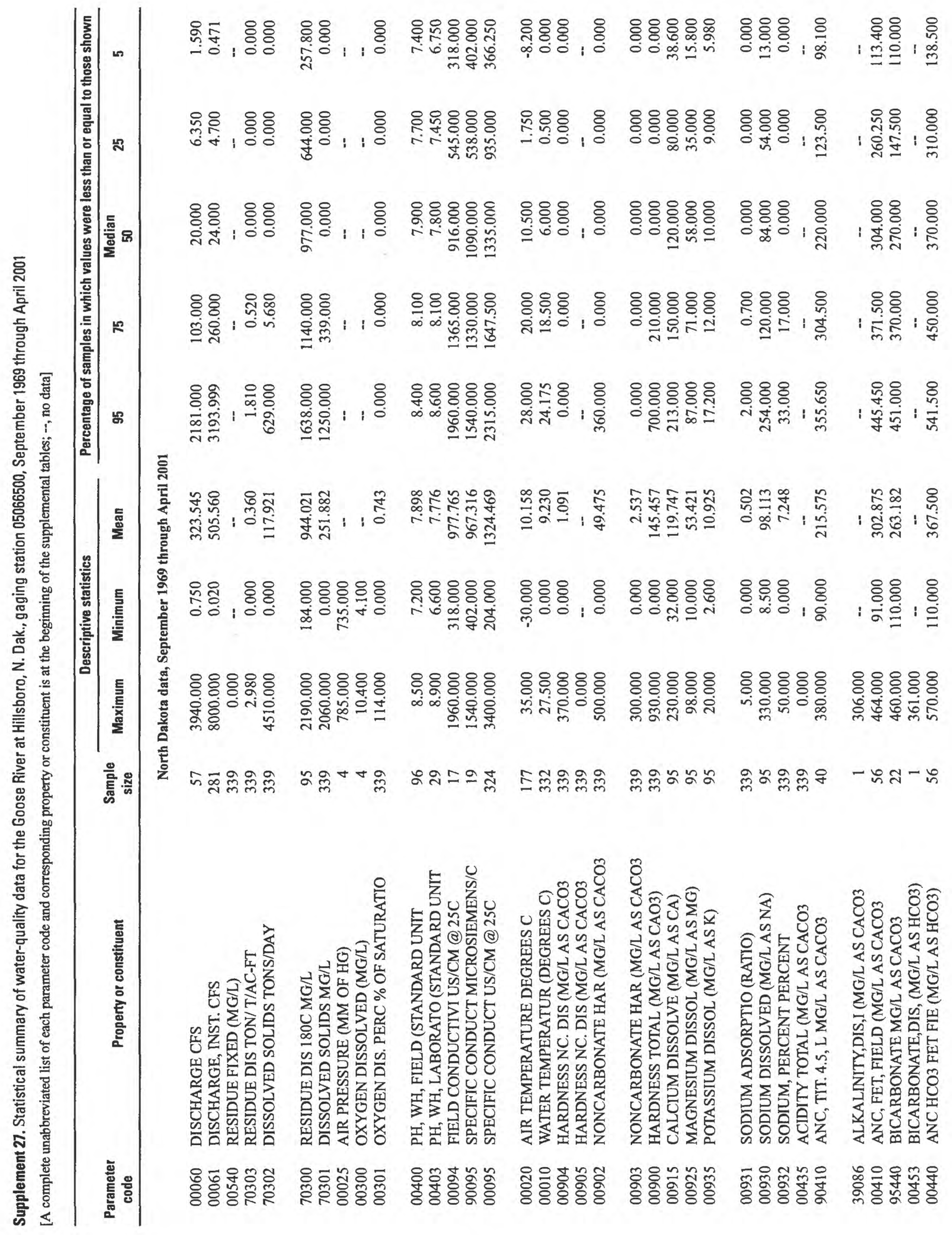




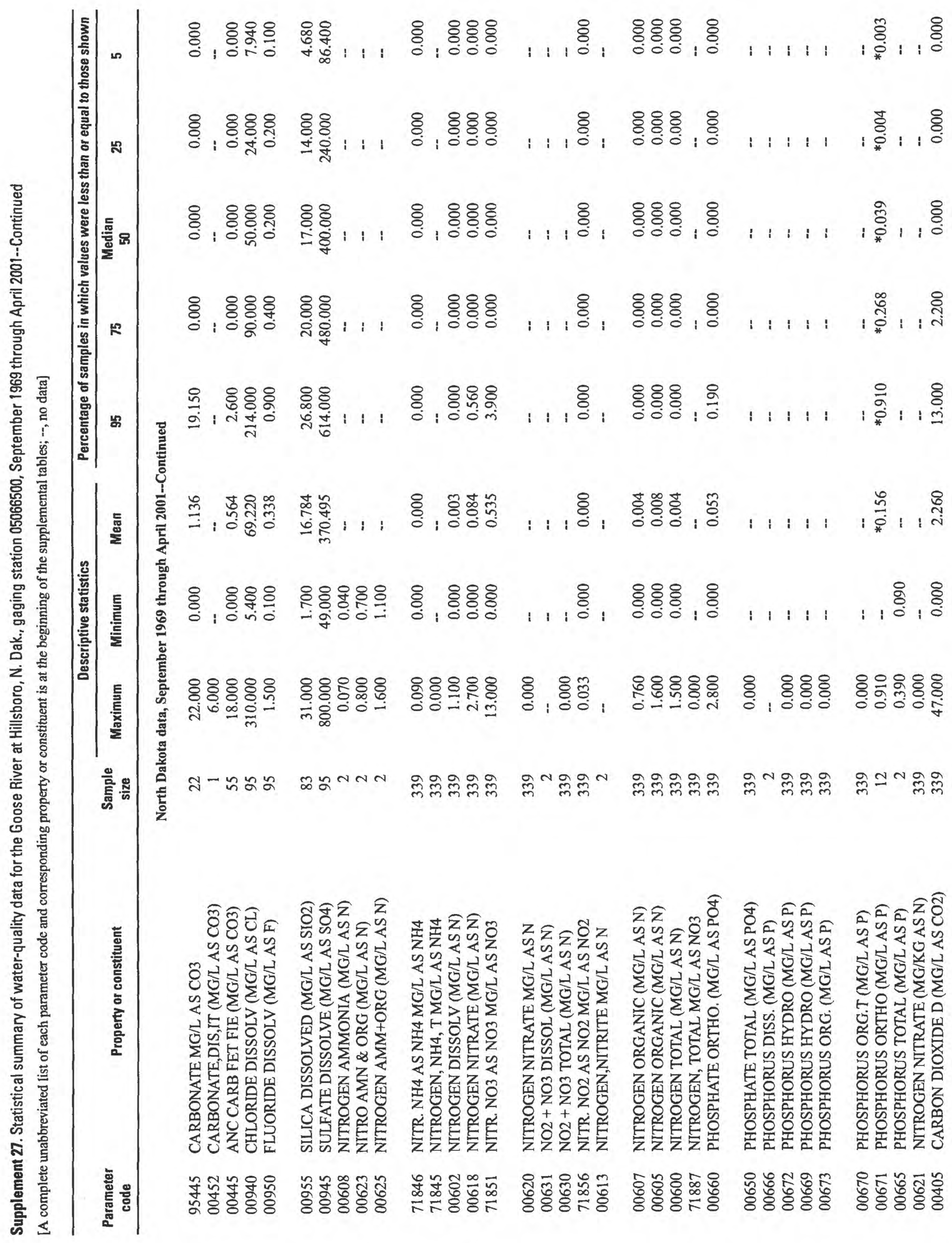




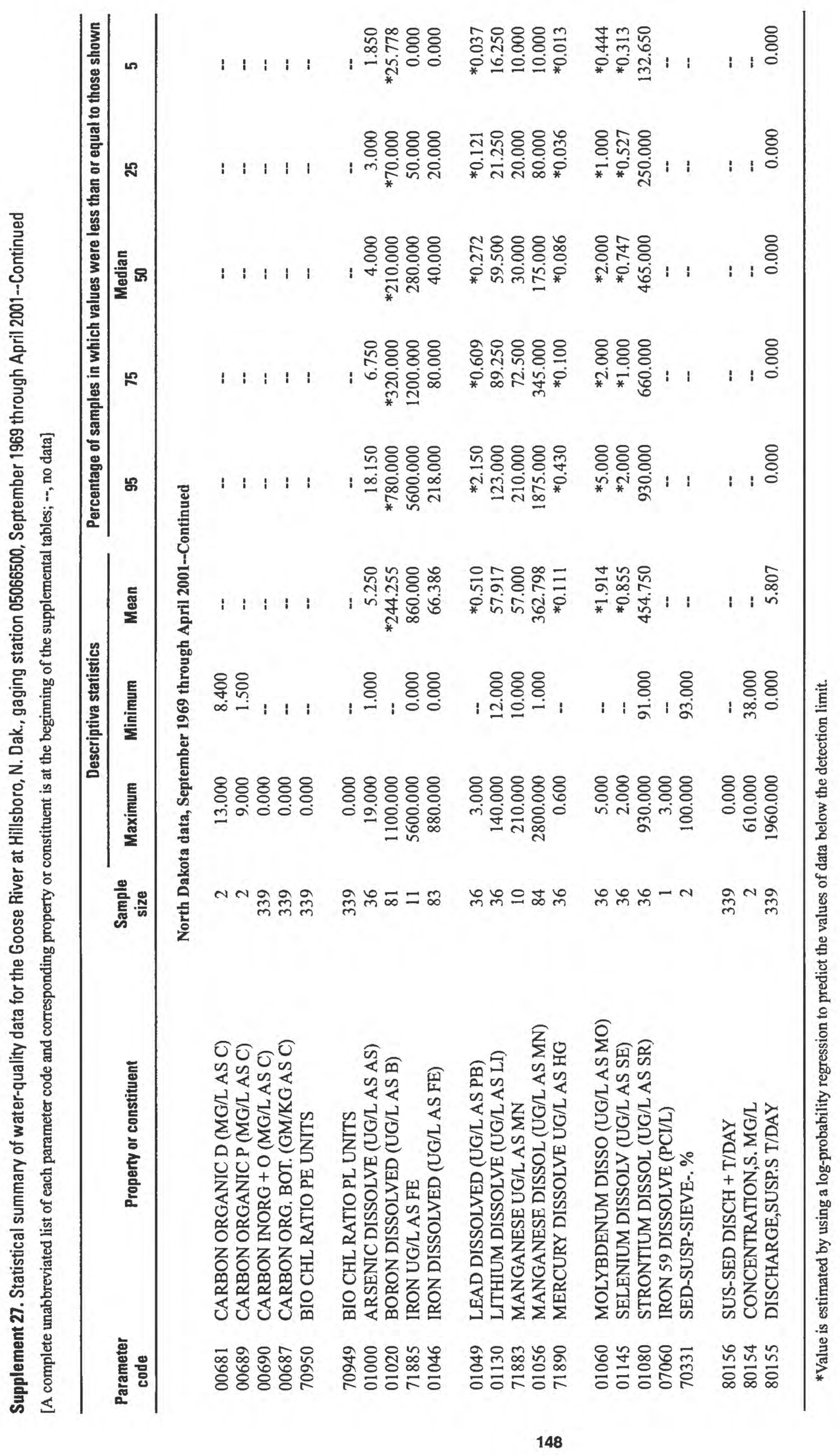




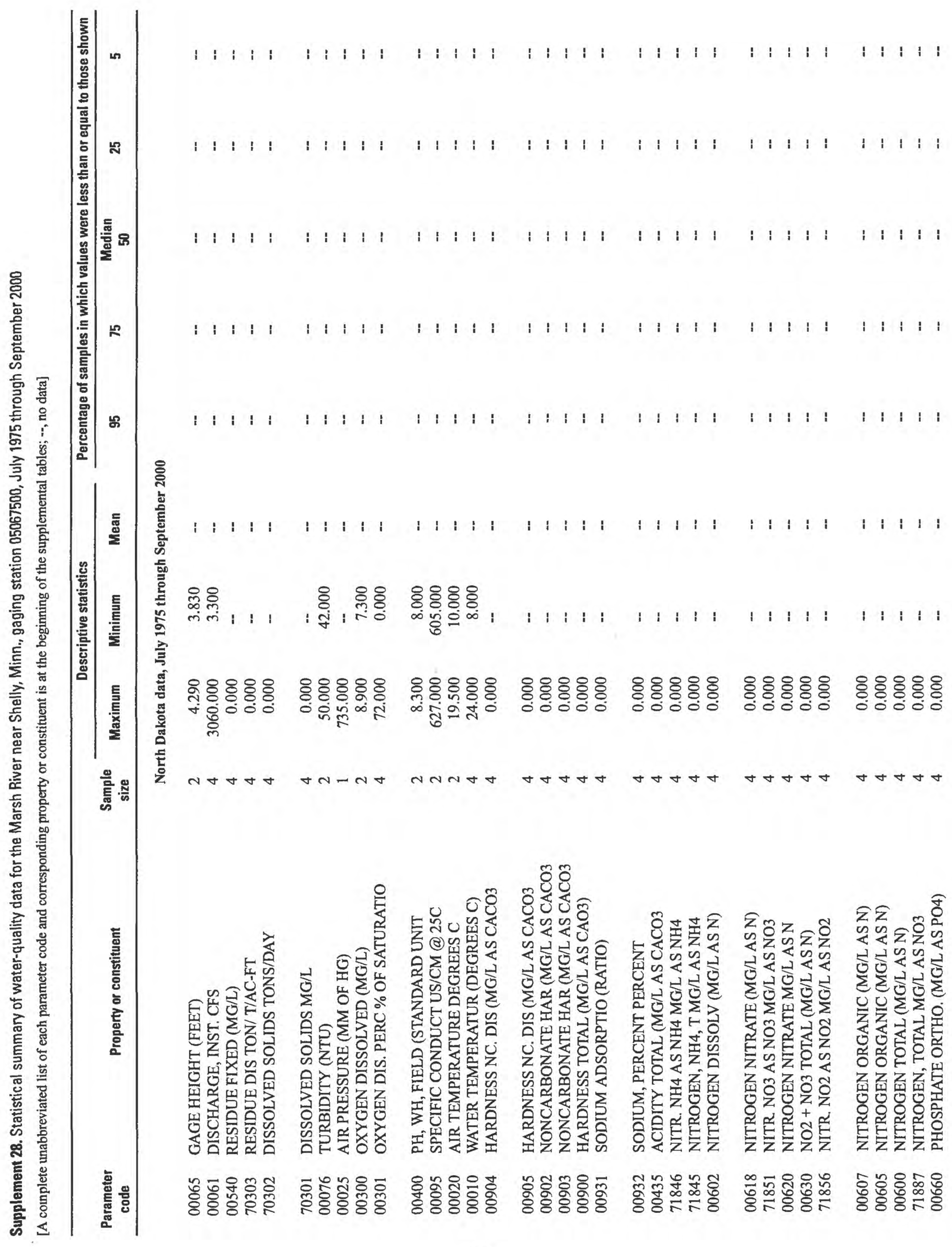




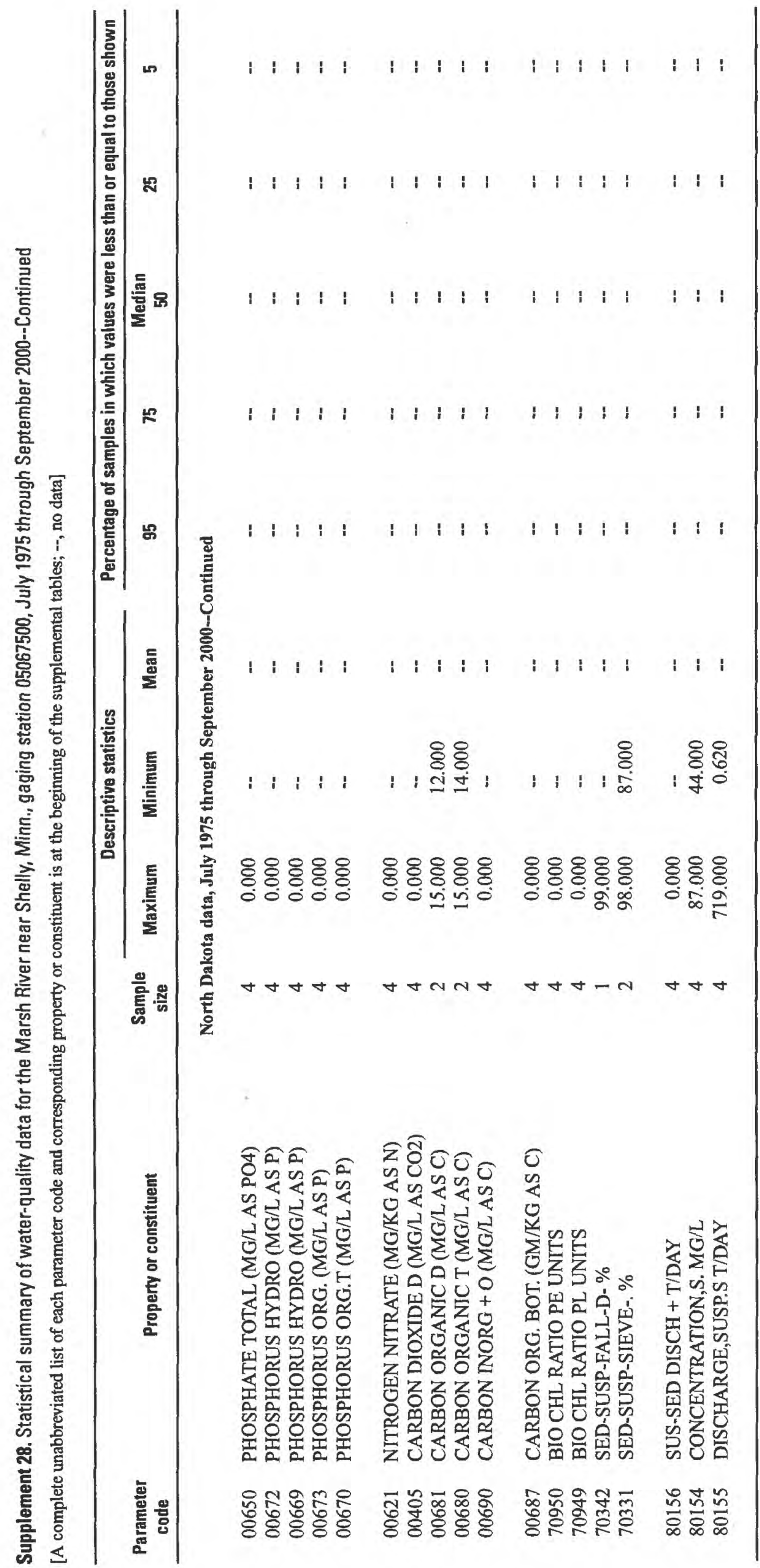




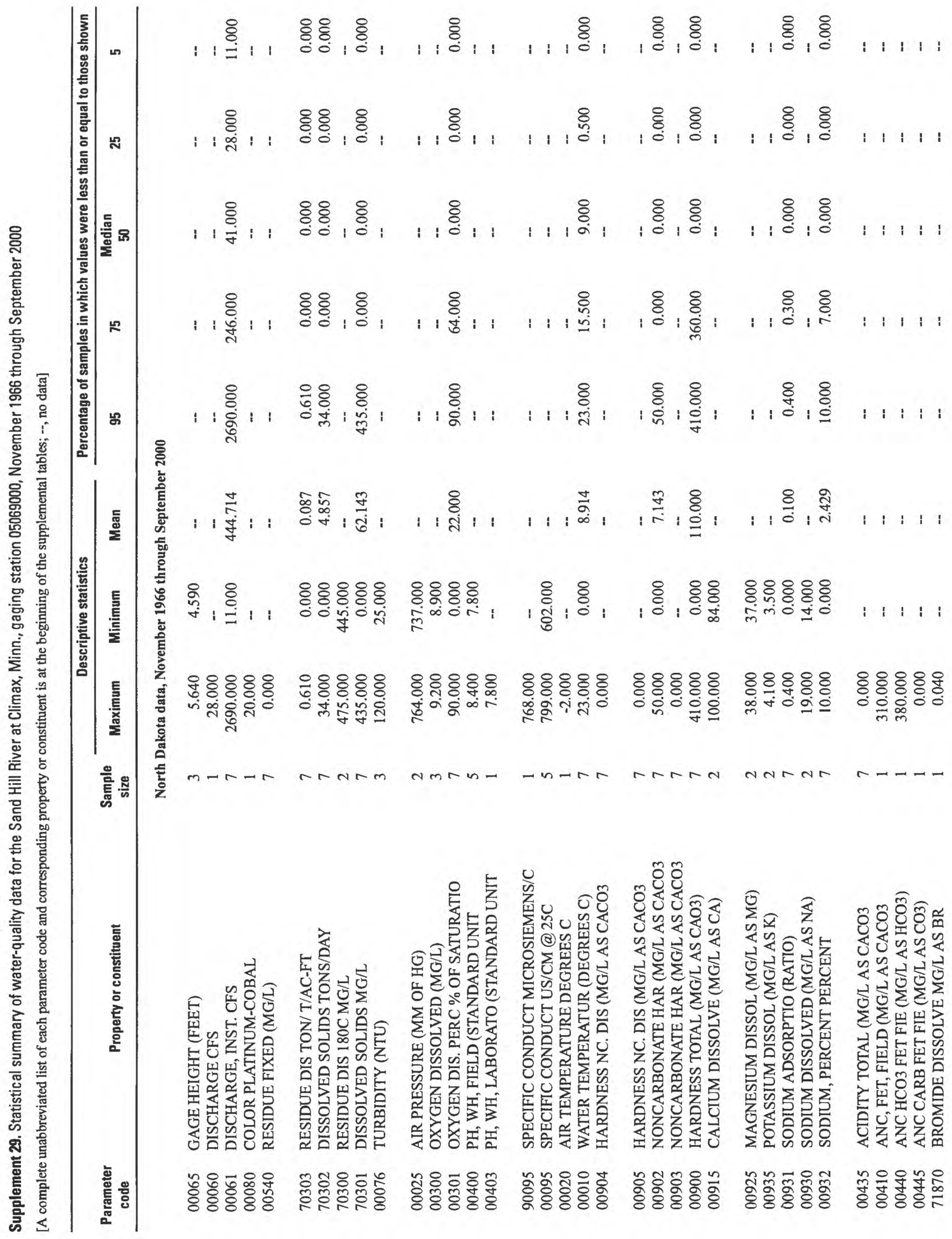




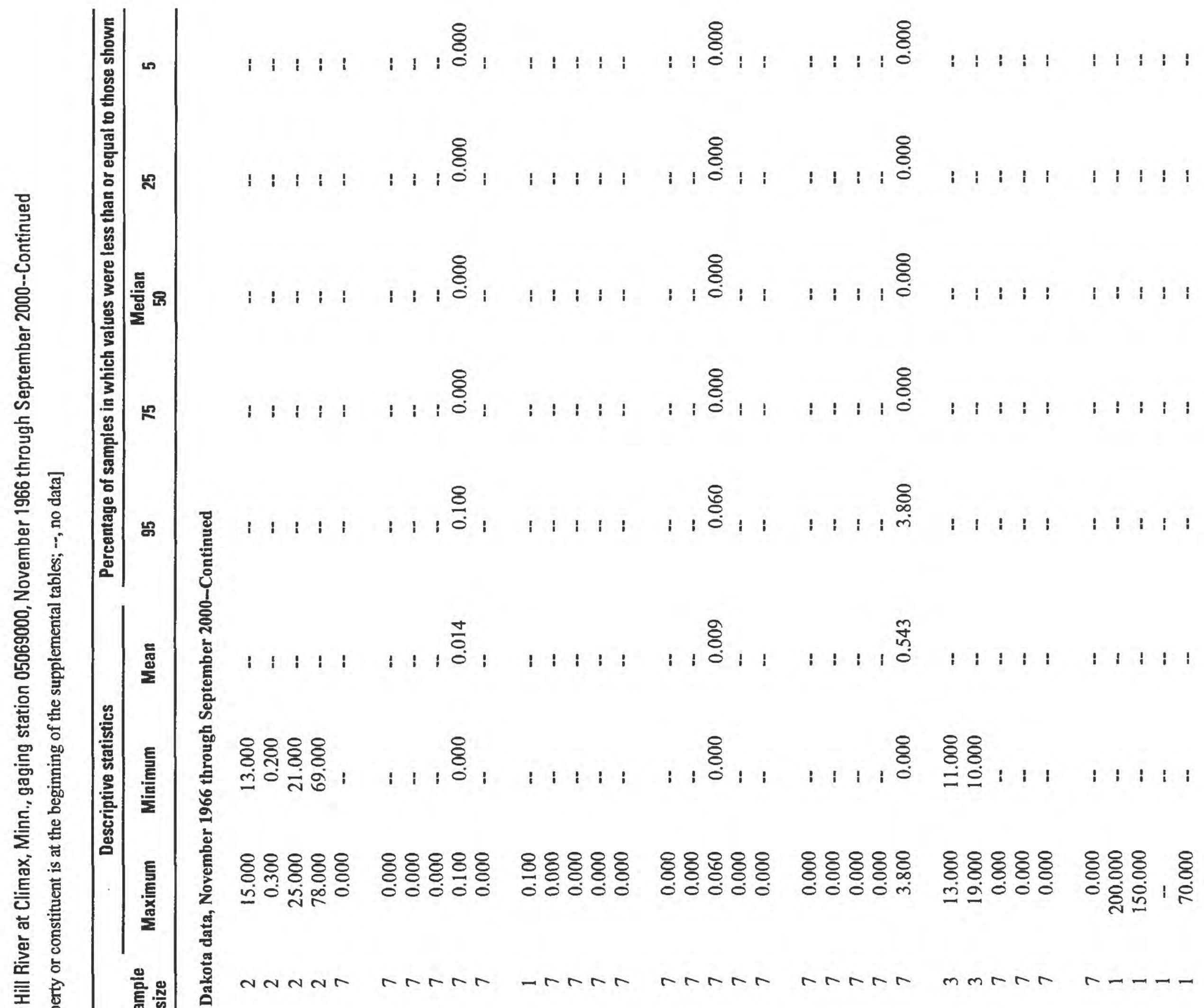

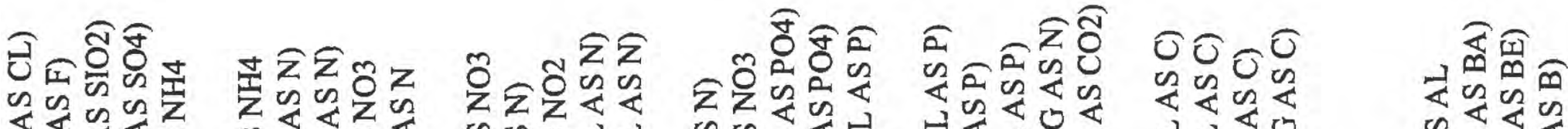

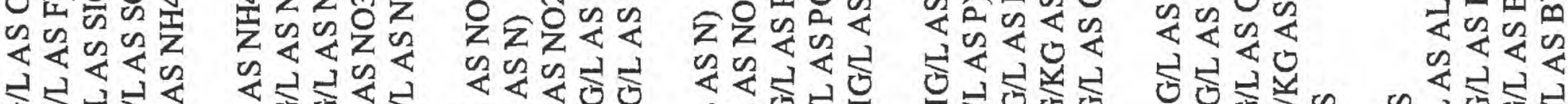
성거엉

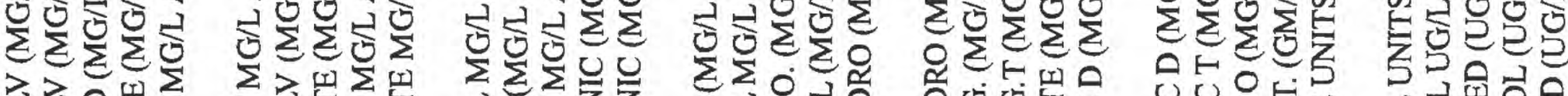

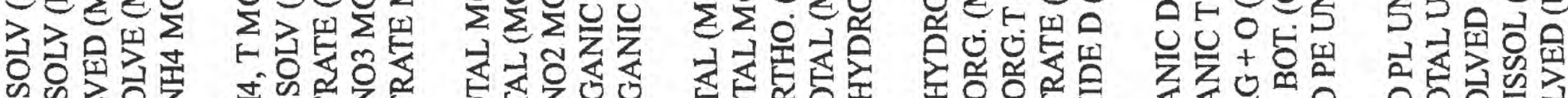

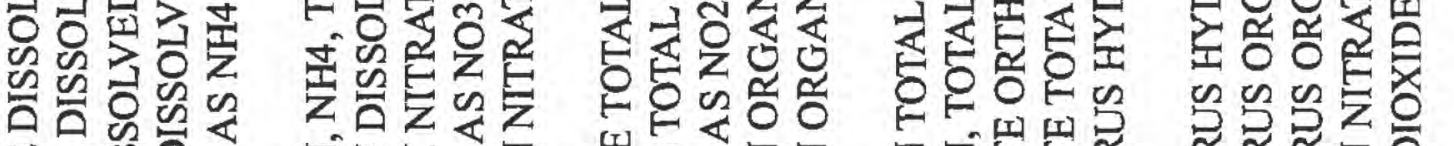

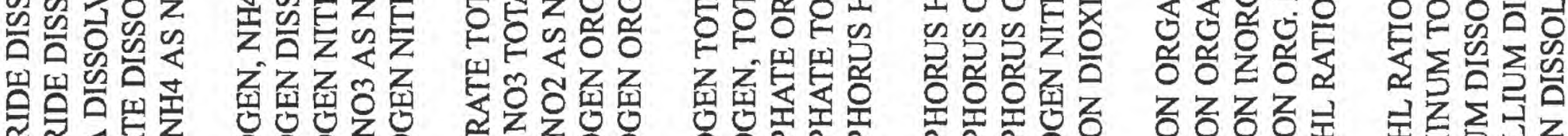
欲息

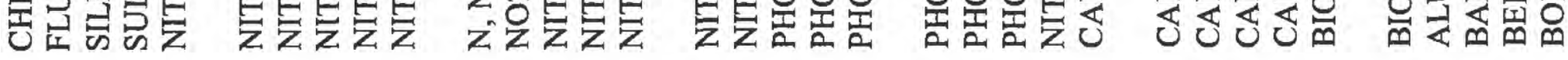




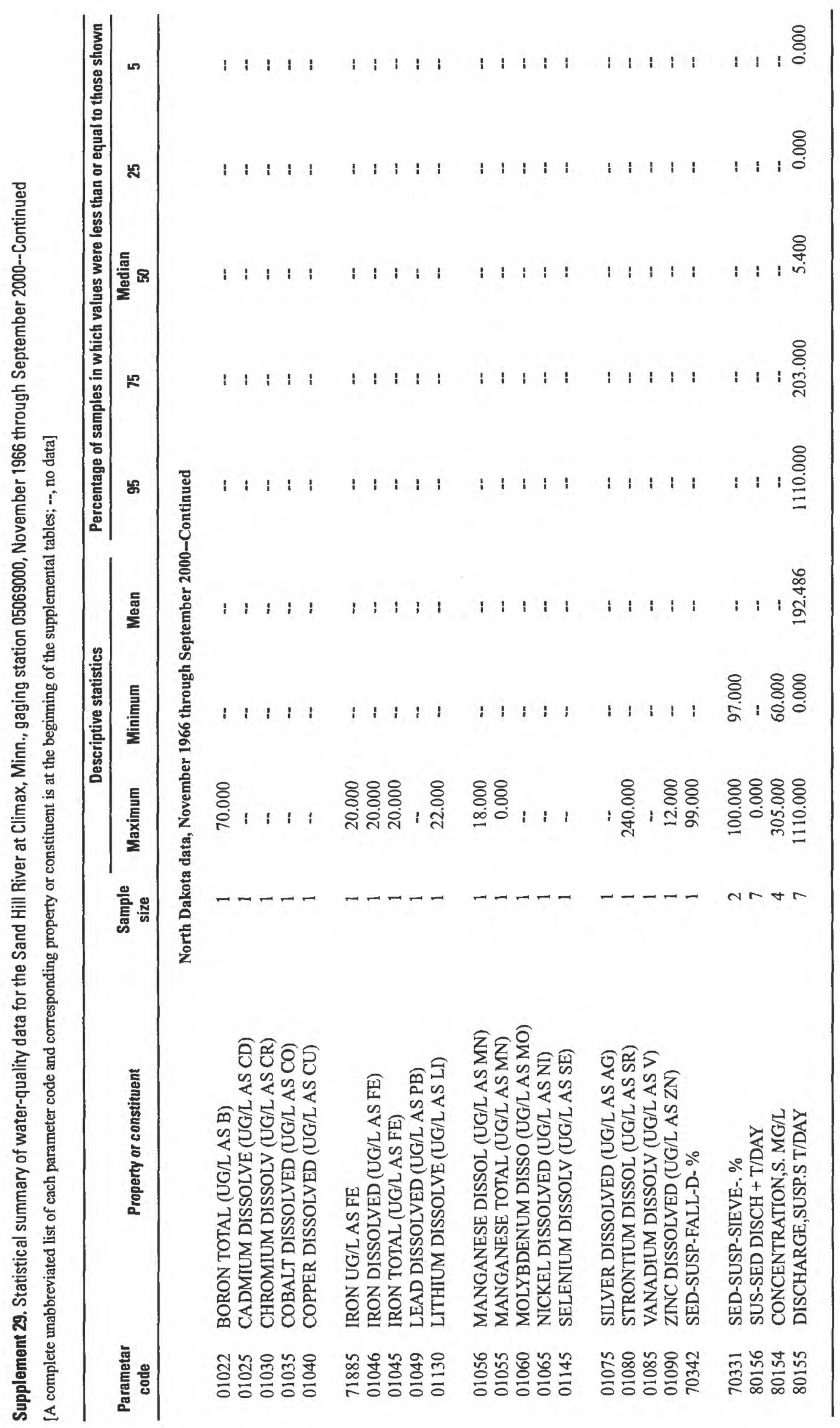




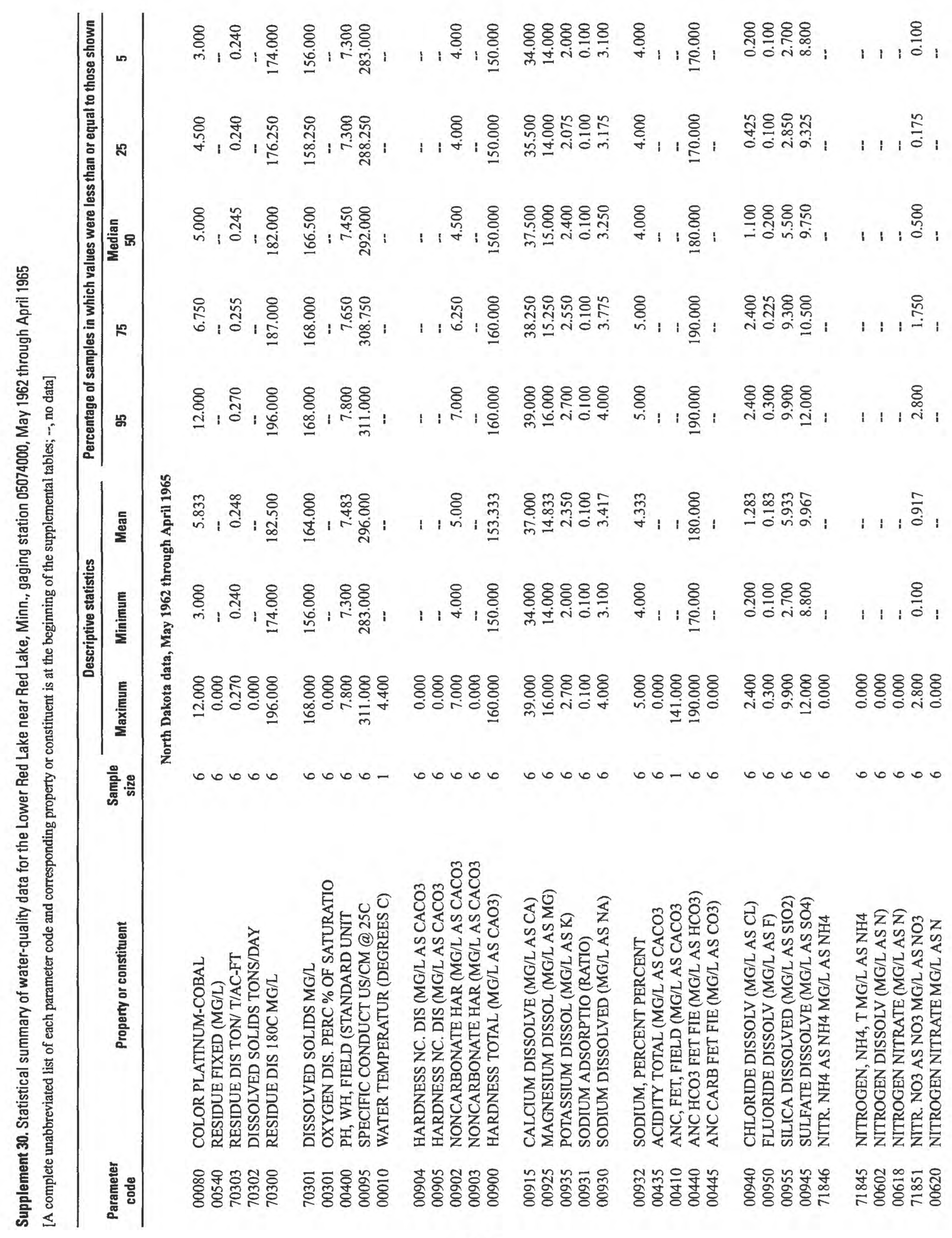




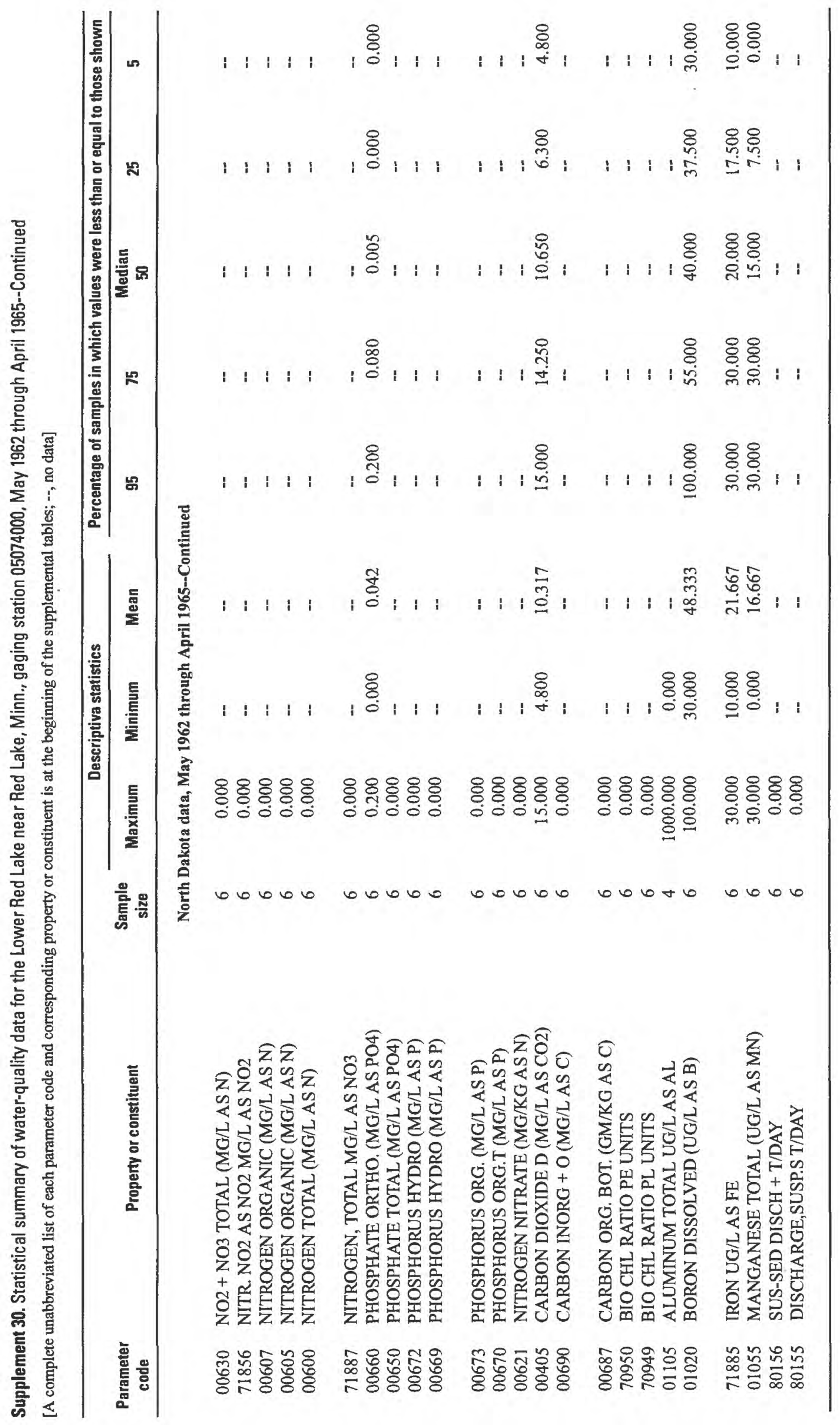




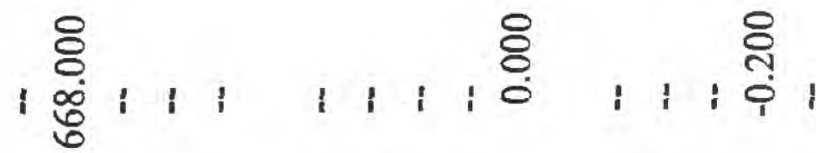

;

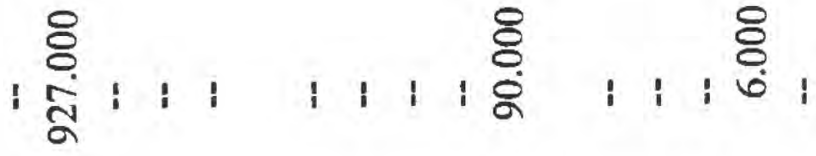

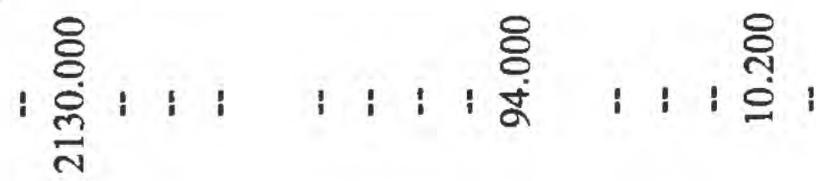

,

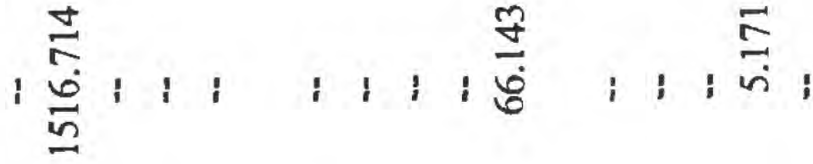

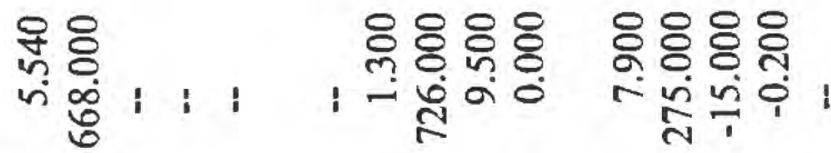

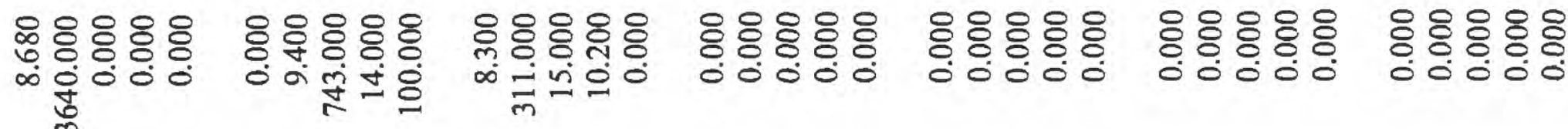

untror renenent mentror

$\operatorname{rar}$

$n-a r$

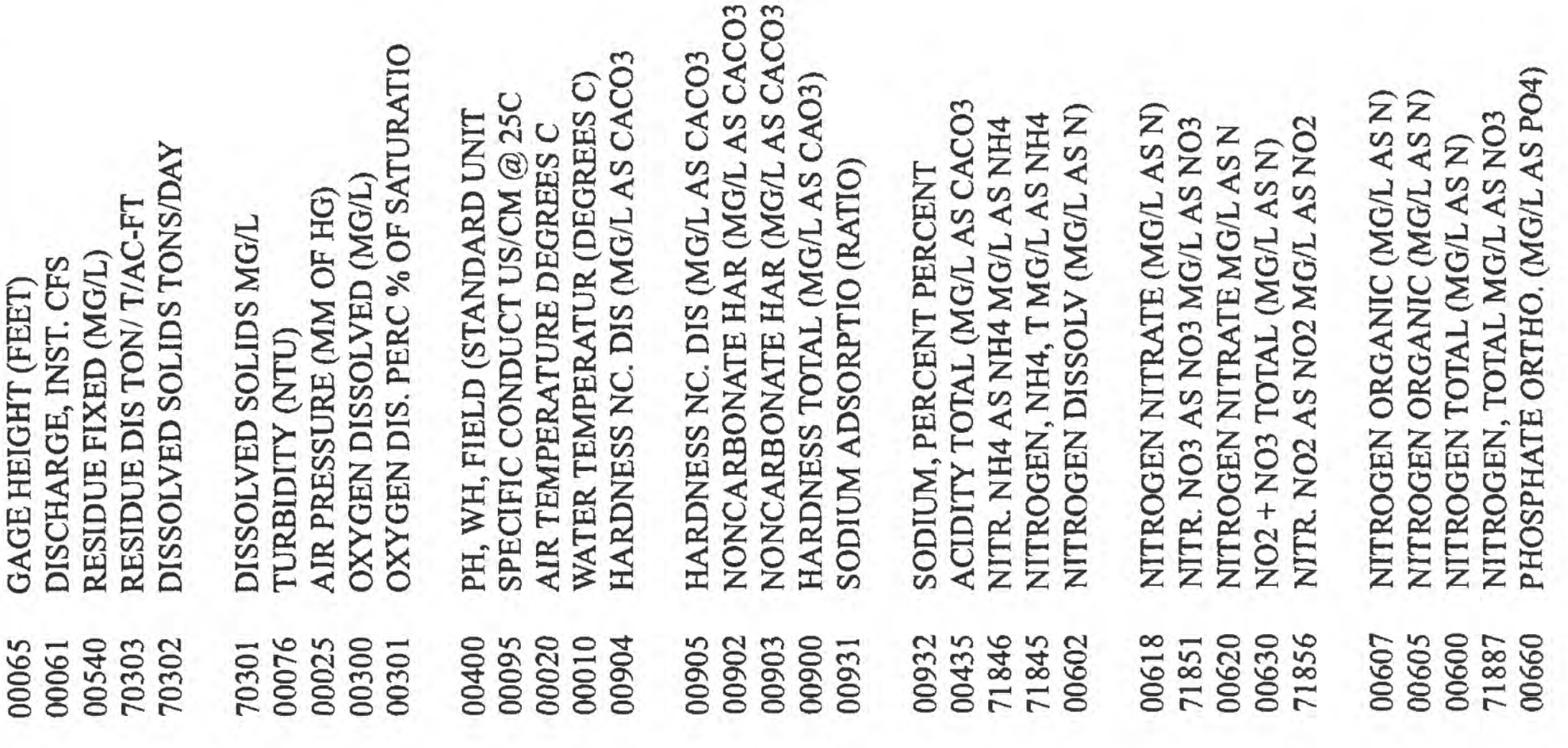




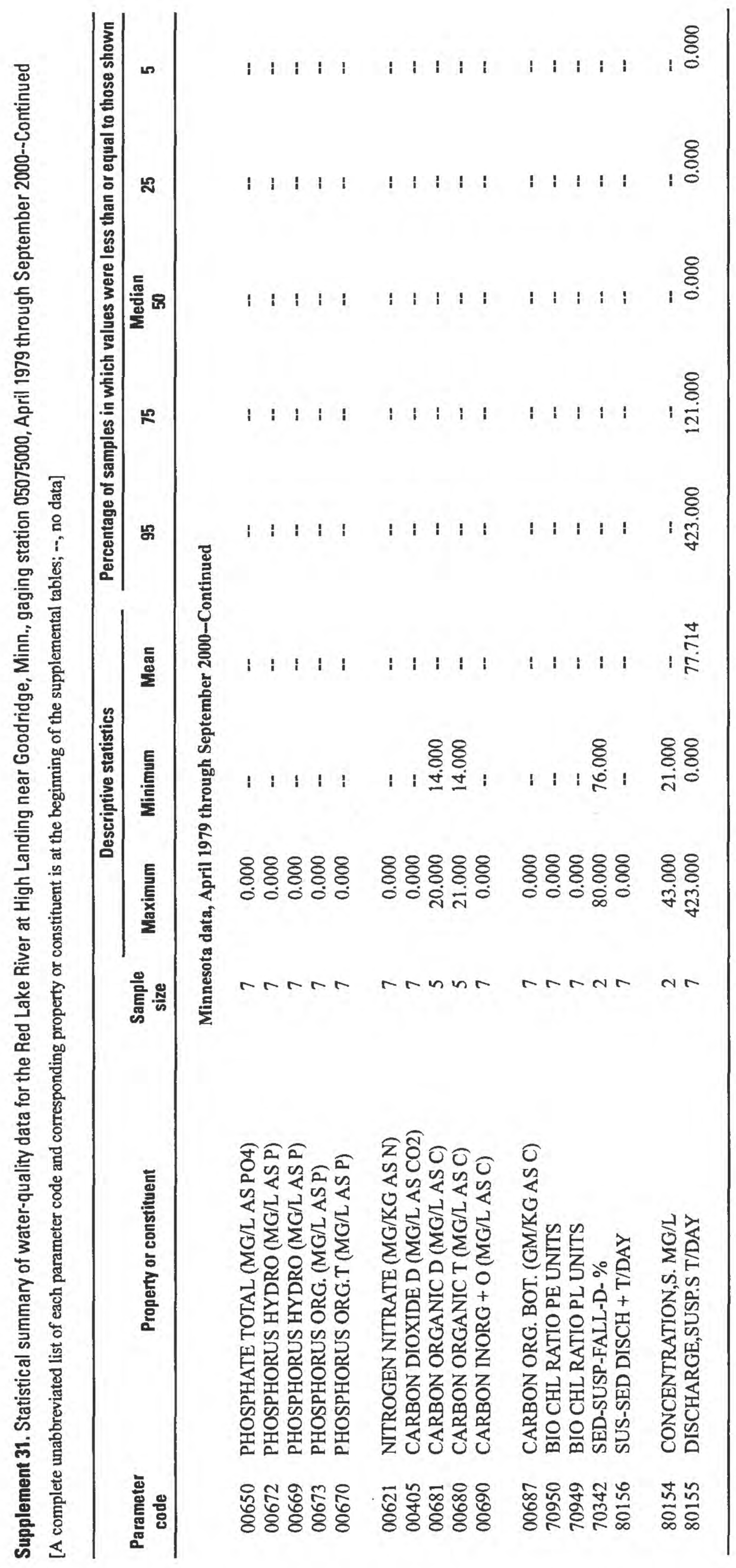




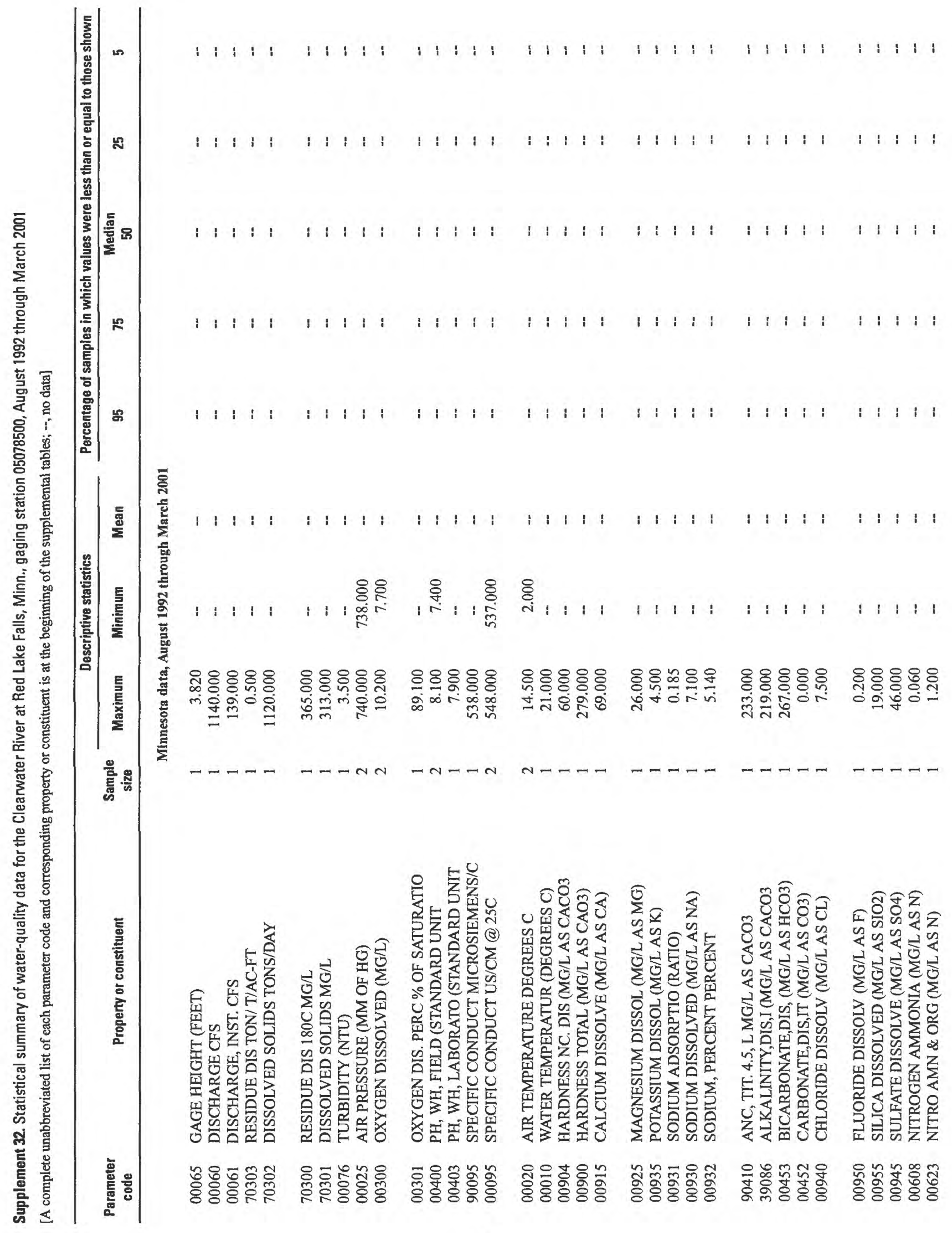


i i i i

1 1 1

1 1 1 1

1 1 1

1 1 1 :

i : : i



i i i

; i i

1 1 1

I I I

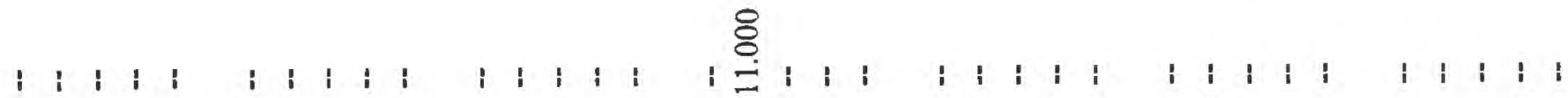

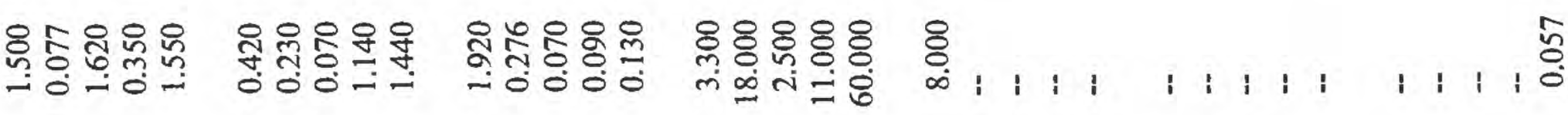

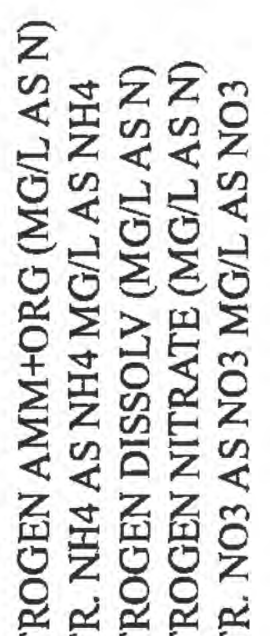

운

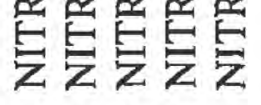

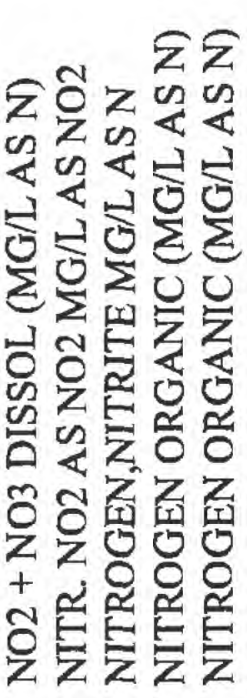

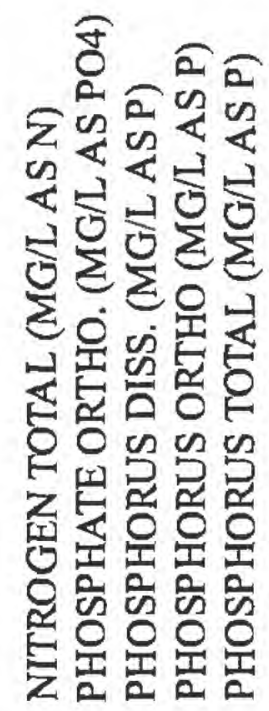

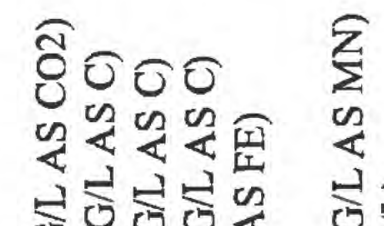

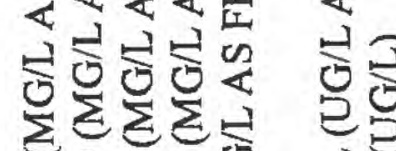

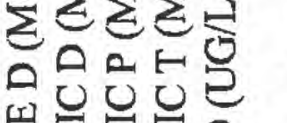

된브ㄴㅡㅡㄹ

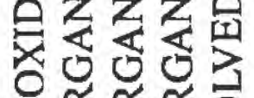

응응응

毛乐员

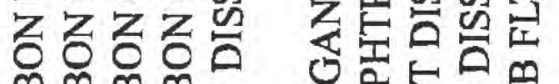

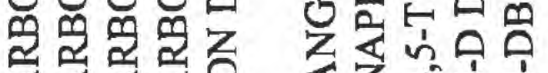

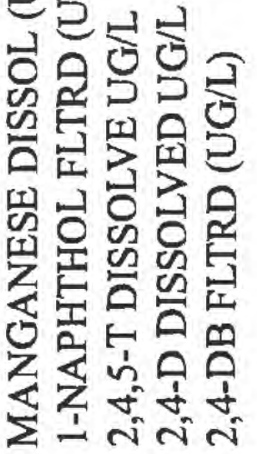

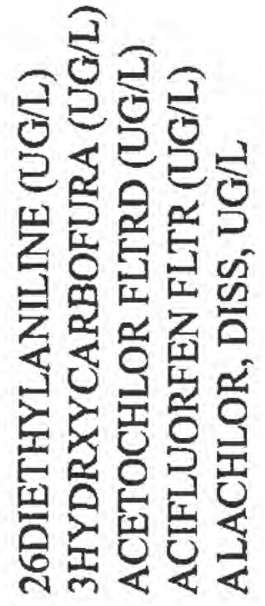

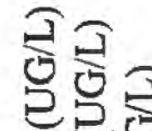

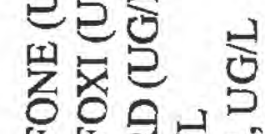

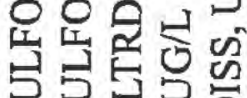

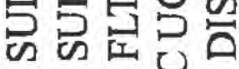

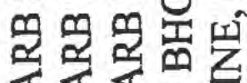

过《其

㧗吉 


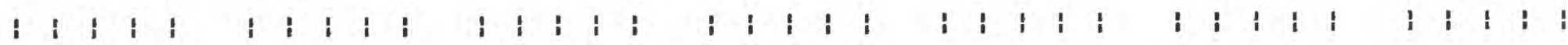

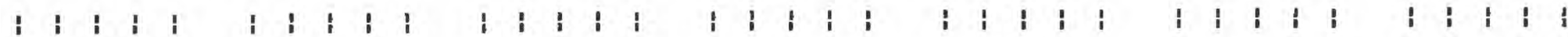

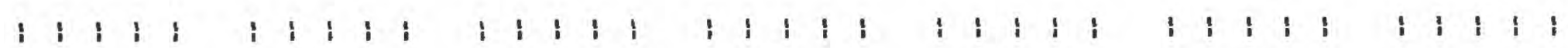

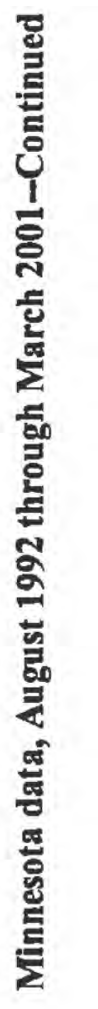

i i i i i

i i i i i i ठ ठి

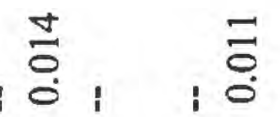

$---$

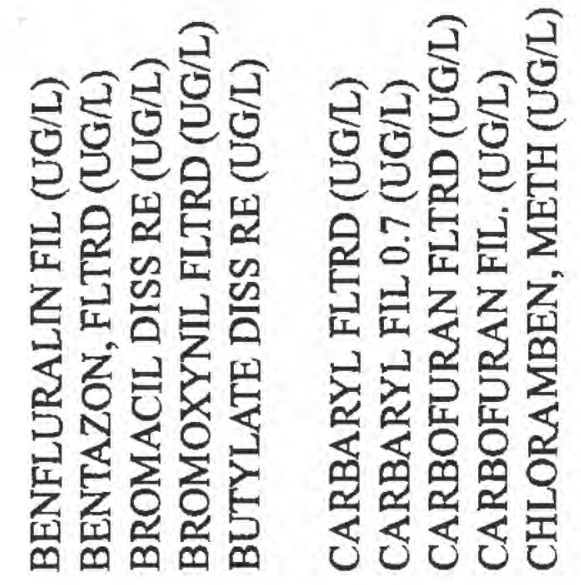

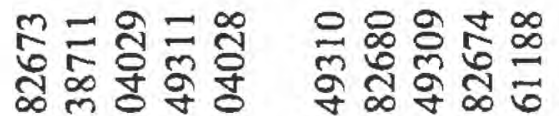

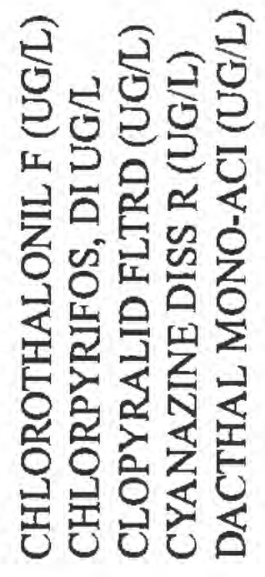

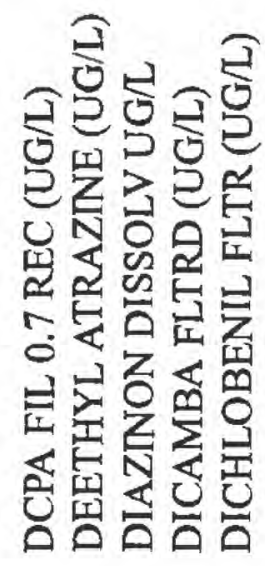

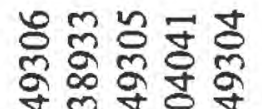

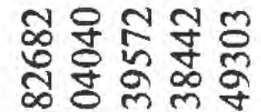
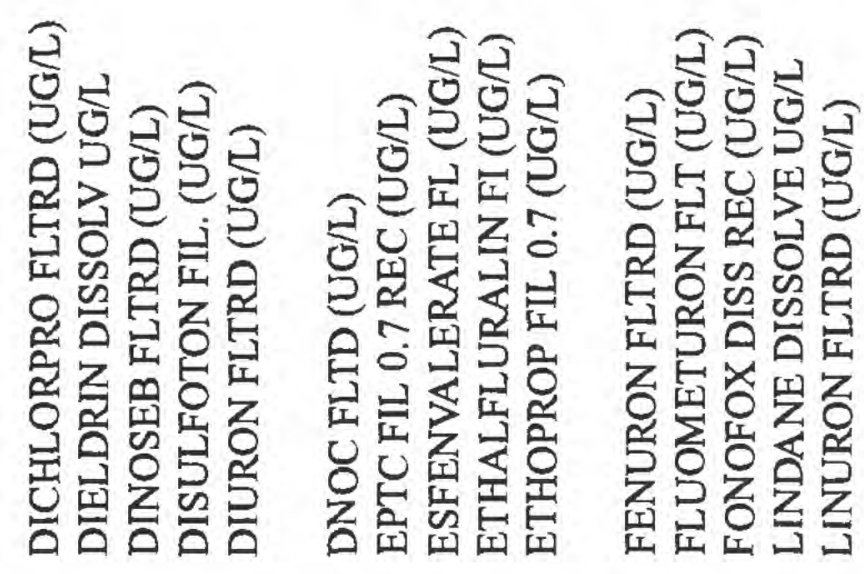

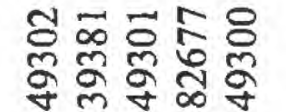




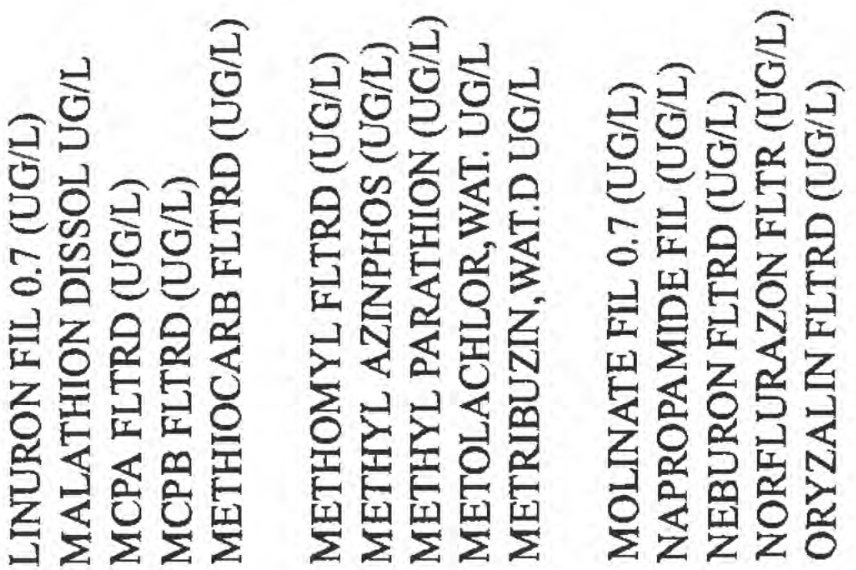

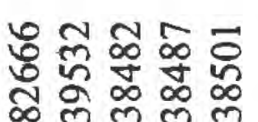

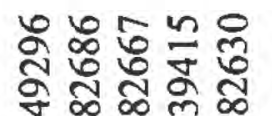

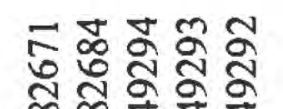

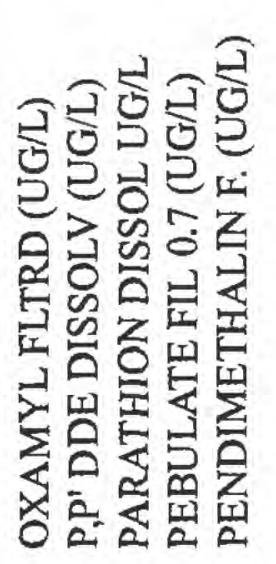

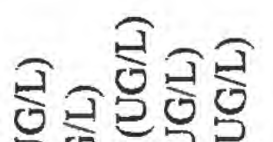
过空远 i

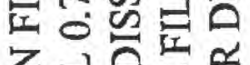
是星的

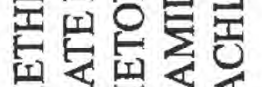

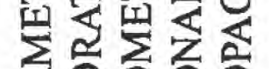

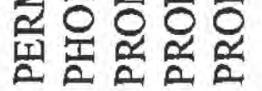

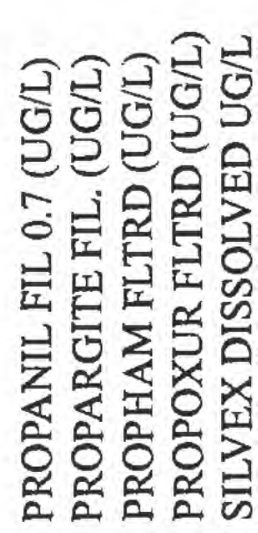

ิㅗㅇํํ오ํ엉 包论定

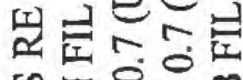

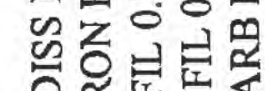

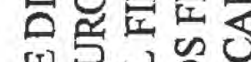
닌분욜 곤

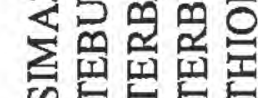

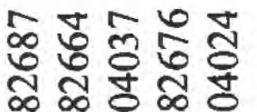

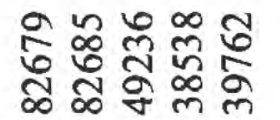




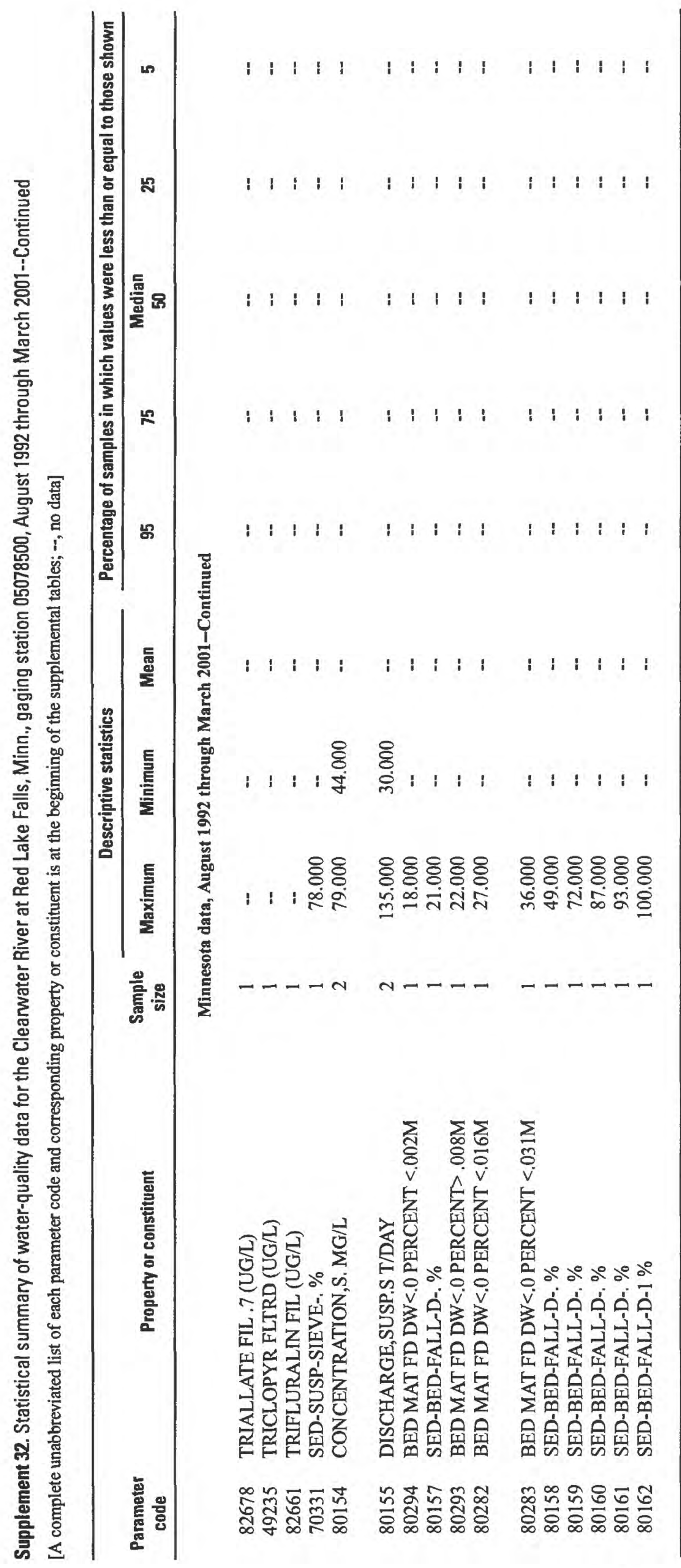




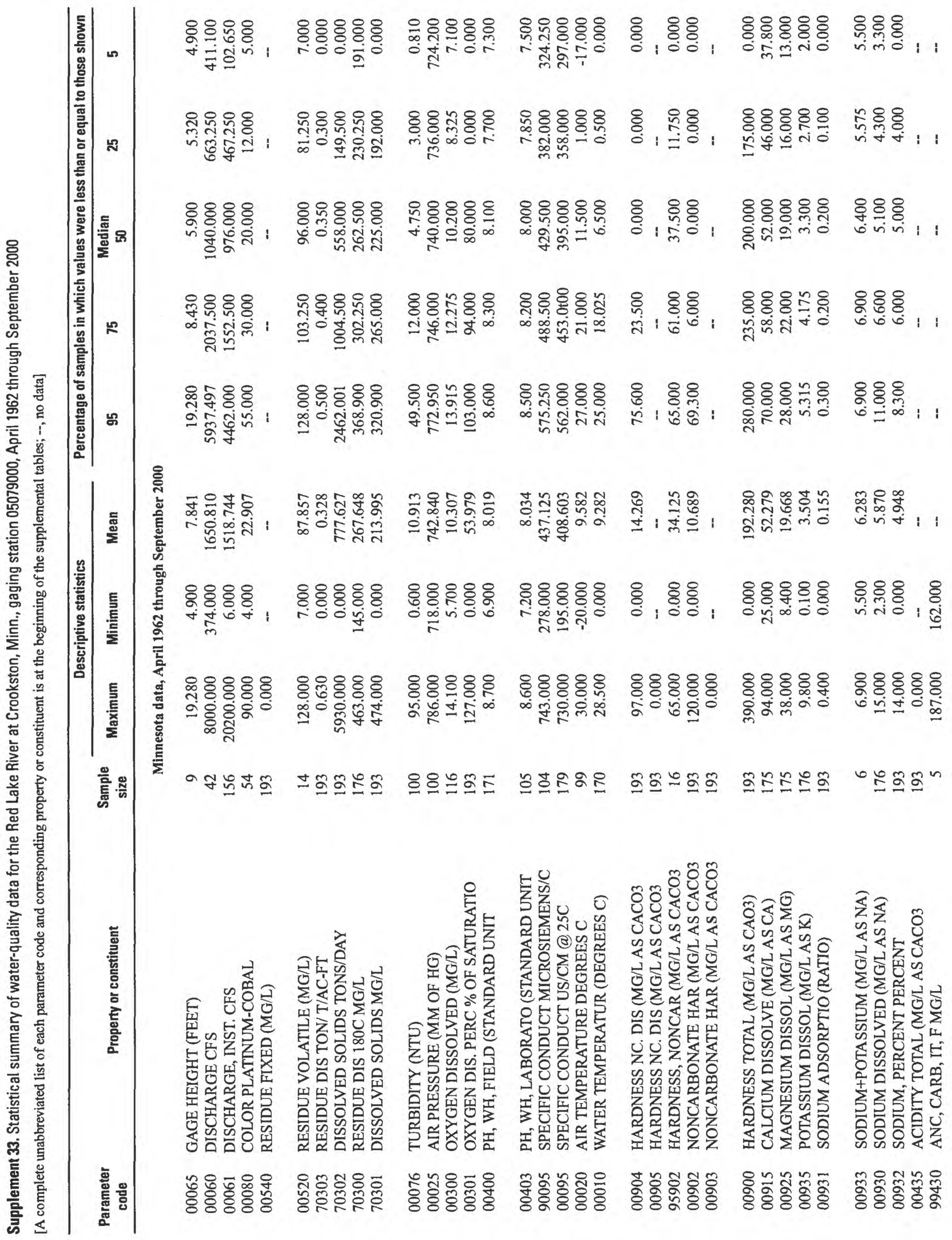




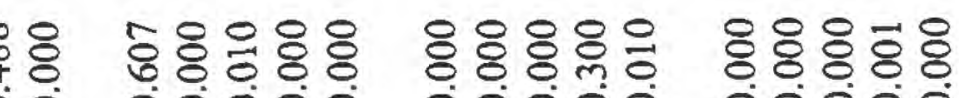

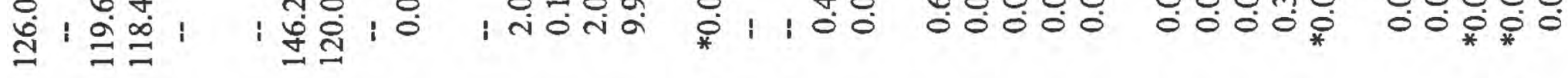

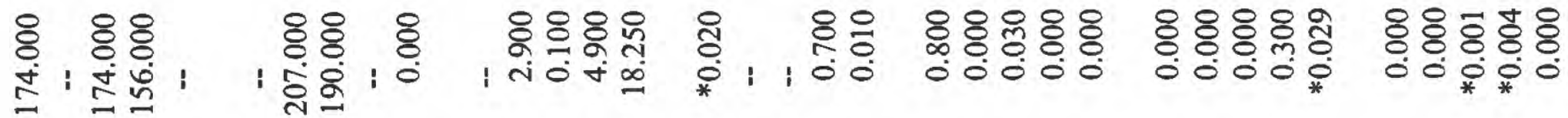

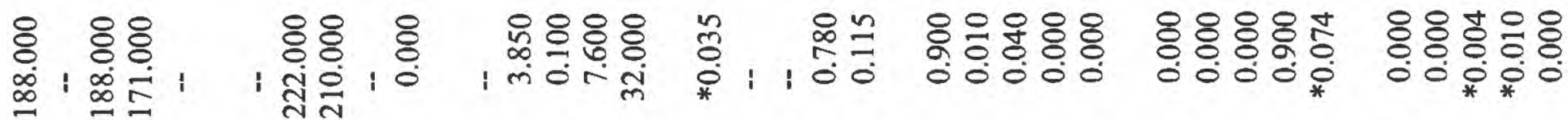

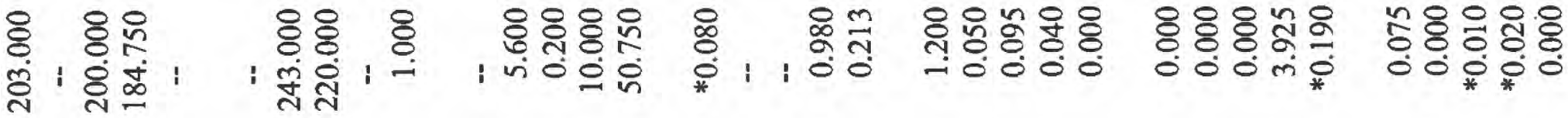

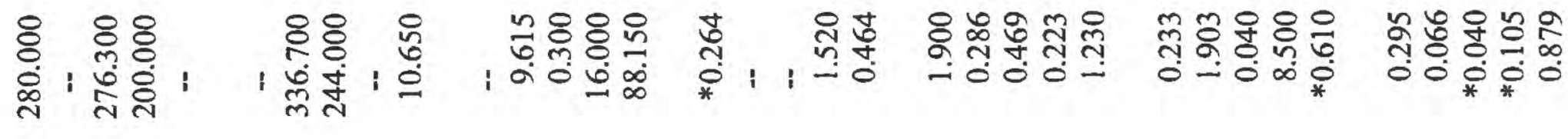

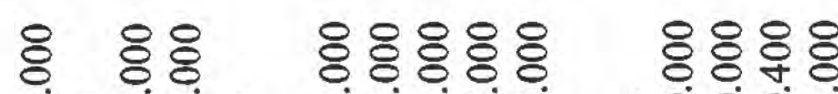

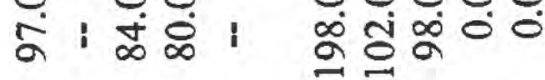

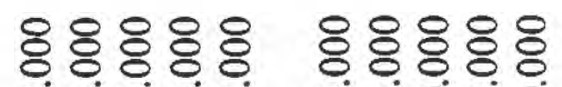

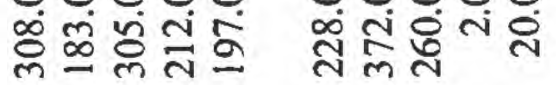

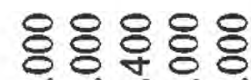
iñ

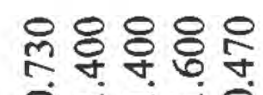

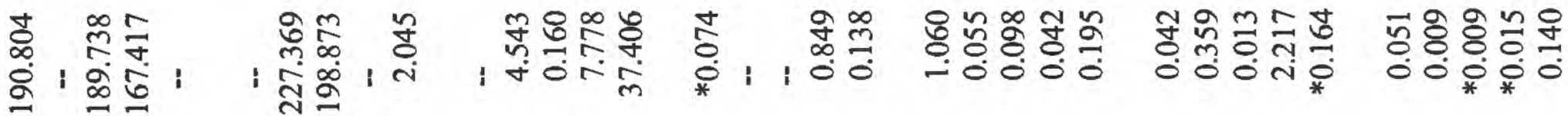

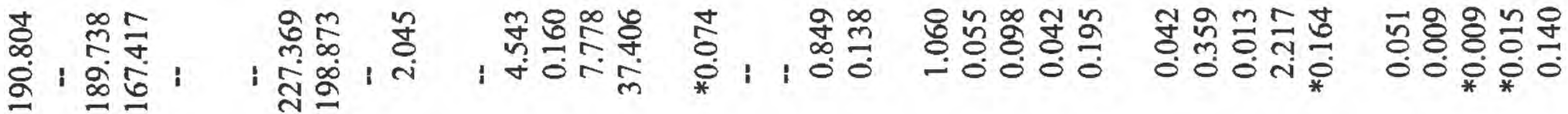
m-

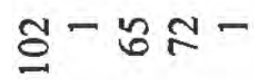

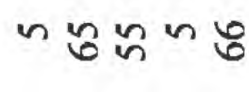

子ํํㄴ돈 $\stackrel{\Xi}{\mathfrak{y}}$

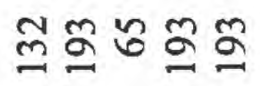

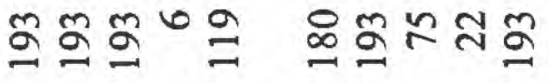
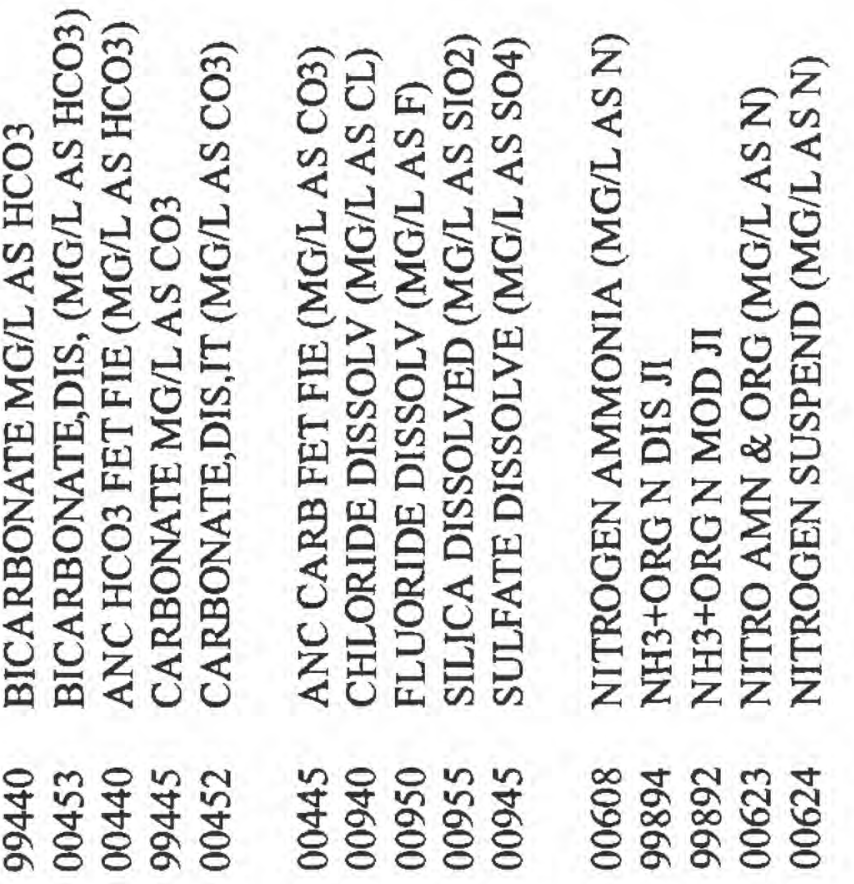

z

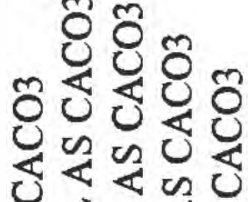

का

实

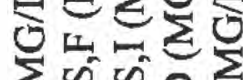

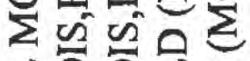

成足是

量是至

E是牙

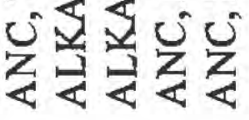

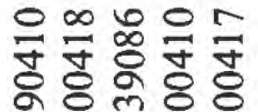

प⿺廴

요유

융융융

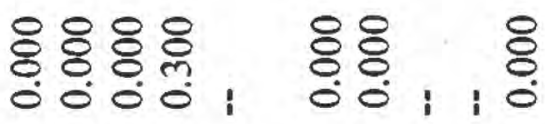

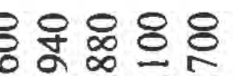

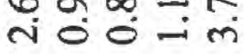

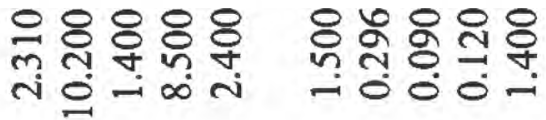




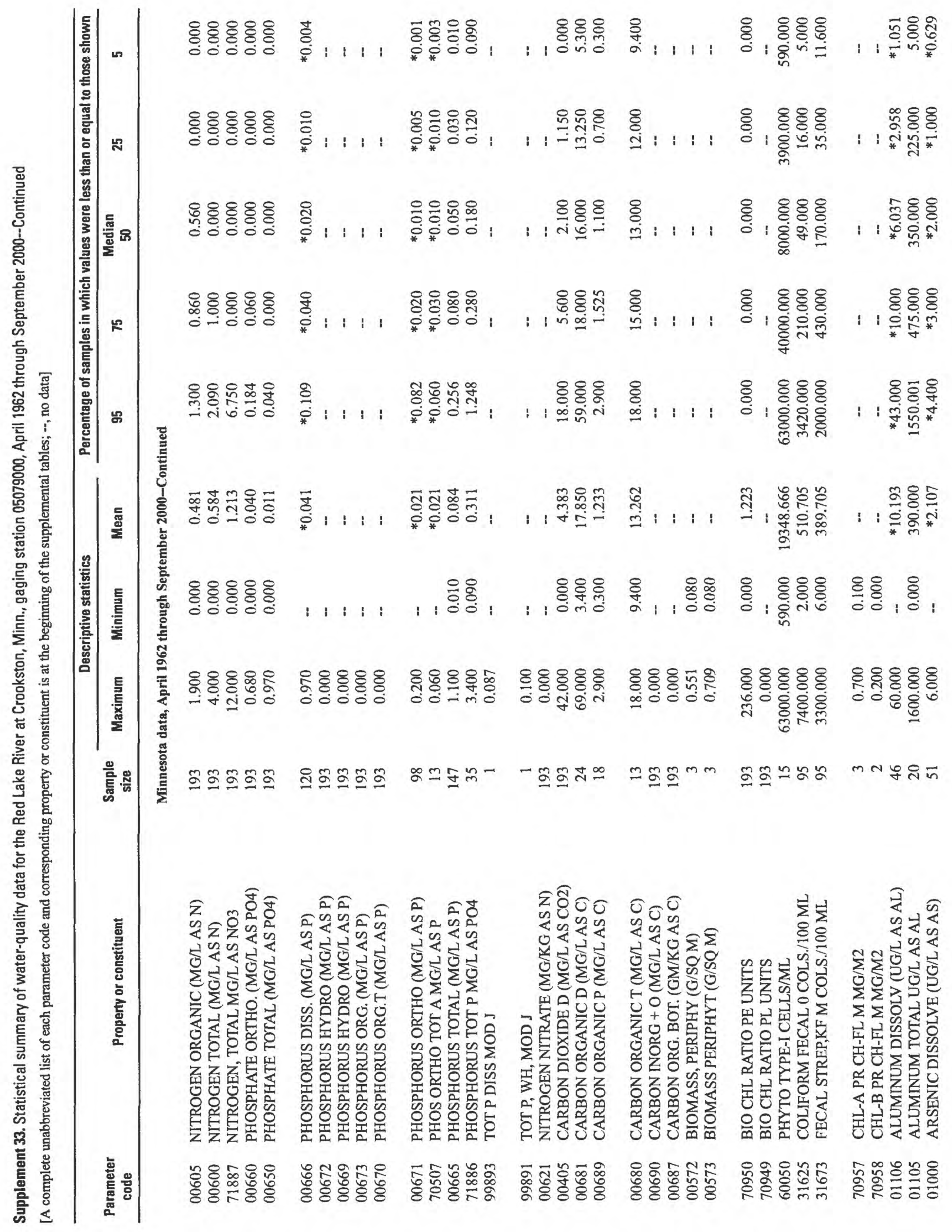




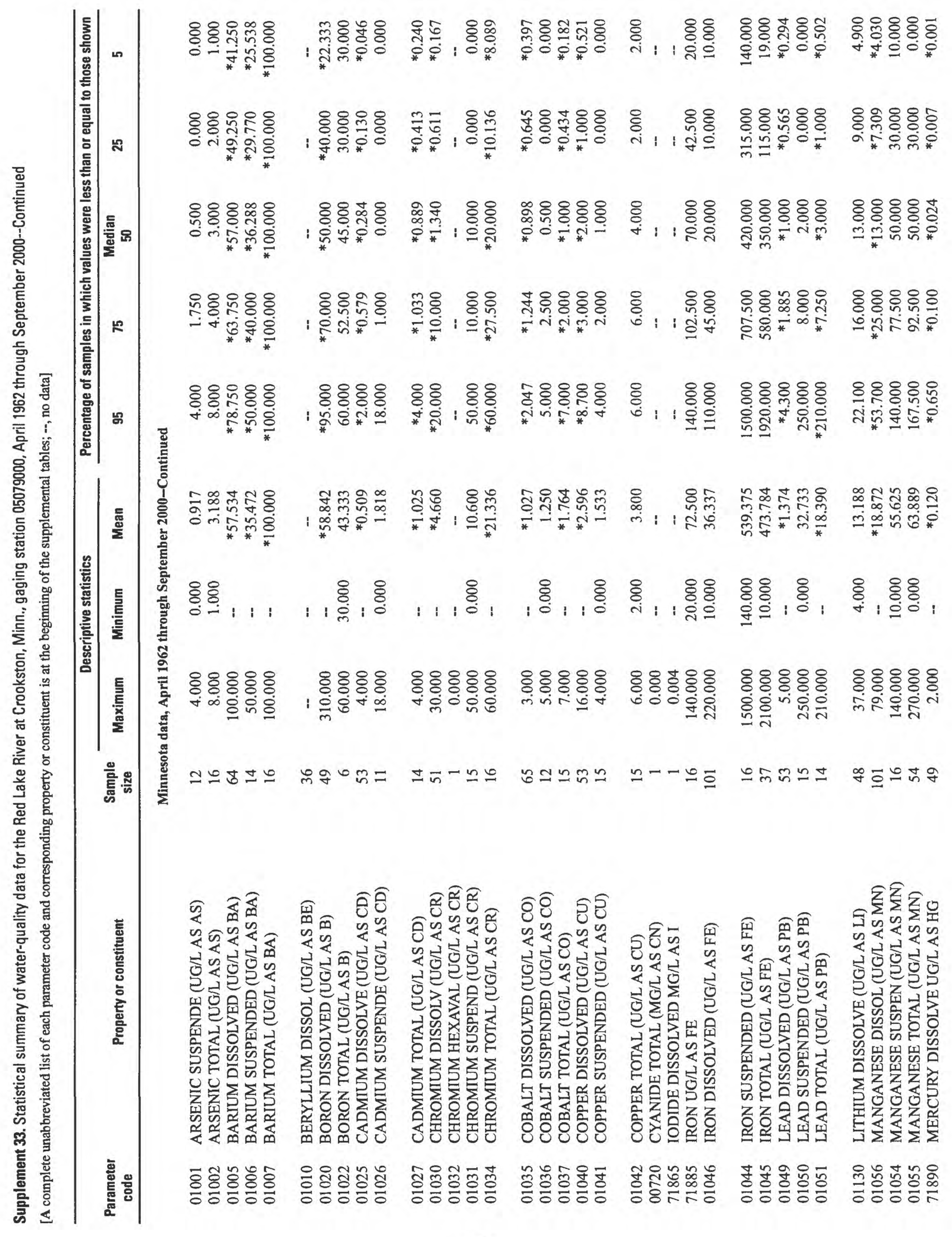




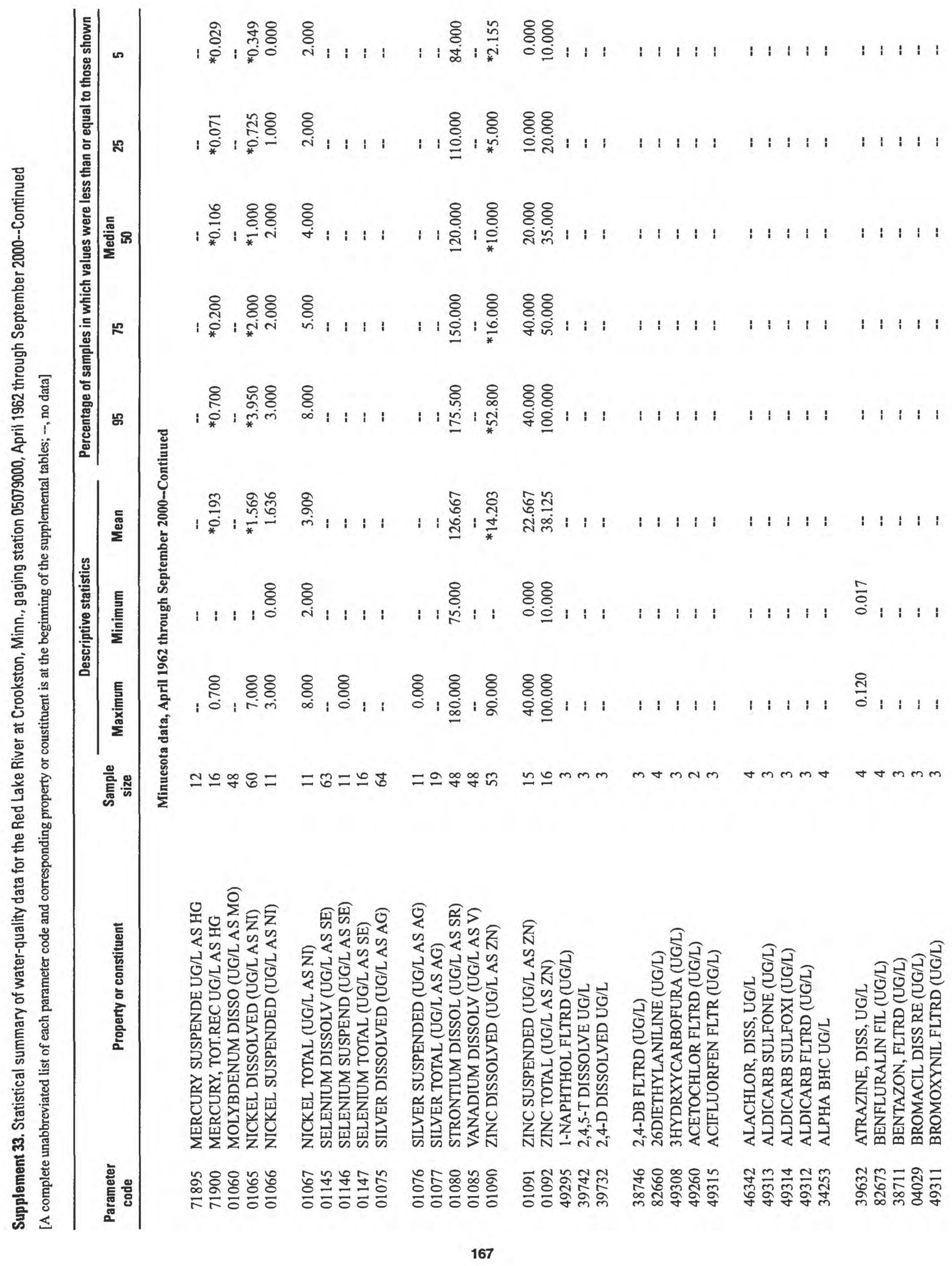




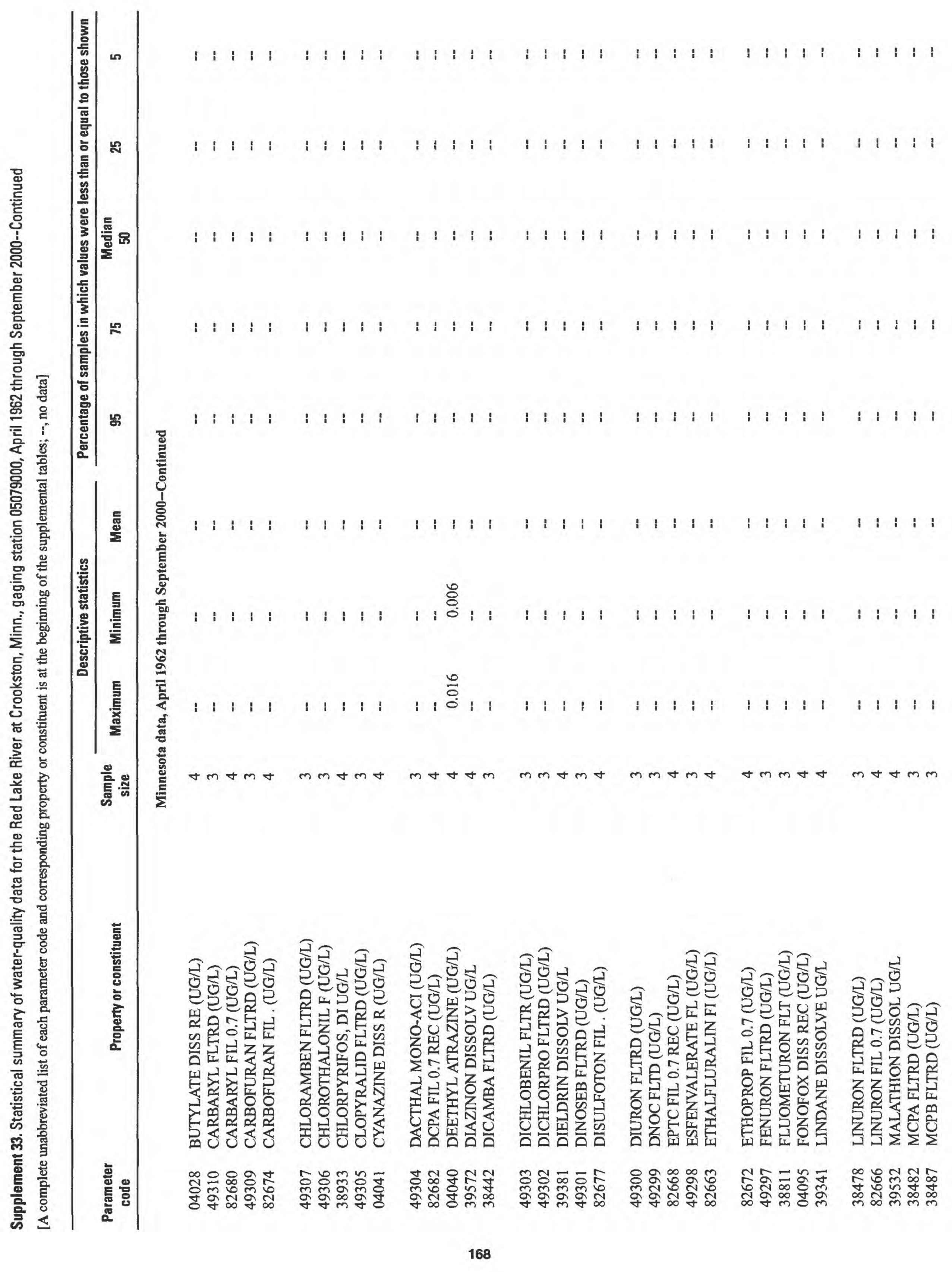




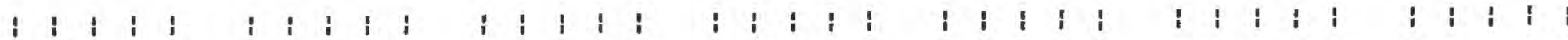

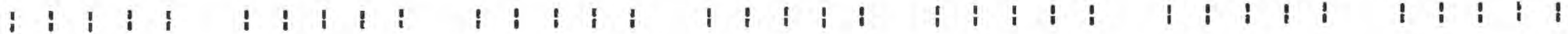

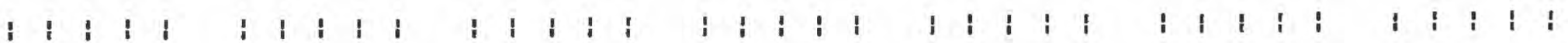

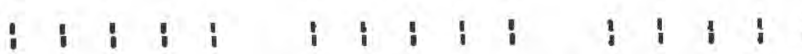

i i i i

$1 ; i: 1$

$1,1+1$

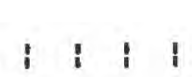

$i \quad i \quad 1$

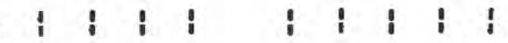

i i i

i i i i

i 1 i i i

i i i i

i 1 i 1

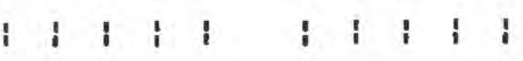

1 i 11

i i i

i i i i

i i i i

i i

1 1 i i 1 i 1 i

$m m+\forall \forall$

$\forall+m m$

$m m+\forall+$

$A+t 4 t$

オナナナm

$m m+\theta$

$A t \theta m A$

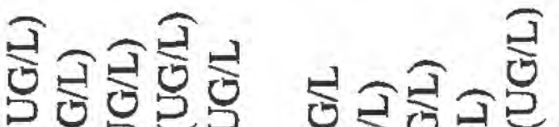

謧
얼름

论洝

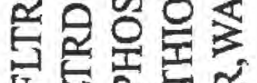

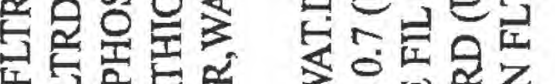

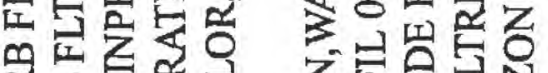

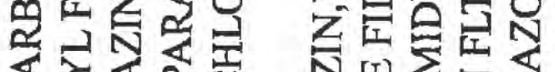

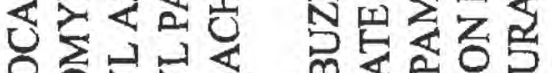

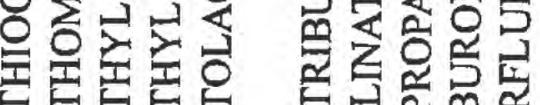

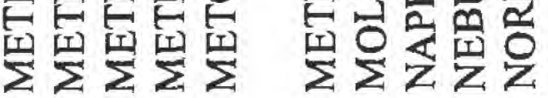

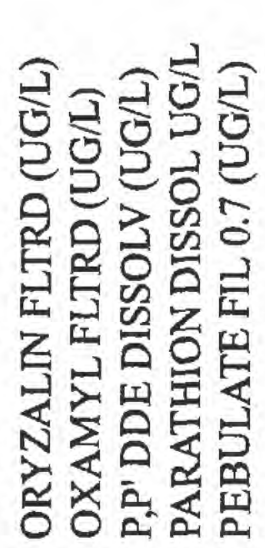

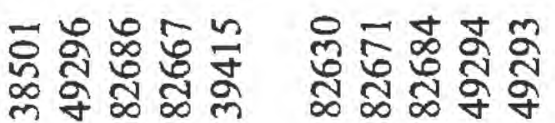

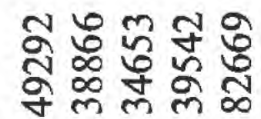

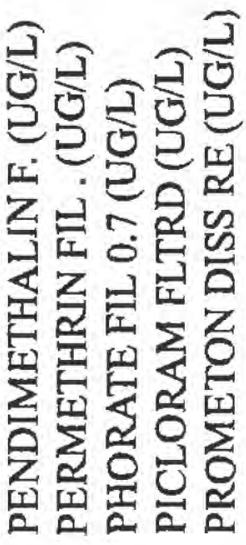

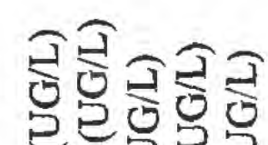

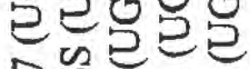

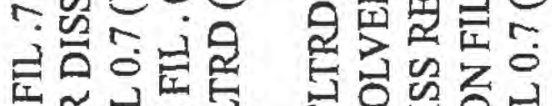

떠일

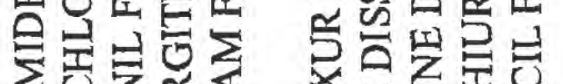

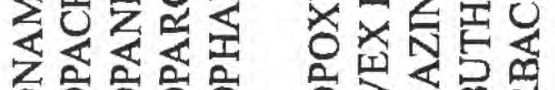

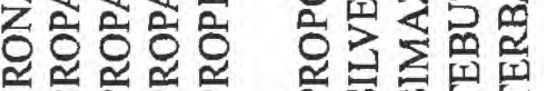

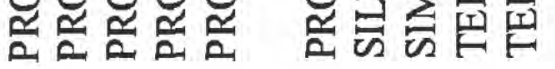

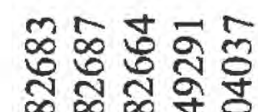

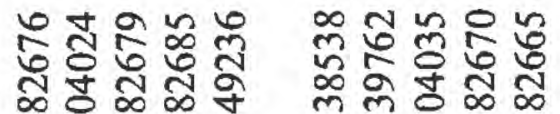

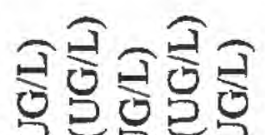

ट

。屋里国

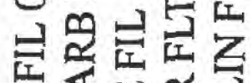

㺷四

눈

舟皮题

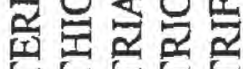

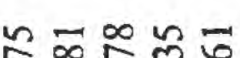

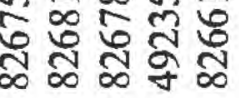




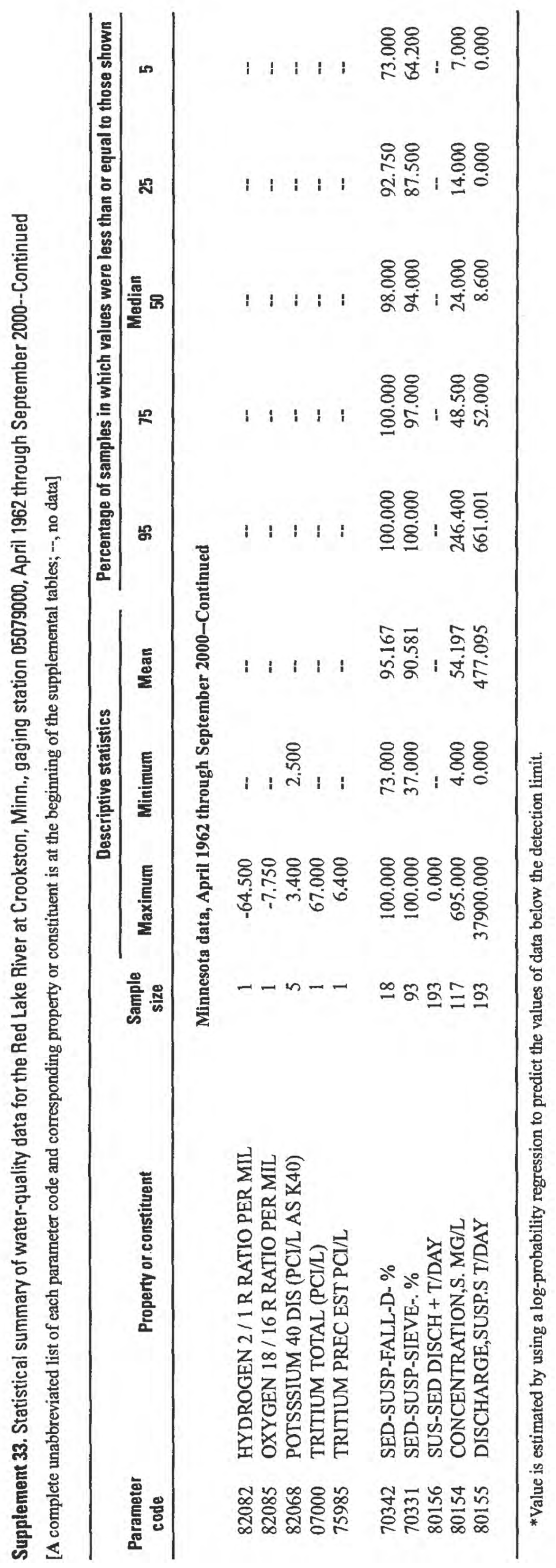




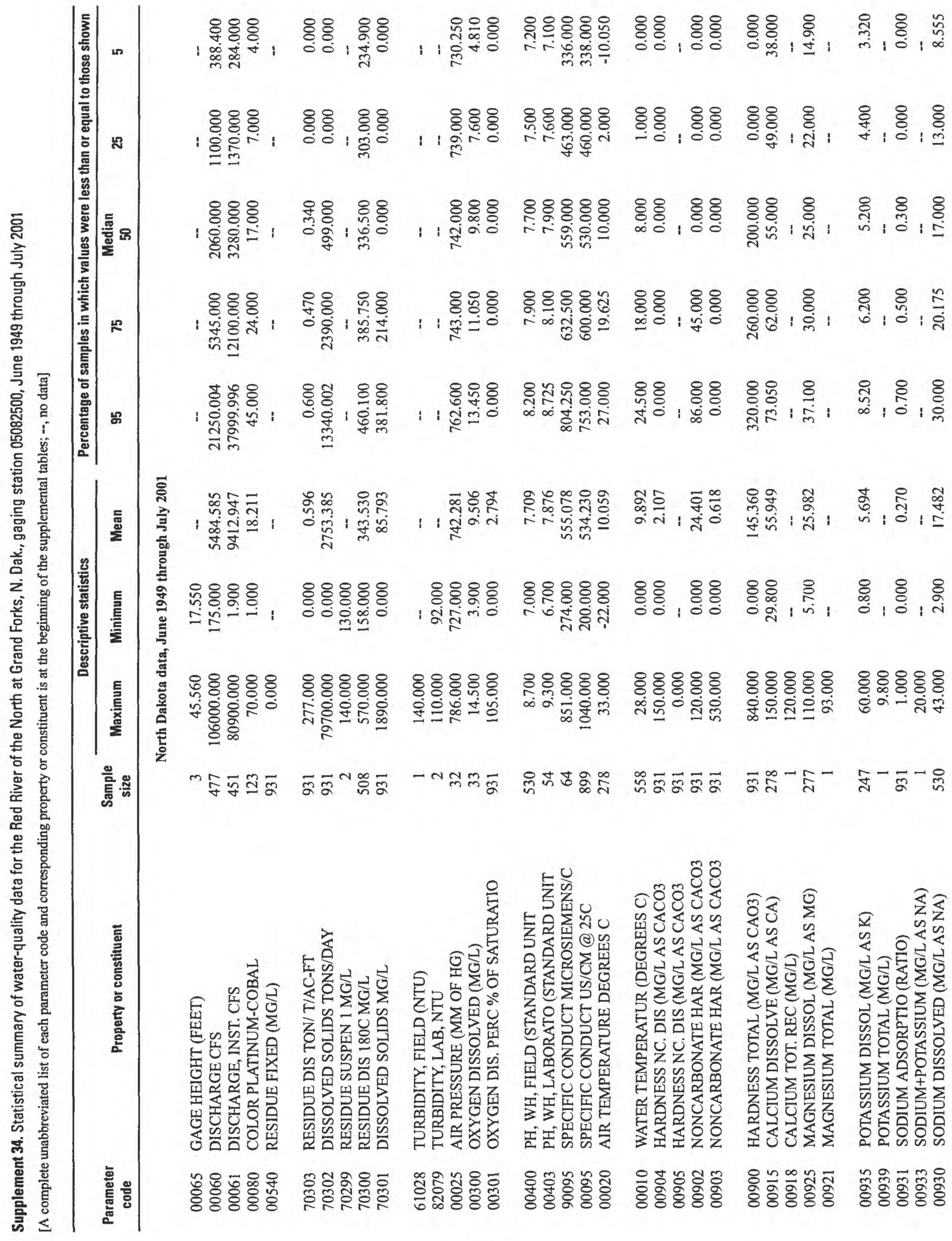




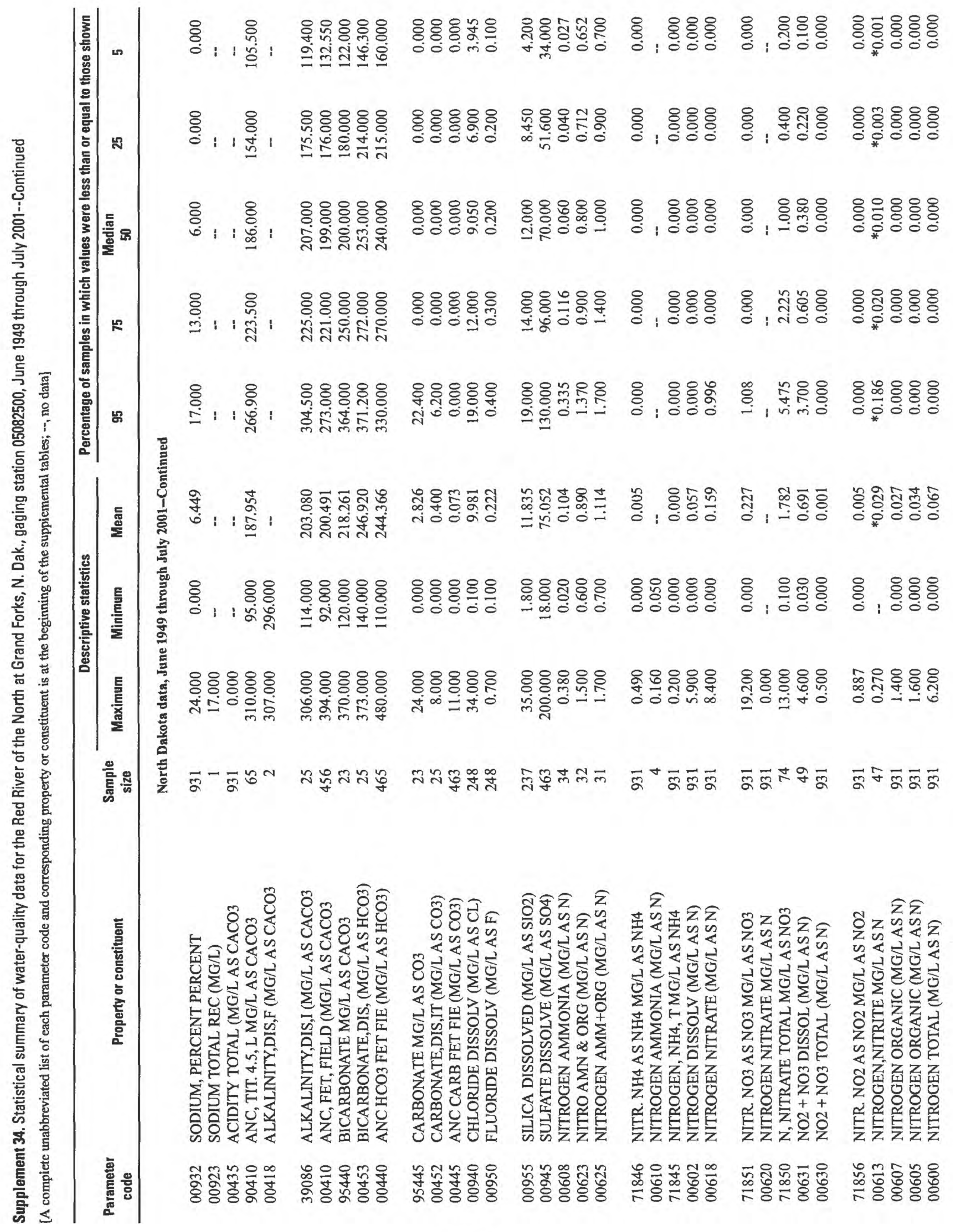




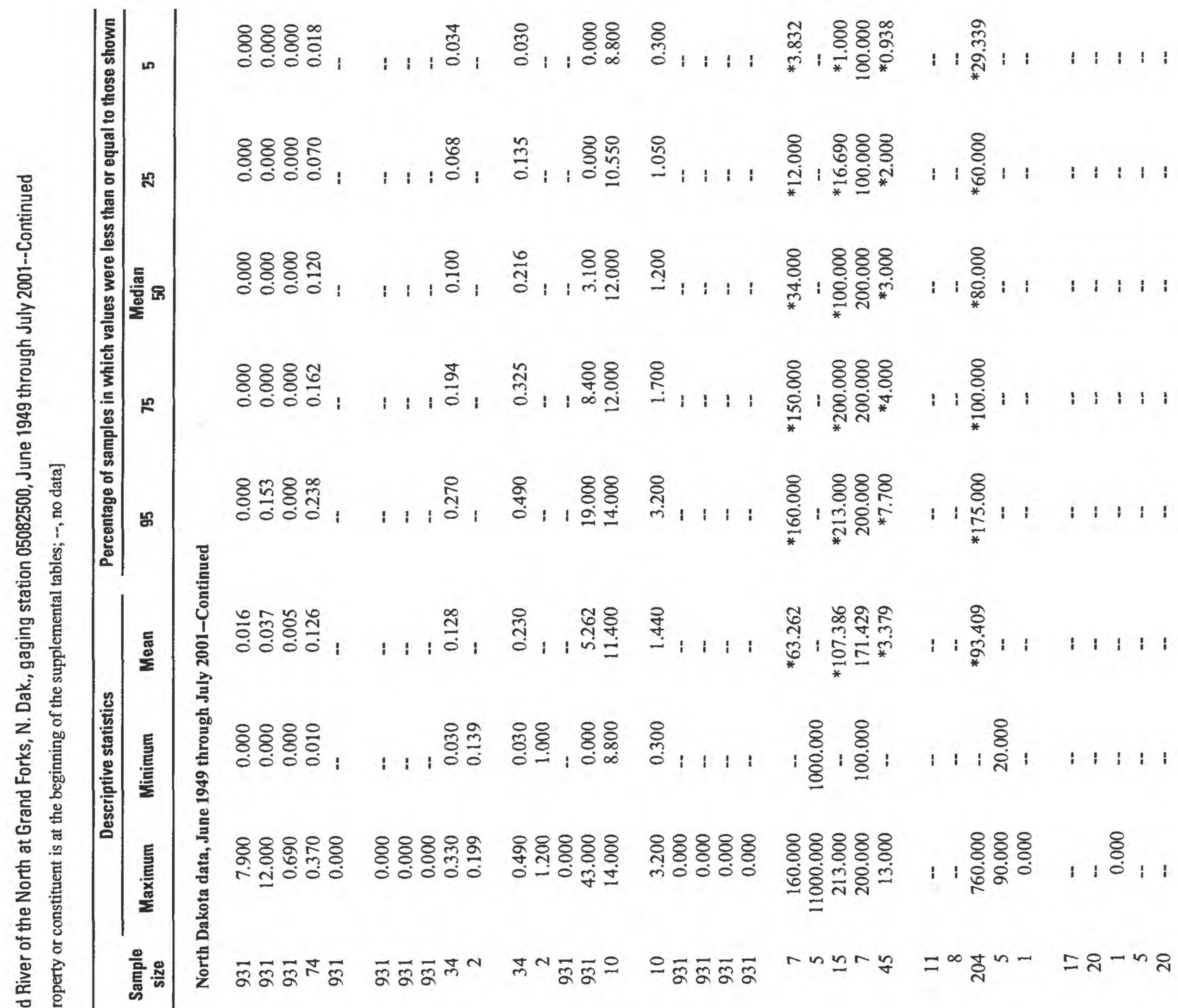

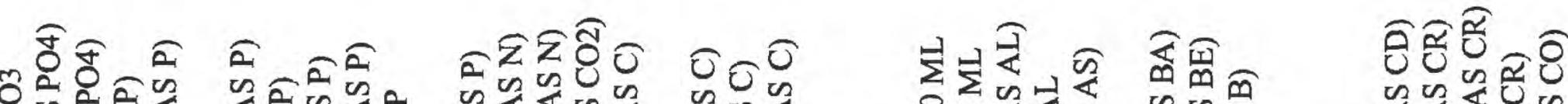

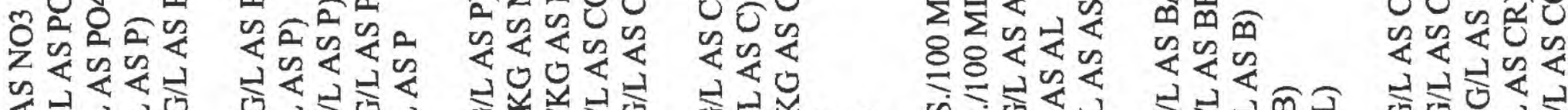

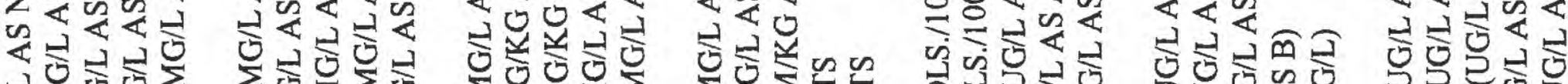

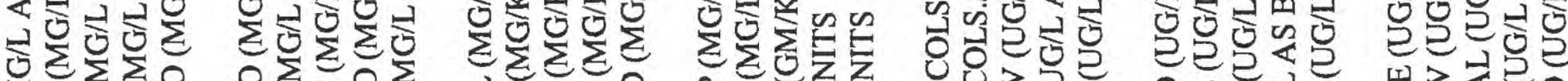

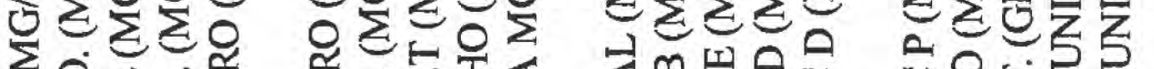

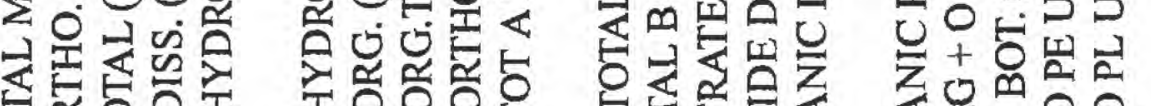

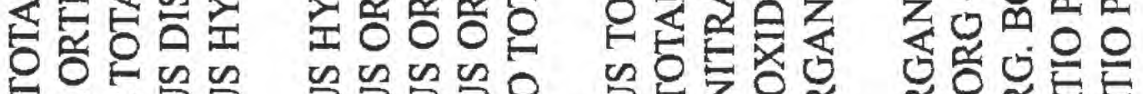

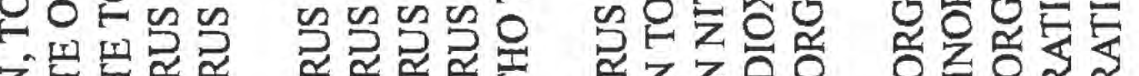

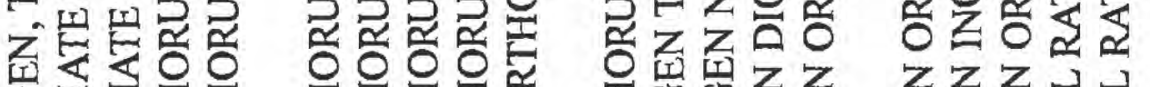
논

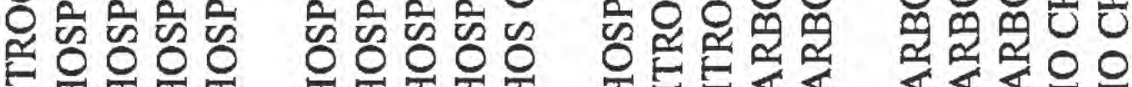

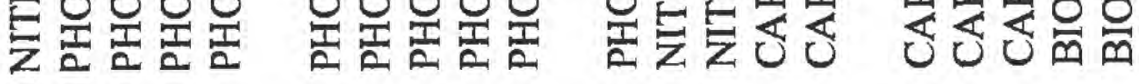
०

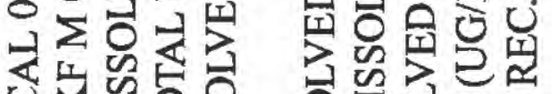
幽等

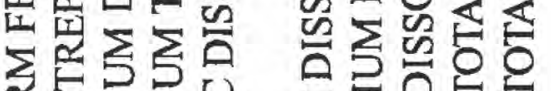
的是证

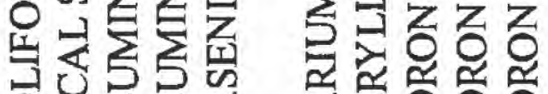

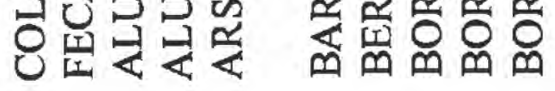

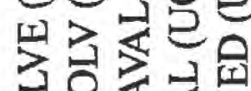

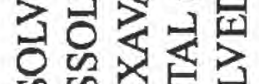
员产宫

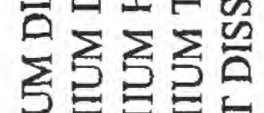
就星

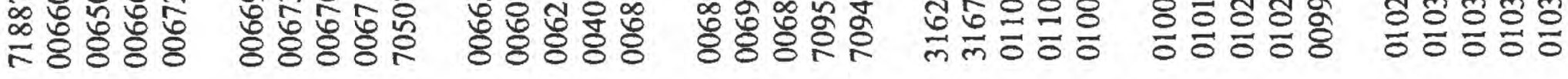




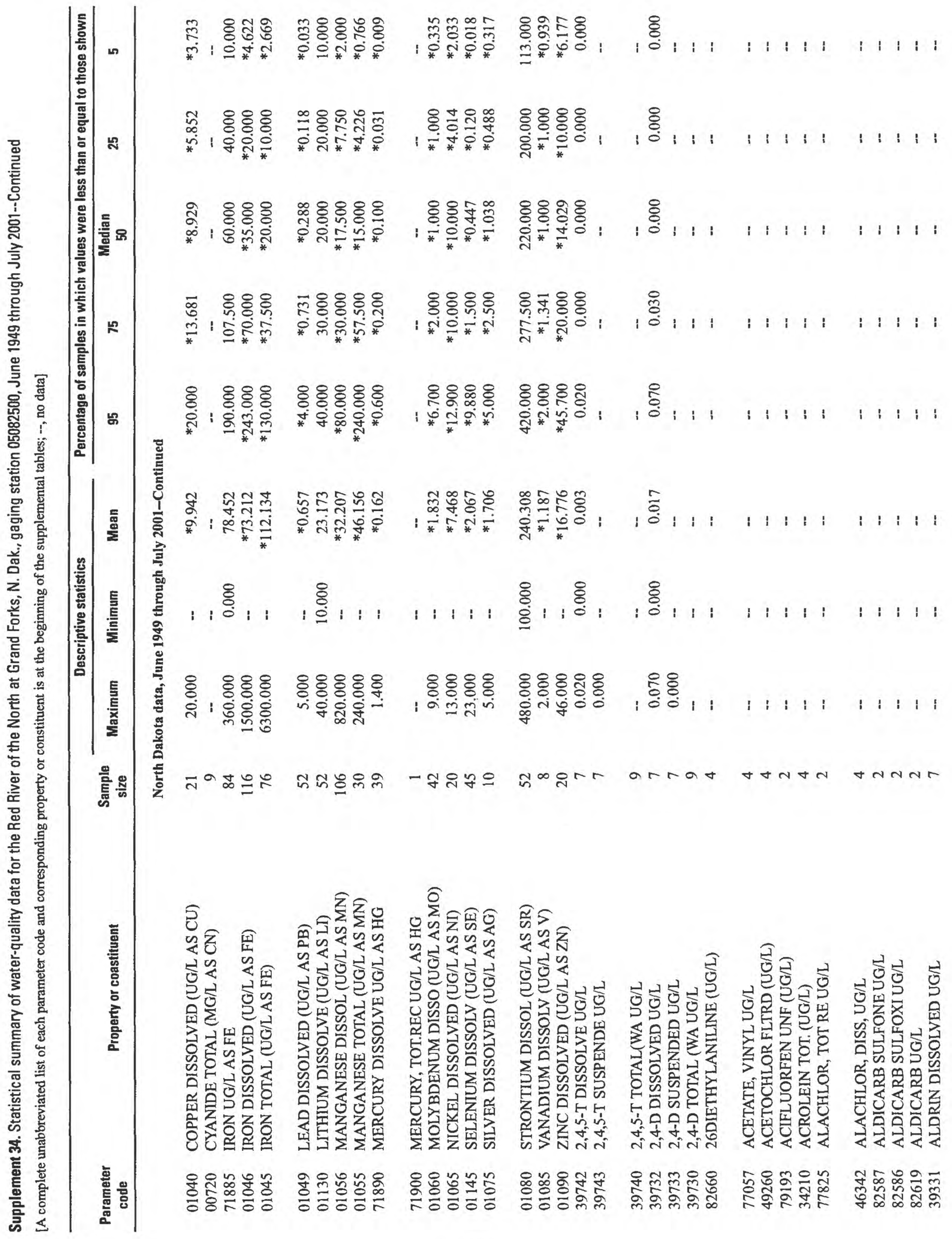



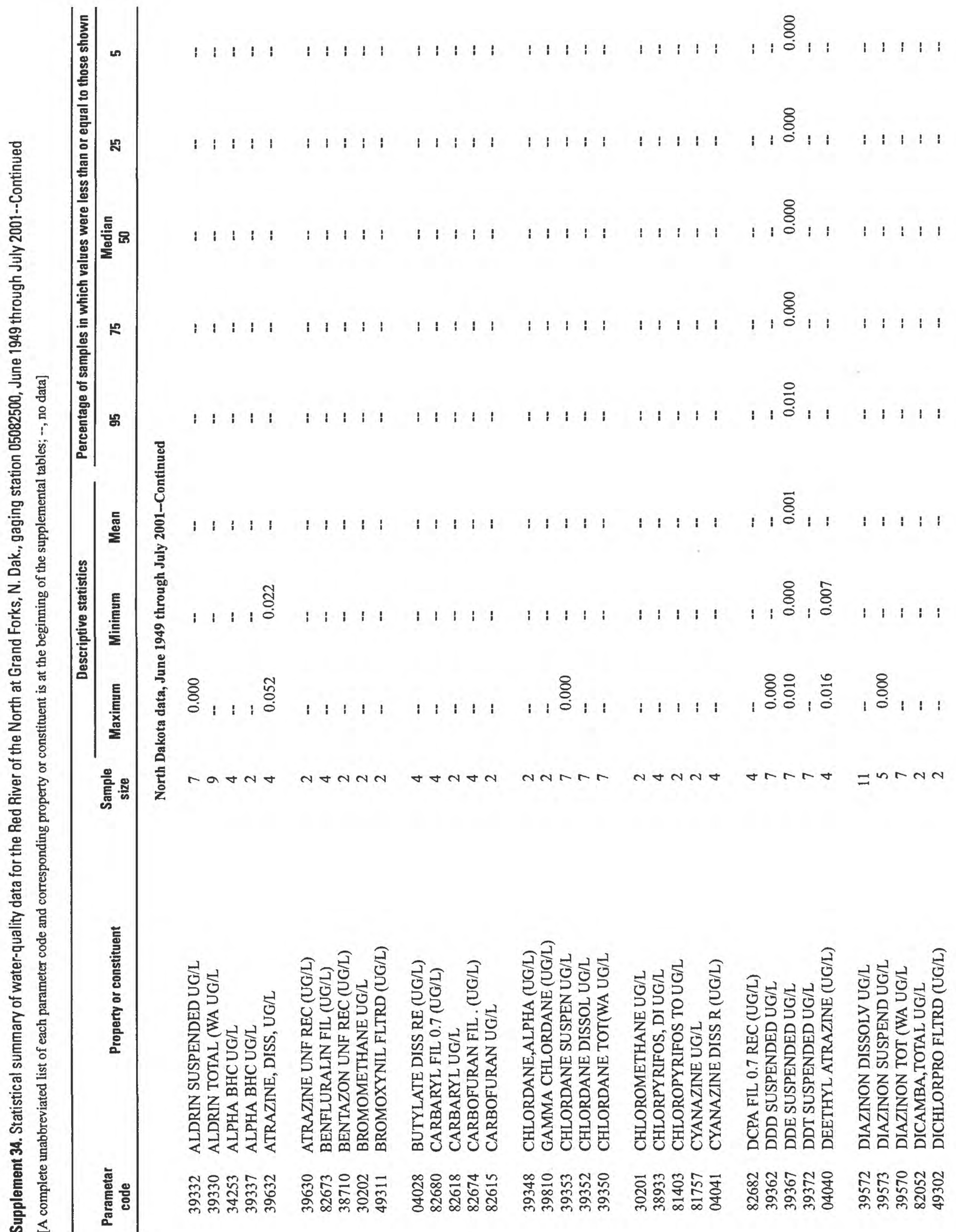

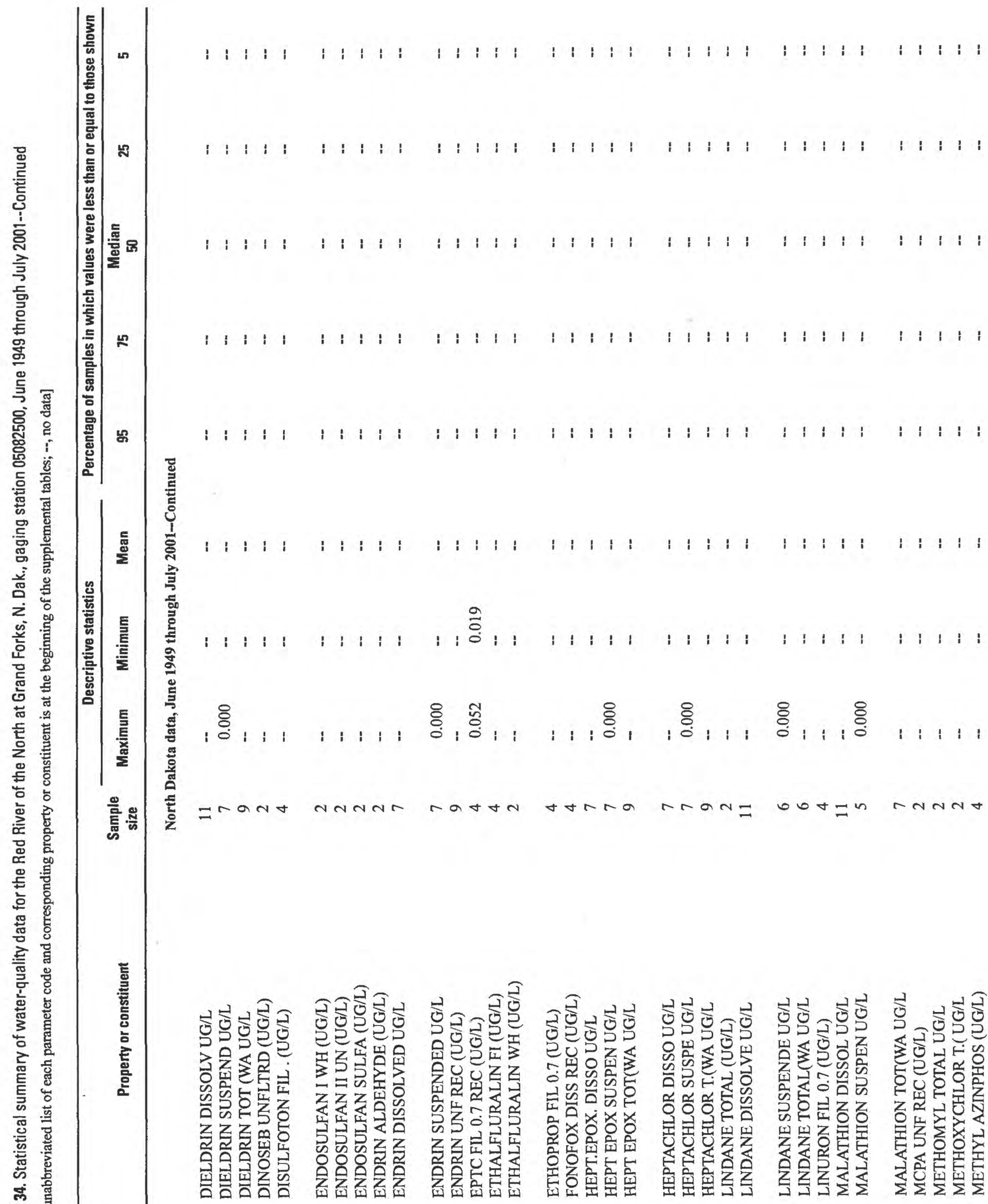

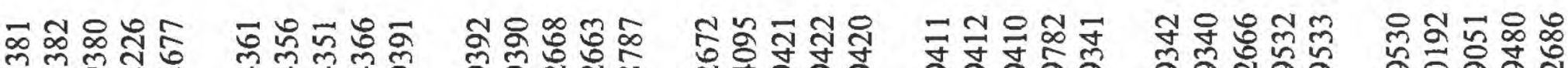

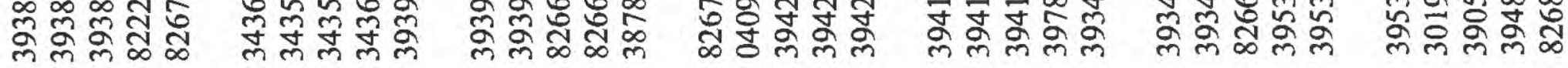




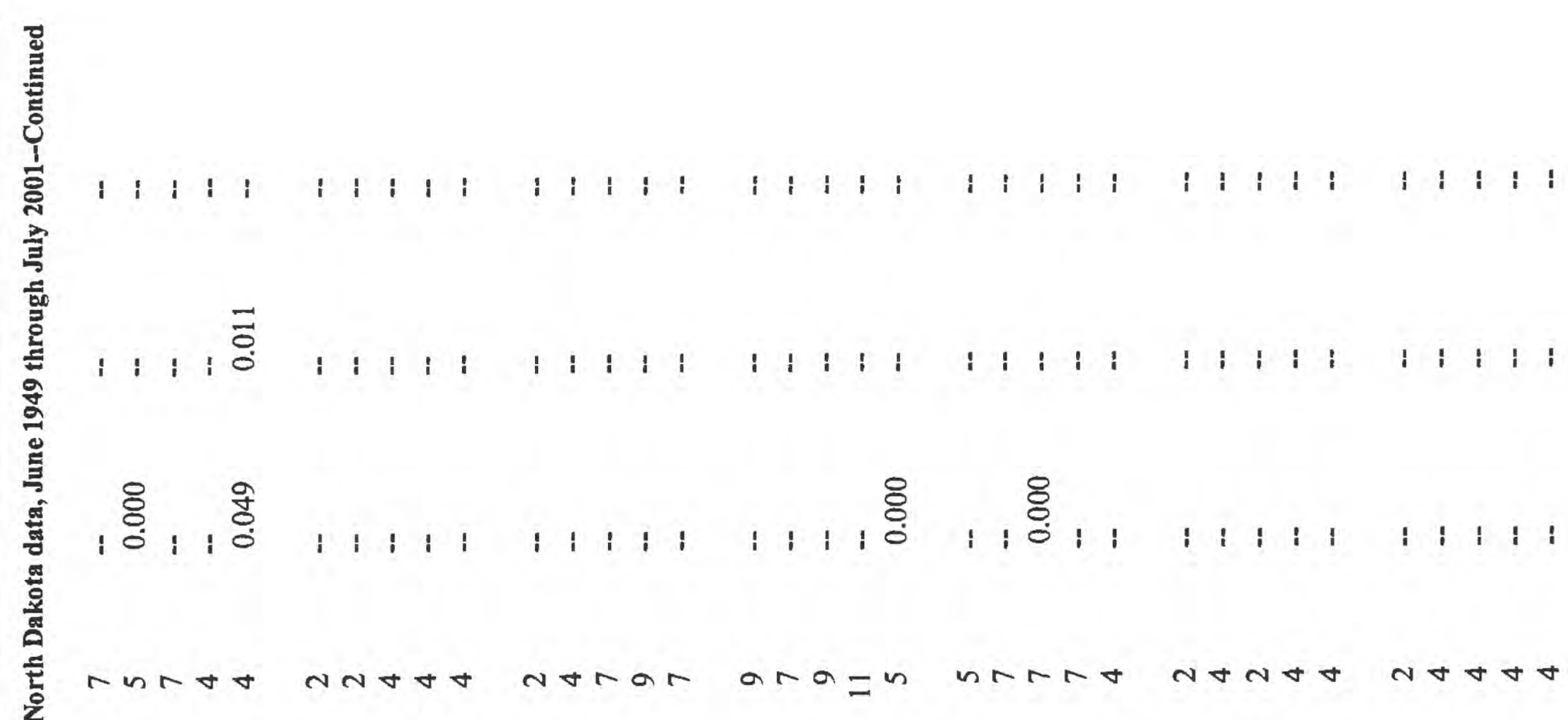

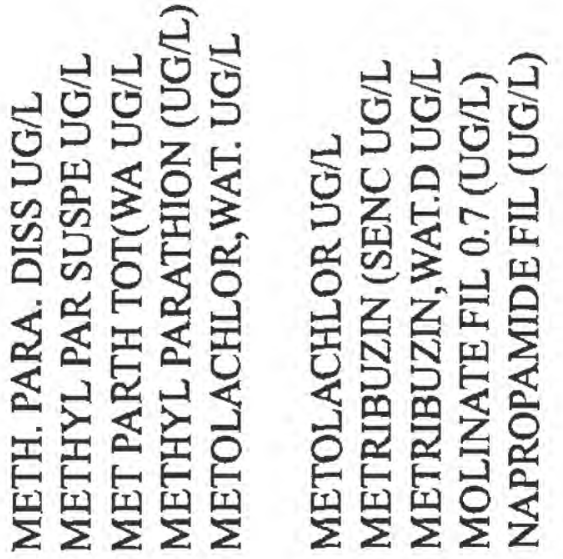

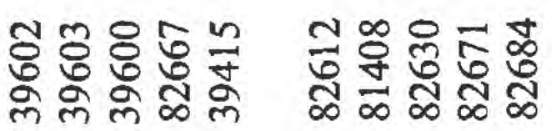

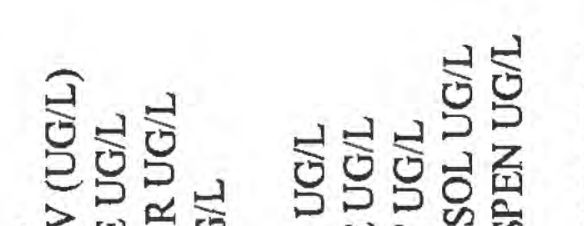

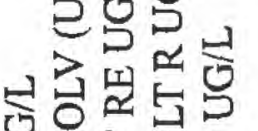

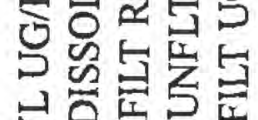

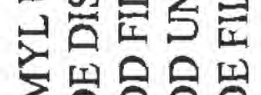

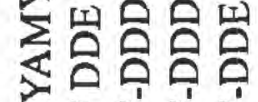

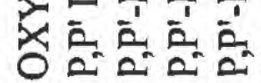

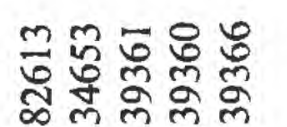
这最点

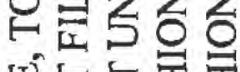

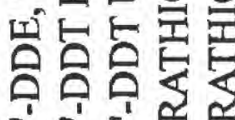

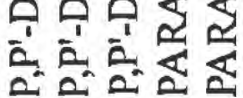

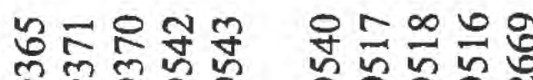

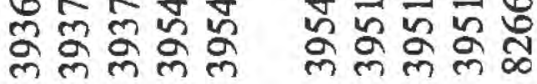

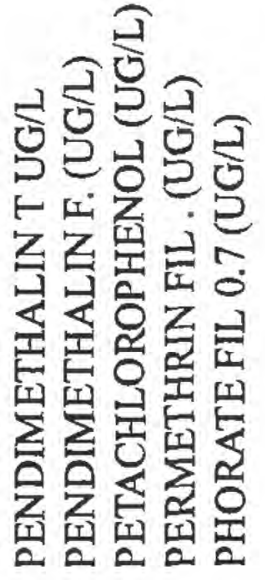

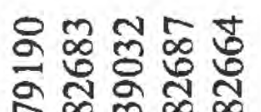

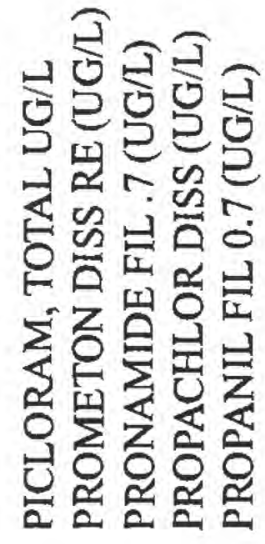

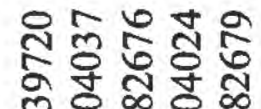


$\begin{array}{llllllllllllllllllllllllllllllll} & 1 & 1 & 1 & 1 & 1 & 1 & 1 & 1 & 1 & 1 & 1 & 1 & 1 & 1 & 1 & 1 & 1 & 1 & 1 & 1 & 1 & 1 & 1 & 1 & 1 & 1 & 1 & 1 & 1 & 1 & 1\end{array}$

$\begin{array}{llllllllllllllllllllllllllllllllllll} & 1 & 1 & 1 & 1 & 1 & 1 & 1 & 1 & 1 & 1 & 1 & 1 & 1 & 1 & 1 & 1 & 1 & 1 & 1 & 1 & 1 & 1 & 1 & 1 & 1 & 1 & 1 & 1 & 1 & 1 & 1 & 1 & 1 & 1\end{array}$

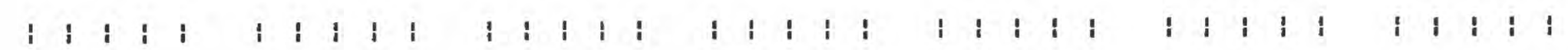

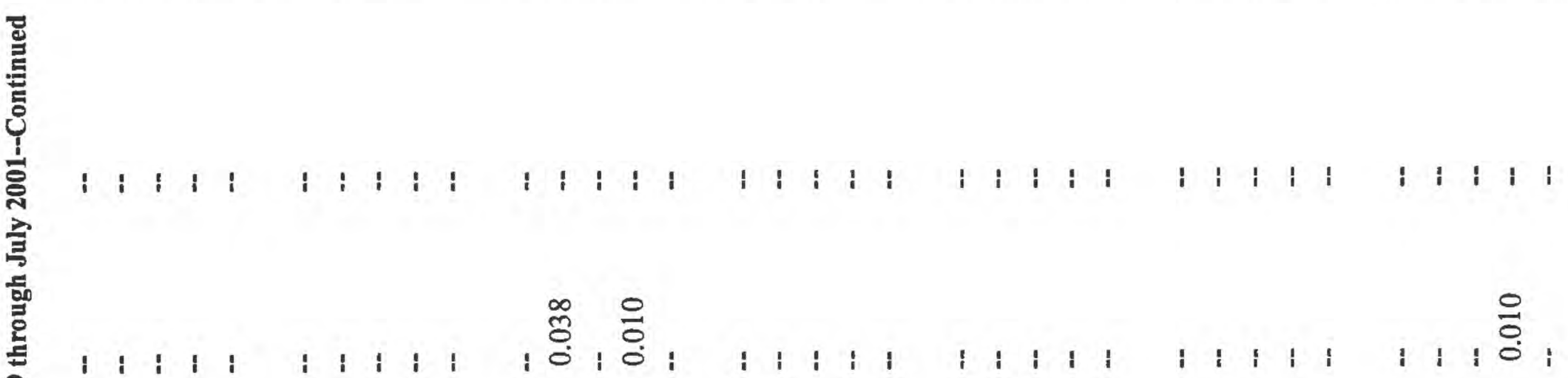
要

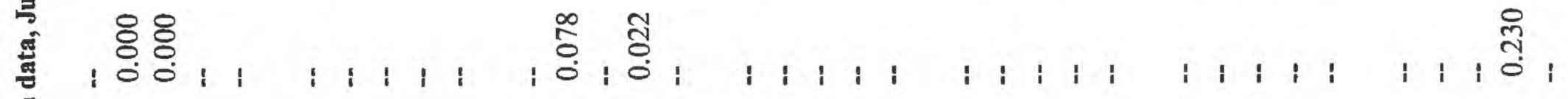
要

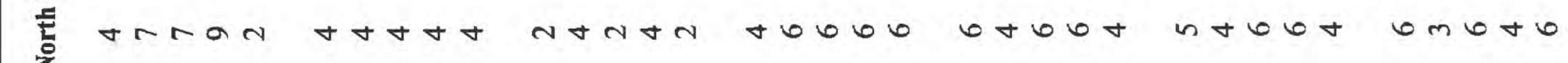

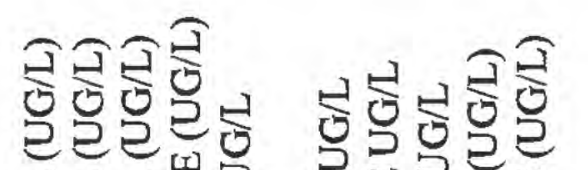

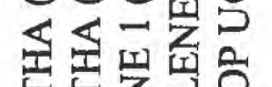

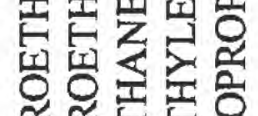

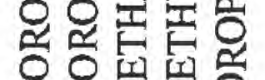

空完䂞是

을을

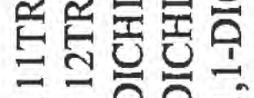

띡복

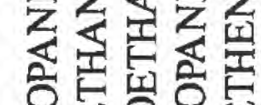

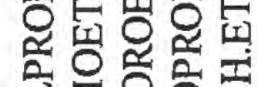

焉是是员

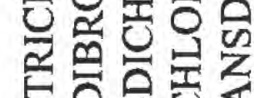

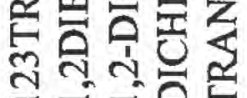

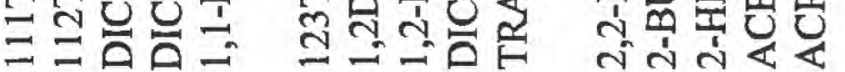

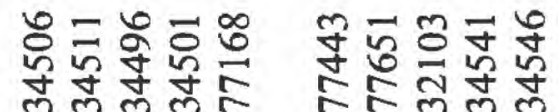

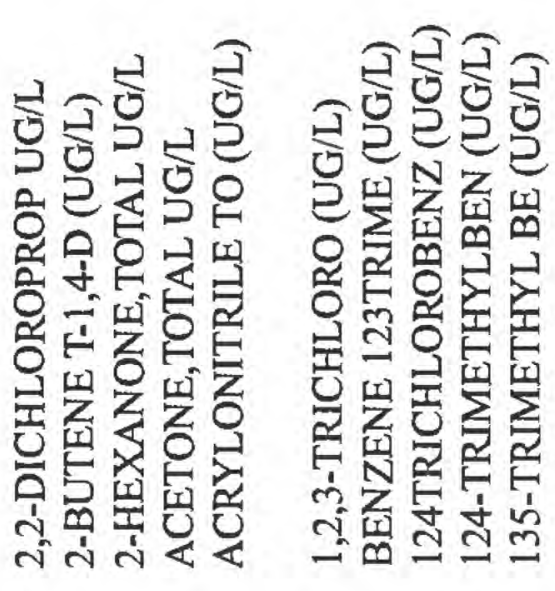

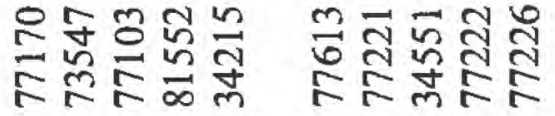




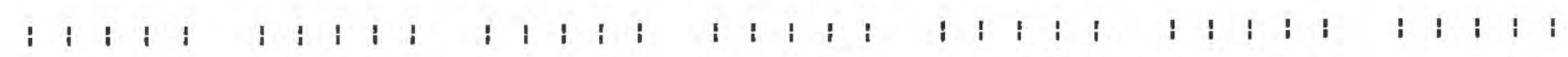




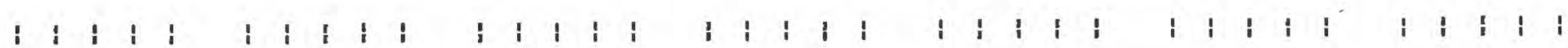

$\begin{array}{lllllllllllllllllllllllllllllll} & 1 & 1 & 1 & 1 & 1 & 1 & 1 & 1 & 1 & 1 & 1 & 1 & 1 & 1 & 1 & 1 & 1 & 1 & 1 & 1 & 1 & 1 & 1 & 1 & 1 & 1 & 1 & 1 & 1 & 1\end{array}$

$\begin{array}{llllllllllllllllllllllllllllllllll} & 1 & 1 & 1 & 1 & 1 & 1 & 1 & 1 & 1 & 1 & 1 & 1 & 1 & 1 & 1 & 1 & 1 & 1 & 1 & 1 & 1 & 1 & 1 & 1 & 1 & 1 & 1 & 1 & 1 & 1 & 1 & 1 & 1\end{array}$



i i i i

i i i

i i i i

$1 ; 1$

i i i i

I I I I I I I I I

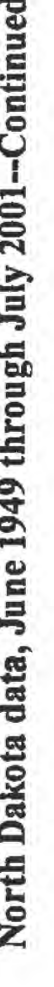

i 1 i

i i i i

i i i

i i i i

용,,$\frac{0}{0}$

i i i

i i i i i i i

ำ 융

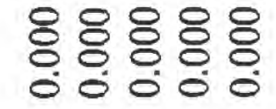

i

$1 ; 1 ; 1,1$,

$\frac{8}{0}$

i i i i

i i i i

(6)

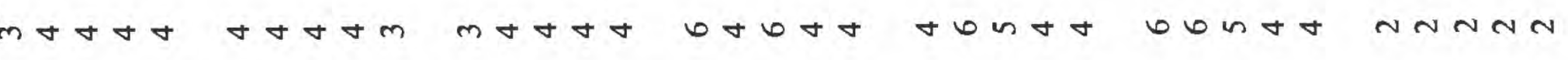

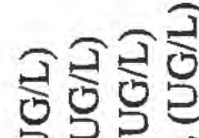

包已承

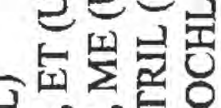

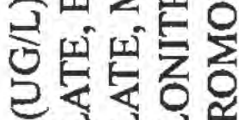

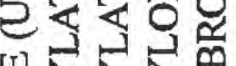

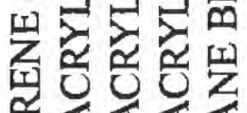

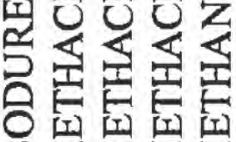

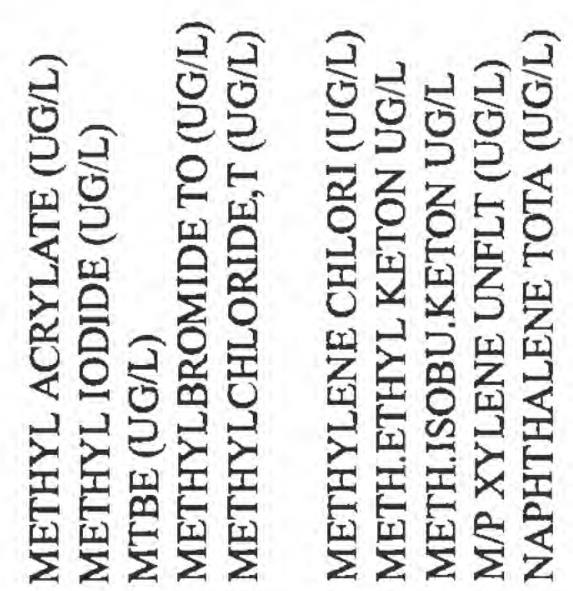

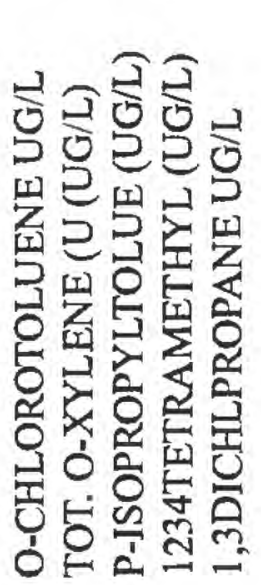

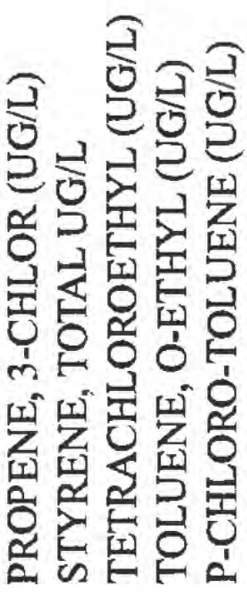

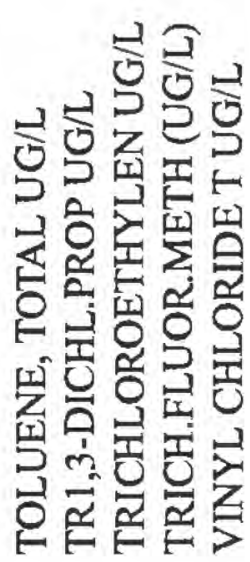

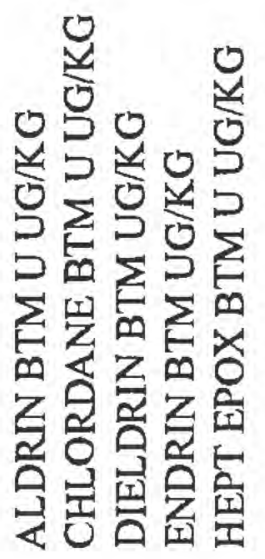

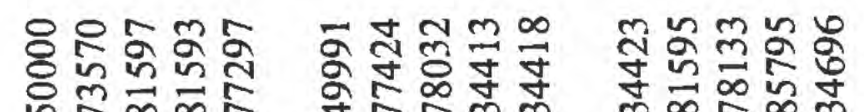

는

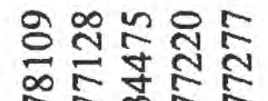

응요요요

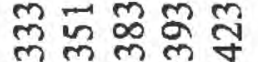

๙ू. 


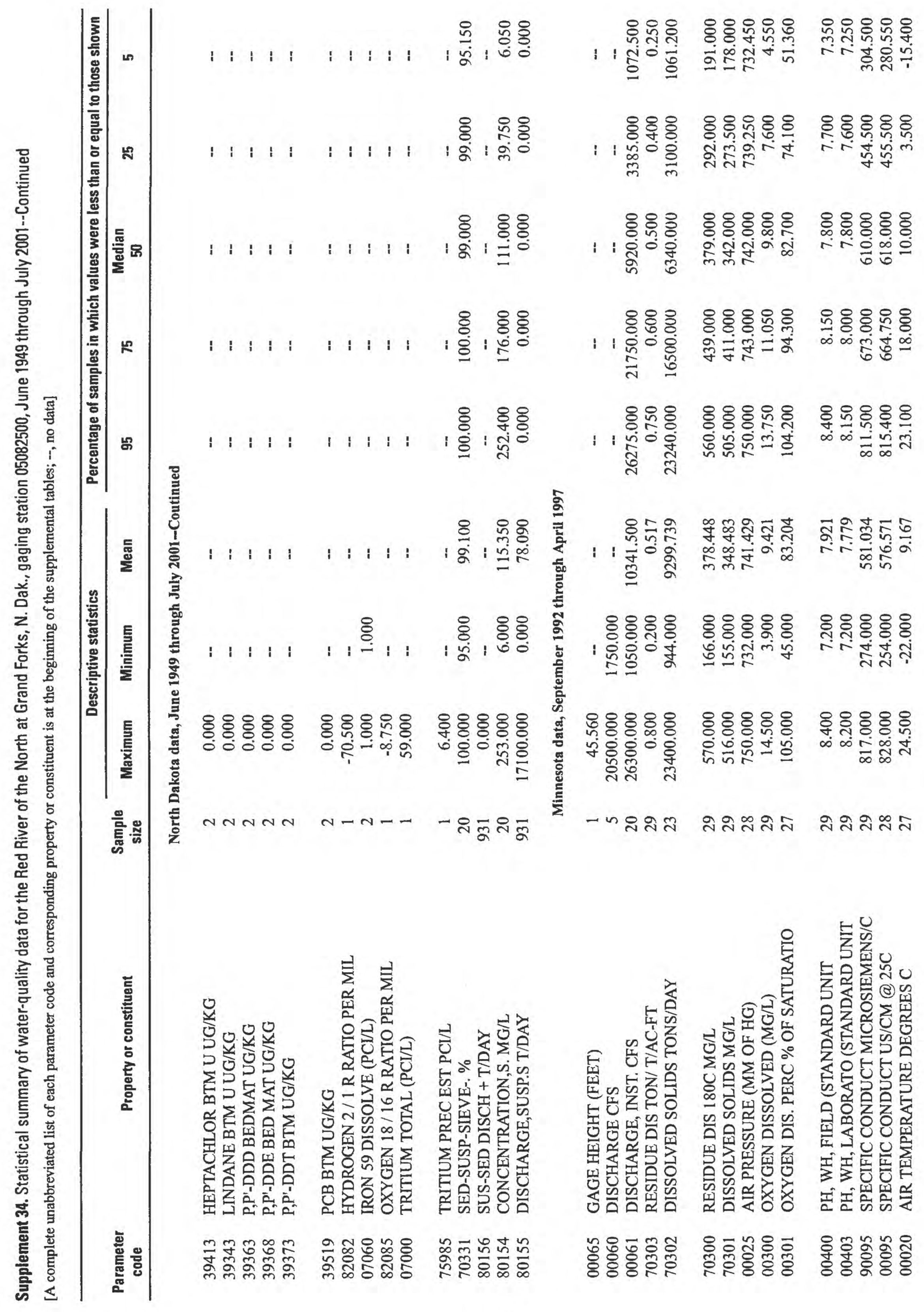




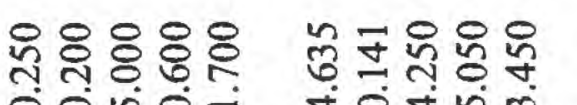

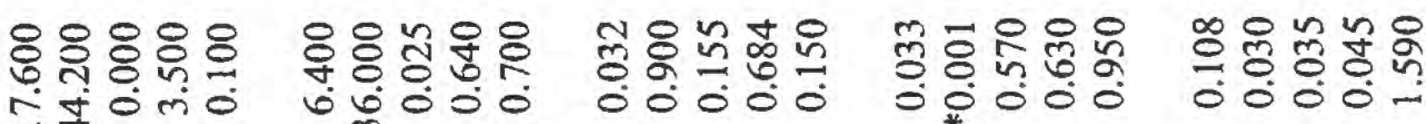

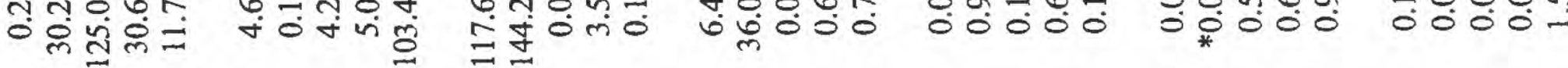

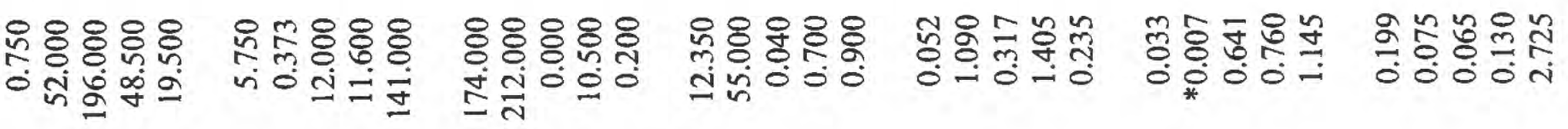

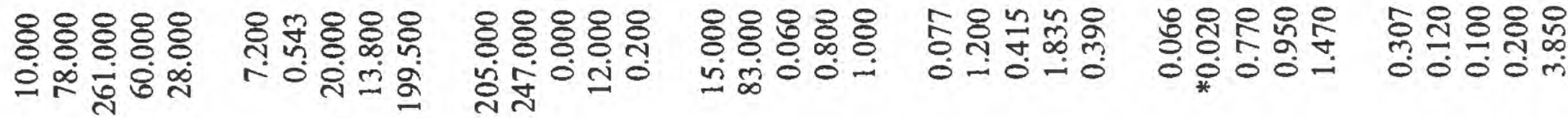

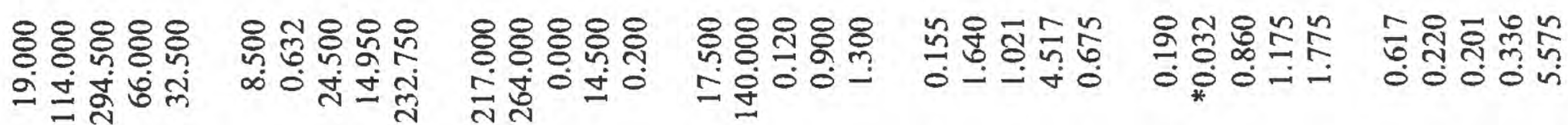

$888: 80$

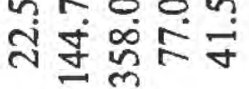

ㅇํ용

8888

녕

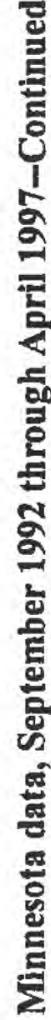

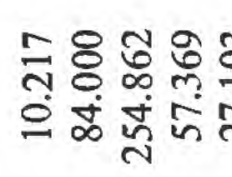

유요요

$\infty$

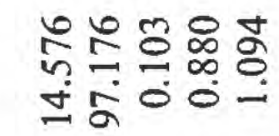

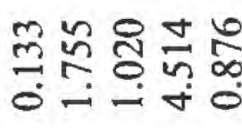

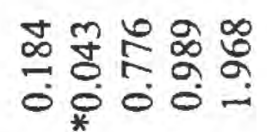

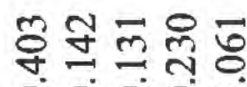

$\therefore \circ 9 \Xi$ 융

$8: 8 \%$

ํ. 잉ำ

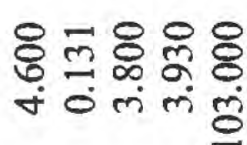

$8: 88: 8$

응융융

0
0
0
0

\% 용용

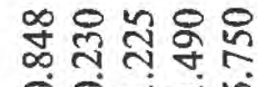

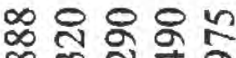

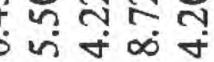

o

$0000=$

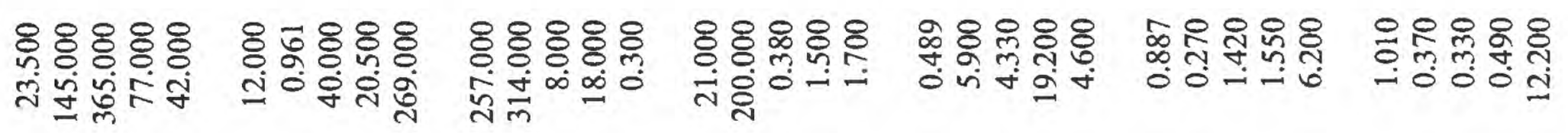

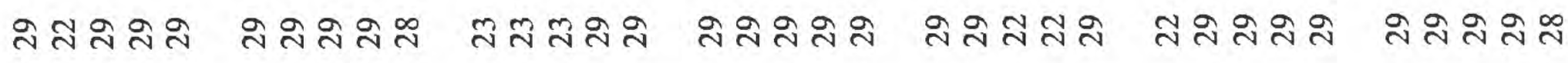

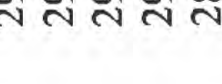

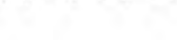

\section{(n)}

产

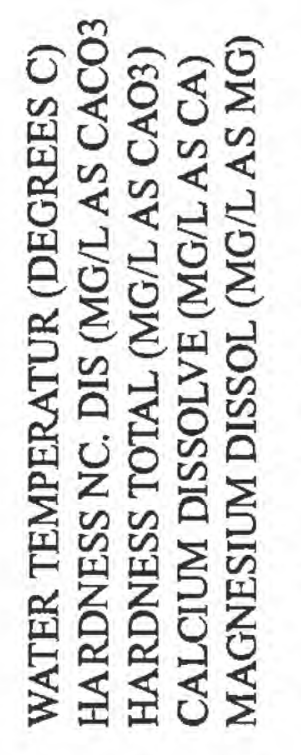

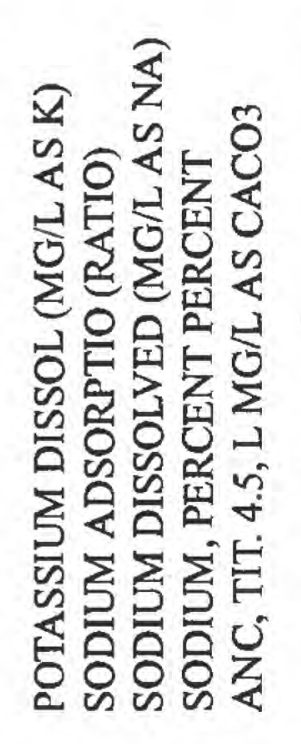

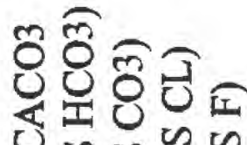

पर

너ㅇㅓㅓ엃얼

¿ $\sum_{i} \sum_{i} \sum_{i}$

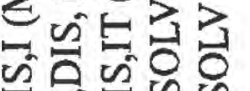

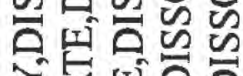

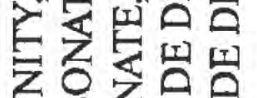

zo

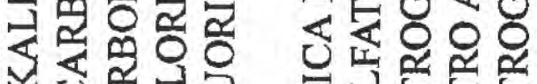

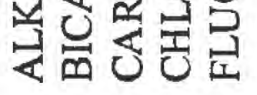

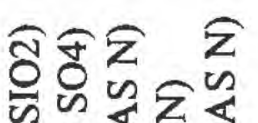

पर्ष这

겅성류엉로

$\sum \sum \leq \sum 0$

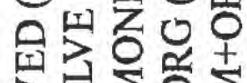

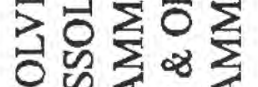

员象安安

《文员

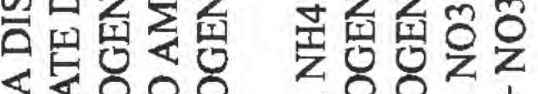

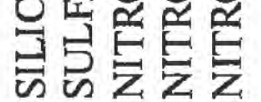

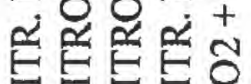

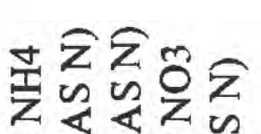

踣

웅

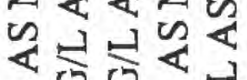

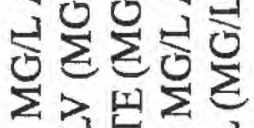

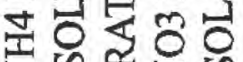

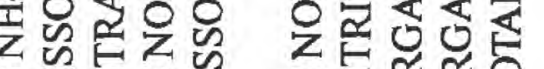

는융용

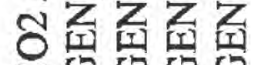

Z Oㅇㅇㅇ

象急急

करसस्थ

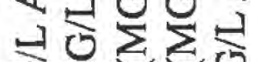

坛U

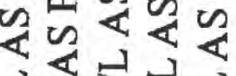

넝성허엉엉

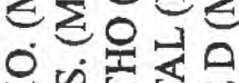

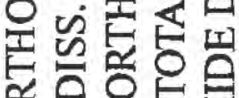

on w

四类品

소눅오

ते के फे क्ष

존운월윰원

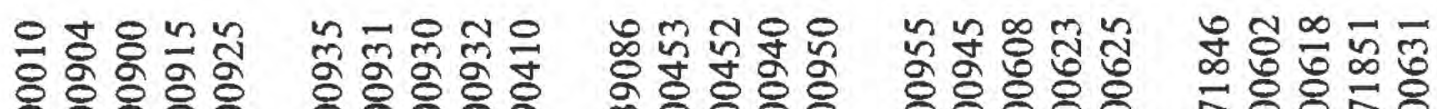

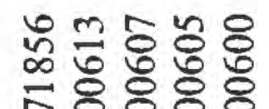

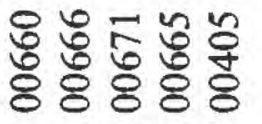




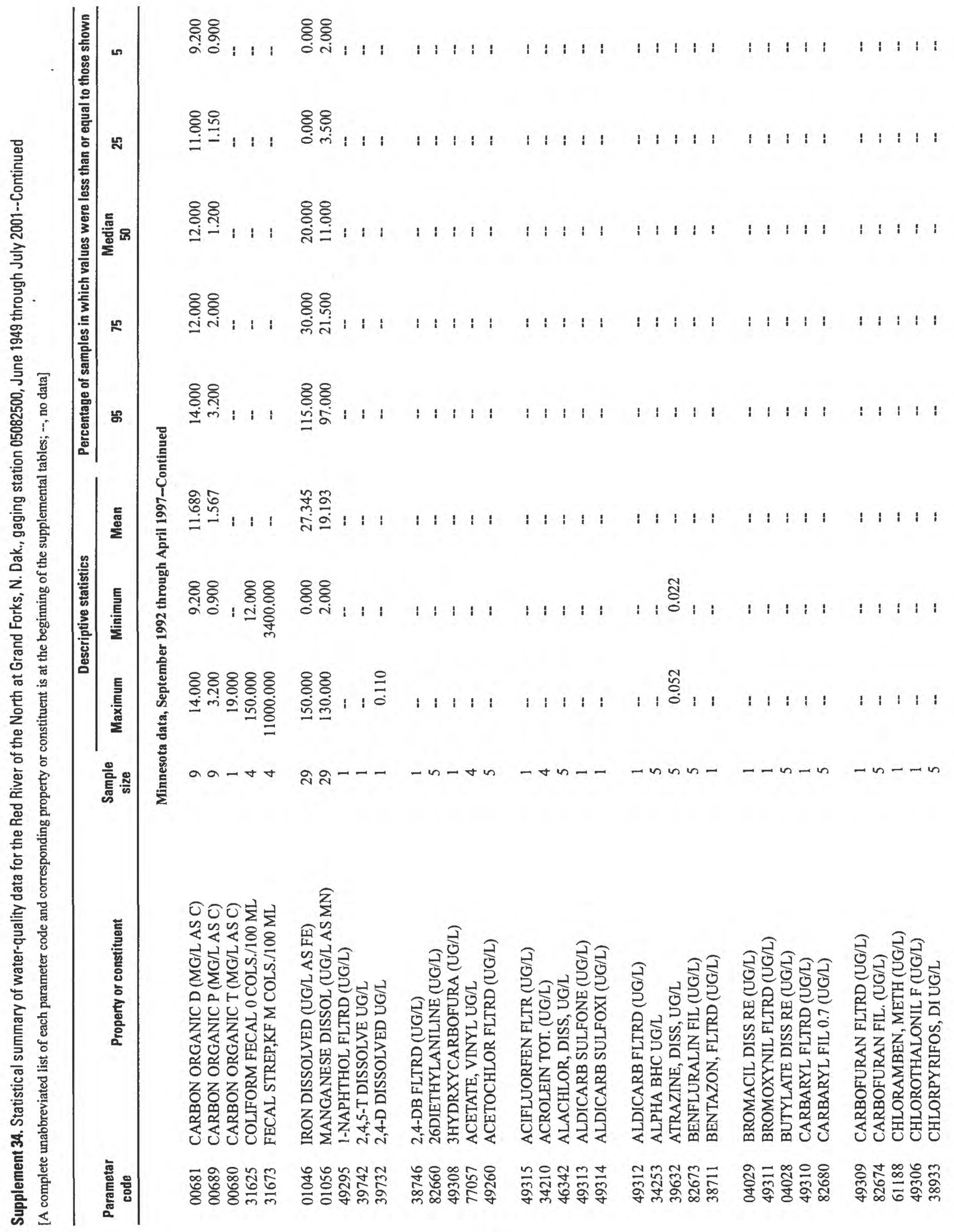


$\begin{array}{llllllllllllllllllllllllllll} & 1 & 1 & 1 & 1 & 1 & 1 & 1 & 1 & 1 & 1 & 1 & 1 & 1 & 1 & 1 & 1 & 1 & 1 & 1 & 1 & 1 & 1 & 1 & 1 & 1 & 1 & 1\end{array}$

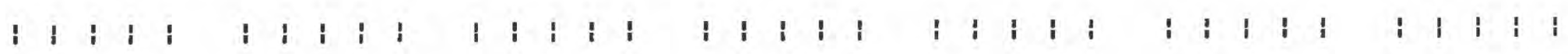

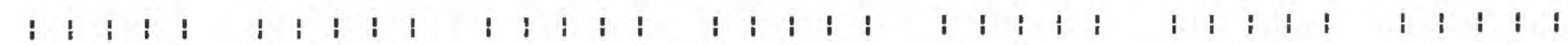

远

i : i i

i i i

1 1 1 i i

1 1 1 1

1 1 i i i

i i i i I i i i i (1)

1 1 i i

1 1 1 1

i i i i

i i i

i i i i

1 i i i i i i i i

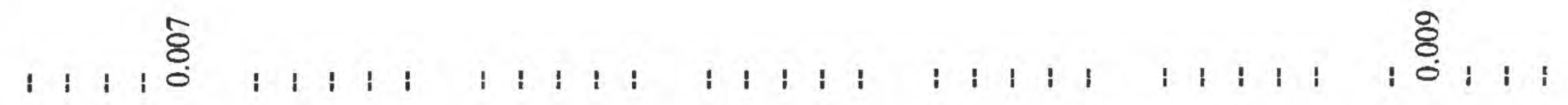

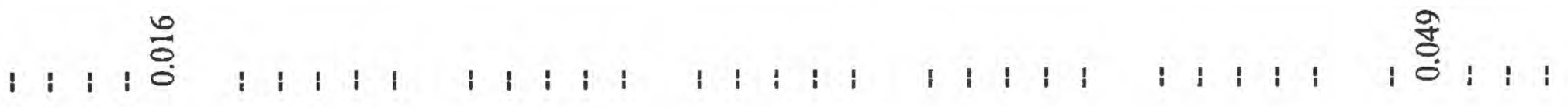

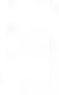

$-n-m n$

$n-m-n$

$-n--n \quad-n n-7$

$n n \rightarrow \infty n$

$-\rightarrow-n$

non $n \pi n$

엉종

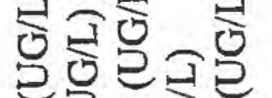

包论罗

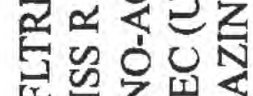

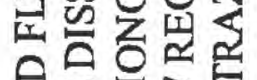

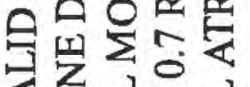

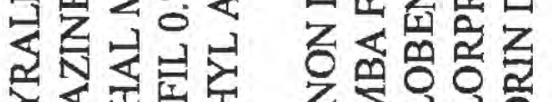

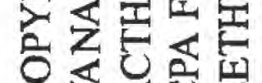

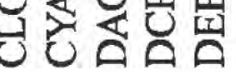

离

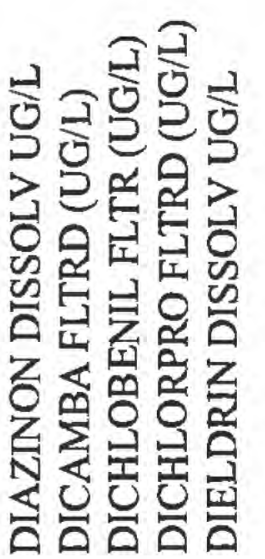

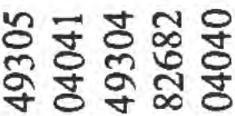

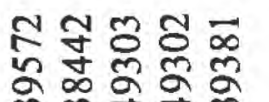

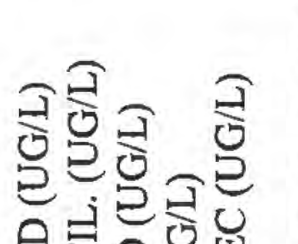

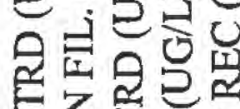

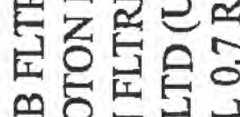

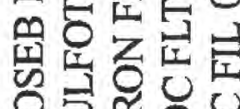

o 50

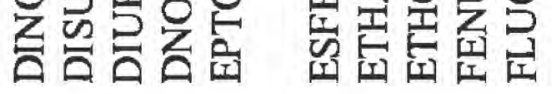

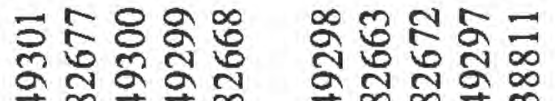

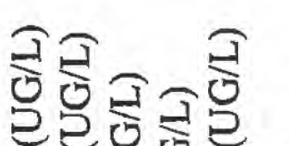

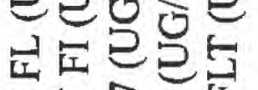

늡으을

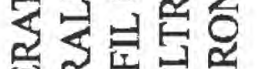

웍옹

起是是

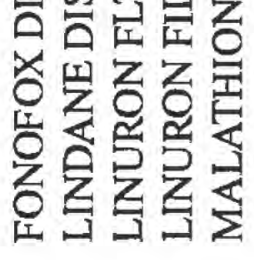

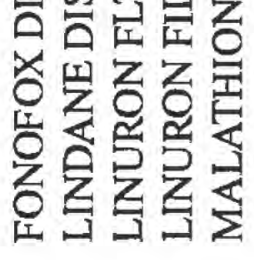

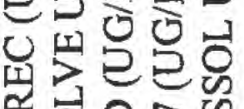

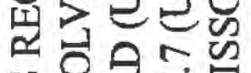

क 舟完

它目古

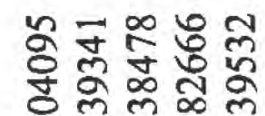

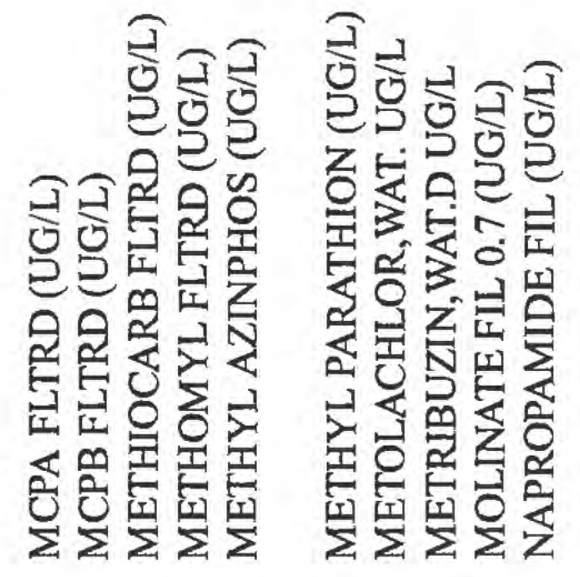

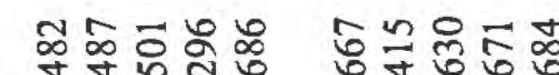

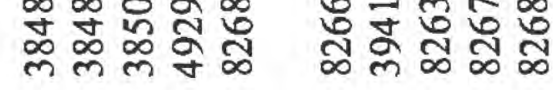




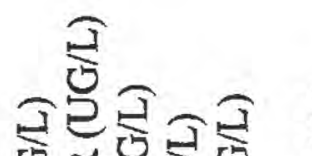

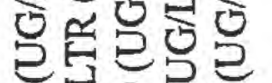

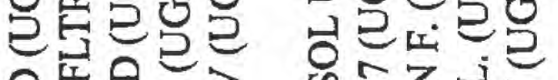

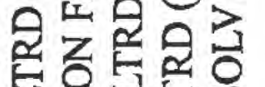

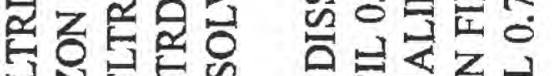

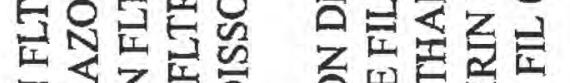

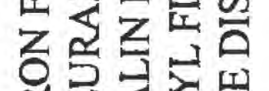
号运是

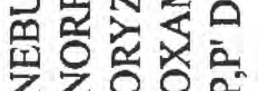

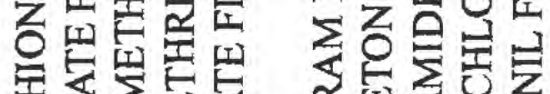

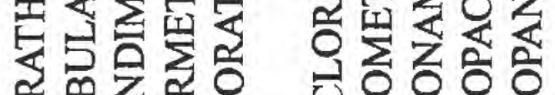

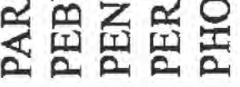

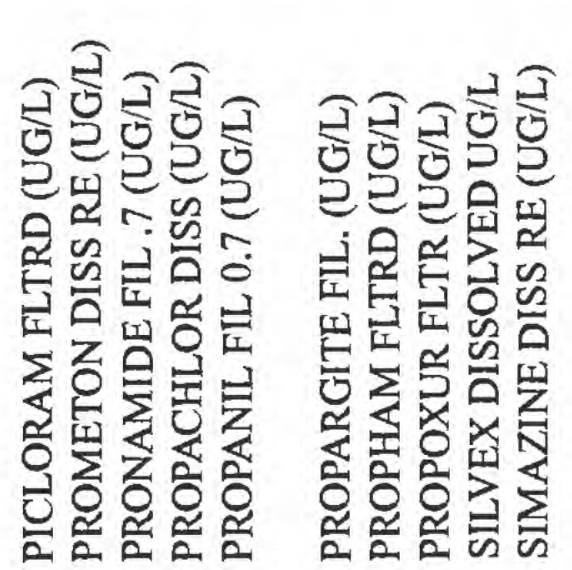

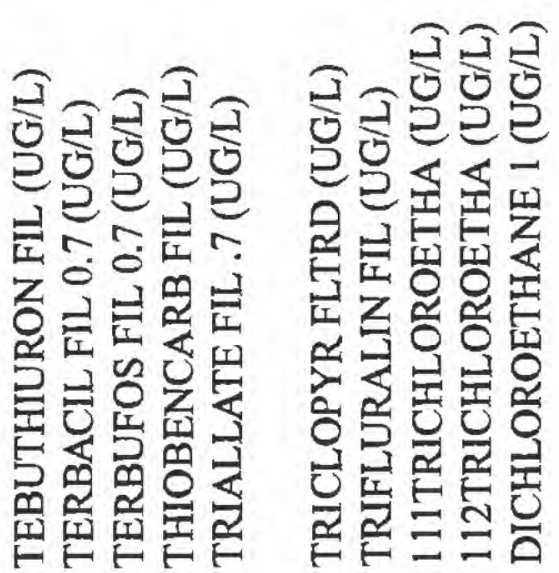
롱러엉엄엉

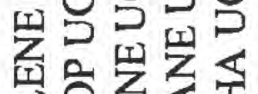

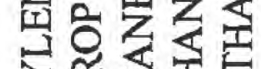
造氙牙 농녕 造完完

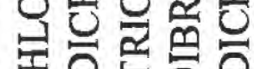

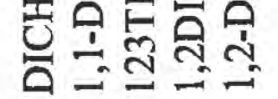

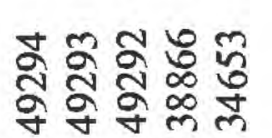

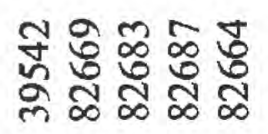

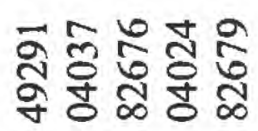

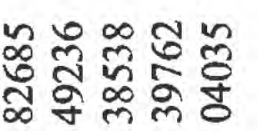

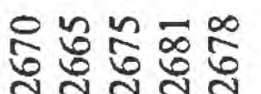

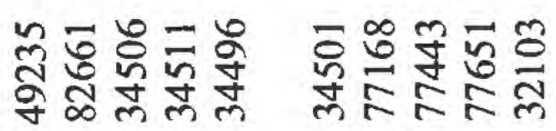




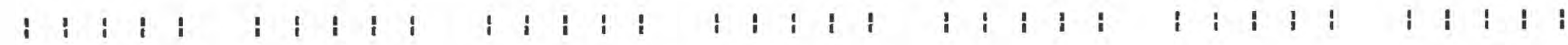

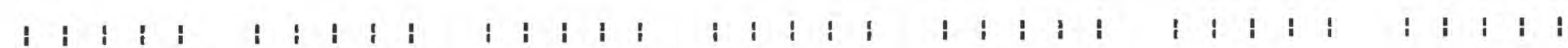

i 1 i

i i i i

i i i i

i i i :

i i i i

i 1 i 1 i 1 i 1 i 1

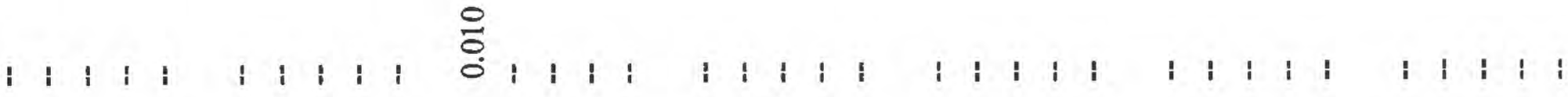

กั?

స్ర

I I

1 1 1 1 2

i 1

1 1

i 1 i i

i i i i i i i i

$\forall \forall \forall \forall$

$\theta \theta \theta$

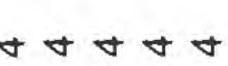

$\theta \theta \theta \theta \theta$

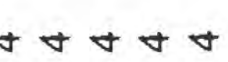

$\theta \forall \forall \forall$

$\forall \forall \forall \forall \forall$

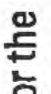

要

墨

옹얼

ㄹㄹㄹ엉옴

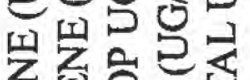

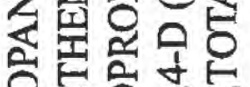

牙施

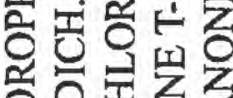

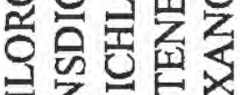

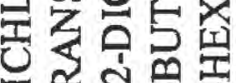

鱼瓷究

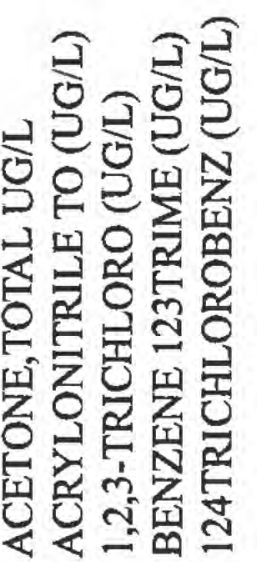

궁송엉엉

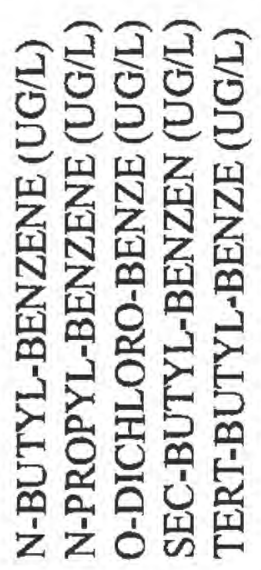

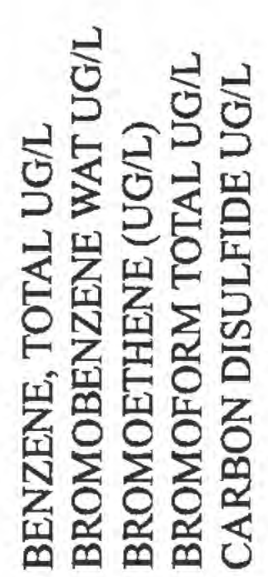

농 串造

컹엉옹

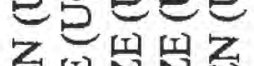

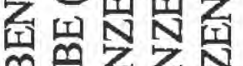

닐

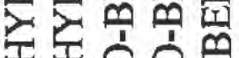

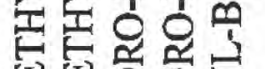

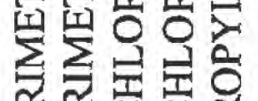

象要专里

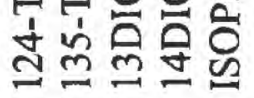

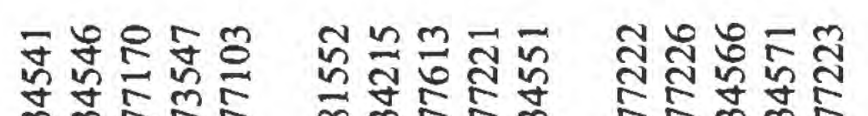

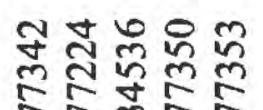

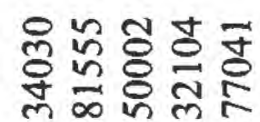

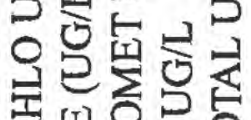

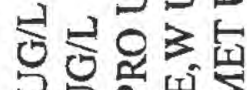

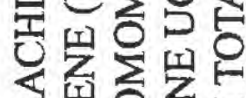

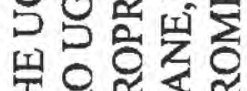

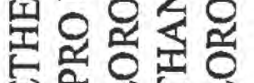

N这空

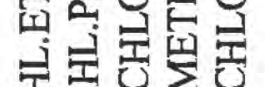

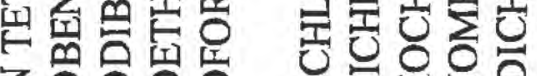

žo

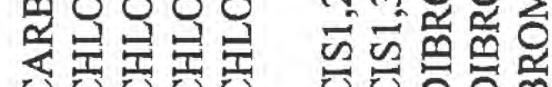

पूलि

두유

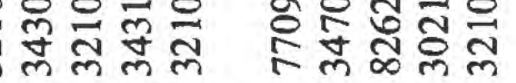




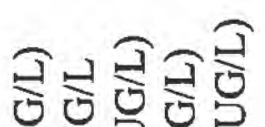
可包它

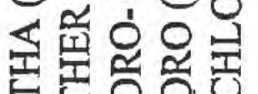

분일

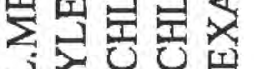

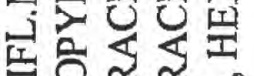

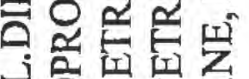
宁员点留 它芯恣志 亜 음
วงิํํำ 얼런로

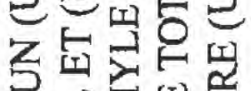
在四罟 至部

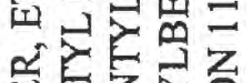
到空总 密息密密

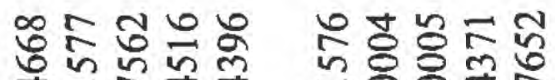

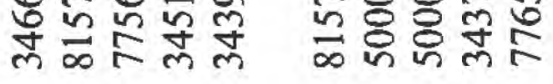

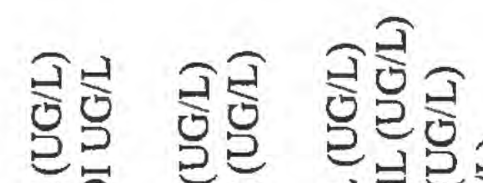

记

质牙

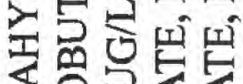

sos

凷罢到

觉勻这

离融

분로옹

츤 띤롤

칭멩

놌원옹

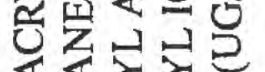

这空开

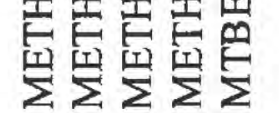

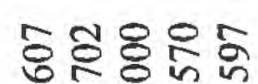

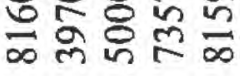

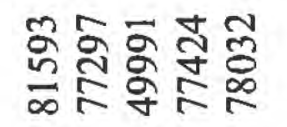

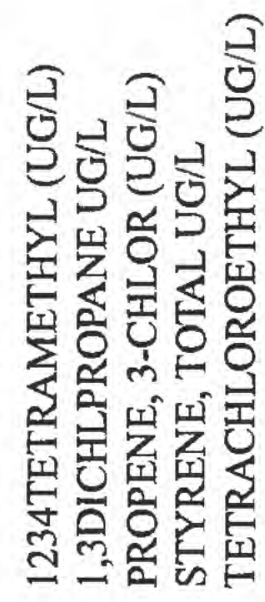

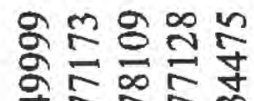




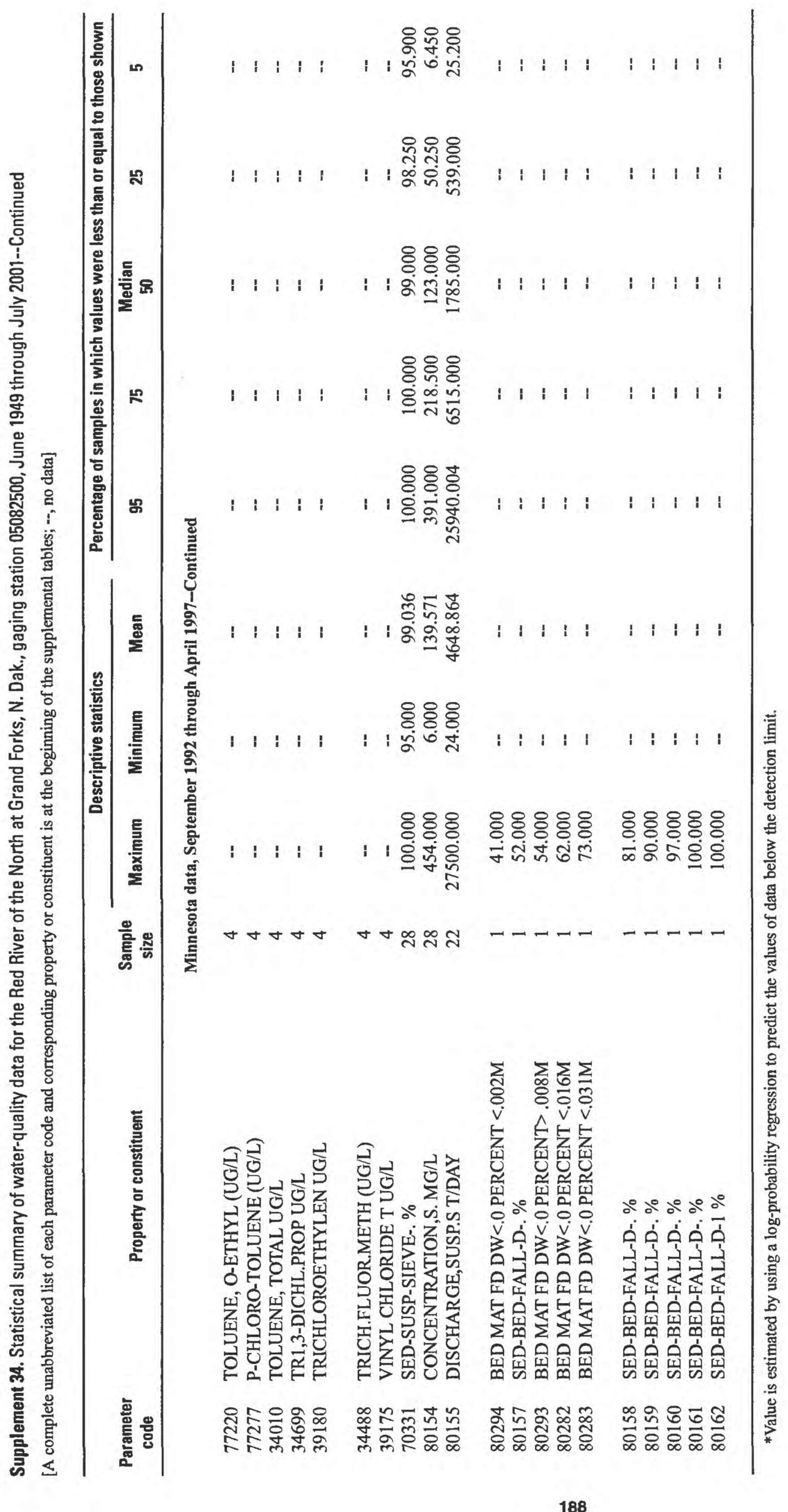




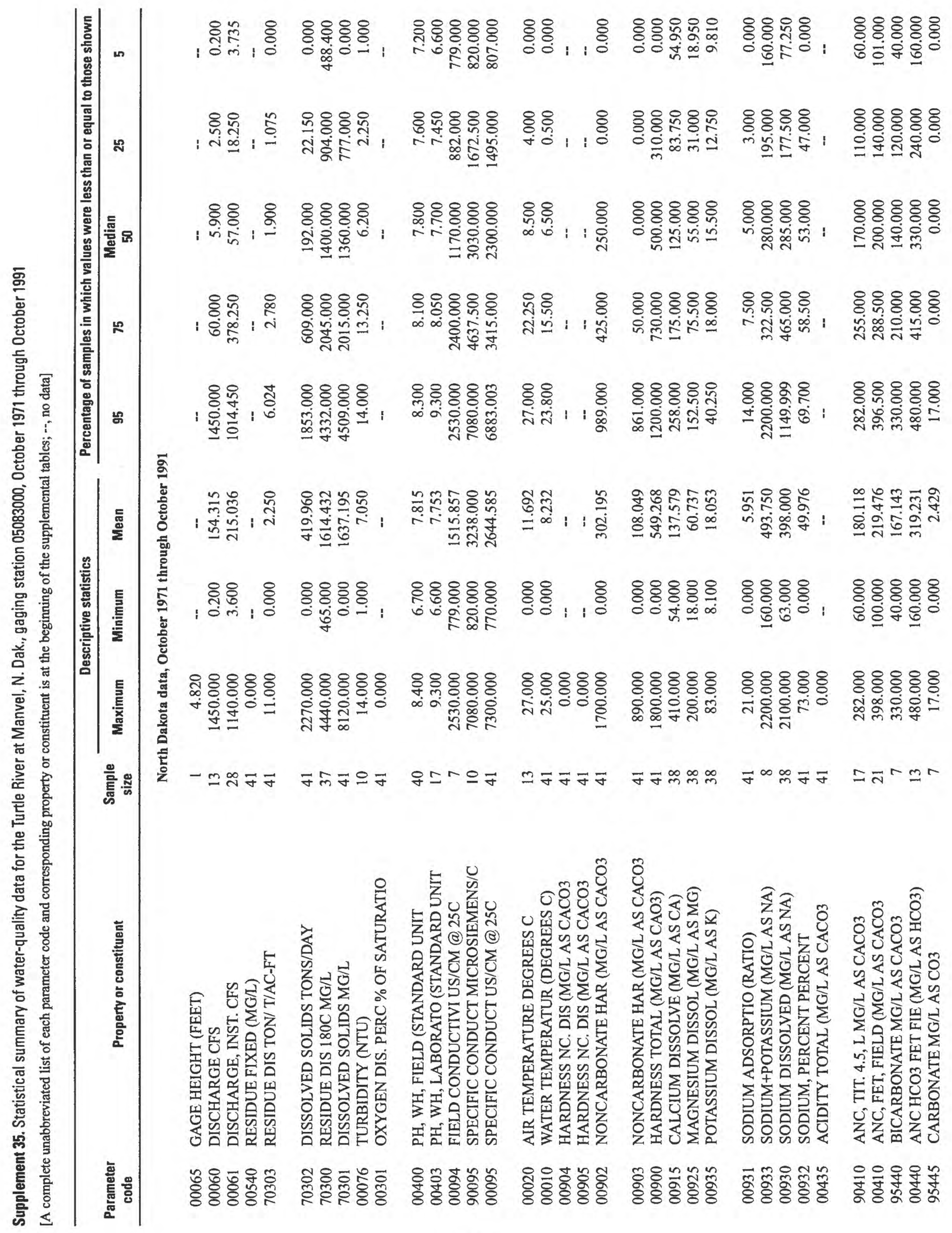




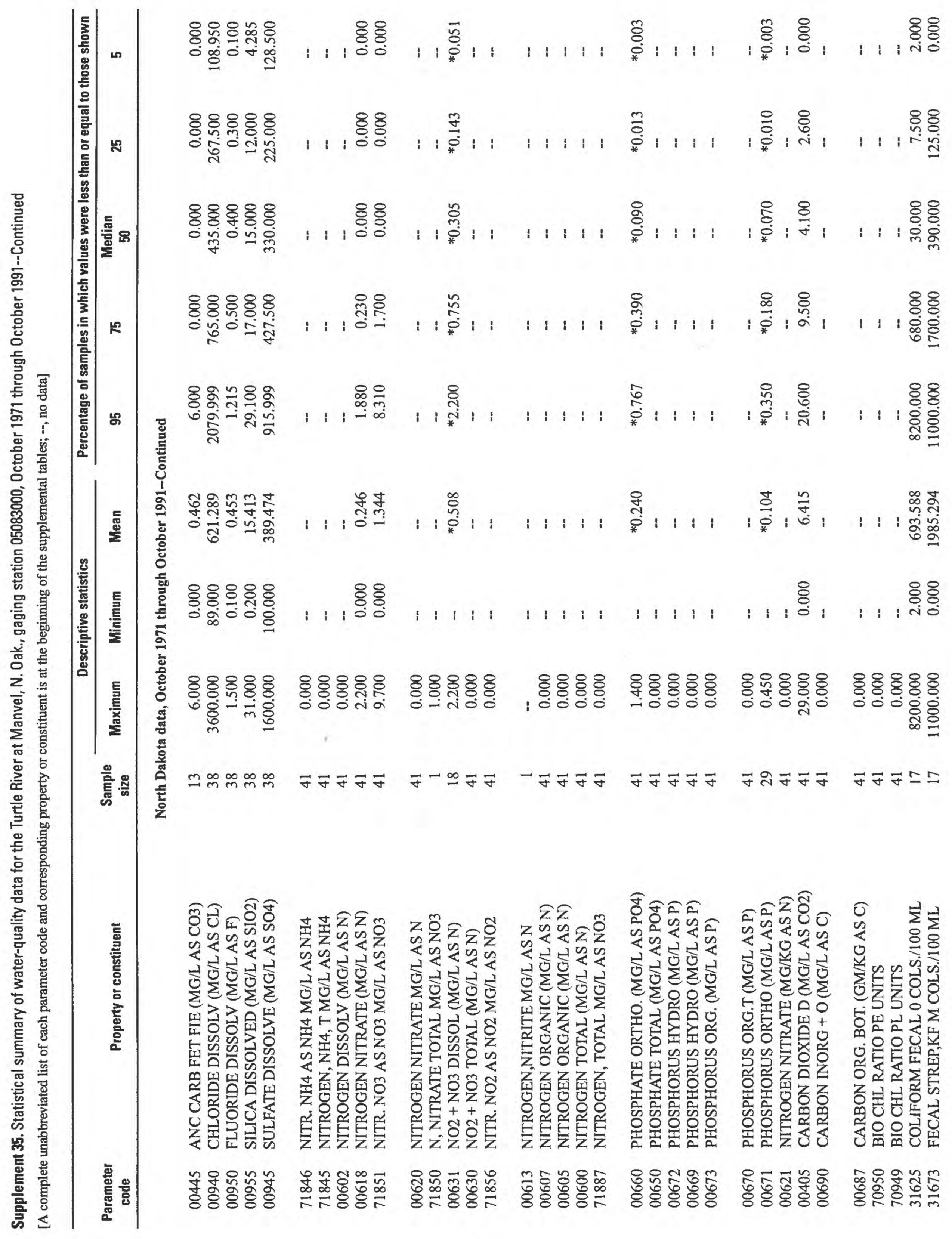




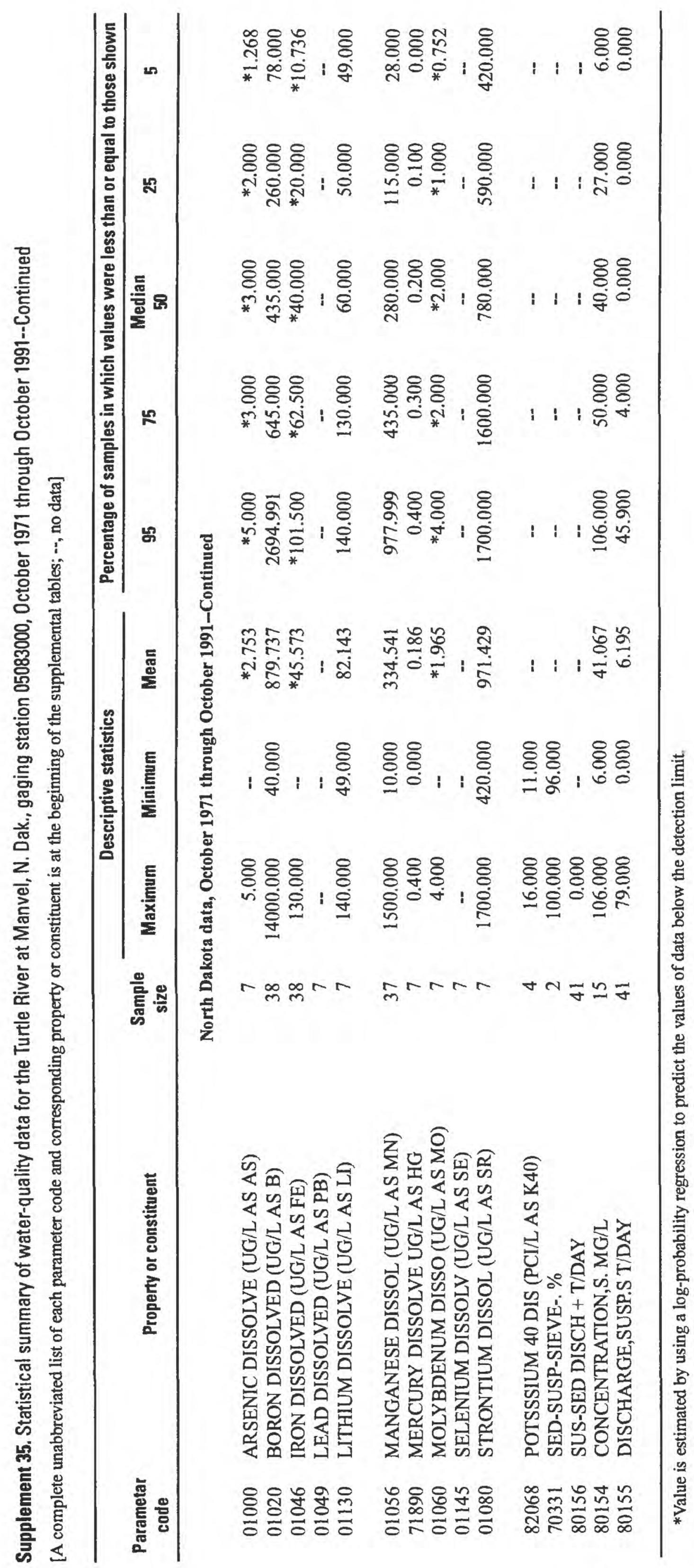




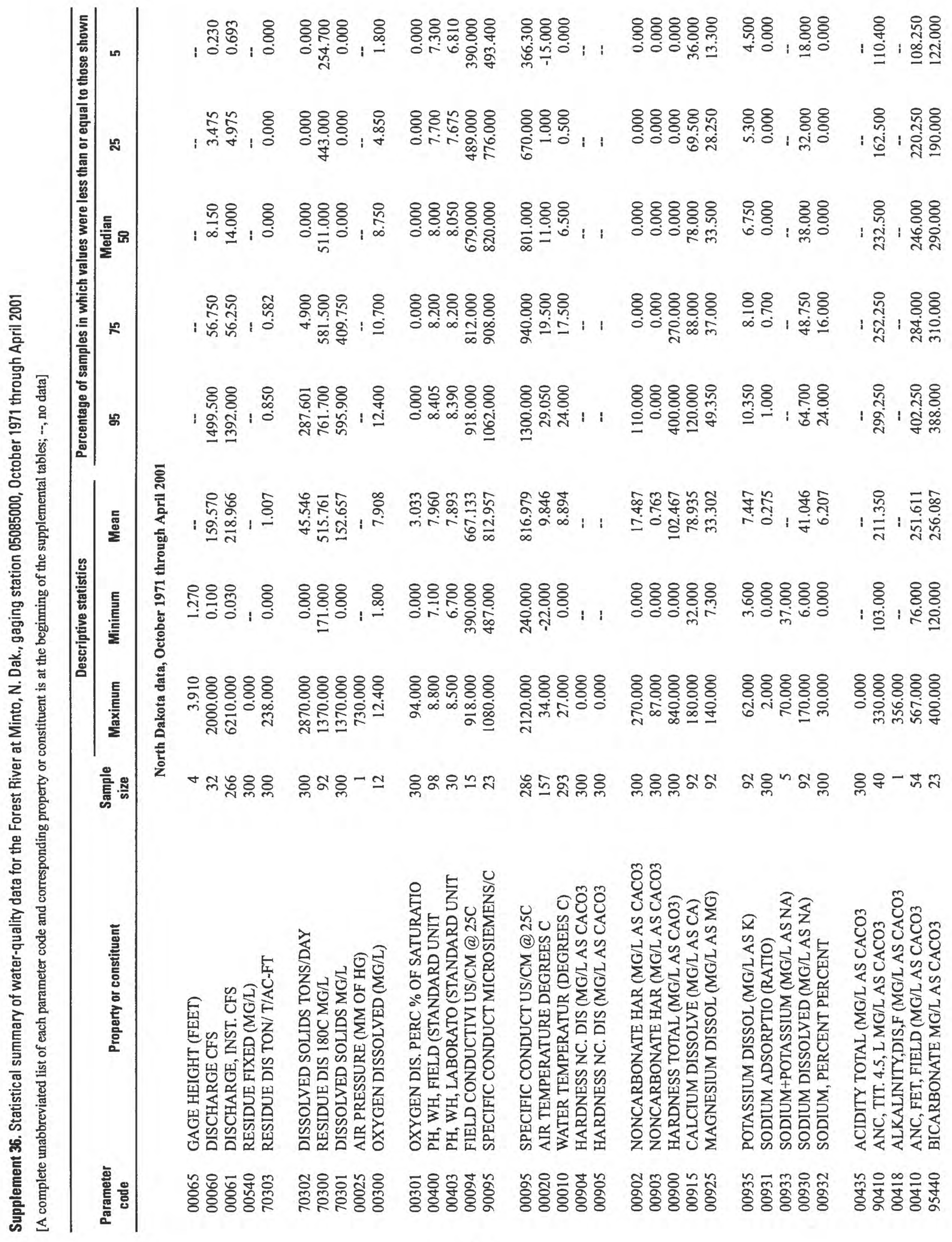




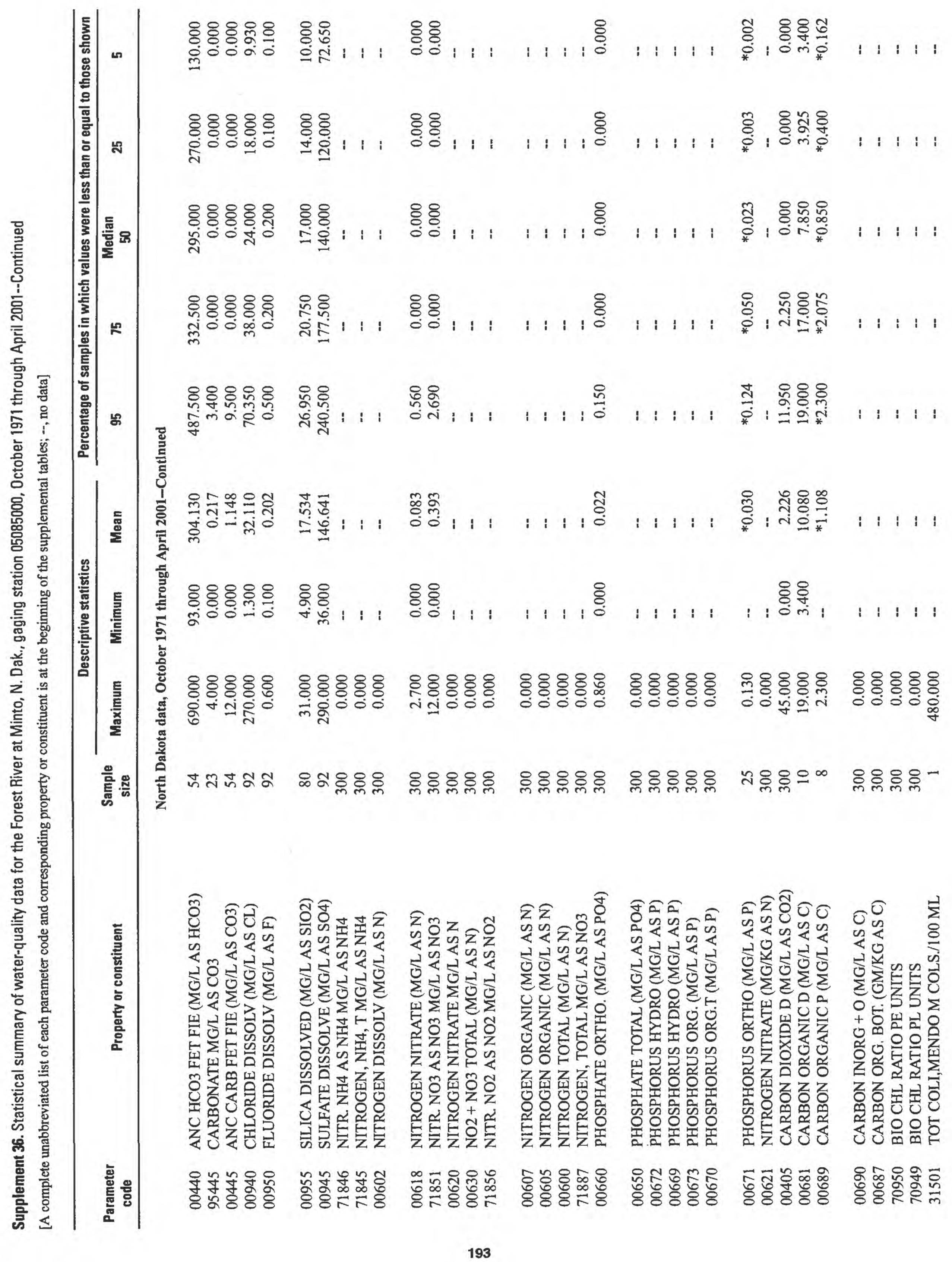




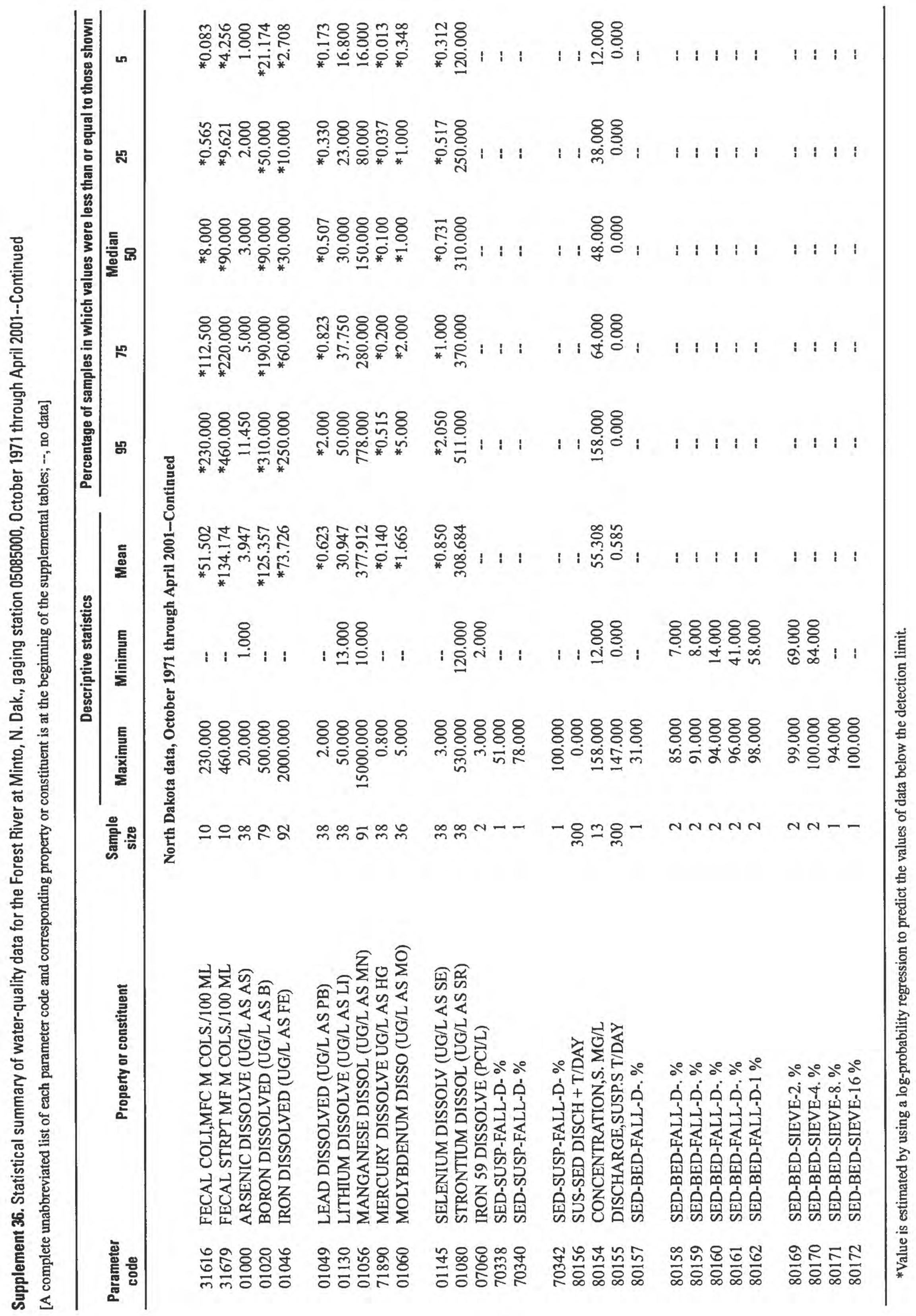




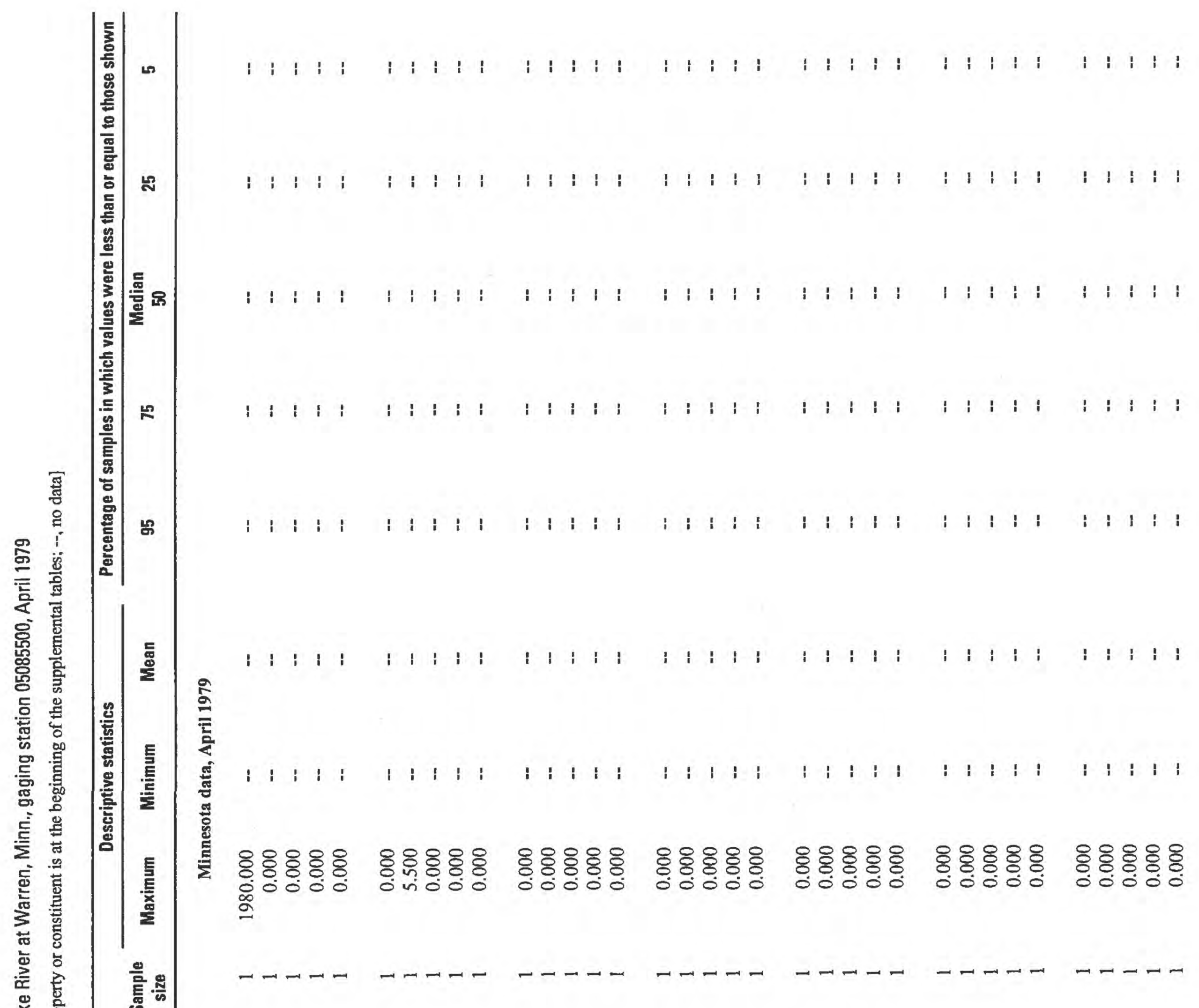

융요용

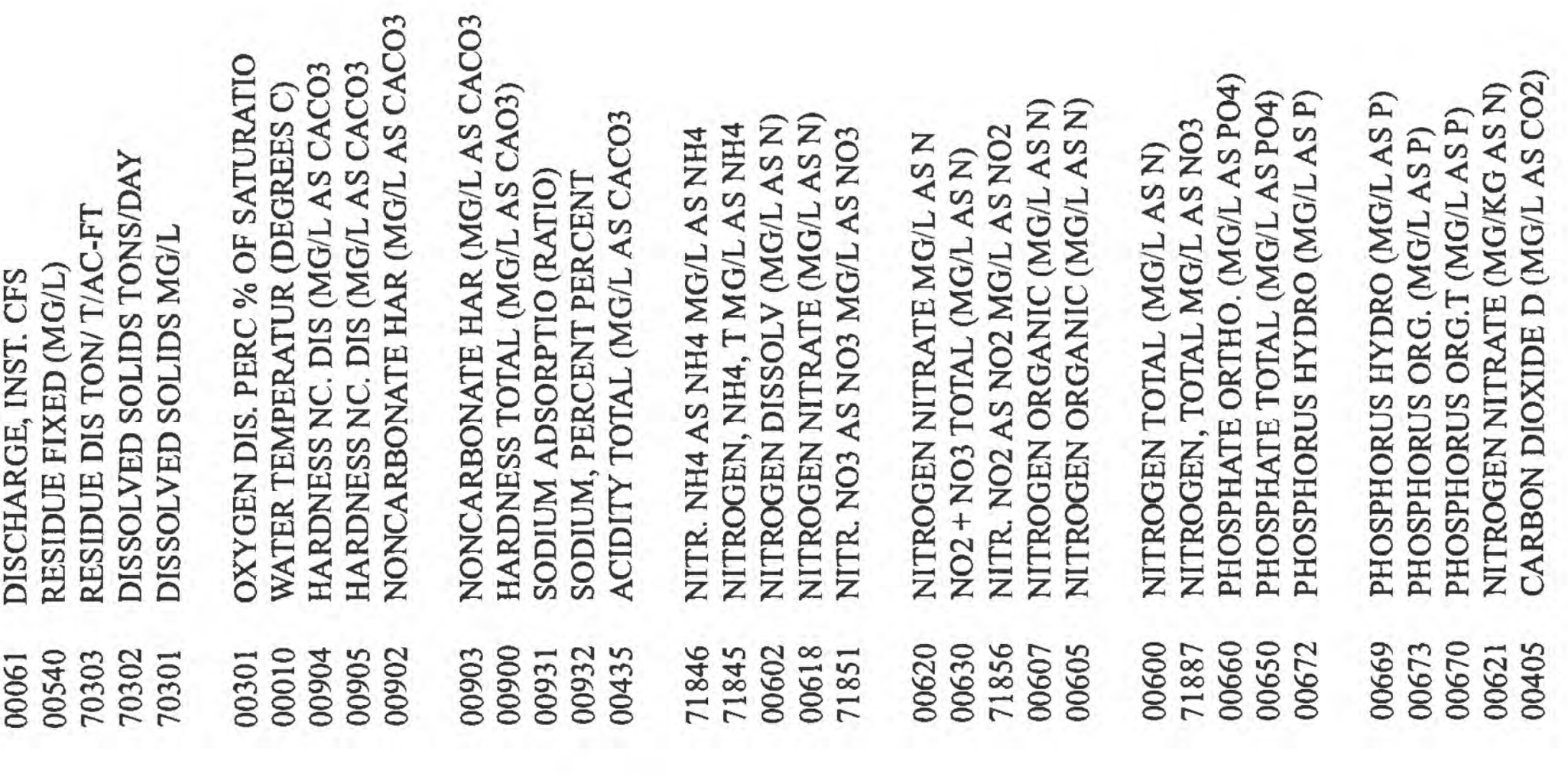




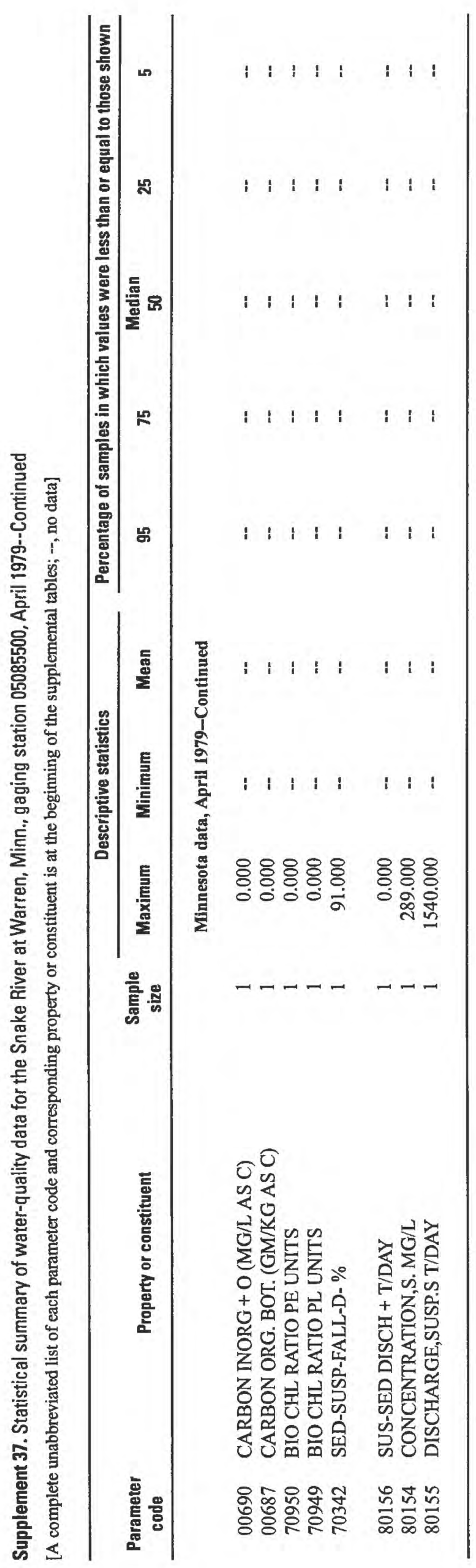




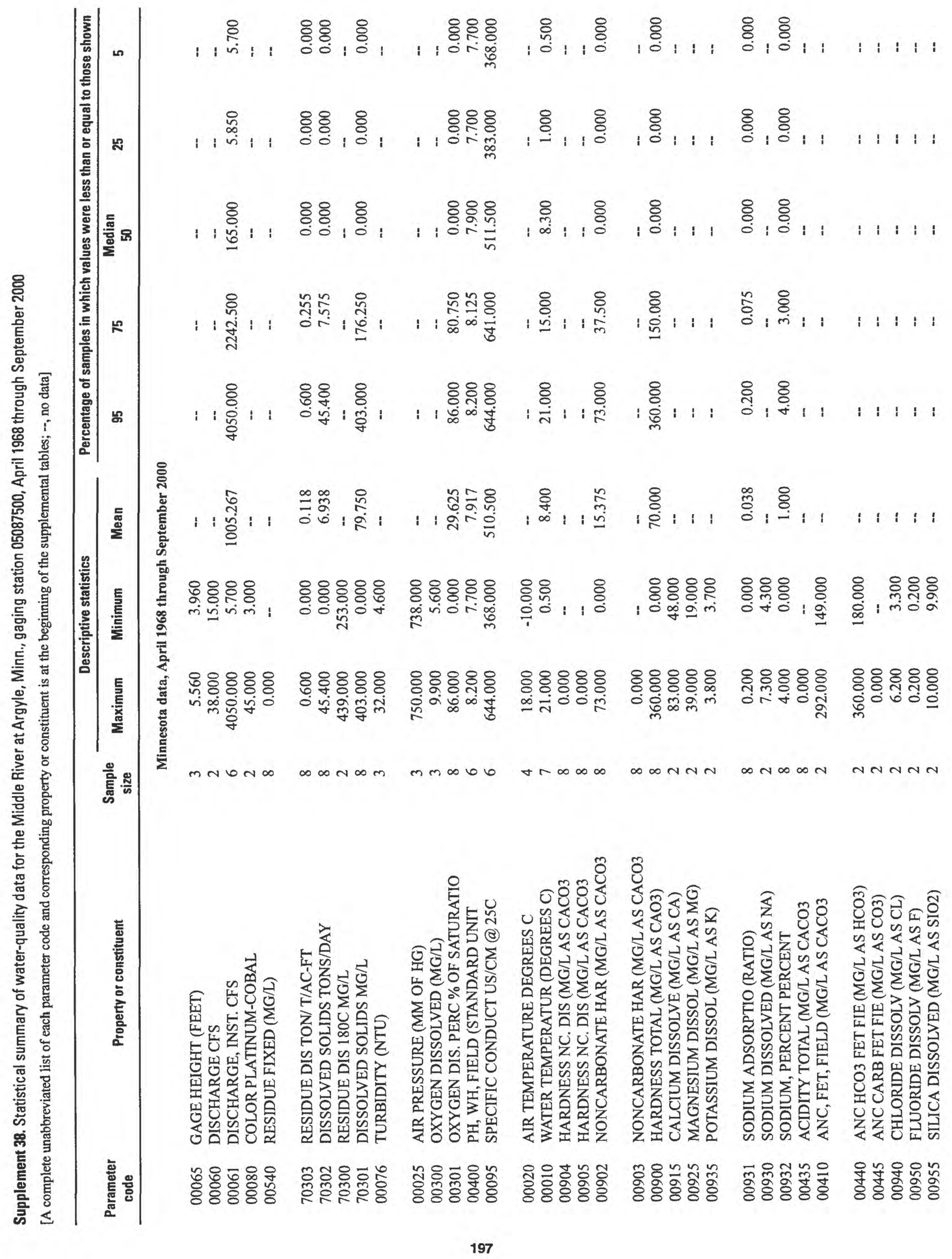




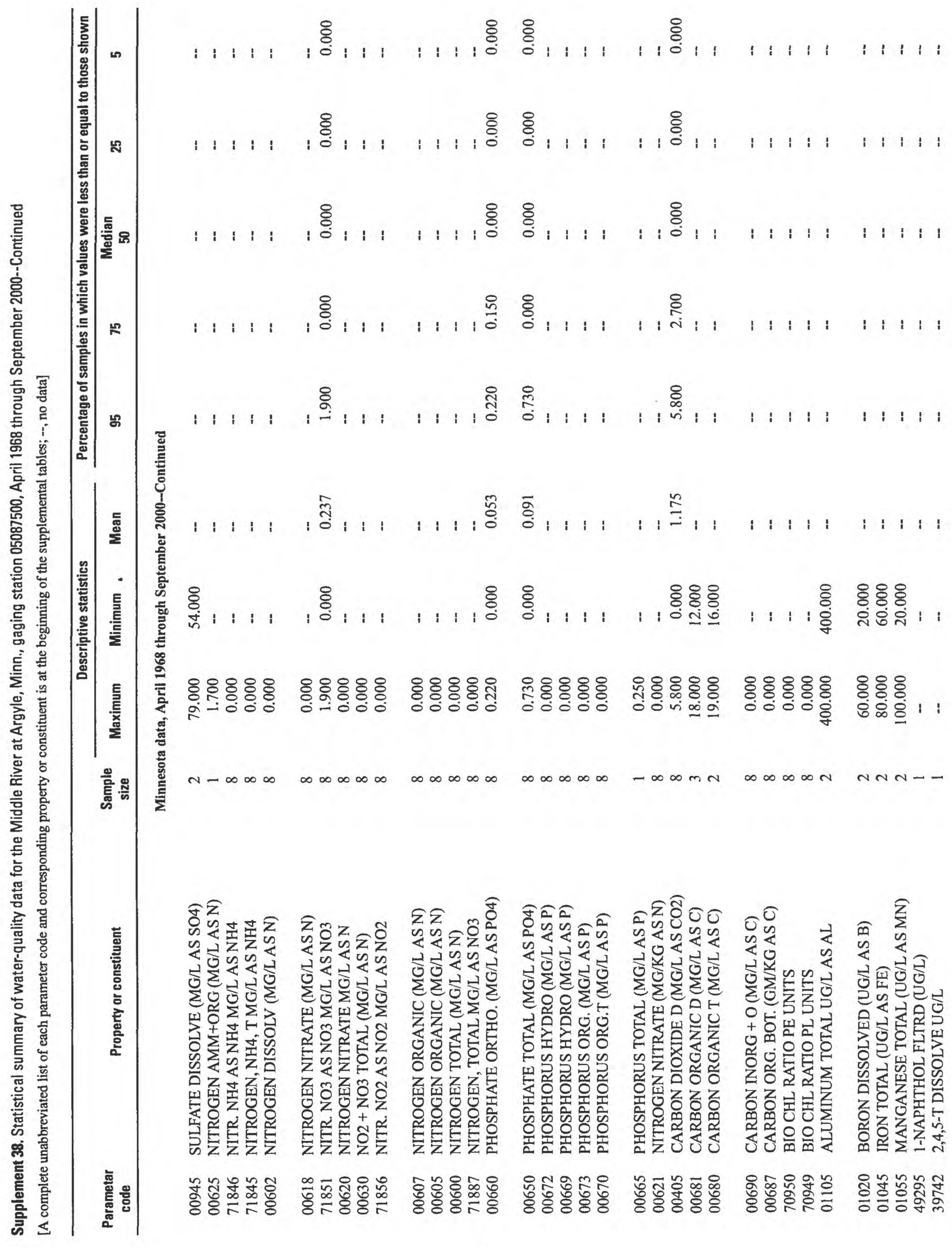




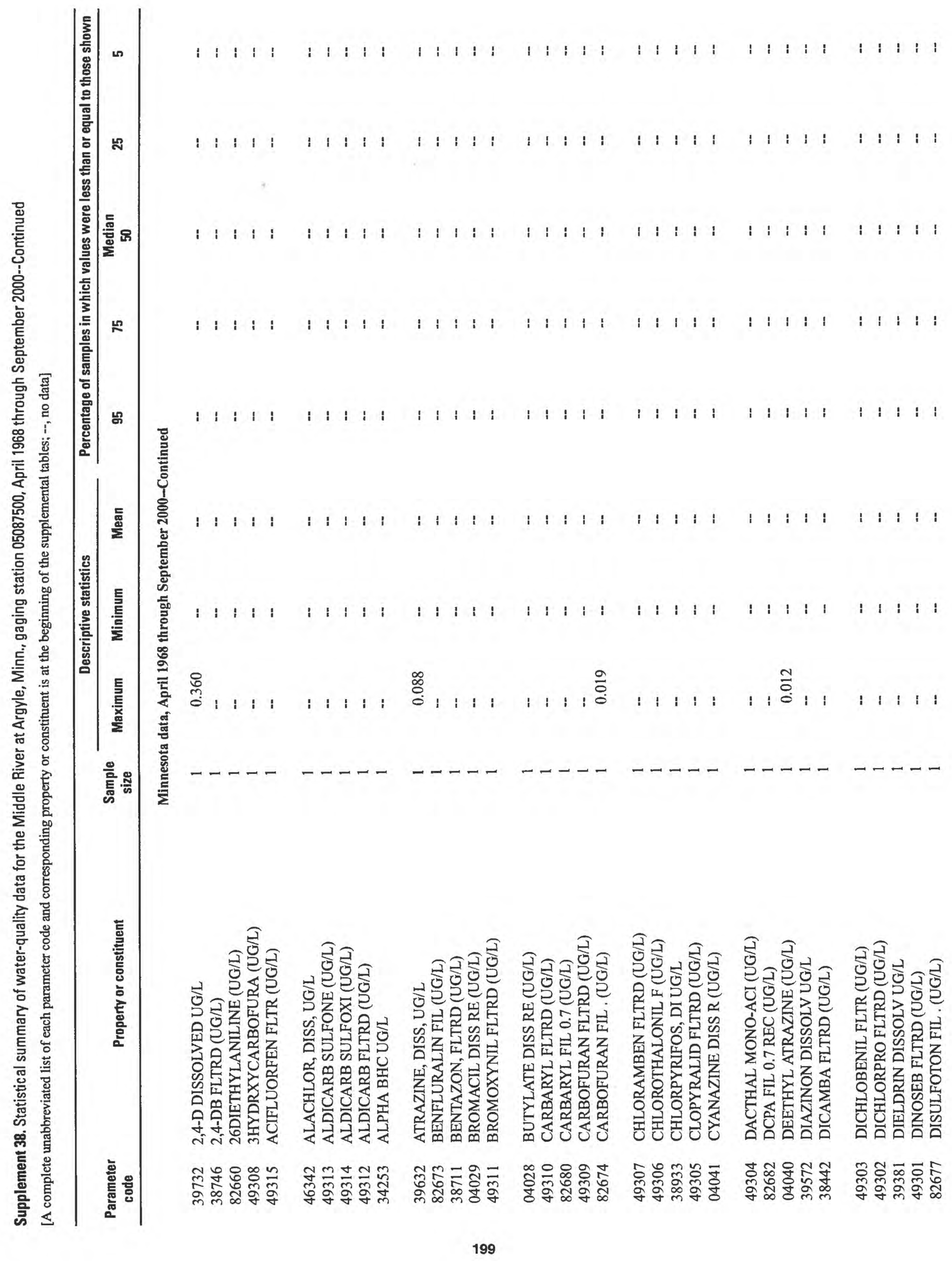




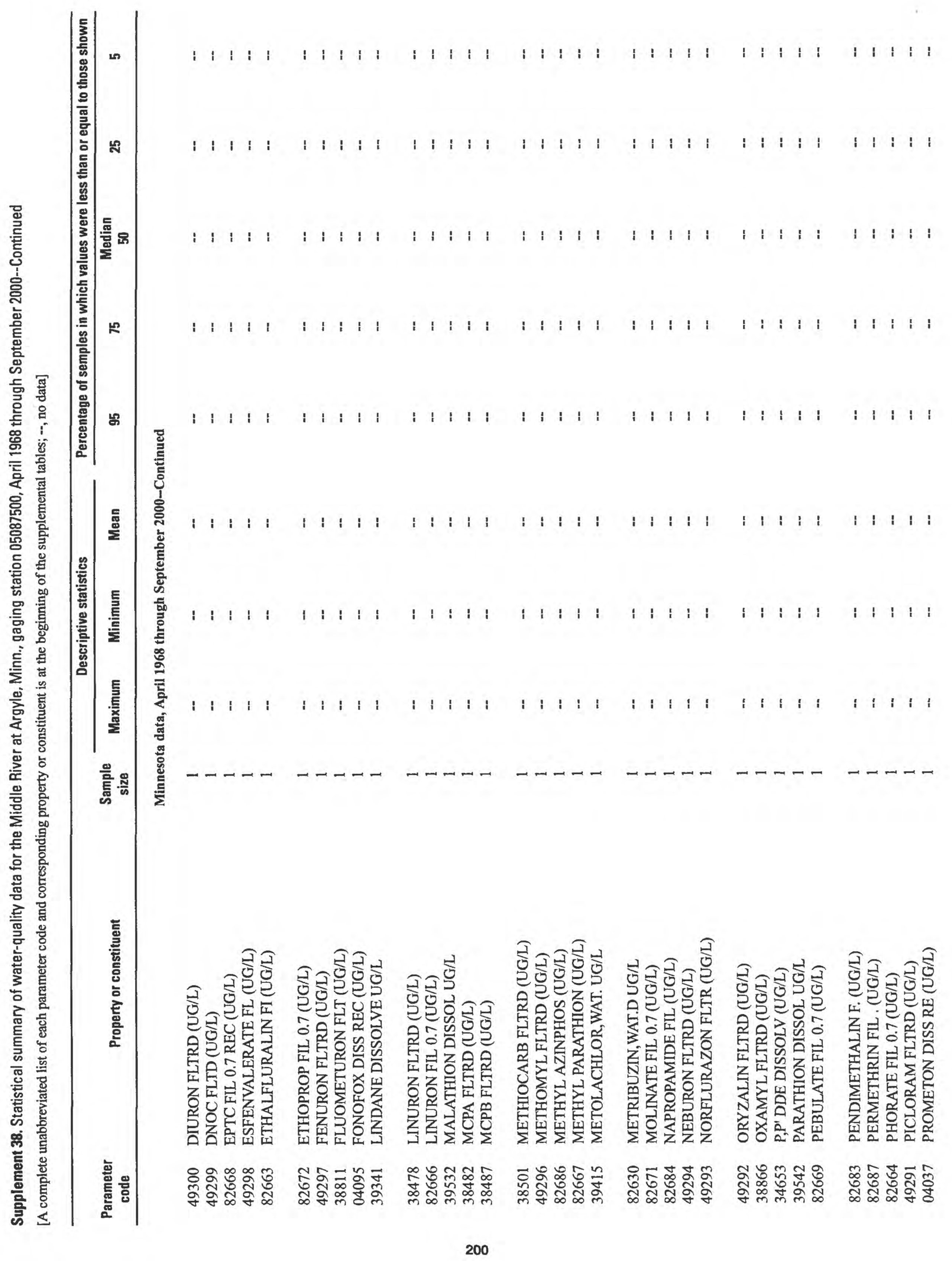




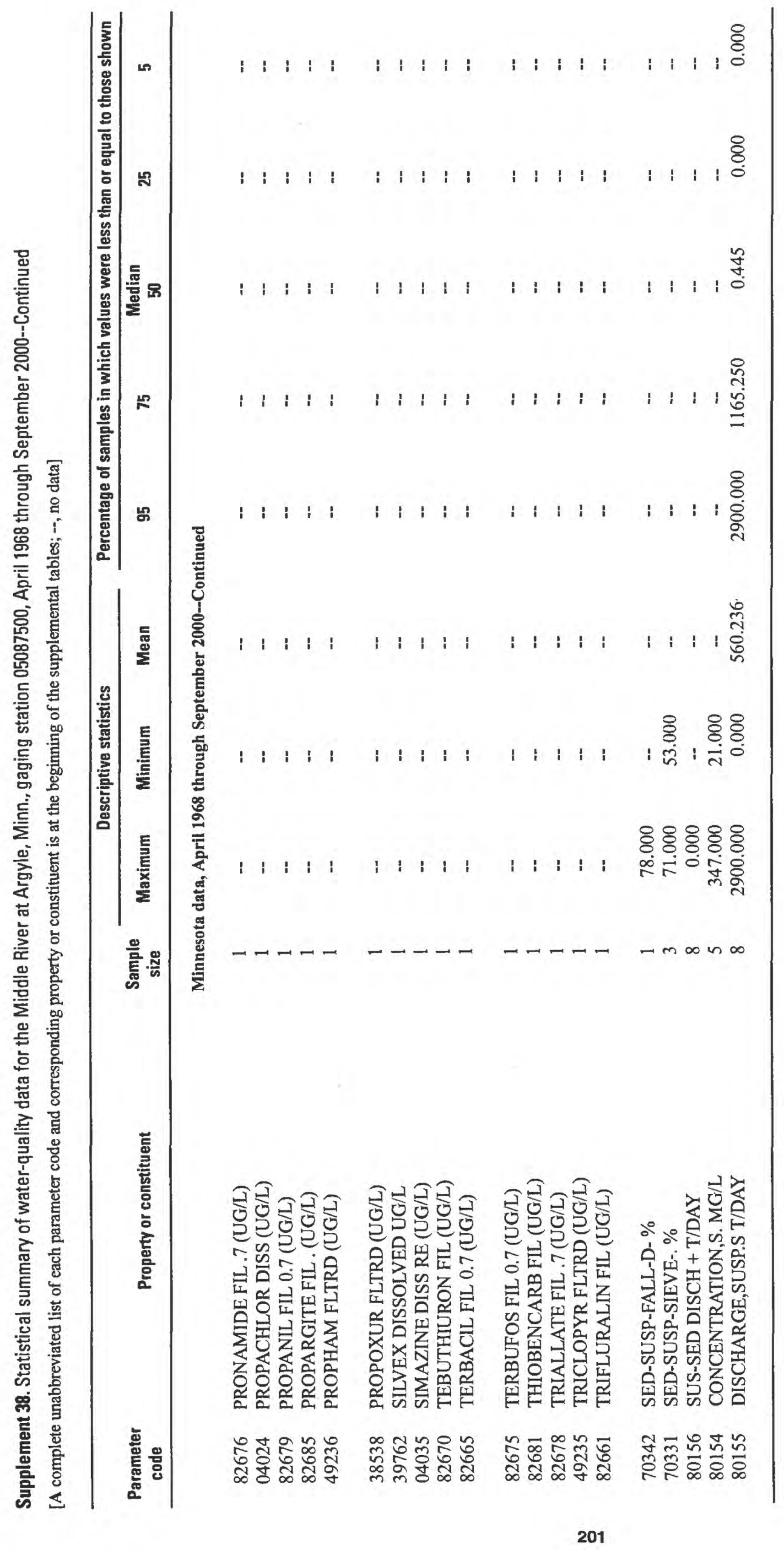




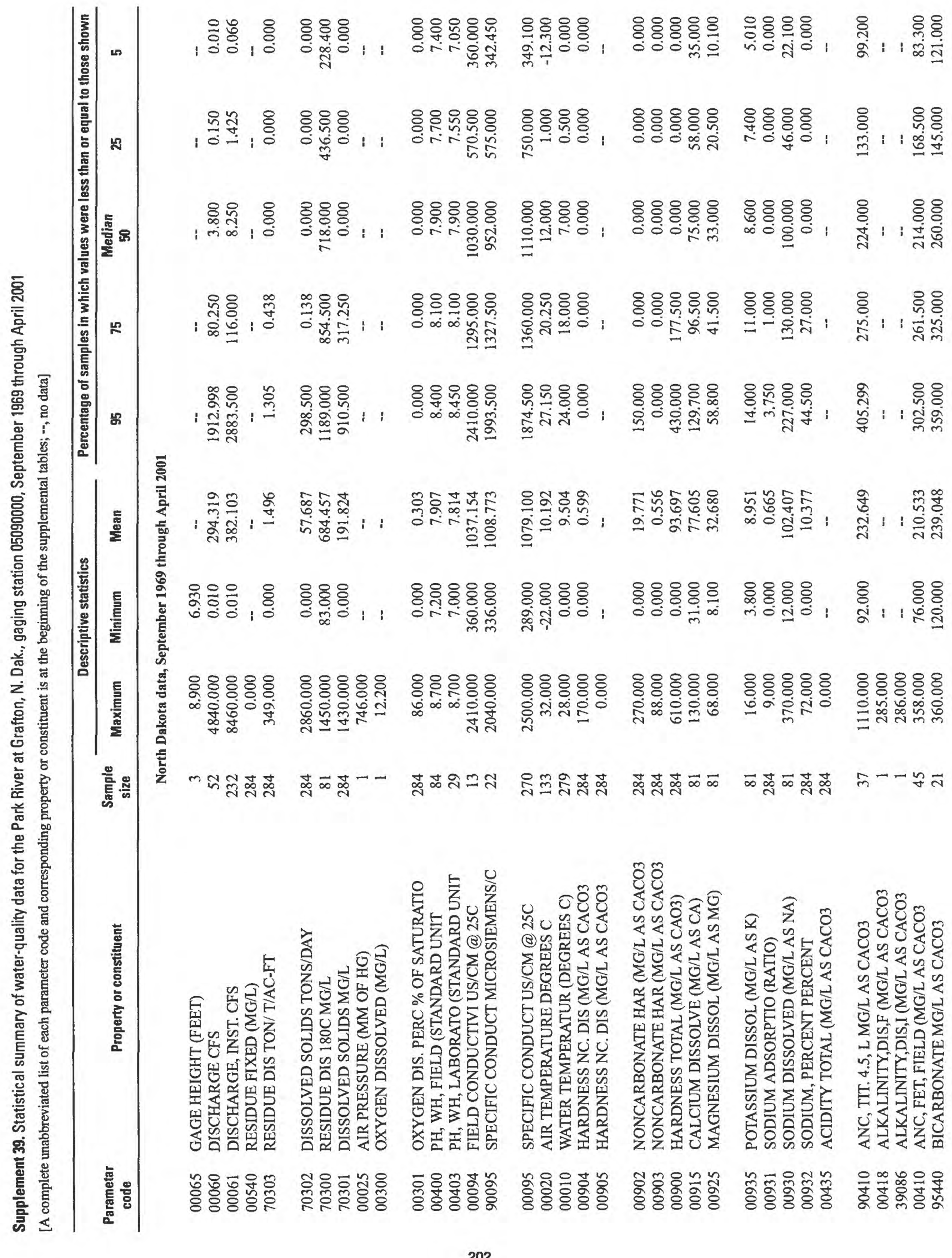




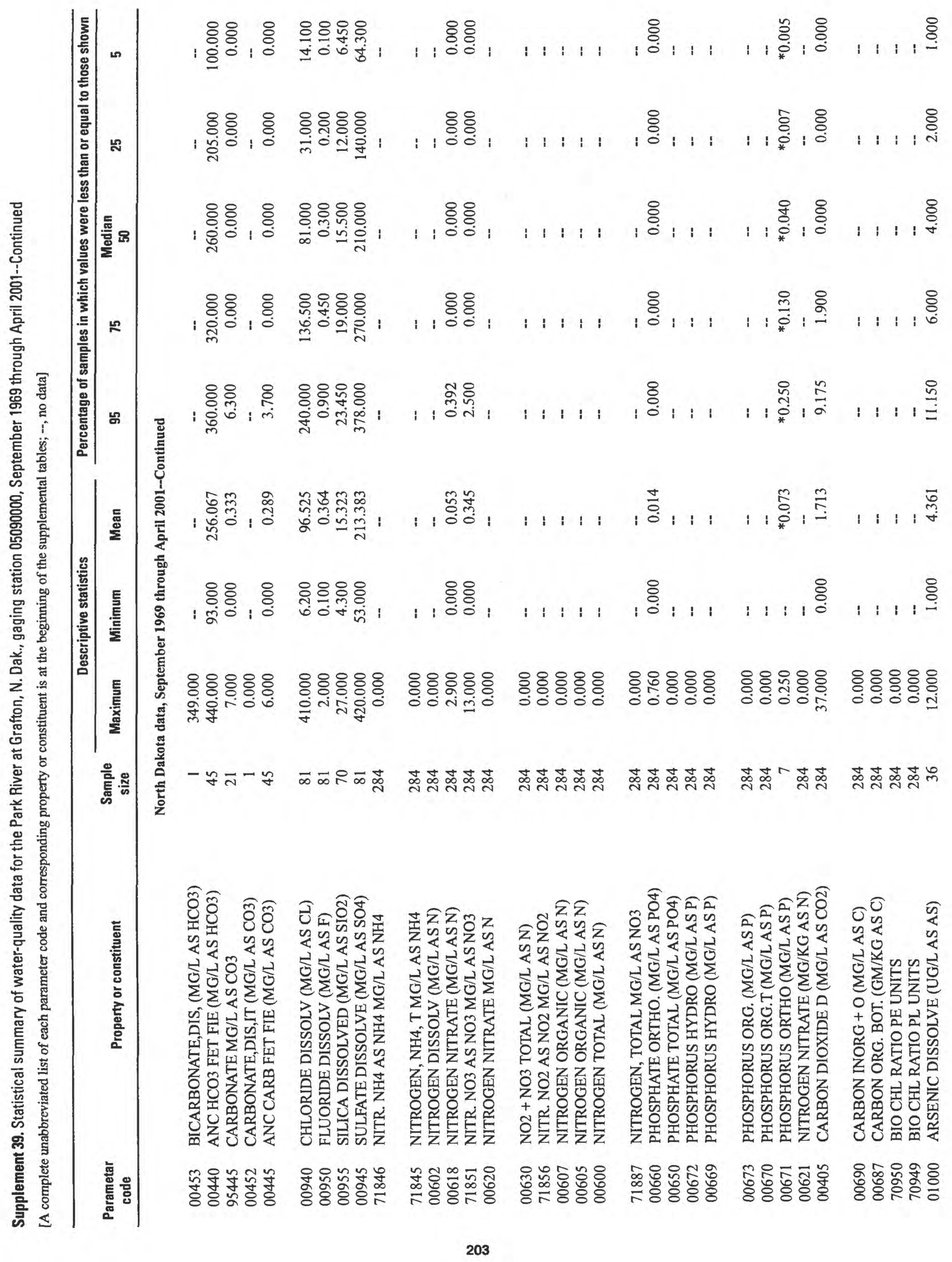




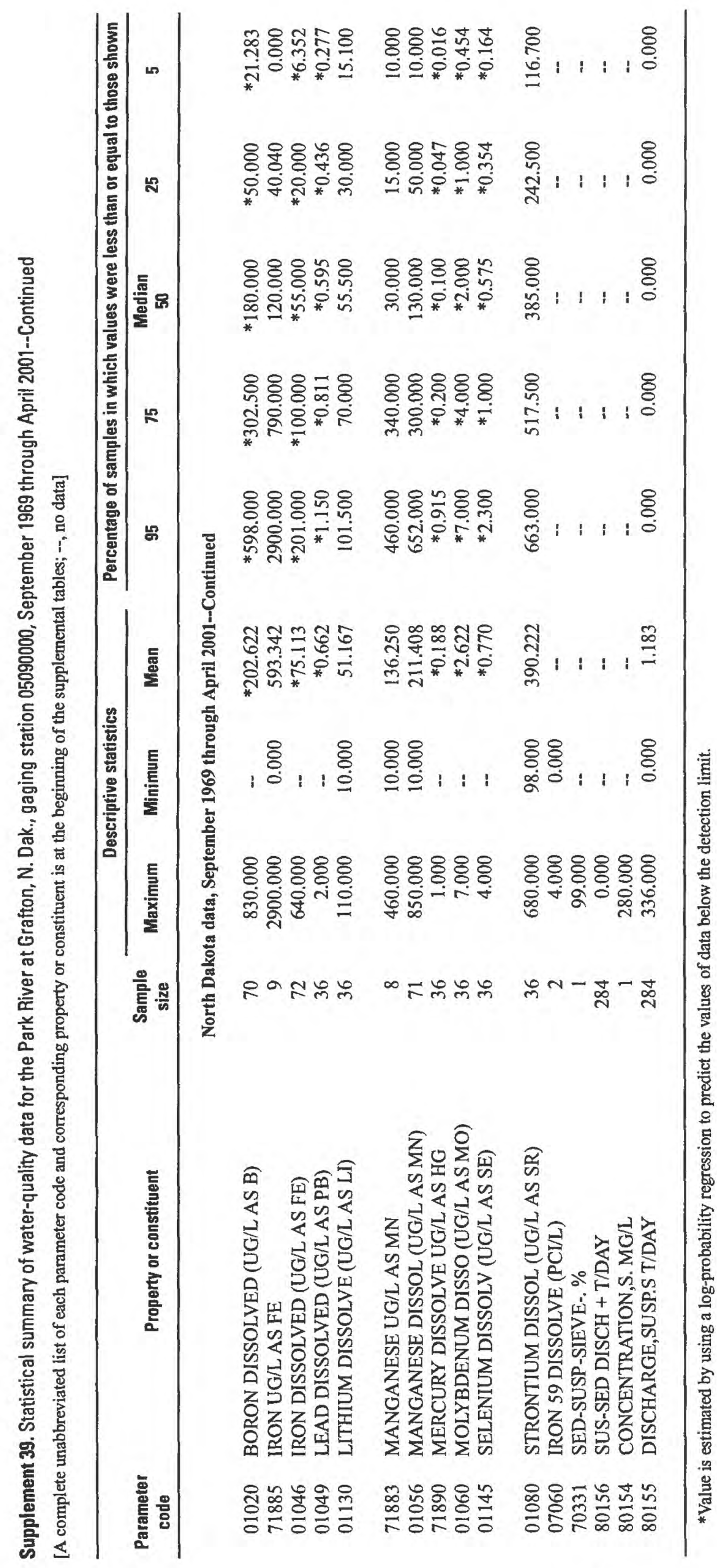




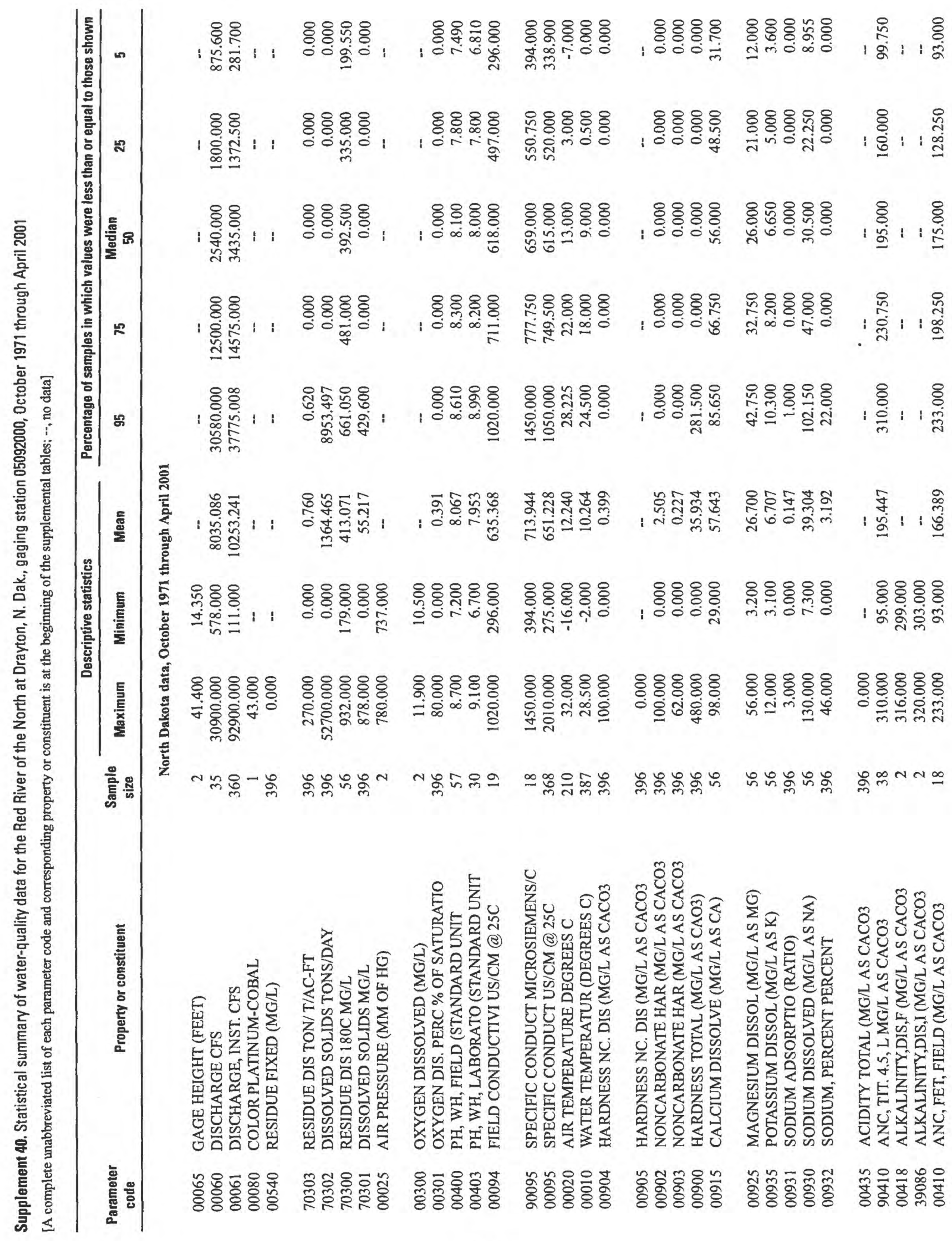




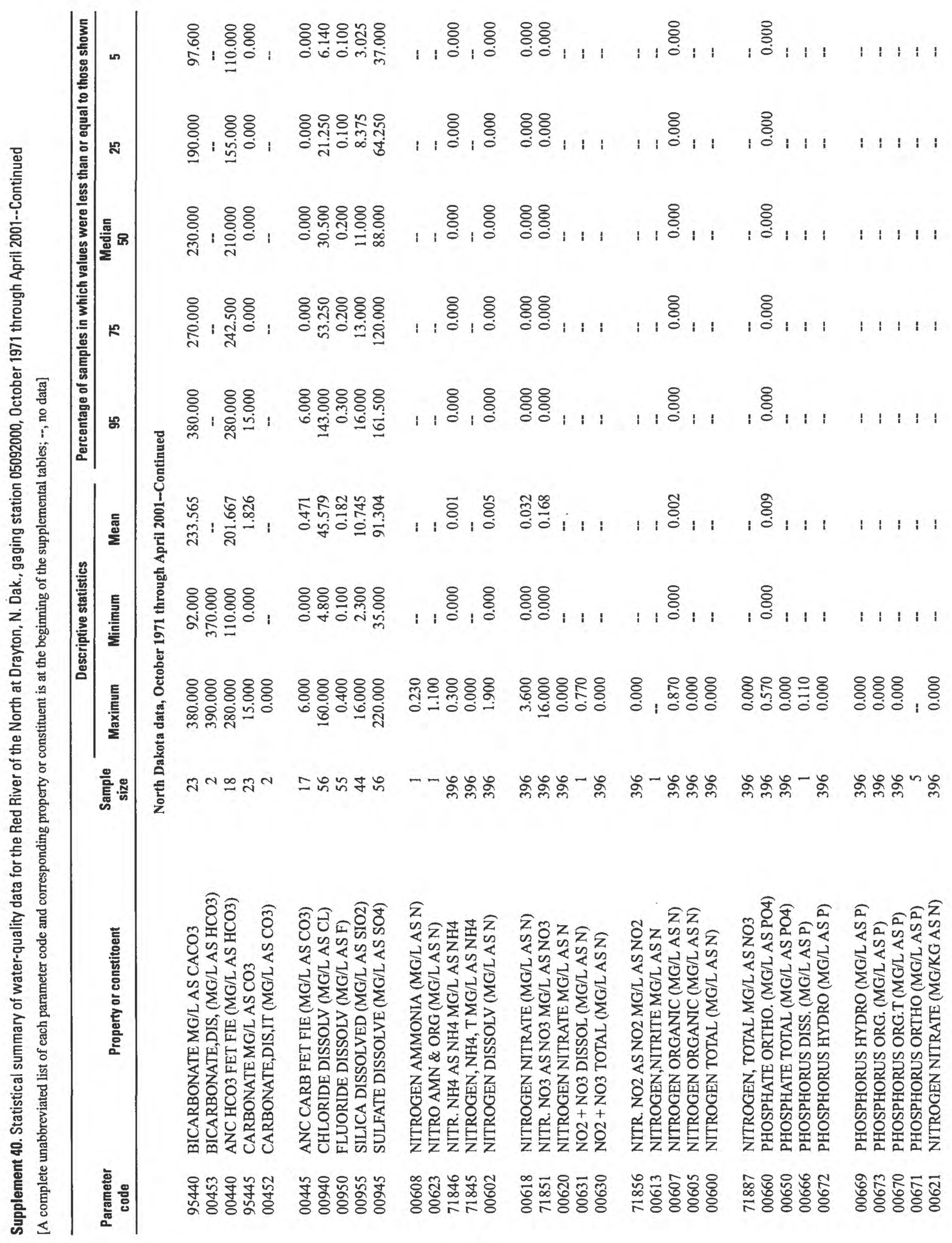




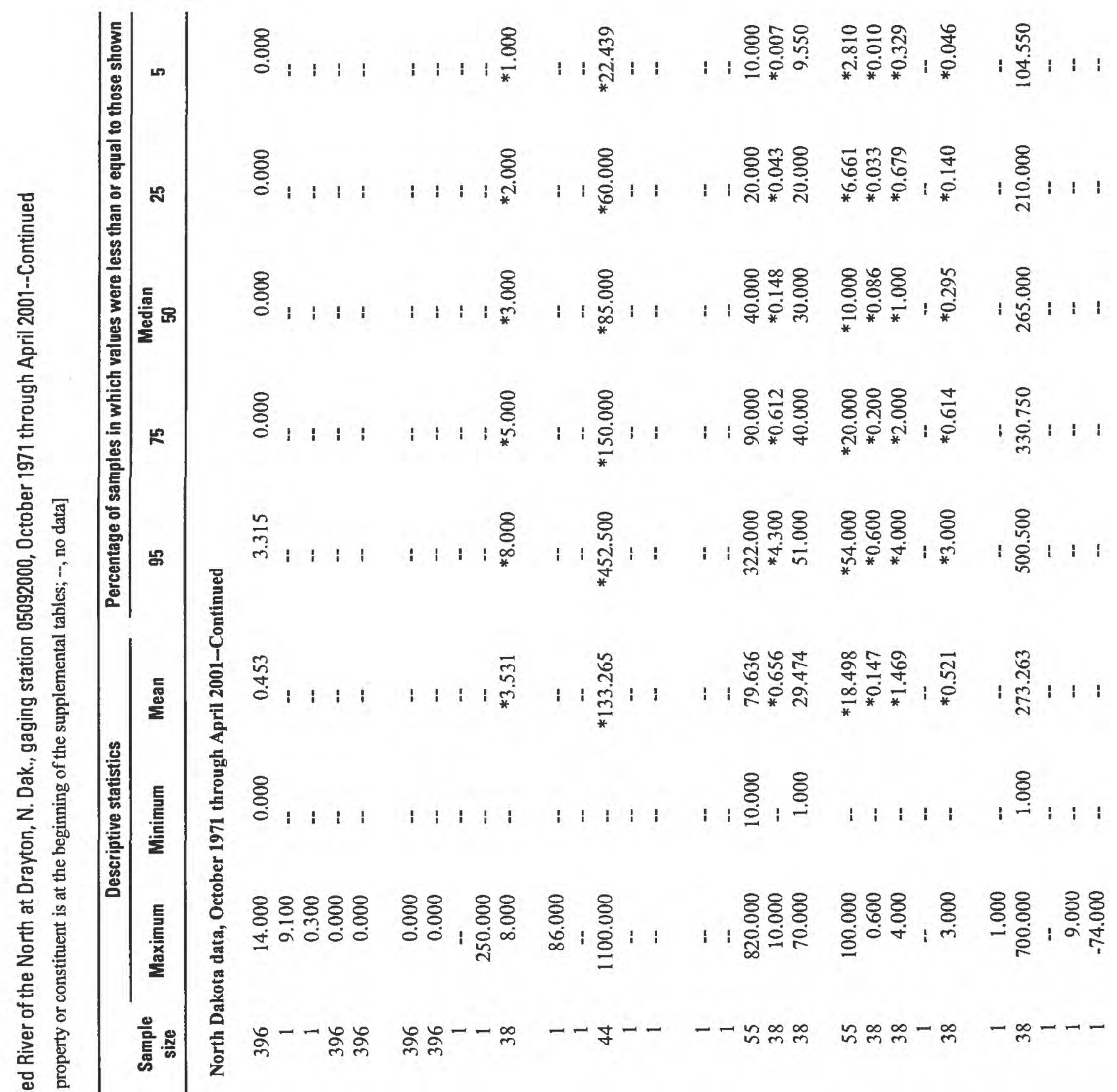

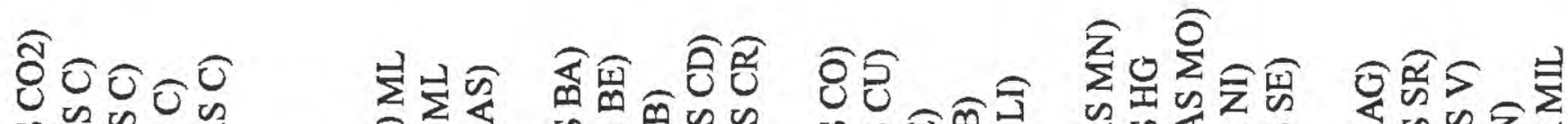

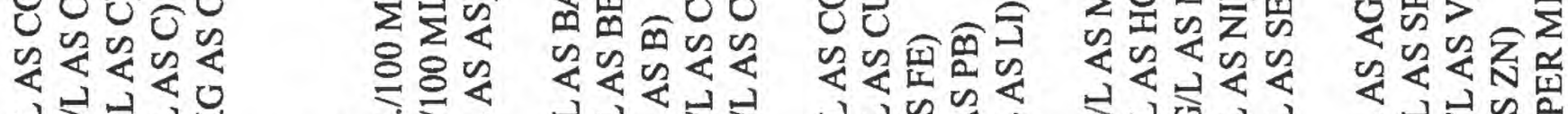

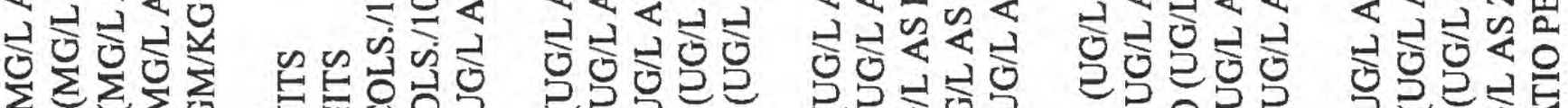

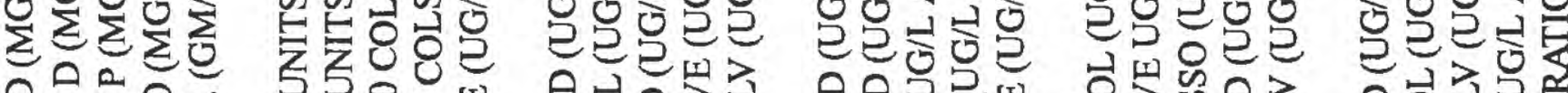
只司 50

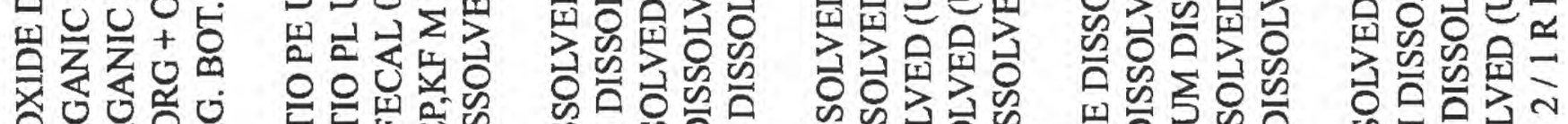

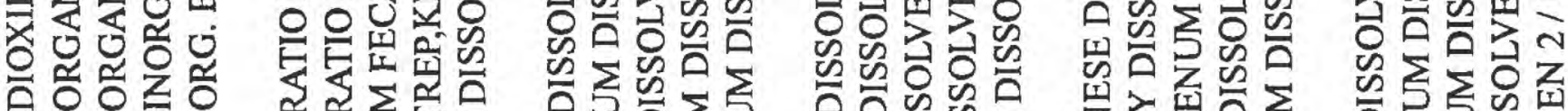

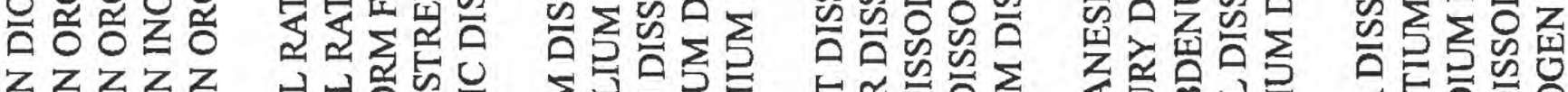

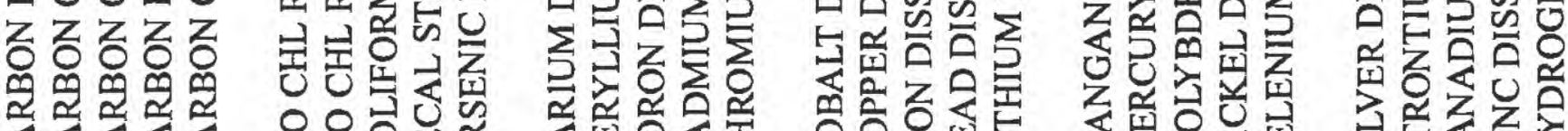

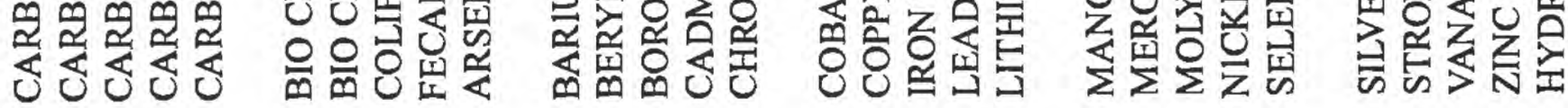

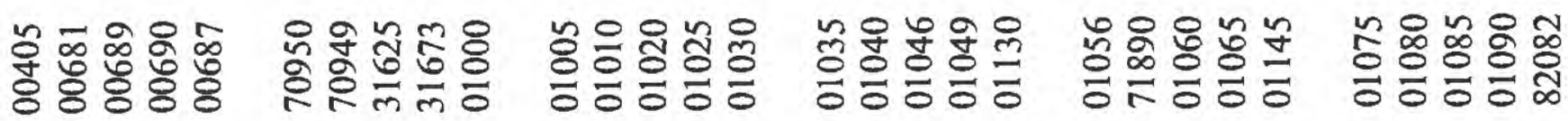




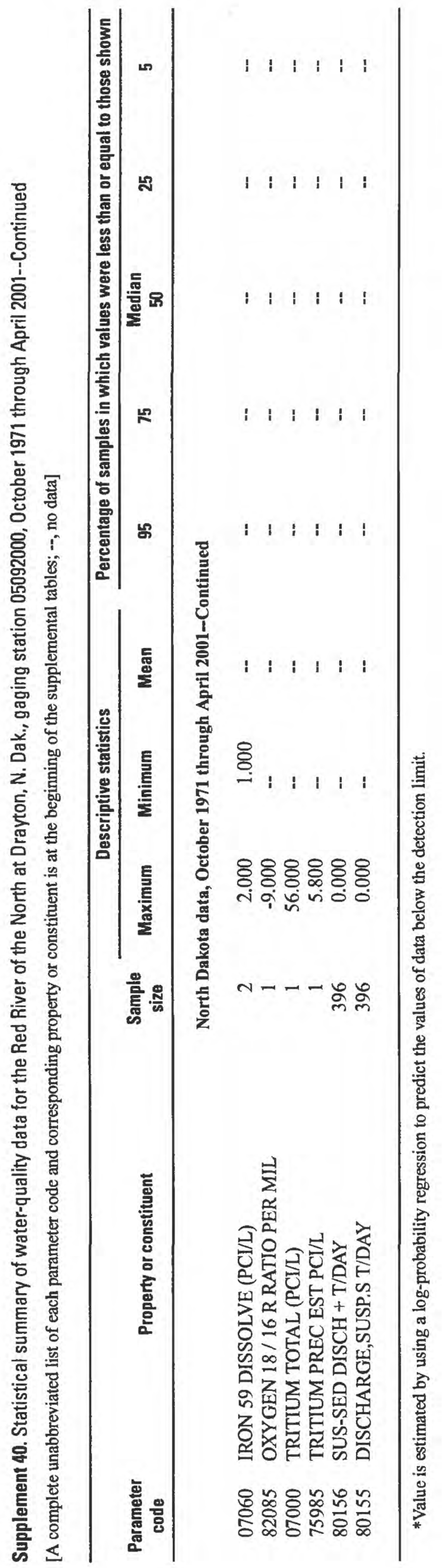




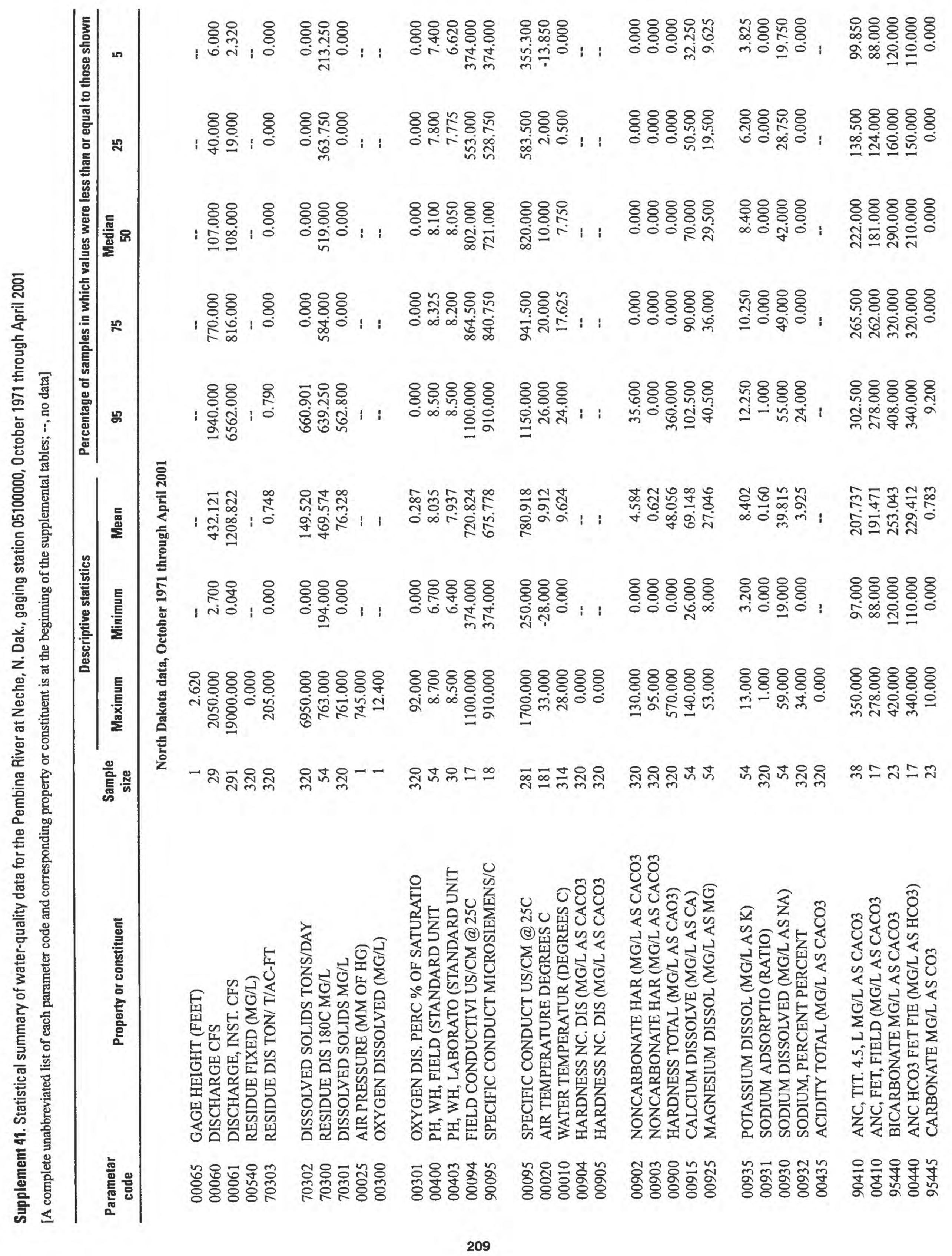




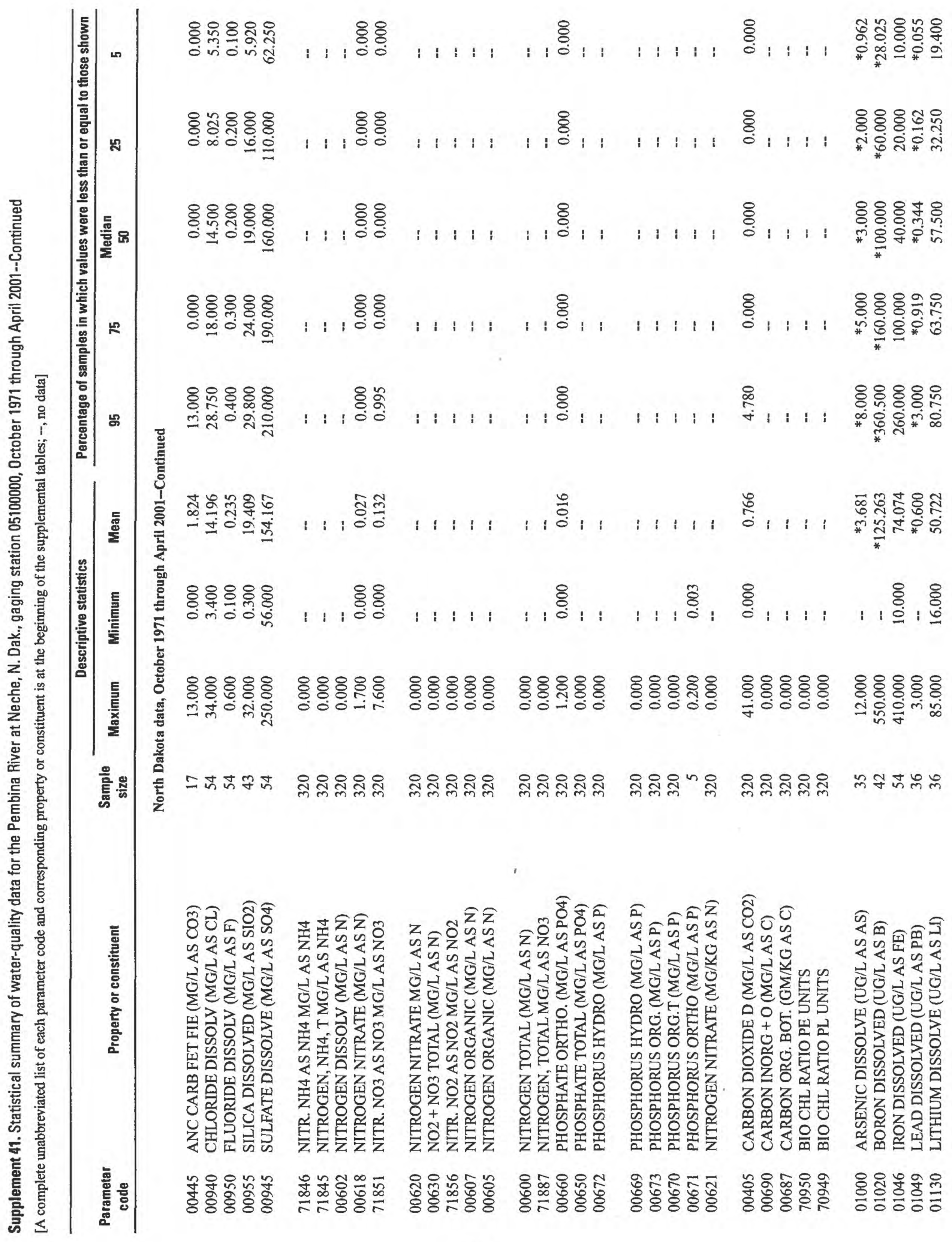




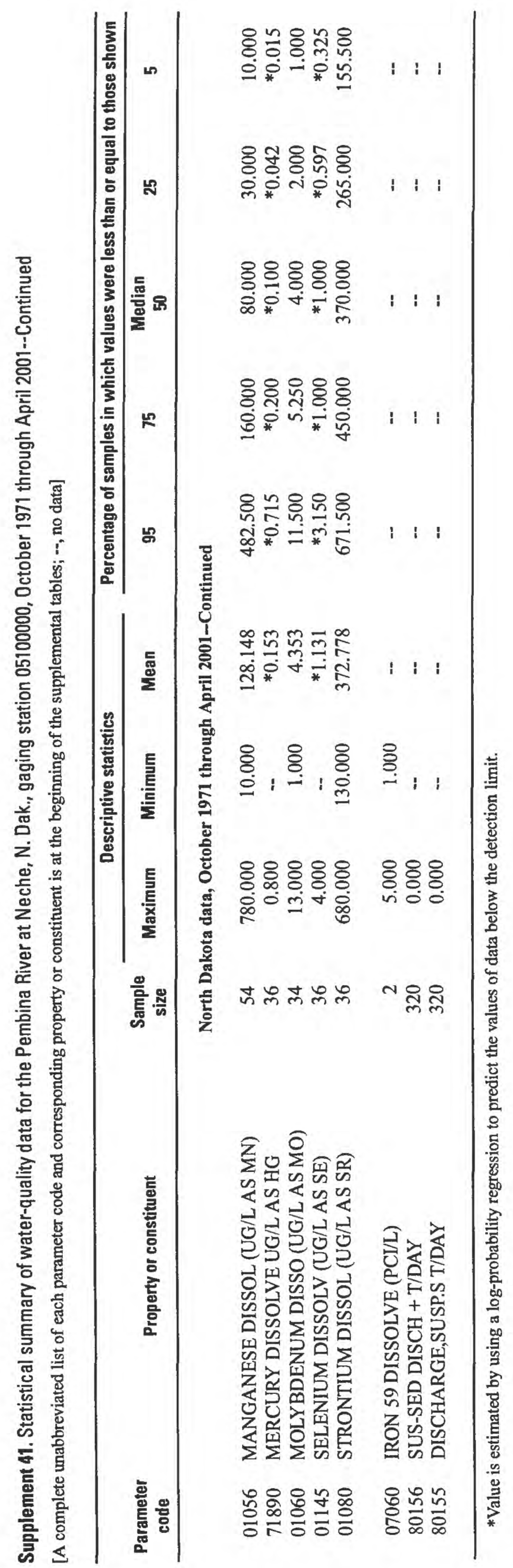




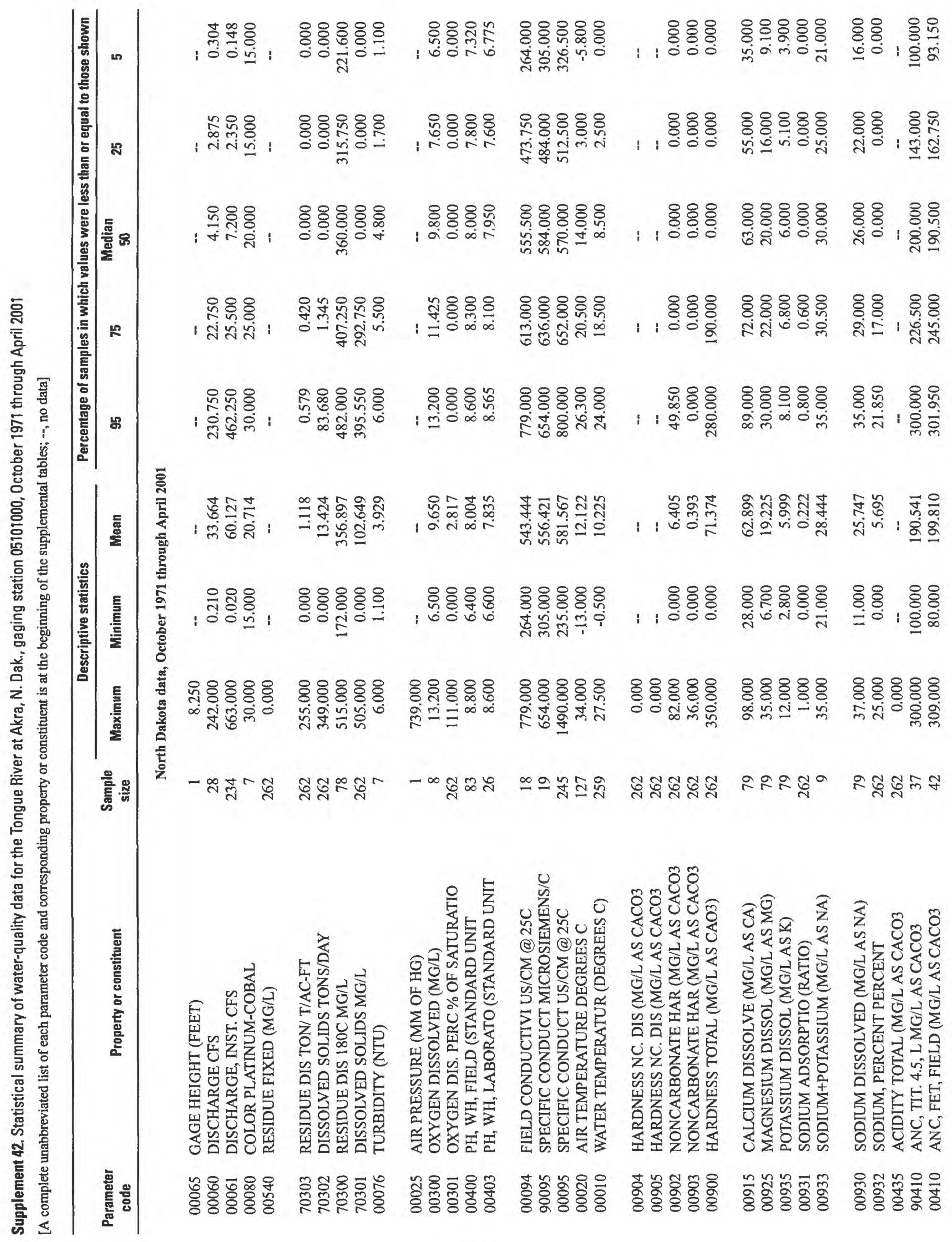




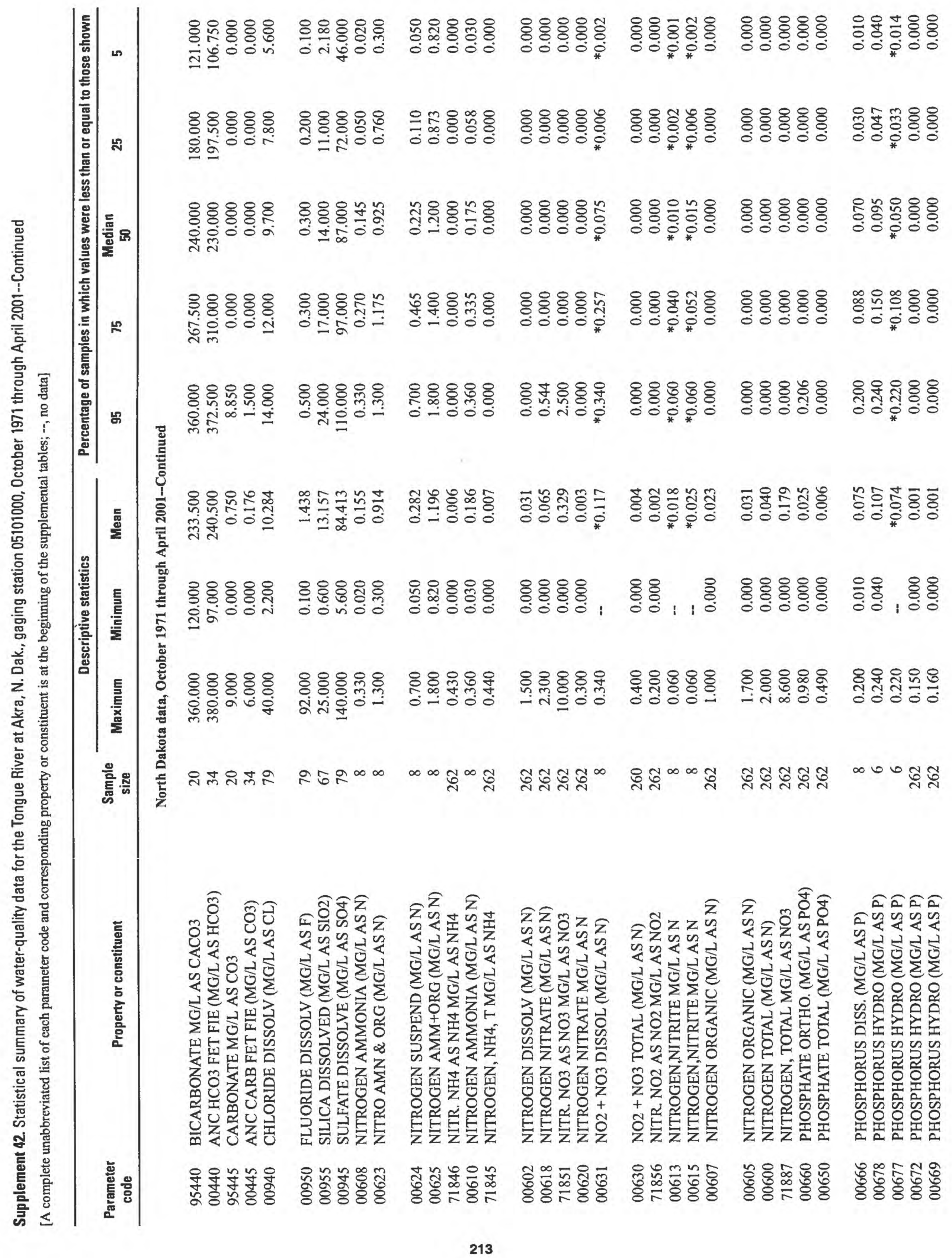




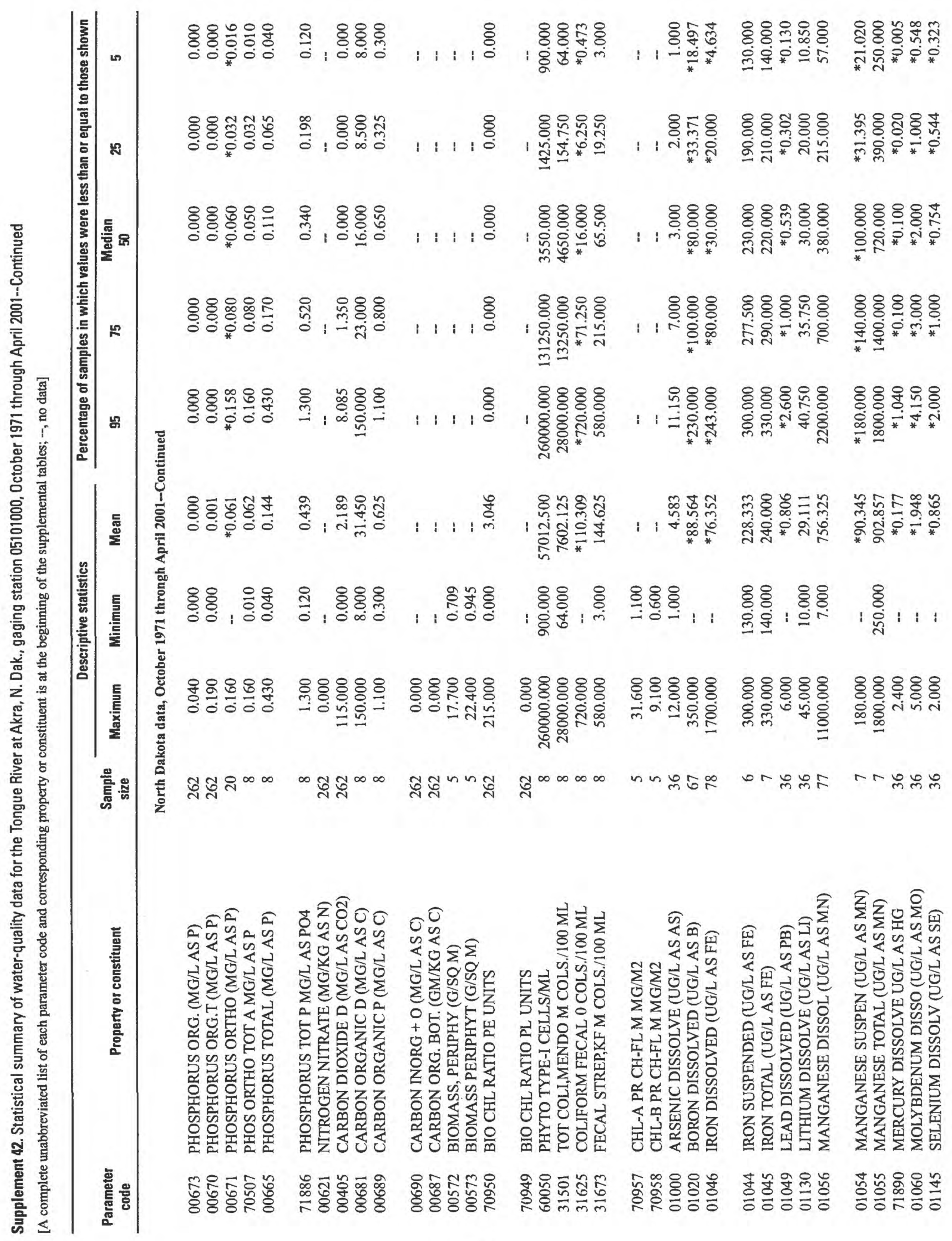




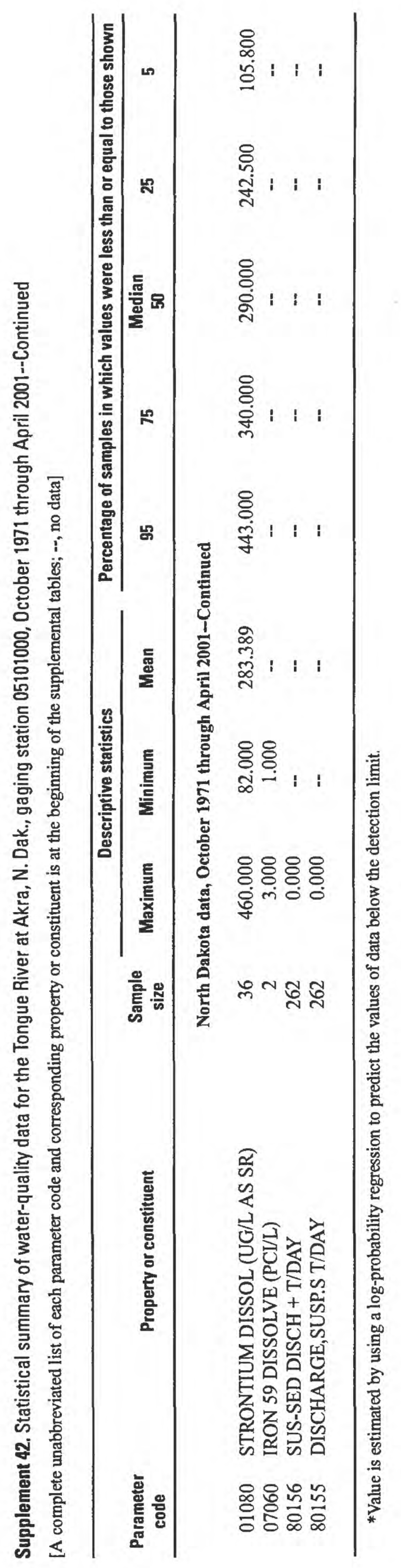




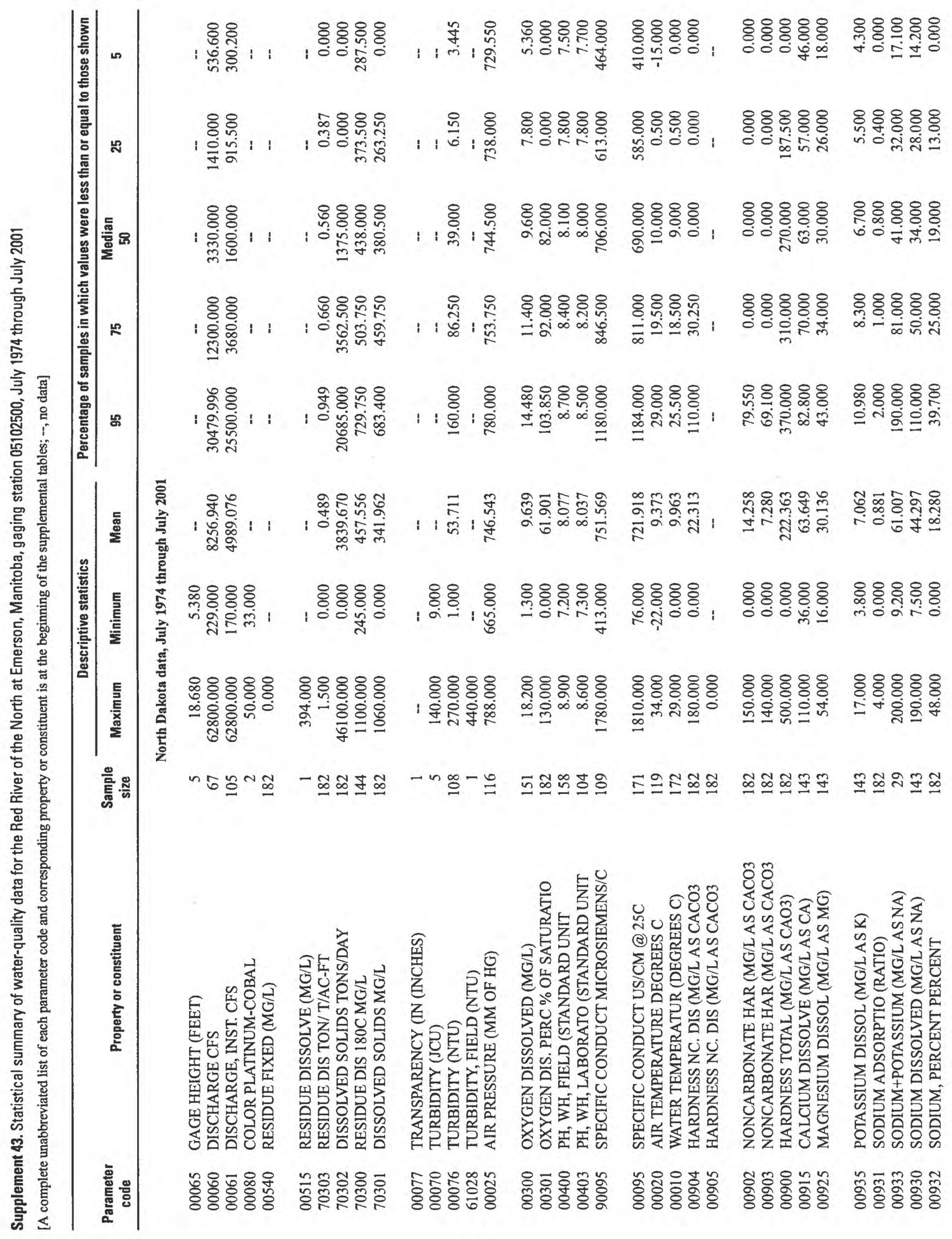




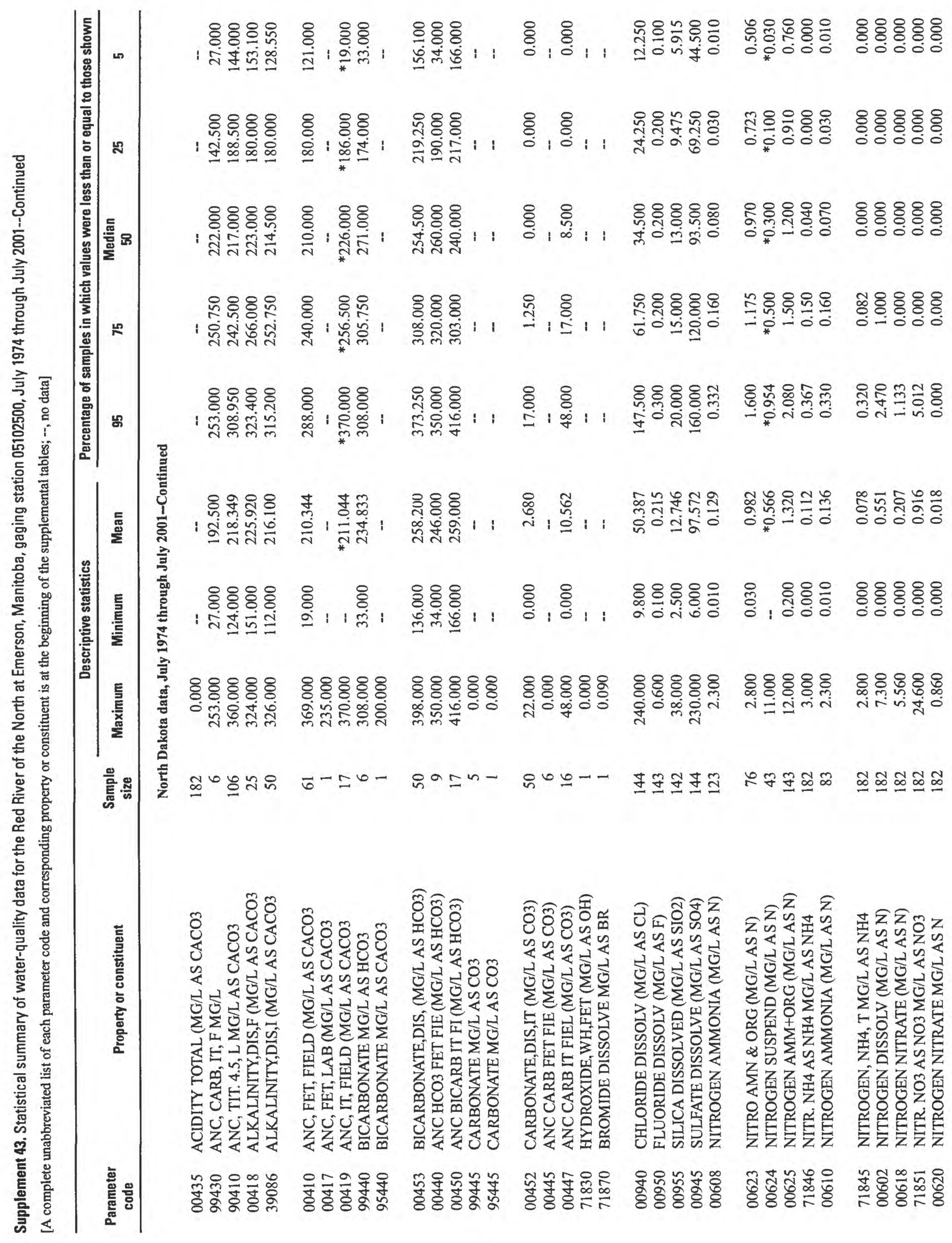




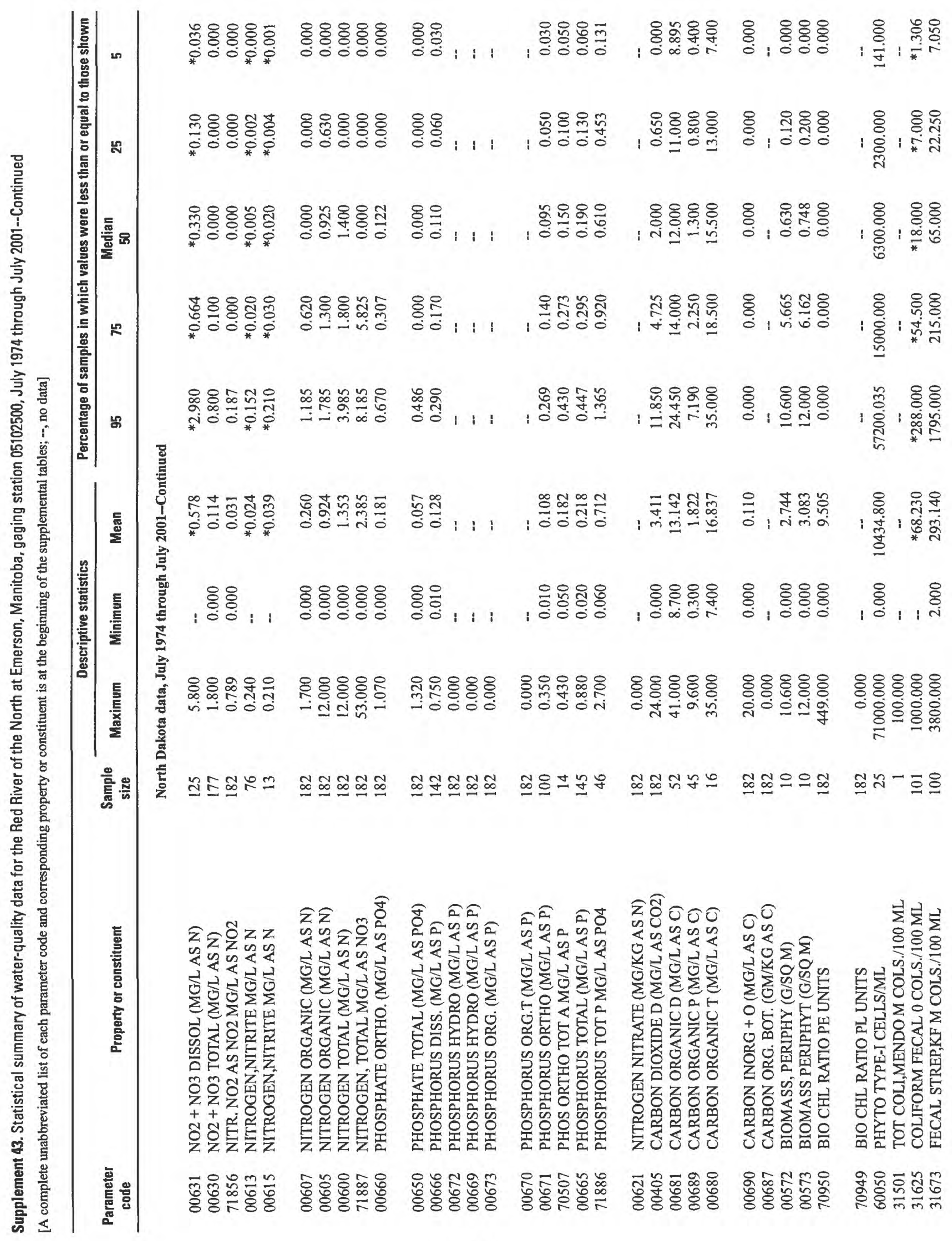




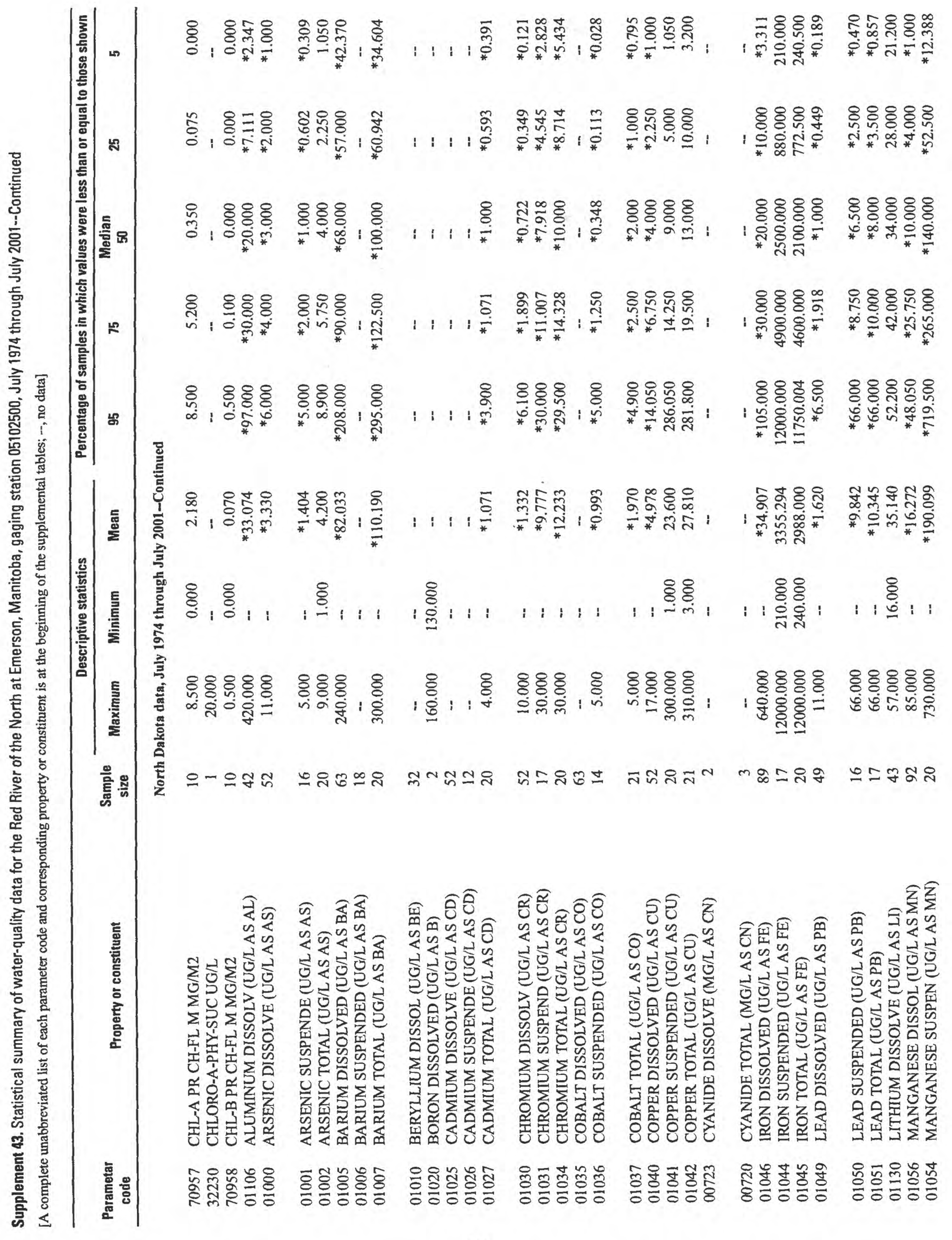




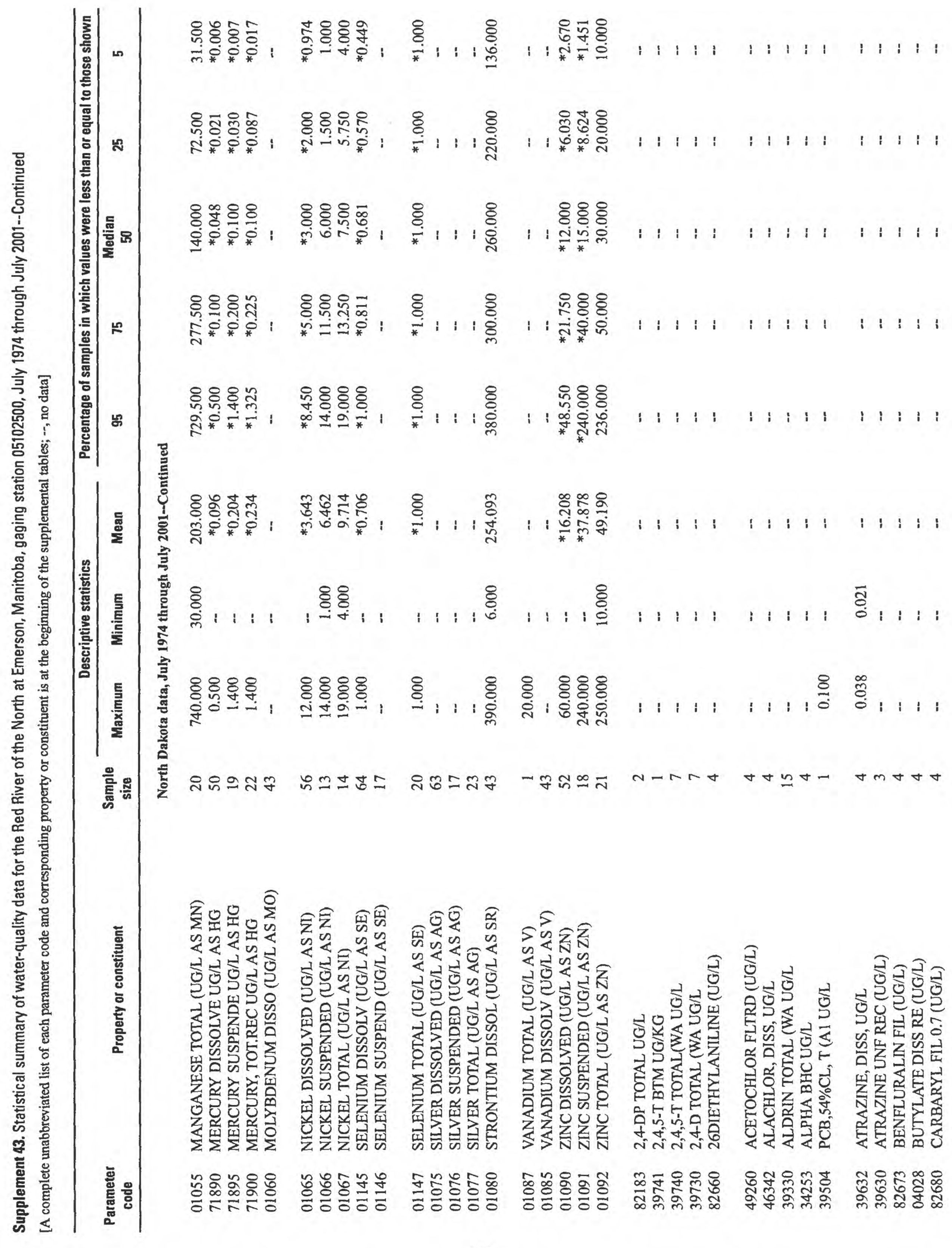



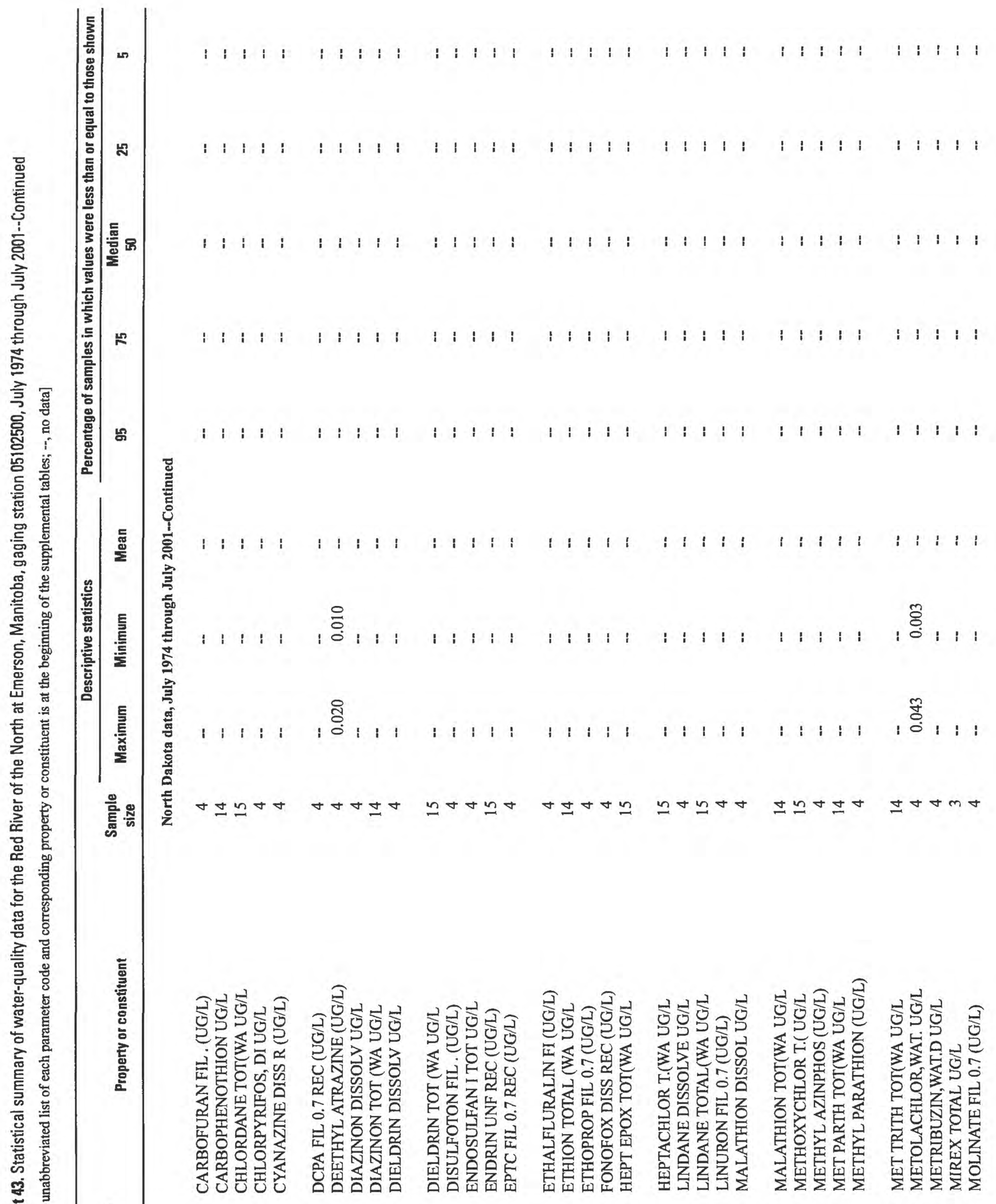

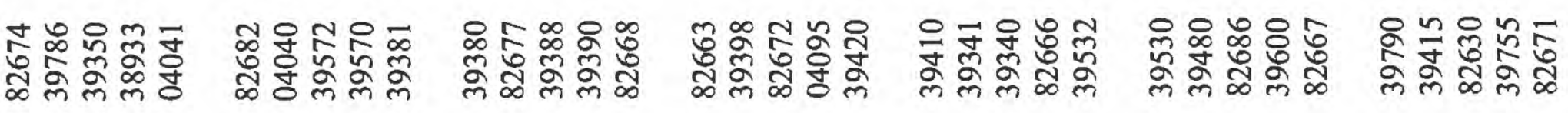




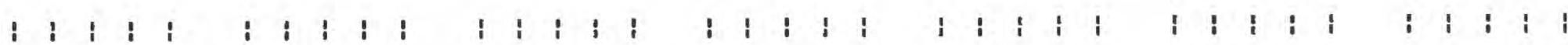

i i 1 i 1 1 1 1 1

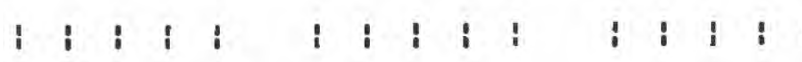

i i i i

i i i i

i i i

$1 ;$

i i i i

1 1 i 1 1 : 1 i

: 1 i i

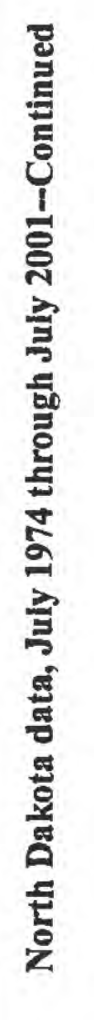

1 1 i i

1 i i i

i 1 i i

i i i i

i 1 i i i

1 i i i i i i i

$1+11$ i

i i i i

i i i i

i i i i

i $\quad$ i $\quad$ i

:

ஜㅇㅇㅇ

i i i

i i

$1 ; i \quad i$

i i i

$\forall \forall n \pm$

$\pm m m \nabla$

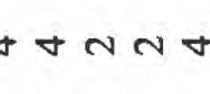

$\dot{\nabla} \nabla \nabla \nabla$

$-6 m \forall \forall$

$\forall \forall \forall \forall \forall-\operatorname{\theta n} m$

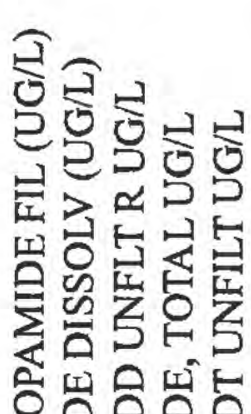

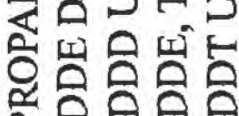

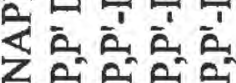

넝

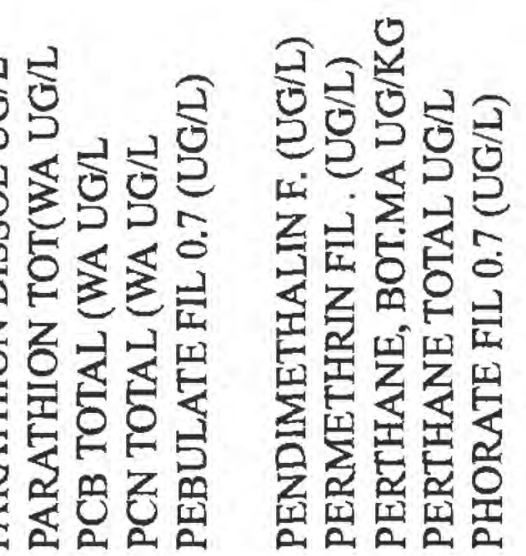

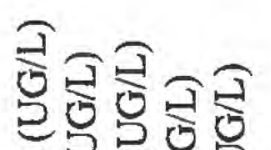

国已已

此官

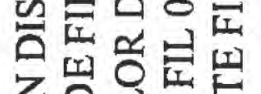

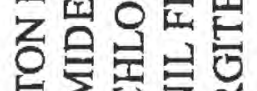

四毜员

之志令

哇旪吕吕吕

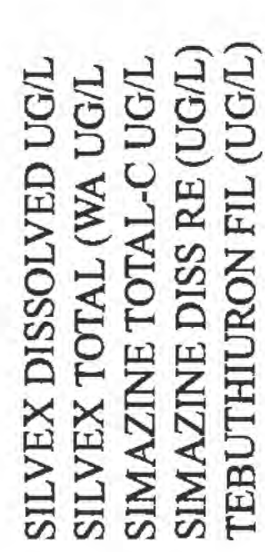

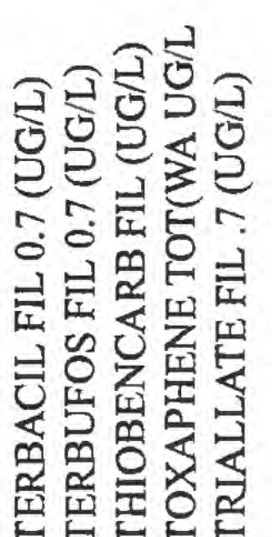

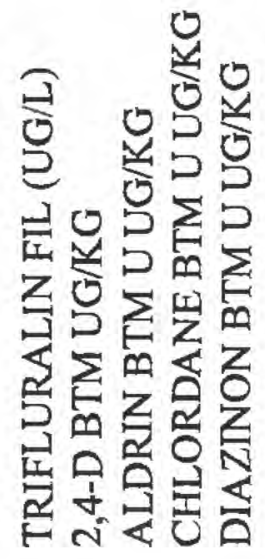

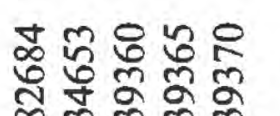

군뉴뉴융

అ心

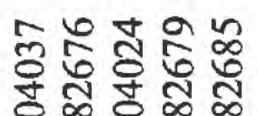

주윤ํํㅇ용

늉유유유

후을

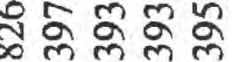




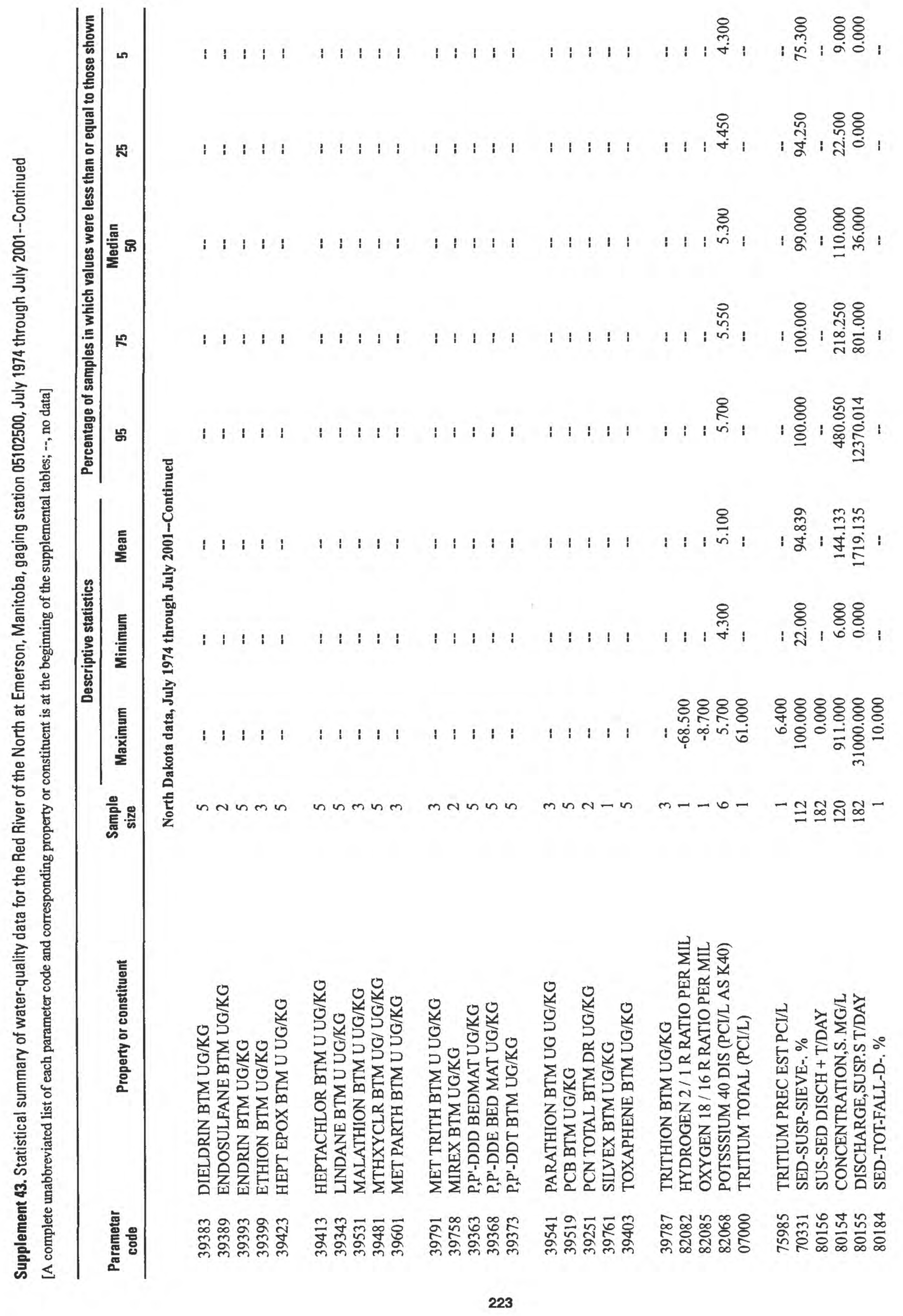




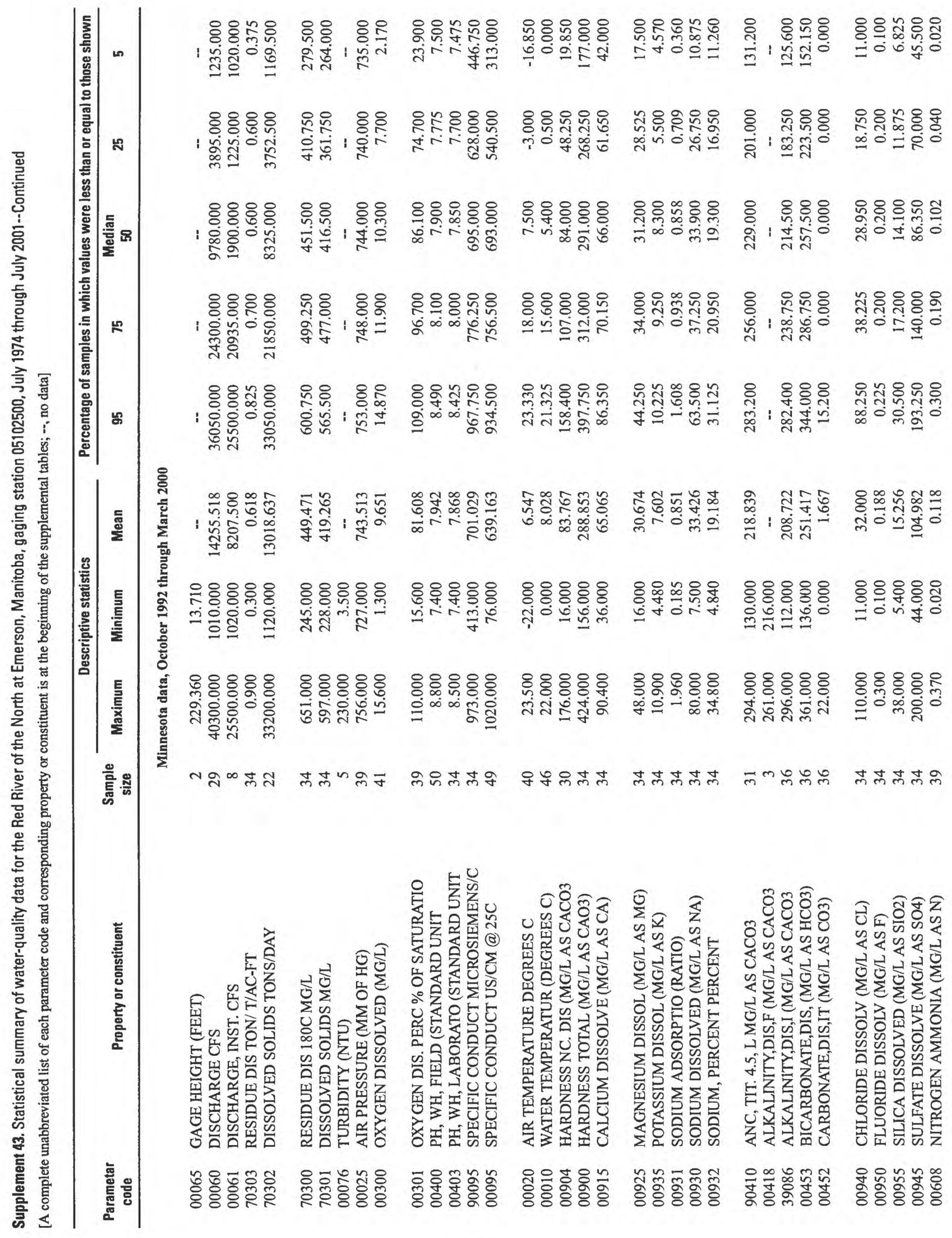




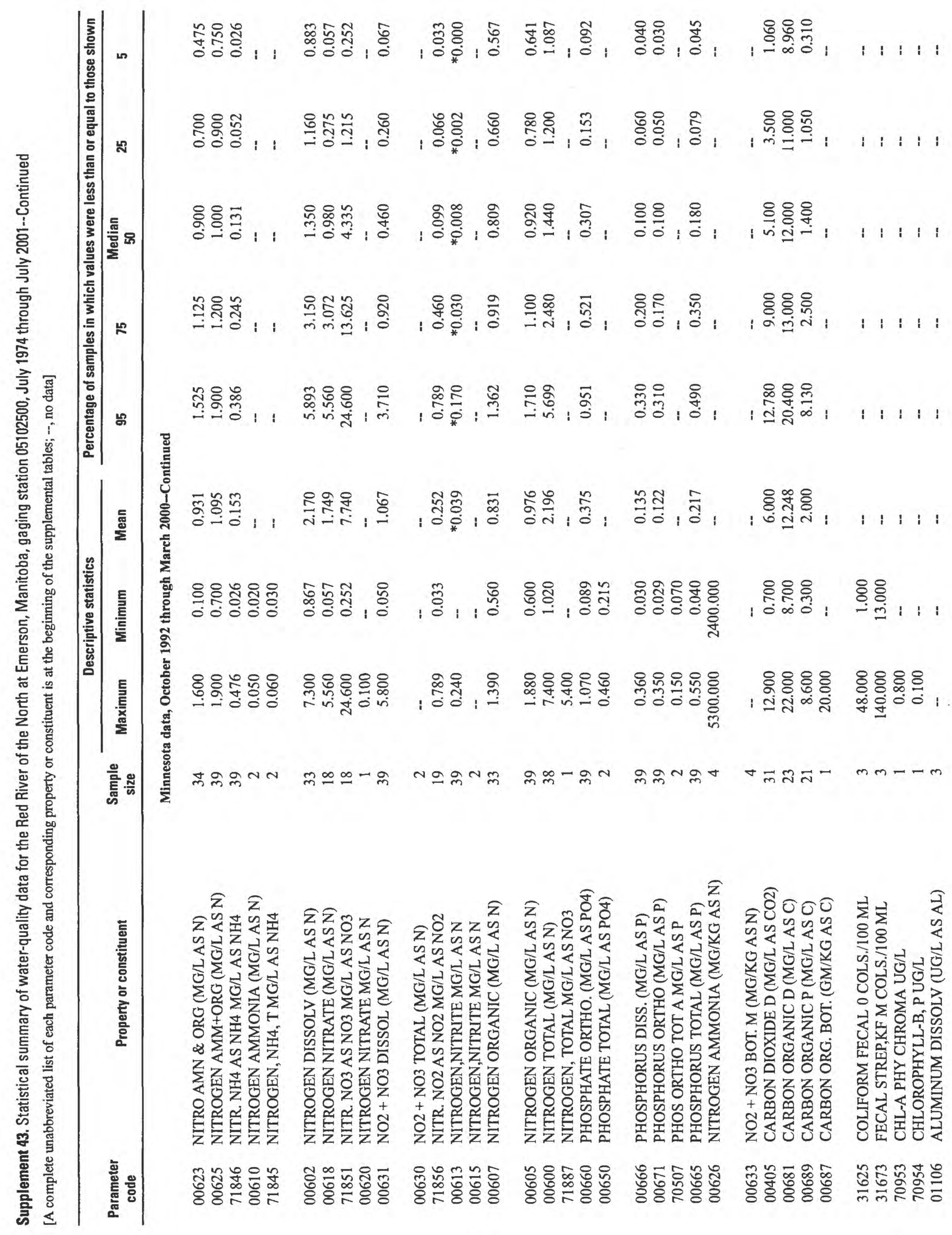




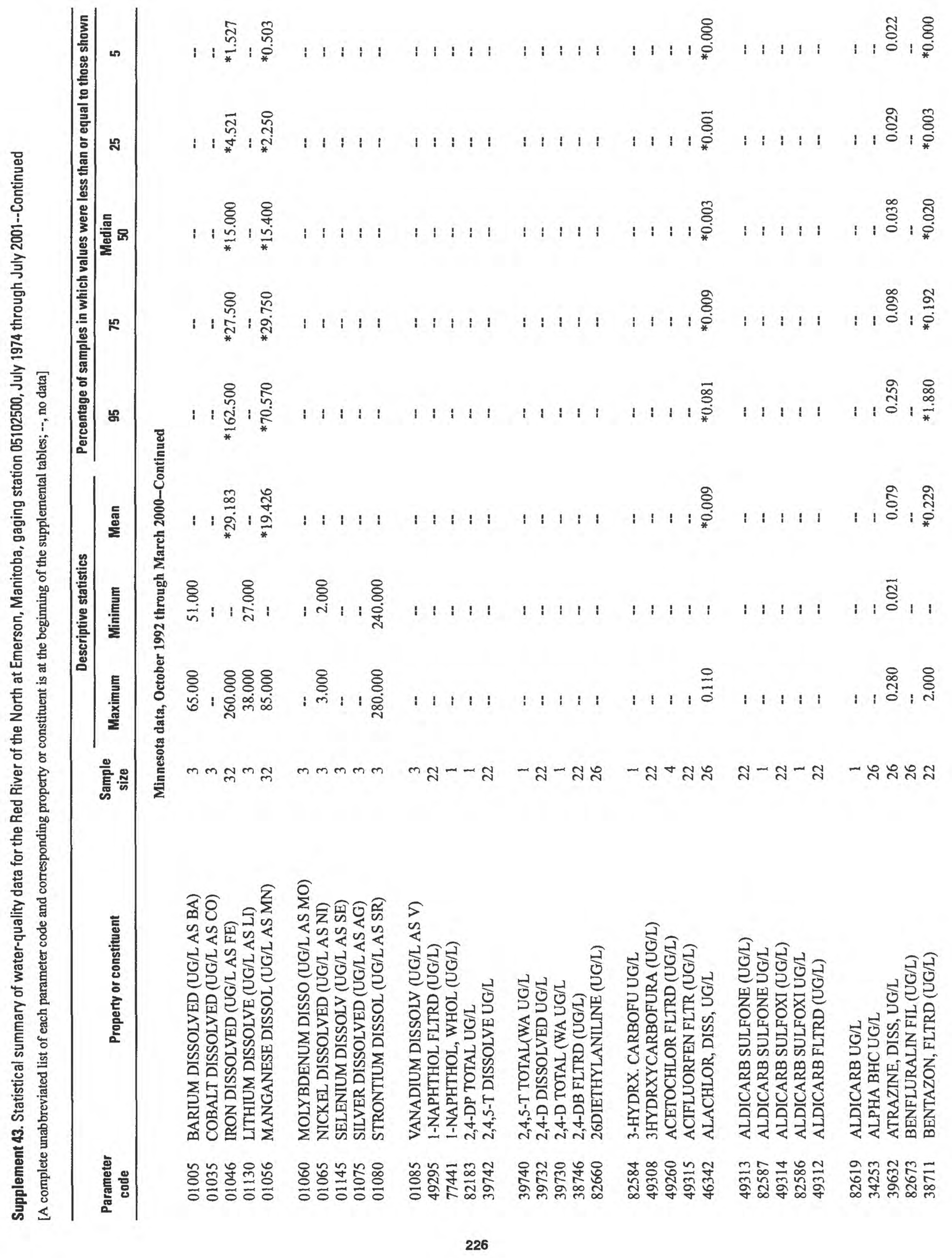




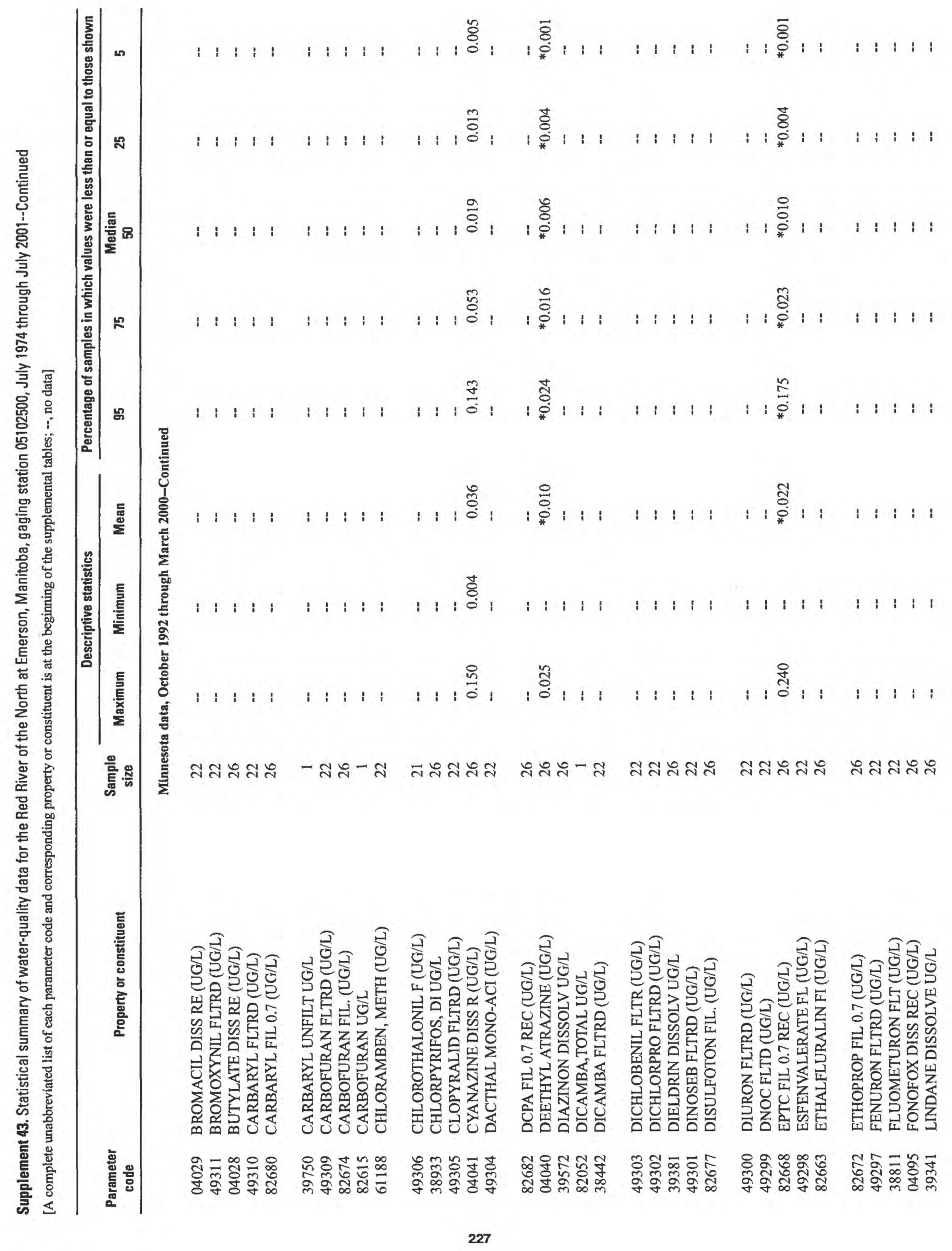




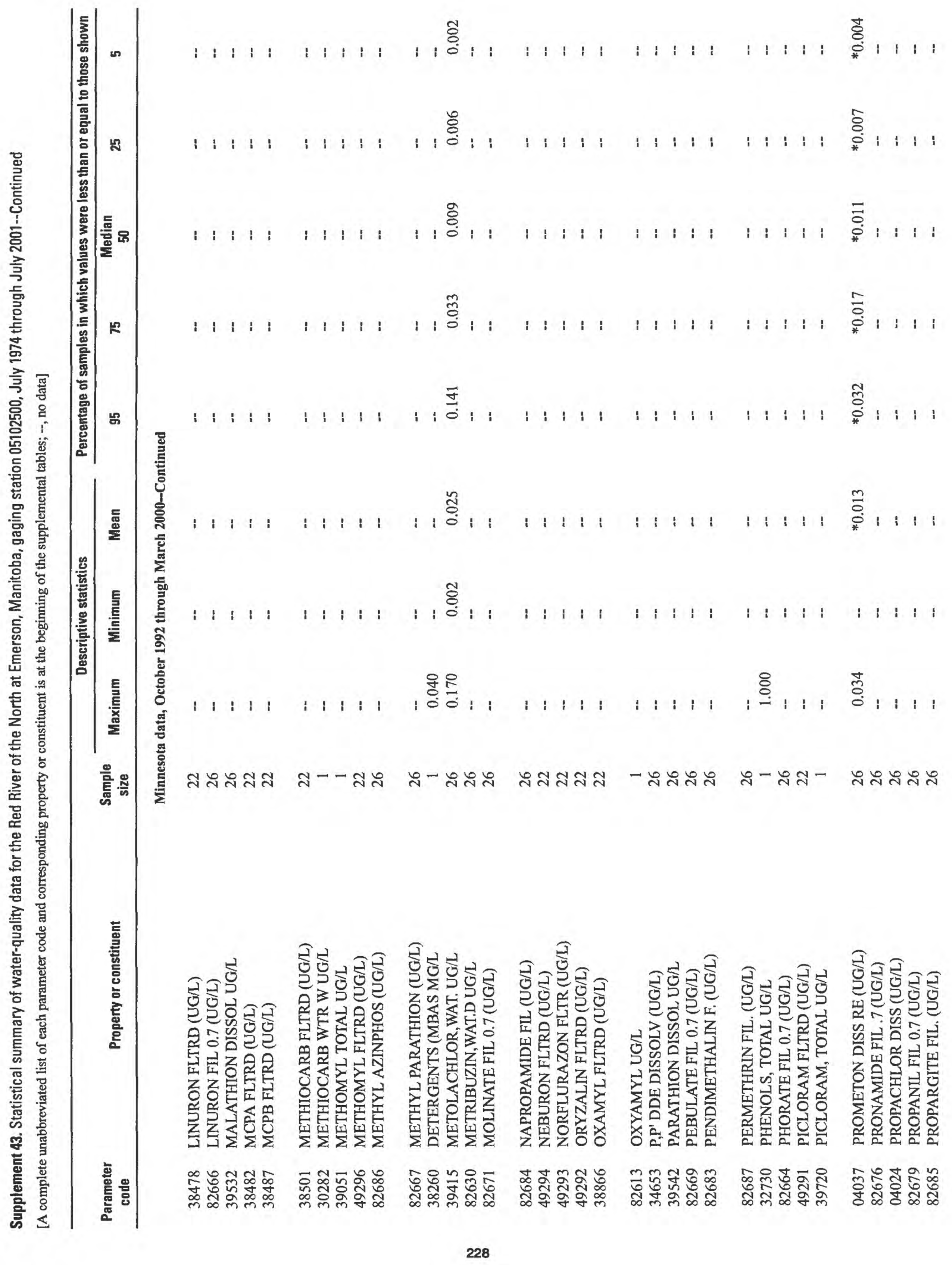




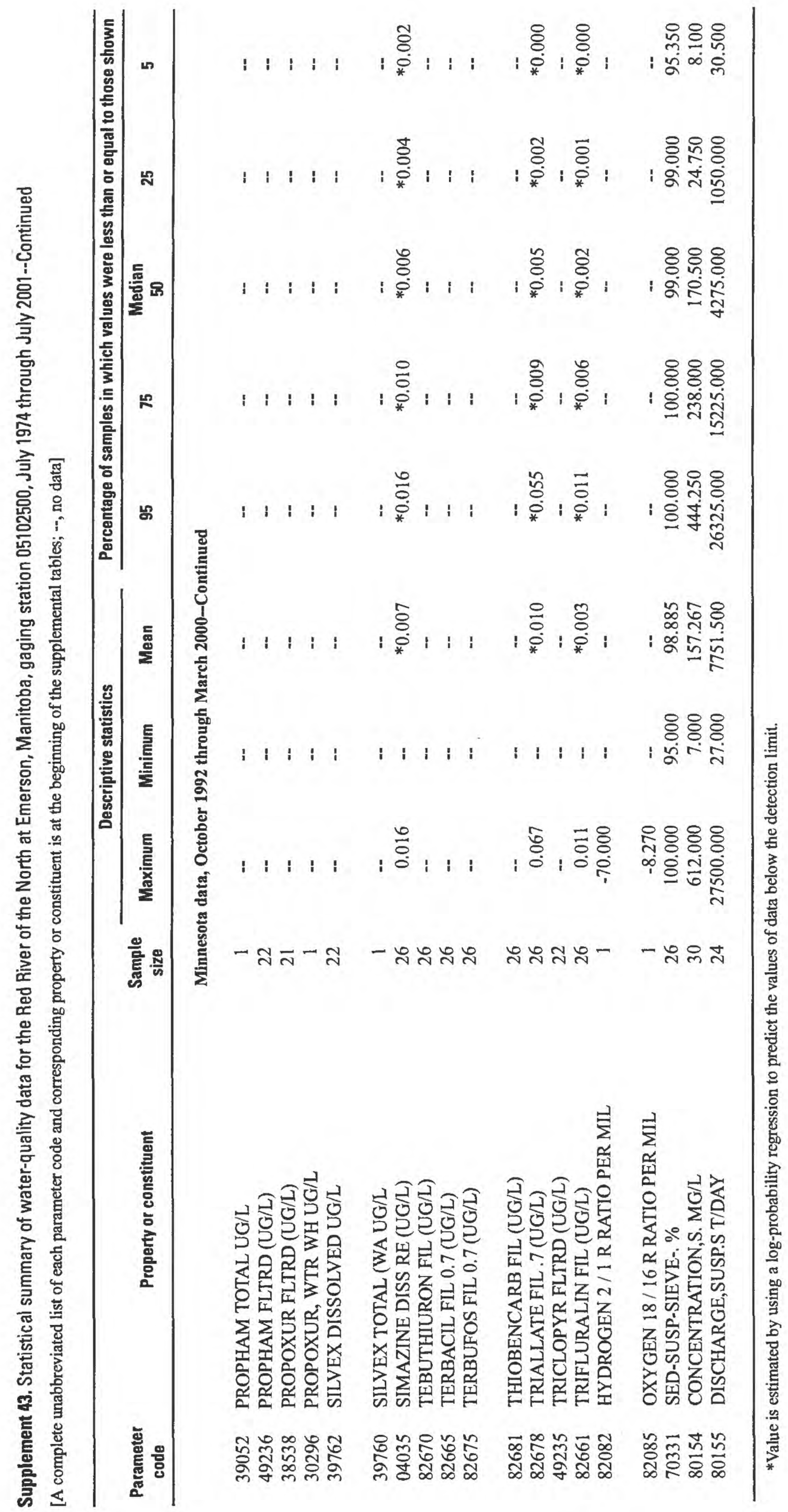

